\title{
Optical Cavities for Optical Atomic Clocks, Atom Interferometry and Gravitational-wave Detection
}

\section{Miguel Dovale Álvarez}

\author{
A thesis submitted to \\ the University of Birmingham \\ for the degree of \\ Doctor of Philosophy \\ Experimental work undertaken at \\ the National Physical Laboratory

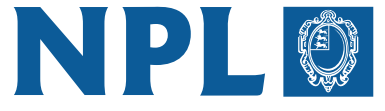 \\ National Physical Laboratory

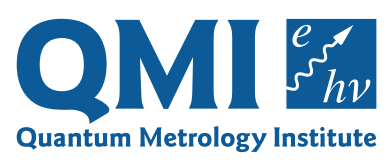

School of Physics and Astronomy College of Engineering and Physical Sciences University of Birmingham Submitted: September 2018 


\section{UNIVERSITYOF BIRMINGHAM}

\section{University of Birmingham Research Archive \\ e-theses repository}

This unpublished thesis/dissertation is copyright of the author and/or third parties. The intellectual property rights of the author or third parties in respect of this work are as defined by The Copyright Designs and Patents Act 1988 or as modified by any successor legislation.

Any use made of information contained in this thesis/dissertation must be in accordance with that legislation and must be properly acknowledged. Further distribution or reproduction in any format is prohibited without the permission of the copyright holder. 

A mis hermanas Ángela e Isabel y a mis abuelas Pepita y Consuelo. 



\section{Abstract}

$\mathrm{T}^{\mathrm{T}}$ is an extremely exciting time for physics. In the last 100 years we have moved

1 from the formulation of Einstein's general relativity to the first direct observation of gravitational waves in late 2015 by the Laser Interferometer Gravitational-wave Observatory (LIGO). Within that time science and technology have come a long way: we have learned to use light to cool atoms to nearly absolute zero temperature, and to use atomic transitions in the microwave and optical regimes to devise the most accurate time and frequency references. We have observed the wave-like behaviour of cold atoms in diffraction experiments using both micro-fabricated structures and the periodic structure of light beams. Exploiting this wave-like behaviour, we have constructed atom interferometers which allow us to test and measure gravity in a new scale. All of these amazing experiments have one thing in common, from LIGO's giant $4 \mathrm{~km}$ arms to the transportable atomic clocks sent to space or the atom interferometers that will someday replace current navigation systems, they all make use of a device that has become essential in many areas of science and technology: the Fabry-Perot optical cavity.

This thesis delves deeply into the application of optical cavities at the forefront of experimental physics, and it is divided into three parts, each pertaining to a different field where optical cavities are a key technology. Part I of the thesis follows the development of a next-generation, thermal-noise-limited, ultra-stable optical cavity for use as the reference oscillator in optical atomic clocks. This is part of the effort being carried out at the National Physical Laboratory in the UK in the field of precision metrology of time and frequency towards redefining the base SI unit of time, the second, in terms of an optical frequency standard. Part II presents work in the application of optical cavities for enhancing the sensitivity of atom interferometers, with an analysis of multi-photon Bragg diffraction inside an optical cavity. This led to an exploration of the true potential and limitations of the technique. The findings were used to aid the design of the MIGA experiment in France, and design a multi-mirror cavity for enhanced atom interferometry that overcomes some of the limitations of two-mirror cavities. Lastly, Part III presents work towards enhancing current and future laser interferometer gravitational-wave detectors by using near-unstable optical cavities in order to reduce the mirror coating thermal-noise floor, and by modelling parametric oscillatory instabilities that arise in the interferometers from the coupling between the optical field and the mechanical resonances of the test masses. The work carried out during the extent of this $\mathrm{PhD}$, and the variety of contexts, accounts for the relevance and versatility of such an elegant setup as the Fabry-Perot optical cavity is. 



\section{Acknowledgements}

This work was realised with the financial support of the Defence Science and Technology Laboratory.

I would like to thank my advisors Prof. Andreas Freise and Dr. Conor Mow-Lory for their patience and support over the last four years. I am enormously indebted to them for their permanent advice, understanding and encouragement to make this project successful.

I am also grateful to my colleagues Dr. Daniel Brown, Dr. Anna Green, Dr. Daniel Töyra and Dr. Haoyu Wang, who have helped me greatly along the way, and to everyone at the School of Physics and Astronomy at the University of Birmingham.

I am most grateful to Dr. Ross Williams for his guidance during my first year here.

I am very thankful to Prof. Patrick Gill and Dr. Marco Schioppo, and to everyone at the National Physical Laboratory, for their continued support.

I would also like to thank my professors at Universidade de Santiago de Compostela for their encouragement and help during difficult times.

Finally, I want to deeply thank my family and friends for their permanent support and patience. My years in the UK were transformed by Ana, Eugenia, Jorge, Luis and Sergio. Great moments with Antón, David, Hugo, Juan, Jean Pierre, Miguel, Ramón, Rubén, Sara, and so may others from back home, have shaped the person who I am now. Unconditional support from my mother and father has been crucial to be able to make my dreams come true. And the transcendental role played by Javier to climb the glass staircase of life has been and shall be fundamental until each of us fulfils his true purpose in life.

Ringo, even though you cannot hear me or talk to me, you help me greatly every day. 



\section{Statement of Originality}

This thesis reports on my own research work conducted during my $\mathrm{PhD}$ at the University of Birmingham between September 2014 and September 2018.

The work described in Chapters 2 and 3 is based on an internal NPL document [1] authored by Dr. Ross Williams and myself as lead author, and it follows the design and construction of an experiment during my research secondment at the National Physical Laboratory. The content reproduced here is my own work, and it has been adapted to suit the format of this thesis.

After my departure from NPL in April of 2016, the experiment was continued by Dr. Ross Williams, who later also left and handed the baton to Dr. Marco Schioppo. Dr. Schioppo has been extremely helpful in both advancing the experiment and providing information and data on its progress. The content reproduced in Chapter 4 is my own analysis of the data graciously provided by Dr. Schioppo.

The work presented in Chapters 5 and 6 was reported in Fundamental limitations of cavity-assisted atom interferometry [2], published in Physical Review A in November 2017, where I am the lead author. I led the analytical and modelling work and wrote the manuscript. My colleagues Dr. Daniel Brown and Mr. Aaron Jones aided the modelling work and provided critical revisions. Figures and text from the paper have been used verbatim; the structure of the content has been modified to suit the format of this thesis.

The work presented in Chapter 7 is based on an internal University of Birmingham document [3] authored by Prof. Andreas Freise and myself as lead author. It was reported in Exploring gravity with the MIGA large scale atom interferometer [4], published in Sci- entific Reports in September 2018. The content reproduced here is my own work, and it has been adapted to suit the format of this thesis.

Chapter 8 describes the design of a novel multi-mirror resonator for enhanced atom interferometry. It is based on an internal University of Birmingham document [5] authored by Dr. Conor Mow-Lowry, Prof. Andreas Freise, and myself as lead author. The content reproduced here is my own work, and it has been adapted to suit the format of this thesis. 
The work presented in Chapter 9 was reported in Feasibility of near-unstable cavities for future gravitational wave detectors [6], published in Physical Review D in January 2018, of which Dr. Haoyu Wang and myself are first and second authors respectively. Dr. Wang and myself worked alongside one another in constructing and carrying out the experiment, analysing the data, and writing the manuscript. The structure of the content has been modified to suit the format of this thesis.

The work presented in Chapter 10 was reported in The influence of dualrecycling on parametric instabilities at Advanced LIGO [7], published in Classical and Quantum Gravity in September 2017, in which I am the third author. I worked alongside Dr. Anna Green and Dr. Daniel Brown in the modelling effort, in particular contributing the finite element analysis of the test masses. 


\section{Contents}

Abstract $\quad$ v

Acknowledgements vii

Statement of Originality ix

List of Figures $\quad$ xviii

Conventions, notations, and acronyms $\quad$ xix

1 Atomic clocks, cold atoms and gravitational waves 1

1.1 The common link: Fabry-Perot resonators . . . . . . . . . . . . 2

1.2 The future of atomic time - Chapters $2-4 \ldots \ldots . \ldots$

1.3 The cold atoms revolution - Chapters $5-8 \ldots \ldots . \ldots 5$

1.4 The dawn of a new era in astronomy - Chapters $9-10 \ldots 6$

1.5 Structure of the thesis . . . . . . . . . . . . . . 7

I Cavities for Optical Atomic Clocks 9

2 Thermal-noise-limited room-temperature ULE cavity 11

2.1 Optical frequency standards: Towards a redefinition of the SI second 12

2.2 Fabry-Perot resonator theory . . . . . . . . . . . . . . . . . 13

2.2.1 Fields in a Fabry-Perot resonator . . . . . . . . . . . . . 13

2.2.2 Static response and resonance condition . . . . . . . . . . 15

2.2 .3 Transverse modes . . . . . . . . . . . . . . . . 17

2.2.4 Fundamental Gaussian beam . . . . . . . . . . . . . 19

2.2 .5 Resonator stability . . . . . . . . . . . . . . . 21

2.3 The art of making ultra-stable optical cavities . . . . . . . . . . . . 24

2.3.1 Thermal noise limit . . . . . . . . . . . . . . . . . 24

2.3.2 Spacer length and mirror ROC . . . . . . . . . . . . . . 28

2.4 Force-insensitive optical cavity . . . . . . . . . . . . . . . . . . 31

2.4.1 Spacer geometry and material . . . . . . . . . . . 31

2.4.2 Spacer structural mechanics . . . . . . . . . . . . . . . 34

2.4.3 Model for the force-induced length fluctuations . . . . . . . . 36

2.5 Optimisation of the support positions . . . . . . . . . . . 38

2.5.1 Probing the $(d x, d z)$ parameter space. . . . . . . . 38 
2.5.2 Transverse and longitudinal accelerations . . . . . . . . . . 43

2.5.3 Further optimisation of the spacer geometry . . . . . . . . . 45

2.5.4 Optimal configuration . . . . . . . . . . . . . . 47

2.5.5 Balanced support forces . . . . . . . . . . . . 51

3 Isolation from external perturbations $\quad \mathbf{5 5}$

3.1 Pressure and temperature fluctuations and vibrations . . . . . . . 56

3.1.1 Pressure sensitivity . . . . . . . . . . . . . . 56

3.1.2 Temperature sensitivity . . . . . . . . . . . 56

3.1.3 Vibration sensitivity . . . . . . . . . . . . . . . 59

3.2 Design of the cavity enclosure . . . . . . . . . . . . . . . . . . . 59

3.2.1 Heat shields . . . . . . . . . . . . . . . . . . 59

3.2.2 Vacuum chamber . . . . . . . . . . . . . . . 60

3.3 Heat transfer in the cavity enclosure . . . . . . . . . . . . . . . 64

3.3.1 Thermal modelling . . . . . . . . . . . . . . . 64

3.3.2 Radiative heat transfer through the ventilation holes . . . . . 67

3.4 CAD drawings and photos of the system . . . . . . . . . . . . . . 74

4 Measuring resonator stability $\quad \mathbf{8 3}$

4.1 Status of the experiment . . . . . . . . . . . . . . . 84

4.2 Three-corner hat measurement (March 31st 2018) . . . . . . . . . . 87

4.3 Comparison over fibre-link (April 23rd 2018) . . . . . . . . . . . . . 91

\section{Cavities for Atom Interferometry 93}

5 Cavity atom optics $\quad \mathbf{9 5}$

5.1 Large momentum transfer beam splitters using optical cavities . . . 96

5.2 Atomic Bragg diffraction . . . . . . . . . . . . . . . . . 98

5.2.1 The atom-field Hamiltonian . . . . . . . . . . . . . . . . . . 98

5.2.2 Adiabatic elimination of the excited state . . . . . . . . . . . 101

5.2.3 Equations of motion of the momentum eigenstates . . . . . . 103

5.2 .4 Numerical model . . . . . . . . . . . . . . . . . . 105

5.2.5 First order Bragg diffraction . . . . . . . . . . . . 106

5.3 Interaction regimes . . . . . . . . . . . . . . . . 106

5.3.1 Raman-Nath regime (fast interaction) . . . . . . . . . . 106

5.3.2 Bragg regime (slow and weak interaction) . . . . . . . . . 109

5.3.3 Channeling regime (slow and strong interaction) . . . . . . 112

5.4 The quasi-Bragg regime . . . . . . . . . . . . . . . . 113

5.4.1 Pulse-shape dependence of the transition probability . . . . . 116

5.5 Cavity-assisted Bragg beam splitter . . . . . . . . . . . . . 119

5.5.1 Time-domain model of the Fabry-Perot resonator . . . . . . . 119

5.5.2 Cavity-induced deformation of the pulse envelope . . . . . . . 123

5.5.3 Effect of finesse and length on the atomic transitions . . . . . 125

6 Fundamental limitations of cavity-assisted atom interferometry 131

6.1 Towards low-power and low-noise large momentum transfer atom

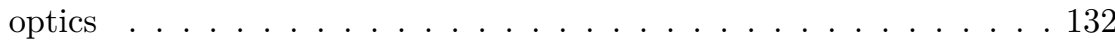


6.2 Cavity bandwidth limits . . . . . . . . . . . . . . . . . 132

6.3 Constraints on spatial filtering and beam size . . . . . . . . . . . . 135

6.4 Atom temperature and cavity length limits for interferometers . . . . 139

6.5 Summary and future work . . . . . . . . . . . . . . . 145

7 Gravitational wave detection with cavity-assisted atom interferometry

7.1 The MIGA large scale atom interferometer . . . . . . . . . . . . . 148

7.2 Beam geometry and mirror radii of curvature in MIGA . . . . . . . . 149

7.3 Cavity-enhanced interrogation in MIGA . . . . . . . . . . . . 156

7.4 MIGA antenna design conclusions . . . . . . . . . . . . . . 163

8 4-mirror large-waist cavity with tuneable stability for enhanced atom interferometry $\quad \mathbf{1 6 5}$

8.1 A novel resonator design for enhanced atom interferometry . . . . . 166

8.2 Eigenmode size and stability . . . . . . . . . . . . . . . . . . . . . . . . . 168

8.3 Transient response . . . . . . . . . . . . . . . . . . . . 173

8.4 Over-coupling and transverse mode filtering . . . . . . . . . . . . . . 175

8.5 Gaussian mode non-degeneracy . . . . . . . . . . . . . . . . 177

8.6 Summary and future work . . . . . . . . . . . . . . . . 177

\section{Cavities for Gravitational-wave Detection 181}

9 Near-unstable cavities for future gravitational wave detectors $\mathbf{1 8 3}$

9.1 The hunt for gravitational wave signals . . . . . . . . . . . . . . . . . 184

9.1.1 Detectors and noise sources . . . . . . . . . . . . . . 184

9.1.2 Thermal noise reduction . . . . . . . . . . . . . . . 186

9.2 Near-unstable cavities . . . . . . . . . . . . . . . . . . . . . . . 188

9.2.1 Thermal noise and resonator stability . . . . . . . . . 188

9.2 .2 Degenerate cavity issues . . . . . . . . . . . . . . . 190

9.3 Tabletop NUC optical design . . . . . . . . . . . . . . . . . . . 191

9.3.1 Plane-concave cavity with tuneable stability . . . . . . . . . . 191

9.3.2 Precision characterisation of cavity stability . . . . . . . . . 193

9.3.3 Alignment and frequency stabilisation systems . . . . . . . 194

9.4 Pushing the cavity over the edge . . . . . . . . . . . . . . 196

9.4.1 Influence of mirror imperfections . . . . . . . . . . . . . . 196

9.4.2 Characterising cavity stability . . . . . . . . . . . . 197

9.4.3 Results and simple astigmatic model . . . . . . . . . . . . 198

9.5 Summary and future work . . . . . . . . . . . . . . . 202

10 Modelling parametric instabilities at Advanced LIGO and ET 205

10.1 Parametric instabilities . . . . . . . . . . . . . . 206

10.1.1 Optomechanical interaction of light and mirrors . . . . . . . . 206

10.1.2 Parametric instability in a Fabry-Perot cavity . . . . . . . . . 207

10.1.3 Observation of parametric instabilities and mitigation strategies 208

10.2 Finite element modelling . . . . . . . . . . . . . . . . . . . . 210 
10.2.1 Introduction to eigenmode analysis using the finite element

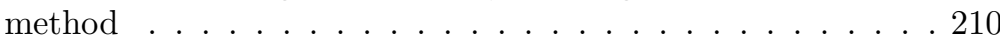

10.2.2 Accuracy of the model . . . . . . . . . . . . . . . . . 212

10.3 Eigenmode analysis of Advanced LIGO and ET test masses . . . . . 218

10.3.1 Advanced LIGO test masses . . . . . . . . . . . . . . . . . . . . . . . 218

10.3 .2 ET test masses . . . . . . . . . . . . . . . . 220

10.4 Summary and future work . . . . . . . . . . . . . . 223

11 Summary and conclusions

$\begin{array}{ll}\text { IV Appendix } & 229\end{array}$

$\begin{array}{ll}\text { A Characterisation of frequency stability } & 231\end{array}$

B Müller-Chiow-Chu method $\quad 235$

$\begin{array}{lr}\text { List of publications and conferences } & 239\end{array}$

$\begin{array}{ll}\text { References } & 244\end{array}$ 


\section{List of Figures}

1.1 Fabry-Perot optical cavity . . . . . . . . . . . . . . . . 3

1.2 The future of atomic time . . . . . . . . . . . . . . . 4

1.3 Light-pulse atom interferometer . . . . . . . . . . . . . . . 5

1.4 The dawn of a new era in astronomy . . . . . . . . . . 7

2.1 Fields in a Fabry-Perot resonator . . . . . . . . . . . . . . . . . . 14

2.2 Intensity response of the Fabry-Perot resonator . . . . . . . . . . . . 16

2.3 Transverse Hermite-Gauss modes . . . . . . . . . . . . . . . . . . . 18

2.4 Gaussian beam profile . . . . . . . . . . . . . . . 20

2.5 Resonances of transverse modes . . . . . . . . . . . . . . . 23

2.6 Thermal noise floor . . . . . . . . . . . . . . . . . . 27

2.7 Higher order mode resonance frequency offsets for different mirror combinations . . . . . . . . . . . . . . . . . . 29

2.8 Higher order mode relative power for different mirror combinations . 30

$2.93 \mathrm{D}$ view of the cavity as modelled in COMSOL . . . . . . . . . . . . . 32

2.10 Geometrical parameters of the cavity . . . . . . . . . . . . . . 32

2.11 Poisson effect deformation . . . . . . . . . . . . . . . 34

2.12 Tilting deformation of the cavity . . . . . . . . . . . . 35

2.13 Deformation of the cavity under a vertical force, 3D view . . . . . . 37

2.14 Deformation of t he cavity under a vertical force, section view . . . . 37

2.15 Relative length fluctuations in the $d x-d z$ parameter space . . . . . 40

2.16 Relative length fluctuations vs $d x \ldots \ldots$. . . . . . . . . . . . . . 41

2.17 Relative length fluctuations vs $d z \ldots \ldots$. . . . . . . . . . . . 41

2.18 Length fluctuations vs $d x$ for offset mirrors (vertical acceleration) . . 42

2.19 Length fluctuations vs $d x$ for offset mirrors (transverse acceleration) 44

2.20 Length fluctuations vs $d x$ for offset mirrors (longitudinal acceleration) 44

2.21 Optimising cutout depth and spacer diameter . . . . . . . . . 45

2.22 Acceleration and tilt sensitivity gradients vs spacer diameter . . . . 46

2.23 Relative length fluctuations in the $d x-d z$ parameter space (optimised) 48

2.24 Length fluctuations vs $d x$ for offset mirrors (vertical acceleration), optimised cavity . . . . . . . . . . . . . . . 4 49

2.25 Length fluctuations vs $d x$ for offset mirrors (transverse acceleration), optimised cavity . . . . . . . . . . . . . . . . 4 49

2.26 Length fluctuations vs $d x$ for offset mirrors (longitudinal acceleration), optimised cavity ..................... 50 50

2.27 Sensitivity to inhomogeneous loading of the supports . . . . . . . . 52

2.28 Photo of the cavity mount system . . . . . . . . . . . . . 53 
3.1 Vibration levels at the National Physical Laboratory . . . . . . . . . 58

3.2 Aluminium heat shield system . . . . . . . . . . . . . . . . 61

3.3 Photo of the aluminium heat shields . . . . . . . . . . . . . . 61

3.4 Wedged laser windows and wedged aluminium annulus . . . . . . . . 62

3.5 Heat conduction between shields through the glass spheres . . . . . . 62

$3.63 \mathrm{D}$ rendering of the vacuum chamber . . . . . . . . . . . 63

3.7 Photo of the top piece of the vacuum chamber . . . . . . . . . 63

3.8 Temperature response of the heat shields . . . . . . . . . . . . . 65

3.9 Thermal impact of the shield plate thickness . . . . . . . . . . 65

3.10 Thermal impact of the beam hole radius . . . . . . . . . . . . . . 66

3.11 Thermal impact of the laser windows . . . . . . . . . . . . . . 66

3.12 Proposed ventilation hole geometries to minimise radiative heat transfer 67

3.13 Particle tracing simulation emission point example . . . . . . . . 68

3.14 Particle tracing simulation examples using the type A geometry . . . 69

3.15 Particle statistics in the type A geometry . . . . . . . . . . . . 70

3.16 Particle tracing simulation example in the type $\mathrm{C}$ geometry . . . . . 71

3.17 Particle statistics for all hole geometries . . . . . . . . . . . . . 72

3.18 Comparison of radiative heat transmissivity in the different geometries 73

$3.193 \mathrm{D}$ view of the cavity and baseplates. . . . . . . . . . . . 74

$3.203 \mathrm{D}$ view of the cavity, shields and vacuum chamber . . . . . . . . 75

3.21 Cross section of the system showing the optical path . . . . . . . . 76

3.22 Top view of the system . . . . . . . . . . . . . . . . 77

3.23 Transverse cross section of the system . . . . . . . . . . . . . 78

3.24 Photo of the cavity assembly . . . . . . . . . . . . . . . . . 79

3.25 Photo of the cavity assembly and heat shields . . . . . . . . . . . . 80

3.26 Photo of the cavity assembly, heat shields and vacuum chamber . . . 81

4.1 Manufacturing defects of the spacer . . . . . . . . . . . . . . 84

4.2 Vacuum pressure stability . . . . . . . . . . . . . . . . 85

4.3 Cavity drift rate . . . . . . . . . . . . . . . . . . 86

4.4 Three-corner hat measurement scheme . . . . . . . . . . . . . 87

4.5 Three-corner hat measurement beat note signals . . . . . . . . . . . 89

4.6 Modified Allan deviations of the three-corner hat measurement . . . 90

4.7 Comparison of NPL's new cavity vs PTB's cryogenic silicon cavity . 92

5.1 Diffraction of atoms by a standing wave light field. . . . . . . . . . . 100

5.2 Diffraction of atoms from an 8-photon beam splitter vs pulse duration 104

5.3 Higher order effects in atomic Bragg diffraction . . . . . . . . . . . 105

5.4 Pendellösung oscillations in first order Bragg diffraction . . . . . . . 107

5.5 The Raman-Nath regime . . . . . . . . . . . . . . . . . . . . . . . . . 108

5.6 The Bragg regime . . . . . . . . . . . . . . . . . . . . 111

5.7 Effective transfer efficiency in the Bragg regime . . . . . . . . . . 112

5.8 The channeling regime . . . . . . . . . . . . . . . . . . . . 114

5.9 Interaction regimes in the $\bar{\Omega}-\delta t$ parameter space . . . . . . . . . . . 115

5.10 Pulse-shape dependence of the transition probability in the quasiBragg regime . . . . . . . . . . . . . . . . 117

5.11 Mirror pulse differences between different pulse shapes . . . . . . . . 119 
5.12 Fields in a Fabry-Perot resonator, time-domain model . . . . . . . . 120

5.13 Transient response of a Fabry-Perot resonator to short pulses . . . . 122

5.14 Transient response of the cavity to ultra short pulses . . . . . . . . . 123

5.15 Cavity-induced deformation of a Gaussian input pulse . . . . . . . . 124

5.16 Transient response of the cavity vs input pulse duration . . . . . . 124

5.17 Effect of cavity finesse on the mirror pulses . . . . . . . . . . . 126

5.18 The cavity bandwidth limit . . . . . . . . . . . . . . 126

5.19 Effect of the cavity on the atomic transitions . . . . . . . . . . . 127

5.20 Mirror pulse parameters vs cavity photon lifetime . . . . . . . . . . . 129

6.1 Cavity-assisted atom interferometry . . . . . . . . . . . . . 133

6.2 Cavity photon lifetime limit vs Bragg order . . . . . . . . . . . . . . 134

6.3 Spatial filtering and beam size under the bandwidth limit . . . . . . 136

6.4 Spatial filtering and geometrical parameters vs beam size . . . . . . 138

6.5 Continued on the following page. . . . . . . . . . . . . . . . . 142

6.5 Geometrical and optical limits of the cavity-assisted atom interferometer 143

7.1 Scheme of the MIGA instrument . . . . . . . . . . . . . . . . . 148

7.2 Bragg diffraction scheme in the MIGA instrument . . . . . . . . . . 150

7.3 Mode non-degeneracy vs mirror radii of curvature . . . . . . . . . . . 153

7.4 Mode non-degeneracy vs mirror radii of curvature, detail . . . . . . . 154

7.5 Relative power on higher order modes for different ROC configurations 155

7.6 Deformation of the input pulse vs MIGA cavity finesse . . . . . . . . 157

7.7 Transient response of the MIGA cavities at different finesses . . . . . 157

7.8 Mirror pulse parameters vs MIGA cavity finesse . . . . . . . . . . . . 159

7.9 First order Bragg diffraction in MIGA for different finesses . . . . . 161

7.10 Second order Bragg diffraction in MIGA for different finesses . . . . 162

8.1 Atom temperature limits in the geometrical limit . . . . . . . . . . 167

8.2 Scheme of the 4 -mirror cavity setup . . . . . . . . . . . . . . 168

8.3 Transfer matrices of the 4-mirror cavity setup . . . . . . . . . . . . 169

8.4 Eigenmode size and stability as a function of the $d_{1}$ length. . . . . . 172

8.5 Fields in the 4-mirror cavity . . . . . . . . . . . . . . . . . 174

8.6 Mode non-degeneracy vs the $d_{1}$ length . . . . . . . . . . . . . . . . 178

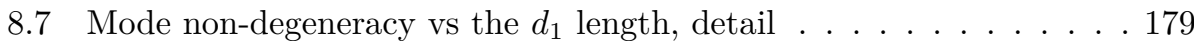

9.1 Advanced LIGO detectors . . . . . . . . . . . . . . . . . . . . 185

9.2 Advanced LIGO noise curves . . . . . . . . . . . . . . . . . . . 187

9.3 Fabry-Perot resonator stability in the $g_{1}-g_{2}$ parameter space . . . . 189

9.4 Separation factors in different experiments . . . . . . . . . . . . 192

9.5 Tabletop near-unstable cavity setup . . . . . . . . . . . . 195

9.6 Cavity scan measurement showing $\mathrm{HG}_{00}, \mathrm{HG}_{02}, \mathrm{HG}_{20}, \mathrm{HG}_{04}, \mathrm{HG}_{22}$, and $\mathrm{HG}_{40} \ldots \ldots \ldots$. . . . . . . . . . . . . . . . . . . . . . . . . . . . . . .

9.7 CCD images of the resonant modes as a function of cavity stability . 200

9.8 Fits of the simple astigmatic mirror model. . . . . . . . . . . . . . 201

10.1 Optomechanical interaction: Stokes and anti-Stokes processes. . . . . 206

10.2 Mesh size in the finite element model of a cylinder. . . . . . . . . . . 211 
10.3 Chree-Lamb coefficients . . . . . . . . . . . . . . . . . . . 214

10.4 Chree-Lamb modes in ideal cylinders . . . . . . . . . . . . . . . . 215

10.5 Accuracy of the FEM model vs eigenfrequency . . . . . . . . . . . . . 217

10.6 Geometry of an Advanced LIGO test mass. . . . . . . . . . . . . . . 218

10.7 Accuracy of the FEM model and number of mesh elements . . . . . 219

10.8 Shape of the $15538 \mathrm{~Hz}$ mechanical mode . . . . . . . . . . . . . 220

10.9 Geometry of the proposed ET test masses . . . . . . . . . . . . . 222

10.10 ET-HF drumhead mode . . . . . . . . . . . . . . . . . . 223 


\section{Conventions, notations, and acronyms}

Units. Numerical results are mostly given in terms of the International System of Units. Two exceptions can be found in Part I of the thesis, where pressure is given in millibar (mbar), and accelerations are given in $g\left(1 g=9.81 \mathrm{~m} / \mathrm{s}^{2}\right)$.

Notation. Vectors in $\mathbb{R}^{3}$ are specified in bold font (e.g., $\left.\boldsymbol{k}, \boldsymbol{r}\right)$. We use Dirac notation for state vectors in Hilbert space (e.g., $|\Psi\rangle,|m\rangle$ or $|\boldsymbol{p}\rangle$ ), and hats for linear operators in vector spaces (e.g., $\widehat{\mathcal{H}}, \widehat{\boldsymbol{p}}$, or $\widehat{M}$ ).

Electric field. We use the complex phasor notation for the electric field, defining the measurable quantity of the field as

$$
\xi(t) \equiv \frac{1}{2} \sqrt{\epsilon_{0} c}\left(E(t)+E(t)^{*}\right)
$$

in $\mathrm{V} / \mathrm{m}$, where $\epsilon_{0}$ is the vacuum permittivity, $c$ is the speed of light, and $E(t)$ is a complex quantity, in units of $\sqrt{\mathrm{W}}$, representing the amplitude and phase of the field. This greatly simplifies the electric field computations. Sometimes we will also drop the variable part of the phase of $E(t)$,

$$
E(t) \equiv a(t) e^{-i(\omega t-\boldsymbol{k} \cdot \boldsymbol{r})},
$$

and use $a(t) \equiv a_{0}(t) e^{i \phi_{0}}$, which contains the amplitude of the field and only the constant part $\phi_{0}$ of the phase.

Atom optics. We follow the conventions of Meystre [1], using the semiclassical formulation of a quantum-mechanical atom in a classical field. As often as possible, we use the adimensional interaction time $\tau \omega_{r}$ and intensity $\Omega / \omega_{r}$, where $\omega_{r}$ is the recoil frequency of the interaction of interest in units of radians per second. This makes all results readily scalable for the atomic transition of interest, with $\omega_{r}=\hbar k^{2} / 2 M$, where $k$ is the effective wave vector of the optical standing wave field, and $M$ is the mass of the atom. Wherever a result is given in terms of seconds or hertz, they will be referenced to either the rubidium- $87 \mathrm{D}_{2}$ transition $\left(5^{2} \mathrm{~S}_{1 / 2} \rightarrow 5^{2} \mathrm{P}_{3 / 2}\right)$ with $\omega_{r}=23694 \mathrm{rad} / \mathrm{s}$, or the caesium-133 $\mathrm{D}_{2}$ transition $\left(6^{2} \mathrm{~S}_{1 / 2} \rightarrow 6^{2} \mathrm{P}_{3 / 2}\right)$ with $\omega_{r}=12983 \mathrm{rad} / \mathrm{s}$. 
Acronyms. Acronyms are defined as they are introduced in the text. See also Table 1.

Notations. This thesis touches on many fields of physics, and sometimes the same notation is used in different contexts. See Table 2 for a list of some recurrent symbols used throughout the thesis. Wherever a different notation is used, or confusion is possible, it is stated clearly.

\begin{tabular}{|r|l|}
\hline Acronym & Meaning \\
\hline AEI & Albert Einstein Institute \\
BS & acousto-optic modulator \\
CAD & computer aided design \\
CTE & coefficient of thermal expansion \\
EOM & electro-optic modulator \\
EM & end mirror \\
ET & Einstein Telescope \\
ETM & end test mass \\
FEM & finite element method \\
FP & Fabry-Perot \\
FWHM & full width at half maximum \\
HG & Hermite-Gauss \\
HOM & higher order mode \\
IM & input mirror \\
IS & International System of Units \\
ITM & input test mass \\
LMT & large momentum transfer \\
LG & Laguerre-Gauss \\
LIGO & Laser Interferometer Gravitational-wave Observatory \\
LISA & Laser Interferometer Space Antenna \\
NIST & National Institute of Standards and Technology \\
NPL & National Physical Laboratory \\
PBS & polarising beam splitter \\
PD & photo-detector \\
PDH & Pound-Drever-Hall \\
PI & parametric instability \\
rf & radio frequency \\
rms & root mean square (quadratic mean) \\
ROC & radius of curvature \\
ULE & ultra-low expansion \\
&
\end{tabular}

Table 1: List of acronyms and their meaning. 


\begin{tabular}{|c|c|}
\hline Notation & Description \\
\hline$\equiv$ & definition \\
\hline$c$ & speed of light $(\mathrm{m} / \mathrm{s})$ \\
\hline$\hbar=h / 2 \pi$ & reduced Planck constant (Js) \\
\hline$T$ & temperature $(\mathrm{K})$, or intensity transmissivity \\
\hline$f$ & Fourier frequency $(\mathrm{Hz})$ \\
\hline$y$ & normalised frequency \\
\hline$\nu, \omega$ & frequency $(\mathrm{Hz})$, frequency $(\mathrm{rad} / \mathrm{s})$ \\
\hline$\Delta \nu, \Delta \omega$ & bandwidth $(\mathrm{Hz})$, bandwidth (rad/s) \\
\hline & phase (rad), or mechanical loss angle \\
\hline$\lambda$ & wavelength $(\mathrm{m})$ \\
\hline$k,(\boldsymbol{k})$ & wave number and wave vector $(1 / \mathrm{m})$ \\
\hline$r$ & position vector $(\mathrm{m})$ \\
\hline$\xi(t)$ & observable amplitude of the electric field $(\mathrm{V} / \mathrm{m})$ \\
\hline$E(t)$ & complex amplitude phasor $(\sqrt{\mathrm{W}})$ \\
\hline$a(t)$ & complex amplitude phasor without time-varying phase $(\sqrt{\mathrm{W}})$ \\
\hline$a_{\text {in }}, a_{\text {refl }}, a_{\text {trans }}, a_{\text {circ }}$ & input, reflected, transmitted, and circulating phasors $(\sqrt{W})$ \\
\hline$q(z)$ & complex beam parameter \\
\hline$w(z), w_{0}$ & Gaussian beam radius and beam waist $(\mathrm{m})$ \\
\hline$R(z)$ & Gaussian beam radius of curvature $(\mathrm{m})$ \\
\hline$z_{R}$ & Rayleigh range $(\mathrm{m})$ \\
\hline$\zeta(z), \zeta$ & local and roundtrip Gouy phase shifts (rad) \\
\hline $\mathrm{HG}_{n m}$ & $(n+m)$-th order Hermite-Gauss transverse electric mode \\
\hline$L$ & cavity length $(\mathrm{m})$ \\
\hline $\mathcal{R}$ & radius of curvature of lens or mirror $(\mathrm{m})$ \\
\hline$r_{a, b}, t_{a, b}$ & amplitude and transmission reflection coefficients \\
\hline$g$ & cavity $g$-factors \\
\hline$g_{1,2}$ & input and end mirror $g$-factors \\
\hline $\mathcal{F}$ & cavity finesse \\
\hline$\Delta \nu_{\mathrm{FSR}}$ & free spectral range $(\mathrm{Hz})$ \\
\hline$\tau$ & cavity photon transit time (or averaging time) (s) \\
\hline$\tau_{p}$ & cavity photon lifetime $(\mathrm{s})$ \\
\hline$\delta t$ & input pulse duration $(\mathrm{s})$ \\
\hline$\delta t_{\pi}$ & mirror pulse duration for $<5 \%$ losses (s) \\
\hline$\tau_{\pi}$ & in-cavity mirror pulse duration for $<5 \%$ losses (s) \\
\hline$S_{n m}$ & optical suppression ratio of $\mathrm{HG}_{n m}$ \\
\hline$Y$ & Young's modulus \\
\hline$\sigma$ & Poisson's ratio \\
\hline$\rho$ & density \\
\hline$S_{y}$ & power spectral density of $y$ (units of $\left.y^{2} / \mathrm{Hz}\right)$ \\
\hline$\sigma_{y}$ & Allan deviation of $y$ (units of $y$ ) \\
\hline$\widehat{\mathcal{H}}$ & Hamiltonian \\
\hline$\Omega_{0}(t)$ & Rabi frequency $(\mathrm{rad} / \mathrm{s})$ \\
\hline$\Omega(t)$ & 2-photon Rabi frequency ( rad/s) \\
\hline $\bar{\Omega}$ & Peak 2-photon Rabi frequency (rad/s) \\
\hline$f(t)$ & field amplitude envelope function \\
\hline$G(t)$ & field intensity envelope function \\
\hline$\omega_{r}$ & recoil frequency of atomic transition $(\mathrm{rad} / \mathrm{s})$ \\
\hline$|g\rangle,|e\rangle$ & ground and excited electronic eigenstates \\
\hline$|p\rangle$ or $|m\rangle$ & momentum eigenstate \\
\hline$J_{m}(x)$ & $m$-th order Bessel function of the first kind \\
\hline
\end{tabular}

Table 2: Description of the main notations used in this thesis. 



\section{Atomic clocks, cold atoms and gravitational waves}

These are some of the things that hydrogen atoms do, given fifteen billion years of cosmic evolution.

- Carl Sagan.

\section{Contents}

1.1 The common link: Fabry-Perot resonators . . . . . . . 2

1.2 The future of atomic time - Chapters $2-4 \ldots \ldots \ldots$

1.3 The cold atoms revolution - Chapters $5-8 \ldots \ldots \ldots$

1.4 The dawn of a new era in astronomy - Chapters $9-10 \ldots 6$

1.5 Structure of the thesis . . . . . . . . . . . . . 7 


\subsection{The common link: Fabry-Perot resonators}

7 He Fabry-Perot (FP) cavity or resonator (Figure 1.1) is perhaps the most

1 deceptively simple setup in optics. In an idealised form it consists of two spherical or plane mirrors separated by a distance. This instrument of seemingly simple design, devised at the end of the 19th century by french physicists Charles Fabry and Alfred Perot [2,3], is today a key resource in experimental physics.

The distinctive feature of the FP resonator - simply resonator or cavity hereafter - is its narrow resonances (or, equivalently, its ability to store coherent light), which makes them extremely useful in metrology, high-resolution spectroscopy, and interferometry, and its the reason for their adoption as laser oscillators.

Optical cavities have become an integral part of laser interferometer gravitationalwave detectors [4], and are proposed as an enabling technology for large area atom interferometry [5], and future gravitational-wave detection with atom interferome$\operatorname{try}[6]$.

All of these applications are discussed in this work:

- Part I follows the development of a next-generation, ultra-stable, thermalnoise-limited, room-temperature optical cavity for use as the master local oscillator in the network of microwave and optical atomic clocks at the National Physical Laboratory (NPL), as part of the effort directed towards a future re-definition of the second in the International System of Units (SI).

- Part II explores the use of optical cavities to enhance the sensitivity of atom interferometers based on multi-photon Bragg diffraction, describing the limitations of these devices for assisting large-momentum-transfer atomic beam splitters by using a mixture of numerical and analytical models. Furthermore, the design of a demonstrator experiment for gravitational-wave detection using cavity-assisted atom interferometry is discussed, and the design of a four-mirror, large-waist cavity with tuneable stability for enhanced atom interferometry is presented.

- Part III presents some of the work being carried out in order to enhance current and future laser interferometer gravitational-wave detectors that make use of giant optical cavities. The development and characterisation of a $1 \mathrm{~m}$ tabletop cavity with tuneable stability is put forward, and its behaviour as the cavity is parametrically pushed towards instability is described in an effort to assist the adoption of near-unstable cavities in future gravitationalwave detectors to reduce their thermal-noise floor. Finally, the problem of parametric instabilities in optical cavities with high-power circulating optical fields and massive mirrors is discussed, and the finite-element analysis of the mechanical resonances of such mirrors is described in detail. 


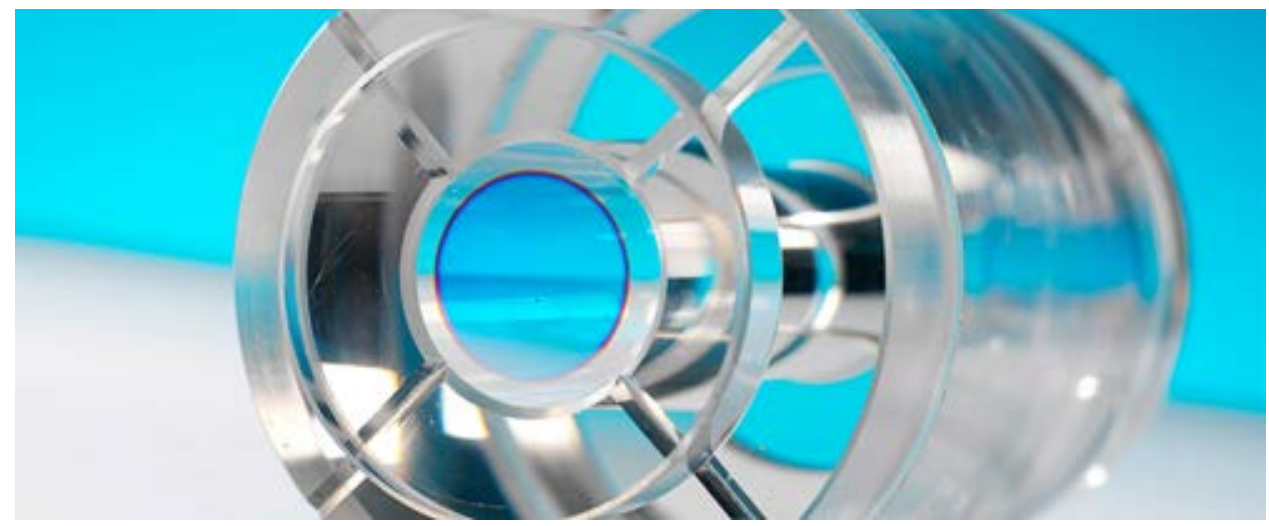

Figure 1.1: Fabry-Perot resonators have become essential in many areas of science and technology. They have found applications across many disciplines: from LIGO's giant $4 \mathrm{~km}$ arm cavities to the world's most accurate clocks. Pictured is the ultrastable cavity that is used as the local oscillator at NPL's strontium ion optical clock. Image courtesy of NPL [7].

\subsection{The future of atomic time - Chapters $2-4$}

In time and frequency metrology we are exploring new ways of achieving ever more accuracy and precision in measurement. Atomic clocks are based on a fundamental property of matter: the quantum absorption or release of electromagnetic energy when an electron "leaps" between the energy levels of an atom. The frequency of the released or absorbed radiation is precisely equal to the energy difference between the two participating quantum states. This makes atomic transitions ideal frequency standards, as nature makes all atoms of the same species identical. In order to measure this frequency a laser locked to the narrow resonance of an ultra-stable FP cavity is used as the local oscillator, much like the mass in a string of a pendulum clock. Today, atomic clocks based on the caesium atom make use of a microwave transition to realise the second to better than 1 part in $10^{15}$, yielding nearly a million times improved accuracy over traditional frequency standards based on astronomical observations, such as the diurnal rotation of the Earth. The time stability of caesium-based atomic clocks is continuously improving. For example, the uncertainty of the NIST-F1 standard was around $10^{-15}$ in 2000 , and was reduced to $3 \cdot 10^{-16}$ in $2013[8,9]$. This is equivalent to an uncertainty of \pm 1 second in roughly 100 million years (Table 1.1).

Atomic time-keeping is at the heart of satellite-based positioning systems, such as GPS, which use these accurate time signals to provide global location tracking with an accuracy of a few metres, as well as the synchronisation of communication and energy networks and the financial trade. We soon expect to be able to redefine the base unit of time, the second, using atomic clocks operating at optical frequencies, rather than microwave $[10,11]$. In optical atomic clocks (Figure 1.2), the local oscillator is a thermal-noise-limited optical cavity based on either a cryogenic silicon spacer or a room-temperature ultra-low-expansion (ULE) glass spacer to which 


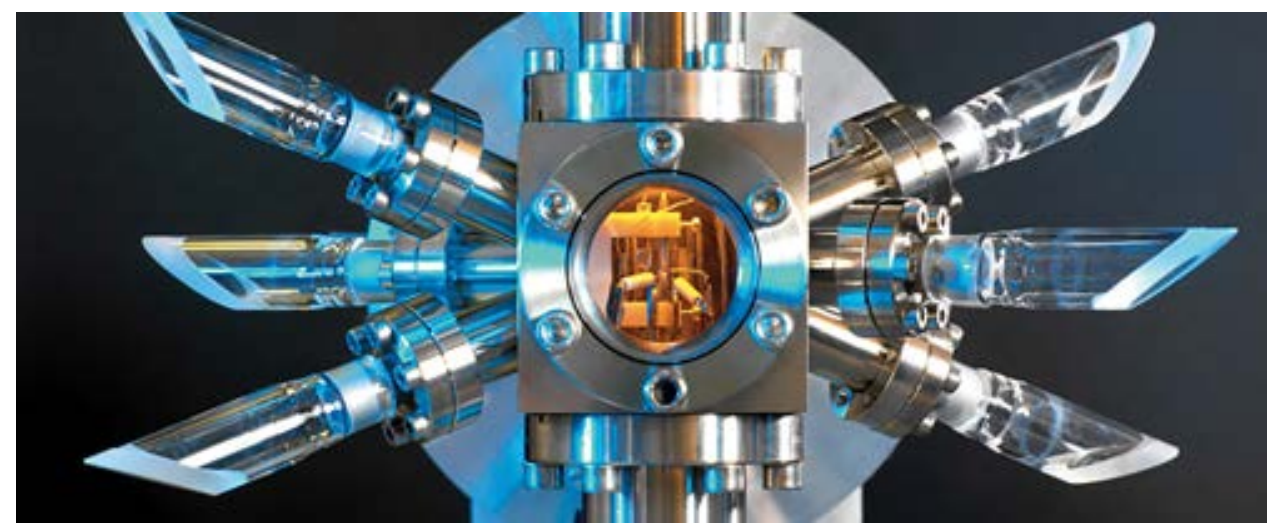

Figure 1.2: Optical atomic clocks provide a time standard with a precision and stability superseding that of the microwave standards which are used today to realise the base unit of time, the second. Pictured is the ion trap at the heart of NPL's strontium ion optical clock. Image courtesy of NPL [7].

\begin{tabular}{lll}
\hline Date & Clock & Uncertainty \\
\hline 17 th century & Pendulum clocks & \pm 10 seconds per day \\
1762 & Harrison's chronometer & \pm 2 seconds per day \\
$1930 ' s$ & Earth's rotation & \pm 1 second in 3 years \\
$1930 ' s$ & Quartz clocks & \pm 1 second in 30 years \\
1955 & First atomic clock & \pm 1 second in 300 years \\
$1980 ' s$ & Improved atomic clocks & \pm 1 second in 300 thousand years \\
2000 & Caesium fountain & \pm 1 second in 30 million years \\
2013 & Improved caesium fountain & \pm 1 second in 100 million years \\
\hline
\end{tabular}

Table 1.1: Evolution of uncertainty in time measurement [7].

high-reflectivity mirrors are optically contacted. The next generation of these devices is expected to achieve a relative stability better than 1 part in $10^{16}$. In Part I of the thesis we give a detailed account of the technical hurdles that need to be overcome to achieve such an amazing stability, and the design and experimental work that is addressing them at NPL, where a new state-of-the-art reference cavity is being developed [12].

Ultra-stable cavities that are only limited by the fundamental thermal motion of its constituents require very careful design and environmental control. These devices are extremely sensitive to pressure and temperature fluctuations and vibrations, and hence great effort is put into isolating them from external perturbations. When a laser's frequency $\nu$ is locked to the narrow resonance of a cavity of length $L$, if the servo electronics are well designed, the structural stability of the cavity is transferred to the frequency stability of the laser, $\frac{\Delta \nu}{\nu}=-\frac{\Delta L}{L}$. An analogy can be made that if the spacer of a cavity were the Earth $\left(L=12.7 \cdot 10^{6} \mathrm{~m}\right)$, a human hair added to the diameter $(\Delta L \approx 15 \mu \mathrm{m})$ would cause a frequency shift $\Delta \nu \approx 300 \mathrm{~Hz}$ (for a laser wavelength of $1064 \mathrm{~nm}$; this analogy is attributed to James Bergquist of 
NIST). In another analogy, if we managed to construct an ultra-stable cavity with a ULE spacer the size of the Earth $\left(\Delta L \approx 8 \cdot 10^{-14}\right.$ for a perfectly isolated ULE spacer of length $L=12.7 \cdot 10^{6} \mathrm{~m}$ and small cross-section), then a laser locked to it would experience frequency fluctuations in the order of $\mu \mathrm{Hz}$ !

\subsection{The cold atoms revolution - Chapters $5-8$}

The advent of atom trapping and cooling has brought about a revolution in atomic and optical physics, a culmination of which is high-precision atom interferometry. By skilfully using laser pulses to manipulate the internal energy state of the atom and/or its external momentum state (Figure 1.3), we have created matter wave interferometers whose signal is linked to the local acceleration and/or rotation experienced by the atom. These devices allow us to test gravity at a new scale, and promise significant advances in applications ranging from geophysical exploration to tests of general relativity $[13,14]$ or gravitational-wave detection $[6,15-18]$. Since the demonstration of the first light-pulse atom interferometer in 1991 [19], the method has made possible new measurements of the gravitational constant $[20,21]$ and the fine structure constant [22-24], and tests of the weak equivalence principle $[25,26]$. It has also been used to measure gravity accelerations [27,28], gravity gradients $[29,30]$, and rotations [31-34]. It also has proposed applications in tests of quantum electrodynamics [35] and quantum-entanglement at macroscopic distances [36].

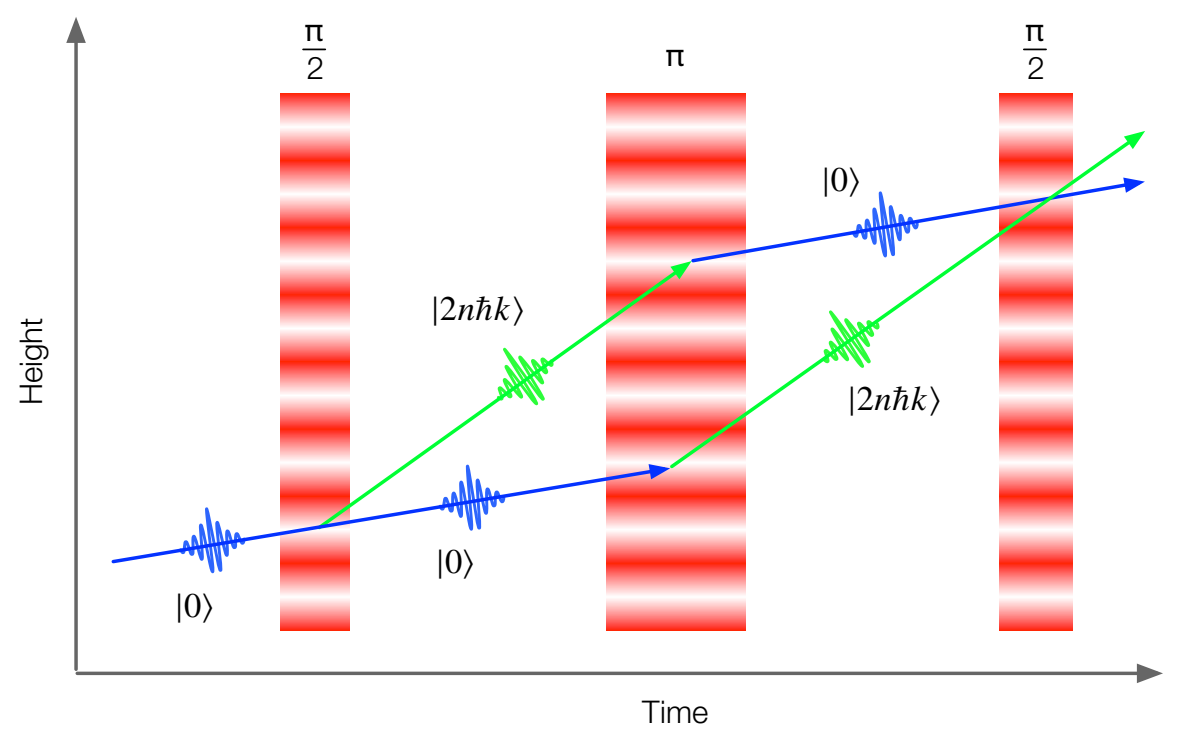

Figure 1.3: In light-pulse atom interferometers a sequence of light pulses is applied to ensembles of cold atoms in order to manipulate their quantum state. Their signals are linked to the local acceleration and/or rotation experienced by the atoms, and their sensitivity scales with the momentum difference between the interfering matter waves. 
Perhaps the most tantalising application of all is in gravitational-wave astronomy, where experiments based on correlating atom interferometer signals are expected to observe gravitational waves with frequencies below $1 \mathrm{~Hz}$, a frequency band where current laser interferometers, such as Advanced LIGO, have poor sensitivity [37].

The sensitivity of atom interferometers scales with the momentum difference between the interfering matter waves. We aim to be able to employ large momentum transfer (LMT) beam splitters, which are expected to ultimately lead to a 1000fold increase or more in the sensitivity of atom interferometers by drastically increasing the number of photon-atom interactions during the interferometric sequence. LMT beam splitters however suffer from two important drawbacks: the increased sensitivity to distortions of the laser wavefronts and increased power requirements. Optical cavities have been proposed as the key enabling technology for LMT beam splitters, and cavity-assisted atom interferometers have already been demonstrated [5,38-40], as running the interferometer sequence inside the cavity can help mitigate the disadvantages of the technique. However no quantitative understanding of how the cavity parameters affected the atomic transitions existed until now. Part II of the thesis answers some of the questions arising from the application of cavities to assist LMT beam splitters based on multi-photon Bragg diffraction [41], elaborates on the feasibility of the technique for gravitational-wave detection based on this technology [6], and presents the design of a multi-mirror resonator to avoid some of the pitfalls encountered by the FP resonator [42].

\subsection{The dawn of a new era in astronomy - Chapters $9-10$}

A billion years ago a system of two black holes merged together in a cataclysmic event that emitted gravitational waves: ripples propagating in the fabric of spacetime. On the 14th of September 2015, the Advanced LIGO detectors in Livingston and Hanford, the most sensitive length sensing devices ever built, detected the signal (Figure 1.4) in what became the first direct observation of gravitational waves [43], codenamed GW150914, and another confirmation of Einstein's general relativity for the first time in the strong field regime. This observation, the culmination of decades of experimental work, marked the beginning of a new era in astronomy, as a new window into the cosmos was opened.

Since the first observation, the Advanced LIGO second-generation detectors have participated in a series of subsequent observations, notably the first joint gravitational and electromagnetic observation of a binary neutron star inspiral [44,45] (GW170817) from the galaxy NGC 4993, located about 140 million light years away. In the near future, with the development of third-generation detectors such as the Einstein Telescope (ET) underground [46], or the Laser Interferometer Space Antenna (LISA) mission in space [47], we expect to widen our view of the gravitational universe.

The Advanced LIGO detectors are the most sensitive length sensing devices ever created [4]. They are modified Michelson interferometers with giant 4-km-long FP cavity arms using $40 \mathrm{~kg}$ fused silica mirrors as test masses. The test masses are suspended using silica fibres and quadruple suspensions systems. The passing 
of a gravitational wave causes the length of the cavities to fluctuate, generating an interferometric signal at the output photodetector that is proportional to the gravitational wave's characteristic strain. A set of fundamental noise sources of seismic, thermal and quantum origin affect the measurement. Part III of the thesis presents the work being carried out towards enhancing the sensitivity of future detectors, particularly in reducing the thermal noise floor by using nearunstable cavities [48], and in simulating parametric instabilities that result from the optomechanical interaction between the cavity's optical field and the test mass' mechanical modes [49].

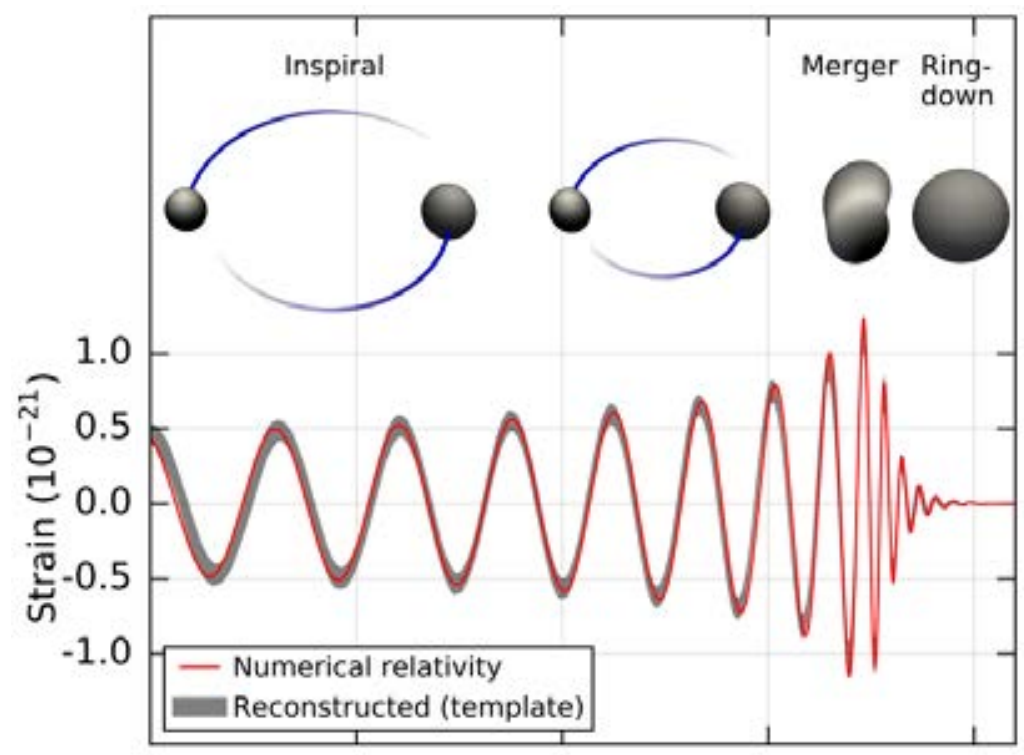

Figure 1.4: GW150914: In late 2015 the LIGO Hanford and Livingston observatories registered the first ever gravitational wave signal originated from a binary black hole merger about one billion years ago. The measurement, with a significance greater than $5 \sigma$, constituted the first confirmation of Einstein's general relativity in the strong-field regime. The peak gravitational wave strain was $1.0 \cdot 10^{-21}$. This detection marked the beginning of the era of gravitational-wave astronomy. Reproduced from [43].

\subsection{Structure of the thesis}

This thesis is structured as follows: Part I (Chapters $2-4$ ) follows the development of a thermal-noise-limited, ultra-stable optical cavity for high-precision time and frequency metrology. The cavity is expected to reach a relative stability of 1 part in $10^{16}$ at a few seconds of integration time. The design of the force-insensitive cavity spacer is presented in Chapter 2, and the design of the cavity enclosure (pressure, temperature, and vibration isolation systems) is presented in Chapter 3. 
Chapter 4 documents the current status of the experiment and a characterisation of the achieved stability.

Part II (Chapters 5-8) explores the application of optical cavities to assist the beam splitter pulses in large momentum transfer atom interferometers based on multi-photon Bragg diffraction. Chapter 5 introduces the theoretical framework as well as the analytical and numerical models that were developed in order to conceive our analysis. Chapter 6 applies this framework to realise the potential and the fundamental limitations of these devices. These findings are then used to assist the design of the MIGA experiment in France in Chapter 7. Lastly, Chapter 8 proposes a multi-mirror cavity for enhanced atom interferometry that circumvents some of the limitations found with FP resonators.

Part III (Chapters $9-10$ ) is devoted to advancement in instrumentation science for laser interferometer gravitational-wave detectors. Chapter 9 follows the development of an optical cavity with tuneable stability to investigate the feasibility of using near-unstable cavities in gravitational-wave detectors in order to reduce the thermal noise floor. Chapter 10 shows the work being carried out in towards modelling parametric instabilities in current and future detectors, and particularly revolves around the development of the finite element models of the test masses that are required in this effort. Lastly, Chapter 11 provides a brief summary of the work presented in the thesis and discusses the main results. 
PART I

\section{Cavities for Optical Atomic Clocks}





\section{Thermal-noise-limited room-temperature ULE cavity}

Never measure anything but frequency!

- Arthur Schawlow [50].

\section{Contents}

2.1 Optical frequency standards: Towards a redefinition of the SI second .................... 12

2.2 Fabry-Perot resonator theory . . . . . . . . . . . . . 13

2.2.1 Fields in a Fabry-Perot resonator . . . . . . . . . . . 13

2.2.2 Static response and resonance condition . . . . . . 15

2.2.3 Transverse modes . . . . . . . . . . . . . . . 17

2.2.4 Fundamental Gaussian beam . . . . . . . . . . . . 19

2.2.5 Resonator stability . . . . . . . . . . . . . . . . 21

2.3 The art of making ultra-stable optical cavities . . . . . . . 24

2.3.1 Thermal noise limit . . . . . . . . . . . . . . . . . 24

2.3.2 Spacer length and mirror ROC . . . . . . . . . 28

2.4 Force-insensitive optical cavity . . . . . . . . . . . . . . . . 31

2.4.1 Spacer geometry and material . . . . . . . . . . . 31

2.4.2 Spacer structural mechanics . . . . . . . . . . . . . 34

2.4.3 Model for the force-induced length fluctuations . . . . 36

2.5 Optimisation of the support positions . . . . . . . . . 38

2.5.1 Probing the $(d x, d z)$ parameter space . . . . . . 38

2.5.2 Transverse and longitudinal accelerations . . . . . . . . 43

2.5.3 Further optimisation of the spacer geometry . . . . . . 45

2.5.4 Optimal configuration . . . . . . . . . . . . 47

2.5.5 Balanced support forces . . . . . . . . . . . . 51 


\subsection{Optical frequency standards: Towards a redefinition of the SI second}

Gor centuries, astronomical observations of the rotation of the Earth and other 1 celestial bodies were used as frequency standards for the definition of the base unit of time. Before 1967 the second was defined in the International System of Units (SI) via observation of the diurnal rotation of the Earth, and since the 1940's quartz oscillators were used to subdivide this measurement (1 second was defined as a fraction 1/86400 of the mean solar day). These observations were corrected for known irregularities and smoothed over long intervals, and their accuracy was generally good to 4 parts in $10^{9}$ during a year [51]. But the Earth's period of rotation is not constant: it is gradually slowing down due to tidal friction, varies with the season, and also fluctuates unpredictably.

In 1955 Louis Essen and Jack Parry produced at NPL the first practical atomic frequency standard, based on the microwave hyperfine transition in ${ }^{133} \mathrm{Cs}$ at $9192631770 \mathrm{~Hz}$ with an accuracy of 1 part in $10^{10}$. Unlike the Earth's rotation, atoms constitute the perfect frequency references because nature makes all atoms of the same species identical. The caesium standard was adopted in 1967 to set the definition of the SI second. The technology around atomic timekeeping has advanced considerably since then, and commercial caesium standards have reached accuracies better than 1 part in $10^{12}$ [10]. The progress on laser cooling and trapping techniques has led to the demonstrations of the first sodium atomic fountain in 1989 [52] and the first caesium fountain clock in 1991 [53], today used to realise the second to better than 1 part in $10^{15}$.

In the years to come new types of atomic clocks operating at optical rather than microwave frequencies promise significant advances in science and technology, and may lead to a redefinition of the SI second [11]. Optical atomic clocks are based on nearly forbidden optical transitions in atoms or ions of frequencies close to $10^{15} \mathrm{~Hz}$ (approximately $10^{5}$ times higher than the microwave frequencies). This offers a significant improvement in clock stability at equivalent averaging times as compared to the microwave standards: all other things being equal, the frequency instability of an atomic clock is proportional to its operating frequency and inversely proportional to the width of the atomic transition (although in practice the stability also depends on the signal-to-noise ratio of the atomic absorption feature).

A key part of any atomic clock is a stable local oscillator that is used to interrogate the absolute frequency reference (i.e., the clock transition of the atom or ion). For optical atomic clocks the local oscillator is an ultra-stable laser servocontrolled to the narrow resonance of a high-finesse ultra-stable Fabry-Perot cavity. The cavity consists of two mirrors with high-reflectivity coatings optically contacted to the ends of a spacer that is either cryogenically cooled or made of an ultra-low expansion material. The laser's frequency is stabilised to the resonance of the cavity using the Pound-Drever-Hall (PDH) method [54]. This procedure uses the light reflected from the cavity to detect the cavity resonance. The light is first phase-modulated so that both a carrier field and rf sidebands are incident on the cavity. The heterodyne beat between the reflected carrier field - in resonance with the cavity - and the reflected off-resonance sidebands is detected and demodulated 
to obtain an error signal that is fed back to the laser to tightly lock its frequency to the cavity resonance. If the $\mathrm{PDH}$ control of the laser frequency is good enough, the frequency stability of the cavity resonance is transferred to the frequency stability of the laser. Since the cavity's resonance frequencies are only dependent on the cavity dimensions, it is the cavity's dimensional stability that is transferred to the laser's frequency stability. Today, cavities limited only by the fundamental thermal fluctuations of its constituent parts are capable of achieving relative instabilities below $10^{-16}[55]$.

The first part of the thesis describes the development of a next-generation thermal-noise-limited ultra-stable reference cavity at NPL. The main feature of this cavity is a long $\sim 0.5 \mathrm{~m}$ spacer that is designed to achieve a thermal-noise performance below the $10^{-16}$ level at room temperature. Long reference cavities present many technical challenges, as they are very sensitive to accelerations, temperature and pressure fluctuations and vibrations. This chapter describes the development of the cavity geometry, which is designed to achieve a certain thermal-noise performance and is optimised to be virtually acceleration-insensitive. Chapter 3 describes the development of the isolation systems designed to keep all external sources of noise below the thermal-noise limit, and Chapter 4 presents the experimental characterisation of the system as well as the results of the achieved performance of frequency stability.

\subsection{Fabry-Perot resonator theory}

It will prove useful to develop the basic theory of optical FP resonators, starting with the expressions for the optical fields inside the cavity. This will allow us to introduce the most important features of these devices, which will be used time and time again, as well as the notation used throughout the thesis.

\subsubsection{Fields in a Fabry-Perot resonator}

The most elemental treatment of FP resonators, reproduced in many textbooks [56], is the geometric multiple-beam interference picture, where the resonator is modelled as a plane parallel plate of thickness $L$ and refractive index $n$, immersed in a medium of refractive index $n^{\prime}$. We first use this intuitive method, which deals only with input excitations of constant amplitude, and is sufficient to describe the static response of the cavity, from which we will derive basic properties such as the resonance condition, the frequency to length coupling, and the resonator bandwidth and finesse. The more modern approach of difference equations will be used in Chapter 5 to describe the transient response of the cavity, which will allow us to deal with the more realistic scenario of input excitations with time-varying amplitudes. The latter treatment will be motivated by our need to compute the cavity's response to the short pulses which are employed in atom interferometry. We will not, however, deal with any variations of the cavity length or the input laser frequency. For a detailed account of those effects, see e.g. [57].

We assume that the optical field is monochromatic with frequency $\omega$ and wave vector $\boldsymbol{k}$. We use the complex phasor notation of the field by introducing the 


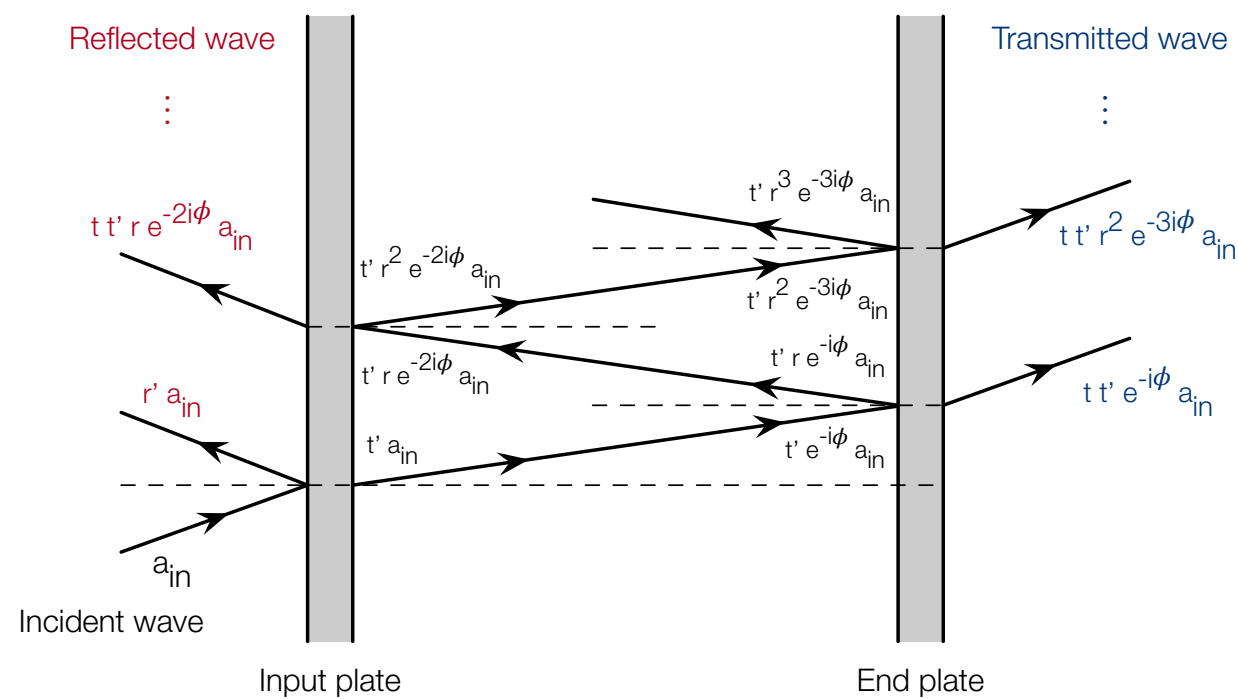

Figure 2.1: The plane parallel plate model is sufficient to describe the static response of a Fabry-Perot resonator to an input plane wave of amplitude $a_{\text {in }}$. This schema depicts the first few reflections and transmissions at the plate's interfaces.

complex amplitude $E(t)$, in units of $\sqrt{\mathrm{W}}$, so that the measurable electric field is given in units of $\mathrm{V} / \mathrm{m}$ by

$$
\xi(t) \equiv \frac{\sqrt{\epsilon_{0} c}}{2}\left(E(t)+E(t)^{*}\right)=\sqrt{\epsilon_{0} c} \operatorname{Re}\{E(t)\},
$$

where $\epsilon_{0}$ is the vacuum permittivity and $c$ is the speed of light. Using the complex phasor $E(t)$, which represents the amplitude and phase of the field, greatly simplifies the electric field computations. When the physical electric field is required, it can easily be obtained from $E(t)$ by casting Equation 2.1. Furthermore, we drop the variable part of the phase of $E(t)$, and use a complex phasor $a(t)=a_{0}(t) e^{i \phi_{0}}$, which contains only the constant part of the phase of the wave function. The variable part can always be retrieved as

$$
E(t)=a(t) e^{-i(\omega t-\boldsymbol{k} \cdot \boldsymbol{r})} .
$$

In the plane parallel plate model, consider that the plate is illuminated by an incident plane wave of constant complex amplitude $a_{\mathrm{in}}$. The plate will respond with a series of reflected and transmitted waves (Figure 2.1). The superposition of all the waves leaving the first surface in the direction opposite to the incident wave forms the reflected field

$$
a_{\mathrm{refl}}(\phi) \equiv a_{\mathrm{in}}\left(r^{\prime}+t t^{\prime} r e^{-2 i \phi}+t t^{\prime} r^{3} e^{-4 i \phi}+t t^{\prime} r^{5} e^{-6 i \phi}+\ldots\right),
$$

where the first term corresponds to the wave reflected at the first surface immediately upon incidence of the incoming wave, the second to the wave transmitted into the 
plate and transmitted out through the first surface after one round-trip (i.e., after covering twice the distance between plates), the third to the wave which underwent two round-trips, and so forth. The quantities $r, t$ and $r^{\prime}, t^{\prime}$ are the amplitude reflection and transmission coefficients for waves travelling from the plate to the surrounding medium and vice versa, and are given by the Fresnel formulas [56]. They are dependent on the angle of incidence, but for our purposes it is safe to consider near normal incidence so that $r=\frac{n-n^{\prime}}{n+n^{\prime}}, r^{\prime}=-r$ and $t \cdot t^{\prime}=\left(1-r^{2}\right)$ are constants (Stoke's equations). We are assuming that there are no losses at the plates, and that the plates are perfectly parallel. Note that using Fresnel's equations implies a phase change of $0^{\circ}$ or $180^{\circ}$ upon reflection, depending on the sign of $\left(n-n^{\prime}\right)$, and a $0^{\circ}$ phase change upon transmission. This is the historical convention, used throughout most of the literature, but we will soon introduce a more convenient one. Note the phasor $e^{-2 i \phi}$ that is accumulated in every resonator round-trip, where $\phi$ is the phase thickness of the plate,

$$
\phi \equiv k n L,
$$

and $k=2 \pi / \lambda$ is the wave number of the incident wave, with $\lambda$ the wavelength of the light. Similarly, the superposition of all the waves leaving the second surface in the direction of the incident wave forms the transmitted field

$$
a_{\text {trans }}(\phi) \equiv a_{\text {in }}\left(t t^{\prime} e^{-i \phi}+t t^{\prime} r^{2} e^{-3 i \phi}+t t^{\prime} r^{4} e^{-5 i \phi}+\ldots\right) .
$$

Finally the waves that by repeated reflection and transmission at the two surfaces remain confined within the cavity form the circulating field. This field has a component travelling in the direction of the incident wave (i.e., from the input plate to the end plate) $a_{\mathrm{circ}, 1}$, and another in the opposite direction $a_{\mathrm{circ}, 2}$,

$$
\begin{aligned}
& a_{\text {circ }, 1}(\phi) \equiv a_{\text {in }}(\phi) t^{\prime} e^{-i \phi}\left(1+r^{2} e^{-2 i \phi}+r^{4} e^{-4 i \phi}+\ldots\right), \\
& a_{\text {circ }, 2}(\phi) \equiv a_{\text {in }}(\phi) t^{\prime} r e^{-2 i \phi}\left(1+r^{2} e^{-2 i \phi}+r^{4} e^{-4 i \phi}+\ldots\right) .
\end{aligned}
$$

It is possible to carry out these sums to obtain closed form expressions for all the fields:

$$
\begin{aligned}
a_{\mathrm{refl}}(\phi)= & a_{\mathrm{in}}(\phi) r^{\prime}+a_{\mathrm{in}}(\phi) \frac{t t^{\prime} r e^{-2 i \phi}}{1-r^{2} e^{-2 i \phi}}, & a_{\mathrm{circ}, 1}(\phi) & =a_{\mathrm{in}}(\phi) \frac{t^{\prime} e^{-i \phi}}{1-r^{2} e^{-2 i \phi}}, \\
& a_{\mathrm{trans}}(\phi)=a_{\mathrm{in}}(\phi) \frac{t t^{\prime} e^{-i \phi}}{1-r^{2} e^{-2 i \phi}}, & a_{\mathrm{circ}, 2}(\phi) & =a_{\mathrm{in}}(\phi) \frac{t^{\prime} r e^{-2 i \phi}}{1-r^{2} e^{-2 i \phi}} .
\end{aligned}
$$

\subsubsection{Static response and resonance condition}

We can now obtain the complex reflection and transmission coefficients of the resonator from the ratios $a_{\text {refl }}(\phi) / a_{\text {in }}(\phi)$ and $a_{\text {trans }}(\phi) / a_{\text {in }}(\phi)$ respectively (using Stoke's equations):

$$
\begin{aligned}
& R_{\mathrm{cav}}(\phi) \equiv \frac{a_{\mathrm{refl}}(\phi)}{a_{\mathrm{in}}(\phi)}=\frac{r\left(e^{-2 i \phi}-1\right)}{1-r^{2} e^{-2 i \phi}} \\
& T_{\mathrm{cav}}(\phi) \equiv \frac{a_{\mathrm{trans}}(\phi)}{a_{\mathrm{in}}(\phi)}=\frac{\left(1-r^{2}\right) e^{-i \phi}}{1-r^{2} e^{-2 i \phi}}
\end{aligned}
$$


These complex coefficients completely characterise the static amplitude and phase response of the resonator. The intensity response functions can be obtained simply by casting $\left|R_{\text {cav }}\right|^{2}$ or $\left|T_{\text {cav }}\right|^{2}$. The transmission intensity response function (known simply as the cavity's transmittance) takes the form of the Airy distribution (Figure 2.2)

$$
\mathcal{T}(\phi) \equiv\left|T_{\text {cav }}\right|^{2}=\frac{1}{1+\frac{4 r^{2}}{\left(1-r^{2}\right)^{2}} \sin ^{2}(\phi)} .
$$

This function peaks (i.e., the transmitted field power to input power ratio peaks) when the phase thickness is an integer multiple of $\pi$, which means that the transmitted waves interfere constructively and the cavity is on resonance with the input excitation. This resonance condition can be expressed in terms of the frequency of the input field as

$$
\nu=m \frac{c}{2 n L} \equiv m \Delta \nu_{\mathrm{FSR}}
$$

where $m \in \mathbb{Z}$, and we have defined the separation in frequency between consecutive resonances as the free spectral range (FSR) $\Delta \nu_{\mathrm{FSR}}=c / 2 n L$. Note that moving one of the plates by $\lambda / 2$ is equivalent changing the input wave frequency by $\Delta \nu_{\mathrm{FSR}}$.

Differentiating Equation 2.11 yields the so called frequency to length coupling, which relates a change in resonator length to the equivalent change in resonance frequency and vice versa,

$$
\frac{\Delta \nu}{\nu}=-\frac{\Delta L}{L}
$$

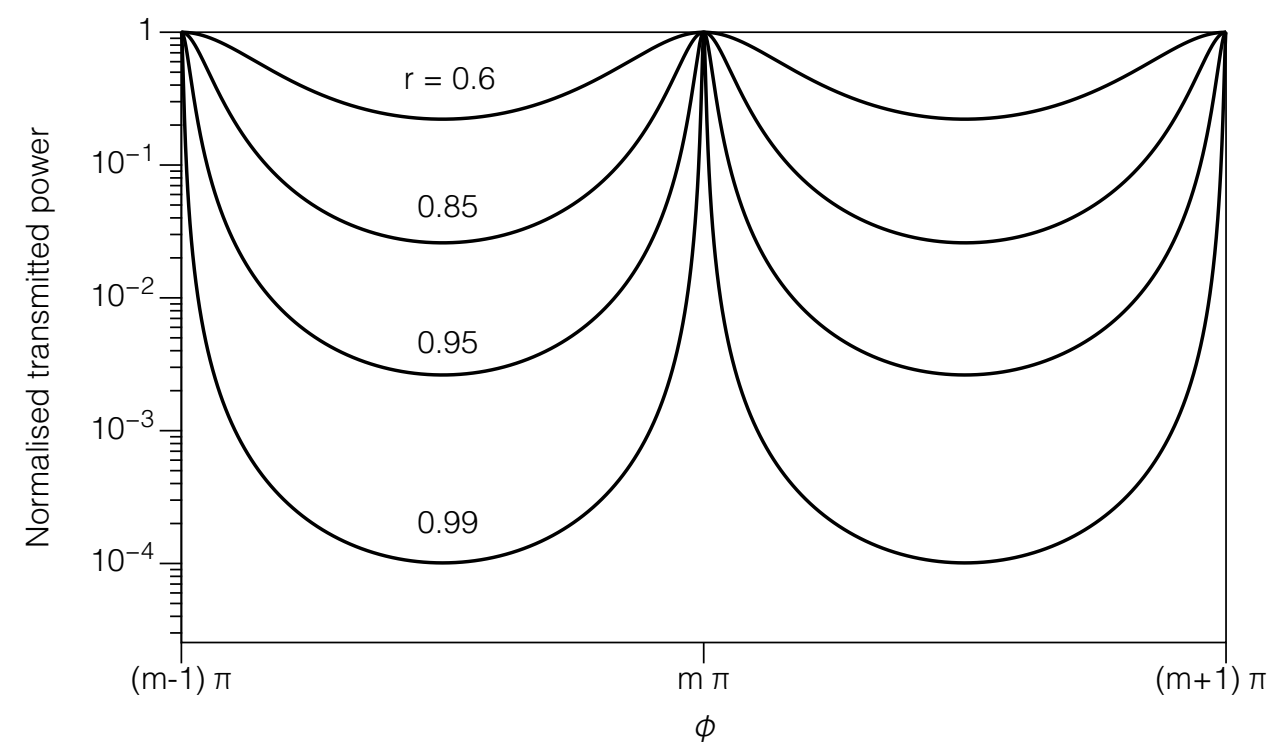

Figure 2.2: Transmission intensity response function as a function of the phase thickness of the Fabry-Perot resonator for different values of the mirror amplitude reflectivity coefficient $r$ indicated next to each curve. 
This equation is the basis for laser frequency stabilisation. By electronically locking the frequency of a laser to the resonance of a cavity using the Pound-Drever-Hall scheme, it is possible to transfer the cavity's length stability to the laser's frequency.

The full width at half maximum (FWHM) frequency of the resonance peaks is known as the resonator's bandwidth,

$$
\Delta \nu_{\mathrm{FWHM}} \approx \frac{c}{2 \pi n L} \frac{1-r^{2}}{r},
$$

and is obtained by solving $\mathcal{T}(\phi)=\frac{1}{2}$ for $\phi=m \pi \pm \frac{\epsilon}{2}$ and expanding $\sin ^{2}(\phi)$ to second order in $\epsilon$, with $\epsilon$ being the phase width of the resonance. The approximation given here is therefore valid to second order in $\epsilon$ (or, equivalently, it is valid for values of $r$ close to 1 ). The ratio of peak separation to peak width is known as the finesse of the resonator

$$
\mathcal{F} \equiv \frac{\Delta \nu_{\mathrm{FSR}}}{\Delta \nu_{\mathrm{FWHM}}} \approx \frac{\pi r}{1-r^{2}}
$$

The cavity transmittance can be re-cast in terms of the cavity finesse as

$$
\mathcal{T}(\phi)=\frac{1}{1+\left(\frac{2 \mathcal{F}}{\pi}\right)^{2} \sin ^{2}(\phi)},
$$

and the resonator bandwidth as

$$
\Delta \nu_{\mathrm{FWHM}}=\frac{c}{2 n L \mathcal{F}} .
$$

It is possible to generalise the method to account for plates having different reflection and transmission coefficients, in which case the finesse will read

$$
\mathcal{F} \equiv \frac{\Delta \nu_{\mathrm{FSR}}}{\Delta \nu_{\mathrm{FWHM}}} \approx \frac{\pi \sqrt{r_{1} r_{2}}}{1-r_{1} r_{2}}
$$

where $r_{1}$ and $r_{2}$ are the amplitude reflection coefficients of the input and end plates respectively. For an overview of the different aspects of the amplitude and intensity response of the FP resonator see [58].

\subsubsection{Transverse modes}

So far we have treated the problem of the fields in an optical cavity as the interference of many plane waves, describing only the on-axis properties of the electric fields and ignoring the spatial variation of the waves in the directions transverse to the optical path. That is, we have considered the optical fields to be functions of time and the location $z$ in the optical axis $a(z, t)$. Furthermore, for now we have only considered fields that are constant in time. This approximation allowed us to carry out an analysis of the basic properties of optical cavities by deriving the resonance condition, the bandwidth, and the finesse. However the optical fields will in general feature transverse variations in amplitude and phase, and it is necessary to take these into account for a more complete description of any optical system.

The cavity's eigenmodes [59-61] are a set of waves whose transverse pattern is self-reproducing after each resonator round-trip and form a basis. Any transverse 


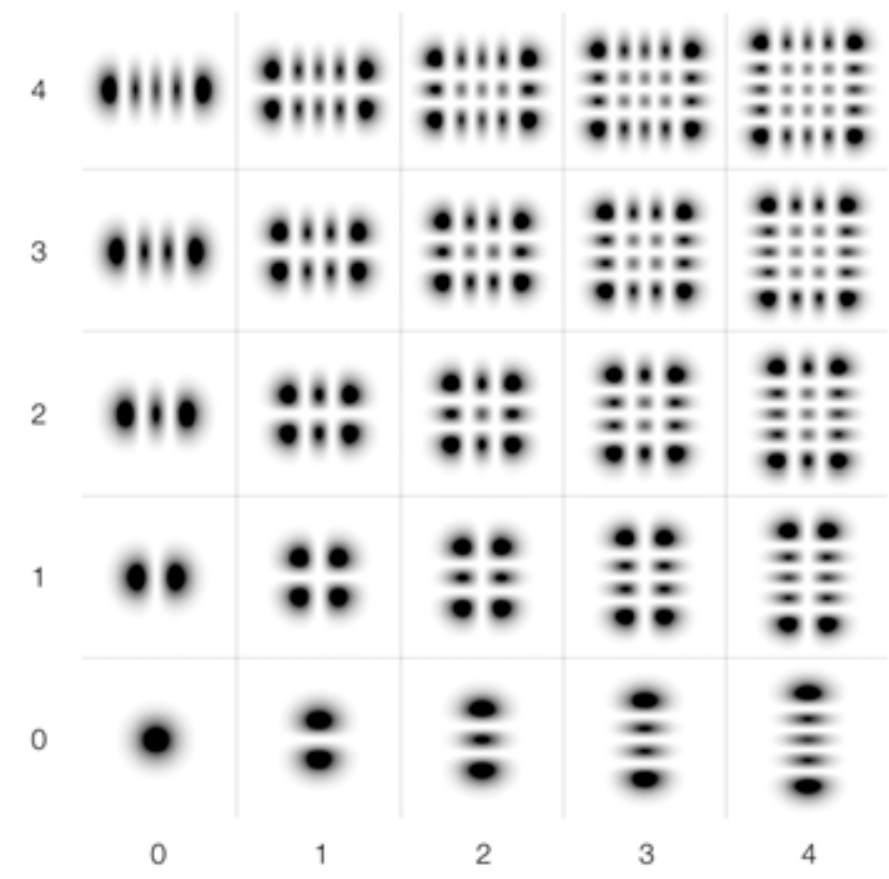

Figure 2.3: Shapes of the first few transverse Hermite-Gauss modes. The numbers indicate the mode numbers $n, m$.

pattern in a realistic beam - no matter how complex - can be expressed in terms of a superposition of such eigenmodes (or simply modes hereafter). These modes are solutions to the paraxial approximation of Helmholtz's wave equation. For a wave propagating in the $z$ direction $a(x, y, z)=u(x, y, z) e^{-i k z}$ this reduces to

$$
\left(\frac{\partial^{2}}{\partial x^{2}}+\frac{\partial^{2}}{\partial y^{2}}-2 k \frac{\partial}{\partial z}\right) u=0
$$

where $u=u(x, y, z)$ is taken to be slowly varying with respect to the wavelength, and thus the term $\partial^{2} u / \partial z^{2}$ has been neglected from the wave equation. The solution set known as the Hermite-Gauss (HG) modes forms an orthogonal basis, and is given by (Figure 2.3)

$$
u_{n m}(x, y, z) \equiv u_{n}(x, z) u_{m}(y, z),
$$

where $n, m \in \mathbb{N}$ are the mode numbers, and the function $u_{n}(x, z)$ is formed by the product of a Hermite function and a complex Gaussian function,

$$
u_{n}(x, z) \equiv\left(\frac{2}{\pi}\right)^{\frac{1}{4}}\left(\frac{e^{i(2 n+1) \zeta(z)}}{2^{n} n ! w(z)}\right)^{\frac{1}{2}} H_{n}\left(\frac{\sqrt{2} x}{w(z)}\right) \exp \left(-i \frac{k x^{2}}{2 R(z)}-\frac{x^{2}}{w^{2}(z)}\right)
$$

where $H_{n}(x)$ is the Hermite polynomial of order $n, w(z)$ and $R(z)$ are the beam's radius and its radius of curvature respectively, and $\zeta(z)$ is an extra longitudinal 
phase lag experienced by the beam known as the Gouy phase shift, which stems from the transverse spatial confinement of the beam and the consequent spread in transverse momenta [62], and will be introduced in the next section. Any laser beam can be described as the sum of all HG modes:

$$
E(x, y, z, t) \equiv \sum_{j} \sum_{n, m} c_{j n m} u_{n m}(x, y, z) \exp \left(i \omega_{j} t-i k_{j} z\right) .
$$

This description is extremely useful to analyse complex optical systems, and the coupling factors $c_{j n m}$ can yield information about the system's intrinsic imperfections [63]. We denote with $\mathrm{HG}_{n m}$ the projection of the field on the eigenfunction defined by the $n m$-th order HG mode (given by $\sum_{j} c_{j n m}$ ). In an ideal experiment the laser field would be a pure fundamental mode, i.e., the optical field would have no components other than $\mathrm{HG}_{00}$. A slight transverse misalignment of the beam can be described as coupling to the first order modes $\mathrm{HG}_{01}$ and $\mathrm{HG}_{10}$ (e.g., a beam having $95 \% \mathrm{HG}_{00}$ and $5 \% \mathrm{HG}_{01}$ ). Modes with a mode number greater than zero are known as higher order modes (HOMs). An alternative choice of basis is spanned by the solution set known as the Laguerre-Gauss (LG) modes, which exhibit cylindrical symmetry.

In closed resonators (i.e., in cavities with closed walls or infinite mirrors where radiation cannot scape the system) it can be rigorously proven that the HG or the LG eigenmodes form a complete set of normal modes, and thus any arbitrary optical field can be expressed using these functions as the basis set. For open resonators, as it will always be the case experimentally, completeness is not guaranteed.

In the early days of lasers, the physical reality as well as the mathematical existence of transverse modes in open resonators was a matter of considerable debate. Even now, in fact, except for a few special situations, rigorous mathematical existence and completeness proofs for optical resonator modes do not exist. Real lasers have never had any difficulty in finding such modes in which to oscillate, however; and from a combination of empirical and experimental evidence, it is now entirely accepted that transverse eigenmodes as we have defined them in the preceding paragraphs do exist, and do provide a physically realistic and meaningful basis for describing laser oscillation in real laser resonators.

- A. E. Siegman [64].

\subsubsection{Fundamental Gaussian beam}

The lowest order Hermite-Gauss mode $\mathrm{HG}_{00}$ is what is usually referred to as a Gaussian beam [61]. Its transverse intensity profile is a Gaussian distribution,

$$
I(r, z) \equiv \frac{2 P}{\pi w^{2}(z)} \exp \left(\frac{-2 r^{2}}{w^{2}(z)}\right)
$$

for a beam propagating in the $z$ direction, where $P$ is the total power in the beam, and $w(z)$ is the radius at $1 / e^{2}$ of the maximum intensity. The beam can be univocally defined by giving the minimum radius $w_{0}$ of the distribution (known as the beam waist) and its location along the propagation direction (Figure 2.4). At a 
distance $z$ from the waist, the radius of the beam and the radius of curvature of its wavefront are given by

$$
w(z)=w_{0} \sqrt{1+\left(\frac{z}{z_{R}}\right)^{2}},
$$

and

$$
R(z)=z+\frac{z_{R}^{2}}{z}
$$

respectively, where $z_{R} \equiv \pi w_{0}^{2} / \lambda$ is known as the Rayleigh range. The longitudinal phase lag experienced by the beam,

$$
\zeta(z)=\arctan \left(\frac{z}{z_{R}}\right)
$$

is known as the Gouy phase shift of the fundamental mode (although often we will refer to it simply as the Gouy phase). A very useful parameter that fully characterises the beam is the so-called complex beam parameter

$$
q(z) \equiv z+i z_{R}=z+q_{0}
$$

where $q_{0} \equiv i z_{R}$, or

$$
\frac{1}{q(z)} \equiv \frac{1}{R(z)}-i \frac{\lambda}{\pi w^{2}(z)} .
$$

All the information about the beam (e.g., its waist size and location) is coded in the complex beam parameter ${ }^{1}$.

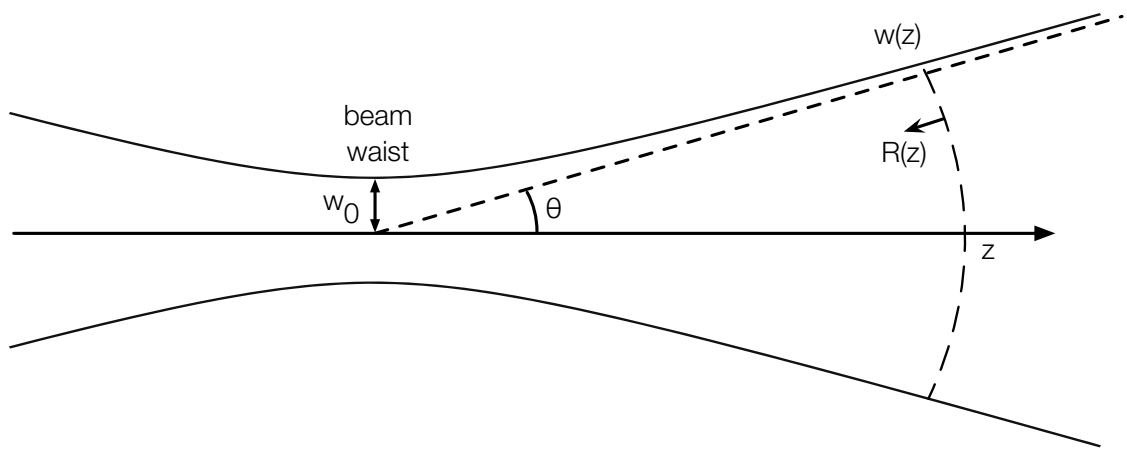

Figure 2.4: Profile of a Gaussian beam propagating in the $z$ direction, showing the waist radius $w_{0}$, the beam radius $w(z)$, the radius of curvature $R(z)$ of the beam's wavefront, and the divergence angle $\theta=\arctan \left(\lambda / \pi w_{0}\right)$ (also known as the diffraction angle, it is the angle between the propagation axis and $w(z)$ for $z \gg z_{R}$.

\footnotetext{
${ }^{1}$ We can recast other parameters in terms of $q(z)$. For example, $w^{2}(z)=\frac{\lambda}{\pi} \frac{|q(z)|^{2}}{\operatorname{Im}[q(z)]}, \quad R(z)=\frac{|q(z)|^{2}}{\operatorname{Re}[q(z)]}, \quad \zeta(z)=\arctan \left(\frac{\operatorname{Re}[q(z)]}{\operatorname{Im}[q(z)]}\right), \quad w_{0}^{2}=\frac{\lambda}{\pi} \operatorname{Im}[q(z)]$.
} 


\subsubsection{Resonator stability}

As stated in Section 2.2.3, the eigenmode of a cavity is self-consistent, i.e., it reproduces itself after one round-trip in the resonator. This applies to resonators of any complexity, made up of an arbitrary number of elements. The self-consistency condition can be expressed as

$$
\widehat{M}(z) \boldsymbol{q}(z)=\boldsymbol{q}(z)
$$

where $\boldsymbol{q}(z)$ is the column vector $(q(z), 1)^{T}$, and $\widehat{M}(z) \equiv M_{i j}$ with $i, j=1,2$ is the resonator's round-trip transfer matrix referenced to a transverse plane at position $z$ in the optical axis. Solving for the complex beam parameter, and imposing a confined solution, yields

$$
\frac{1}{q(z)}=\frac{M_{22}(z)-M_{11}(z)}{2 M_{12}(z)}-\frac{\sqrt{1-m^{2}}}{\left|M_{12}(z)\right|}=\frac{1}{R(z)}-i \frac{\lambda}{\pi w^{2}(z)},
$$

where $m \equiv \frac{\operatorname{tr} \widehat{M}(z)}{2}$ is known as the resonator's $m$ value. If $m$ is real and $|m| \leq 1$, the resonator is said to be real and geometrically stable. That is, the eigenmode is confined, meaning that it has a real and positive radius given by

$$
w^{2}(z)=\frac{\lambda}{\pi} \frac{\left|M_{12}(z)\right|}{\sqrt{1-m^{2}}} .
$$

The cavity $g$ factor is defined as $g \equiv \frac{m+1}{2}$, with the stability criterion reading

$$
0 \leq g \leq 1
$$

Geometrically, the $g$ factor can be thought of as the magnification experienced by the beam after a round-trip in the resonator, with the condition for stability implying that the beam will maintain a finite size given an arbitrary number of round-trips.

For a Fabry-Perot cavity formed by an input mirror at position $z=0$ with radius of curvature $\mathcal{R}_{1}$ and an end mirror at position $z=L$ and radius of curvature $\mathcal{R}_{2}$ (from now on we shall consider that the space between the two mirrors is void), the transfer matrix referenced to an arbitrary point at a distance $z$ from the input mirror is given by

$$
\widehat{M}(z) \equiv\left(\begin{array}{cc}
M_{11}(z) & M_{12}(z) \\
M_{21}(z) & M_{22}(z)
\end{array}\right)
$$

with

$$
\begin{aligned}
& M_{11}(z)=\frac{4 L^{2}-2\left(\mathcal{R}_{1}+2\left(\mathcal{R}_{2}+z\right)\right) L+\mathcal{R}_{1} \mathcal{R}_{2}+2\left(\mathcal{R}_{1}+\mathcal{R}_{2}\right) z}{\mathcal{R}_{1} \mathcal{R}_{2}} \\
& M_{12}(z)=\frac{2\left(-\left(\mathcal{R}_{1}-2 z\right) L^{2}+\mathcal{R}_{1} \mathcal{R}_{2} L-2 z\left(\mathcal{R}_{2}+z\right) L+\left(\mathcal{R}_{1}+\mathcal{R}_{2}\right) z^{2}\right)}{\mathcal{R}_{1} \mathcal{R}_{2}} \\
& M_{21}(z)=-\frac{2\left(-2 L+\mathcal{R}_{1}+\mathcal{R}_{2}\right)}{\mathcal{R}_{1} \mathcal{R}_{2}} \\
& M_{22}(z)=\frac{\mathcal{R}_{1}\left(\mathcal{R}_{2}-2 L\right)-2\left(-2 L+\mathcal{R}_{1}+\mathcal{R}_{2}\right) z}{\mathcal{R}_{1} \mathcal{R}_{2}}
\end{aligned}
$$


When referenced to the position of the input or end mirror the transfer matrix is greatly simplified

$$
\widehat{M}(0)=\left(\begin{array}{cc}
\frac{4 L^{2}-2\left(\mathcal{R}_{1}+2 \mathcal{R}_{2}\right) L+\mathcal{R}_{1} \mathcal{R}_{2}}{\mathcal{R}_{1} \mathcal{R}_{2}} & \frac{2 L\left(\mathcal{R}_{2}-L\right)}{\mathcal{R}_{2}} \\
-\frac{2\left(-2 L+\mathcal{R}_{1}+\mathcal{R}_{2}\right)}{\mathcal{R}_{1} \mathcal{R}_{2}} & 1-\frac{2 L}{\mathcal{R}_{2}}
\end{array}\right) .
$$

We can now obtain an explicit expression for the $g$ factor of the Fabry-Perot cavity, thus parametrising its geometrical stability, as

$$
g \equiv g_{1} g_{2}=\left(1-\frac{L}{\mathcal{R}_{1}}\right)\left(1-\frac{L}{\mathcal{R}_{2}}\right),
$$

where we have defined the $g$ factors of the mirrors $g_{i} \equiv 1-L / \mathcal{R}_{i}$. For a more in-depth discussion of Fabry-Perot resonator stability as parametrised by $g_{1}$ and $g_{2}$, see Chapter 9 .

It is useful to derive an expression for the radius of the beam at the position of the mirrors, sometimes referred to as the beam spot. The size of the beam at the mirrors is closely related to the geometrical stability of the cavity, and it is a key parameter in any experiment (e.g., to determine the thermal noise floor of the mirror, or to determine the right mirror size for a particular application). Using Equations 2.30 and 2.34 and expressing the results in terms of the mirror $g$ factors yields

$$
w_{1}^{2} \equiv \frac{\lambda L}{\pi} \sqrt{\frac{g_{2}}{g_{1}\left(1-g_{1} g_{2}\right)}} \quad \text { and } \quad w_{2}^{2} \equiv \frac{\lambda L}{\pi} \sqrt{\frac{g_{1}}{g_{2}\left(1-g_{1} g_{2}\right)}},
$$

where $w_{1}$ and $w_{2}$ are the beam spots at the input and end mirrors respectively. The beam waist can also be cast in terms of $g_{1}$ and $g_{2}$ as

$$
w_{0}^{2} \equiv \frac{\lambda L}{\pi} \sqrt{\frac{g_{1} g_{2}\left(1-g_{1} g_{2}\right)}{\left(g_{1}+g_{2}-2 g_{1} g_{2}\right)^{2}}} .
$$

The total phase shift acquired by the beam after a resonator round-trip, known as the round-trip Gouy phase shift, is given by

$$
\zeta \equiv \operatorname{sgn}\left[M_{12}(z)\right] \arccos (m) .
$$

Note that $m$ and $\zeta$ are functions of half the trace of the round-trip transfer matrix, and therefore are invariant under some transformations, such as the position $z$ in the optical axis. For a Fabry-Perot cavity it is given by

$$
\zeta=2 \arccos \left( \pm \sqrt{g_{1} g_{2}}\right)
$$

where the sign \pm is determined by the sign of $g_{1}$. Higher order modes accumulate an additional phase shift with respect to the fundamental mode,

$$
\Delta \phi_{n m} \equiv(n+m) \zeta
$$

where $n$ and $m$ are the mode numbers. Thus, the offset in resonance frequency of the $n m$-th order mode with respect to the fundamental mode is given by

$$
\Delta \nu_{n m}=\frac{\Delta \phi_{n m}}{2 \pi} \Delta \nu_{\mathrm{FSR}}=(n+m) \frac{\zeta}{2 \pi} \Delta \nu_{\mathrm{FSR}}
$$




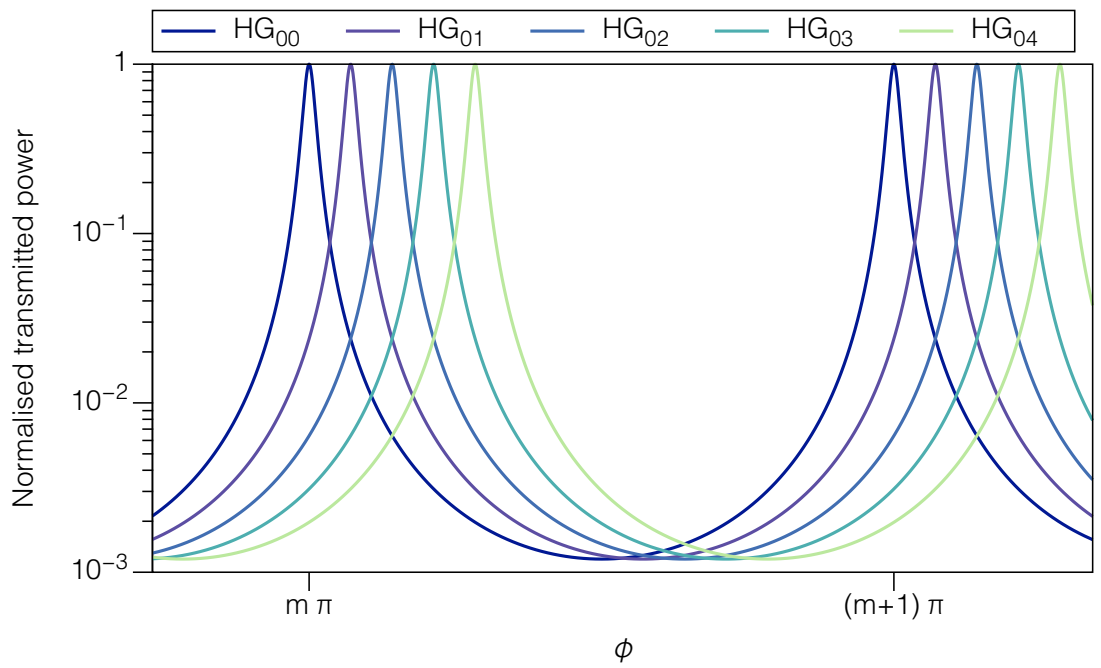

Figure 2.5: Transmission intensity response function as a function of the phase thickness of a Fabry-Perot cavity showing the resonance peaks of the fundamental mode $\mathrm{HG}_{00}$ and the first four higher order transverse modes $\mathrm{HG}_{01-04}$ (in this example the finesse of the cavity is $\sim 45$ ).

Therefore, for a Fabry-Perot cavity the separation in resonance frequency between successive transverse modes, known as the transverse mode separation frequency, is given by

$$
\Delta \nu \equiv \frac{\zeta}{2 \pi} \Delta \nu_{\mathrm{FSR}}=\frac{1}{\pi} \arccos \left[ \pm \sqrt{\left(1-\frac{L}{\mathcal{R}_{1}}\right)\left(1-\frac{L}{\mathcal{R}_{2}}\right)}\right] \Delta \nu_{\mathrm{FSR}}
$$

This is an important quantity with profound experimental implications, and will be discussed further in Section 2.3.2 and Chapter 9. The extra phase accumulated by transverse modes in a resonator round-trip means that the cavity can discriminate between modes of different $n+m$ order. That is, the cavity can act as a spatial filter of the input beam due to the frequency dependency of its resonance condition (Figure 2.5). If a certain mode or a set of modes becomes co-resonant with the fundamental mode - an effect known as higher order mode degeneracy - the cavity can have the effect of resonantly enhancing the power on those modes (thus possibly enhancing a beam distortion). Cavities that are near the boundary of geometrical instability will do this for all higher order modes (near $g=1$ ) or for all even order modes (near $g=0$ ). 


\subsection{The art of making ultra-stable optical cavities}

\subsubsection{Thermal noise limit}

When a laser is locked to the resonance of a Fabry-Perot cavity, the length stability of the cavity is transferred to the frequency stability of the laser, obeying Equation 2.12,

$$
\frac{\Delta \nu}{\nu}=-\frac{\Delta L}{L},
$$

where $\frac{\Delta \nu}{\nu}$ is the relative stability of the laser frequency and $\frac{\Delta L}{L}$ is the relative stability of the cavity length. Our ability to produce an ultra-stable laser is thus determined by our ability to keep the distance between the cavity mirrors (as well as their parallelism) as stable as possible. Hence, great effort is directed towards isolating the cavity from external perturbations, such as pressure and temperature fluctuations, and vibrations, in order to reduce cavity length fluctuations in experiments aiming to realise an ultra-stable frequency reference.

In today's most advanced optical cavities, the ultimate limit imposed on the cavity's length stability is set by fundamental thermal processes. The next generation of ultra-stable cavities is expected to reach a relative length stability $\frac{\Delta L}{L}<10^{-16}$. The most common cavity configuration consists of two mirrors with high-reflectivity dielectric coatings optically contacted to the ends of a spacer made with ultra-low expansion ${ }^{2}$ glass, such as $\mathrm{ULE}^{3}$. Thermal fluctuations of the mirrors and spacer result in random modifications of the optical path length in the cavity, and hence unavoidable thermal noise. The fluctuation dissipation theorem [66] relates the spectrum of random motion (i.e., Brownian motion of the atoms of each component) to thermodynamical loss in a system, which depends on material properties such as the mechanical loss angle and also on the size of the optical mode on the mirrors. For a Fabry-Perot resonator whose mirrors are subject to a force of amplitude $F_{0}$ and frequency $f$, the spectral density of the thermal fluctuations of the averaged cavity length $L$, as probed by the laser field, is given by

$$
S_{L}(f) \equiv \frac{2 k_{B} T}{\pi^{2} f^{2}} \frac{W_{\text {diss }}}{F_{0}^{2}}
$$

where $k_{B}$ is Boltzmann's constant, $T$ is the temperature of the system, and $W_{\text {diss }}$ is the time-averaged dissipated power in the system. For a uniformly distributed internal loss, $W_{\text {diss }}=2 \pi f U \phi$, where $U$ is the maximum elastic strain energy and $\phi$ is the loss angle of the system. The resulting relative displacement noise is converted into relative frequency noise by

$$
\frac{\sqrt{S_{\nu}(f)}}{\nu}=\frac{\sqrt{S_{L}(f)}}{L}
$$

\footnotetext{
${ }^{2}$ The coefficient of thermal expansion (CTE) describes how the length of an object changes with temperature. Typical values for materials at room temperature are: Aluminium $23 \mathrm{ppm} / \mathrm{K}$, Iron $12 \mathrm{ppm} / \mathrm{K}$, Glass $9 \mathrm{ppm} / \mathrm{K}$, ULE $0 \pm 0.02 \mathrm{ppm} / \mathrm{K}$ [65].

${ }^{3} \mathrm{ULE}$ (ultra-low-expansion glass) is a brand of glass from Corning Inc. made of $\mathrm{TiO}_{2}$-doped silica. ULE's CTE has a zero crossing at room temperature.
} 
where $S_{L}(f)$ and $S_{\nu}(f)$ are the displacement and frequency fluctuations power spectral densities respectively ${ }^{4}$. The contribution to the displacement thermal noise of the cavity mirrors has two terms: a contribution from the substrate and another from the coating. The total thermal displacement noise is

$$
S_{L}(f) \equiv S_{\mathrm{sp}}+S_{\mathrm{sb}, \mathrm{IM}}+S_{\mathrm{ct}, \mathrm{IM}}+S_{\mathrm{sb}, \mathrm{EM}}+S_{\mathrm{ct}, \mathrm{EM}}
$$

assuming that all contributions are completely uncorrelated. The subscripts sp, sb and ct refer to the spacer, substrate and coating contributions respectively, and the subscripts IM and EM distinguish between the input and end mirrors of the cavity respectively.

When a laser is locked to the cavity, the mirrors are subject to the radiation pressure force exerted by the circulating laser beam in the fundamental Gaussian mode, which has the form

$$
P(r)=\frac{2 F_{0}}{\pi w^{2}} e^{-2 r^{2} / w^{2}}
$$

where $r$ denotes the radial position within the optic referenced to the optical axis, and $w$ is the radius of the beam at the position of the mirror. The spacer is also subject to the radiation pressure force, as the mirrors are rigidly attached to its end faces.

The contributions to the cavity's thermal noise spectral density have been derived analytically as [67-69]

$$
\begin{aligned}
& S_{\mathrm{sp}}(f)=\frac{4 k_{B} T}{2 \pi f} \frac{L}{A_{\mathrm{sp}} Y_{\mathrm{ULE}}} \phi_{\mathrm{ULE}}, \\
& S_{\mathrm{sb}}(f)=\frac{4 k_{B} T}{2 \pi f} \frac{1-\sigma_{\mathrm{FS}}^{2}}{\sqrt{\pi} Y_{\mathrm{FS}} w} \phi_{\mathrm{FS}}, \\
& S_{\mathrm{ct}}(f)=\frac{4 k_{B} T}{2 \pi f} \frac{2\left(1+\sigma_{\mathrm{FS}}\right)\left(1-2 \sigma_{\mathrm{FS}}\right) d_{\mathrm{ct}}}{\pi Y_{\mathrm{FS}} w^{2}} \phi_{\mathrm{ct}},
\end{aligned}
$$

where $\phi_{\mathrm{ULE}}, \phi_{\mathrm{FS}}$ and $\phi_{\mathrm{ct}}$ are the loss angles of the ULE spacer, of face area $A_{\mathrm{sp}}$, the fused silica substrate, and the coating's dielectric material, of thickness $d_{\mathrm{ct}}$, respectively; $Y_{\mathrm{ULE}}$ and $Y_{\mathrm{FS}}$ are the Young's modulus of ULE and fused silica, and $\sigma_{\mathrm{FS}}$ is the Poisson ratio of fused silica. These parameters are listed in Table 2.1. Fused silica is chosen for the mirror substrate material, as the order of magnitude lower mechanical loss angle compared to ULE significantly reduces the thermal noise floor. The three contributions are depicted in Figure 2.6.

The spacer is modelled as a cylinder of radius $R_{\mathrm{sp}}$ and length $L$, with a central bore of radius $R_{\text {bore }}$. The radiation pressure force of the laser is taken to be uniformly distributed across the face of the spacer of area $A_{\mathrm{sp}}=\pi\left(R_{\mathrm{sp}}^{2}-R_{\mathrm{bore}}^{2}\right)$, so that $P=F_{0} / A_{\mathrm{sp}}$. The mirror is modelled as an infinite half-space, i.e., as $(x, y, z) \in \mathbb{R}^{3}: x \geq x_{0}$, where $x_{0}$ is the position in the optical axis at which the laser interacts with the mirror surface, which is an accurate approximation as long as

\footnotetext{
${ }^{4}$ The power spectral density $S_{\zeta}(f)$ of a quantity $\alpha$ has units (dimensions of $\left.\alpha\right)^{2} / \mathrm{Hz}$ and measures the spread of signal energy in the Fourier frequency spectrum. From the spectral density it is possible to calculate the rms variation of the quantity using $\alpha_{\mathrm{rms}}^{2}=\int_{0}^{\infty} S_{\alpha}(f) d f$.
} 
the size of the mirror is much larger than the size of the beam at its location [70]. Treating the mirror as a finite cylindrical mass of radius $R$ would introduce a correction of order $O(w / R)$. The coating is modelled as a thin layer of thickness $d_{\text {ct }}$ and elasticity similar to the substrate (as in the case of $\mathrm{Ta}_{2} \mathrm{O}_{5} / \mathrm{SiO}_{2}$ coatings on ULE or fused silica [71]) on the substrate's front face.

\begin{tabular}{llr}
\hline$L$ & Cavity length & $0.5 \mathrm{~m}$ \\
$A_{\mathrm{sp}}$ & Spacer face area & $\pi\left(R_{\mathrm{sp}}^{2}-R_{\mathrm{bore}}^{2}\right)$ \\
$R_{\mathrm{sp}}$ & Spacer radius & $5 \mathrm{~cm}$ \\
$R_{\mathrm{bore}}$ & Bore radius & $0.75 \mathrm{~cm}$ \\
$d_{\mathrm{ct}}$ & Coating thickness & $5.24 \mu \mathrm{m}$ \\
$\phi_{\mathrm{ULE}}$ & ULE loss angle & $1.6 \cdot 10^{-5}$ \\
$\phi_{\mathrm{FS}}$ & Fused silica loss angle & $1 \cdot 10^{-6}$ \\
$\phi_{\mathrm{ct}}$ & Dielectric coating loss angle & $4 \cdot 10^{-4}$ \\
$\sigma_{\mathrm{FS}}$ & Fused silica Poisson's ratio & 0.17 \\
$Y_{\mathrm{ULE}}$ & ULE Young's modulus & $67.7 \cdot 10^{9} \mathrm{~Pa}$ \\
$Y_{\mathrm{FS}}$ & Fused silica Young's modulus & $73.1 \cdot 10^{9} \mathrm{~Pa}$ \\
\hline
\end{tabular}

Table 2.1: Geometrical and mechanical parameters needed to compute the thermal displacement noise contributions of the spacer, the mirror substrates and the mirror coatings. The cavity length and the spacer radius listed here are preliminary design values and will be adjusted in Sections 2.3.2 and 2.5.3 respectively.

The contribution to the thermal noise of the mirror coatings scales with $1 / w^{2}$, and that of the substrate with $1 / w$. Therefore choosing configurations with large beam spots on the mirrors helps reduce the thermal noise. Of course, the cavity cannot support an arbitrarily large beam and at the same time be geometrically stable, as discussed in Section 2.2.5.

The scaling with spacer radius is very weak as the spacer face area only affects the spacer contribution to the thermal noise, which is small compared to the substrate and coating contributions. For a long cavity with a ULE spacer, fused silica substrates, and dielectric mirror coatings, it is the coatings which give the dominant contribution to the thermal noise, despite their thickness of only a few micrometres.

To characterise the cavity's length instability we use the Allan deviation $\sigma_{y}(\tau)$ of the normalised relative frequency fluctuations $y$. The spectral density of the normalised relative frequency fluctuations is (see Appendix A for more information)

$$
S_{y}(f) \equiv \frac{S_{\nu}(f)}{\nu^{2}}=\frac{S_{L}(f)}{L^{2}} .
$$

Because of the $1 / f$ noise of the three contributions, the resulting Allan deviation is constant $\sigma_{y}(\tau)=\sigma_{y}$, and is given by

$$
\sigma_{y}=\sqrt{2 \ln (2) S_{y}(f) f}
$$

For the parameters listed in Table 2.1, the thermal noise limit at $1 \mathrm{~Hz}$ is listed in Table 2.2 for different mirror radii of curvature combinations. 

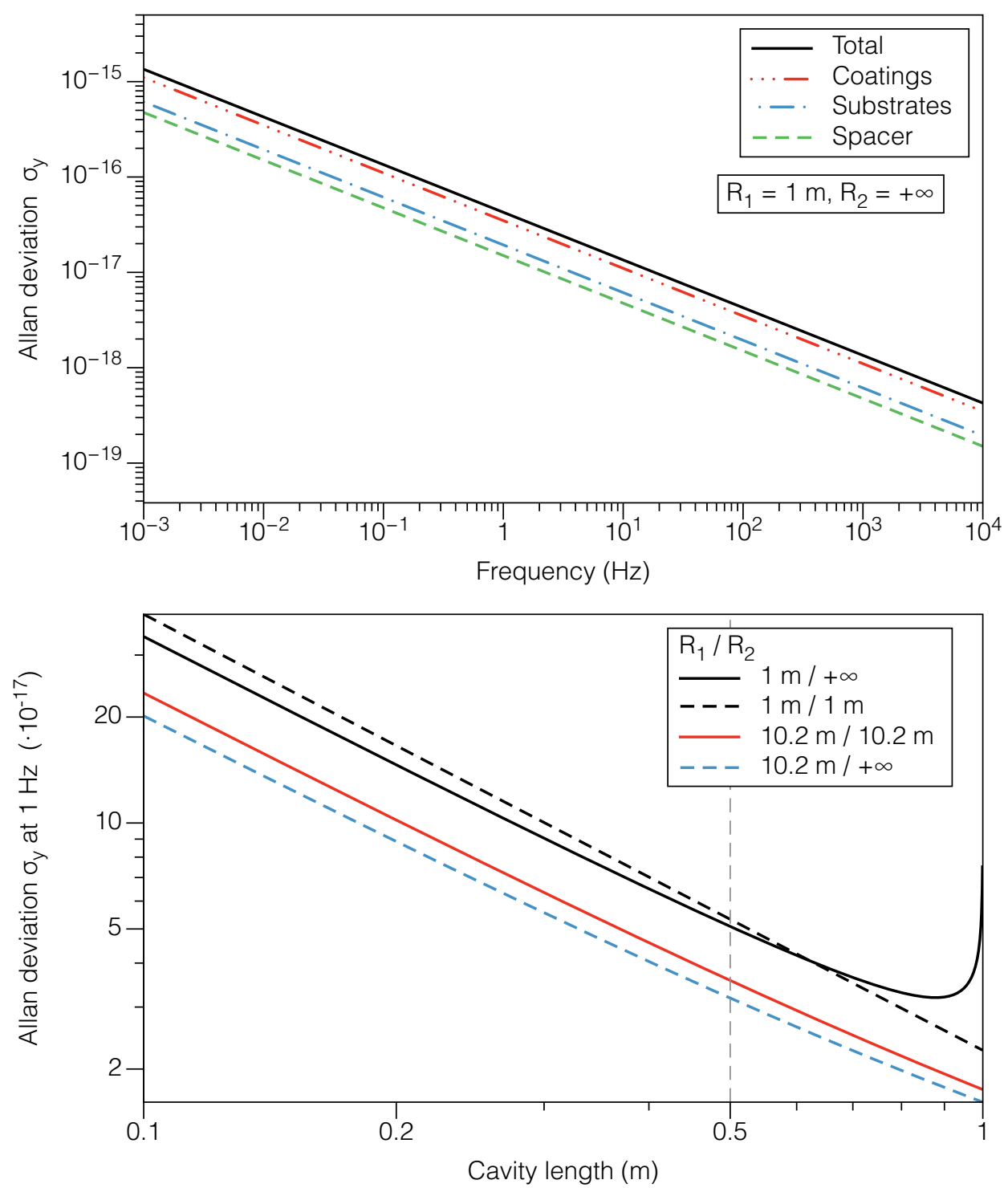

Figure 2.6: Thermal noise floor in a cavity made with a ULE spacer and fused silica mirrors with thin $(5.24 \mu \mathrm{m})$ dielectric coatings. For a $0.5 \mathrm{~m}$ spacer with a $1 \mathrm{~m} /+\infty$ mirror combination, the mirror coatings give the dominant contribution to the thermal noise floor over the contributions from the spacer and the mirror substrates (a); The noise at $1 \mathrm{~Hz}$ for different mirror combinations as a function of cavity length (b) gives an indication of the effect of the size of the optical mode on the mirrors on the resulting thermal noise floor. Note that the cavity with a $1 \mathrm{~m} /+\infty$ mirror combination (black curve in lower graph) is hemispherical at $L=1$ (i.e., it is critically stable with $g_{1}=0$ and $g_{2}=1$ ), where the Allan deviation of the thermal noise presents a singularity (note that the assumption of the thermal-noise model that the size of the beam at the mirror is much smaller than the mirror size is not valid in this case). 


\begin{tabular}{cc}
\hline $\mathcal{R}_{1} / \mathcal{R}_{2}$ & $\sigma_{y}$ at $1 \mathrm{~Hz}$ \\
\hline $1 \mathrm{~m} /+\infty$ & $5.65 \cdot 10^{-17}$ \\
$1 \mathrm{~m} / 1 \mathrm{~m}$ & $5.32 \cdot 10^{-17}$ \\
$10.2 \mathrm{~m} / 10.2 \mathrm{~m}$ & $3.57 \cdot 10^{-17}$ \\
$10.2 \mathrm{~m} /+\infty$ & $3.16 \cdot 10^{-17}$ \\
\hline
\end{tabular}

Table 2.2: Relative displacement thermal noise at $1 \mathrm{~Hz}$ for different mirror ROC configurations and a $0.5 \mathrm{~m}$ cavity length, assuming a temperature of $300 \mathrm{~K}$ and $\lambda=1064 \mathrm{~nm}$.

\subsubsection{Spacer length and mirror ROC}

The beam spots $w$ at the mirrors are determined by the cavity length $L$ and the mirror's radii of curvature (ROC) $\mathcal{R}_{1}$ and $\mathcal{R}_{2}$. A stable configuration must be chosen and thus a constraint is set on the three parameters,

$$
0 \leq\left(1-\frac{L}{\mathcal{R}_{1}}\right)\left(1-\frac{L}{\mathcal{R}_{2}}\right) \leq 1 .
$$

Additionally we have to check that the resulting cavity configuration does not suffer from higher order mode degeneracy, i.e., we have to ensure that no higher order transverse modes up to a certain order are co-resonant with the fundamental mode. The resonant frequencies of the cavity's eigenmodes are determined by the resonator's round-trip Gouy phase shift $\zeta$. The resonant frequency of the $l$-th longitudinal mode, $\mathrm{nm}$-th transverse mode is given by

$$
\nu_{l n m} \equiv l \Delta \nu_{\mathrm{FSR}}+(n+m+1) \frac{\zeta}{2 \pi} \Delta \nu_{\mathrm{FSR}},
$$

where $l \in \mathbb{Z}, n, m \in \mathbb{N}$, and $\Delta \nu_{\mathrm{FSR}}$ is the cavity's free spectral range. The transverse mode spacing is therefore $(\zeta / 2 \pi) \Delta \nu_{\mathrm{FSR}}$. For the Fabry-Perot cavity the round-trip Gouy phase is given by (see Section 2.2.5)

$$
\zeta=2 \arccos \left( \pm \sqrt{g_{1} g_{2}}\right),
$$

where $g_{i}=1-L / \mathcal{R}_{i}$, and the sign \pm is given by the sign of $g_{1}$. Since $\zeta \in[0,2 \pi]$, we can write $\zeta=2 \pi / p$ with $p \in[1,+\infty)$. The number $p$ rounded to the lower integer is the number of transverse modes from one longitudinal resonance that lie within one free spectral range. There will be some degree of higher order mode degeneracy if there are transverse modes which satisfy that $\nu_{q n m}$ is an integer multiple of the free spectral range, i.e., if $(m+n+1) / p \rightarrow k$ with $k \in \mathbb{Z}$. In addition, if $p \in \mathbb{N}_{>0}$, all modes with $m+n+1=p, 2 p, 3 p \ldots$ would be co-resonant with the fundamental mode. For example, for a cavity of length $0.5 \mathrm{~m}$ and mirror ROCs of $1 \mathrm{~m}$ the free spectral range is $300 \mathrm{MHz}$ and the mode separation frequency is $100 \mathrm{MHz}$, exactly $(1 / 3) \Delta \nu_{\mathrm{FSR}}{ }^{5}$, therefore all modes with $m+n+1=3,6,9 \ldots$ will be degenerate with the fundamental mode.

Initially, we choose a $\mathcal{R}_{1}=1 \mathrm{~m}, \mathcal{R}_{2}=+\infty$ configuration that is affordable and offers good ROC tolerances, making the cavity hemispherical. Also of interest is

\footnotetext{
${ }^{5}$ For $L=0.5 \mathrm{~m}$ and $\mathcal{R}_{1,2}=1 \mathrm{~m}$, we have $\zeta / 2 \pi=\arccos (0.5) / \pi=1 / 3$.
} 

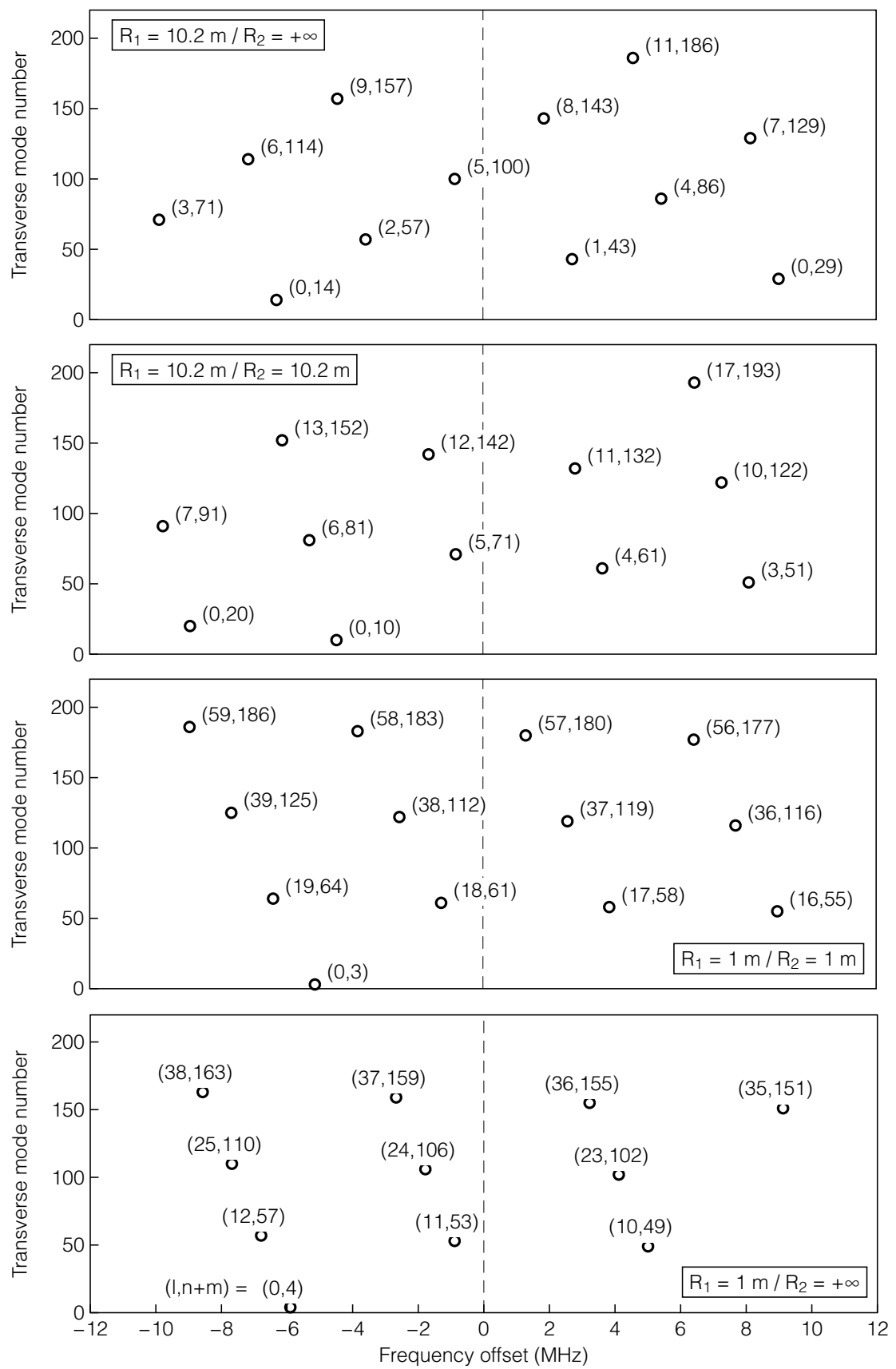

Figure 2.7: Higher order mode resonance frequency offsets for a cavity with $L=$ $0.4850125 \mathrm{~m}$ and the four mirror combinations under consideration. The labels indicate (longitudinal resonance, transverse mode number). 

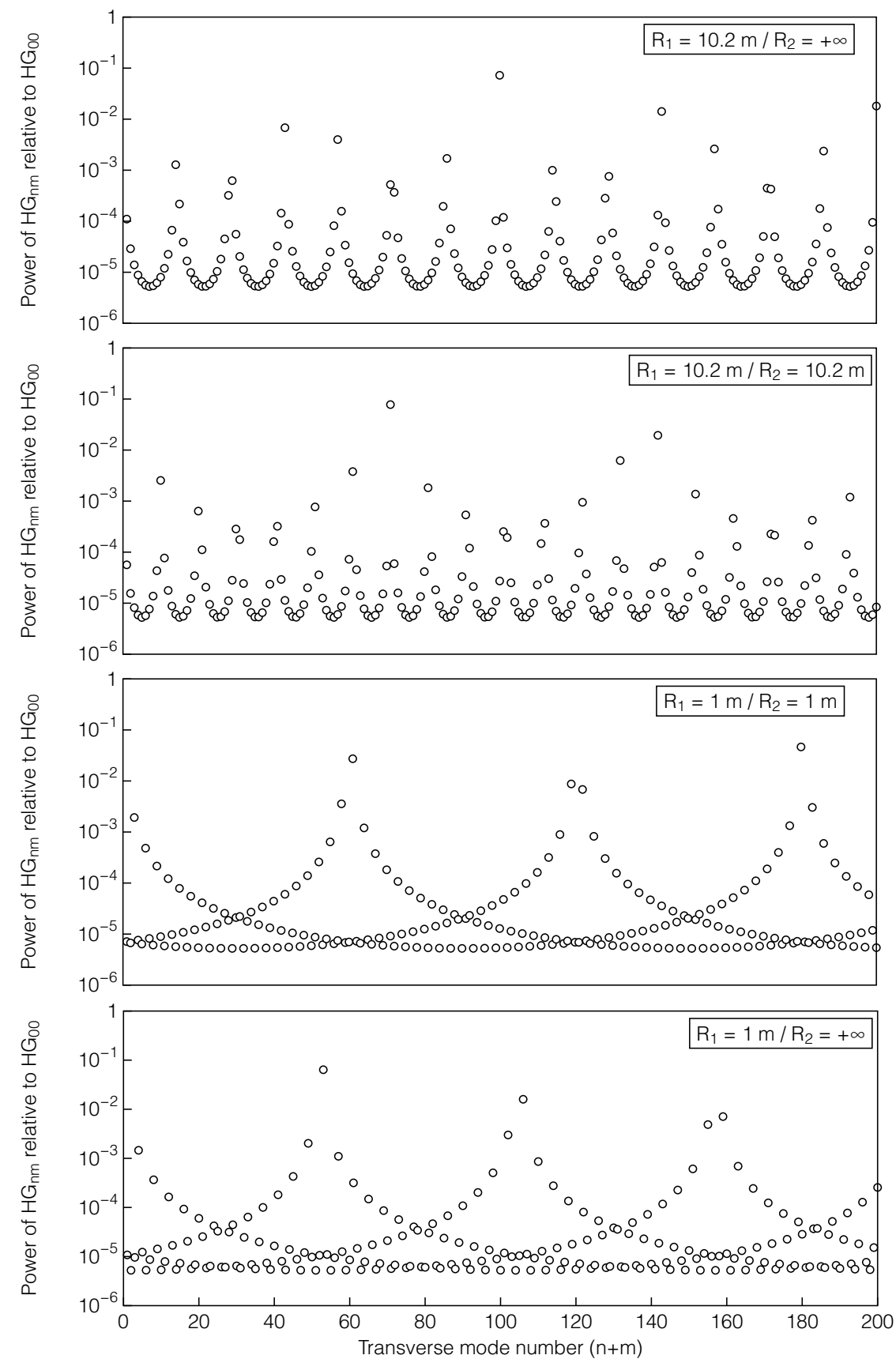

Figure 2.8: Transmitted power of the first 200 Hermite-Gauss modes relative to the power of the fundamental mode, for a cavity with $L=0.4850125 \mathrm{~m}$ and the four mirror combinations under consideration, assuming a finesse of $\mathcal{F}=1.5 \cdot 10^{5}$. 
a $\mathcal{R}_{1,2}=10.2 \mathrm{~m}$ symmetric configuration, which we may opt for in the future, as well as the $\mathcal{R}_{1}=1 \mathrm{~m}, \mathcal{R}_{2}=1 \mathrm{~m}$ and $\mathcal{R}_{1}=10.2 \mathrm{~m}, \mathcal{R}_{2}=+\infty$ combinations. The displacement thermal noise for each of the four configurations being considered is depicted in Figure 2.6.

The length of the cavity is then fine tuned to avoid coupling to HOMs up to a certain order (typically considering HOMs up to order 15 is sufficient). Figure 2.8 shows the modes up to $n+m=200$ that are within $\pm 10 \mathrm{MHz}$ of resonance for the different mirror combinations and a spacer length ${ }^{6}$ of $0.485 \mathrm{~m}$. For the chosen $1 \mathrm{~m} /+\infty$ mirrors, the modes closest to resonance are the $n+m=53$ modes, with a $0.89 \mathrm{MHz}$ offset, while the $n+m=4$ modes have a $5.9 \mathrm{MHz}$ offset.

Amairi et al [69] estimate the effect on the error signal in the case of the $n m=01$ mode lying inside the modulation frequency. They consider the worst case scenario of $10 \%$ fluctuations in the $10 \%$ coupling to the 01 mode (lying at half the modulation frequency away from the 00 mode) for a $L=0.1, \mathcal{F}=10^{5}$ cavity, and find a frequency fluctuation of the order of $75 \mathrm{mHz}\left(\approx 3 \cdot 10^{-16}\right.$ relative instability). For a longer cavity $(L \approx 0.5 \mathrm{~m})$ with a higher finesse $\left(\mathcal{F} \approx 2.5 \cdot 10^{5}\right)$ the effect should be over an order of magnitude less severe due to the narrower cavity linewidth and consequently sharper error signal.

\subsection{Force-insensitive optical cavity}

\subsubsection{Spacer geometry and material}

In order to achieve thermal-noise-limited performance with a fractional length instability below $10^{-16}$, we set out to design a long optical cavity with a spacer length of approximately $0.5 \mathrm{~m}$. With this length and the chosen spacer and mirror parameters, the cavity's thermal noise floor at $1 \mathrm{~Hz}$ is approximately $6 \cdot 10^{-17}$. The long cavity length means that the cavity will be very sensitive to external perturbations. Previous long reference cavities at NPL were of the 'mushroom' spacer kind $[72,73]$ or the rectangular spacer kind [69] (with spacer lengths ranging from 10 to $28 \mathrm{~cm}$ ). Shorter cavities for transportable applications have been designed using cubic spacers [74]. For the next generation of long reference cavities for ultra-high performance applications, we employ a cylindrical spacer with four small rectangular cutouts for support (Figure 2.9). The cylindrical shape offers enhanced symmetry for non-axial accelerations. The rectangular cutouts are made in order to support the structure at four points using $3.9 \mathrm{~mm}$ diameter Viton ${ }^{7}$ spheres or semi-spheres; the contact surface between the Viton and the spacer is a circle of $\sim 2 \mathrm{~mm}$ diameter. This system can be made effectively insensitive to accelerations in all directions, as those detailed in $[69,72,74]$. The design has to ensure that the optical path length remains unchanged when a force is applied uniformly to the structure. Several geometrical parameters are investigated and

\footnotetext{
${ }^{6}$ Note that this spacer length yields a total cavity length (optical path length) of $L=0.485012$ $\mathrm{m}$. This is due to the extra axial length introduced by a concave mirror with radius of curvature $\mathcal{R}$, and a concave region of radius $r$, given by $\mathcal{R}-\sqrt{\left(\mathcal{R}^{2}-r^{2}\right)}$. For example, for $\mathcal{R}=1 \mathrm{~m}$ and $r=5 \mathrm{~mm}$, the extra axial length is $12.5 \mu \mathrm{m}$.

${ }^{7}$ Viton is the most common brand of fluoroelastomers. We use Fluoroelastomer 70 Shore A.
} 


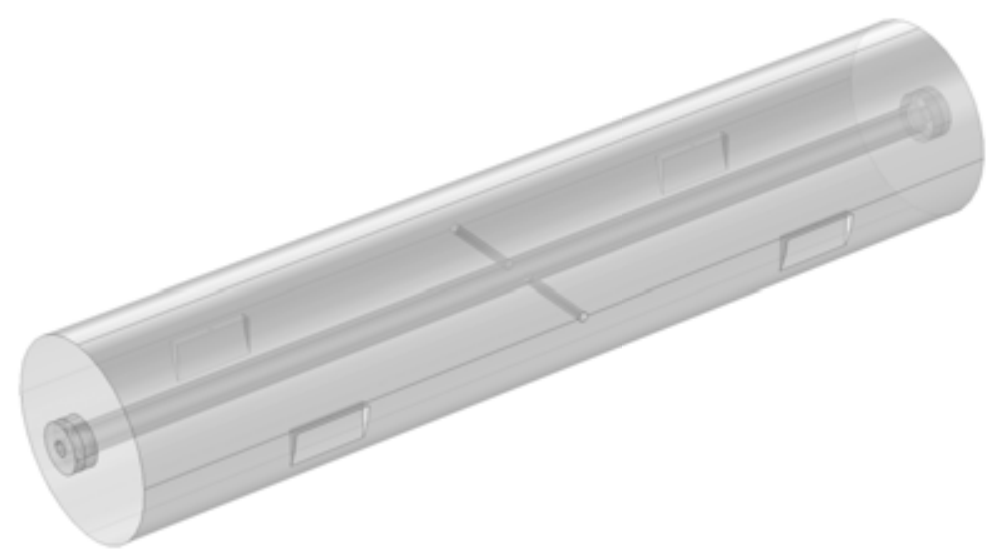

Figure 2.9: Full 3D view of the cavity as modelled in COMSOL. The cavity features a cylindrical $0.485 \mathrm{~m}$ ULE spacer with a $15 \mathrm{~mm}$ bore to which fused silica mirrors with high reflectivity coatings are optically contacted. A ULE annulus is optically contacted to the back of the mirror to compensate the offset in CTE introduced by the mirror substrates. Four cutouts are made to the spacer cylinder in order to support the structure at four points.

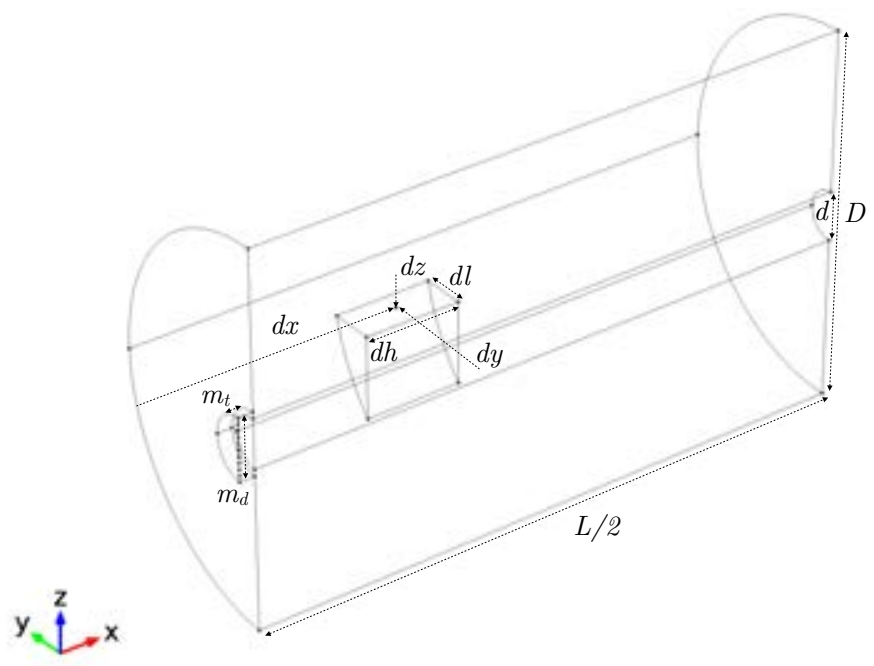

Figure 2.10: 3D view of one quarter of the cavity as modelled for vertical accelerations, depicting the basic geometrical design parameters. The planes of symmetry which define this "slice" of the whole geometry are the middle $x y$ plane and the middle $y z$ plane. The support positions $d x, d y$ and $d z$ are referenced with respect to the spacer's face, the middle $x z$ plane, and the middle $x y$ plane respectively. 
optimised for this purpose, and a cavity design virtually insensitive to external forces is achieved.

Doing these investigations requires developing an structural mechanics model of the cavity. To this end we develop a finite element model of the cavity using COMSOL Multiphysics [75] (see Chapter 10 for more details on finite element analysis). A static stress-strain model is used, because the frequency of accelerations that make a significant contribution to the frequency noise are less than $10 \mathrm{~Hz}$ and therefore, to a good approximation, can be considered to be at dc relative to the first structural resonance of the cavity at approximately $10 \mathrm{kHz}$. The following analysis is therefore restricted to the quasi-static response of the cavity to an applied force. The model solves for the displacement field of the structure under external loads,

$$
\boldsymbol{\psi}(x, y, z) \equiv\left(\begin{array}{l}
u(x, y, z) \\
v(x, y, z) \\
w(x, y, z)
\end{array}\right)
$$

The cavity's length fluctuation $\Delta L$ can then be calculated by measuring the longitudinal displacements of the input and end mirrors, $u_{\mathrm{IM}}$ and $u_{\mathrm{EM}}$ respectively, and subtracting them,

$$
\Delta L \equiv u_{\mathrm{EM}}-u_{\mathrm{IM}} .
$$

Note that $\Delta L$ is positive if the cavity expands (e.g., if $u_{\mathrm{IM}}<0$ and $u_{\mathrm{EM}}>0$, or in general $u_{\mathrm{EM}}>u_{\mathrm{IM}}$ ) and negative if the cavity contracts (e.g., if $u_{\mathrm{IM}}>0$ and $u_{\mathrm{IM}}<0$, or in general $\left.u_{\mathrm{IM}}>u_{\mathrm{EM}}\right)$.

We consider constant accelerations in all three directions ( $z$, vertical; $x$, longitudinal (optical); and $y$, transverse). For vertical accelerations we only need to model a $1 / 4$ section of the cavity, exploiting the symmetry of the geometry in order to reduce computation time (Figure 2.10). For longitudinal and transverse accelerations, $1 / 2$ models are sufficient (with $z y$ and $z x$ middle symmetry planes respectively). The cavity is constrained not to move normal to the surfaces defined by the symmetry boundary conditions, thus only symmetric solutions are obtained. The $2 \mathrm{~mm}$ support points located at the rectangular cutouts are constrained not to move in the vertical direction. The relevant material properties for the cavity are listed in Table 2.3.

The displacement of the mirrors and their tilting angle are evaluated from a set of points defined along vertical and horizontal cut lines in the front of the mirror (the surface contacted to the spacer). Tilting angle is important because it has a second order effect on the cavity length change. Figure 2.10 depicts the geometrical parameters of the cavity.

\begin{tabular}{ccc}
\hline & ULE & Fused Silica \\
\hline Poisson's ratio & 0.17 & 0.17 \\
Young's modulus & $67.7 \mathrm{GPa}$ & $73.1 \mathrm{GPa}$ \\
Density & $2210 \mathrm{~kg} / \mathrm{m}^{3}$ & $2203 \mathrm{~kg} / \mathrm{m}^{3}$ \\
\hline
\end{tabular}

Table 2.3: Mechanical properties of ULE (ultra-low expansion $\mathrm{TiO}_{2}$-doped silica glass) and fused silica at room temperature. 
(a)

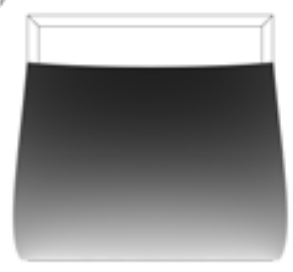

(b)

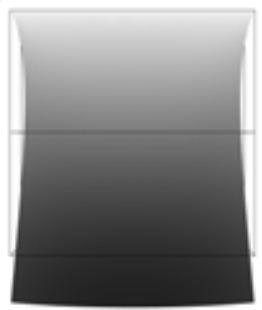

(c)

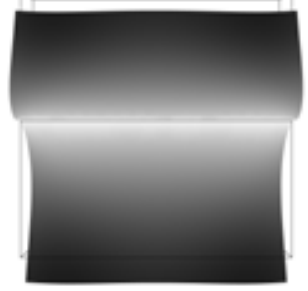

Figure 2.11: Deformation of a cube subject to a vertical force for different positions of a vertical supporting plane: (a) supported at the bottom; (b) supported at the top; (c) supported at the middle. The original shape of the cube is indicated by the frame lines. Shade indicates normalised displacement in either of the two directions transverse to the applied acceleration, from 0 (white) to 1 (black).

\subsubsection{Spacer structural mechanics}

The behaviour of the cavity structure under the action of external forces is explained through the combination of two effects: the Poisson effect and mirror tilt.

The Poisson effect consists on the expansion or contraction of the structure under load in the two directions orthogonal to the direction of the applied force. For example, when the spacer is compressed along $z$ due to a vertical force, it expands along $x$ and $y$, therefore directly modifying the cavity length in the optical axis $x$. The Poisson's ratio measures the ratio of transverse expansion to expansion in the direction of the applied acceleration (its value is 0.17 in all directions for both ULE and fused silica). Under a vertical acceleration the spacer contracts vertically if it is resting on its base (expanding on $x$ and $y$ ), or expands if it is being held at the top (contracting on $x$ and $y$ ). If the spacer is supported at an arbitrary $z$ plane, it will contract above said plane ( $x$ and $y$ expansion) and expand below it ( $x$ and $y$ contraction).

See Figure 2.11 for an example Poisson deformation of a cube being held at a plane normal to the direction of the applied acceleration. There is a certain position of the supporting plane along $z$ for which the expansion and contraction in the $x$ axis cancel out, resulting in an overall zero displacement of the cube in that axis. The cylindrical spacer, being supported at four points lying on a plane normal to the vertical, will behave similarly to the cube. The position $d z$ of this plane relative to the middle $x y$ plane can be adjusted to cancel the relative displacement of the mirror in the optical axis due to this effect.

The structure also bends about the supporting points, as depicted in Figure 2.12, which causes a tilt of the end faces of the spacer and hence of the mirrors. Depending on the longitudinal positions of the supports, the mirrors will tilt outwards or inwards, and depending on their radii of curvature this will cause an overall expansion or contraction of the optical path length. There exists a certain position of the supports along the optical axis for which the length change due to mirror tilt cancels out. This effect is well-known in beam physics [76], and the positions of the supports at 
(a)

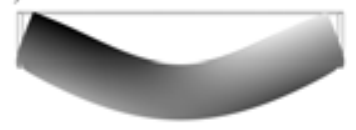

(b)

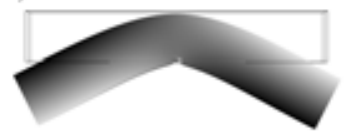

(c)

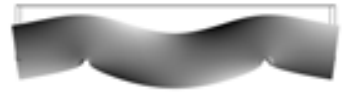

Figure 2.12: Deformation of a rectangular spacer under a vertical force for different axial positions of the supports: (a) supported at the ends; (b) supported at the middle; (c) supported at the Airy points where tilt-induced length changes cancel. The original shape of the block is indicated by the frame lines. Shade indicates normalised longitudinal displacement from 0 (white) to 1 (black).

which the effect cancels are known as the Airy points [77].

The induced mirror tilt causes a second order cavity length change [64]. A tilt $\theta_{1}$ of the input mirror and $\theta_{2}$ of the end mirror with respect to the vertical causes the centre of the optical axis to shift by $\Delta z_{1}$ and $\Delta z_{2}$ with respect to the input and end mirrors respectively, with the optical axis shifting by an angle $\Delta \theta=\left(\Delta z_{2}-\Delta z_{1}\right) / L$ overall. The modified cavity length will be

$$
L^{\prime}=L+\Delta L_{\mathrm{tilt}}=\frac{\Delta z_{2}-\Delta z_{1}}{\sin (\Delta \theta)}
$$

with

$$
\begin{aligned}
\Delta z_{1} & =\frac{g_{2}}{1-g_{1} g_{2}} L \theta_{1}+\frac{1}{1-g_{1} g_{2}} L \theta_{2}, \\
\Delta z_{2} & =\frac{1}{1-g_{1} g_{2}} L \theta_{1}+\frac{g_{1}}{1-g_{1} g_{2}} L \theta_{2}, \\
\Delta \theta & =\frac{\Delta z_{2}-\Delta z_{1}}{L}=\frac{\left(1-g_{2}\right) \theta_{1}-\left(1-g_{1}\right) \theta_{2}}{1-g_{1} g_{2}} .
\end{aligned}
$$

Note that for fixed $g_{1,2}$ the sensitivity to mirror tilt scales with $L$. Also note that if one of the mirrors is plane $\left(g_{1}=1\right.$ or $\left.g_{2}=1\right)$, the length fluctuation becomes sensitive only to the tilt of that mirror. We can get an idea of the order of magnitude of the length fluctuations due to tilt by using a small angle approximation on $\sin (\Delta \theta)$, which yields

$$
\Delta L_{\mathrm{tilt}} \simeq \frac{L \theta^{2}\left(g_{2}-g_{1}\right)^{2}}{2\left(1-g_{1} g_{2}\right)^{2}} .
$$

As $\Delta L_{\mathrm{tilt}} \propto \theta^{2}$, and $\theta$ is typically in the order of $10^{-9} \mathrm{rad}$, this effect in itself is of no concern as the resulting length fluctuation lies below the cavity's thermal noise floor. However, the problem resides in our ability to perfectly align the mirrors within the faces of the spacer to begin with. If one of the mirrors is offset from the centre of the spacer face by $\Delta r$, then a tilt $\theta$ of one of the mirrors will cause a first order length fluctuation $\Delta L_{\text {tilt }} \sim \theta \Delta r$, which is a more severe effect that will be investigated further. In order to make the cavity insensitive to accelerations 
at the required levels, we need a configuration which results in nearly zero net displacement of the mirrors taking into account possible offsets of the mirror with respect to the centre of the spacer face.

\subsubsection{Model for the force-induced length fluctuations}

We can express the cavity's relative length fluctuations due to an external force as the sum of two terms: one accounting for the deformation due to the Poisson effect, and another due to mirror tilt,

$$
\frac{\Delta L}{L} \equiv\left(\frac{\Delta L}{L}\right)_{\text {Poisson }}+\left(\frac{\Delta L}{L}\right)_{\text {Tilt }} \equiv \mathcal{P}+\mathcal{T} .
$$

where the terms $\mathcal{P}$ and $\mathcal{T}$ represent the contributions of the two effects to the overall relative length change of the cavity when it is subject to a small acceleration. This description is a useful picture for the treatment that follows. It should be noted, however, that ultimately cross terms must exist. For example, the displacement induced by the Poisson effect will also produce a small tilt of the mirrors.

For small accelerations the induced deformations are linear and proportional to the magnitude of the acceleration. Let $\left(a_{x}, a_{y}, a_{z}\right)$ be the acceleration vector applied to the cavity. The two contributions to the cavity's length change are, to first order [78]

$$
\begin{aligned}
& \mathcal{P} \approx a_{x} k_{x}+a_{y} k_{y}+a_{z} k_{z} \\
& \mathcal{T} \approx a_{x} \kappa_{x} \Delta r_{z}+a_{y} \kappa_{y} \Delta r_{y}+a_{z} \kappa_{z} \Delta r_{z}
\end{aligned}
$$

where $k_{x, y, z}$ and $\kappa_{x, y, z}$ are the acceleration sensitivity and tilt sensitivity coefficients respectively, and $\Delta r_{j}$ is the small displacement of the mirror from the centre of the spacer face in the $j$ direction (note that mirror tilt only contributes to the overall length change if $\Delta r_{y}>0$ or $\left.\Delta r_{z}>0\right)$.

Let $d x$ and $d z$ be the longitudinal and vertical position of the supports respectively (see Figure 2.10). Through the intuition presented in the previous section we know that, under a vertical acceleration, adjusting $d z$ helps compensate for the Poisson effect contribution, while adjusting $d x$ helps compensate for mirror tilt. We confirm this via simulations in which we vary $d x$ and $d z$ and measure the distance between the displaced mirror centres and the mirror tilt angles. We also see that under longitudinal and transverse accelerations, the Poisson effect has a negligible contribution to the overall length change $\left(k_{x, y} \approx 0\right)$. As for the tilt-induced length changes, we see that for vertical and transverse accelerations they can be compensated by adjusting $d x$, while for longitudinal accelerations they can be compensated by adjusting $d z$. We treat each coefficient as approximately dependent only on its primary compensating parameter (see Table 2.4). This is not an unreasonable assumption as far as we can tell from all the support configurations we simulated.

These coefficients determine the shape of the relative length fluctuations $\frac{\Delta L}{L}$ in the $(d x, d z)$ parameter space. As we will soon see, there is a region in this space where $\Delta L$ has a zero crossing. In that region we venture to write $k_{x, y, z} \approx 0$ and $\kappa_{x, y, z} \approx 0$, as the cavity becomes virtually insensitive to forces in all directions, even for offset mirrors. Moreover we are interested in the gradients of the coefficients near 


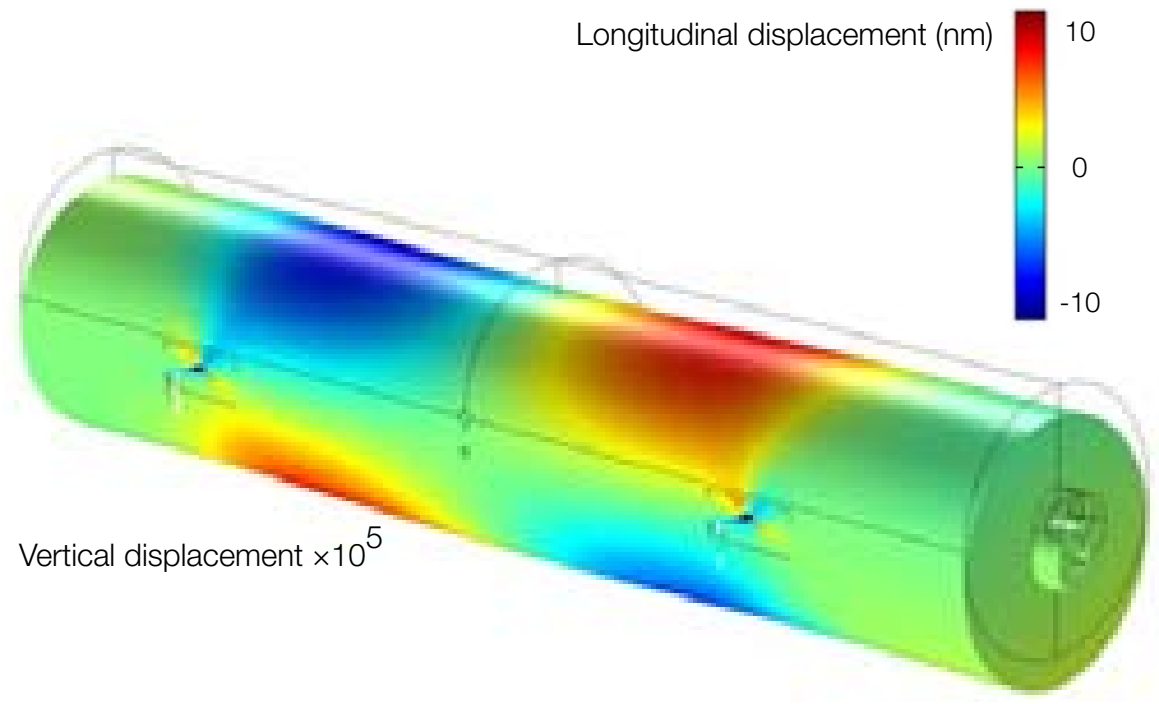

Figure 2.13: Cavity structure deformed under a vertical acceleration of $1 \mathrm{~g}$ (vertical displacement scaled by $10^{5}$ ). Colour indicates longitudinal displacement.

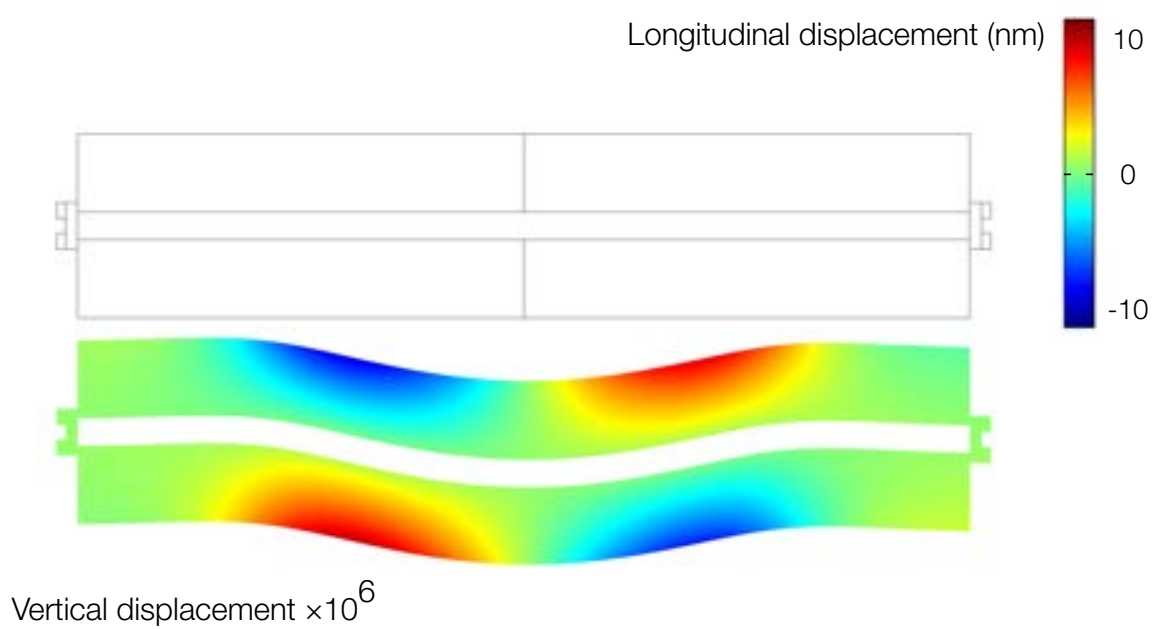

Figure 2.14: Section view of the cavity's middle $x z$ plane showing the deformation due to a vertical acceleration of $1 \mathrm{~g}$ (vertical displacement scaled by $10^{6}$ ). Colour indicates longitudinal displacement. Note how the longitudinal length change at the location of the mirrors is very low due to the optimised support positions. 


\begin{tabular}{ccc}
\hline & Acceleration & Tilt \\
\hline Vertical & $k_{z}(d z)$ & $\kappa_{z}(d x)$ \\
Transverse & $k_{y} \approx 0$ & $\kappa_{y}(d x)$ \\
Longitudinal & $k_{x} \approx 0$ & $\kappa_{x}(d z)$ \\
\hline
\end{tabular}

Table 2.4: Acceleration and tilt sensitivity coefficients in each direction. These coefficients measure the cavity's relative length change response to applied forces. The acceleration sensitivity coefficients measure the cavity's response to the deformation induced by the Poisson effect, which is negligible for longitudinal and transverse accelerations. The tilt terms only result in a first order length change if the mirror is offset from the centre of the spacer face. We take each coefficient as approximately dependent only on the parameter that is used to compensate the respective effect.

this region of null sensitivity, i.e., on the slopes of $k_{z}(d z), \kappa_{z}(d x), \kappa_{y}(d x)$ and $\kappa_{x}(d z)$, which provide information about the system's sensitivity to $d x$ and $d z$ deviations. The acceleration sensitivities $k_{j}$ have units $\left(\mathrm{g}^{-1}\right)$, and their gradients have units $\left(\mathrm{g}^{-1} \mathrm{~m}^{-1}\right)$. The tilt sensitivities $\kappa_{j}$ have units $\left(\mathrm{g}^{-1} \mathrm{~m}^{-1}\right)$ and their gradients have units $\left(\mathrm{g}^{-1} \mathrm{~m}^{-2}\right)$. In its final, optimised configuration, the cavity's support positions $d x$ and $d z$ will be chosen so that they give nearly zero length change and also minimise the acceleration and tilt sensitivity gradients.

Note that despite the fact that it is possible to achieve a virtually force-insensitive configuration due to the $\Delta L=0$ region in the $(d x, d z)$ space, in reality the cavity will have a residual acceleration sensitivity owing to machining tolerances of the spacer and imperfections of the mounting system. The residual acceleration sensitivity in all directions should be characterised in the final system, and its impact on the relative fluctuations of the cavity's resonances assessed given the vibration noise levels the cavity is subject to. Assuming a typical vibration noise at the cavity of $1 \mu \mathrm{g}$, the acceleration sensitivity should be below $10^{-10}$ to achieve thermal-noiselimited performance. An active vibration isolation system needs to be implemented to procure such a low-vibration-noise environment for the cavity (see Section 3.1.3).

\subsection{Optimisation of the support positions}

\subsubsection{Probing the $(d x, d z)$ parameter space}

In order to find a force insensitive configuration, the position of the supports is varied in the simulations. Table 2.5 lists the geometrical parameters of the model. See Figures 2.13 and 2.14 for example simulations under vertical forces (note that the vertical displacement has been scaled). The model solves for the 3D displacement field in the cavity, as the assembly (spacer, mirrors and annuli) is elastically deformed due to an applied force of $1 \mathrm{~g}$ in the vertical direction.

To study the response of the cavity in the $(d x, d z)$ parameter space, a 2D parameter sweep is performed in COMSOL (Figure 2.15). The black points correspond to 
simulations where the relative length fluctuations lay within the interval

$$
\left[-5 \cdot 10^{-11}, 5 \cdot 10^{-11}\right]\left(g^{-1}\right) \text {. }
$$

In this region the specific deformation of the cavity assembly results in an overall zero change of cavity length, i.e., a zero crossing of $\Delta L$. Mounting the cavity using values of the support positions $d x$ and $d z$ within this region make it insensitive to vertical forces.

The results shown in Figure 2.15 suggest that the region $\Delta L=0$ is a curve in this space. As anticipated, simulations show that for perfectly centred mirrors the tilt contribution is negligible and thus adjusting $d z$ is paramount to compensate for length change: no matter what value of $d x$ is chosen, the cavity can be made force insensitive by choosing $d z$ adequately, while the opposite is not true. Thus, the value of $d z$ has to be carefully chosen within a certain interval so that obtaining a zero crossing of $\Delta L$ is guaranteed.

Figures 2.16 and 2.17 are different representations of the same information presented in Figure 2.15, but it is helpful to visualise the behaviour of $\Delta L$ with $d x$ and $d z$ separately. Note how adjusting $d x$ for a given $d z$ does not necessarily make the cavity force insensitive (Figure 2.16), while adjusting $d z$ for a given $d x$ does it. Unfortunately the parameter $d z$ will be fixed in the final cavity design, while $d x$ can be adjusted after the fact by changing the position of the supporting Viton spheres within the spacer cutouts. Fortunately, choosing a value of $d z$ within a certain interval $\left(d z_{0}, d z_{1}\right)$ will guarantee that $\Delta L$ has a zero crossing for the design range of $d x$.

\begin{tabular}{lr}
\hline Spacer length & $0.485 \mathrm{~m}$ \\
Spacer diameter $(D) \dagger$ & $15 \mathrm{~cm}$ \\
Cutout depth $(d l) \dagger$ & $20 \mathrm{~mm}$ \\
Cutout width $(d h) \dagger$ & $40 \mathrm{~mm}$ \\
Bore diameter $(d)$ & $15 \mathrm{~mm}$ \\
Substrate thickness $\left(d_{\mathrm{sb}}\right)$ & $6.35 \mathrm{~mm}$ \\
Mirror diameter $(d)$ & $25.4 \mathrm{~mm}$ \\
Annuli outer diameter $\dagger$ & $25.4 \mathrm{~mm}$ \\
Annuli inner diameter $\dagger$ & $14 \mathrm{~mm}$ \\
Annuli thickness $\dagger$ & $5 \mathrm{~mm}$ \\
Support area diameter & $2 \mathrm{~mm}$ \\
Vent hole diameter & $5 \mathrm{~mm}$ \\
Longitudinal support position $(d x) \dagger$ & unknown \\
Vertical support position $(d z) \dagger$ & unknown \\
\hline
\end{tabular}

Table 2.5: Geometrical configuration of the cavity for initial modelling (See Figure 2.10). The ULE annuli that is optically contacted to the back surfaces of the mirrors is explained in Section 3.1.2. Some of these geometrical parameters are inherited from previous reference cavities at NPL, and others are new design choices (e.g., the initial spacer length and diameter). Parameters followed by a $\dagger$ dagger symbol will be explored and optimised in the model. The length of the spacer has already been optimised in Section 2.3.2. 


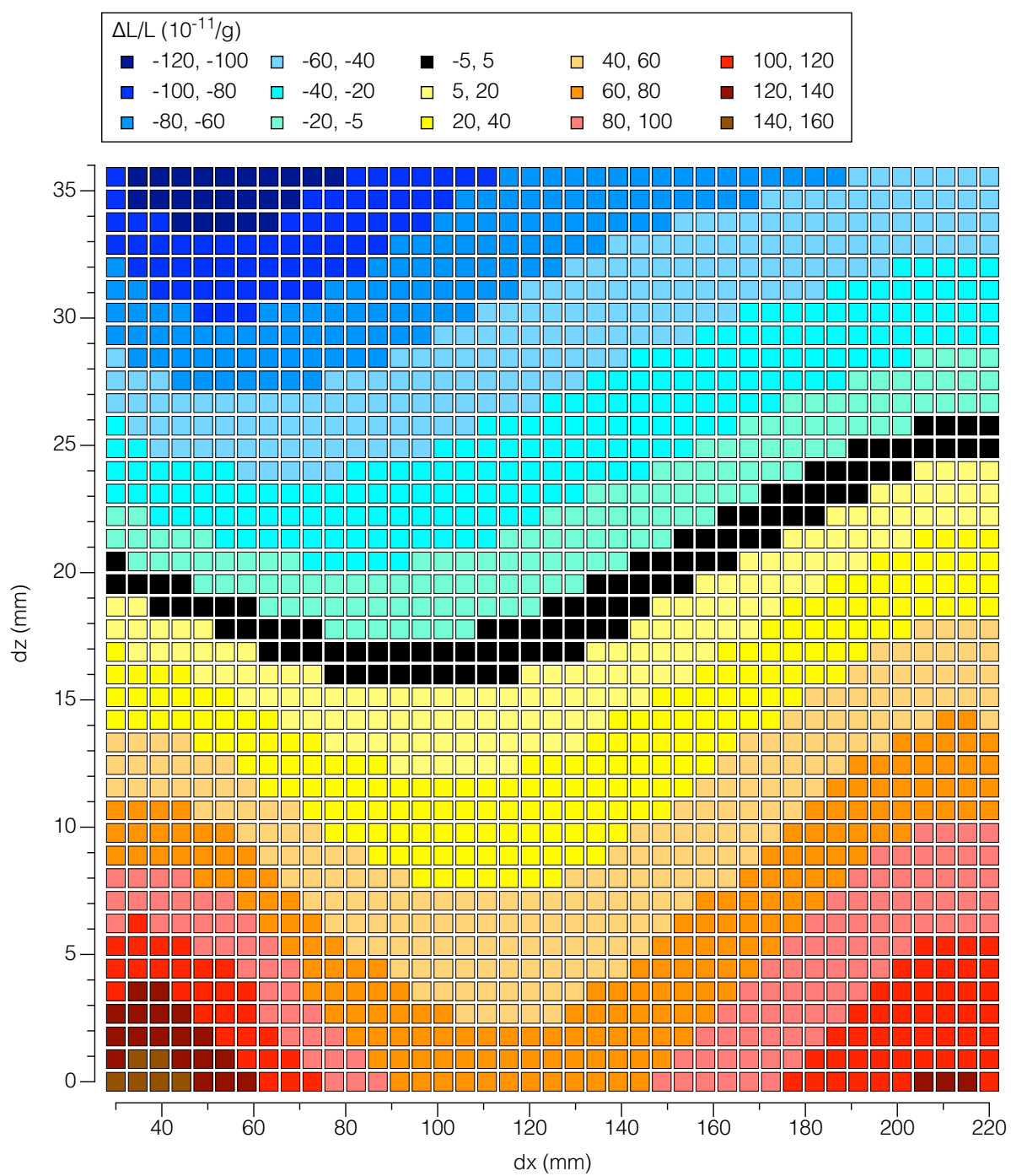

Figure 2.15: Relative length fluctuations $\frac{\Delta L}{L}$ due to a vertical force of $1 \mathrm{~g}$ (colour) in the parameter spacer of the longitudinal $d x$ and vertical $d z$ support positions. The length fluctuation $\Delta L$ has a zero crossing in the black coloured region. Below the black region (warm colours) the applied acceleration induces a positive length change (the cavity expands), whereas above the black region (cool colours) it induces a negative length change (the cavity contracts).

To estimate the effect of mirror tilt we simulate cases in which the mirrors are not centred in the faces of the spacer, but displaced by a certain vertical offset $\Delta r_{z}$, which causes a shift of the optical axis and induces a cavity length change. We do this for a fixed $d z \in\left(d z_{0}, d z_{1}\right)$ as we change $d x$ (Figure 2.18). We can see that as $d x$ approaches what we shall call the Airy point $d x_{\text {Airy }}$ the tilt effect cancels. 


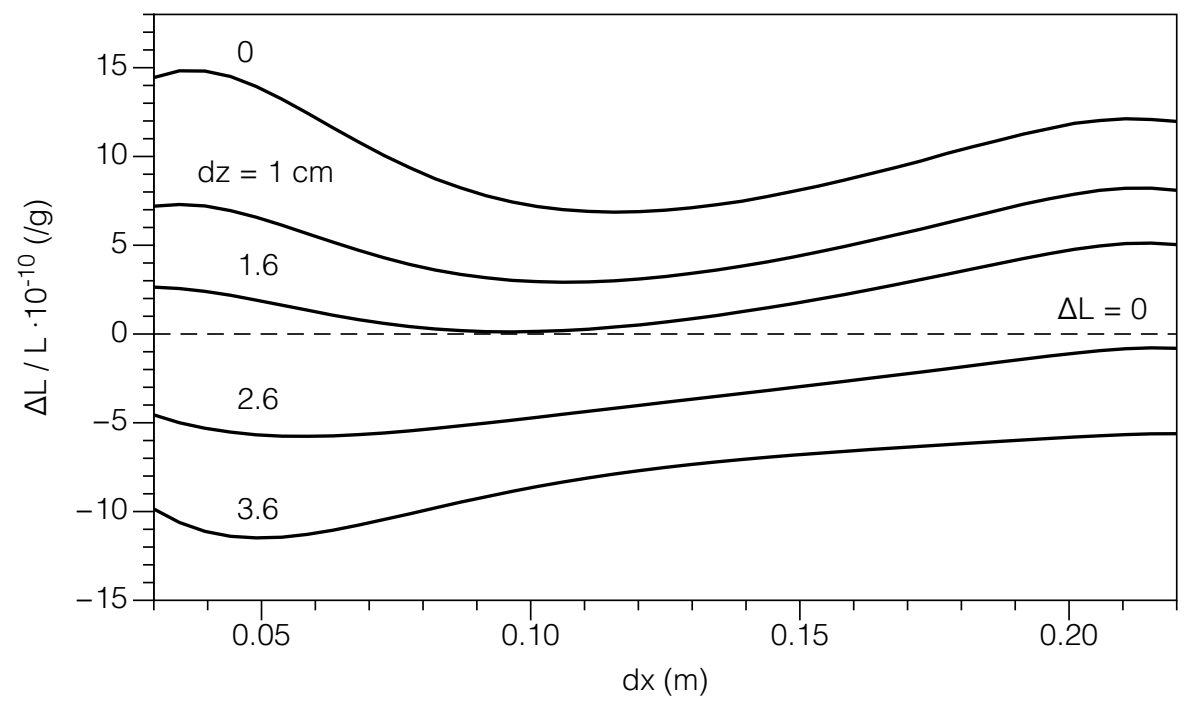

Figure 2.16: Relative length fluctuations $\frac{\Delta L}{L}$ due to a vertical force of $1 g$ as a function of the longitudinal support position $d x$ for different values of the vertical support position $d z$. Changing $d x$ for a given $d z$ does not guarantee that the cavity can be made insensitive to accelerations.

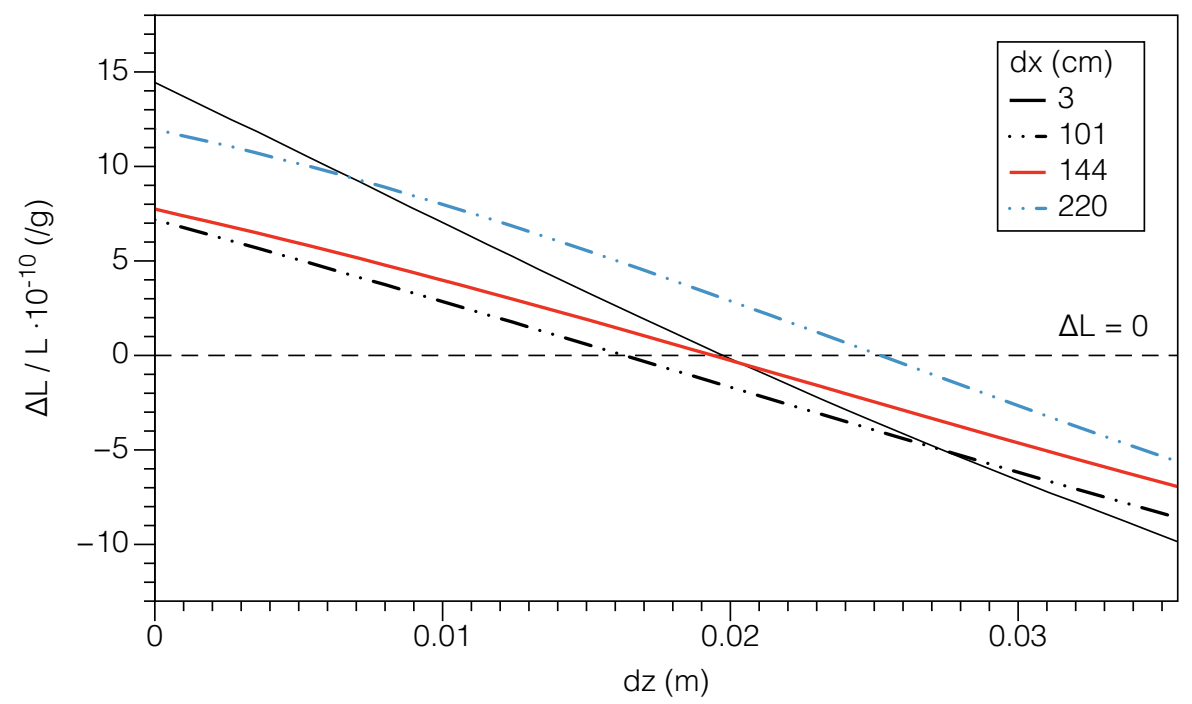

Figure 2.17: Relative length fluctuations $\frac{\Delta L}{L}$ due to a vertical force of $1 \mathrm{~g}$ as a function of the vertical support position $d z$ for different values of the longitudinal support position $d x$. By changing $d z$ for a given $d x$, the cavity can always be made insensitive to accelerations. 


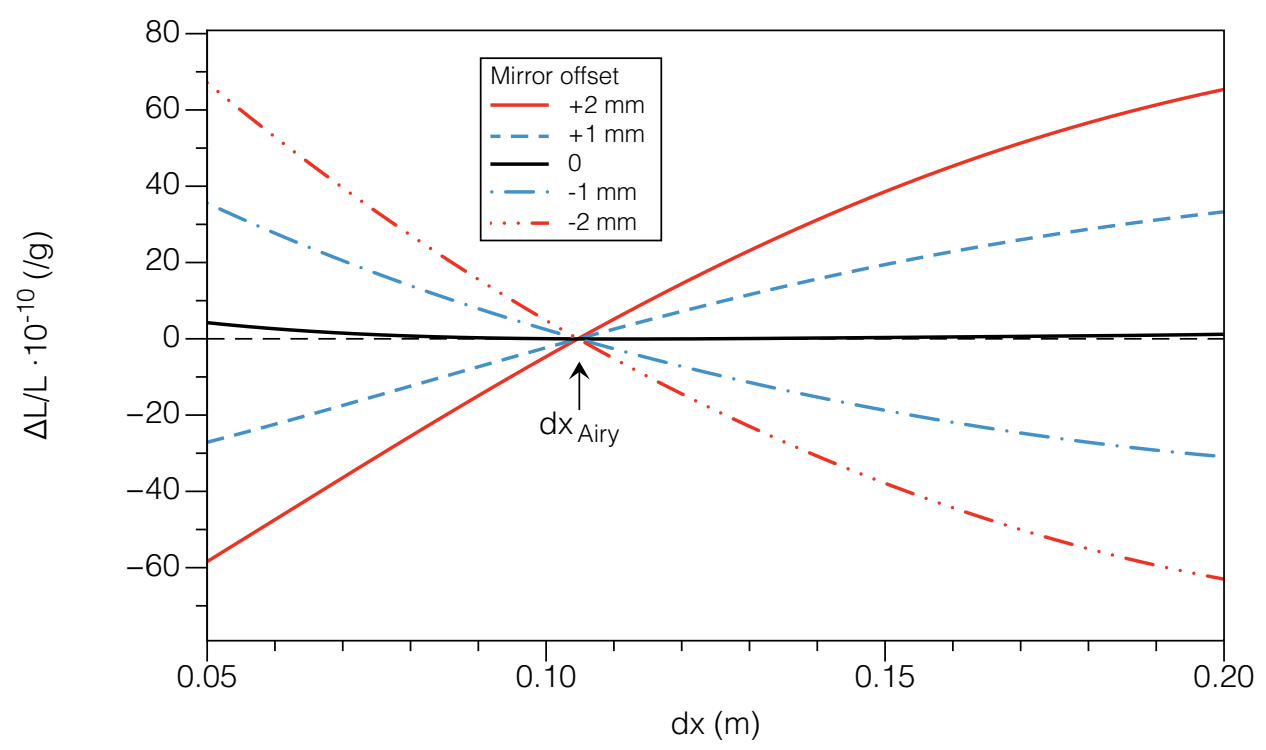

Figure 2.18: Relative length fluctuations $\frac{\Delta L}{L}$ due to a vertical force of $1 \mathrm{~g}$ as a function of the longitudinal support positions for different vertical offsets $\Delta r_{z}$ of the mirrors with respect to the centre of the spacer face. The tilt-induced length change is compensated at the so-called Airy point $d x=d x_{\text {Airy }}$.

This is the case for both vertical and transverse accelerations, i.e., $\kappa_{z}\left(d x_{\text {Airy }}\right) \approx 0$ and $\kappa_{y}\left(d x_{\text {Airy }}\right) \approx 0$. Unfortunately, as we will see in the next section, the Airy point changes slightly depending on the direction of the applied acceleration. This means that when we fix $d x$ to the Airy point for vertical forces, there will be an unavoidable length change due to transverse forces if the optical axis is not perfectly centred. The same goes for longitudinal accelerations, where tilt is compensated for with $d z$ instead of $d x$.

The Airy point for vertical forces can be represented as a vertical line $d x=d x_{\text {Airy }}$ in Figure 2.16. We know that this line will eventually intersect the $\Delta L=0$ curve for some value $d z_{\Delta L=0} \in\left(d z_{0}, d z_{1}\right)$. And in fact, we know that at this point the vertical tilt sensitivity coefficient will be nearly zero. The support positions $\left(d x_{\text {Airy }}, d z_{\Delta L=0}\right)$ will make the cavity insensitive to vertical forces, as it is compensated for both the Poisson effect and the mirror tilt contributions to length change. 


\subsubsection{Transverse and longitudinal accelerations}

In the previous section we determined optimal support positions which make the cavity insensitive to vertical forces, $\left(d x_{\text {Airy }}, d z_{\Delta L=0}\right)$. In this section we carry out simulations to confirm that the resulting configuration is also insensitive to forces in the transverse and longitudinal directions to the desired levels. For transverse accelerations the model considers only one half of the cavity geometry as divided by the middle symmetry $y z$ plane, with a symmetry boundary condition defined on that plane. Figure 2.19 shows the resulting cavity length fluctuations when the system is subject to a transverse acceleration of $1 \mathrm{~g}$. As we can see, the length fluctuations become significant only if the optical axis is shifted. For perfectly centred mirrors the cavity is virtually insensitive to transverse forces at the level we are concerned with. With this, we confirm that transverse accelerations only contribute to the fractional length change through the respective tilt term $k_{y} \approx 0$.

As expected, the tilt cancels at the Airy point, however we find that the position of the Airy point is slightly dependent on the direction of the applied force. Our simulations show a difference of about $2.5 \mathrm{~mm}$ when considering vertical and transverse forces, which will inevitably result in some length change for transverse forces if $d x$ is set to the Airy point obtained for vertical forces (as will be the case) and if $\Delta r_{y}>0$. This may be due to how the cutouts break the cylindrical symmetry of the spacer, and we suspect this effect would be much more severe in rectangular or mushroom spacer cavities. We also find a small dependence of the Airy point with spacer diameter $D$, which might be due to the same cause. Note that the slope of $\frac{\Delta L}{L}(d x)$ near $d x_{\text {Airy }}$ is larger for the smaller spacer diameter $D$, suggesting that the transverse tilt sensitivity gradient decreases with $D$.

For longitudinal accelerations the model consists of one half of the cavity as divided by the middle $x z$ plane, plus a symmetry boundary constraint on that plane. Similarly to transverse accelerations, Figure 2.20 confirms that the resulting length fluctuations when the system subject to a longitudinal force of $1 g$ are nearly zero unless there is some vertical offset $\Delta r_{z}$ of the mirrors. In this case tilt cannot be cancelled by placing $d x$ at the Airy point, as this tilt can only be compensated by adjusting $d z$. However the effect is very small and in fact the simulations reach numerical error with the mesh size used. 


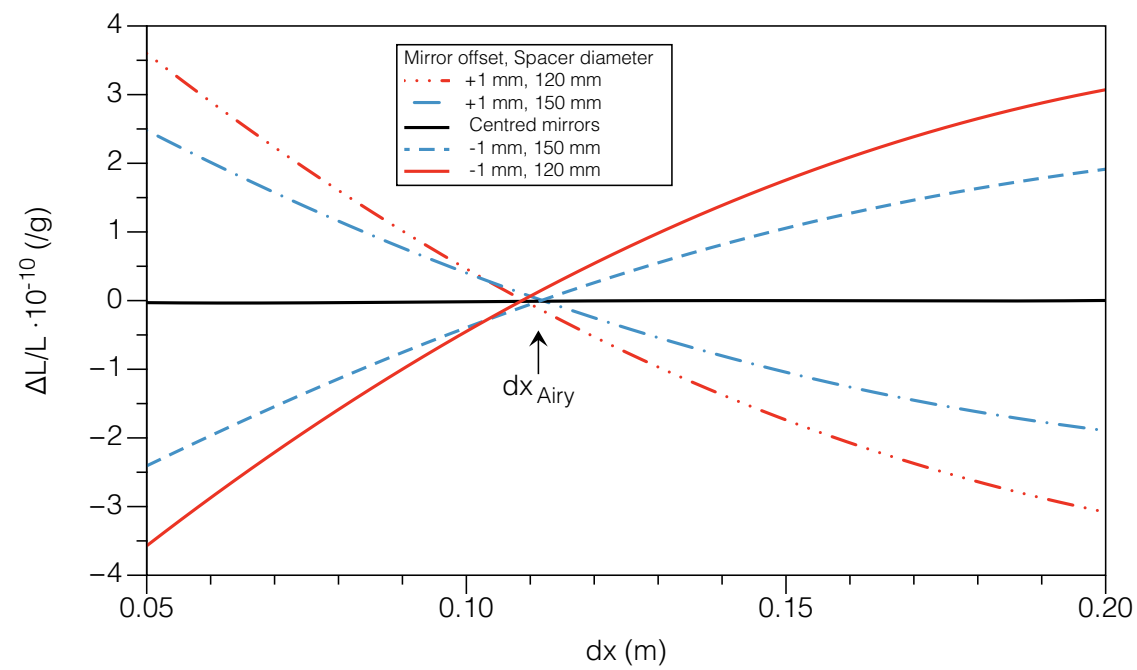

Figure 2.19: Relative length fluctuations $\frac{\Delta L}{L}$ due to a transverse acceleration of $1 \mathrm{~g}$ as a function of axial support positions for different horizontal offsets $\Delta r_{y}$ of the mirrors and two spacer diameters. The tilt-induced length change cancels at the Airy point $d x=d x_{\text {Airy }}$, which is slightly different depending on the direction of the applied acceleration and the diameter of the spacer.

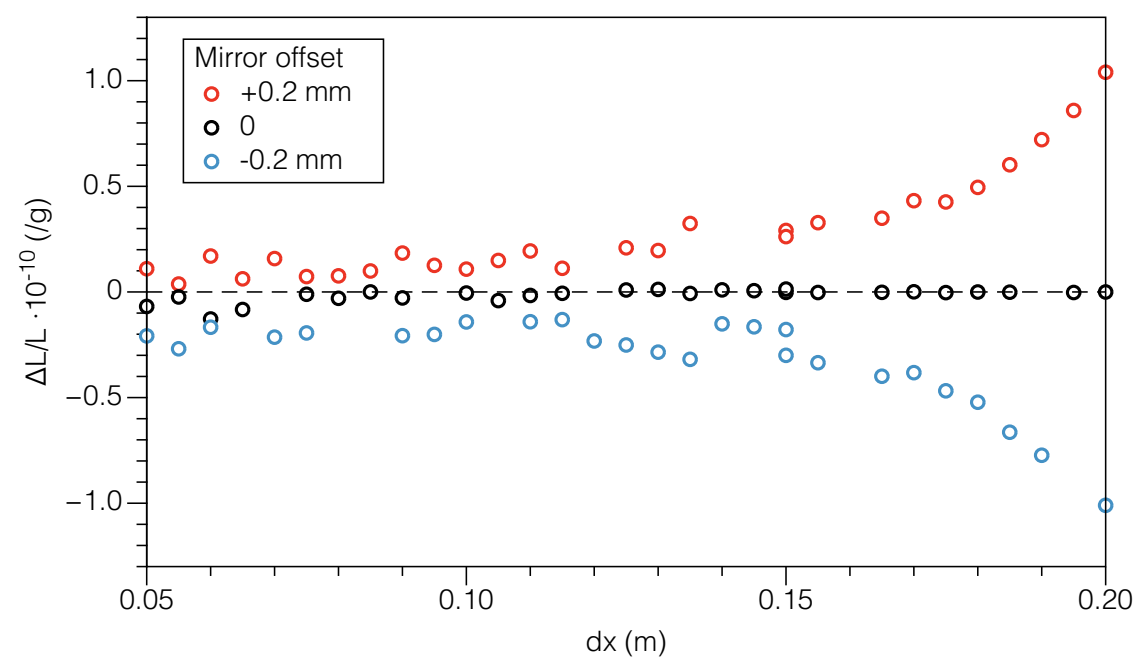

Figure 2.20: Relative length fluctuations $\frac{\Delta L}{L}$ due to a longitudinal acceleration of $1 \mathrm{~g}$ as a function of the longitudinal support positions for different vertical offsets $\Delta r_{z}$ of the mirrors. This simulation reaches numerical error for the mesh size used. It is repeated in Section 2.5.4 with a higher density mesh. 


\subsubsection{Further optimisation of the spacer geometry}

Having achieved a force insensitive configuration through the optimisation of the support positions, we are now interested in learning what the optimal spacer diameter and cutout dimensions are. To optimise these parameters, we look at the gradient of $\frac{\Delta L}{L}$ with $d x$ and $d z$ (i.e., the gradients of $k_{z}(d z), \kappa_{z}(d x), \kappa_{y}(d x)$ and $\left.\kappa_{x}(d z)\right)$. This will allow us to establish a configuration that is not only force insensitive, but also has low susceptibility to $d x$ and $d z$ deviations. After each step of this second-stage optimisation, the acceleration and tilt sensitivities change, and thus the optimal support positions $\left(d x_{\text {Airy }}, d z_{\Delta L=0}\right)$ have to be re-computed.

First we obtain the gradient of $k_{z}$ by performing fits of $\frac{\Delta L}{L}$ vs $d z$ for perfectly centred mirrors. We then obtain the gradients of $\kappa_{x, y, z}$ by measuring mirror tilt as a function of the corresponding compensating parameter ( $d x$ in the vertical and transverse directions and $d z$ in the longitudinal). In each optimisation step the procedure is: $(i)$ build the geometry with the relevant parameter values; $(i i)$ find the vertical Airy point $d x_{\text {Airy }}$ by introducing a small vertical optical axis shift; (iii) fix $d x$ to $d x_{\text {Airy }}$ and find the corresponding $d z_{\Delta L=0} ;(i v)$ fix the supports at $\left(d x_{\text {Airy }}, d z_{\Delta L=0}\right)$ and measure the gradients.

The simulations show that changing $D$ contributes to both the acceleration and tilt sensitivity gradients, but changing $d l$ has negligible impact on mirror tilt and only contributes to the acceleration sensitivity gradient. Additionally, we see that changing the cutout width, $d h$, has a negligible effect overall. Thus, we will first optimise $d l$ to the value that results in the lowest $k_{z}$ gradient and is feasible, and then investigate how the gradients of both $k_{z}$ and $\kappa_{j}$ change with $D$.

Figure 2.21 a shows that the gradient of $k_{z}$ increases approximately linearly with cutout depth $d l$, and thus smaller cutouts will make the system less sensitive
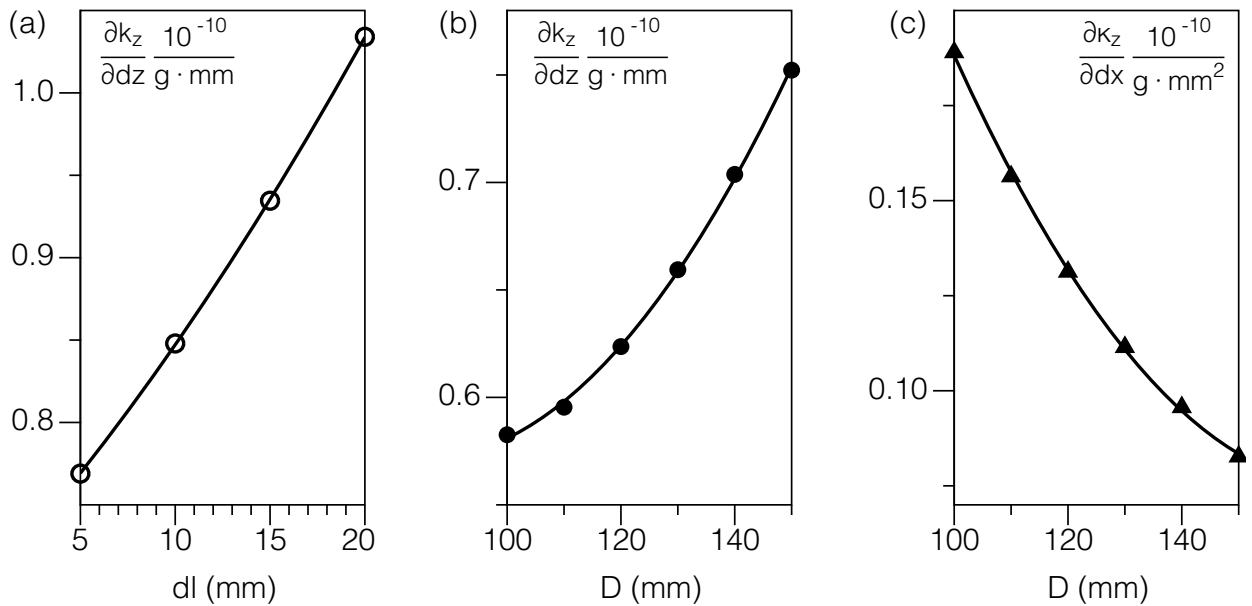

Figure 2.21: Vertical acceleration sensitivity gradient for different values of the spacer cutout depth (a); the smaller the cut the less susceptible the system is to $d z$ deviations. Vertical acceleration (b) and tilt (c) sensitivity gradients for different values of the spacer diameter $D$. 


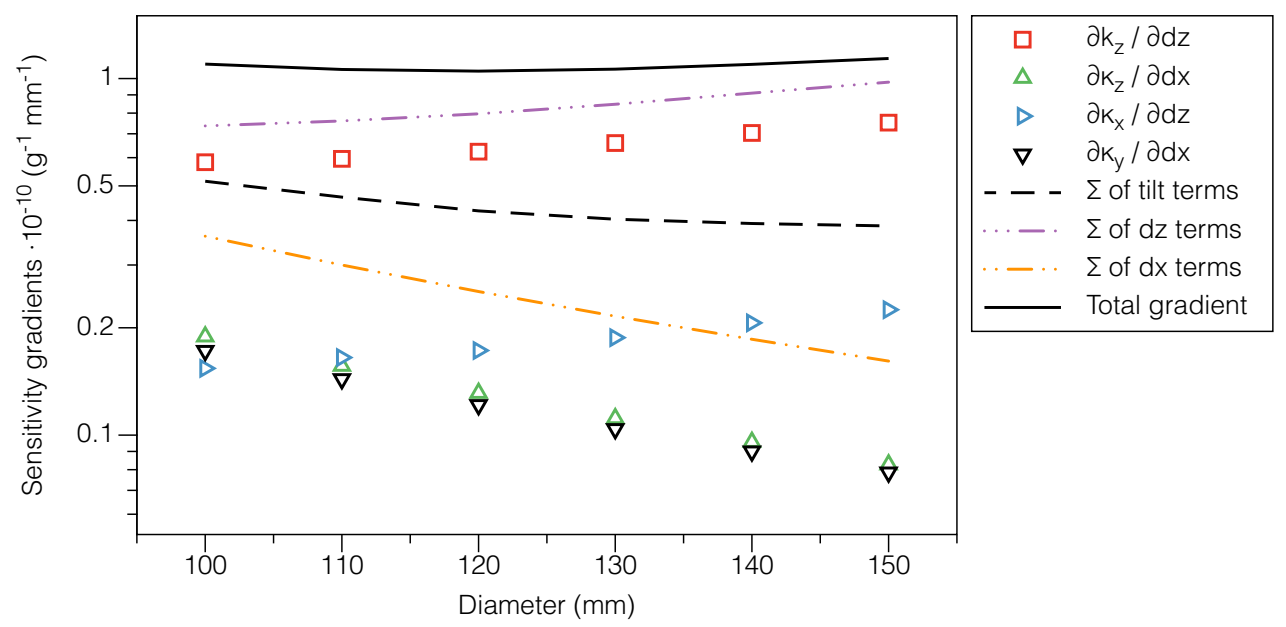

Figure 2.22: Gradients of the vertical acceleration sensitivity and the tilt sensitivities (the latter multiplied by $\Delta r=1 \mathrm{~mm}$ to so that they can be compared against the former).

to deviations in $d z$ or machining imperfections affecting the vertical positions of the supports. We set $d l=5 \mathrm{~mm}$, which we regard to as the minimal cutout depth required to be able to safely and easily mount the cavity in its supports by hand; and set the cutout width to $20 \mathrm{~mm}$ in order to allow enough space for adjustment of the longitudinal support positions, as it is not a sensitive parameter. We also find that the value of $d z_{\Delta L=0}$ increases linearly with cutout depth.

For the chosen cutout dimensions we carry out simulations changing the diameter of the spacer $D$ and measure the gradients of $k_{z}$ and $\kappa_{x, y, z}$ (Figure $2.21 \mathrm{~b}-\mathrm{c}$ ). For the gradients of the tilt sensitivities we measure the longitudinal displacement of an array of points along a vertical cut line at the surface of the mirror as we change $d x$ (or $d z$ in the case of longitudinal accelerations), or along a horizontal cut line in the case of transverse accelerations. We then perform linear fits for each set of points and extract the angle of inclination of the mirror from the slope of the fit. A second fit of the angle of inclination with respect to $d x$ (for vertical or longitudinal accelerations) or $d z$ (for transverse accelerations) will yield the corresponding tilt sensitivity gradient.

To be able to compare the acceleration and tilt sensitivity gradient terms in one plot, we assume a rather large offset of the optical axis of $\Delta r=1 \mathrm{~mm}$ (Figure 2.22). The gradients of $k_{z}$ and $\kappa_{x}$ increase with $D$, while the gradients of $\kappa_{z}$ and $\kappa_{y}$ decrease. Of course, the system is more sensitive to $d z$ deviations due to the direct length change induced by $k_{z}$ compared to the indirect length change caused by tilt. Even for a large mirror offset of $1 \mathrm{~mm}$, the tilt contribution to the total sensitivity is small. The system is overall more sensitive to tilt the smaller the spacer diameter is, as expected. In this case, with $\Delta r=1 \mathrm{~mm}$, the total sensitivity gradient remains approximately constant at $\approx 10^{-10}\left(\mathrm{~g}^{-1} \mathrm{~mm}^{-1}\right)$. In the end we choose a spacer diameter of $10 \mathrm{~cm}$ where we believe there will be a good trade off between sensitivity 
to $d x$ and to $d z$ deviations.

\subsubsection{Optimal configuration}

We have established a final spacer diameter of $10 \mathrm{~cm}$, as well as a final cutout depth of $5 \mathrm{~mm}$. In this section we carry out final simulations using these parameters and more realistic cutouts with rounded edges due to the particular machining technique that will be used. We also employ higher density meshes (i.e., meshes with a larger number of nodes) in order to increase the accuracy of the simulations and to determine the final support positions and the resulting acceleration and tilt sensitivities and sensitivity gradients.

We repeat the $(d x, d z)$ parameter sweep in COMSOL (Figure 2.23). In order to find the vertical Airy point we introduce a vertical force and vertical offsets $\Delta r_{z}$ of the mirrors (Figure 2.24), to obtain

$$
d x_{\text {Airy }}=101.32 \mathrm{~mm}
$$

about $1 \mathrm{~mm}$ away from the nominal position for a rectangular bar, given by $d x_{\text {Airy }}=L\left(\frac{1}{2}-\frac{\sqrt{3}}{6}\right)[77]$. We fix the longitudinal support positions to $d x_{\text {Airy }}$ and vary $d z$ to find

$$
d z_{\Delta L=0}=2.20 \mathrm{~mm}
$$

and an acceleration sensitivity gradient of $60.7 \times 10^{-12}\left(\mathrm{~g}^{-1} \mathrm{~mm}^{-1}\right)$.

We then fix the vertical support position to $d z_{\Delta L=0}$ and vary $d x$ as we evaluate the tilt angle of the mirror, finding that it cancels at $d x=101.13 \mathrm{~mm}$, a deviation of $190 \mu \mathrm{m}$ with respect to the previous step. This yields a residual vertical acceleration sensitivity of $\pm 7 \cdot 10^{-12}\left(g^{-1}\right)$.

The system is second-order sensitive to transfer forces through the tilt term, due to the asymmetry between the vertical Airy point (to which we set the longitudinal support positions) and the transverse Airy point (Figure 2.25). We measure an offset of the Airy points of $2.53 \mathrm{~mm}$, which yields a transverse sensitivity of $\pm 1.8 \times 10^{-11}\left(g^{-1}\right)$ at $\Delta r_{y}= \pm 0.1 \mathrm{~mm}$ (the estimated precision of the alignment of the mirror with the optical contacting technique). Similarly, the system is second-order sensitive to longitudinal forces, with a longitudinal sensitivity of $\pm 5.1 \times 10^{-12}\left(g^{-1}\right)$ at $\Delta r_{z}= \pm 0.1 \mathrm{~mm}$. See Table 2.6 for a summary of the final mechanical properties of the optimised force-insensitive cavity. 


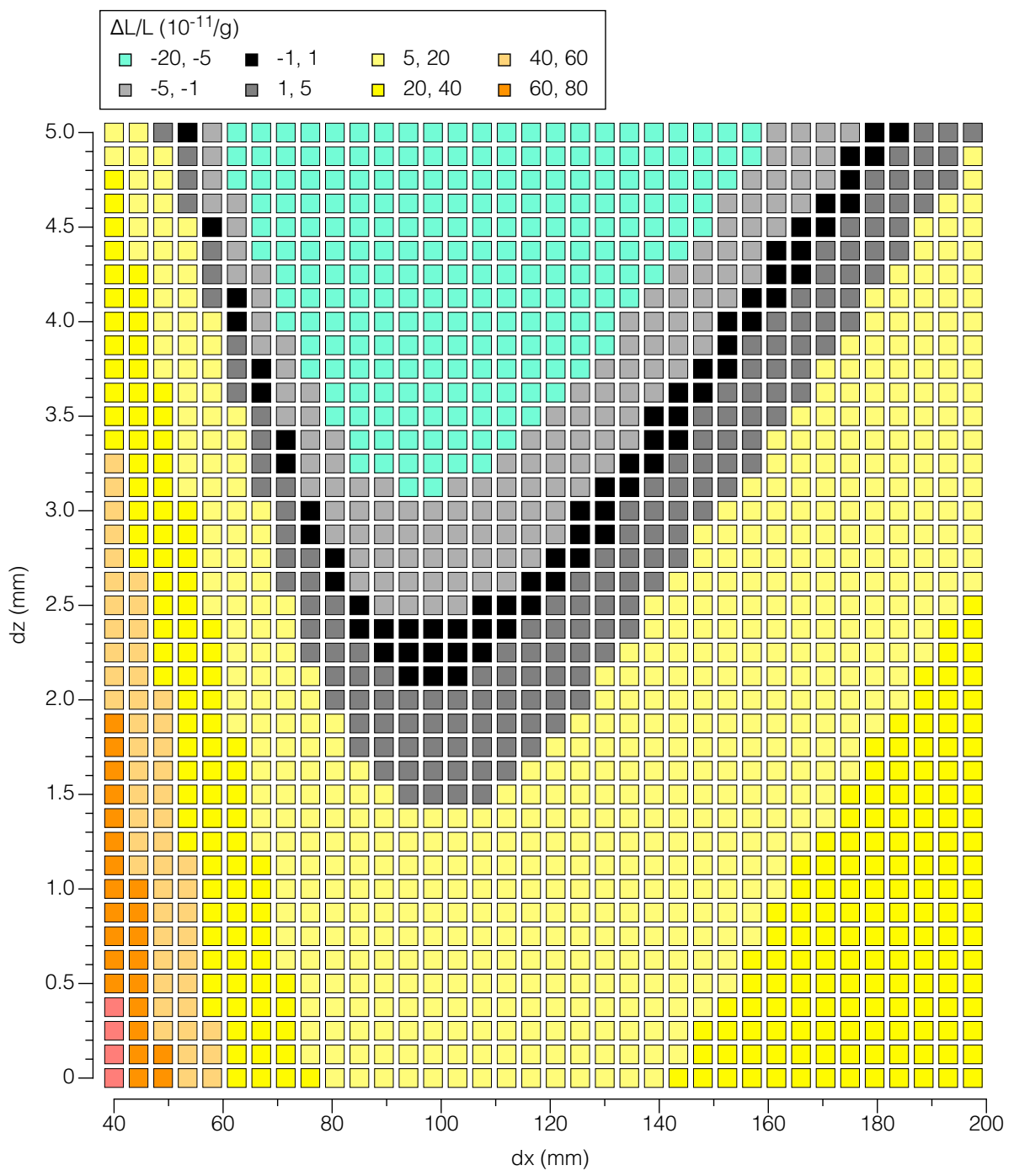

Figure 2.23: Relative length fluctuations $\frac{\Delta L}{L}$ due to a vertical force of $1 \mathrm{~g}$ (colour) in the $d x-d z$ parameter spacer. This model features a $10 \mathrm{~cm}$ spacer with $5 \mathrm{~mm}$ cutouts with rounded edges, $5 \mathrm{~mm}$ vent holes, mirrors and mirrors annuli. In each simulation the mesh used consisted of a starting free tetrahedral mesh using COMSOL's physics-controlled Normal mesh option, plus three iterations of adaptive mesh refinement. 


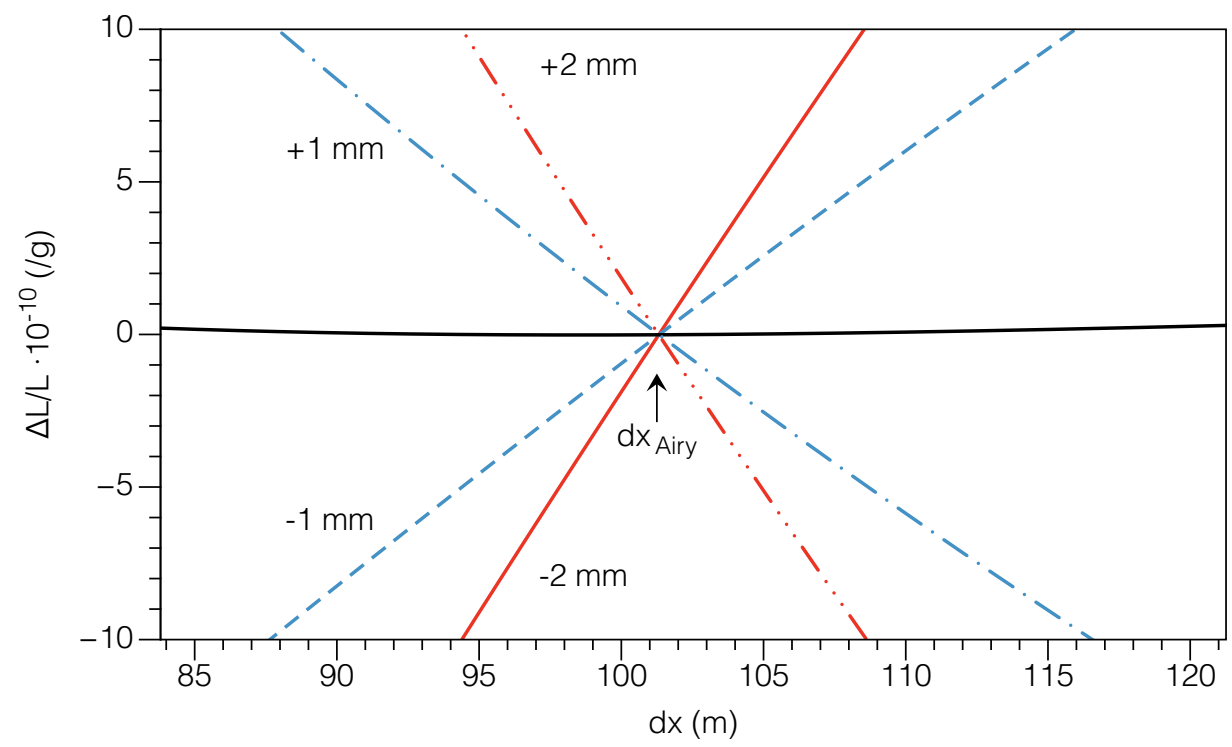

Figure 2.24: Relative length fluctuations $\frac{\Delta L}{L}$ under a vertical force of $1 \mathrm{~g}$ as a function of the axial support position and for different vertical offsets $\Delta r_{z}$ of the mirrors. The curves intersect at the vertical Airy point $d x_{\text {Airy }}=101.32 \mathrm{~mm}$ where the tilt contribution to the overall cavity length change cancels.

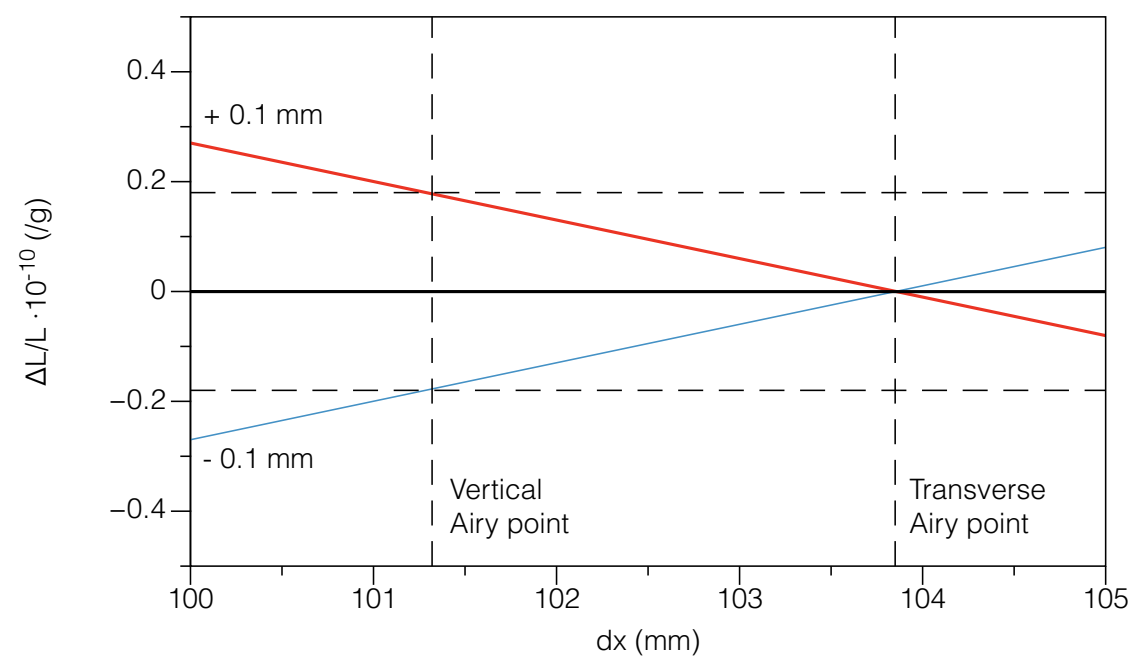

Figure 2.25: Relative length fluctuations $\frac{\Delta L}{L}$ under a transverse force of $1 \mathrm{~g}$ as a function of the axial support position and for typical horizontal offsets of the mirror of $\pm 0.1 \mathrm{~mm}$. The transverse tilt contribution cancels at the transverse Airy point, which we estimate is $2.53 \mathrm{~mm}$ away from the vertical Airy point to which we set the cavity. 


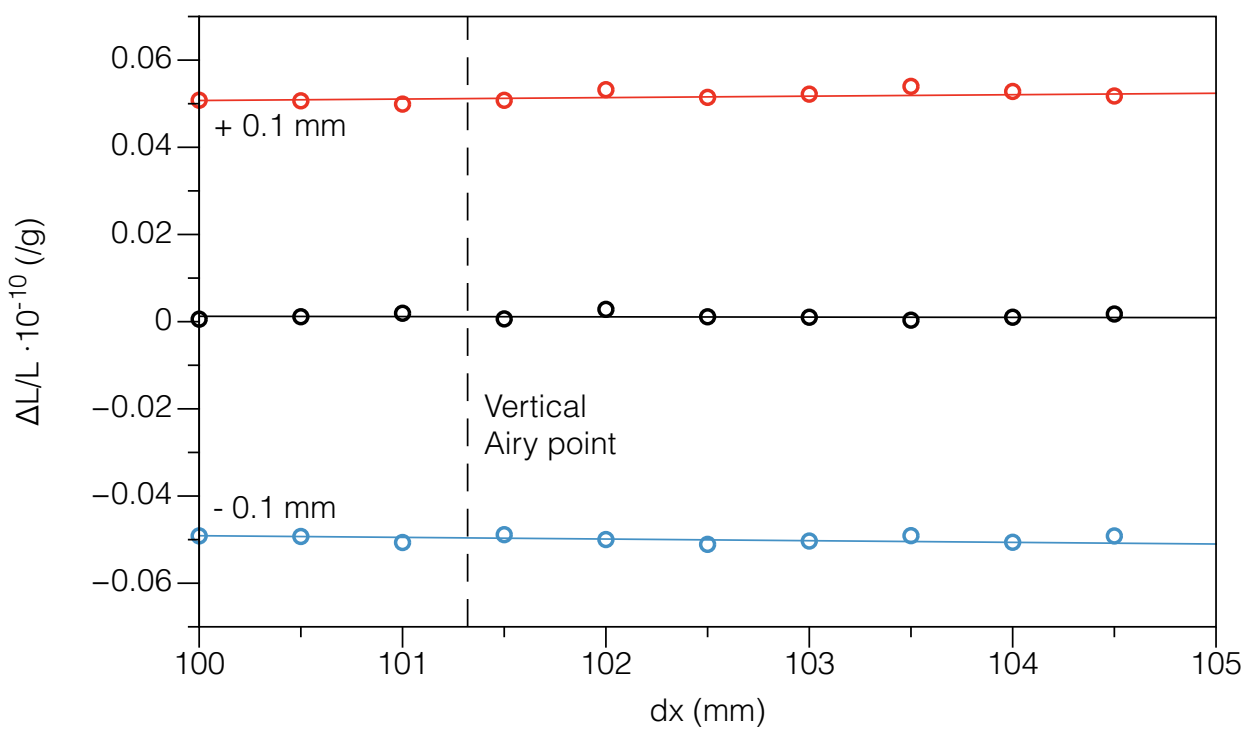

Figure 2.26: Relative length fluctuations $\frac{\Delta L}{L}$ under a longitudinal force of $1 \mathrm{~g}$ as a function of the axial support position and for typical vertical offsets of the mirror of $\pm 0.1 \mathrm{~mm}$. The longitudinal tilt contribution cannot be compensated for by adjusting $d x$.

\begin{tabular}{cc}
\hline$d x_{\text {Airy }}$ & $101.32 \mathrm{~mm}$ \\
$d z_{\Delta L=0}$ & $2.20 \mathrm{~mm}$ \\
$k_{z}$ residual & $\pm 7 \cdot 10^{-12}\left(g^{-1}\right)$ \\
$\kappa_{y} \cdot 0.1 \mathrm{~mm}$ & $\pm 1.8 \cdot 10^{-11}\left(g^{-1}\right)$ \\
$\kappa_{x} \cdot 0.1 \mathrm{~mm}$ & $\pm 5.1 \cdot 10^{-12}\left(g^{-1}\right)$ \\
$\partial k_{z} / \partial d z$ & $60.7 \cdot 10^{-12}\left(g^{-1} \mathrm{~mm}^{-1}\right)$ \\
$\partial \kappa_{z} / \partial d x$ at $\Delta r_{z}=0.1 \mathrm{~mm}$ & $1.9 \cdot 10^{-12}\left(g^{-1} \mathrm{~mm}^{-1}\right)$ \\
$\partial \kappa_{y} / \partial d x$ at $\Delta r_{y}=0.1 \mathrm{~mm}$ & $1.7 \cdot 10^{-12}\left(g^{-1} \mathrm{~mm}^{-1}\right)$ \\
$\partial \kappa_{x} / \partial d z$ at $\Delta r_{z}=0.1 \mathrm{~mm}$ & $1.5 \cdot 10^{-12}\left(g^{-1} \mathrm{~mm}^{-1}\right)$ \\
\hline
\end{tabular}

Table 2.6: Values of the acceleration and tilt sensitivities and their gradients at the final support positions $\left(d x_{\text {Airy }}, d z_{\Delta L=0}\right)$. All the coefficients tend to zero at this point as $\Delta L \rightarrow 0$. Their gradients measure the sensitivity to $d x$ and $d z$ deviations. $k_{y}$ and $k_{x}$ are given for a $1 \mathrm{~mm}$ offset of the optical axis. 


\subsubsection{Balanced support forces}

An additional challenge that rises when designing long cavities are the effects of inhomogeneous mounting forces. Since the cavity is supported by four points the support plane is over-constrained, and thus the distribution of the forces depends sensitively on mechanical tolerances. Due to its dimensions ( $L=0.485 \mathrm{~mm}$ and $D=100 \mathrm{~mm})$ and high mass $(8.2 \mathrm{~kg})$, the system becomes very sensitive to small differences of the loading forces acting on each support.

Ideally when the system is subject to a longitudinal, transverse or vertical force, all supports experience the same load and exert an equal reaction force. This homogeneous loading leads to the deformation of the structure that we have studied so far. But in practice there will be force differences between the mounting points (e.g., due to height imperfections of the support posts or machining imperfections of the spacer). As a result the Viton spheres will be compressed differently, which will lead to an asymmetric deformation of the spacer, and hence to an additional length change.

To determine quantitatively how a force imbalance induces cavity length fluctuations, we simulate the system under accelerations in each direction for different supporting configurations. We can simulate a $100 \%$ force imbalance between two pairs of supports by setting a fixed constraint on one pair and no constraints on the other, and obtain different imbalance ratios by applying different boundary loads to the free pair with direction opposite to the applied acceleration. There are two extreme cases: $100 \%$ force imbalance between rear and front supports (preserving symmetry in the $x z$ plane) and $100 \%$ imbalance between the lateral pairs of supports (preserving symmetry in the middle $y z$ plane).

For a longitudinal acceleration of $1 \mathrm{~g}$ and a $100 \%$ force imbalance between front and rear supports, we obtain a relative length change of $45.49 \times 10^{-9}\left(g^{-1}\right)$. This is the worst case scenario. Examining the other two directions or creating the imbalance between other pairs of supports yields much lower length fluctuations. Figure 2.27 shows the results for different force imbalance ratios.

We see that $\frac{\Delta L}{L}$ scales linearly with $\frac{\Delta F}{F}$, where $\Delta F=F_{1}-F_{2}$, with $F_{i}$ the total force acting on a pair of supports, and $F$ the total force acting on the system. Assuming a typical vibration noise of $1 \mu \mathrm{g}$, the reaction forces need to be balanced to 1 part in $10^{3}$ in order for the cavity to reach a fractional instability below $10^{-16}$. See Table 2.7 for a comparison of these results against past references. Our results are in good agreement with those from Nazarova et al [79] who estimated the effect using the same method, and in rough agreement with Amairi et al [69] who do not explain how they estimated their values.

The effect is more severe in the longitudinal direction, followed by the vertical and the transverse directions. For longitudinal accelerations a $100 \%$ imbalance between the front and rear pairs induces the maximum length change, whereas for transverse and vertical accelerations the maximum length change is achieved for a $100 \%$ imbalance between the lateral pairs of supports (these are the worst case scenarios), as expected.

Häfner et al [55] use an effective three point support system by placing the foursupport structure into a self-balancing mount that consists of three glass spheres; two on one end located symmetrically at a distance from the $z x$ plane; one on the 


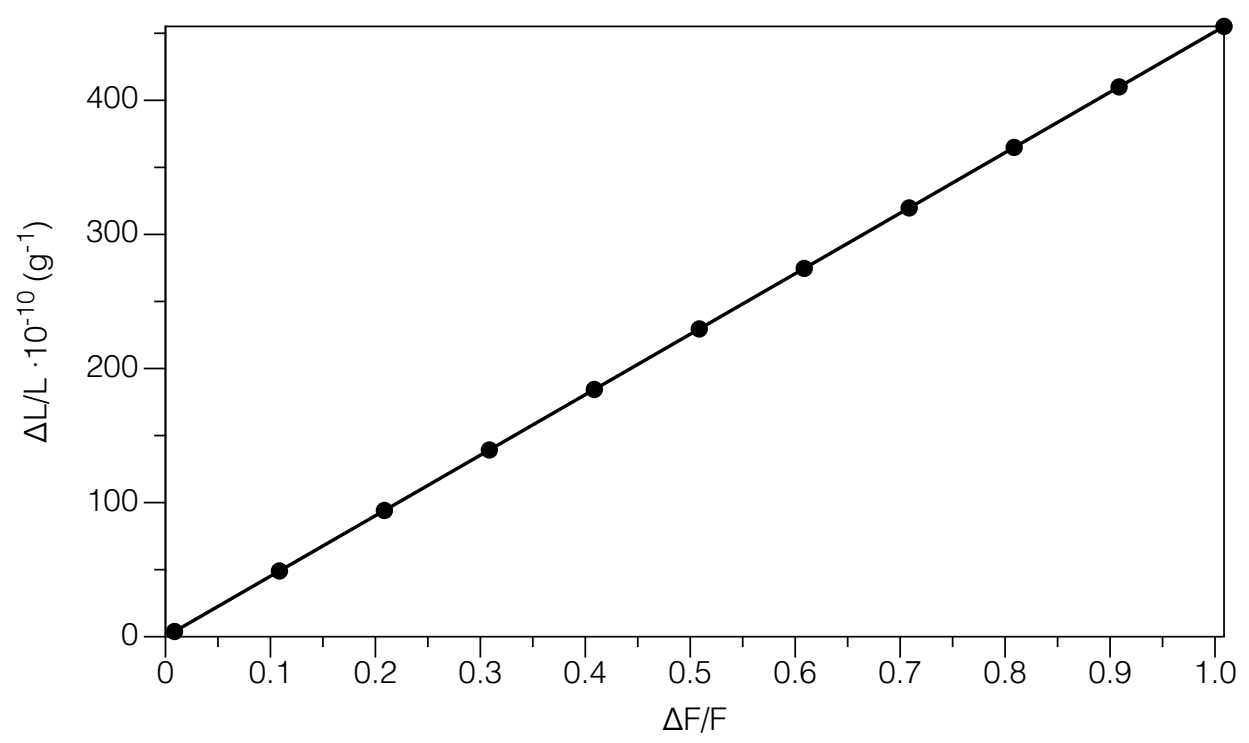

Figure 2.27: Relative length change $\frac{\Delta L}{L}$ under a longitudinal acceleration of $1 \mathrm{~g}$ as a function of the relative force imbalance.

\begin{tabular}{ccccc}
\hline & \multicolumn{4}{c}{ Effect of inhomogeneous loading $\frac{\Delta F}{F} \cdot 10^{-9}\left(g^{-1}\right)$} \\
& This work & Häfner 2015 & Amairi 2013 & Nazarova 2006 \\
\hline Longitudinal & 45.49 & 86.72 & 7.4 & 7.01 \\
Vertical & 1.44 & - & 0.24 & - \\
Transverse & 1.84 & - & - & - \\
\hline Mass $(\mathrm{kg})$ & 8.2 & 6.8 & 4.7 & 1.1 \\
\hline
\end{tabular}

Table 2.7: Acceleration sensitivity induced by inhomogeneous loading of the supports in different experiments.

other end located in the $z x$ plane. The end with two spheres prevents the structure from rotating along the $x$ axis, while the end with one sphere allows rotation, achieving automatic levelling of all four mounting posts and eliminating the effect of any loading differences, equalising the reaction forces of the supports. We adopt a very similar support system to that detailed in [55], with the exception that we use Viton pads instead of Viton spheres between the ultra low expansion rods and the aluminium clamps, which provides better stiffness of the support mechanism (Figure 2.28). For more details on the mounting mechanism see [55]. 


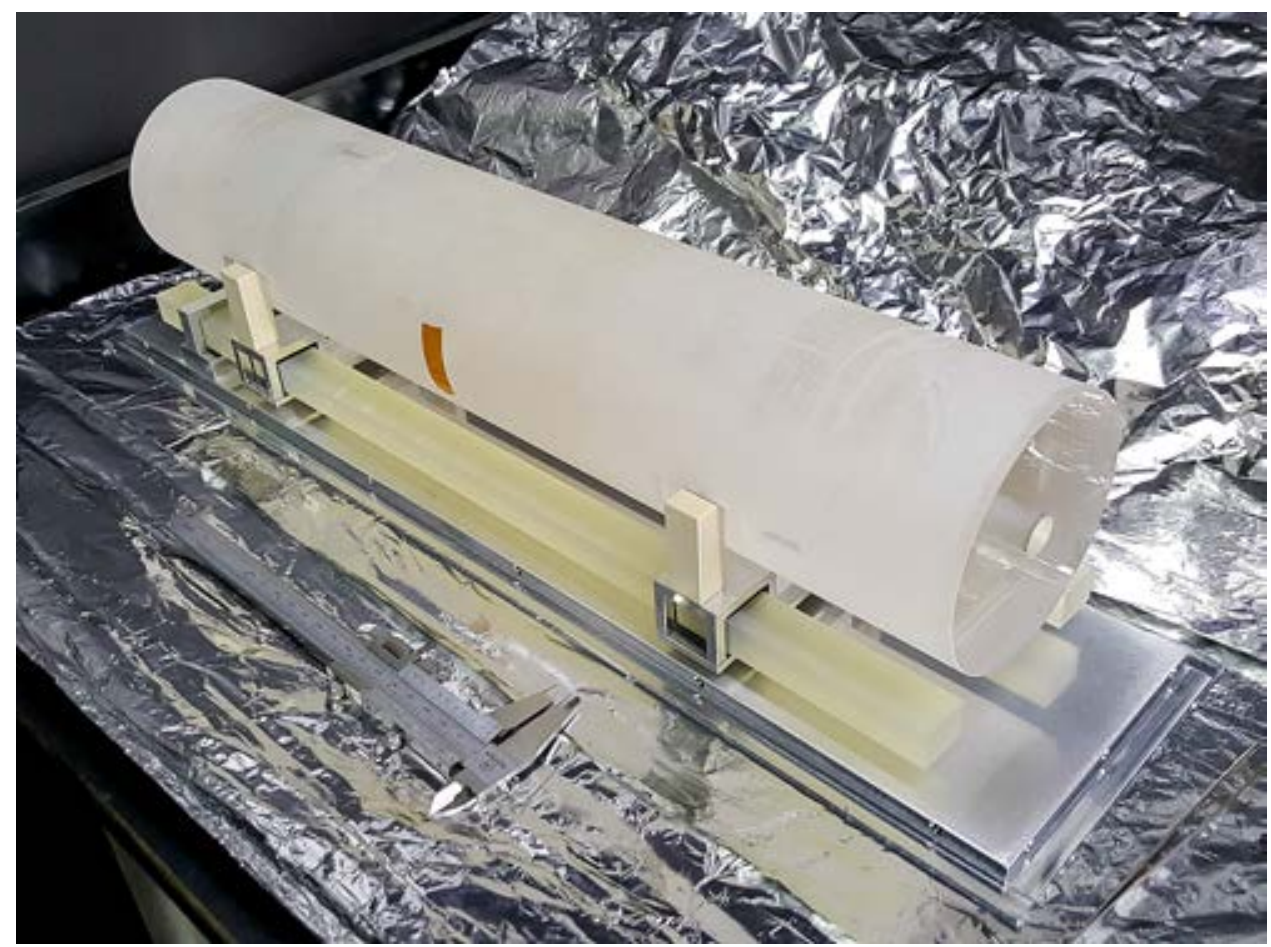

Figure 2.28: Photo of the cavity spacer and the cavity mount system. The mount is very similar to that detailed in [55], with the cavity spacer being supported at four points via Viton spheres that are resting on PEEK posts. The PEEK posts are attached to a pair of aluminium clamps that are fastened to a pair of Clearceram rods for spacing (Clearceram $(\mathrm{CCZ})$ is a brand of ultra low expansion glass, similar to ULE or Zerodur). To fasten the clamps to the rods we use Viton pads. The rods experience nearly zero thermal expansion at around room temperature, and decouple the cavity support points from the thermal expansion of the aluminium base plate. One of the aluminium clamps rests on a pair of glass spheres, while the other rests on just one glass sphere in the middle and is able to rotate about the optical axis, which helps balance the inhomogeneous mounting forces acting on the spacer at the support points. The glass spheres rest on conical cuts made to the aluminium base-plate. 



\section{Isolation from external perturbations}

\section{Contents}

3.1 Pressure and temperature fluctuations and vibrations . . . . 56

3.1 .1 Pressure sensitivity . . . . . . . . . . . . 56

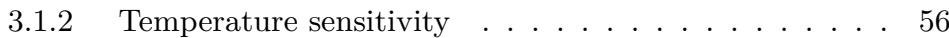

3.1.3 Vibration sensitivity . . . . . . . . . . . . 59

3.2 Design of the cavity enclosure . . . . . . . . . . . . 59

3.2.1 Heat shields . . . . . . . . . . . . . . . . . . . 59

3.2 .2 Vacuum chamber . . . . . . . . . . . . . . 60

3.3 Heat transfer in the cavity enclosure . . . . . . . . . . . . . 64

3.3.1 Thermal modelling . . . . . . . . . . . . . . . 64

3.3.2 Radiative heat transfer through the ventilation holes . 67

3.4 CAD drawings and photos of the system . . . . . . . . 74 


\subsection{Pressure and temperature fluctuations and vibrations}

$\mathrm{T}^{\mathrm{N}}$ addition to the fundamental noise floor of thermodynamical origin discussed 1 in the previous chapter, the cavity's length stability is challenged by pressure changes, temperature fluctuations and vibrations. In this chapter we detail the design of several isolation systems to keep these sources of noise below the cavity's thermal noise floor.

\subsubsection{Pressure sensitivity}

Changes in the local pressure $P$ in the cavity bore produce fluctuations of the cavity's optical path length $n L$ via a change of the local refractive index $n$. Consequently, the cavity's length and resonance frequency fluctuate. A pressure change $\Delta P$ couples to relative cavity length change as [80]

$$
\frac{\Delta L}{L}=-\frac{d n}{d P} \Delta P
$$

For dry air at room temperature, $d n / d P \approx 3 \cdot 10^{-7} \mathrm{mbar}^{-1}$, leading to a relative frequency fluctuation of $\frac{\Delta \nu}{\nu}=\left(3 \cdot 10^{-7} \mathrm{mbar}^{-1}\right) \Delta P$. Therefore, in order to achieve a relative length instability below $10^{-16}$ in air, the local air pressure has to be stabilised to better than $\approx 3.3 \cdot 10^{-10}$ mbar. The effects of pressure fluctuations are greatly minimised by mounting the cavity in vacuum. In a completely sealed vacuum system the gas mixture inside the cavity bore consists of mainly $\mathrm{N}_{2}, \mathrm{H}_{2} \mathrm{O}$, and $\mathrm{O}_{2}$. For an ideal gas, a pressure change $\Delta P$ causes a fractional change of the index of refraction given by [81]

$$
\frac{\Delta n}{n} \approx \frac{a}{2 k_{B} T} \cdot 100 \cdot \Delta P,
$$

where $a$ is the gas particle polarisability, $k_{B}$ is Maxwell's constant, $T$ is the temperature of the system in $\mathrm{K}$, and $\Delta P$ is in mbar. For the common vacuum gases, $a \equiv a_{\text {vacuum }} \approx 1.4 \cdot 10^{-30} \mathrm{~m}^{3}$. Therefore, to achieve a relative length instability below $10^{-16}$ for a cavity mounted in vacuum, the pressure has to be stabilised to $\Delta P<1.5 \cdot 10^{-9}$ mbar (a requirement five times less strict than mounting the cavity in air). A vacuum system is designed for this purpose, as described in Section 3.2.2.

\subsubsection{Temperature sensitivity}

Temperature fluctuations contribute to the cavity's length change by directly modifying the distance between the two mirrors through thermal expansion and deformation of the spacer and mirror substrates. A temperature change $\Delta T$ couples to the cavity's relative length change as

$$
\frac{\Delta L}{L}=-\alpha(T) \Delta T
$$

where $\alpha(T)$ is the system's coefficient of thermal expansion (CTE), and is in general a function of the system's temperature $T$. While a cavity spacer typically consists 
of ULE, with its characteristically small CTE with a zero crossing value at around room temperature, the fused silica substrates (required for acceptable thermal noise performance, as stated in the previous chapter) have a considerably larger CTE. If the system were comprised of the ULE spacer only (or a ULE spacer and ULE mirror substrates), it would be possible to cancel any length changes due to temperature fluctuations by fixing the temperature of the system to the zero crossing value $T_{0}$ of ULE, hence achieving a cavity design that is virtually insensitive to temperature changes (i.e., with its $\Delta T$-induced length instability determined by the performance of the temperature control and isolation systems).

In our system, with a ULE spacer and fused silica mirror substrates, the mirrors experience a much larger thermal expansion than the spacer they are contacted to. The front face of the mirror is constrained by the optical contact to the spacer face, while the back face is unconstrained and expands outwards, resulting in a bulging of the mirror. The effective CTE $\alpha_{\text {eff }}$ of the composite system is given by [82]

$$
\alpha_{\mathrm{eff}} \approx \alpha_{\mathrm{ULE}}+\delta \frac{d_{\mathrm{sb}}}{L}\left(\alpha_{\mathrm{FS}}-\alpha_{\mathrm{ULE}}\right)
$$

where $\alpha_{\mathrm{ULE}}$ and $\alpha_{\mathrm{FS}}$ are the CTEs of the ULE spacer and the fused silica substrates respectively, $d_{\mathrm{sb}}$ is the substrate diameter, $L$ is the spacer length, and $\delta$ is a geometry-dependent coefficient relating the radial expansion of the mirrors to their longitudinal displacement due to the thermal deformation. Typical CTE values for ULE and fused silica around room temperature are $\alpha_{\mathrm{ULE}}=2.4 \cdot 10^{-9}\left(T-T_{0}\right)$ and $\alpha_{\mathrm{FS}}=500 \cdot 10^{-9}+2.2 \cdot 10^{-9}(T-294.15 \mathrm{~K})$ respectively, in units of $\mathrm{K}^{-1}$.

Combining Equations 3.3 and 3.4 we find the relative cavity length change due to the thermal expansion of the system, with a term given by the expansion of the spacer and an additional term due to the thermal deformation of the mirrors. Contacting ULE annuli to the back of a fused silica mirror is a well-known method used to reduce this effect [82]. The effectiveness of this technique is dependent on various dimensions: the size of the mirror relative to the spacer, the thickness of the annulus, and the inner diameter of the annulus.

Our simulations show that the ability of the annulus to shift the zero-crossing temperature back towards that of the spacer increases with its thickness up to a certain point at which the effect levels off. Similarly, decreasing the inner diameter of the annulus shifts the zero-crossing temperature back to higher values, but the minimum inner diameter is limited by the extent of the anti-reflection coating on the back surface of the mirror. For long cavities such as this, however, the largest contribution to the total $\Delta T$-induced length change comes from the expansion of the spacer, and not from the deformation of the mirror. Hence, contacting the ULE annuli to the back of the mirrors provides only a minor improvement.

The effects of temperature fluctuations are greatly minimised by $(i)$ using heat shields to isolate the cavity from external temperature changes, and (ii) mounting the system (cavity and heat shields) in vacuum, as heat transfer via convection is largely eliminated. Great effort is also put into minimising conductive and radiative heat transfer inside the cavity enclosure, as described in Section 3.2.1. 


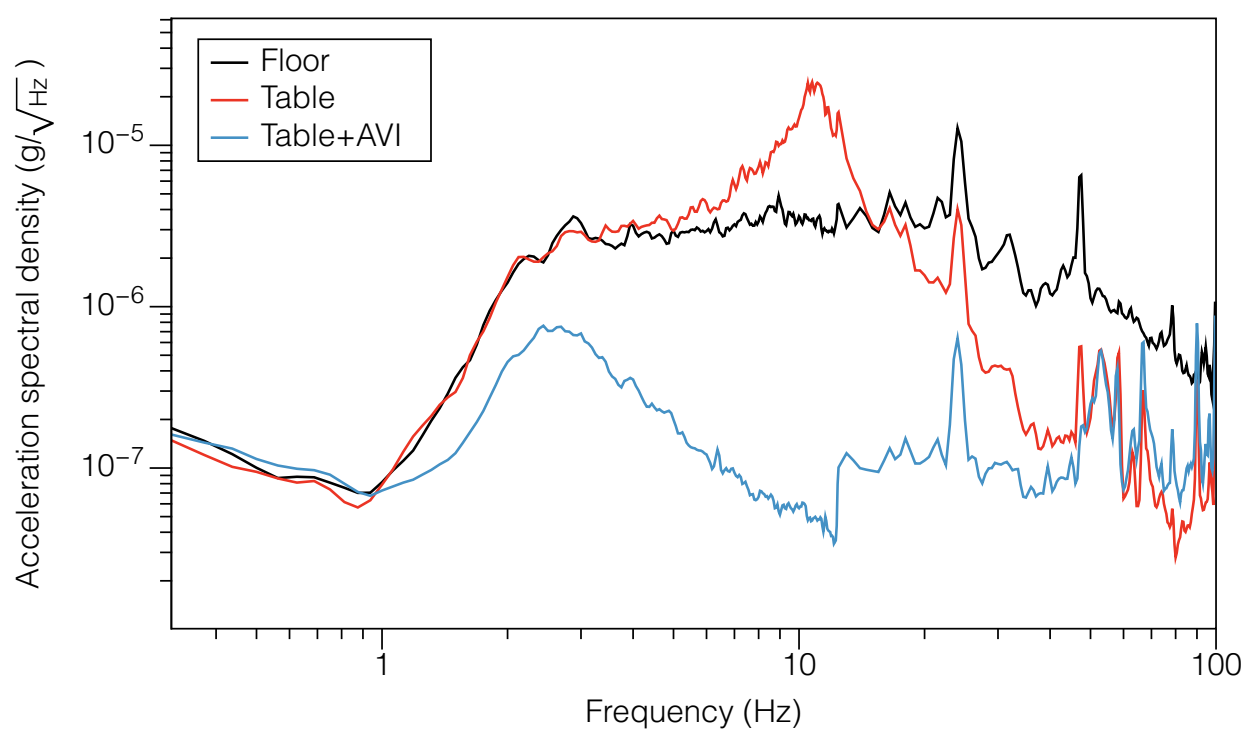

Figure 3.1: Vibration levels measured at the National Physical Laboratory in Teddington (UK), at the laboratory floor, at an optical table, and at an optical table with an active vibration isolation system. The AVI system provides an improvement in the $1-50 \mathrm{~Hz}$ range, and works best at around $11 \mathrm{~Hz}$, where we see the fundamental resonance of the optical table.

The spectrum of seismic noise is very dependent on site location and time. Below $50 \mathrm{mHz}$, the main source of noise is atmospheric pressure fluctuations caused by wind turbulence and infrasonic waves. From 50 to $500 \mathrm{mHz}$, the main source of noise is ocean waves generating surface and body waves in the earth's crust at coastal areas with most of the energy coming from coastlines in the vicinity of large storms. In this frequency range, there are two dominant features, one at roughly $60-90 \mathrm{mHz}$, corresponding to the fundamental ocean wave frequency. Another is at twice the fundamental $120-160 \mathrm{mHz}$, coming from the interaction of the reflected and incoming ocean waves, known as the microseismic peak. Above $500 \mathrm{mHz}$, along with oceanic and coastal body waves, the main sources of noise are local, such as wind-blown vegetation and human activity.

— D.B. Newell et al [83]. 


\subsubsection{Vibration sensitivity}

The cavity is subject to vibrations in all directions, resulting in a source of noise that needs to be studied, and consequent attempts at vibration isolation need to be made. Different sources of vibration (e.g., atmospheric pressure fluctuations, ocean waves, and human activity) have effects in different spectral regions, typically falling below $100 \mathrm{~Hz}$. Since low frequency components of the spectral density have a bigger influence on laser linewidth than high frequency components, the system is mounted on an active vibration isolation (AVI) system.

Active vibration isolation is a powerful way to reduce vibration noise levels, therefore extending the low frequency isolation capabilities of the cavity. An AVI system consists of a passive isolation system (typically a block or array of springs that serves as mount for an optical table) to which a feedback circuit is applied. The feedback circuit is composed of a sensor (e.g., a piezoelectric accelerometer), a controller, and an actuator. The acceleration signal detected in the sensor is processed by the controller, amplified and fed back to the system via the actuator. The cavity is rigidly coupled to the AVI system through the heat shields and vacuum chamber (the AVI system can support up to $400 \mathrm{~kg}$ of load). The AVI system is in turn resting on the floor to avoid low frequency mechanical resonances introduced by any additional supporting structures.

Measurements of the vibration levels in a laboratory at the National Physical Laboratory were performed and the results are shown in Figure 3.1. The measurements show the acceleration spectral densities measured at the laboratory floor, at the surface of an optical table, and at the same table with an AVI system engaged. Notice that the table has its fundamental resonance at around $11 \mathrm{~Hz}$, where it enhances the vibration levels, and begins to provide suppression above $\sim 15 \mathrm{~Hz}$. The AVI system offers considerable improvement at low frequencies starting at $1 \mathrm{~Hz}$, specially in the $3-20 \mathrm{~Hz}$ range, and works best at around $11 \mathrm{~Hz}$, achieving vibration levels almost two orders of magnitude lower than the floor levels. While it is possible to damp out most of these vibrations actively and passively, even greater suppression can be achieved by optimising the way in which the cavity is mounted, as we have seen in the previous chapter.

\subsection{Design of the cavity enclosure}

\subsubsection{Heat shields}

In order to minimise temperature fluctuations the cavity is surrounded by a system of three aluminium heat shields and an aluminium vacuum chamber, greatly minimising the impact of exterior temperature variations. Initially we planned for the temperature of the system to be servo-controlled to the system's null CTE temperature by placing peltiers on the outside of the outermost shield (shield 1). This would make the cavity virtually insensitive to temperature changes. Following thermal modelling and tests in vacuum, it is decided that the amount of thermal isolation provided by the heat shields and the vacuum chamber is sufficient to allow the system to reach thermal-noise-limited performance without having to risk placing active heating elements inside the vacuum system. Instead, the temperature 
of the system is controlled from outside of the vacuum chamber. In addition, the laboratories at NPL are temperature controlled to $\pm 100 \mathrm{mK}$.

The shields are manufactured by Atlas Technologies, and their surfaces treated with the Emissivac surface polishing technology ${ }^{1}$ by the same company [84]. The polished plates have a very low emissivity, which helps slow radiative heat transfer in the cavity enclosure (see Section 3.3.1). Each shield consists of six $10 \mathrm{~mm}$ plates made of aluminium, joined together by a series of vented screws (Figures 3.2, 3.3). The company polished all the surfaces of the shield plates and the interior surfaces of the vacuum chamber. The finished surfaces provide less outgassing surface area and hence reduced outgassing rates (thus minimising both pressure and temperature fluctuations due to outgassing), achieving a surface emissivity of $\epsilon \approx 0.04$, with $\mathrm{rms}$ surface imperfections in the 0.2 to $0.4 \mu \mathrm{m}$ level.

The shields are designed so that the sideplates are fastened to the baseplate first, then the endplates and lastly the topplate. The topplates can then be removed with ease for quick access to the contents. Shield 1's sideplates and endplates have a lip that protrudes out $1 \mathrm{~cm}$ and allows for vertical fastening of these plates to the baseplate. This is designed so that shield 1 can be taken apart and put together without having to remove its baseplate from the interior of the vacuum chamber. The baseplates of shields 2 and 3 have a small lip that protrudes out and serves as a base for mounting the rest of the shield plates.

A small circular hole is drilled to the six endplates so that the laser beam can enter and exit the cavity (beam holes). All six beam holes have wedged laser windows attached to them to help prevent back reflections and parasitic etalons. Thorlabs's wedged laser windows have a wedged face and a flat face at an angle of $0.5^{\circ}[85]$. A simple solution to obtain a double wedged element is attaching the window to an aluminium annulus that has a wedged face and a flat face at a certain angle (Figure 3.4). The flat face of the annulus can then be attached to a socket on the endplate so that the angle of incidence of the beam in the optical surfaces is never zero. A rotatable mount is designed to attach a zero-order quarter-waveplate to the endplates of shield 3 .

Each shield is supported by three $10 \mathrm{~mm}$ glass spheres that rest on $5 \mathrm{~mm}$-deep conical cuts that are made to each of the baseplates. The mechanical contact between the aluminium and the glass spheres is a very thin ring where the spheres touch the walls of the conical cuts as they are elastically compressed against them due to gravity (Figure 3.5). As a result the thermal contact between successive shields is greatly reduced, and heat transfer via conduction is minimised. The gap between successive shields is $15 \mathrm{~mm}$, except for the baseplates where it is $4.14 \mathrm{~mm}$, given by the radius of the glass spheres and the depth of the conical cuts. All the conical cuts are vented to avoid the generation of pockets of air underneath the glass spheres. The shields need to have additional ventilation holes drilled for vacuum purposes (see Section 3.3.2).

\subsubsection{Vacuum chamber}

The vacuum chamber for this system is designed to be made of two pieces that are fastened together vertically: the bottom piece and the top piece. Each piece

\footnotetext{
${ }^{1}$ We used Atlas Technologies' Emissivac Grade 2 surface polish.
} 


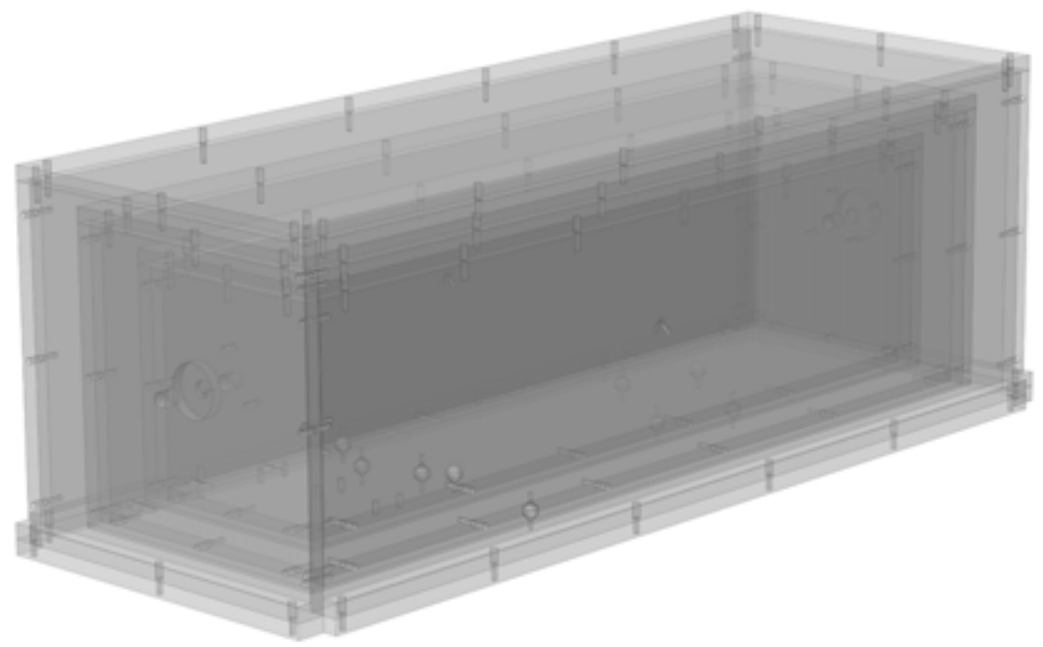

Figure 3.2: $3 \mathrm{D}$ view of the aluminium heat shields that surround the cavity using a transparency. The shields provide a great amount of passive thermal isolation. Each shield is supported by three small glass spheres that rest on conical cuts made to each of the baseplates, hence greatly reducing thermal contact between shields and minimising conductive heat transfer in the system.

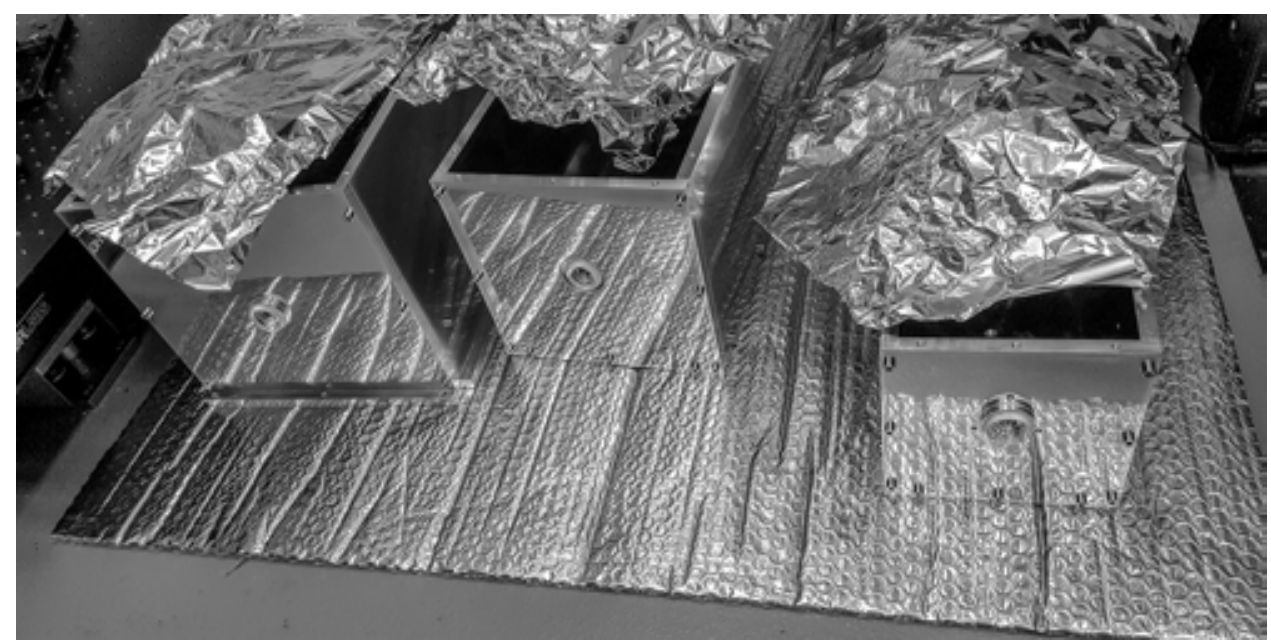

Figure 3.3: Photo of the three heat shields (without topplates nor the shield 1 baseplate) with the wedged laser windows attached to the endplates. Note the large specular reflectivity of the aluminium surfaces due to the special polishing technique to bring the surface emissivity down. 

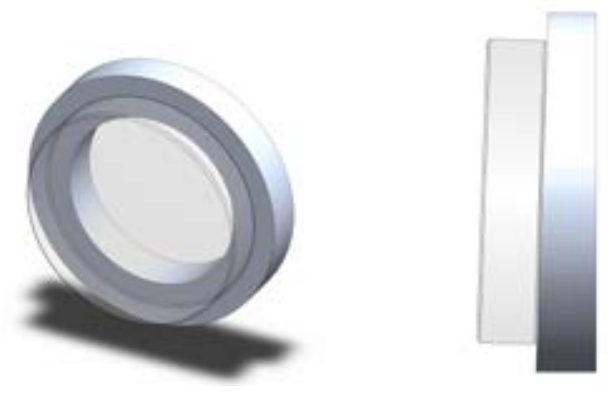

Figure 3.4: Wedged laser windows attached to a wedged aluminium annulus. These are then attached to a socket at each of the heat shields' endplates to prevent back reflections or parasitic etalons.
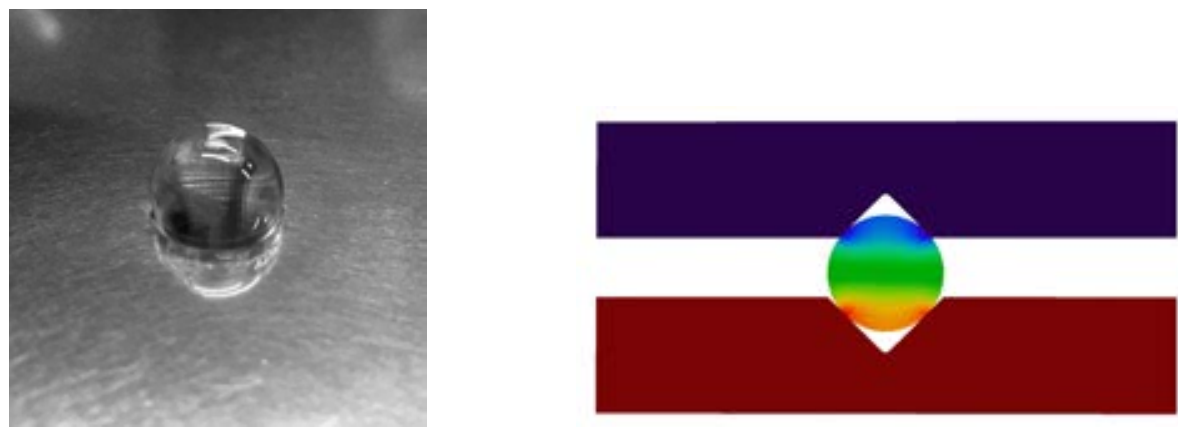

Figure 3.5: Photo of a glass sphere sitting on its conical cut (left) and a simulation showing heat transfer between the baseplates via conduction through the glass sphere (right). The only thermal contact between successive shields is through the glass spheres. Heat transfer between shields is primarily due to surface-to-surface radiation. The gap between shields is $15 \mathrm{~mm}$ for all surfaces except baseplates, where the gap is $4.14 \mathrm{~mm}$.

is manufactured from a single block of aluminium and the interior surfaces are polished to the same grade as the heat shield plates. The chamber is sealed with lead wire and pumped by a noble diode ion pump to avoid argon instability. The ion pump current is recorded and converted to pressure. As stated in Section 3.1.1, in order to achieve thermal-noise-limited performance the pressure of the system has to be stable to below the $3 \cdot 10^{-9}$ level.

The bottom piece is short and the top piece is tall so that when the top piece is removed the contents of the chamber are easily accessible. The bottom piece has three rectangular islands protruding from the floor to provide elevation for the contents and allow the feedthrough connections to go underneath. Four Atlas flush-mount bimetallic DN16CF flanges of $16 \mathrm{~mm}$ of inner diameter are located at each side of the bottom piece for feedthroughs and other connections. The bottom piece weighs approximately $23 \mathrm{~kg}$ and rests on the AVI system, which in turn rests on the floor. 


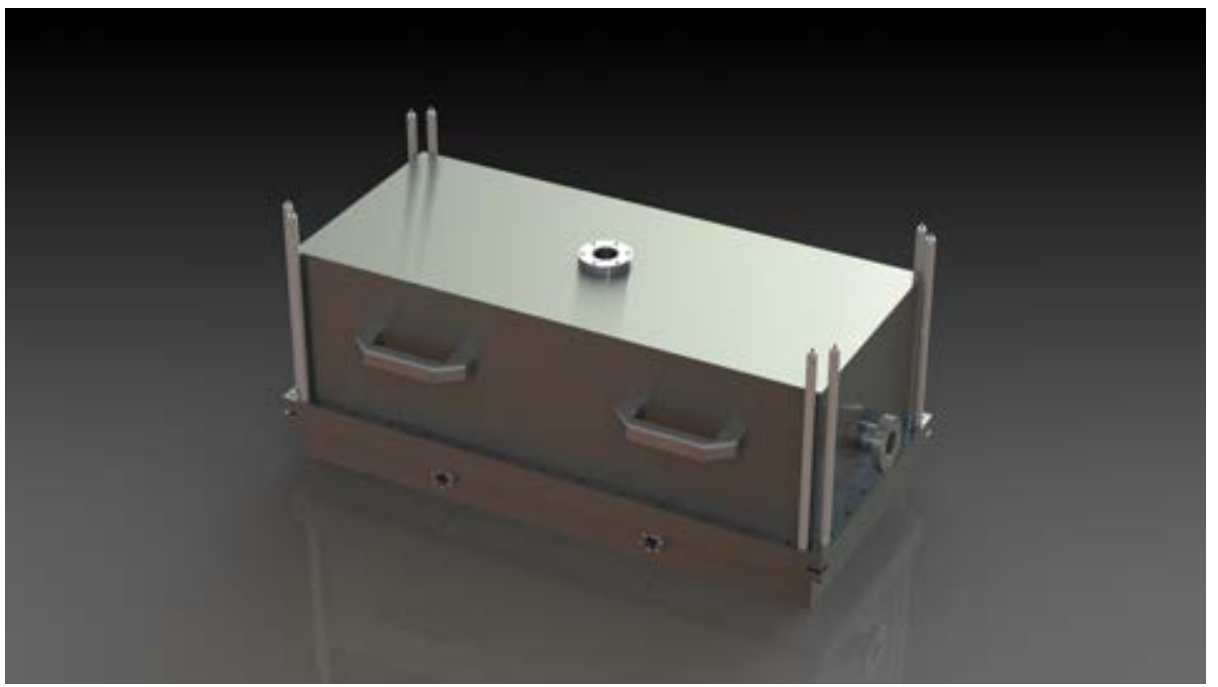

Figure 3.6: 3D rendering of the vacuum chamber as modelled in SolidWorks. The chamber is made of two pieces, each machined from a single block of aluminium. The interior walls of the chamber are polished with the same technique as the heat shield plates to reduce the surface's emissivity.

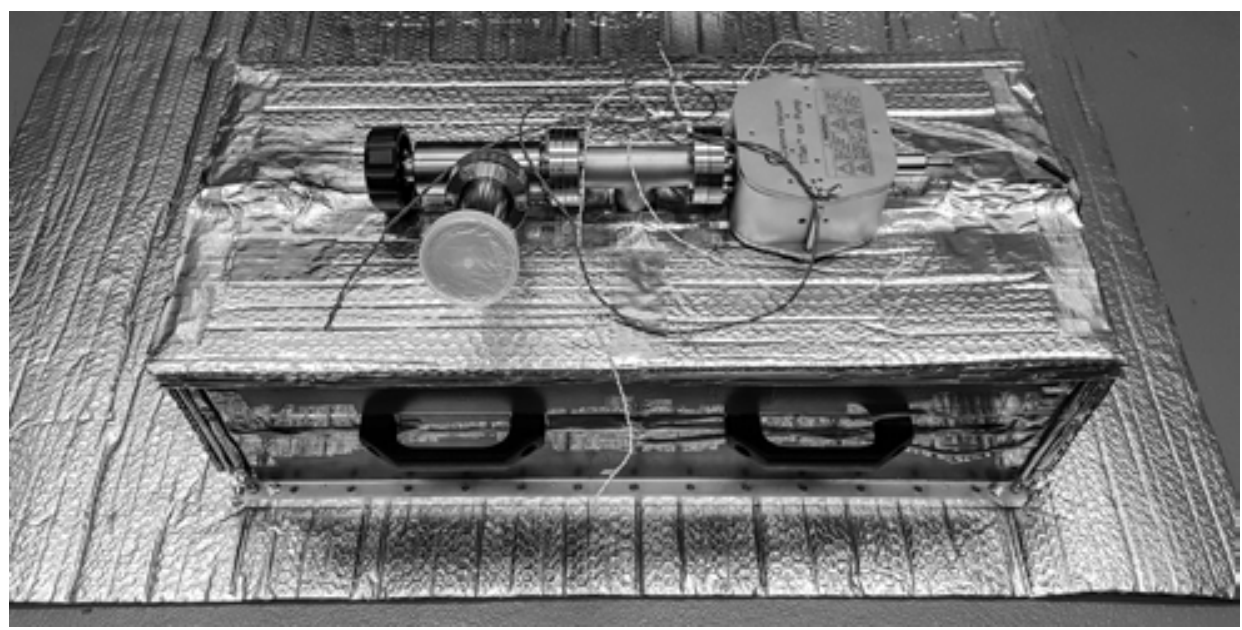

Figure 3.7: Photo of the top piece of the vacuum chamber, featuring a Gamma Vacuum TiTan 10S ion pump. The vacuum chamber is completely surrounded by a heating jacket to actively control the temperature of the system.

The lid features a flush-mount bimetallic DN40CF flange on top for vacuum pump connections. It also features two weld-neck bimetallic DN40CF flanges for the optical viewports, with the necks at a 3 degree angle to the chamber face to avoid back reflections. When lowering the lid to close the chamber, eight $30 \mathrm{~cm}$ long 
Thorlabs posts serve as a guide to align the two pieces together and avoid contact with the contents (two posts at each corner are fastened to the bottom piece and go through clearance holes in the top piece). The top piece weighs around $40 \mathrm{~kg}$ and requires two people to handle it; two handles at each side are included for this purpose.

The inside corners of both pieces have a $1.05^{\prime \prime}$ radius to facilitate material removal during machining. Both pieces have a $2 \mathrm{~cm}$ lip for fastening with M6 screws, and the gasket-lay is polished for a lead wire seal. The top piece's lip has an M6 tapped hole in every corner for force-separation of the two pieces; a small cut into the corner of the bottom piece's lip is made so that a piece of stainless steel can be placed to protect the aluminium during a force-separation procedure.

In a completely sealed vacuum system, changes in pressure are attributable to outgassing of elements inside the system. To reduce this problem the components inside the vacuum system are cleaned in order to reduce outgassing rates. Each shield plate, screw, glass sphere, and all parts of the cavity, cavity mount and

\subsection{Heat transfer in the cavity enclosure}

\subsubsection{Thermal modelling}

A finite element model of the heat shield system is developed using COMSOL Multiphysics [75]. To greatly simplify the simulations, no screws or screw holes are considered; the model consists only of the three aluminium shields and the nine glass spheres that make up the thermal isolation system. Due to the vacuum system and the minimal thermal contact between the shields, radiative heat transfer from surface to surface becomes the predominant means of heat transfer in the system.

To test this, we run a simulation in which we apply a temperature change to the outer shield (shield 1), and measure the response of the inner shields (shields 2 and 3). We ran simulations including and ignoring radiative effects (Figure 3.8). The shield 1 to shield 3 time constant in the simulation with no radiative heat transfer (only conduction) is $\approx 21$ days, whereas when considering both radiative and conductive heat transfer, the time constant is $\approx 6$ days, more than 3 times shorter. Surface to surface heat radiation is evidently playing the major role in heating the system. See Figure 3.9 for the variation of the time constant with shield plate thickness. The $10 \mathrm{~mm}$ shield plates are already quite thick, and the system is very heavy, so we decided to stick with that value.

With the system being dominated by radiative heat transfer, we have two concerns: $(i)$ heating on the mirror via radiative transmission through the beam holes, and ( $i$ ) radiative transmission through the shields' ventilation holes. The second concern is addressed in the next section. To investigate the first concern we include the vacuum chamber in the thermal model and measure the average temperature on the back surface of the mirrors after a temperature of $+1 \mathrm{~K}$ is applied to the outer surface of the $2 \mathrm{~cm}$ thick chamber walls. We do this as we vary the beam hole radius to enlarge or reduce the aperture the radiation needs to pass through to impinge on the mirror. As expected, the mirrors heat up faster as the beam hole diameter increases, but the effect is rather slow compared to the overall heating of the cavity (Figure 3.10). The beam hole radius should then be 


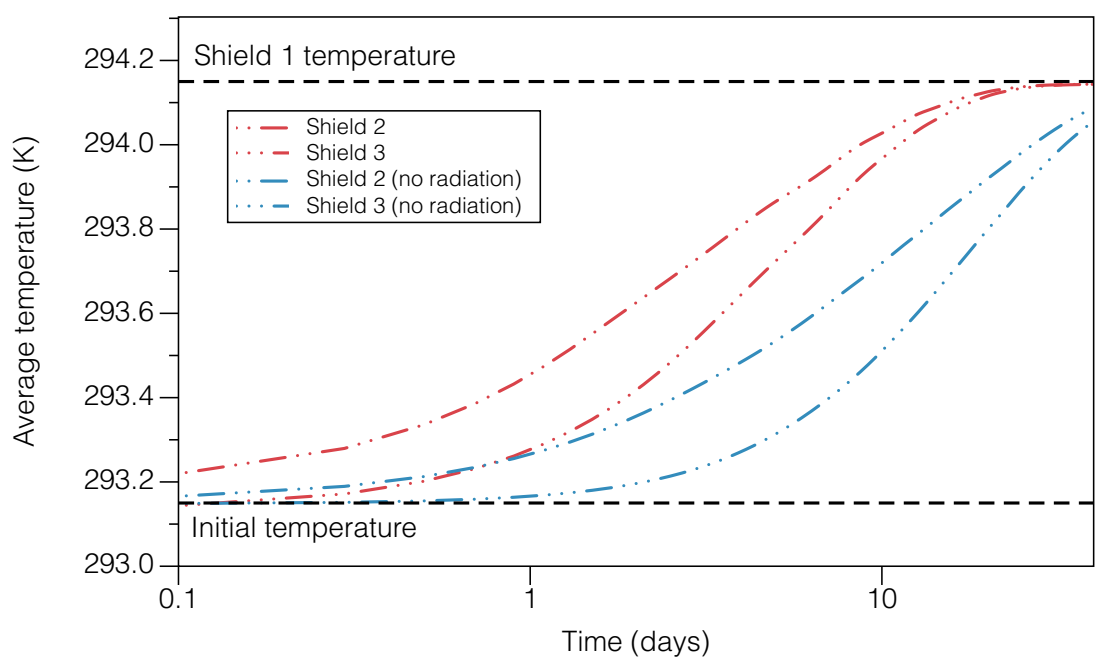

Figure 3.8: Simulated temperature response of shields 2 and 3 over time after a temperature difference of $+1 \mathrm{~K}$ is applied to the outer surface of shield 1 . The blue curves represent a simulation considering only heat conduction through the thermal contacts between shields (i.e., through the glass spheres). The red curves include the effect of surface-to-surface radiative heating, leading to a much faster response.

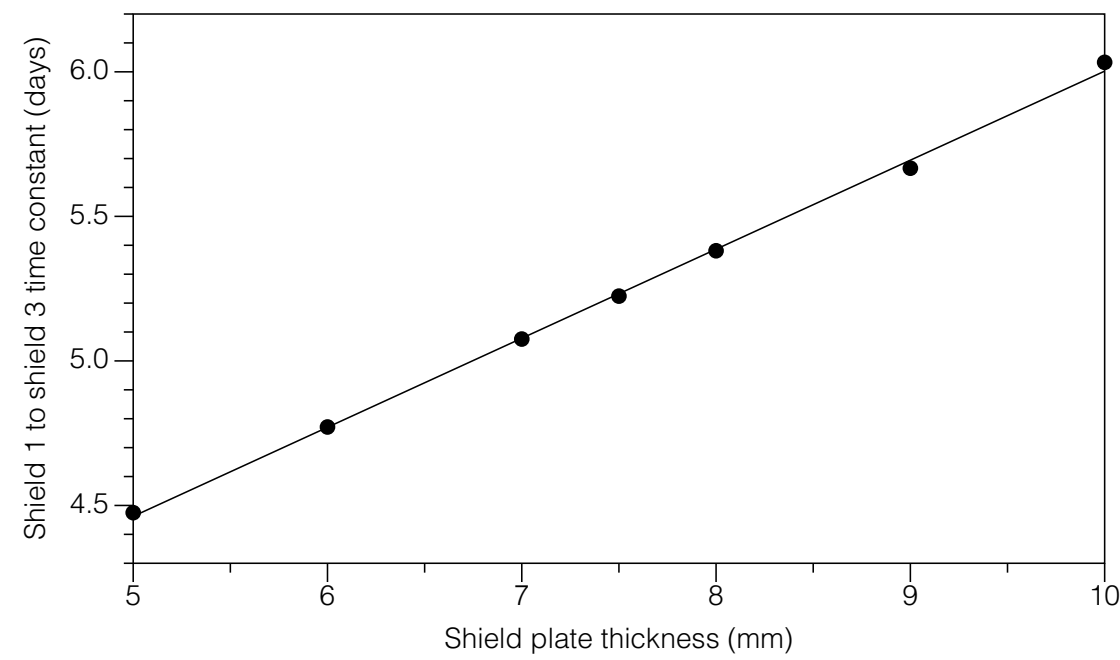

Figure 3.9: Simulated variation of the shield 1 to shield 3 time constant with the thickness of the aluminium plates, showing a linear trend. We use $10 \mathrm{~mm}$ thick plates, resulting in a time constant of about 6 days. 


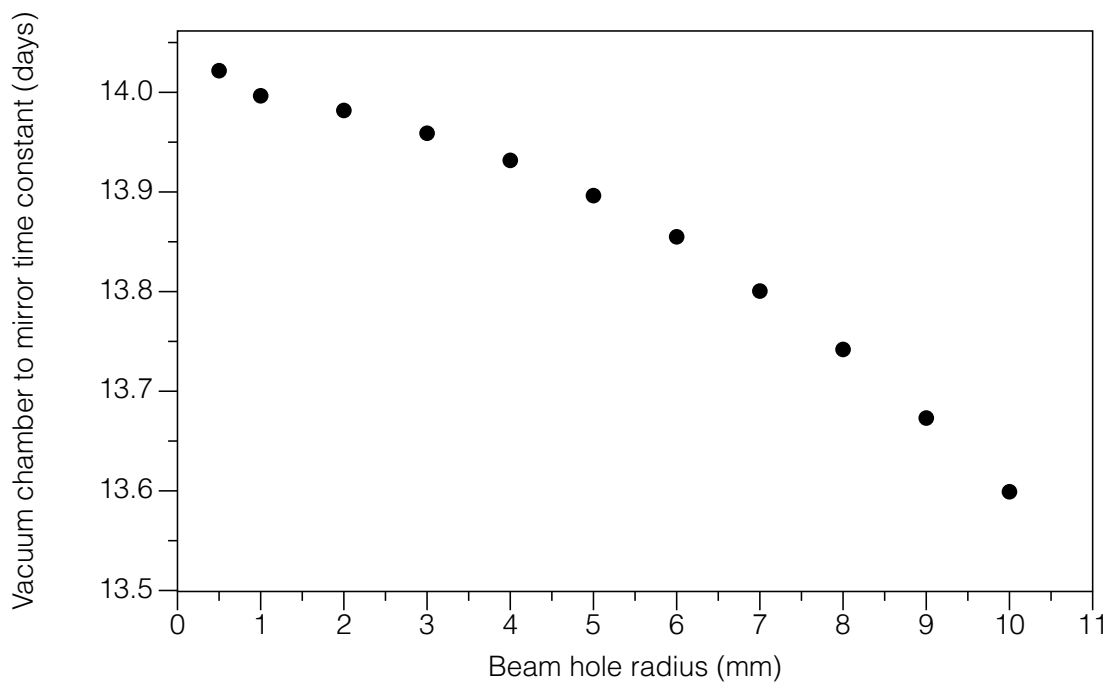

Figure 3.10: Simulated variation of the vacuum chamber to mirror time constant as a function of the beam hole radius. One of the main concerns while designing the heat shield system is that the radiative heating of the cavity would not be uniform, but rather faster on the cavity mirrors due to the beam holes at each of the shields' endplates. We find, however, that this is not the case, even for rather large beam holes.

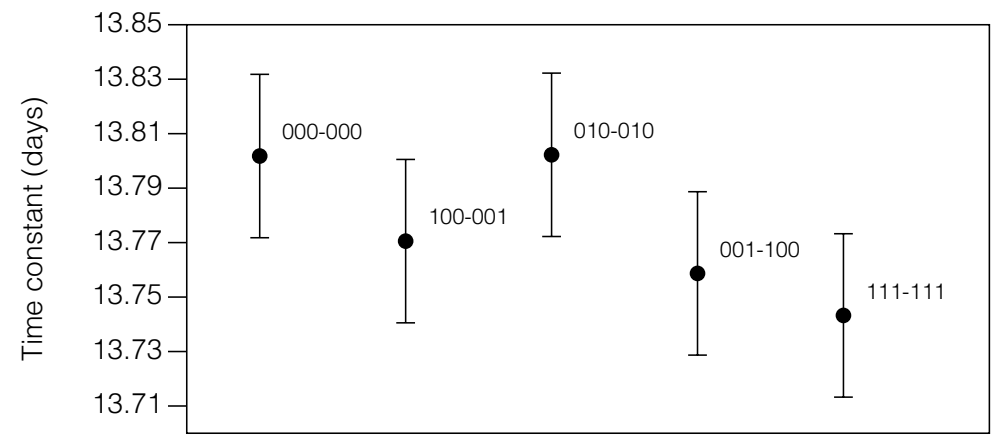

Figure 3.11: Simulated variation of the vacuum chamber to mirror time constant for different configurations of the laser windows. The number next to each point indicates the window configuration: each digit represents an endplate socket, with a 0 indicating that there is no window in the socket, and a 1 indicating otherwise. For example, $100-001$ represents a simulation where the windows are placed only in the outer shield's endplates; $001-100$ a simulation with the windows placed only in the inner shield, etc. The error bars are obtained from fitting the temperature response of the back surface of the mirror. The variation of the time constant is negligible in all cases. 
made as small as possible, but we decide to go with $5 \mathrm{~mm}$ in order to be safe from the tolerances of the cavity mounting mechanism. We are also interested in the effect that including the wedged laser windows has on the heating of the mirror. We simulate the following configurations: $(i)$ windows in shield 1 only, $(i i)$ windows in shield 2 only, (iii) windows in shield 3 only, ( $i v)$ no windows, $(v)$ windows in all shields. In order to reduce computation time the windows are modelled as simple N-BK7 cylinders filling the endplate holes. The simulations show negligible variation in all 5 configurations, indicating that the windows are effectively invisible to the passing radiation (Figure 3.11 ).

\subsubsection{Radiative heat transfer through the ventilation holes}

The three heat shields need additional ventilation holes drilled to them for vacuum purposes. This will of course have an impact on the system's thermal response, which is dominated by radiative heat transfer. Therefore, in order to obtain the best possible passive thermal isolation, the amount of vent holes and their size should be minimised, as far as the system meets the pressure requirements described in Section 3.1. We wish to determine, however, if it is possible to minimise radiative heat transfer through the vent holes by having them drilled in ways other than simply straight through. We suspect that this optimisation will not make a significant improvement to our specific system's overall thermal response, as our shields will not need many holes in order to be properly vented, and their size will be small anyway. However, we carry on to answer this question for the sake of other thermal isolation systems currently being developed at NPL that might benefit from the results of this investigation.

A

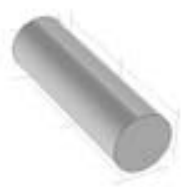

B

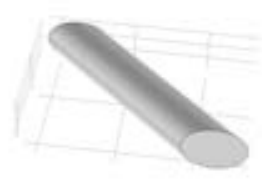

C

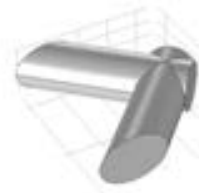

D

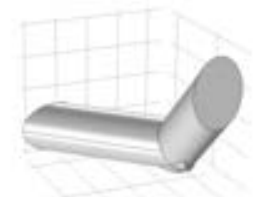

Figure 3.12: Hole geometries under consideration. Type A: straight hole; type B: 45 degree hole; type C: 45 degree hole with 90 degree horizontal turn; type D: 45 degree hole with 90 degree vertical turn. Note that the type D hole has its entry and exit boundaries at different heights of the heat shield plate it is drilled to, as opposed to the other geometries.

We consider four different hole geometries that can be easily be machined inhouse to any of the shield plates (Figure 3.12). Geometries A (straight hole) and B (45 degree hole) involve just one drilling direction. Geometries C (45 degree hole with 90 degree horizontal turn) and D (45 degree hole with 90 degree vertical turn) involve drilling from two directions, one from each side of the plate. We develop finite element models of each of the proposed holes in COMSOL using the Mathematical Particle Tracing module.

The model solves for the spacetime trajectories of a set of particles emitted 


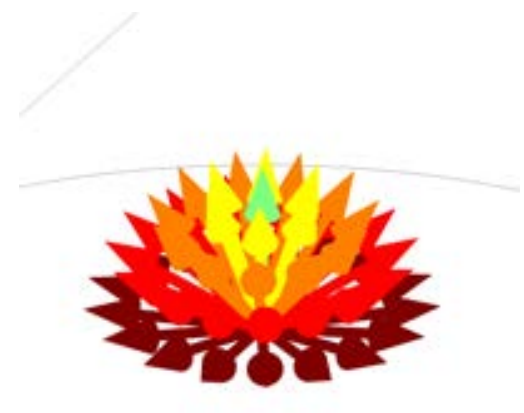

Figure 3.13: Emission of 50 particles from a single point (colour represents emission angle). In the average simulation $10^{4}$ particles are emitted from a grid of 100 points $\left(10^{6}\right.$ total particles).

diffusely from the hole's entry boundary. A total $N_{T}=N_{v} \cdot N$ non-interacting particles are emitted from $N$ points on a regular grid at this boundary. At each launch point, $N_{\mathrm{v}}$ particles are emitted in all directions of a hemisphere at constant speed (Figure 3.13). As a result, the model simulates the worst case scenario of the hole being thermally radiated by an infinite-plane source.

The boundary conditions at the hole walls are mixed specular and diffuse reflection, with surface emissivity $\epsilon$ and specular reflectivity $\gamma_{s}$. The emissivity equals the absorptivity by Kirchhoff's law [86]. Since the material has non-zero emissivity, it has a non-zero absorptivity as well, and a number of particles will be absorbed at the hole surface, hence contributing to the heating of the shield where the hole is located. Therefore, for hole walls with emissivity $\epsilon$ and specular reflectivity $\gamma_{s}$, a fraction $\epsilon$ of the incident radiation is absorbed and the rest reflected or transmitted. Out of the amount of radiation that is reflected, a fraction $\gamma_{s}$ will be reflected specularly and the rest will be reflected diffusely. The particles that are not absorbed will either be transmitted through the hole's exit boundary, contributing to the heating of the next shield, or back through the hole's entry boundary, hence contributing to the heating of the previous stage of the thermal isolation system. The simulation terminates when all the particles have been either absorbed at the hole walls or transmitted through the exit or entry boundaries. The hole geometry which minimises transmission through its exit boundary will be the optimal in terms of isolation, as it will help slow heat transfer in the system.

Figure 3.14 presents results of four particle tracing simulations for the type A hole using different values of $\epsilon$ and $\gamma_{s}$. Each particle's position is registered once it has frozen, meaning that it has either been transmitted out of the hole or it has been absorbed. The small "comet tail" of each particle represents its momentum vector at the time it is frozen, and the colour represents the emission angle with respect to the normal of the entry boundary, from $0^{\circ}$ (green) to $>80^{\circ}$ (dark red). Figure 3.14 a depicts a realistic scenario in which the emissivity is small, $\epsilon=0.05$, and the specular reflectivity is high, $\gamma_{s}=0.9$. Figure $3.14 \mathrm{~b}$ depicts a case with zero 
(a)

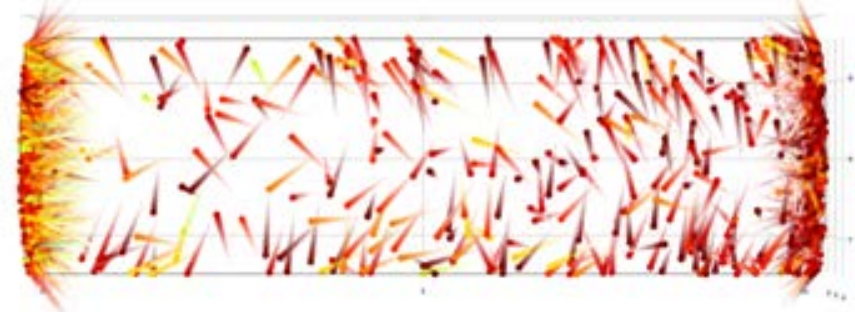

(b)

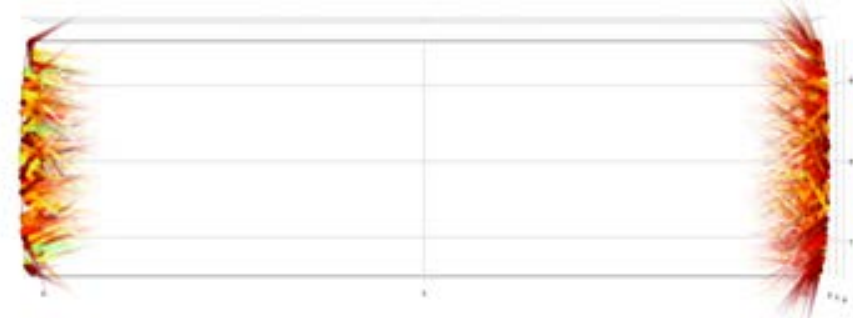

(c)

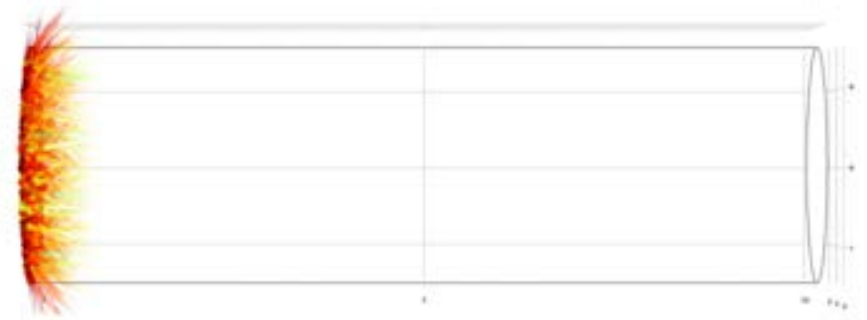

(d)

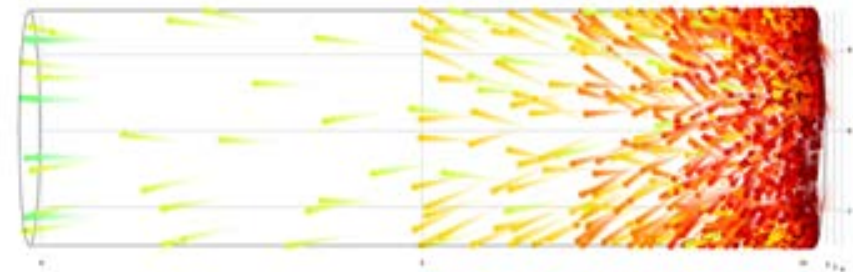

Figure 3.14: Particle tracing simulations for the type A hole geometry (dummy simulation with $N=10$ and $N_{v}=100$ for demonstration purposes only) for different values of the hole walls' emissivity $\epsilon$ and specular reflectivity $\gamma_{s}$. (a) $\epsilon=0.05, \gamma_{s}=0.9$; (b) $\epsilon=0, \gamma_{s}=0$; (c) $\epsilon=0, \gamma_{s}=1$; (d) $\epsilon=1, \gamma_{s}=$ (any). Note that in the $\epsilon=1$ case the specular reflectivity plays no role in the problem, as the probability of absorption at the hole wall is 1 . 

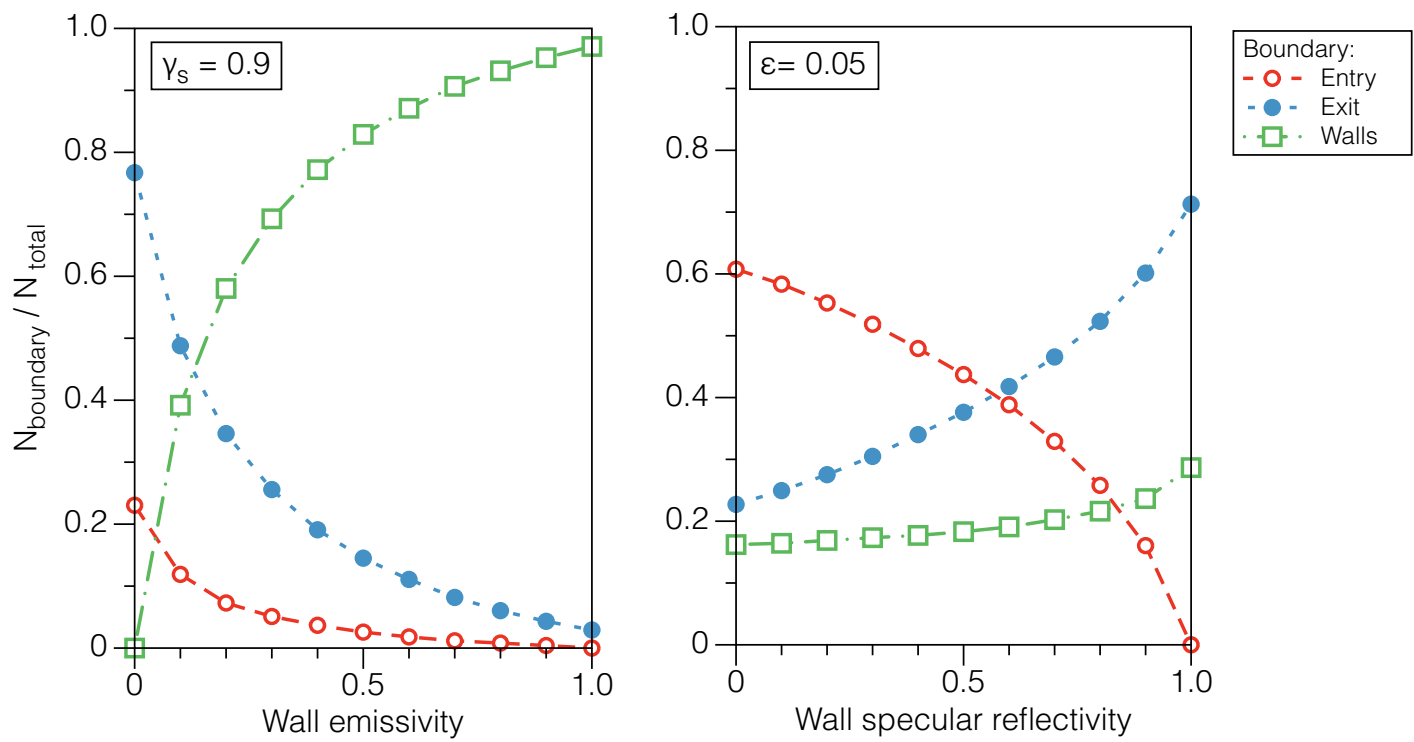

Figure 3.15: Particle statistics in the type A hole. Probabilities as a function of wall emissivity for $\gamma_{s}=0.9$ (left) and as a function of wall specular reflectivity for $\epsilon=0.05$ (right).

emissivity and where all reflections are diffuse. In this case, since the emissivity of the hole walls is zero, there is no absorption, and all the particles make it out of the hole either through the exit boundary or back through the entry. Figure $3.14 \mathrm{c}$ depicts a case with zero emissivity and where all reflections are specular, meaning that no radiation is absorbed at the walls, and because of the particularity of this geometry no particles return to the entry boundary; hence, in this case the probability of transmission at the exit boundary is $100 \%$. Figure $3.14 \mathrm{~d}$ depicts a case with emissivity 1 . In this case the probability of absorption at the hole walls is $100 \%$, i.e., whenever a particle hits the hole walls it is absorbed, and only the particles that are emitted with angles such that they travel directly towards the exit boundary make it out of the hole. Figure 3.15 shows the statistics for the type A hole simulations as a function of $\epsilon$ and $\gamma_{s}$. Figure 3.16 shows the result of a simulation of the type $\mathrm{C}$ hole.

We can see that the probability of a particle ending up being absorbed at the hole walls increases with the emissivity from 0 at $\epsilon=0$ to nearly $100 \%$ at $\epsilon=1$, as expected. The reason for this probability not reaching $100 \%$ at $\epsilon=1$ is the small probability of the particles taking a trajectory that leads directly to the hole's exit (this is specially high in the type A geometry). As the probability of absorption at the hole walls increases, the probability of transmission decrease. The particles originated in a previous stage of the system that are absorbed at the hole walls contribute to the heating of the shield where the hole is located, just as though they hit the shield directly without entering the hole. The hole with the least exit transmission probability will be the one which contributes the least to the radiative 


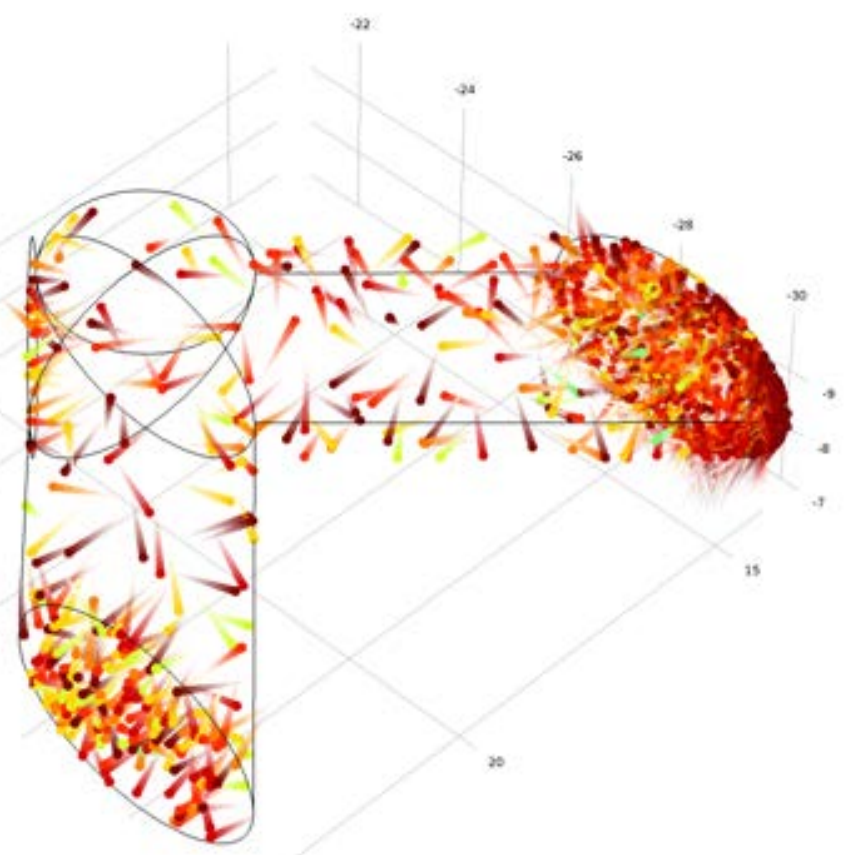

Figure 3.16: Particle tracing simulation in the type $\mathrm{C}$ hole geometry (dummy simulation with $N=10$ and $N_{v}=100$ for demonstration purposes only) with $\epsilon=0.05$ and $\gamma_{s}=0.9$.

heating of the next stage of the system.

The irradiance at a boundary within an enclosure is proportional to the specular view factor of that boundary with all other boundaries within the enclosure, integrated over the entire surface. We thus expect that the hole geometry whose exit boundary has the smallest specular view factor will be the one with the minimal transmission probability. The irradiance $H$ at a surface $A$ can be expressed as [86]

$$
H \equiv \int_{A} J(\mathbf{r}) d F_{d A d S}^{\mathrm{s}}
$$

where $J(\mathbf{r})$ is the surface radiosity and $d F^{\mathrm{s}}{ }_{d A d S}$ is the specular view factor of surface $A$, given by

$$
d F_{d A d S}^{\mathrm{s}} \equiv \frac{\left\{\begin{array}{l}
\text { Diffuse energy leaving } d S \text { intercepted by } d A \text { by } \\
\text { direct travel or any number of specular reflections }
\end{array}\right\}}{\text { Total diffuse energy leaving } d S} .
$$

where $d A$ is a surface element in surface $A$ and $d S$ is an arbitrary surface element in the enclosure. The specular view factor can be expressed as an infinite sum of diffuse view factors with different contributions for each possible direct path 


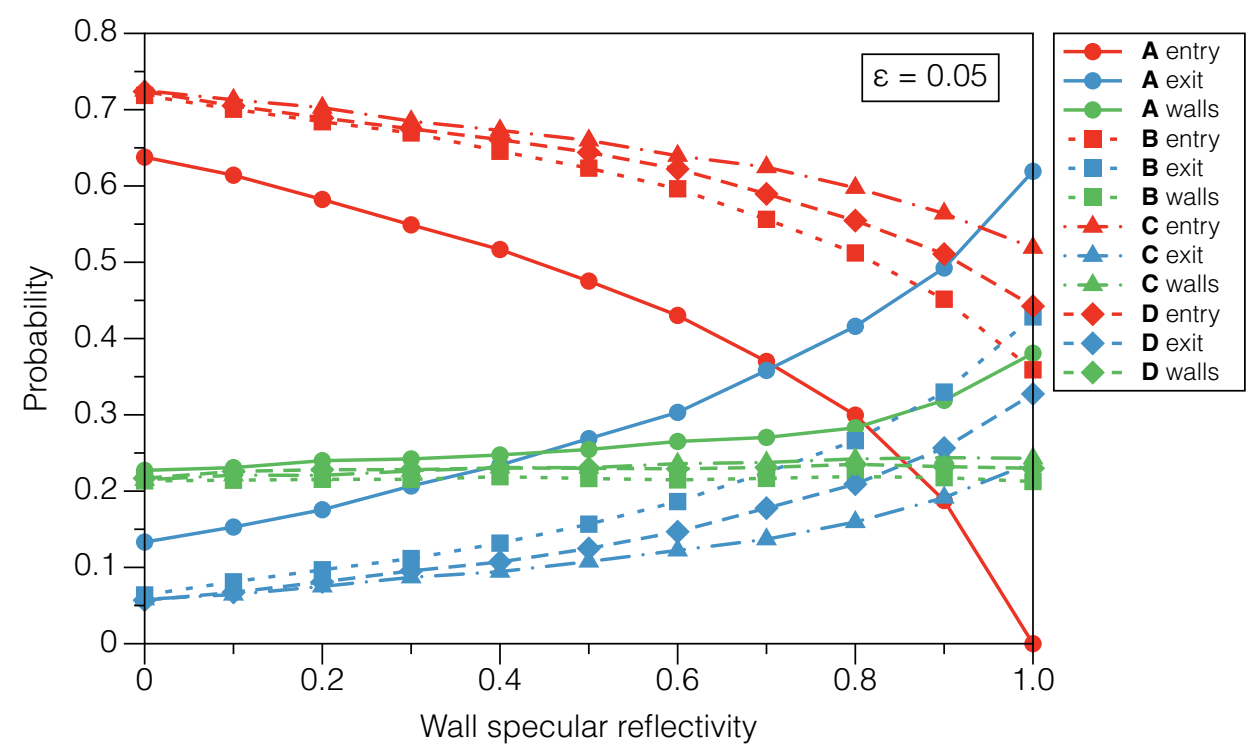

Figure 3.17: Transmission probability at the exit (blue) and entry (red) boundaries, and absorption probability at the hole walls (green) as a function of specular reflectivity, $\gamma_{s}$, for the different hole geometries with emissivity $\epsilon=0.05$. The three probabilities add up to 1 .

or reflection path. Our particle tracing method can be seen as a brute force way of determining which hole's exit boundary has the smallest specular view factor. It is possible to derive the specular view factor of simple enclosures analytically. However, our method is fast and can be applied to ever more complex geometries. A first approximation considering only the first diffuse term would point to the type $\mathrm{C}$ as the geometry whose exit boundary has the smallest specular view factor.

Figure 3.17 shows the particle statistics results for the four geometries. The probability of a particle ending its trajectory at each of the three hole boundaries (entry, walls, and exit) is presented for each hole geometry as a function of the specular reflectivity for a typical emissivity of polished aluminium $\epsilon=0.05$. Exit transmission probabilities are depicted in blue.

Note that when $\gamma_{s}$ increases the exit transmission probability increases too, and less particles make it back through the hole's entry surface. This is due to the fact that the specular view factor $d F^{\mathrm{s}} d A d S$ of the exit boundary increases with $\gamma_{s}$. Therefore, the "rougher" the walls of the holes are, the better the hole will be in blocking incoming radiation, as expected. The probability of absorption at the hole walls remains more or less constant with $\gamma_{s}$ due to the constant emissivity. The only exception to this is the type A geometry, which exhibits an increase in absorption probability for large values of $\gamma_{s}$. In this geometry when $\gamma_{s}=1$ the particles never make it back to the entry boundary, and they are either directly transmitted through the exit or will eventually arrive at the exit after a number of specular reflections. That is, the type A geometry is the only one which does not 


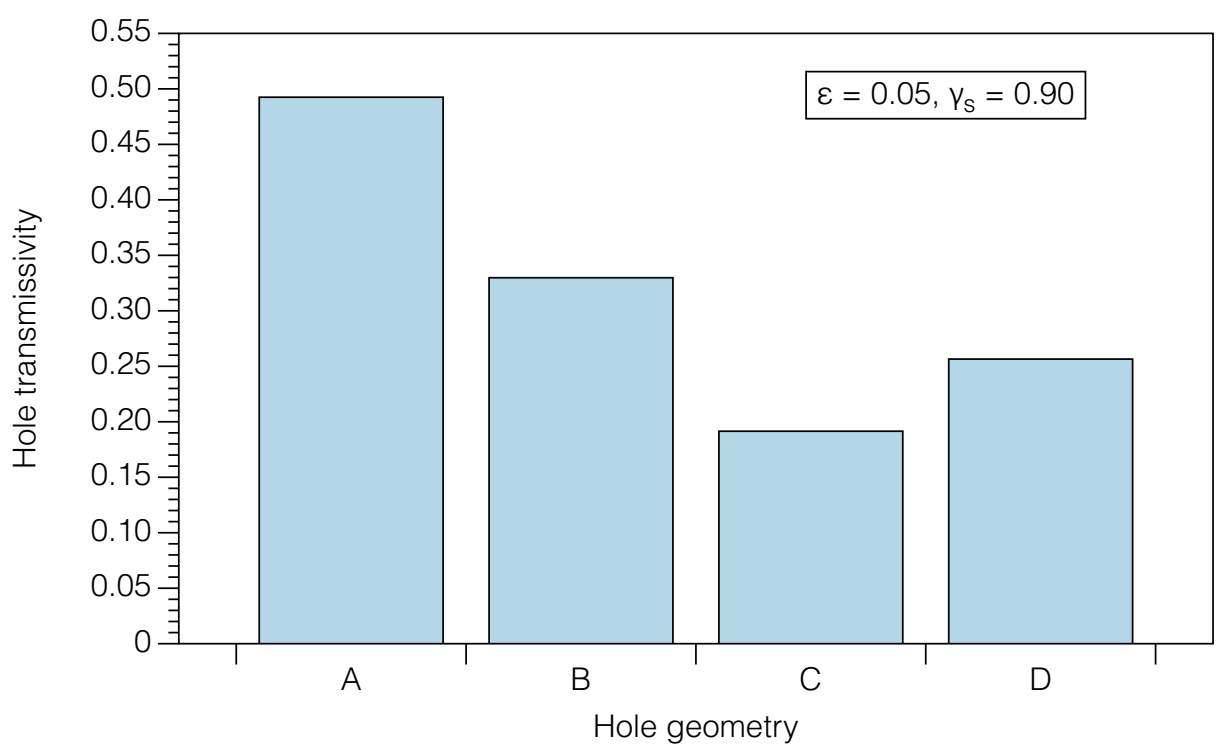

Figure 3.18: Radiative heat transmissivity for the different hole geometries.

allow specular back reflections. For $\gamma_{s}=0$ the type B, C, and D geometries have the same probabilities of exit and entry boundary transmission respectively. This is due to the effective mean distance between the entry and exit boundaries being the same in those geometries, but a bit shorter for the type A hole, which also explains why exit transmission probability is always higher for the straight hole than for any of the other types.

The type $\mathrm{C}$ geometry presents the lowest exit transmission probability throughout the whole range of specular reflectivities, and therefore is the best option for minimising radiative heat transfer in the cavity enclosure. These probabilities are compared in Figure 3.18 for the case $\epsilon=0.05$ and $\gamma_{s}=0.9$, in which the type $\mathrm{C}$ hole provides a factor $\approx 2.5$ less transmitted radiation than the straight through hole. Note that in order to drill this type of hole, the size of the drill head has to be carefully chosen. The maximum drill size that can be used to make a type $\mathrm{C}$ hole in our $10 \mathrm{~mm}$ thick plates is $3.5 \mathrm{~mm}$. Many type $\mathrm{C}$ holes could be drilled to the plates in order to vent them, and the impact on the system's thermal performance would be minimised with respect to the other hole types. 


\subsection{CAD drawings and photos of the system}

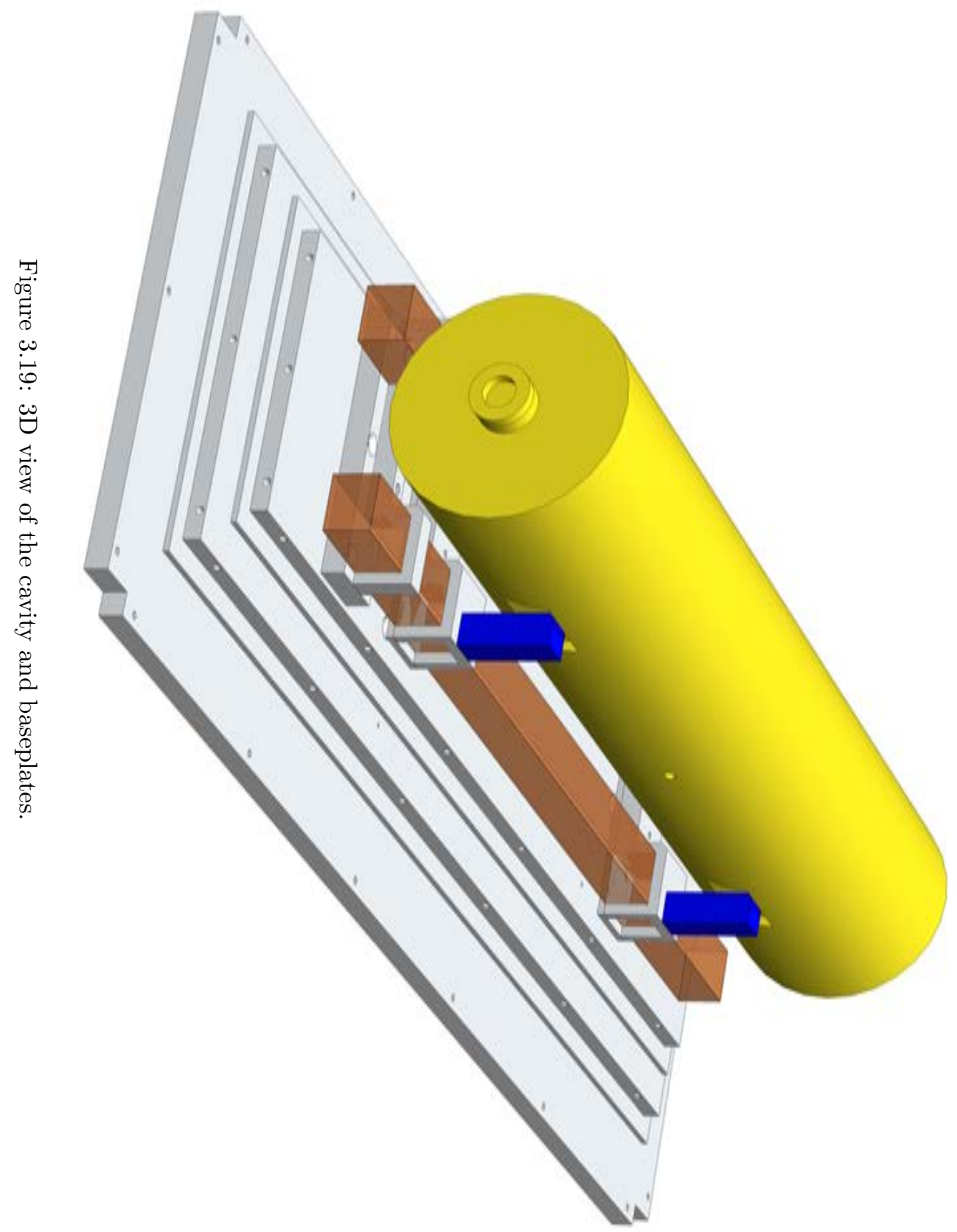




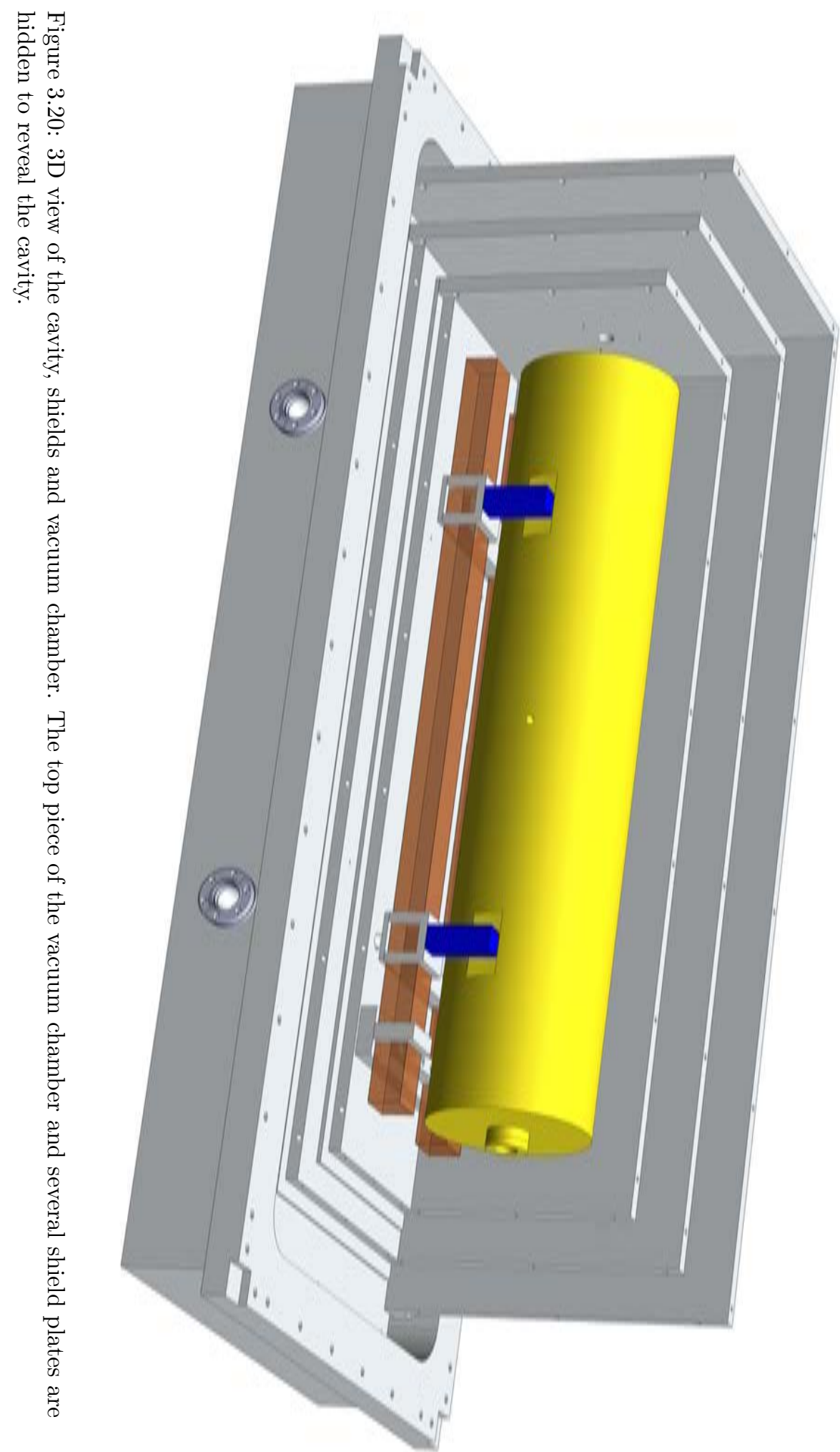




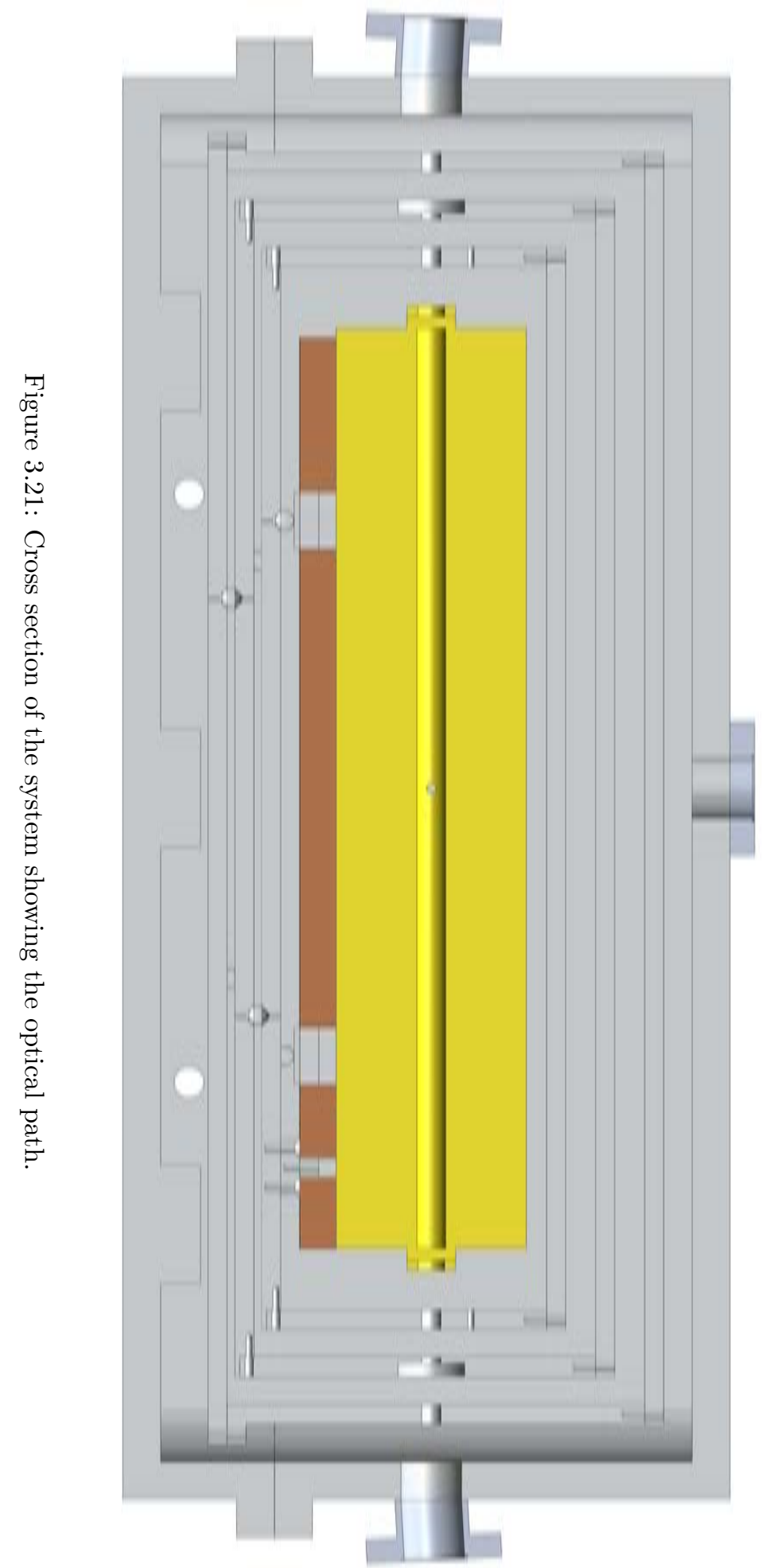




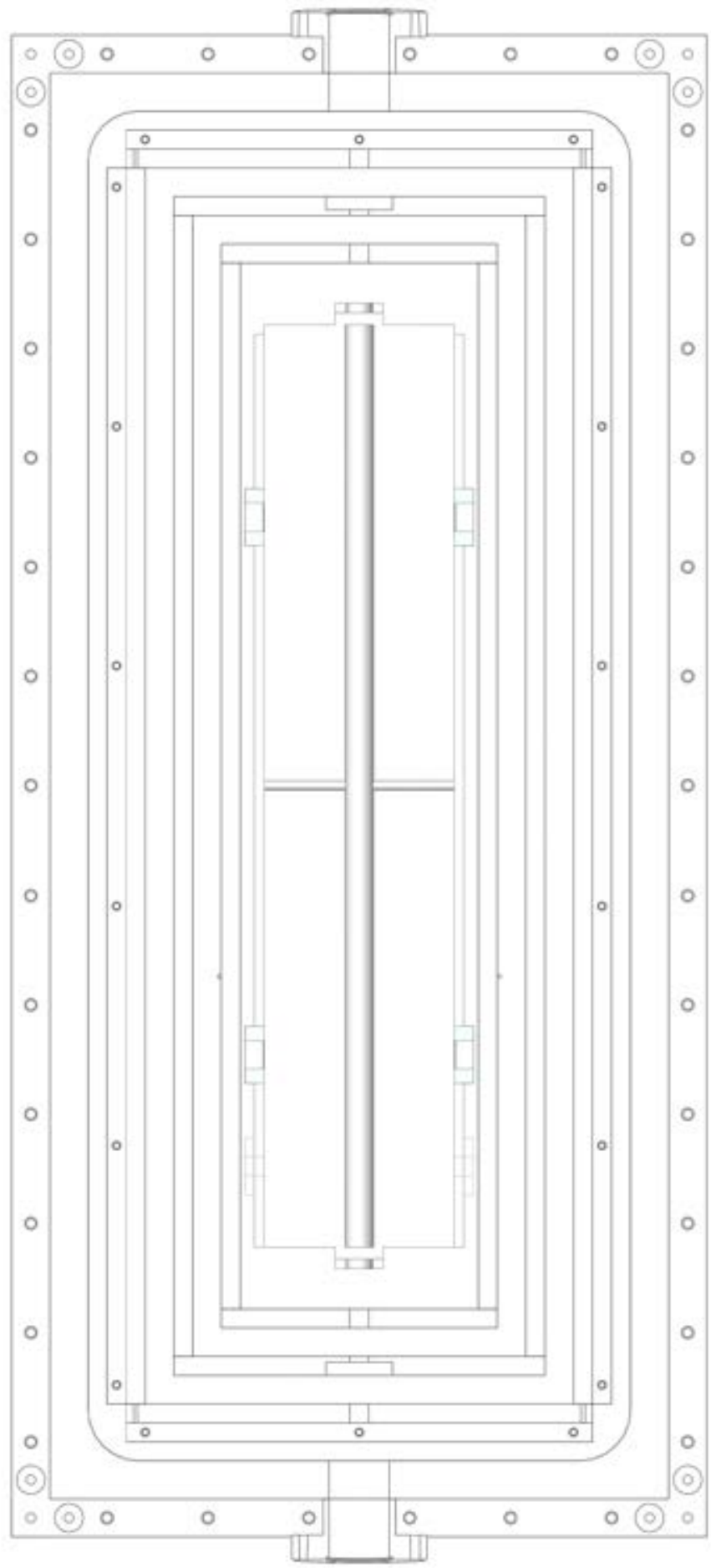

Figure 3.22: Top view of the system. 


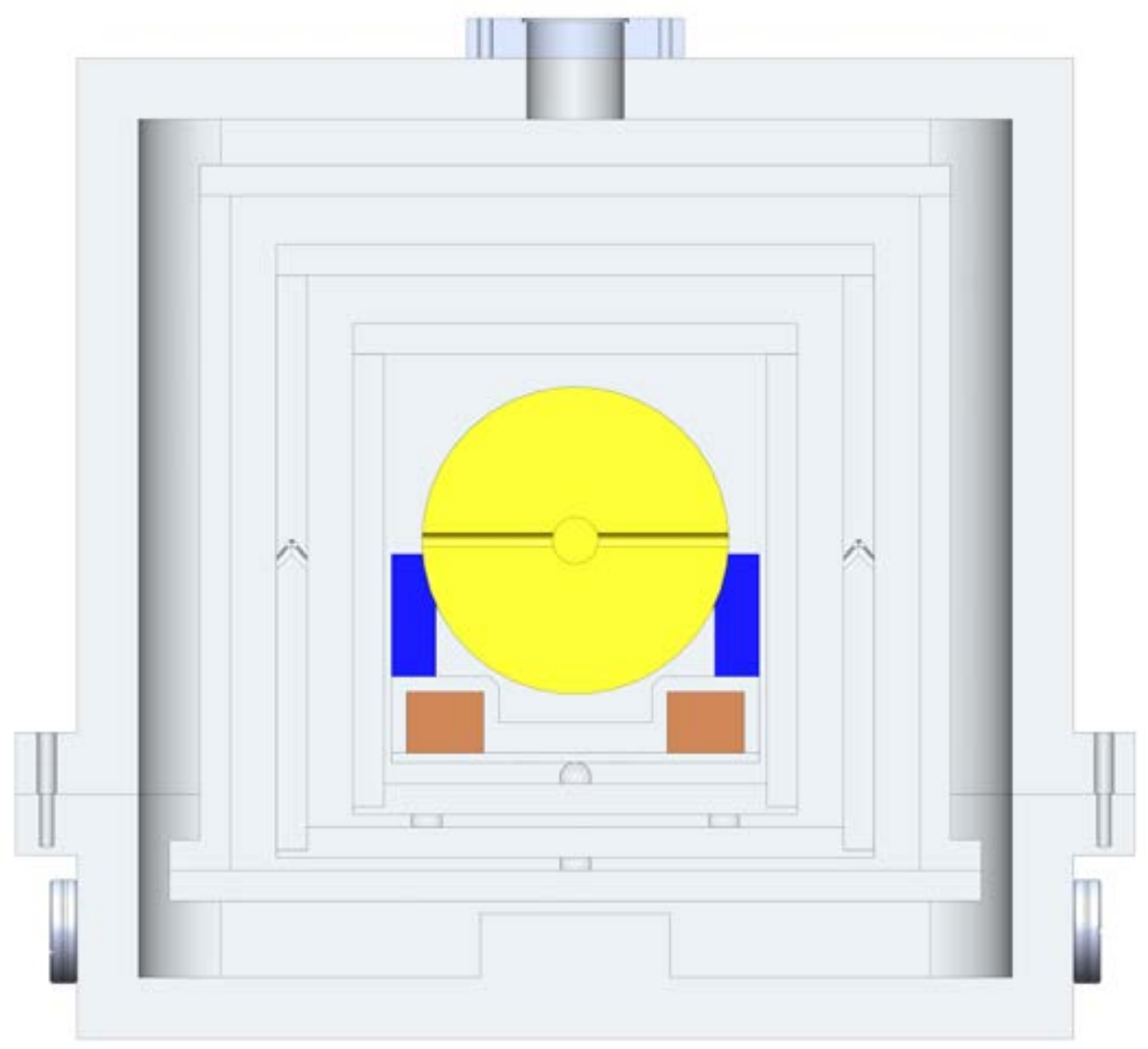

Figure 3.23: Transverse cross section of the system. 


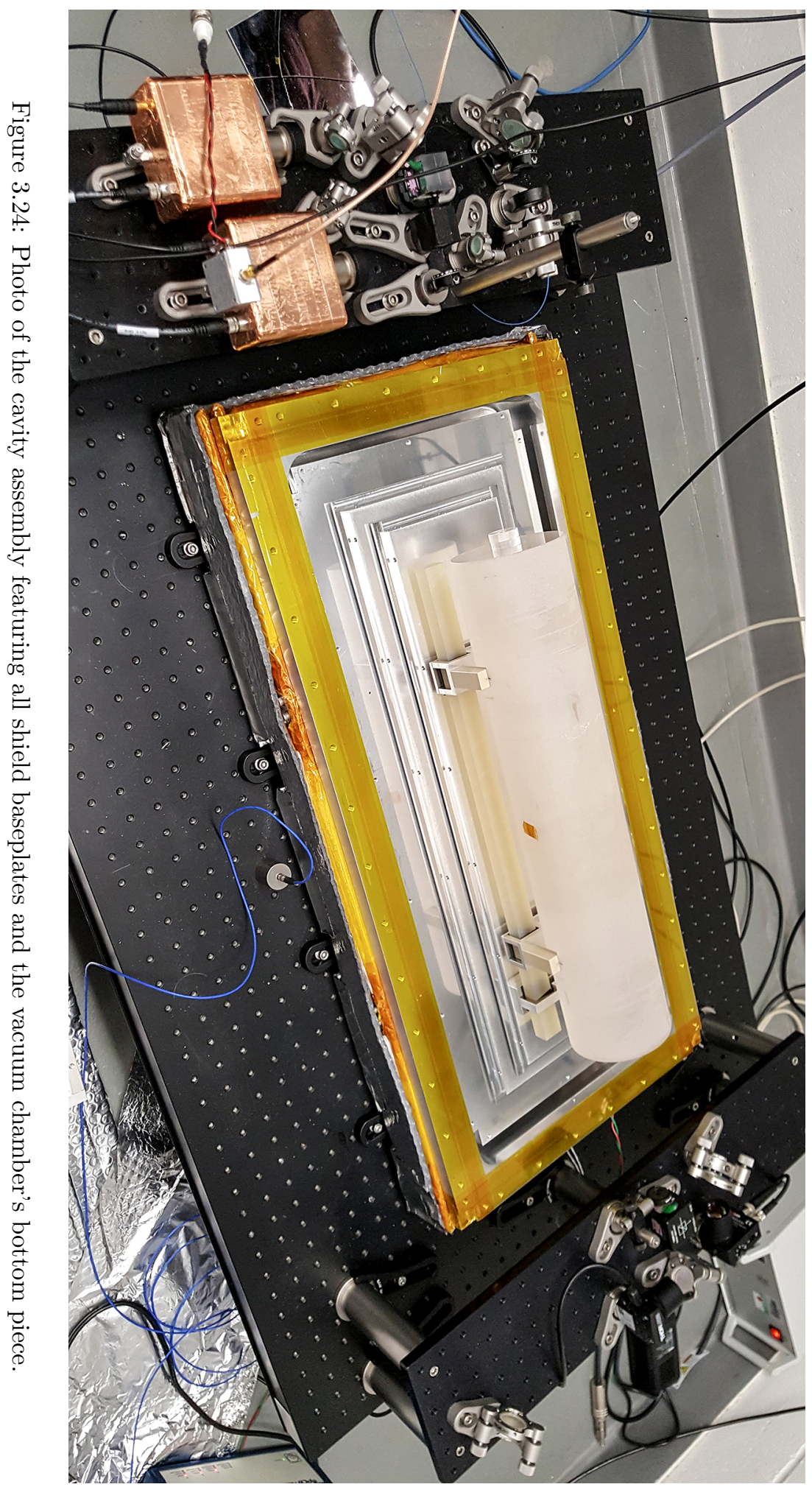




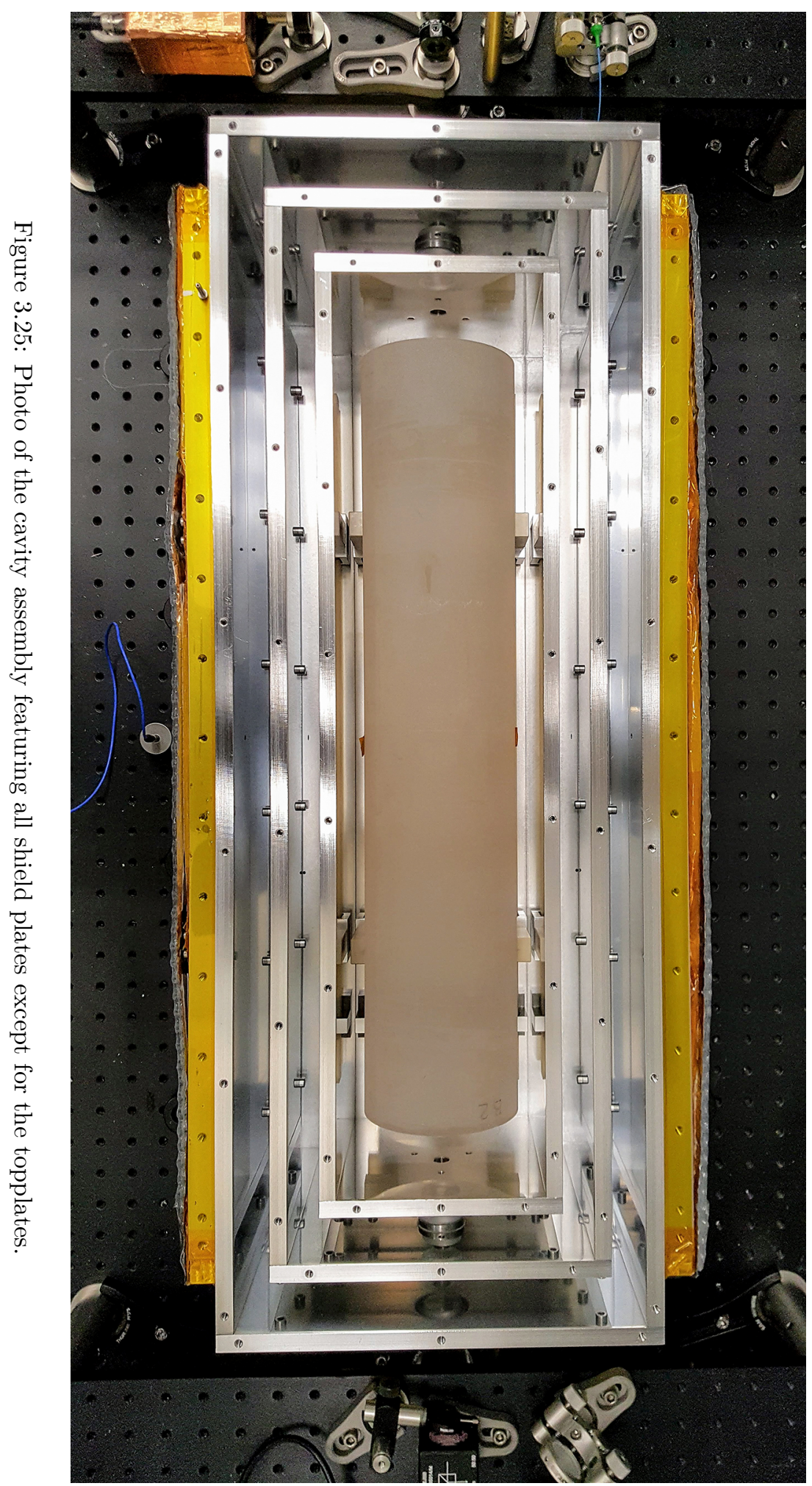




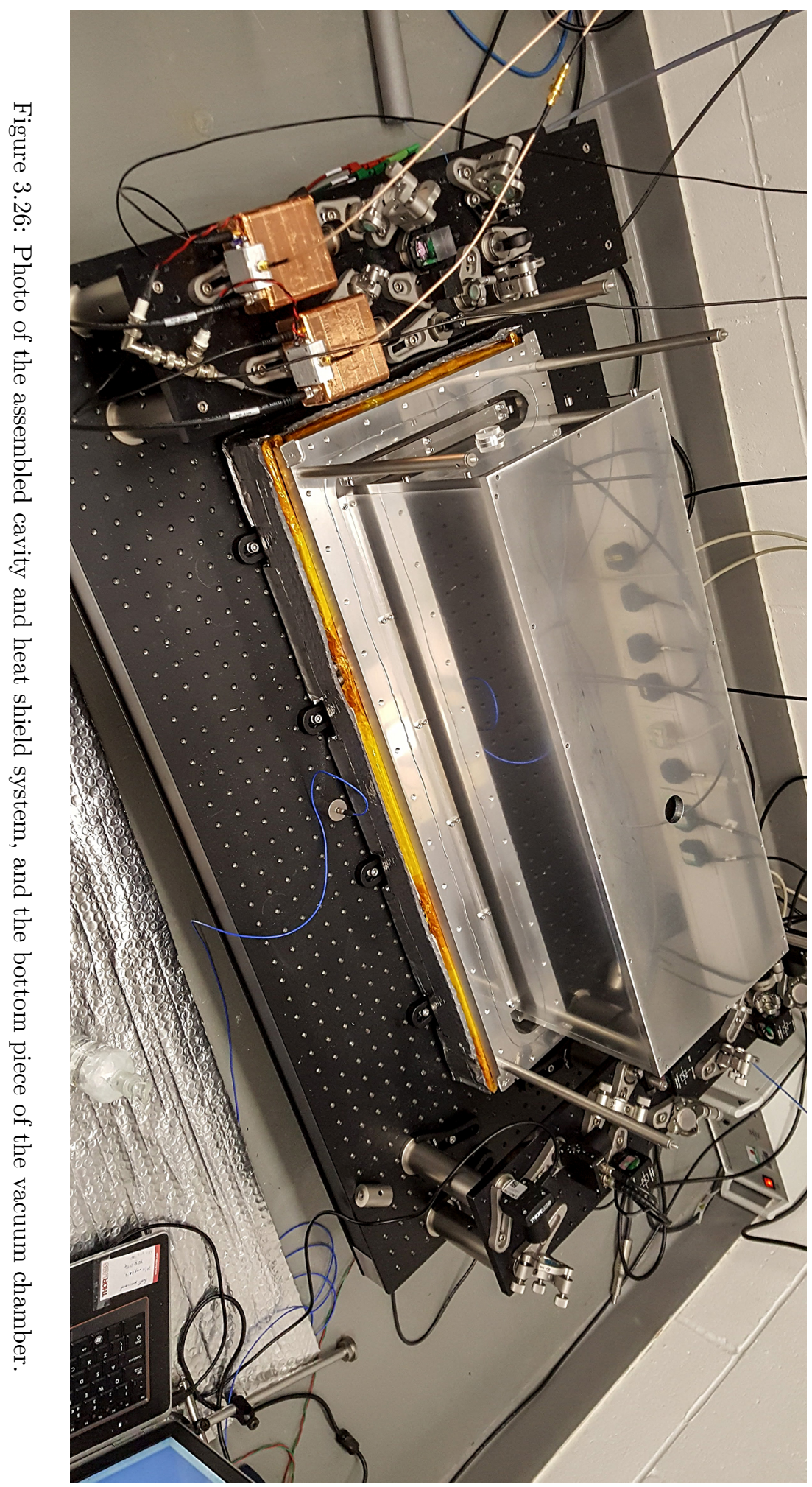



CHAPTER 4

\section{Measuring resonator stability}

Of course, the real test of the achieved performance can only be accomplished with a second, independent detector system. Disappointment is the experimenters' first reward for this measurement.

— John L. Hall [87].

\section{Contents}

4.1 Status of the experiment . . . . . . . . . . 84

4.2 Three-corner hat measurement (March 31st 2018) . . . . . 87

4.3 Comparison over fibre-link (April 23rd 2018) . . . . . . . . 91 


\subsection{Status of the experiment}

7 HE first build of the new optical reference cavity is complete and the cavity

1 is operational at NPL. A commercial Nd:YAG laser is stabilised to it, and the ultra-stable laser system is serving as a master oscillator to transfer stability through a frequency comb to the multiple microwave and optical clocks at NPL [88].

The major problem during construction of the cavity was the machining of the long spacer. We sent two blocks of ULE from Corning Inc. to Swedish company Microbas Precision AB for machining of the spacers for two identical systems. Unfortunately, the first spacer suffered from a few small cracks and dents, while the second spacer - although not imperfection-free — was returned in much better shape. We decided to build the first system using the slightly more damaged spacer, and reserve the pristine spacer for the second system. Despite the defects, the geometry of both spacers seemed to meet the dimension requirements and machining tolerances that we specified, and Microbas offered the job for free. However, with two significant chips of several millimetres in size at one of the end faces of the spacer, one near the central bore and another near the edge of the face (Figure 4.1), our main concern was that the acceleration sensitivity of the system - which was carefully investigated in Chapter 2 - would be off and unable to be compensated for experimentally.

While we have numerically determined a residual vertical acceleration sensitivity of $7 \cdot 10^{-12}\left(g^{-1}\right)$ in Section 2.5.4, by driving the AVI system at $2 \mathrm{~Hz}$ we measure a vertical acceleration sensitivity of $5.9 \cdot 10^{-10}\left(g^{-1}\right)$. This is not far off from PTB's $1.5 \cdot 10^{-10}\left(g^{-1}\right)$ obtained on a similar system [55]. We have not optimised the positions of the supports experimentally (as they do in [55]), but instead we fixed the positions to the optimal configuration obtained from the model. For a spacer

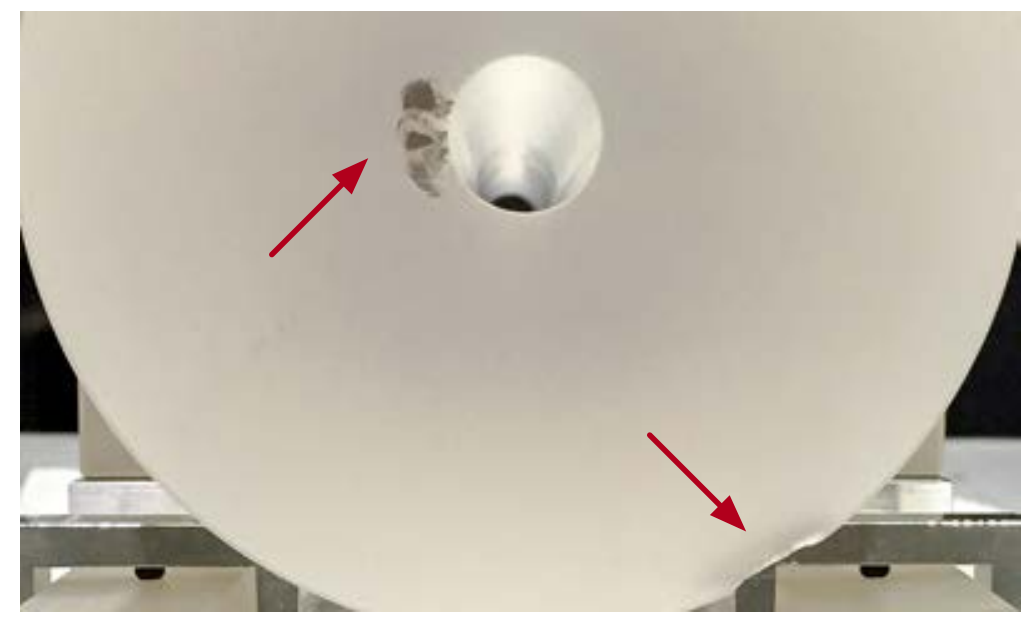

Figure 4.1: The long cavity spacer was machined from a single block of ULE by Microbas Precision AB. One of the spacers was returned with several millimetre-size imperfections. Despite this the cavity achieved acceptable acceleration sensitivity performance, allowing it to reach its thermal noise floor. 


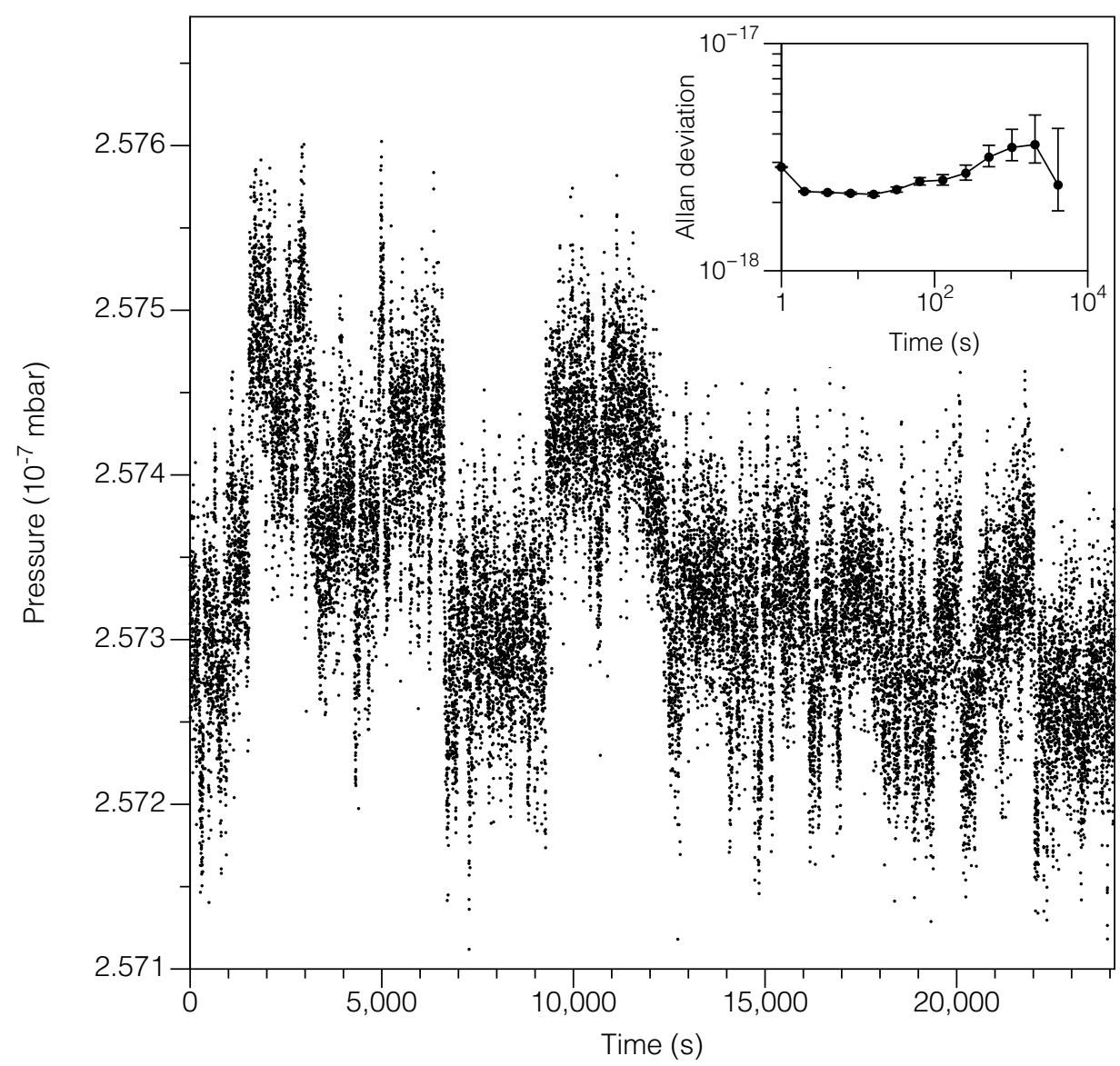

Figure 4.2: Absolute pressure at the ion pump as a function of time, converted from ion pump current. Inset: Resulting relative frequency fluctuations.

with a few un-modelled cracks and dents, and without any further experimental optimisation, we regard this measured sensitivity as a success.

The ion pump current is continuously measured and recorded, and the readings converted into pressure (Figure 4.2). The pressure at the ion pump was $\sim 2.574$. $10^{-7}$ mbar at the time of these measurements, and we measure a pressure instability of less than $3 \cdot 10^{-11}$ mbar from 1 to $1000 \mathrm{~s}$ of integration, yielding a relative frequency instability below $5 \cdot 10^{-18}$, an order of magnitude lower than the thermal noise floor. The system's temperature is stabilised to $302.970 \pm 0.001 \mathrm{~K}$ by actuating on the outside surface of the vacuum chamber, which is surrounded by a heat blanket. The vacuum chamber and heat shields act as a low pass filter for external temperature fluctuations, providing a great amount of passive thermal insulation with a characteristic response time of $\sim 21$ days.

A commercial monolithic Nd:YAG $1064 \mathrm{~nm}$ laser (Mephisto by Coherent) is frequency-stabilised to the cavity using a PDH loop. An electro-optic modulator 
is used to generate the rf sidebands for the PDH loop, with a locking bandwidth of $40 \mathrm{kHz}$ (limited by the first resonance of the laser's piezo electric transducer). The performance of the PDH loop is characterised by analysing the stability of the locking signal, yielding an Allan deviation of $1.0 \cdot 10^{-17}$, a few parts below the cavity's thermal noise floor of $\sim 6 \cdot 10^{-17}$. The residual amplitude modulation is actively stabilised [89] so that the induced relative frequency fluctuations remain below the thermal noise floor. The finesse of the fundamental mode of the cavity is approximately $1.5 \cdot 10^{5}$, as measured from optical ring down. This is lower than the $2.5 \cdot 10^{5}$ expected from the specifications of the mirror coatings, and it is thought to be due to micro-imperfections or dust in the mirror surfaces.

The absolute frequency of the laser is continuously measured and recorded using the femtosecond frequency comb systems that are used to realise the UK time scale at NPL [90]. The drift rate of the laser was monitored from the beginning of March 2018 until the end of August of the same year, and the results are presented in Figure 4.3. The drift rate is positive and is continuously and exponentially slowing down, which is consistent with the stress relaxation of the cavity spacer and optical contacts that result in an overall shrinking of the cavity. The drift rate was $700 \mathrm{mHz} / \mathrm{s}$ (slowing down by $10 \mathrm{mHz} / \mathrm{s} /$ day) at the end of March, and $150 \mathrm{mHz} / \mathrm{s}$ (slowing down by $1 \mathrm{mHz} / \mathrm{s} /$ day) at the end of August. The temperature control system experienced issues between the 15th of June and 12th of July, during which the cavity and laser system suffered some performance fluctuations.

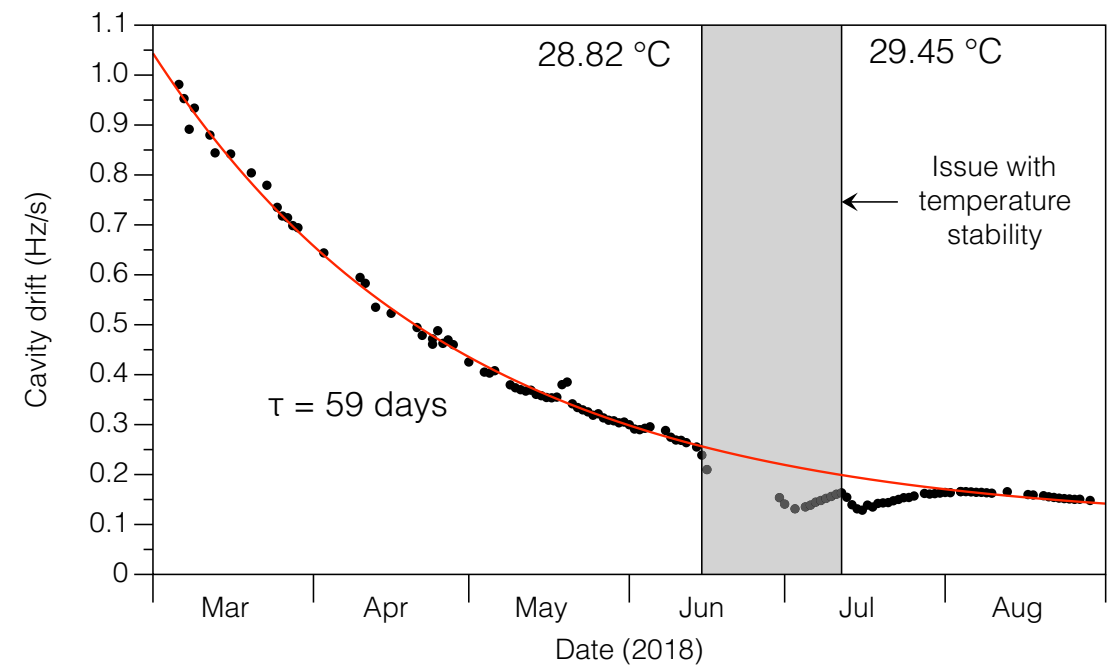

Figure 4.3: Frequency drift rate of the laser locked to the ultra-stable cavity. The rate is slowing down with a decay time of approximately 2 months, consistent with an overall shrinking of the cavity due to the stress relaxation of the ULE spacer. The temperature control system suffered issues between June and July, leading to disruptions of the drift rate. 


\subsection{Three-corner hat measurement (March 31st 2018)}

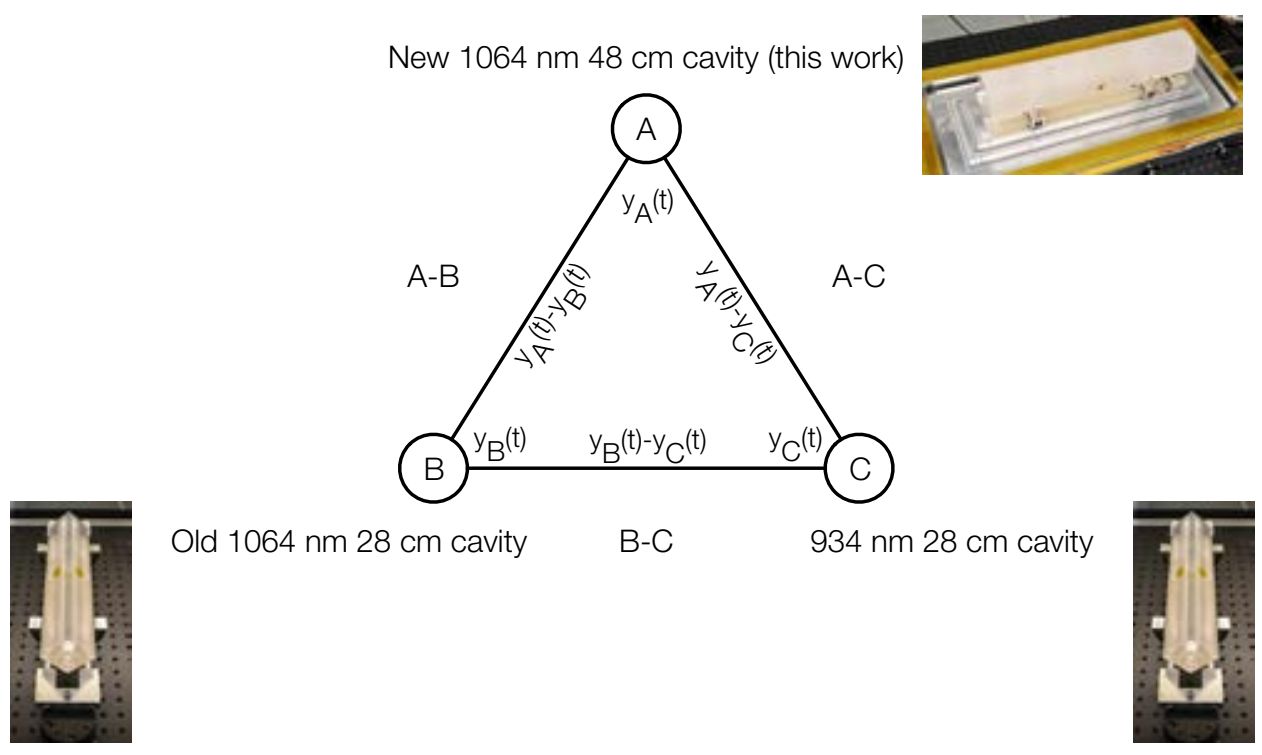

Figure 4.4: Scheme of the three-corner hat measurement performed using the new cavity and two older reference cavities. In order for this method to yield meaningful results, the beat notes have to be taken simultaneously and the three systems have to be completely un-correlated (i.e., independent vibration isolation platforms, electronics, actuators, etc).

Originally we planned to build two identical systems and measure their relative stability through beat note analysis. With identical systems we know that each system contributes half of the measured variance, so obtaining their individual variances is straightforward. Due to time restrictions this could not be achieved yet. An alternative to this is to use the so-called three-corner hat method [91], which allows us to measure the relative stability of our system by comparing it with two other reference cavities already operational at NPL (Figure 4.4). Assuming total un-correlation between the three systems, the individual variances can be separated algebraically. Let us denote with ' $A$ ' the system under test - our new reference cavity - , and with ' $\mathrm{B}$ ' and ' $\mathrm{C}$ ' the two older reference cavities. Since the three systems are completely different, and they are mounted on completely independent vibration isolation platforms, we can assume that their signals are completely un-correlated, therefore

$$
\begin{aligned}
\sigma_{A-B}^{2}(\tau) & \equiv \sigma_{A}^{2}(\tau)+\sigma_{B}^{2}(\tau), \\
\sigma_{A-C}^{2}(\tau) & \equiv \sigma_{A}^{2}(\tau)+\sigma_{C}^{2}(\tau), \\
\sigma_{B-C}^{2}(\tau) & \equiv \sigma_{B}^{2}(\tau)+\sigma_{C}^{2}(\tau),
\end{aligned}
$$

where $\sigma_{A-B}, \sigma_{A-C}$, and $\sigma_{B-C}$ are the Allan deviations of the beat notes of system A with $\mathrm{B}$, system $\mathrm{A}$ with $\mathrm{C}$, and system $\mathrm{B}$ with $\mathrm{C}$, respectively. The individual 
variances may be obtained algebraically as

$$
\begin{aligned}
\sigma_{A}^{2}(\tau) & =\frac{1}{2}\left(\sigma_{A-B}^{2}(\tau)+\sigma_{A-C}^{2}(\tau)-\sigma_{B-C}^{2}(\tau)\right), \\
\sigma_{B}^{2}(\tau) & =\frac{1}{2}\left(\sigma_{A-B}^{2}(\tau)+\sigma_{B-C}^{2}(\tau)-\sigma_{A-C}^{2}(\tau)\right), \\
\sigma_{C}^{2}(\tau) & =\frac{1}{2}\left(\sigma_{A-C}^{2}(\tau)+\sigma_{B-C}^{2}(\tau)-\sigma_{A-B}^{2}(\tau)\right) .
\end{aligned}
$$

A three-corner hat measurement is performed using an older $1064 \mathrm{~nm}$ cavity and a $934 \mathrm{~nm}$ cavity as the two additional references, both $28 \mathrm{~cm}$ ULE cavities. The frequency difference between the two $1064 \mathrm{~nm}$ references and the $934 \mathrm{~nm}$ reference is bridged using a fibre-based optical frequency comb as a transfer oscillator, which does not introduce noise into the measurement [90,92]. Fibre phase-noise cancellation [93] is used in all three links. These three reference cavities are the best in terms of stability at NPL to date. Three beat notes are extracted simultaneously as shown in Figure 4.4. The frequency instabilities $y_{A}(t)-y_{B}(t), y_{A}(t)-y_{C}(t)$, and $y_{B}(t)-y_{C}(t)$ of the three signals (with linear frequency drifts removed) are presented in Figure 4.5. The unit $\mathrm{C}$ seemed to introduce a number of spikes for reasons that are still unknown. In order to carry out this measurement, a short time window of $2000 \mathrm{~s}$ is selected. The short time window will result in a large uncertainty at large values of the averaging time in the Allan deviations of the beat notes, and through propagation of error this will incur even greater uncertainty in the individual deviations. Therefore, this measurement will only provide useful results at low averaging times, but this will be sufficient to have a first indication of the new system's performance. In a three-corner hat measurement, one of the beat notes can be derived algebraically from the other two. We used this fact to obtain a cleaner A-C beat note,

$$
y_{A}(t)-y_{C}(t)=\left[y_{A}(t)-y_{B}(t)\right]+\left[y_{B}(t)-y_{C}(t)\right] .
$$

which smoothes some irregularities introduced by unit C. Figure 4.6 shows the Allan deviations of the beat notes $\sigma_{A-B}, \sigma_{A-C}$, and $\sigma_{B-C}$, and the resulting individual deviations $\sigma_{A}, \sigma_{B}$, and $\sigma_{C}$, corresponding to the new $1064 \mathrm{~nm}$ cavity, the old $1064 \mathrm{~nm}$ cavity, and the $934 \mathrm{~nm}$ cavity respectively. Note the large uncertainty at averaging times $\tau>100 \mathrm{~s}$ due to the reduced number of such samples in the $2000 \mathrm{~s}$ time window used. Finally Figure 4.7 compares the relative instability of the new cavity obtained from the three-corner hat method with the relative instability of the beat note between the new cavity and PTB's cryogenic silicon cavity (measured over fibre link). The relative instability of the new cavity at averaging times between $1 \mathrm{~s}$ and $100 \mathrm{~s}$ looks very good, and the cavity seems to be operating just above its thermal noise floor. The new cavity's best performance is found at $\tau=2 \mathrm{~s}$, where the measured Allan deviation is

$$
\sigma_{y}(\tau=2 \mathrm{~s})=8.86_{-2.19}^{+2.37} \cdot 10^{-17}
$$

just a few parts in $10^{17}$ above its estimated thermal noise floor of $6 \cdot 10^{-17}$, corresponding to a laser linewidth of $\sim 25 \mathrm{mHz}$, offering a considerable improvement over previous reference cavities at NPL. The instability is below $1.5 \cdot 10^{-16}$ from 1 to 100 seconds. 

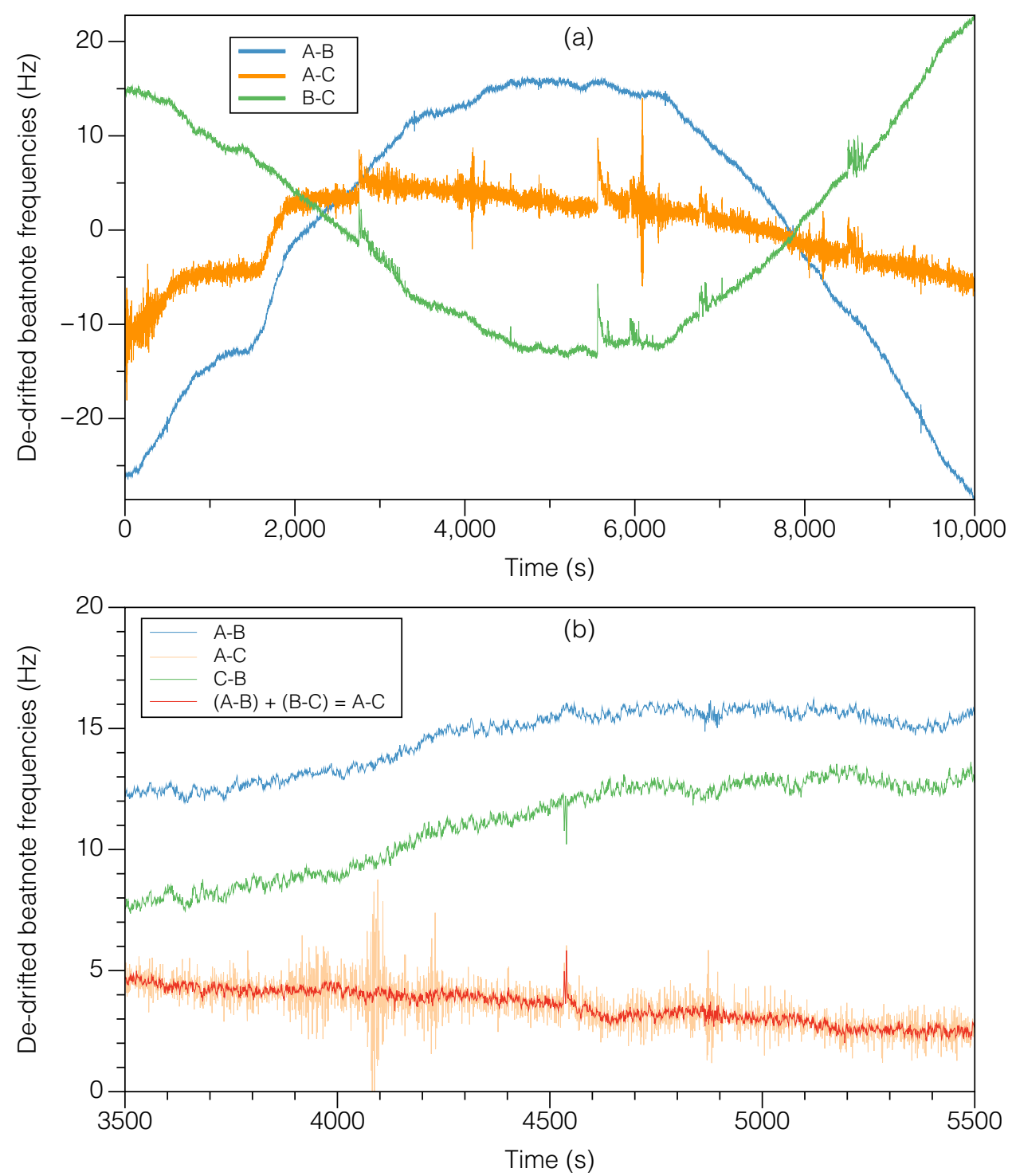

Figure 4.5: Beat notes between the three reference cavities being compared in the three-corner hat measurement (a). A $2000 \mathrm{~s}$ window is used for the measurement due to the spikes of unknown origin introduced by unit C (b). A cleaner A-C beat note can be obtained by adding the other two beat notes to create a virtual signal that is algebraically equivalent, which smoothes some irregularities introduced by unit C. 

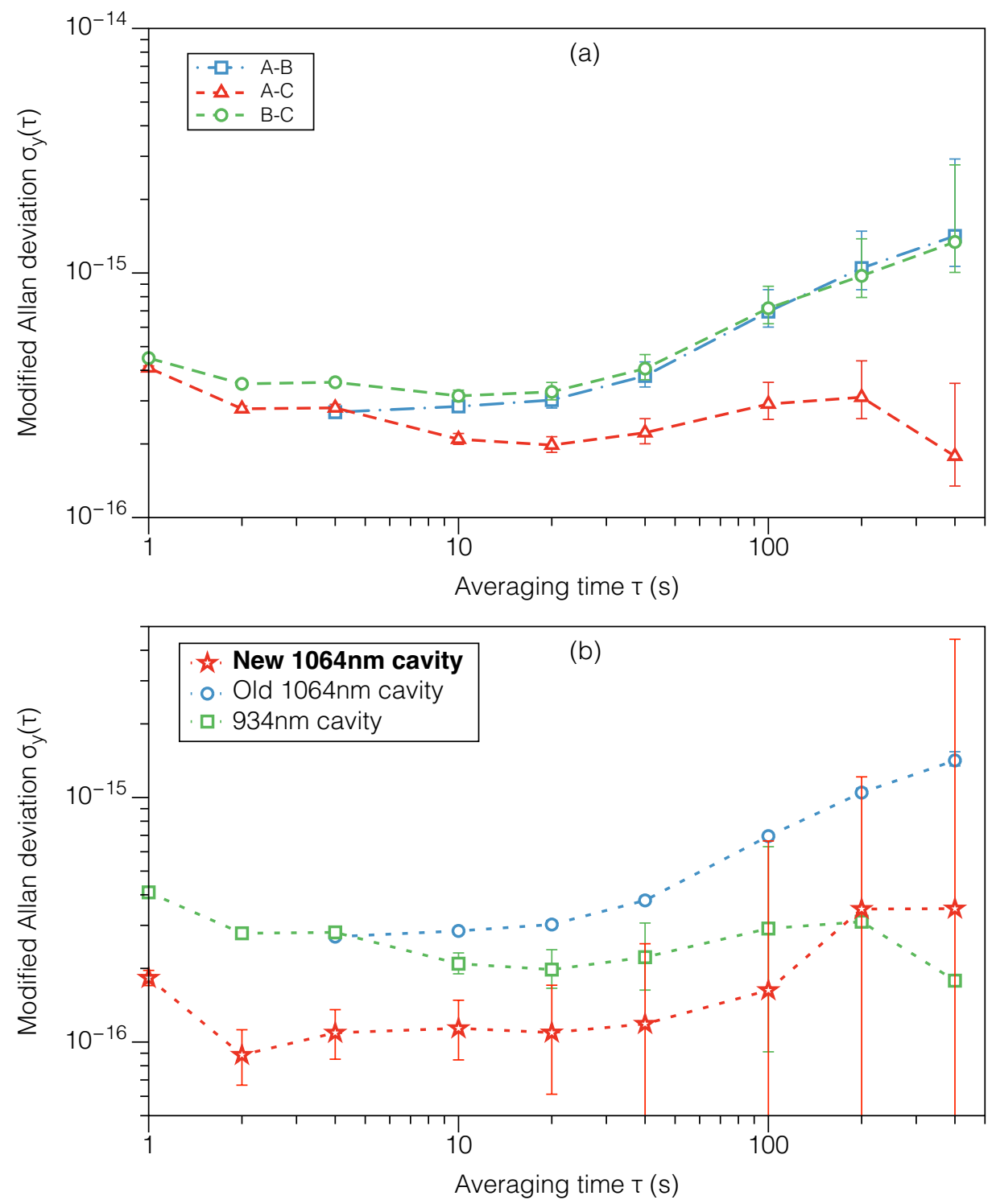

Figure 4.6: Modified Allan deviations of the three beat notes used in the three-corner hat measurement (a) and calculated Modified Allan deviations of each reference cavity. The error bars are obtained through propagation of the error of averaging. The errors are large at long averaging times due to the reduced number of samples in the small $2000 \mathrm{~s}$ time window that is used in the measurement. 
It should be noted that this fractional instability has been achieved without experimental optimisation of the support positions to minimise the system's response to vibrations. The support positions are fixed to those obtained in the simulations presented in Section 2.5.4. When building the second system, there will be room for improvement if the support positions are further optimised experimentally. Additional improvement may also be gained from using an alternative mirror combination yielding larger spot sizes on the mirrors, with the consequent improvement in thermal noise floor as detailed in Section 2.3.1. The second system will also feature a spacer with less machining imperfections.

\subsection{Comparison over fibre-link (April 23rd 2018)}

Another method to assess the stability of the system is to compare it against a known reference, i.e., to perform a beat note analysis with another reference whose stability has been independently characterised. Our system is compared against PTB's silicon sub-10 mHz cryogenic cavity system [94] via the NPL-SYRTE-PTB fibre-link [95] (Figure 4.7). The fibre-link comparison results are inaccurate at low averaging times $(\tau \sim 1 \mathrm{~s})$ due to the link's noise, and accurate at longer averaging times $(\tau \sim 100 \mathrm{~s}$, where, in turn, the three-corner-hat measurement has a large uncertainty), based on the link noise investigation that is currently underway. At averaging times of around $\tau=10 \mathrm{~s}$, both the fibre-link comparison and the threecorner-hat are in good agreement within the measured uncertainty. Knowing that the PTB cavity has a stability of $4 \cdot 10^{-17}$ at $\tau=10 \mathrm{~s}$, we can estimate the stability of our system from the measured comparison, to obtain

$$
\sigma_{y}(\tau=10 \mathrm{~s})=1.1 \cdot 10^{-16} .
$$

With the first build of this new ultra-stable length reference cavity we have achieved the best frequency stability ever procured at NPL. With this new system we have been able to improve the performance of the universal synthesiser, as demonstrated by the reduction of frequency instability of NPL's first generation strontium-87 lattice clock (Sr1) by a factor of two to $1 \cdot 10^{-15} / \sqrt{\tau}$. The improved instability is visible on frequency comparisons with other optical clocks within the European fibre network, enabling us to perform chronometric geodesy and more precise tests of relativity. The development of a second generation strontium- 87 lattice clock (Sr2) will allow implementation of synchronous interrogation to reject Dick noise, and quantum non-destructive detection schemes to minimise dead-time and detection noise [96].

The second build of the system is underway. It will feature a spacer with far less machining imperfections, and there are plans for further improvements: $(i)$ employing low-noise accelerometers to extend the range of the AVI system to frequencies lower than $2 \mathrm{~Hz}$; (ii) placing the AVI system in a higher rigidity breadboard to shift mechanical resonances to higher frequency; (iii) experimentally optimising the position of the cavity support points to further improve the acceleration sensitivity; and $(i v)$ designing a rigid monolithic ULE support structure to mount the cavity on. The second system will provide a definitive way of characterising the achieved performance of the new ultra-stable lasers at NPL. 

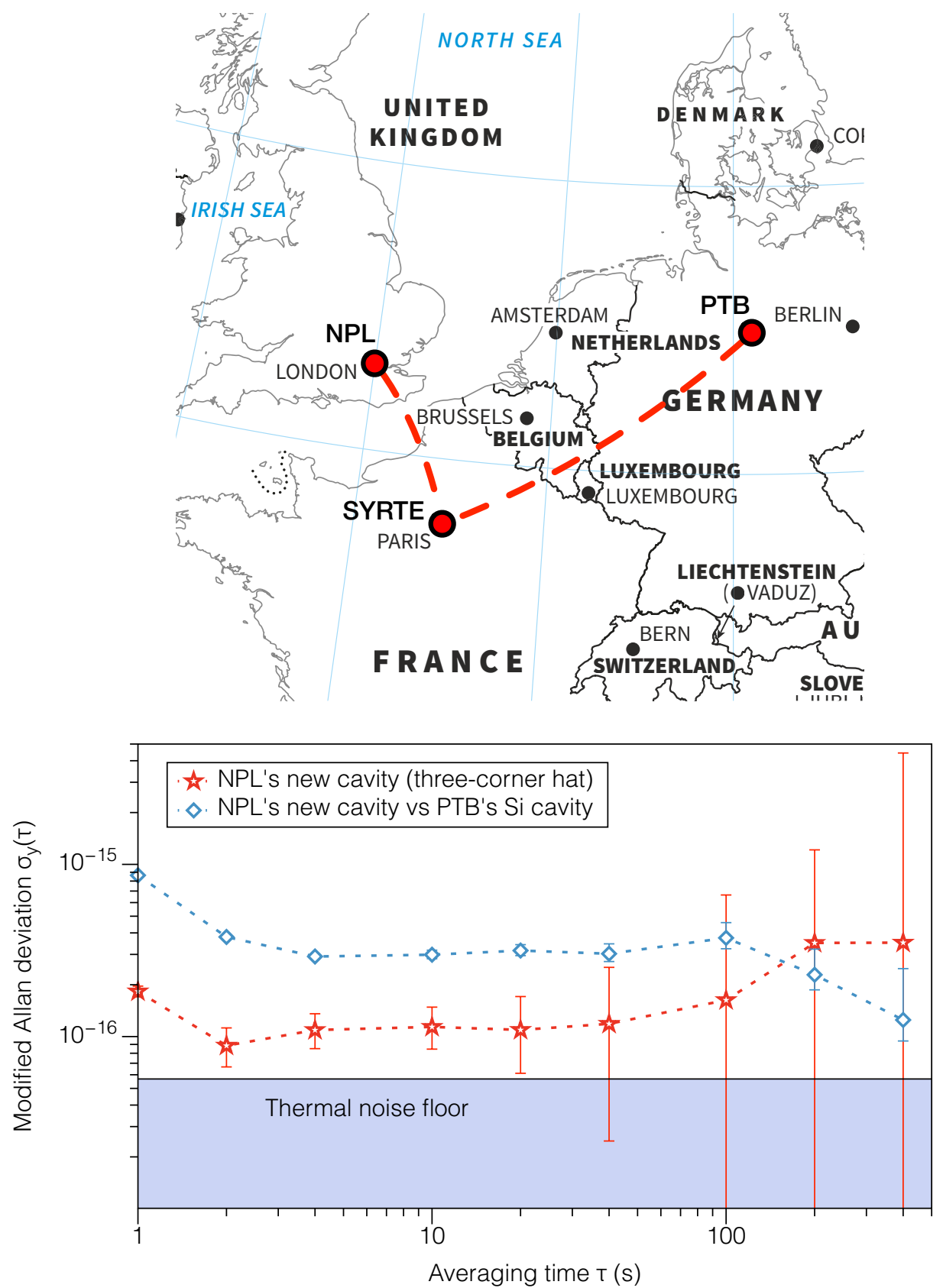

Figure 4.7: Modified Allan deviation of the new cavity and of the beat note between the cavity and PTB's cryogenic silicon cavity (Top: the NPL-SYRTE-PTB fibre-link). The link is currently known to introduce noise at low averaging times $(\tau<10 \mathrm{~s})$. 


\section{PART II}

Cavities FOR

Atom Interferometry 



\section{Cavity atom optics}

\section{Contents}

5.1 Large momentum transfer beam splitters using optical cavities $\mathbf{9 6}$

5.2 Atomic Bragg diffraction . . . . . . . . . . . . . . . 98

5.2 .1 The atom-field Hamiltonian _. . . . . . . . . . . 98

5.2.2 Adiabatic elimination of the excited state . . . . . . 101

5.2.3 Equations of motion of the momentum eigenstates . . 103

5.2 .4 Numerical model . . . . . . . . . . . . . . . . . 105

5.2.5 First order Bragg diffraction . . . . . . . . . . . . . 106

5.3 Interaction regimes $\ldots \ldots \ldots \ldots \ldots$. . . . . . . . . . . . .

5.3.1 Raman-Nath regime (fast interaction) . . . . . . 106

5.3.2 Bragg regime (slow and weak interaction) . . . . . . 109

5.3.3 Channeling regime (slow and strong interaction) . . . . 112

5.4 The quasi-Bragg regime . . . . . . . . . . . . . . . 113

5.4.1 Pulse-shape dependence of the transition probability . 116

5.5 Cavity-assisted Bragg beam splitter . . . . . . . . . . 119

5.5.1 Time-domain model of the Fabry-Perot resonator . . . 119

5.5.2 Cavity-induced deformation of the pulse envelope . . . 123

5.5.3 Effect of finesse and length on the atomic transitions . 125 


\title{
5.1 Large momentum transfer beam splitters using optical cavities
}

\begin{abstract}
$A \begin{gathered}\text { TOM interferometers employing optical cavities to enhance the beam splitter } \\ \text { pulses [5] promise significant advances in science and technology, notably for }\end{gathered}$ future gravitational-wave detectors. Long cavities, on the scale of hundreds of meters, have been proposed in experiments aimed at becoming demonstrators for gravitational-wave detection at frequencies below $1 \mathrm{~Hz}[6]$, where current laser interferometers, such as LIGO [4], have poor sensitivity. Alternatively, short cavities have also been proposed for enhancing the sensitivity of more portable atom interferometers.
\end{abstract}

In light pulse atom interferometry [19], atomic beams are coherently split and later recombined using laser pulses as beam splitters $[97,98]$. The atoms, in freefalling motion under the action of the gravitational field, are generally described as the superposition $|\Psi\rangle$ of two states (e.g., a ground and an excited state), typically hyperfine levels of the ground state of alkali atoms, or just different momentum states of the same internal energy state. In reality, the atom is hardly a two-state system, but under certain conditions it behaves like one. For example, as we will see later in this chapter, in adiabatic diffraction experiments (i.e., in the regime of very long photon-atom interaction times and low interaction strength), the atom can be treated as the superposition of the initial and final momentum eigenstates coupled with an effective interaction strength that is a function of the physical interaction strength (i.e., the laser intensity).

Along its trajectory, the atom is made to interact with a pair of light pulses with opposite propagation vectors that form an optical standing wave. The light pulses are typically obtained by modulating the amplitude of a pair of phase-locked lasers that are retro-reflected off a mirror. The frequency difference between the two lasers should match the frequency splitting between the two states of the atom, plus the Doppler detuning due to the motion of the atom. It is also possible to use just one laser that is also frequency-modulated to create the two frequencies needed for the interferometer. During the interaction, the atomic wave function oscillates between the two states, an effect known as Rabi oscillations, with the duration and intensity of the pulses determining the outcome of the interaction, i.e., determining the final shape of the atom's wave function $\left|\Psi^{\prime}\right\rangle$.

A beam splitter pulse, with a duration of $1 / 4$ of a Rabi oscillation period $\left(\frac{\pi}{2}\right.$ pulse), will put an atom that is initially in one of the two states, into a balanced mixture of the two. The Rabi oscillation period (or, equivalently, the Rabi frequency) is a function of the interaction strength. Beam splitter pulses are used to split and recombine the atomic beams. During the interaction, the excited atom acquires momentum due to the absorption and emission of photons from the optical fields. The amount of momenta exchanged in the interaction is always an integer multiple of $2 \hbar k$, where $\hbar k$ is the photon momentum, as the atom immediately emits a photon into one beam after absorbing another from the anti-parallel beam. Due to the opposed propagation of the light fields, the momentum acquired by the atom is equivalent to having absorbed a photon with an effective wave vector $\boldsymbol{k}=\boldsymbol{k}_{1}-\boldsymbol{k}_{2}=2 \boldsymbol{k}_{1}$, where $\boldsymbol{k}_{1}$ and $\boldsymbol{k}_{2}=-\boldsymbol{k}_{1}$ are the wave vectors of the anti-parallel 
fields. Due to the momentum acquisition after the interaction with a beam splitter pulse, the atom is physically split into two waves with different momentum that follow spatially different paths. A mirror pulse, on the other hand, with a duration of $1 / 2$ of the Rabi period ( $\pi$ pulse), will put an atom that is initially in either of the two states, into the other. Mirror pulses are therefore used to deflect the atomic beams such that the two separate paths converge at a point for recombination.

Inertial forces change the relative phase of the atom with respect to the phase of the optical field. This phase shift manifests as a change in the shape of the final wave function $\left|\Psi^{\prime}\right\rangle$ of the atom after the recombination pulse. Atom interferometers measure this phase shift, e.g., by detecting the number of atoms from the beam that are found in the excited state at the end of the pulse sequence. Atoms are easy to isolate from external perturbations, and this makes them very reliable inertial references.

The sensitivity of atom interferometers is determined by how well we can measure the phase difference between the matter waves at the output of the recombination beam splitter. In a Mach-Zehnder configuration this phase shift is given by

$$
\Delta \phi \equiv(2 \Phi \cdot[\boldsymbol{k} \times(\boldsymbol{v}+\boldsymbol{g} T)]+\boldsymbol{k} \cdot \boldsymbol{g}) T^{2}+\Delta \phi_{L},
$$

where $\boldsymbol{k}$ is the effective wave vector of the photons, $\boldsymbol{v}$ is the initial velocity of the atoms, $\boldsymbol{g}$ is the acceleration due to gravity, $\boldsymbol{\Phi}$ is the angular velocity, and $T$ is the time between pulses. The term $\Delta \phi_{L}$ represents the relative laser phase imprinted on the atoms during the interferometric sequence. If we consider a typical $\frac{\pi}{2}-\pi-\frac{\pi}{2}$ pulse sequence, this takes the form

$$
\Delta \phi_{L} \equiv n\left(\Delta \phi_{1}-2 \Delta \phi_{2}+\Delta \phi_{3}\right)
$$

where $\Delta \phi_{i}$ is the relative phase of the lasers at the position of the atoms during the atom-field interactions, and $n$ is the order of the scattering process. The time between pulses $T$ is limited by the free fall time in atomic fountains, which are as high as the Stanford [99] and the Wuhan [100] $10 \mathrm{~m}$ towers with $T \approx 1 \mathrm{~s}$. The wave vector $\boldsymbol{k}$ scales with the relative recoil momentum between the arms of the interferometer

$$
k \equiv \frac{\Delta p}{\hbar} .
$$

Using conventional atom optics $(n=1), \Delta \boldsymbol{p}$ is limited to two photon recoils, $2 \hbar k$. In recent years, new techniques have been developed where the beam splitters transfer multiple times that amount of momentum to the atoms. In these large momentum transfer (LMT) methods the atoms are coherently scattered by $2 n$ photons from the laser beams and acquire a momentum difference of $2 n \hbar k$. However, the increased number of photon-atom interactions means that the sensitivity to inhomogeneities of the relative laser phase is $n$ times higher than that of a conventional interferometer. LMT methods include sequential Raman pulses [101], sequential two-photon Bragg diffraction [102], and multi-photon Bragg diffraction [103]. The latter has the advantage of achieving large momentum transfer using a single laser pulse while leaving the internal energy state of the atom unchanged, leading to the cancellation of important systematic effects. In addition to the increased sensitivity to the relative laser phase, this method is limited by the available laser power. 
Optical cavities are proposed as the key enabling technology for LMT beam splitters, as performing the interferometric sequence inside the cavity can help mitigate the disadvantages of the technique: cavities provide spatial filtering of the interferometry beam, thus "cleaning" the optical wavefronts, and resonant enhancement in the cavity means that a high intra-cavity power may be achieved using a relatively low input power.

In Chapters 5 and 6 we explore the fundamental limitations of two-mirror cavities for atomic beam splitting, and establish upper bounds on the temperature of the atomic ensemble as a function of cavity length and three design parameters: the cavity $g$ factor, the bandwidth, and the optical suppression factor of the first and second order spatial modes. A lower bound to the cavity bandwidth is found that avoids elongation of the interaction time and maximises power enhancement. An upper limit to cavity length is found for symmetric two-mirror cavities, restricting the practicality of long baseline detectors. For shorter cavities, an upper limit on the beam size is derived from the geometrical stability of the cavity. These findings aim to aid the design of current and future cavity-assisted atom interferometers.

Note that two frequencies, $\omega_{1}$ and $\omega_{2}$, are needed in order to drive Raman or Bragg atomic transitions. To perform the transition inside an optical cavity, both of the required frequencies should be co-resonant in the cavity. To achieve this, the length of the cavity must be stabilised using an auxiliary laser far off resonance with the atomic transition. The length of the cavity could then be chosen so that it is nearly an integer number of half wavelengths of the rf splitting between the initial and final atomic states, so that both frequencies $\omega_{1}$ and $\omega_{2}$ are simultaneously resonant in the cavity. In the presence of external inertial forces, it may be necessary to tune the frequency difference $\omega_{1}-\omega_{2}$ to compensate the Doppler frequency detuning due to the motion of the atom. For example, for a constant acceleration $a$ along the optical axis, the Doppler detuning for the last interrogation pulse is given by $\Delta \omega_{D}=\left(\boldsymbol{k}_{1}-\boldsymbol{k}_{2}\right) \cdot \boldsymbol{v} \approx 2 \boldsymbol{k}_{1} \cdot \boldsymbol{v}=4 n k a T$. If the detuning is small compared to the cavity bandwidth $\Delta \omega$, it may be neglected. The ultimate detuning is thus given by $\Delta \omega$, and the maximum range of the instrument for the measured acceleration results in $\Delta a=\Delta \omega / 4 n k T$. For simplicity, for the reminder of this chapter we consider an interferometer in the absence of external inertial forces.

\subsection{Atomic Bragg diffraction}

\subsubsection{The atom-field Hamiltonian}

In this chapter we deal with a particular type of atomic diffraction scheme, atomic Bragg diffraction, which is the atomic version of the Kapitza-Dirac effect with electrons [104]. In this scheme, the atom is deflected by a standing wave of light. As the atom exchanges energy with the light field, the momentum of the absorbed or emitted light must be compensated by the mechanical motion of the atom. In our description of these phenomena we treat the atoms as quantum systems with an associated quantum vector $|\Psi\rangle$, and the light as a classical field $\boldsymbol{E}$ (a wave in physical space, i.e., not a shower of photons). Moreover we deal only with single particle interference: each atom interferes with itself only. In our semiclassical 
treatment, we neglect the entanglement between the atomic degrees of freedom (i.e., the electronic and the centre-of-mass degrees of freedom) and the degrees of freedom of the electromagnetic field. A full quantum treatment of atomic Bragg diffraction is given in $[105,106]$; however, most Bragg beam splitters demonstrated to date are adequately modelled using the semiclassical picture. Where possible, we use the notation given in $[1,107]$. The treatment developed in this chapter is summarised in Appendix B, along with information on the adiabatic expansion method of Müller, Chiow and Chu.

The quantum state of our system is given by the tensor product of the Hilbert space describing the electronic state of the atom and the Hilbert space describing its centre-of-mass state,

$$
|\Psi\rangle \equiv \sum_{n, m} c_{n m}|n\rangle \otimes|m\rangle
$$

where the electronic eigenstates are $|n\rangle=|g\rangle,|e\rangle$ for a two-level atom (the 'ground' and 'excited' states respectively), and $|m\rangle$ are the momentum eigenstates. However, we start by treating the atom as a two state system with its electronic state decoupled from the external degrees of freedom,

$$
|\Psi\rangle \equiv \sum_{n} c_{n}|n\rangle=c_{g}|g\rangle+c_{e}|e\rangle
$$

The quantisation of the centre-of-mass motion of the atom will be introduced later on through the action of the momentum shift operator,

$$
e^{-i \boldsymbol{k} \cdot \boldsymbol{r}}|\boldsymbol{p}\rangle=|\boldsymbol{p}-\hbar \boldsymbol{k}\rangle
$$

where $|\boldsymbol{p}\rangle$ is an eigenstate of the momentum operator. In this interaction picture, a periodic potential of wave vector $\boldsymbol{k}$ exerts a momentum kick $\pm \hbar \boldsymbol{k}$ on the centre-ofmass motion of the atom.

The applied optical field $\boldsymbol{E}$ is treated as a standing wave of frequency $\omega$ and amplitude $\mathcal{E}(\boldsymbol{r}) f(t)$, and can be written as

$$
\boldsymbol{E}(\boldsymbol{r}, t) \equiv \boldsymbol{\epsilon}(\boldsymbol{r}) \mathcal{E}(\boldsymbol{r}) f(t) \cos (\boldsymbol{k} \cdot \boldsymbol{r}) \cos (\omega t),
$$

where $\boldsymbol{k}$ is the effective wave vector of the field, $\boldsymbol{\epsilon}(\boldsymbol{r})$ is the polarisation of the field, and $f(t)$ is the envelope function describing the temporal profile of the field. It is assumed that $f(t)$ and its derivatives are continuous functions of $t, f(t)=f(-t)$, and $\frac{d}{d t} f(t)$ is zero only at $t=0, \pm \infty$.

The system is described by the total Hamiltonian

$$
\widehat{\mathcal{H}} \equiv \widehat{\mathcal{H}}_{A}+\widehat{\mathcal{H}}_{A F}
$$

where $\widehat{\mathcal{H}}_{A}$ describes the dynamics of the two-level atom, and $\widehat{\mathcal{H}}_{A F}$ describes the dynamics of the atom-field interaction. For an atom of mass $M, \widehat{\mathcal{H}}_{A}$ takes the form

$$
\widehat{\mathcal{H}}_{A}=\frac{\widehat{\boldsymbol{p}}^{2}}{2 M}+\hbar \omega_{g}|g\rangle\left\langle g\left|+\hbar \omega_{e}\right| e\right\rangle\langle e|,
$$



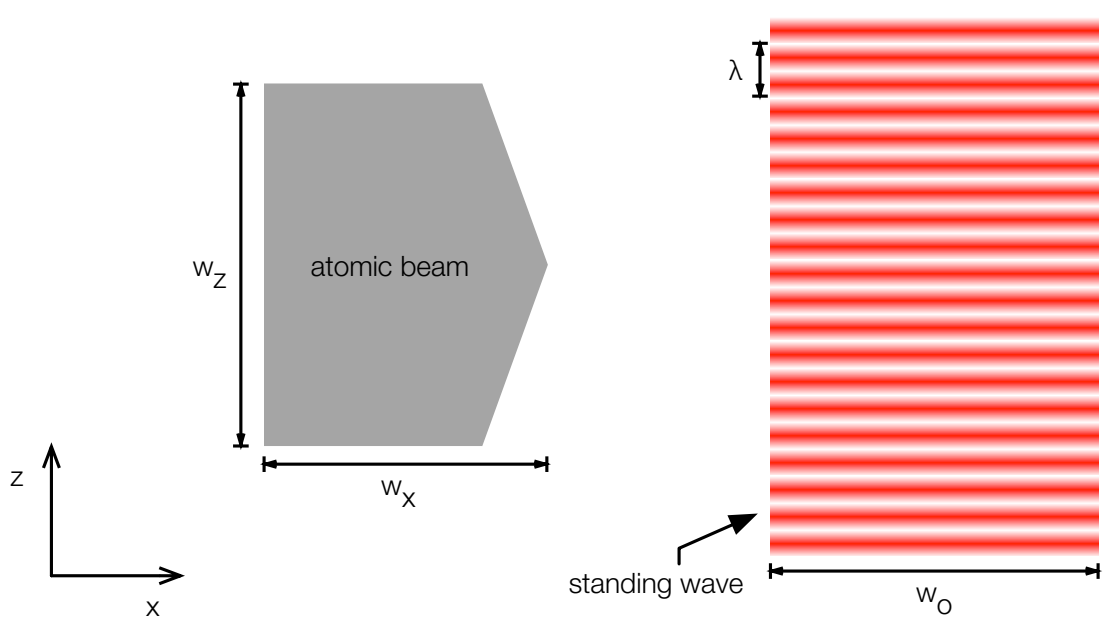

Figure 5.1: Diffraction of atoms by a standing wave light field. The velocity of the atoms along the $x$ direction is large enough that it can be treated classically, whereas the velocity along $z$ is quantised and changes due to the atom-field interaction. The width $w_{z}$ of the atomic beam must be much larger than the period $\lambda / 2$ of the potential.

where $\omega_{g}$ and $\omega_{e}$ are the atomic frequencies of the electronic ground and excited states respectively. We make the following two considerations: $(i)$ that the electric dipole approximation is valid (i.e., it is assumed that the wavelength of the optical field is much larger than the size of the atom, so that $\boldsymbol{E}$ is expected to be homogeneous over the location $\boldsymbol{r}$ of the atomic centre of mass), and ( $i i$ ) that the rotating wave approximation is valid (i.e., it is assumed that the optical field is nearly resonant with the atomic transition and therefore the rapidly oscillating terms in the interaction Hamiltonian can be neglected). Working under these assumptions, in the frame rotating at the laser frequency, and setting the zero of energy to the energy of state $|g\rangle$, yields

$$
\widehat{\mathcal{H}}_{A}=\frac{\widehat{\boldsymbol{p}}^{2}}{2 M}-\hbar \delta|e\rangle\langle e|,
$$

where $\boldsymbol{p}$ is the momentum operator and $\delta \equiv \omega-\omega_{0}$ is the laser detuning from the atomic transition of frequency $\omega_{0} \equiv \omega_{e}-\omega_{g}$. The applied light field $\boldsymbol{E}$ enters the problem through the interaction Hamiltonian as

$$
\widehat{\mathcal{H}}_{A F}=-\widehat{\boldsymbol{d}} \cdot \boldsymbol{E}(\boldsymbol{r}, t),
$$

where $\boldsymbol{d}$ is the atomic dipole moment operator, orientated along the quantisation axis $\epsilon_{\zeta}$,

$$
\widehat{\boldsymbol{d}} \equiv d \boldsymbol{\epsilon}_{\zeta}(|e\rangle\langle g|+| g\rangle\langle e|)
$$

Therefore, the interaction Hamiltonian takes the form

$$
\widehat{\mathcal{H}}_{A F}=\hbar \Omega_{0}(\boldsymbol{r}, t) \cos (\boldsymbol{k} \cdot \boldsymbol{r})(|e\rangle\langle g|+| g\rangle\langle e|),
$$


where we have introduced the quantity known as the Rabi frequency

$$
\Omega_{0}(\boldsymbol{r}, t) \equiv-\frac{1}{\hbar} d \mathcal{E}(\boldsymbol{r}) f(t)\left(\boldsymbol{\epsilon}(\boldsymbol{r}) \cdot \boldsymbol{\epsilon}_{\zeta}\right)
$$

We consider a beam of atoms travelling along the $x$ direction and being scattered by a standing wave light field (Figure 5.1). The field can be treated as having two components, one travelling in the $+z$ direction, and another travelling in the $-z$ direction. The field is polarised in the $x$ direction $\left(\boldsymbol{\epsilon}(\boldsymbol{r})=\boldsymbol{\epsilon}_{\zeta}=\boldsymbol{x}\right)$. The velocity of the atom along $x$ is high enough that it can be treated classically, however its velocity along $z$ is treated quantum mechanically $\left(p_{z}=i \hbar(\partial / \partial z),\left[z, p_{z}\right]=i \hbar\right)$, and will be modified due to the action of the optical field. The width $w_{z}$ of the atomic beam is taken to be much larger than the period $\lambda / 2$ of the potential. In addition we treat the electric field as uniform over the width $w_{x}$ of the atomic beam, $\mathcal{E}(\boldsymbol{r}) \equiv \mathcal{E}_{0}$. The resulting Rabi frequency is written as

$$
\Omega_{0}(t)=-\frac{1}{\hbar} d \mathcal{E}_{0} f(t) .
$$

The total Hamiltonian, ignoring the effect of spontaneous emission ${ }^{1}$, is thus given by

$$
\widehat{\mathcal{H}}=-\frac{\hbar^{2}}{2 M} \frac{\partial^{2}}{\partial z^{2}}-\hbar \delta|e\rangle\langle e|+\hbar \Omega_{0}(t) \cos (k z)(|e\rangle\langle g|+| g\rangle\langle e|) .
$$

\subsubsection{Adiabatic elimination of the excited state}

We now invoke Schrödinger's equation and work in coordinate representation. Let $g(z, t)$ and $e(z, t)$ be the complex amplitudes of states $|g\rangle$ and $|e\rangle$ respectively. Applying the Schrödinger equation yields

$$
\left\{\begin{array}{l}
i \hbar \dot{e}(z, t)=-\frac{\hbar^{2}}{2 M} \frac{\partial^{2} e(z, t)}{\partial z^{2}}+\hbar \Omega_{0}(t) \cos (k z) g(z, t)-\hbar \delta e(z, t), \\
i \hbar \dot{g}(z, t)=-\frac{\hbar^{2}}{2 M} \frac{\partial^{2} g(z, t)}{\partial z^{2}}+\hbar \Omega_{0}(t) \cos (k z) e(z, t) .
\end{array}\right.
$$

For detunings much larger than the linewidth of the excited state (and thus also $\delta \gg \Omega_{0}, \omega_{r}$, with $\Omega_{0}$ the peak Rabi frequency), and if the atoms are prepared initially in the ground state $(g(z, 0)=1, e(z, 0)=0)$, the excited state can be adiabatically eliminated,

$$
e(z, t) \approx \frac{\Omega_{0}(t)}{\delta} \cos (k z) g(z, t)
$$

Substituting in Equation 5.17 yields

$$
i \hbar \dot{g}(z, t)=-\frac{\hbar^{2}}{2 M} \frac{\partial^{2} g(z, t)}{\partial z^{2}}+\hbar \frac{\Omega_{0}^{2}(t)}{\delta} \cos ^{2}(k z) g(z, t) .
$$

\footnotetext{
${ }^{1}$ For long enough times the random momentum kicks experienced by the atoms due to spontaneous decays result in the "washing out" of the interference pattern. The interaction then transitions into the diffusive regime (for more details see [108]).
} 
This is a Mathieu equation [109] that has no analytical solution in general. We look for approximate solutions by expanding $g(z, t)$ in series and transforming Equation 5.19 into an infinite set of coupled differential equations which we then truncate,

$$
g(z, t)=\sum_{m=-\infty}^{+\infty} g_{m}(t) e^{i m k z}
$$

where $m$ labels the transverse momentum state of the atom, i.e., the diffraction order. The atom is now described as an infinite superposition of momentum states $|g, m \hbar k\rangle \equiv|m\rangle$ with plane wave amplitudes $g_{m}(t) e^{i m k z}$. The time and second position derivatives of Equation 5.20 are:

$$
\begin{aligned}
\frac{\partial g(z, t)}{\partial t} & =\sum_{m=-\infty}^{+\infty} \dot{g}_{m}(t) e^{i m k z}, \\
\frac{\partial^{2} g(z, t)}{\partial z^{2}} & =-k^{2} \sum_{m=-\infty}^{+\infty} m^{2} g_{m}(t) e^{i m k z} .
\end{aligned}
$$

Substituting Equations 5.20, 5.21 and 5.22 into Equation 5.19 yields

$i \hbar \sum_{m=-\infty}^{+\infty} \dot{g}_{m}(t) e^{i m k z}=\hbar \omega_{r} \sum_{m=-\infty}^{+\infty} m^{2} g_{m}(t) e^{i m k z}+\hbar \frac{\Omega_{0}^{2}(t)}{\delta} \cos ^{2}(k z) \sum_{m=-\infty}^{+\infty} g_{m}(t) e^{i m k z}$

where we have defined the recoil frequency as

$$
\omega_{r} \equiv \frac{\hbar k^{2}}{2 M}
$$

The cosine operator $\cos (k z)$ acts as a 1-photon momentum kick over the momentum state

$$
\cos (k z)|p\rangle=\frac{1}{2}\left(e^{i k z}+e^{-i k z}\right)|p\rangle=\frac{1}{2}(|p+\hbar k\rangle+|p-\hbar k\rangle),
$$

which effectively introduces the quantisation of the centre-of-mass motion of the atom in the $z$ direction. When the atom absorbs a photon from the field propagating in the $+z$ direction, its momentum changes by $+\hbar k$; when it emits a photon in the same direction, its momentum changes by $-\hbar k$. In the same fashion, the $\cos ^{2}(k z)$ operator acts as a 2-photon momenta kick,

$$
\cos ^{2}(k z)|p\rangle=\frac{1}{2}|p\rangle+\frac{1}{4}(|p+2 \hbar k\rangle+|p-2 \hbar k\rangle) .
$$

Therefore,

$$
\cos ^{2}(k z)\left(\sum_{m=-\infty}^{+\infty} g_{m}(t) e^{i m k z}\right)=\sum_{m=-\infty}^{+\infty}\left[\frac{1}{2} g_{m}(t)+\frac{1}{4} g_{m+2}(t)+\frac{1}{4} g_{m-2}(t)\right] e^{i m k z} .
$$


Substituting into Equation 5.23 yields

$$
\begin{aligned}
i \hbar \sum_{m=-\infty}^{+\infty} \dot{g}_{m}(t) e^{i m k z}= & \sum_{m=-\infty}^{+\infty}\left(\hbar m^{2} \omega_{r}+\hbar \frac{\Omega_{0}^{2}(t)}{2 \delta}\right) g_{m}(t) e^{i m k z}+ \\
& \hbar \frac{\Omega_{0}^{2}(t)}{4 \delta} \sum_{m=-\infty}^{+\infty}\left[g_{m+2}(t)+g_{m-2}(t)\right] e^{i m k z}
\end{aligned}
$$

The action of the field on the atom hence results in the splitting of its wave function into all momentum eigenstates separated by $2 \hbar k$.

\subsubsection{Equations of motion of the momentum eigenstates}

Equation 5.28 holds if and only if

$$
i \dot{g}_{m}(t)=\left[m^{2} \omega_{r}+\Omega(t)\right] g_{m}(t)+\frac{1}{2} \Omega(t)\left[g_{m+2}(t)+g_{m-2}(t)\right]
$$

for all $m$, where we have defined the 2-photon Rabi frequency as

$$
\Omega(t) \equiv \frac{\Omega_{0}^{2}(t)}{2 \delta} .
$$

The resulting equations of motion are described by a tridiagonal Hamiltonian, with the diagonal elements corresponding to the kinetic energy terms, and the off-diagonal elements describing the cross-couplings between momentum states given by the optical field. We can write this Hamiltonian, $\widehat{\mathcal{H}}_{\text {Bragg }}$, as:

$$
\begin{aligned}
\left\langle m\left|\widehat{\mathcal{H}}_{\text {Bragg }}\right| m\right\rangle & =m^{2} \hbar \omega_{r}, \\
\left\langle m \pm 2\left|\widehat{\mathcal{H}}_{\text {Bragg }}\right| m\right\rangle & =\frac{1}{2} \hbar \Omega(t) .
\end{aligned}
$$

Odd and even states are coupled separately and thus, if the initial conditions allow it, we can look at solutions with either all even or all odd terms zero. The system can be truncated to a finite subset of equations expanding states $|-m\rangle$ to $|+m\rangle$ as long as energy conservation is satisfied. For a system with only even or odd states populated, we use the reduced wave function

$$
|\Psi\rangle=\left(\ldots g_{-m-2}, g_{-m}, g_{-m+2} \ldots g_{+m-2}, g_{+m}, g_{+m+2} \ldots\right) .
$$

The reduced Hamiltonian matrix with only even $m$ states populated $\left(g_{m}(t)=0\right.$ for odd $m$ ) takes the form

$$
\widehat{\mathcal{H}}_{\text {even }} \equiv \hbar\left(\begin{array}{ccccccc}
\ddots & \ddots & & & & & \\
\ddots & 16 \omega_{r} & \Omega / 2 & & & & \\
& \Omega(t) / 2 & 4 \omega_{r} & \Omega(t) / 2 & & & \\
& & \Omega(t) / 2 & 0 & \Omega(t) / 2 & & \\
& & & \Omega(t) / 2 & 4 \omega_{r} & \Omega(t) / 2 & \\
& & & & \Omega(t) / 2 & 16 \omega_{r} & \ddots \\
& & & & & \ddots & \ddots
\end{array}\right) \text {, }
$$


and with only odd $m$ states populated $\left(g_{m}(t)=0\right.$ for even $\left.m\right)$ it takes the form

$$
\widehat{\mathcal{H}}_{\text {odd }} \equiv \hbar\left(\begin{array}{cccccc}
\ddots & \ddots & & & & \\
\ddots & 9 \omega_{r} & \Omega(t) / 2 & & & \\
& \Omega(t) / 2 & \omega_{r} & \Omega(t) / 2 & & \\
& & \Omega(t) / 2 & \omega_{r} & \Omega(t) / 2 & \\
& & & \Omega(t) / 2 & 9 \omega_{r} & \ddots \\
& & & & \ddots & \ddots
\end{array}\right) .
$$

The 2-photon Rabi frequency $\Omega(t)$ is in general an arbitrary function of time. For a pulsed field we generally write

$$
\Omega(t) \equiv \bar{\Omega} G(t)
$$

where $\bar{\Omega}$ is the peak 2-photon Rabi frequency - proportional to the peak laser intensity - and $G(t)$ is the envelope function of the field intensity, of full width at half maximum (FWHM) $\delta t$. The parameter space of the interaction for a given $G(t)$ function is therefore spanned by the interaction strength $\bar{\Omega}$ and duration $\delta t$. Furthermore, the shape of $G(t)$ can play a major role in the evolution of the atomic states in certain regions of the parameter space.

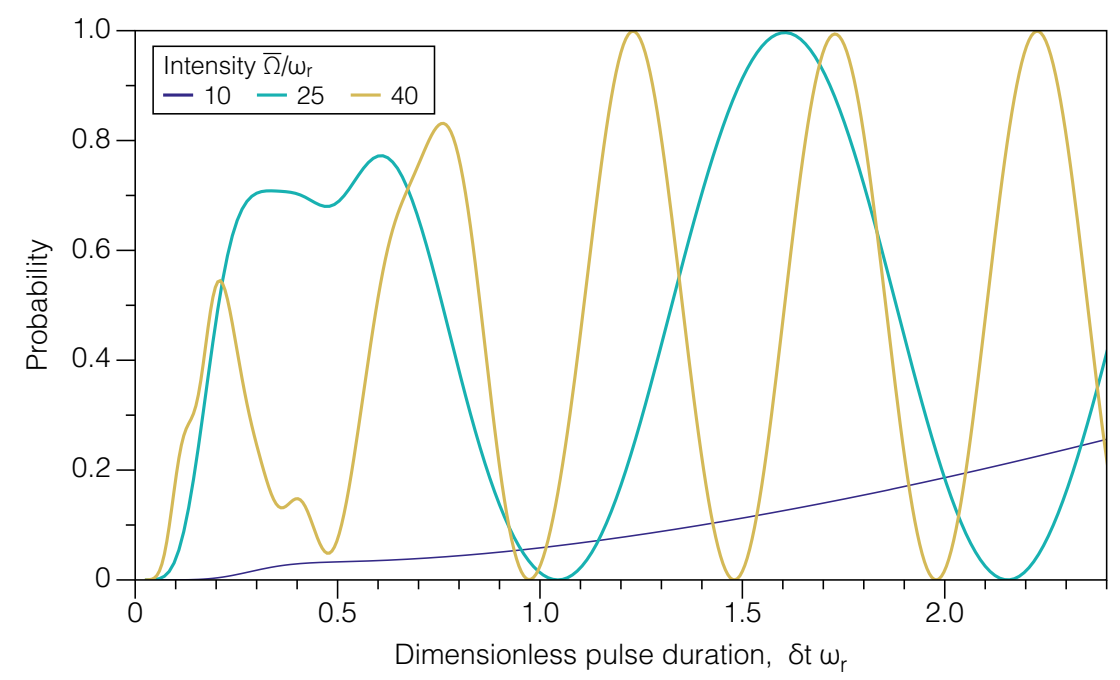

Figure 5.2: Probability of excitation $|0\rangle \rightarrow|+8\rangle$ as a function of the input pulse duration for different values of the laser intensity (using pulses with a Gaussian envelope function). When the interaction time is short $\left(\delta t \omega_{r} \lesssim 1\right)$ high loss of the population into the intermediate states occurs. At longer interaction times the system performs Pendellösung oscillations between the initial and final states. 


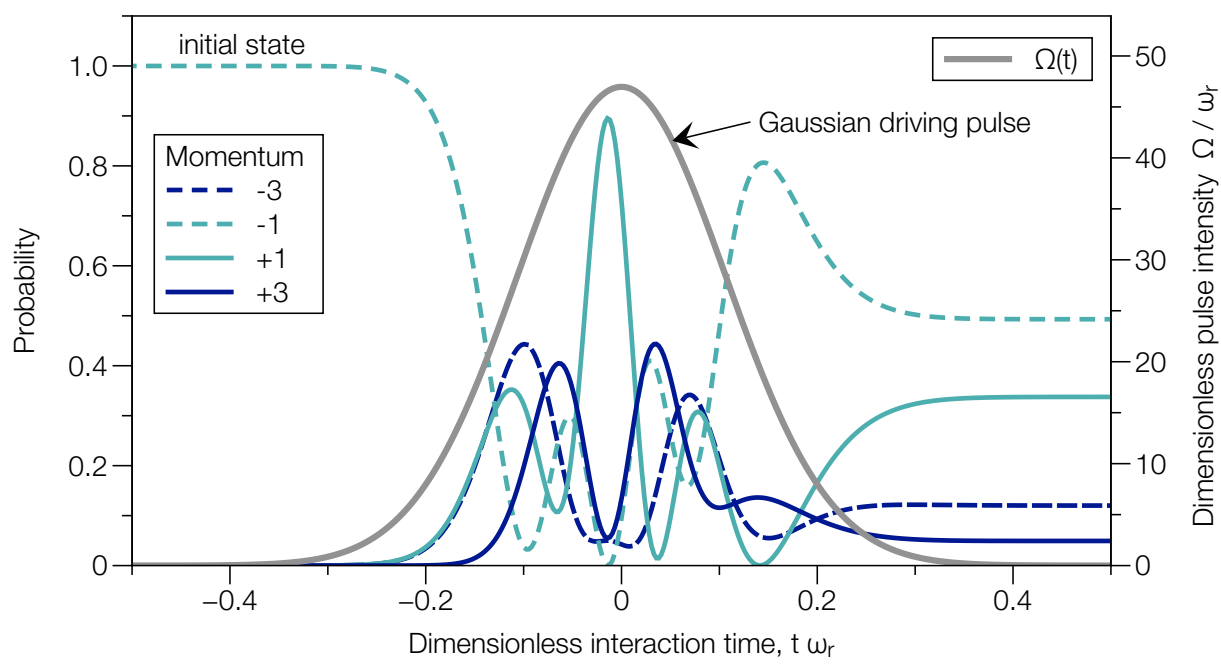

Figure 5.3: Evolution of the probabilities of the momentum eigenstates $|-3\rangle,|-1\rangle$, $|+1\rangle$, and $|+3\rangle$ for an atom initially in state $|-1\rangle$, driven by a Gaussian pulse with the depicted intensity profile. Depending on the interaction parameters of intensity and duration, the shape of the intensity profile $\Omega(t)$ can play a major role in the evolution of the eigenstates.

\subsubsection{Numerical model}

We build a numerical model to solve the equations of motion of the amplitudes of the momentum eigenstates of the atom. Our model solves Equation 5.29 by numerical integration for a truncated set of states $\left|-m_{0}-m\right\rangle \ldots\left|+m_{0}+m\right\rangle$, where $m$ is the order of the $2 m$-photon process and $m_{0}$ is the number of additional outer states considered. We find that considering four outer states is usually sufficient for $m \leq 10$, i.e., the solution is not altered by considering more (allowing a discrepancy of up to $10^{-3}$ in the probability distribution of the final state of the atom). As we will see in the next few sections, the model is checked against the known analytical solutions for first order Bragg diffraction and Bragg diffraction in the Raman-Nath regime, and it also reproduces the results presented in [110]. Since Equation 5.29 couples even and odd states separately, we look only at solutions with either all even or odd terms zero.

See Figure 5.2 for an example $m=4$ process in which we scan the pulse width at fixed intensity and plot the population of the final state, $|+8 \hbar k\rangle$. Throughout this work, we present most results in terms of the dimensionless interaction time $t \omega_{r}$ (or pulse width $\delta t \omega_{r}$ ), and the dimensionless interaction strength or intensity $\bar{\Omega} / \omega_{r}$. This makes all results readily scalable for the atomic transition of interest, with $\omega_{r}=\hbar k^{2} / 2 M$, where $M$ is the mass of the atom. For example, $\omega_{r}=23694 \mathrm{rad} / \mathrm{s}$ for the rubidium- $87 \mathrm{D}_{2}$ transition $\left(5^{2} \mathrm{~S}_{1 / 2} \rightarrow 5^{2} \mathrm{P}_{3 / 2}\right)$, and $\omega_{r}=12983 \mathrm{rad} / \mathrm{s}$ for the caesium-133 $\mathrm{D}_{2}$ transition $\left(6^{2} \mathrm{~S}_{1 / 2} \rightarrow 6^{2} \mathrm{P}_{3 / 2}\right)$. 


\subsubsection{First order Bragg diffraction}

In the case of first order Bragg diffraction, with $|\Psi\rangle=g_{-1}(t)|-1\rangle+g_{+1}(t)|+1\rangle$, the equations of motion reduce to

$$
\left\{\begin{array}{l}
i \dot{g}_{-1}(t)=\left[\omega_{r}+\Omega(t)\right] g_{-1}(t)+\frac{1}{2} \Omega(t) g_{+1}(t), \\
i \dot{g}_{+1}(t)=\left[\omega_{r}+\Omega(t)\right] g_{+1}(t)+\frac{1}{2} \Omega(t) g_{-1}(t),
\end{array}\right.
$$

which have analytical solution. For a constant driving field $(\Omega(t)=\Omega=$ const) and an atom initially in state $|-1\rangle$, the system performs Pendellösung oscillations with frequency $\frac{\Omega}{2}$, with the occupation probabilities given by

$$
P_{|-1\rangle}(t)=\cos ^{2}\left(\frac{\Omega t}{2}\right), \quad P_{|+1\rangle}(t)=\sin ^{2}\left(\frac{\Omega t}{2}\right) .
$$

We can generalise this result for a time dependent driving field $\Omega(t)$ as

$$
P_{|-1\rangle}(t)=\cos ^{2}\left(\frac{1}{2} \int_{-\infty}^{t} \Omega\left(t^{\prime}\right) d t^{\prime}\right), \quad P_{|+1\rangle}(t)=\sin ^{2}\left(\frac{1}{2} \int_{-\infty}^{t} \Omega\left(t^{\prime}\right) d t^{\prime}\right) .
$$

We are now in a position to define the conditions for $\pi$ and $\frac{\pi}{2}$ pulses, which correspond to population transfers of $100 \%$ and $50 \%$ respectively to the target state,

$$
\frac{1}{2} \int_{-\infty}^{+\infty} \Omega(t) d t=\frac{\pi}{2} \quad(\pi \text { pulse }), \quad \frac{1}{2} \int_{-\infty}^{+\infty} \Omega(t) d t=\frac{\pi}{4} \quad\left(\frac{\pi}{2} \text { pulse }\right) .
$$

Since these pulses can be used to split (or deflect) the atomic wave function into two waves that follow spatially different paths due to the momentum difference, they are referred to as beam splitter (or mirror) pulses. The quantity $\int_{-\infty}^{+\infty} \Omega(t) d t$ is often referred to as the pulse area $S$, which is $S=\pi$ and $S=\frac{\pi}{2}$ for $\pi$ and $\frac{\pi}{2}$ pulses respectively. For an atom initially in state $|-1\rangle$, the probability of excitation to state $|+1\rangle$ after interacting with a pulse of area $S$ is

$$
P_{|+1\rangle}=\sin ^{2}\left(\frac{S}{2}\right)
$$

This offers a good opportunity to check our numerical model against a known analytical solution (Figure 5.4). However, the interesting behaviour of our system is displayed only once additional momentum eigenstates are considered (Figure 5.3), and will be discussed in the next section.

\subsection{Interaction regimes}

\subsubsection{Raman-Nath regime (fast interaction)}

For a pulsed driving field with 2-photon Rabi frequency $\Omega(t)=\bar{\Omega} G(t)$, the evolution of the atomic state $|\Psi\rangle$ displays different behaviour on the parameter space of the 

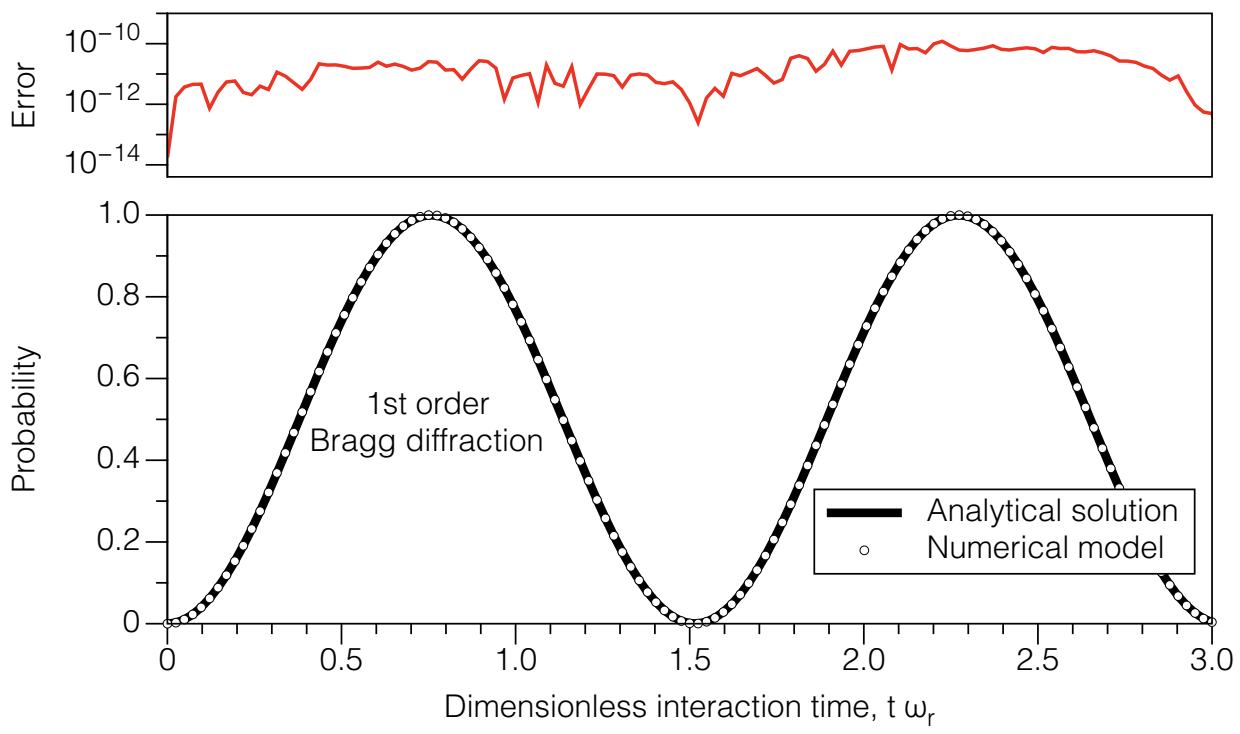

Figure 5.4: Pendellösung oscillations between momentum eigenstates $|-1\rangle$ and $|+1\rangle$ in a two level system driven by a constant potential. The probability of occupation of state $|+1\rangle$ is depicted as a function of time as given by the analytical solution (Equation 5.38, solid line) and as obtained from numerical integration of the equations of motion (Equation 5.37, dashed line). The red curve indicates the absolute error between the numerical result and the analytical solution. The potential used here is $\Omega=4.15 \omega_{r}$.

pulse width $\delta t$ and intensity $\bar{\Omega}$, influenced by the time-energy uncertainty principle. Moreover, in certain regions of the parameter space the shape of $G(t)$ can play a major role in the outcome of the interaction.

The equation of motion of the momentum eigenstates, in the case of a constant driving field $\Omega(t)=\bar{\Omega}=$ const, is

$$
i \dot{g}_{m}(t)=\left[m^{2} \omega_{r}+\bar{\Omega}\right] g_{m}(t)+\frac{1}{2} \bar{\Omega}\left[g_{m+2}(t)+g_{m-2}(t)\right]
$$

In the limit of very short interaction time, the well-defined time places a high energy uncertainty with respect to the kinetic energy term $m^{2} \omega_{r}$, which can be neglected as a result. The equations of motion reduce to

$$
i \dot{g}_{m}(t)=\frac{1}{2} \bar{\Omega}\left[g_{m+2}(t)+g_{m-2}(t)\right]
$$

where we have shifted the total energy scale by $-\hbar \bar{\Omega}$ for simplicity. These equations have analytical solution in the form of $m$-th order Bessel functions of the first kind [1]

$$
g_{2 m}(t)=(-i)^{m} J_{m}(\bar{\Omega} t) .
$$


This interaction limit is known as the Raman-Nath regime. This solution can be easily generalised to account for a time dependent driving field $\Omega(t)$ as

$$
g_{2 m}(t)=(-i)^{m} J_{m}\left(\int_{-\infty}^{t} \Omega\left(t^{\prime}\right) d t^{\prime}\right) .
$$

See Figure 5.5 for an example interaction in the Raman-Nath regime of an atom initially prepared in state $|0\rangle$. The transfer efficiency to any particular state is limited, and the atomic population quickly spreads over many momentum states. In order for this approximation to be valid, the interaction energy must be much greater than the kinetic energy, which translates into the condition

$$
t \ll \frac{1}{\sqrt{2 \bar{\Omega} \omega_{r}}}
$$

on the interaction time. Due to the diffusion of the population into many states, this interaction regime is in principle not suitable for interferometry. For example, the maximum transfer efficiency of the $|0\rangle \rightarrow|+2\rangle$ transition for an atom initially in state $|0\rangle$ is just $\approx 0.336$.

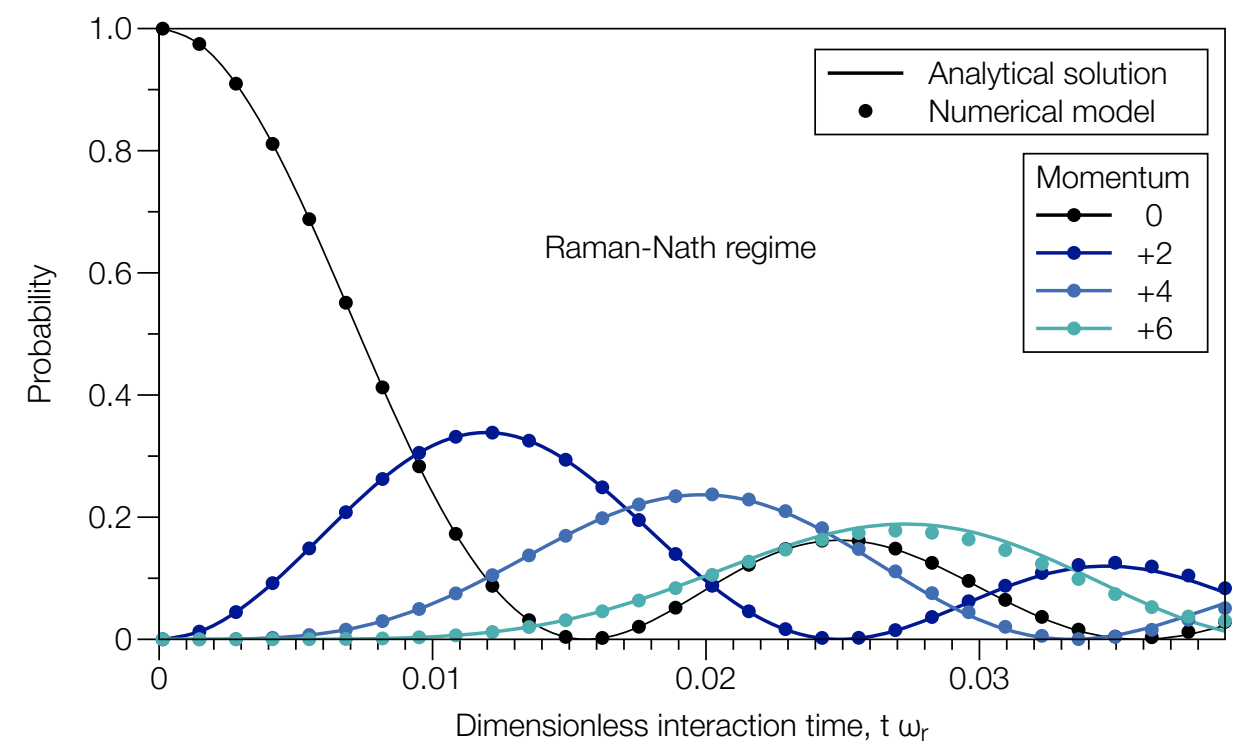

Figure 5.5: Evolution of the probabilities of the momentum eigenstates $|0\rangle,|+2\rangle$, $|+4\rangle$, and $|+6\rangle$ for an atom initially in state $|0\rangle$, driven by a constant potential $\Omega=154 \omega_{r}$. The very short interaction time results in a large energy uncertainty with respect to the kinetic energy of the atoms, thus operating in what is known as the Raman-Nath regime. For longer interaction times the approximation no longer holds and the numerical solution deviates from the Raman-Nath regime prediction. 


\subsubsection{Bragg regime (slow and weak interaction)}

On the opposite extreme of the time-energy uncertainty relation, if the diffraction process is sufficiently slow, energy conservation will favour transitions $|-m\rangle \rightarrow|+m\rangle$, with the intermediate states being adiabatically eliminated. Considering once again a constant driving field, shifting the total energy scale by $m^{2} \hbar \omega_{r}+\hbar \bar{\Omega}$, and having the atoms prepared initially in state $|-m\rangle$ (i.e., $\left.g_{-m}(0)=1, g_{k \neq-m}(0)=0\right)$, the evolution of the initial state is dictated by

$$
i \hbar \dot{g}_{-m}=\frac{1}{2} \hbar \bar{\Omega}\left(g_{-m+2}+g_{-m-2}\right),
$$

and the evolution of an arbitrary intermediate state $g_{-m+2 k}$ with $0<k<m$ is given by

$$
i \hbar \dot{g}_{-m+2 k}=\left(4 k^{2}-4 k m\right) \hbar \omega_{r} g_{-m+2 k}+\frac{1}{2} \hbar \bar{\Omega}\left(g_{-m+2 k+2}+g_{-m+2 k-2}\right) .
$$

The intermediate state can be adiabatically eliminated if $\dot{g}_{-m+2 k} \approx 0$, that is, if

$$
\left|4 k^{2}-4 k m\right| \hbar \omega_{r} \gg \frac{1}{2} \hbar \bar{\Omega},
$$

in which case the slowly varying amplitude of the intermediate state is determined at any given time by the amplitude of the initial state as

$$
g_{-m+2 k} \approx-\frac{1}{8} \hbar \bar{\Omega} \frac{1}{\left(m k-k^{2}\right) \hbar \omega_{r}} g_{-m}
$$

The system can therefore be reduced to an effective two level system of states $|-m\rangle$ and $|+m\rangle$, coupled with an effective Rabi frequency,

$$
\left\{\begin{array}{l}
i \dot{g}_{-m}=\frac{1}{2} \Omega_{\mathrm{eff}} g_{+m}, \\
i \dot{g}_{+m}=\frac{1}{2} \Omega_{\mathrm{eff}} g_{+m},
\end{array}\right.
$$

where we have defined

$$
\Omega_{\mathrm{eff}} \equiv \frac{\bar{\Omega}^{m}}{\left(8 \omega_{r}\right)^{m-1}} \prod_{k=1}^{m-1} \frac{1}{m k-k^{2}}=\frac{\bar{\Omega}^{m}}{\left(8 \omega_{r}\right)^{m-1}} \frac{1}{[(m-1) !]^{2}} .
$$

This system can be solved analytically, yielding

$$
g_{-m}(t)=\cos \left(\frac{\Omega_{\mathrm{eff}} t}{2}\right), \quad g_{+m}(t)=-i \sin \left(\frac{\Omega_{\mathrm{eff}} t}{2}\right),
$$

and the solution can be generalised to account for a time dependent driving field $\Omega(t)$ with effective Rabi frequency $\Omega_{\text {eff }}(t) \propto \Omega^{m}(t)$,

$$
g_{-m}(t)=\cos \left(\frac{1}{2} \int_{-\infty}^{t} \Omega_{\mathrm{eff}}\left(t^{\prime}\right) d t^{\prime}\right), \quad g_{+m}(t)=-i \sin \left(\frac{1}{2} \int_{-\infty}^{t} \Omega_{\mathrm{eff}}\left(t^{\prime}\right) d t^{\prime}\right) .
$$


In this regime the system performs Pendellösung oscillations between states $|-m\rangle$ and $|m\rangle$, with small losses into the intermediate states. The adiabacity condition (Equation 5.48) that is used to eliminate the intermediate states can be alternatively written as

$$
\bar{\Omega} \ll 4|m-1| \omega_{r},
$$

which translates into the requirement of a very long interaction time. The well defined energy means that the pulse (with a Fourier linewidth much smaller than the recoil frequency) will be able to resolve the velocity spread of the cloud (that is typically on the order of 10 times the recoil velocity), hence selecting a fraction of atoms from the source. Moreover, the interaction times required in the Bragg regime may exceed the maximum measurement time available in the experiment, that can be very limited in some cases.

Example transitions in the Bragg regime are presented in Figure 5.6 for different diffraction orders, as obtained from numerical simulation. As made evident by the fast oscillating evolution of the probabilities of the atomic states, the approximation provided by adiabatic elimination of the intermediate states is not always valid. However, these oscillations are much faster than the Rabi flopping frequency between the initial and final states, and they may be difficult to observe experimentally. In practice, Rabi flopping between the initial and final states will not reach $100 \%$ efficiency as predicted in Equation 5.53 for an ideal adiabatic elimination of the intermediate states. Instead, it will follow the behaviour shown in Figure 5.7, and even though the transition probability to the target state may be very close to 1 for short periods of time, the actual observable probability will be closer to the result of applying a low pass filter to the solution. The frequency and amplitude of these fast oscillations depend on the amplitude of the driving field. That is, the oscillations can be dampened by reducing the amplitude of the driving field, hence making the process more adiabatic. Of course, this will come at the expense of the requirement of longer interaction times for the beam splitter pulses.

In the two extreme cases of very short transitions (Raman-Nath regime) and adiabatic transitions (Bragg regime), the outcome of the interaction is independent of the shape of $G(t)$, and only depends on the pulse area $S$. We have previously defined the pulse area in Section 5.2.5 for a two level system as

$$
S \equiv \int_{-\infty}^{+\infty} \Omega(t) d t=\bar{\Omega} \int_{-\infty}^{+\infty} G(t) d t .
$$

This definition of pulse area is still useful to determine the outcome of a transition in the Raman-Nath regime. That is, for a system initially in state $|0\rangle$, the probability of excitation to state $|2 m\rangle$ after interacting with a very short pulse of peak intensity $\bar{\Omega}$ and envelope function $G(t)$ is given by

$$
P_{|2 m\rangle}=J_{m}^{2}(S) \quad \text { (Raman-Nath regime). }
$$

In the Bragg regime, however, with the initial and final states $|-m\rangle$ and $|+m\rangle$ coupled with an effective Rabi frequency proportional to the $m$-th power of $\Omega(t)$, we must introduce another definition of pulse area. We therefore define the " $2 m$-photon 

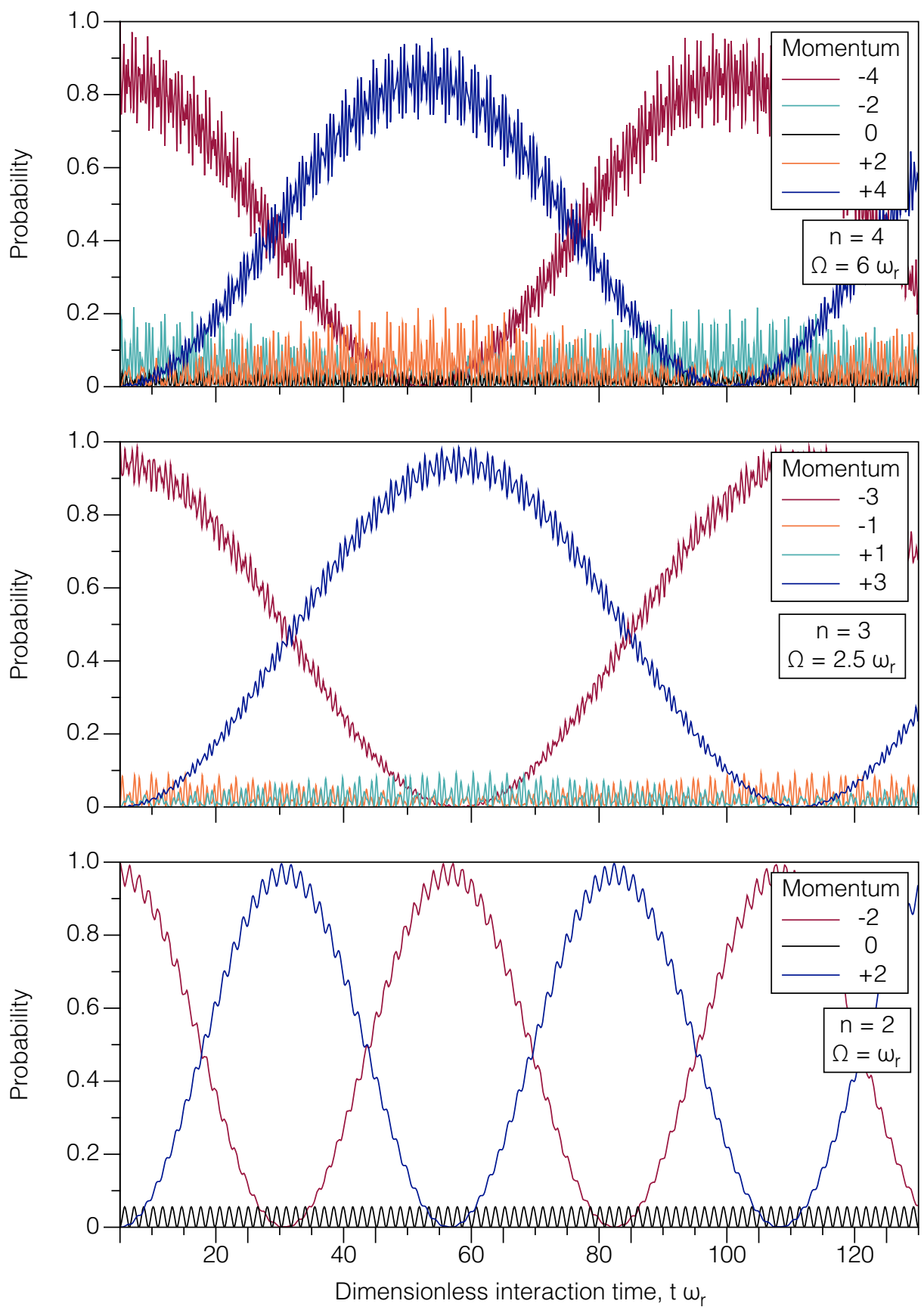

Figure 5.6: Atomic diffraction in the Bragg regime, showing the evolution of the atomic population under a constant driving field for diffraction orders $m=2,3,4$ (4, 6 and 8 photon transitions respectively). The atom performs Pendellösung oscillation between the initial and final states. A small amount of population is lost into intermediate states in a realistic scenario (depicted), but if the interaction time can be made arbitrarily long, virtually lossless transitions can be achieved. 
pulse area" as

$$
\mathcal{S}^{m} \equiv \frac{\int_{-\infty}^{+\infty} \Omega^{m}(t) d t}{\left(8 \omega_{r}\right)^{m-1}[(m-1) !]^{2}}=\frac{\bar{\Omega}^{m} \int_{-\infty}^{+\infty} G^{m}(t) d t}{\left(8 \omega_{r}\right)^{m-1}[(m-1) !]^{2}}
$$

Then, for a system initially in state $|0\rangle$, the probability of excitation to state $|2 m\rangle$ after interacting with a very long pulse of intensity $\bar{\Omega} \ll 4|m-1| \omega_{r}$ is given by

$$
P_{|2 m\rangle}=\sin ^{2}\left(\frac{\mathcal{S}^{m}}{2}\right) \quad \text { (Bragg regime). }
$$

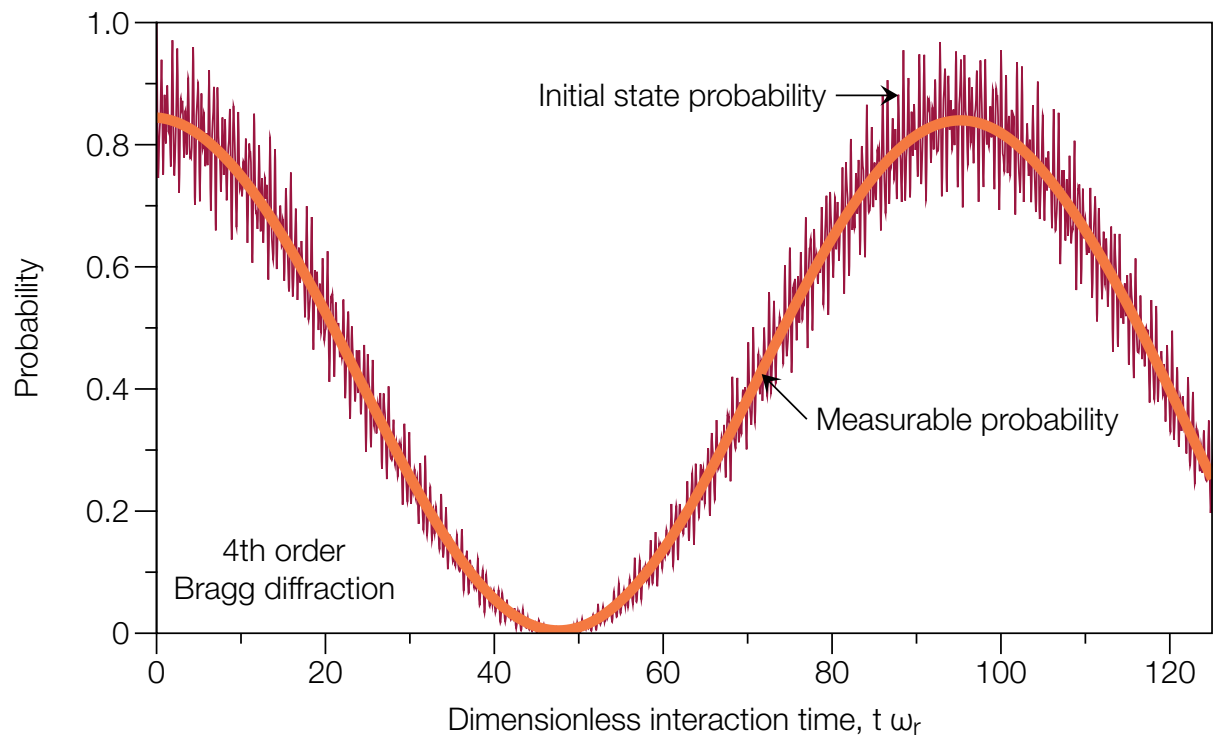

Figure 5.7: In the Bragg regime the probabilities of the momentum eigenstates evolve oscillating quickly about the mean. The amplitude and frequency of the oscillations depend on the amplitude of the driving pulse. Smaller pulse amplitudes lead to smaller and slower oscillations. Experimentally these fast oscillations are difficult to observe due to the strict requirement on the laser pulse's amplitude and timing. Hence, even though the transfer efficiency gets very close to 1 in some cases, the actual measurable probability will be closer to the result of applying a low pass filter to the solution.

\subsubsection{Channeling regime (slow and strong interaction)}

There is a third interaction limit, the channeling regime, where both the interaction time and energy are large. The channeling regime can be thought of as an extension of the slow Bragg regime, where the price has been paid in terms of input energy to drive transitions which violate the adiabacity condition. In this regime, however, the equations of motion have no analytical solution in general, and the evolution of the 


\begin{tabular}{cccc}
\hline Regime & $\begin{array}{c}\text { Pulse shape } \\
\text { dependence }\end{array}$ & Solution $|\langle m \mid \Psi\rangle|^{2}$ & Transition features \\
\hline Raman-Nath & No & $J_{m}^{2}\left(\int_{-\infty}^{t} \Omega\left(t^{\prime}\right) d t^{\prime}\right)$ & $\begin{array}{c}\text { Fast interaction: large energy } \\
\text { uncertainty, high losses. }\end{array}$ \\
Bragg & No & $\sin ^{2}\left(\frac{1}{2} \int_{-\infty}^{t} \Omega_{\mathrm{eff}}\left(t^{\prime}\right) d t^{\prime}\right)$ & $\begin{array}{c}\text { Slow and weak interaction: } \\
\text { with } \Omega_{\mathrm{eff}}(t) \propto \Omega^{m}(t)\end{array}$ \\
$\begin{array}{c}\text { Requires numerical } \\
\text { integration. }\end{array}$ & $\begin{array}{c}\text { Slow and strong interaction: losses } \\
\text { depend on the shape of } \Omega(t) .\end{array}$ \\
\hline
\end{tabular}

Table 5.1: Summary of the different interaction regimes of atomic Bragg diffraction. The three regimes introduced so far are not ideal for interferometry: in the short interaction Raman-Nath regime the transfer efficiency to any particular state is very limited, whereas in the Bragg and channeling regimes the requirement of long interaction times will degrade the performance of the interferometer.

system can only be predicted by numerical methods. The shape of $G(t)$ is known to play a major role in the evolution of the system in the channeling regime [111], with pulses having smooth envelopes (e.g., a Gaussian envelope) yielding low losses of the population into the intermediate states, and pulses having abrupt envelopes (e.g., a square pulse) yielding high losses (Figure 5.8).

For smooth driving pulses, the atom performs Pendellösung oscillations between the initial and final states, much like in the Bragg regime, but with one major difference: during the interaction, the intermediate states participate significantly, despite the fact that by the end of the process the atom will have transitioned to either the initial or final state with relatively low losses to the intermediate ones. If the driving pulse has abrupt amplitude changes the behaviour of the system changes dramatically, and high loss of the population into the intermediate states takes place. The requirement of a long interaction time and intensity makes operation in the channeling regime unsuitable for atom interferometry.

Some properties of the interaction regimes discussed so far are presented in Table 5.1. The three regimes are depicted in Figure 5.9 in the parameter space of the interaction, along with the quasi-Bragg regime, which will be discussed in-depth in the next section.

\subsection{The quasi-Bragg regime}

Müller et al. in 2008 [112] were the first to introduce the theory of atomic diffraction in the quasi-Bragg regime: the transition region between the short interaction Raman-Nath regime and the long interaction Bragg and channeling regimes. Unfortunately in this regime, as in the channeling regime, the equations of motion have no analytical solution in general. In [112] approximate solutions for the population of the initial and final states (i.e., $|-m\rangle$ and $|+m\rangle$ ) are obtained by a method of successive approximations, where the atomic population is assumed to be slowly varying in time and mainly consist of states $|-m\rangle$ and $|+m\rangle$. With this method 

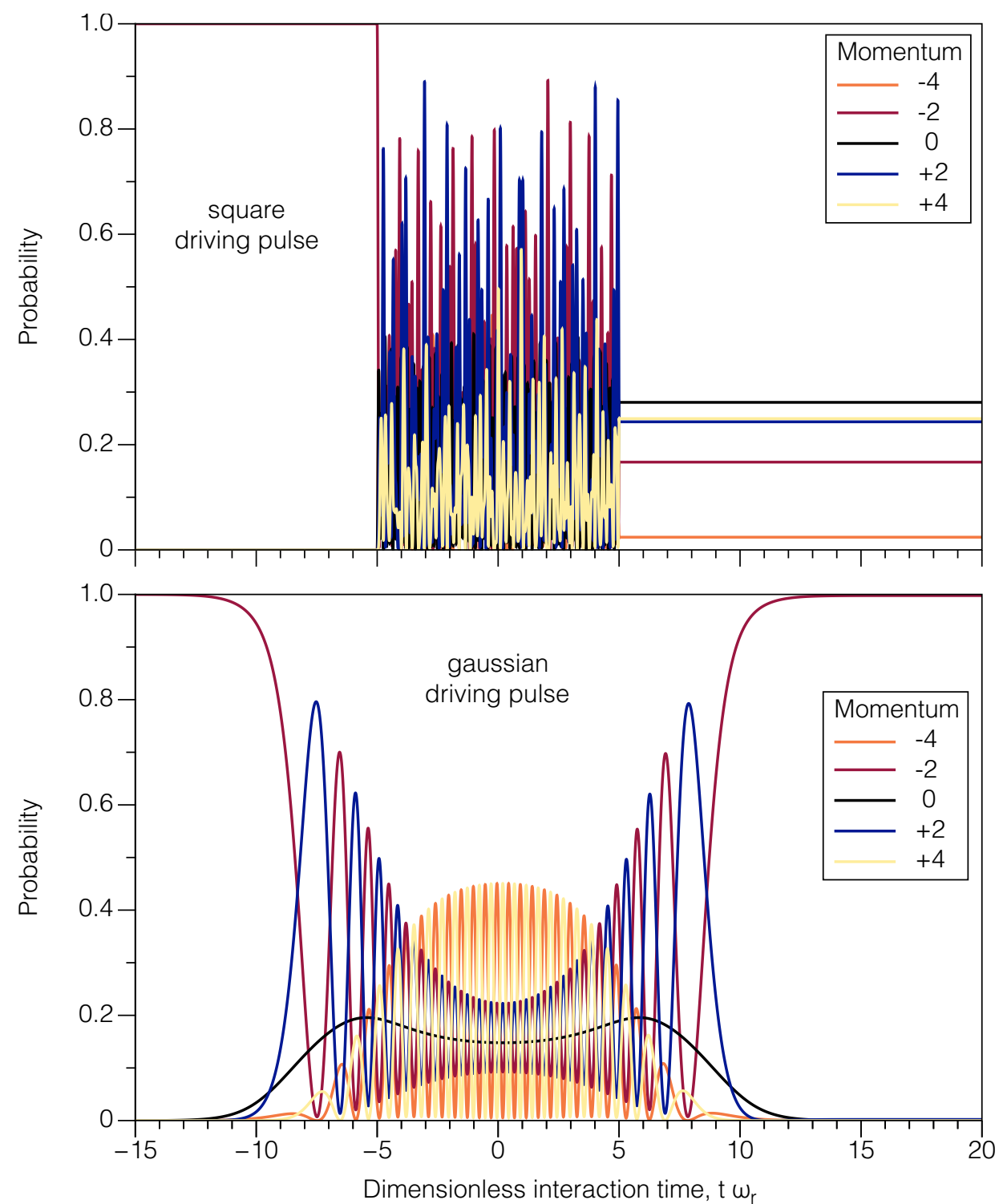

Figure 5.8: Atomic diffraction in the channeling regime, showing the evolution of the atomic population under square and gaussian driving pulses. In this interaction regime, unlike in the Bragg regime, the shape of the envelope function of the driving pulse plays a major role in the outcome of the interaction. Also unlike in the Bragg regime, the intermediate states participate significantly during the interaction. For smooth driving pulses (e.g., a gaussian pulse) the atom will transition into either the initial or final state after the interaction (shown is a $2 \pi$ pulse), whereas for pulses having abrupt changes in amplitude (e.g., a square pulse) the atomic population is lost into the intermediate states and the transfer efficiency to any particular state limited. 
they demonstrate that highly-efficient $|-m\rangle \rightarrow|+m\rangle$ transitions are still possible even in situations which substantially violate the adiabacity condition. In other words, it will be possible to transfer the atomic population into a target state with low losses using relatively short interaction times, thereby using short pulses which will not be able to resolve the velocity spread of the atomic ensemble.

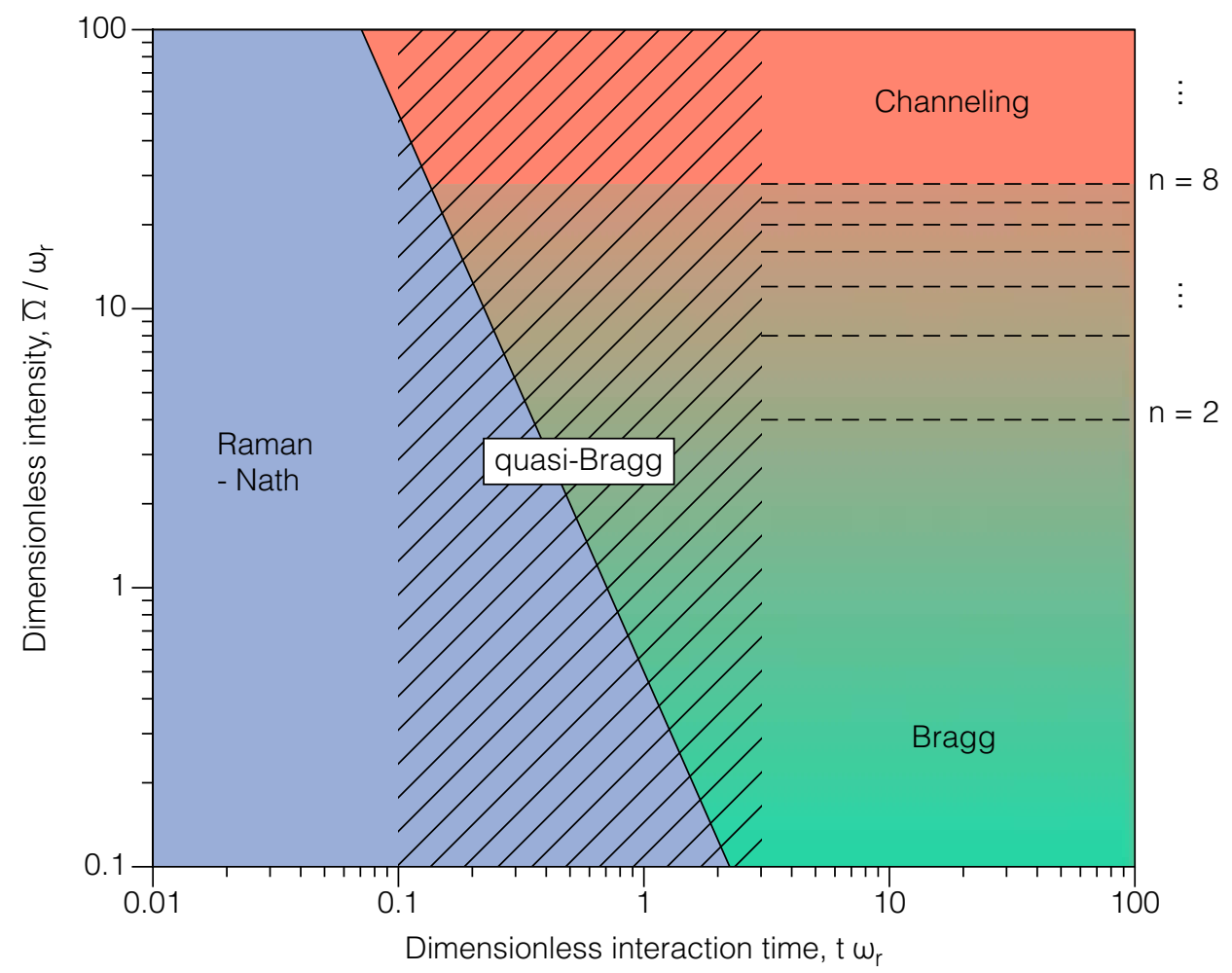

Figure 5.9: Different regimes of atomic Bragg diffraction in the interaction time and intensity parameter space. The solid line separating the Raman-Nath and the Bragg and channeling regimes represents the time-energy relation that permits neglecting the kinetic energy term in the Hamiltonian (Equation 5.45). The horizontal dashed lines show the adiabacity condition (Equation 5.54) for $m=2,3, \ldots$, separating the Bragg and channeling regimes. Between the short interaction Raman-Nath regime and the long interaction Bragg and channeling regimes, a transition regime known as the quasi-Bragg regime exists with no clear boundaries. 


\subsubsection{Pulse-shape dependence of the transition probability}

To demonstrate the effect of pulse shape on the transition probabilities of the system in the quasi-Bragg regime, we simulate the atomic interaction with the three different smooth and symmetric pulse envelope functions shown in Figure 5.10 a: the Gaussian, the hyperbolic secant squared and the Lorentzian. These envelope functions have already been compared in the past in the context of beam splitters based on 2-photon Raman transitions $[113,114]$. Here we extend the comparison to on-resonance multi-photon Bragg beam splitters. The probability of excitation to the excited state in a 10-photon Bragg diffraction process is shown in Figure $5.10 \mathrm{~b}$ for the three envelopes, as we scan the pulse width.

In order to compare interactions with pulses having different envelope functions, we normalise their $2 m$-photon pulse area $\mathcal{S}^{m}$ (Equation 5.57). Let us recall that for a pulsed driving field $\Omega(t)=\bar{\Omega} G(t)$ this new definition of pulse area takes the form

$$
\mathcal{S}^{m}=\frac{\bar{\Omega}^{m}}{\left(8 \omega_{r}\right)^{m-1}[(m-1) !]^{2}} \int_{-\infty}^{+\infty} G^{m}(t) d t .
$$

In addition, we normalise the envelope functions so that the integral of their $m$-th power is equal to their width,

$$
\int_{-\infty}^{+\infty} G^{m}(t) d t=\delta t
$$

Hence, the $2 m$-photon pulse area becomes a dimensionless quantity given by

$$
\mathcal{S}^{m}=\frac{\bar{\Omega}^{m} \delta t}{\left(8 \omega_{r}\right)^{m-1}[(m-1) !]^{2}} .
$$

Pulses with identical $\mathcal{S}^{m}$ will produce Pendellösung oscillations with the same frequency in the adiabatic limit (i.e., in the Bragg regime), independent of the shape of the envelope, as given by equation 5.53. In the quasi-Bragg regime, on the other hand, the shape of $G(t)$ will influence the evolution of the atomic states, as can be seen in Figure 5.10 b.

The following expressions for the Gaussian, hyperbolic secant squared and Lorentzian pulse envelopes verify Equation 5.59:

$$
\begin{aligned}
& G_{\text {Gaussian }}(t, \delta t) \equiv\left[\frac{m \log (16)}{\pi}\right]^{\frac{1}{2 m}} \exp \left[-4 t^{2} \log 2 / \delta t^{2}\right], \\
& G_{\text {sech }}(t, \delta t) \equiv\left[\frac{2 \sinh ^{-1}(1)\left(m-\frac{1}{2}\right) !}{\sqrt{\pi}(m-1) !}\right]^{\frac{1}{m}} \operatorname{sech}^{2}\left[\frac{2 t \log (1+\sqrt{2})}{\delta t}\right], \\
& G_{\text {Lorentzian }}(t, \delta t) \equiv \frac{\left[\frac{(1+\sqrt{2})^{-2 m-\frac{3}{2}}\left(\frac{16}{3+2 \sqrt{2}}\right)^{m}(2 m) !}{\sqrt{\pi}\left(\sqrt{2}(12-8 \sqrt{2})^{2 m}-(2[\sqrt{2}-1])^{4 m}\right) m\left(2 m-\frac{3}{2}\right) !}\right]^{\frac{1}{m}}}{\left(1+4(\sqrt{2}-1)\left(\frac{t}{\delta t}\right)^{2}\right)^{2}} .
\end{aligned}
$$

As a side note, when a pulse resonates inside an optical cavity, depending on the cavity parameters, the pulse's power might be enhanced due to the buildup effect 

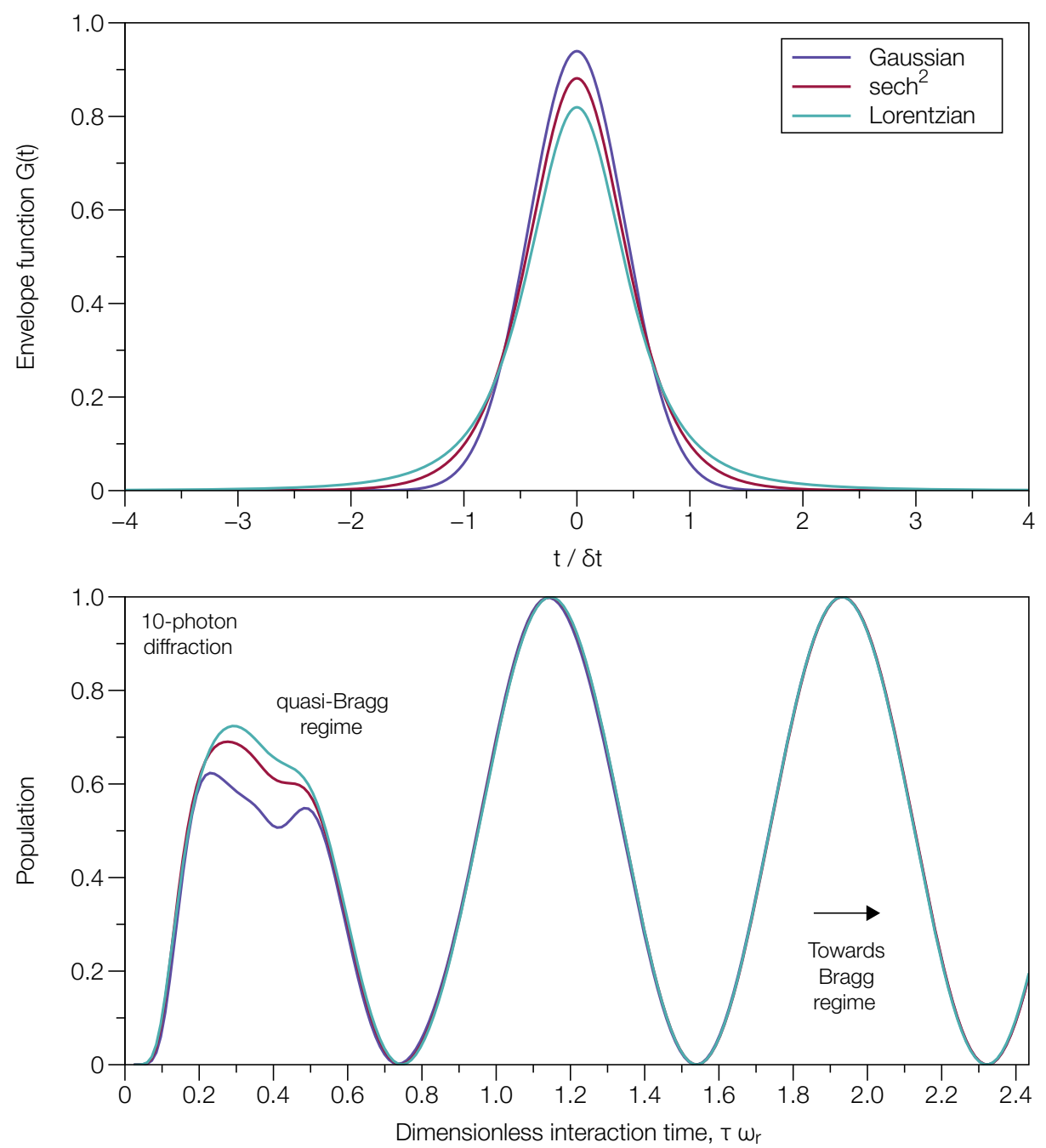

Figure 5.10: Pulse-shape dependence of the transition probabilities in the quasiBragg regime. Pulses having different envelope functions (a), such as the Gaussian, hyperbolic secant squared, and Lorentzian functions, produce transitions with different probabilities in the quasi-Bragg regime (b). All three envelope functions have the same width $\delta t$ and $2 m$-photon pulse area $\mathcal{S}^{m}$. The occupation probability of the excited state in a 10-photon Bragg diffraction process $(m=5)$ is shown as a function of the pulse width $\delta t$. As the pulse duration grows, the transitions become more adiabatic and all three envelopes yield Pendellösung oscillations with the same frequency. In this simulation $\bar{\Omega}=41.5 \omega_{r}$. 
of the resonator. In other words, the cavity might enhance the $2 m$-photon pulse area. As we will see in the next section, for a given set of cavity parameters the area of the cavity's circulating field is a function of the input pulse width,

$$
\int_{-\infty}^{+\infty} G^{m}(t, \delta t) d t \equiv \mathcal{C}(\delta t)
$$

If the pulse width is much longer than the average time a photon spends trapped within the resonator, $\mathcal{C}(\delta t) \rightarrow \mathcal{O} \cdot \delta t$, where $\mathcal{O}$ is the cavity's optical gain, which depends on the electromagnetic properties of the mirrors. Therefore, the $2 m$-photon pulse area of the intra-cavity interrogation field will be given by

$$
\mathcal{S}^{m}=\frac{\bar{\Omega}^{m} \mathcal{C}(\delta t)}{\left(8 \omega_{r}\right)^{m-1}[(m-1) !]^{2}} .
$$

Seeing how the different envelopes will yield different transition probabilities within the first few population cycles, we might be interested in finding if a particular pulse envelope yields more efficient transitions at short interaction times.

For a given envelope function $G(t)$ of the driving field, the probability of exciting the atom to the target state $|m\rangle$ by scattering $2 m$ photons is a function of the peak 2-photon Rabi frequency $\bar{\Omega}$ and the pulse duration $\delta t$

$$
\lim _{t \rightarrow+\infty}|\langle m \mid \Psi(t)\rangle|^{2} \equiv \mathcal{P}(\bar{\Omega}, \delta t) .
$$

Efficient population transfer is possible at short pulse lengths for all three envelope functions considered: $\pi$ pulses with losses below $10^{-10}$ are achievable, assuming perfect control of the pulse's amplitude and timing, at pulse lengths and peak intensities that substantially violate the adiabacity condition of the Bragg regime, as predicted in [112]. We determine the mirror pulse durations $\delta t_{\pi}$ to transfer the ensemble to the target state with losses below $5 \%$

$$
\delta t_{\pi}(\bar{\Omega}) \equiv \min \{\delta t: \mathcal{P}(\bar{\Omega}, \delta t)>0.95\} .
$$

Interestingly, despite the differences in the shape of $\mathcal{P}_{m}$ in the quasi-Bragg regime for each of the three envelope functions considered, the three reproduce virtually the same $\delta t_{\pi}(\bar{\Omega})$ curve within the first Rabi cycle (Figure 5.11).

This means that at the level of transfer efficiency considered $(\mathcal{P}>0.95)$ there is no advantage of choosing one envelope over the others if using the first Rabi cycle. In all cases a minimum $\pi$ pulse duration $\delta t_{\pi}=0.68 \omega_{r}^{-1}$ is achieved at $\bar{\Omega}=32.3 \omega_{r}$.

As we consider the second, third and fourth cycles the different behaviour of each envelope on the transition probabilities starts to reflect on $\delta t_{\pi}(\bar{\Omega})$. Starting on the second Rabi cycle with $\delta t<1 \omega_{r}^{-1}$ and $\bar{\Omega}>40 \omega_{r}$, the transfer efficiency with the Lorentzian envelope function decays less rapidly with increasing $\bar{\Omega}$ or decreasing $\delta t$, followed by the hyperbolic secant squared and the Gaussian envelopes. The Lorentzian therefore allows for the shortest $\pi$ pulses in the second Rabi cycle $\left(\delta t_{\pi}=0.74 \omega_{r}^{-1}\right.$ at $\left.\bar{\Omega}=52.0 \omega_{r}\right)$, followed by the hyperbolic secant squared $\left(0.78 \omega_{r}^{-1}\right.$ at $\left.50.8 \omega_{r}\right)$ and the Gaussian $\left(0.83 \omega_{r}^{-1}\right.$ at $\left.49.2 \omega_{r}\right)$. The differences between the envelopes grow with each consecutive Rabi cycle as the interaction strength grows 


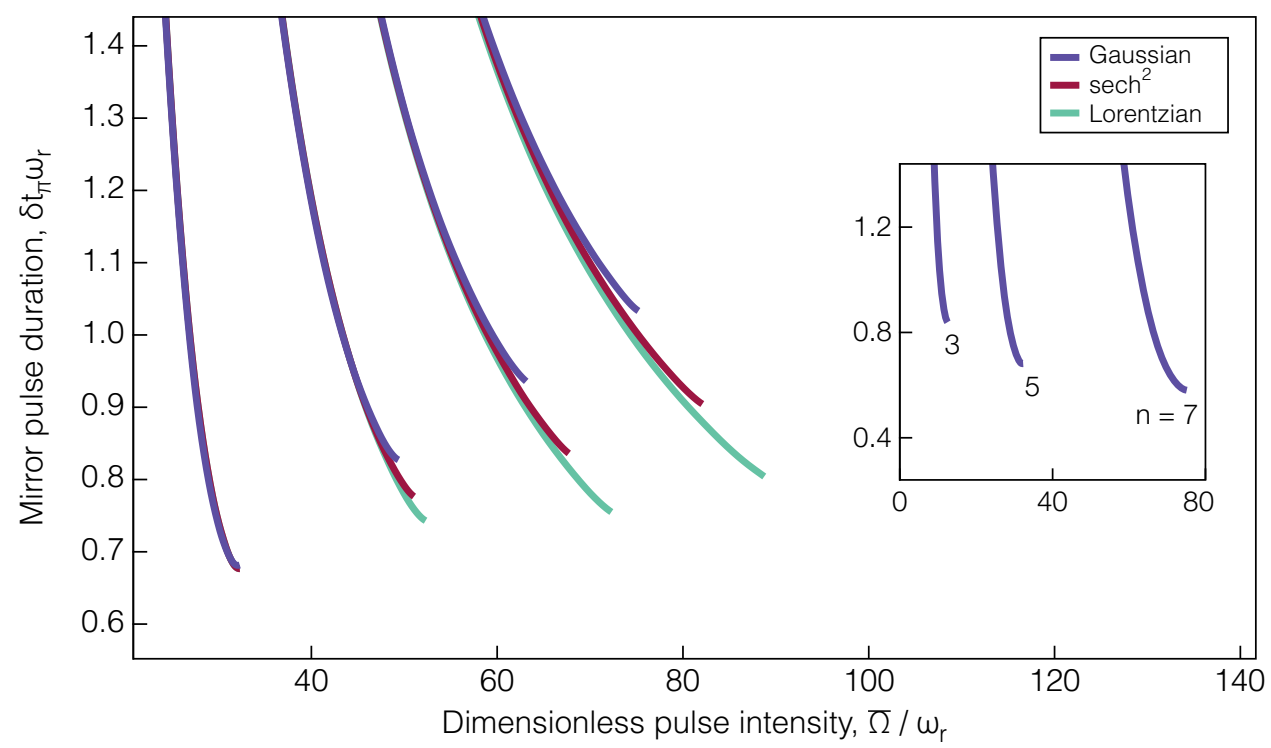

Figure 5.11: Duration and intensity of the $\pi$ pulses within the first four Rabi cycles for $m=5$, for the different envelope functions: Gaussian (blue), hyperbolic secant squared (red) and Lorentzian (green). Inset: First Rabi cycle for $m=3,5,7$. Note that the three pulse envelopes considered yield the same result in the first Rabi cycle, and therefore none provides an advantage for an interferometer operating in this regime.

larger and approaches the channeling regime. Extending the computation to additional diffraction orders confirms the same pattern, with the minimum $\pi$ pulse widths decreasing with increasing order as shown in the inset of Figure 5.11 for $m=3,5,7$.

For the remainder of this work we use the Gaussian envelope function (Equation 5.61) as the envelope function of choice for the interrogation laser. From a computational standpoint the Gaussian envelope has the advantage of decaying more rapidly with time, thus requiring significantly less computational power.

\subsection{Cavity-assisted Bragg beam splitter}

\subsubsection{Time-domain model of the Fabry-Perot resonator}

We now develop a simple time-domain model of an optical cavity in order to compute the transient response of the cavity to an input pulse of time-varying amplitude $a_{\text {in }}(t)$ (Figure 5.12). This will allow us to calculate the intra-cavity interrogation potential $\Omega(t)$ as a function of the cavity parameters.

Let us denote with $r_{a, b}$ and $t_{a, b}$ the amplitude reflection and transmission coefficients of the input $(a)$ and end $(b)$ mirrors respectively, which are both real and positive. We assume from now on that the cavity medium is vacuum. We 
also adopt the convention of $0^{\circ}$ phase shift on reflections and $90^{\circ}$ phase shift on transmission, which is the common notation used today in the analysis of modern optical systems due to its symmetry [63].

Using the phasor notation introduced in Chapter 2, let $a_{1}(t)$ be the amplitude of the total intra-cavity wave travelling in the direction of the incident wave at the position of the input mirror, $a_{2}(t)$ the same at the end mirror, $a_{3}(t)$ the total wave travelling in the opposite direction at the position of the end mirror and $a_{4}(t)$ the same at the input mirror. We can write difference equations for all the complex amplitudes, by following the excitation through a resonator round-trip,

$$
\begin{aligned}
a_{1}(t) & =i t_{a} a_{\mathrm{in}}(t)+r_{a} a_{4}(t), \\
a_{2}(t) & =a_{1}(t-\tau) e^{-i k L}, \\
a_{\mathrm{trans}}(t) & =i t_{b} a_{2}(t), \\
a_{3}(t) & =r_{b} a_{2}(t), \\
a_{4}(t) & =a_{3}(t-\tau) e^{-i k L}, \\
a_{\mathrm{refl}}(t) & =r_{a} a_{\mathrm{in}}(t)+i t_{a} a_{4}(t),
\end{aligned}
$$

where $\tau \equiv L / c$ is the photon transit time between the mirrors. The nominal length of the cavity can be treated as being an integer multiple of the laser wavelength, so that $e^{-2 i k L}=1$. We can then combine these expressions to obtain, e.g., the circulating field at the position of the input mirror in terms of the input field and of itself in the previous round-trip

$$
a_{1}(t)=i t_{a} a_{\mathrm{in}}(t)+r_{a} r_{b} a_{1}(t-2 \tau) \text {. }
$$

Each field can be expressed as a linear combination of itself and of the input pulse at previous times. We can do the same for all the fields inside the resonator, as

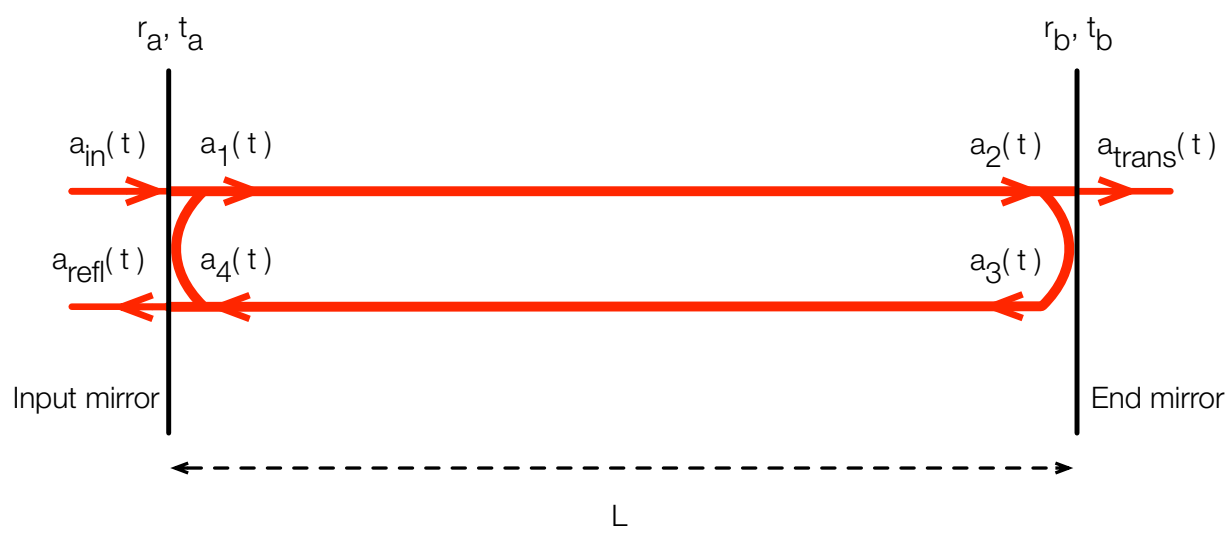

Figure 5.12: Fields in a Fabry-Perot resonator. 
well as the reflected $a_{\text {refl }}(t)$ and transmitted $a_{\text {trans }}(t)$ fields. Hence, we can easily recover the expressions for the static response of the cavity obtained in Chapter 2 by setting $a_{\mathrm{in}}(t)=a_{\mathrm{in}}=$ const. The field at any point inside the resonator can be obtained by writing difference equations with respect to an arbitrary plane between the two mirrors. However, except for a very small delay that can be neglected, the temporal envelope function of the circulating field is the same at any point inside the cavity as long as the width of the input pulse verifies $\delta t \gg \tau$.

These equations can be easily implemented in a computer program using a for-loop. The time step $\Delta \tau$ of the simulation should be at most equal to $\tau$, if not smaller by an integer fraction $\tau / n$. The reason for making $\Delta \tau$ smaller than $\tau$ is that in order to properly sample the input pulse it should verify $\Delta \tau \ll \delta$, where $\delta t$ is the duration of the input pulse, which may in extreme cases be of the order of $\tau$ or smaller (e.g., a $1 \mathrm{~ns}$ pulse in a $1 \mathrm{~m}$ resonator; note that such short pulses are not of interest for atom interferometry where the atomic transitions typically require $\mu \mathrm{s}$ long pulses). When the cavity is injected with a pulse having a smooth envelope function, the cavity response is smooth too (Figure 5.13 a). For a smooth envelope input pulse we can always attain greater resolution in the model by decreasing the size of the time step. However if the injected pulse presents any abrupt changes in amplitude, the circulating field will present the step-wise changes in power of duration $2 \tau$ associated with the photon round-trips in the cavity (Figure $5.13 \mathrm{~b}$ ).

From Equation 5.69 it is possible to derive an ordinary differential equation for the cavity field assuming that the variation of the input field's amplitude over the round-trip time is negligible [115]. Differentiating Equation 5.69 yields

$$
\frac{d a_{1}(t)}{d t}=\frac{r_{a} r_{b}-1}{2 \tau} a_{1}(t)+\frac{i t_{a}}{2 \tau} a_{\text {in }}(t) .
$$

This equation is not useful when considering short input pulses where the width of the pulse is of the order of the cavity photon lifetime or lower. The time it takes for a photon to complete a round-trip in the resonator is $\tau_{\mathrm{rt}} \equiv \Delta \nu_{\mathrm{FSR}}^{-1}$, and in this time the probability of it remaining confined in the resonator is $r^{4}$ due to two internal reflections. Thus, we can define the resonator photon lifetime as

$$
\tau_{p} \equiv \frac{\tau_{\mathrm{rt}}}{1-r^{4}}=\frac{1}{\Delta \nu_{\mathrm{FSR}}\left(1-r^{4}\right)} .
$$

For large values of the finesse we can approximate $1-r^{4}$ by $2\left(1-r^{2}\right)$ and the finesse by $\frac{\pi}{1-r^{2}}$, yielding

$$
\tau_{p} \approx \frac{F}{2 \pi \Delta \nu_{\mathrm{FSR}}}=\frac{F L}{\pi c} .
$$

The photon lifetime is tightly related to the resonator bandwidth by

$$
\tau_{p} \Delta \nu_{\mathrm{FWHM}}=\frac{1}{2 \pi}
$$

which is known as the time-bandwidth product of the resonator.

For input pulses with durations $\delta t$ of the order of the photon lifetime $\tau_{p}$ or lower, Equation 5.70 fails. There is another ordinary differential equation we can use to 

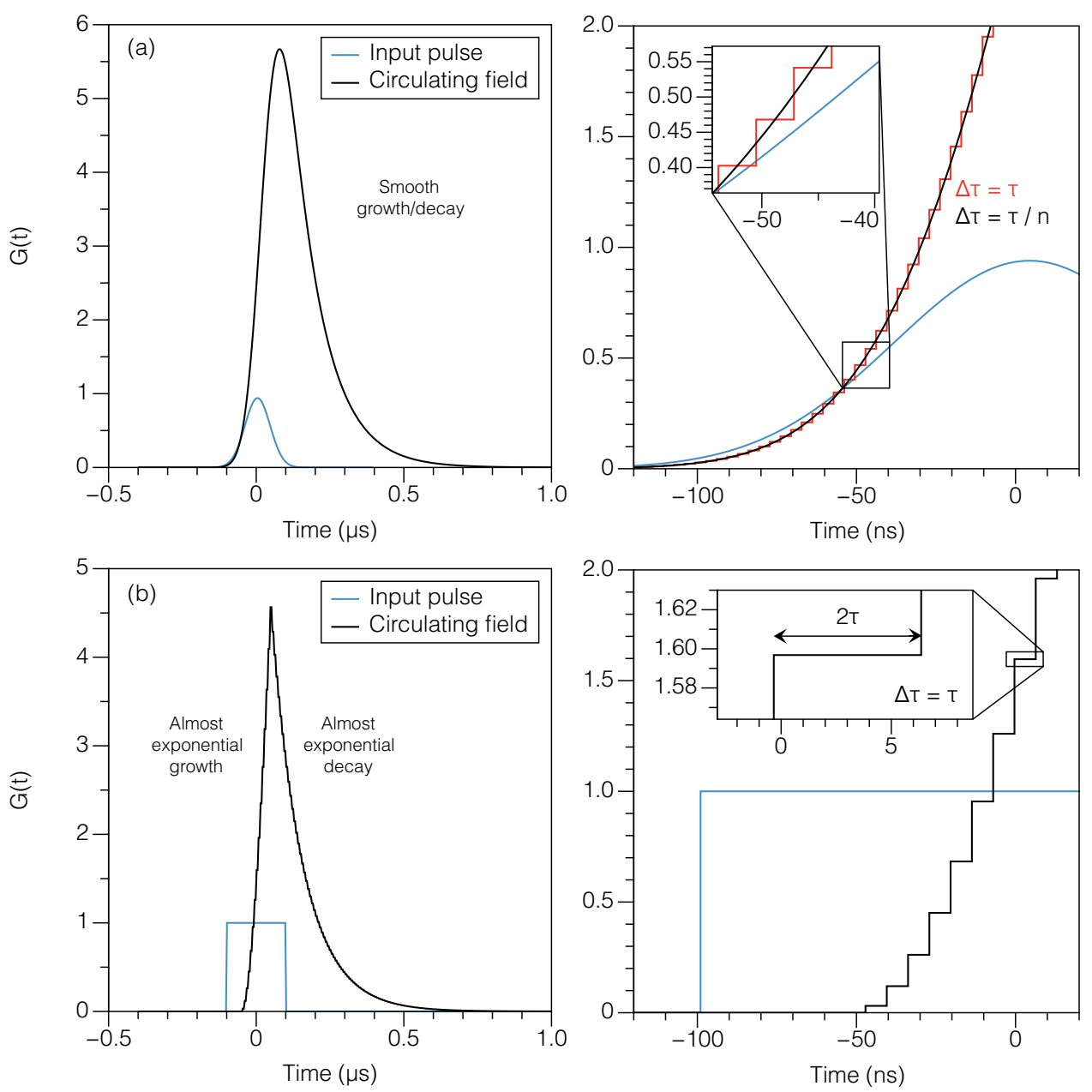

Figure 5.13: Transient response of a Fabry-Perot resonator to short pulses. The envelope functions $G(t)$ of the intensity of the input pulse and the circulating field are shown for a Gaussian (a) and a square (b) input. The increase in pulse area of the circulating field with respect to the input field is a consequence of the optical buildup effect provided by the cavity. For an input pulse with a smooth envelope function, the cavity response is smooth too (a). Our time domain simulation, however, is limited in resolution by the finite step size $\Delta \tau$ : a more accurate reproduction of the cavity response can always be obtained by using a smaller step size. If the input presents abrupt changes (i.e., a vertical step in amplitude), the cavity responds with the step-wise buildup or decay associated with the photon round-trips in the cavity (b). In the square input pulse case, the simulation is $100 \%$ accurate using the minimum time step $\Delta \tau=\tau$, and the circulating field presents steps of duration $2 \tau$. For these simulations a $1 \mathrm{~m}$ cavity with a finesse of $100\left(\tau \approx 3 \mathrm{~ns}, \tau_{p} \approx 100 \mathrm{~ns}\right)$ is illuminated with $100 \mathrm{~ns}$ pulses. 
compute the intra-cavity field, which is derived in quantum optics textbooks [116]

$$
\frac{d a(t)}{d t}=-\gamma a(t)+\sqrt{\frac{\gamma}{2 \tau}} a_{\text {in }}(t) .
$$

where $\gamma$ is the cavity's halfwidth, $\gamma \equiv \pi c / 2 \mathcal{F} L$.

While Equation 5.70 fails for $\delta t \lesssim \tau_{p}$, Equation 5.74 is still accurate, provided that the input pulse duration is much greater than the photon transit time $\delta t \gg \tau$. Both equations however require that the input pulse be smooth, and will fail to reproduce behaviour such as that depicted in Figure $5.13 \mathrm{~b}$. The differential equations may be useful in some situations, e.g., to obtain an explicit form for the circulating field given an explicit and smooth input function (see Section 7.3). However, the only fail-safe way of reproducing the response of the cavity to any accuracy is to implement Equations 5.68 with a sufficiently small step size.

\subsubsection{Cavity-induced deformation of the pulse envelope}

If the pulse duration is comparable to the photon lifetime of the cavity, the circulating field will present a deformed $G(t)$, asymmetric and with a larger area and width than the input pulse (Figure 5.15). For example, for a cavity with a photon lifetime

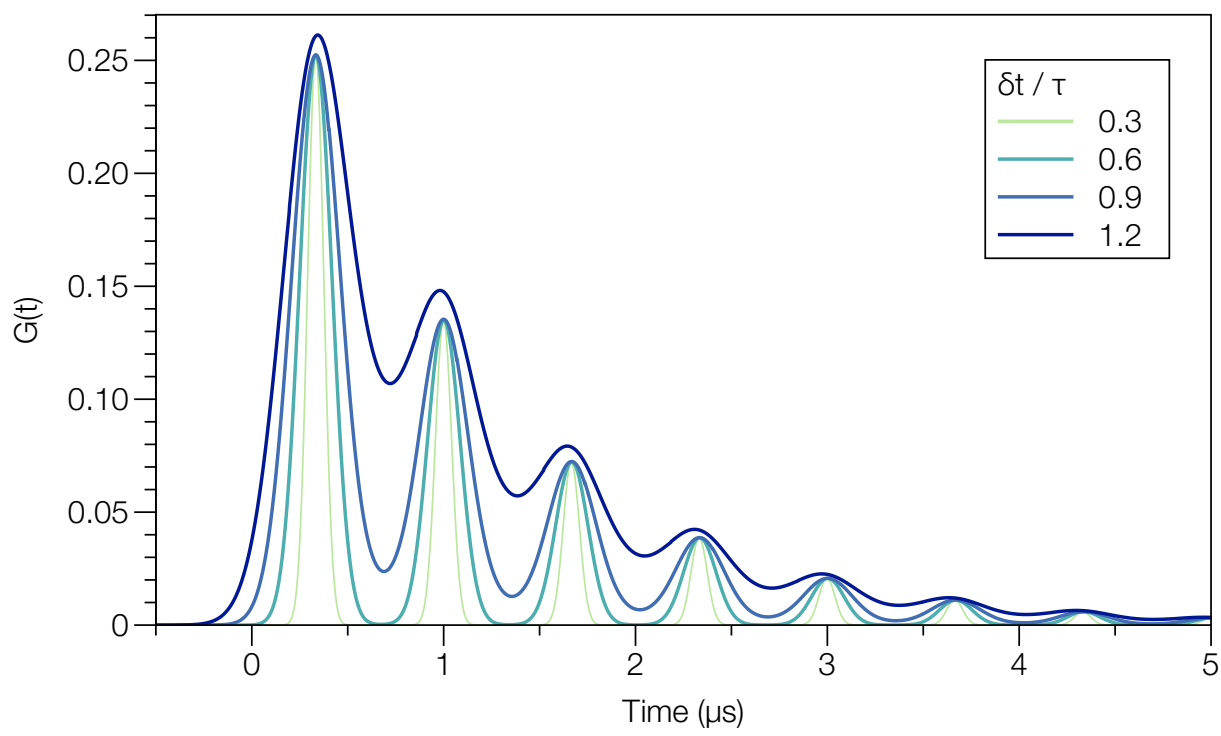

Figure 5.14: Transient response of the cavity to ultra short pulses. When the input pulse duration $\delta t$ is of the order of the photon transit time $\tau$ or lower, the cavity response presents a "train" of pulses decaying exponentially in amplitude according to the storage time of the cavity. In this simulation a $100 \mathrm{~m}$ cavity (photon transit time $\tau \approx 333 \mathrm{~ns}$ ) with a finesse of 10 is illuminated with pulses of different duration according to the ratio $\delta t / \tau$ given. 


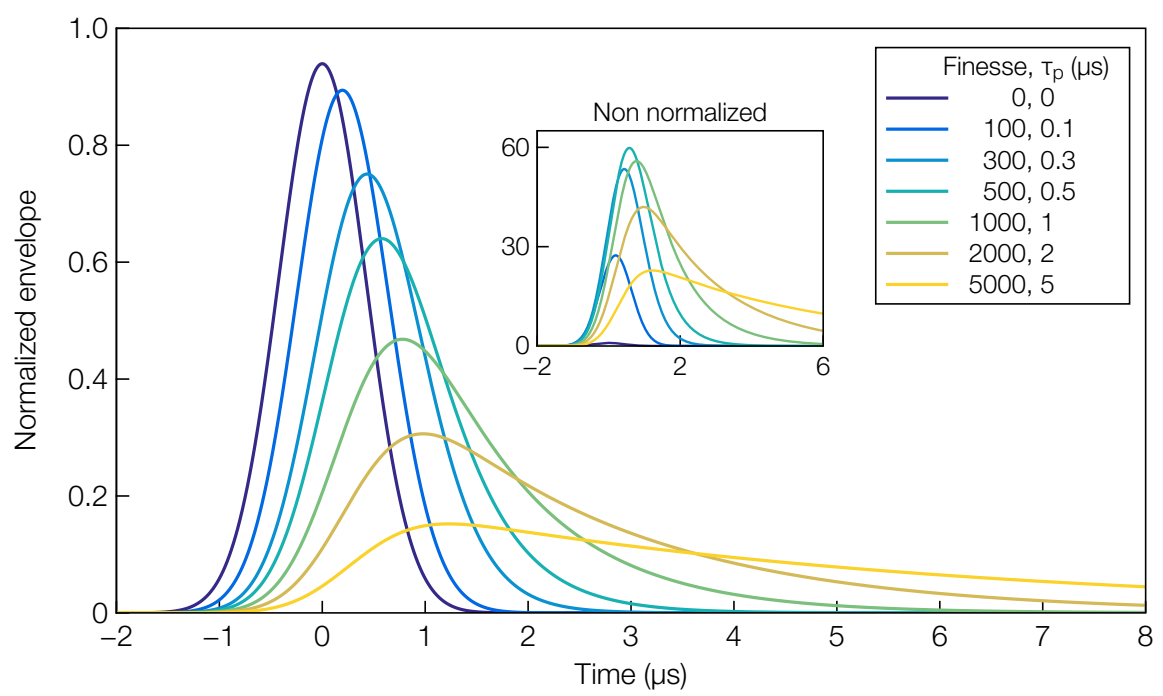

Figure 5.15: Cavity-induced deformation of a Gaussian input pulse. Envelope functions of the intra-cavity field for a $1 \mathrm{~m}$ cavity injected with a $1 \mu$ s pulse for different cavity finesses. All areas are normalised to the input pulse area for comparison. When the pulse duration is comparable to the photon lifetime of the cavity, its envelope function is elongated. Inset: Envelopes without normalisation.

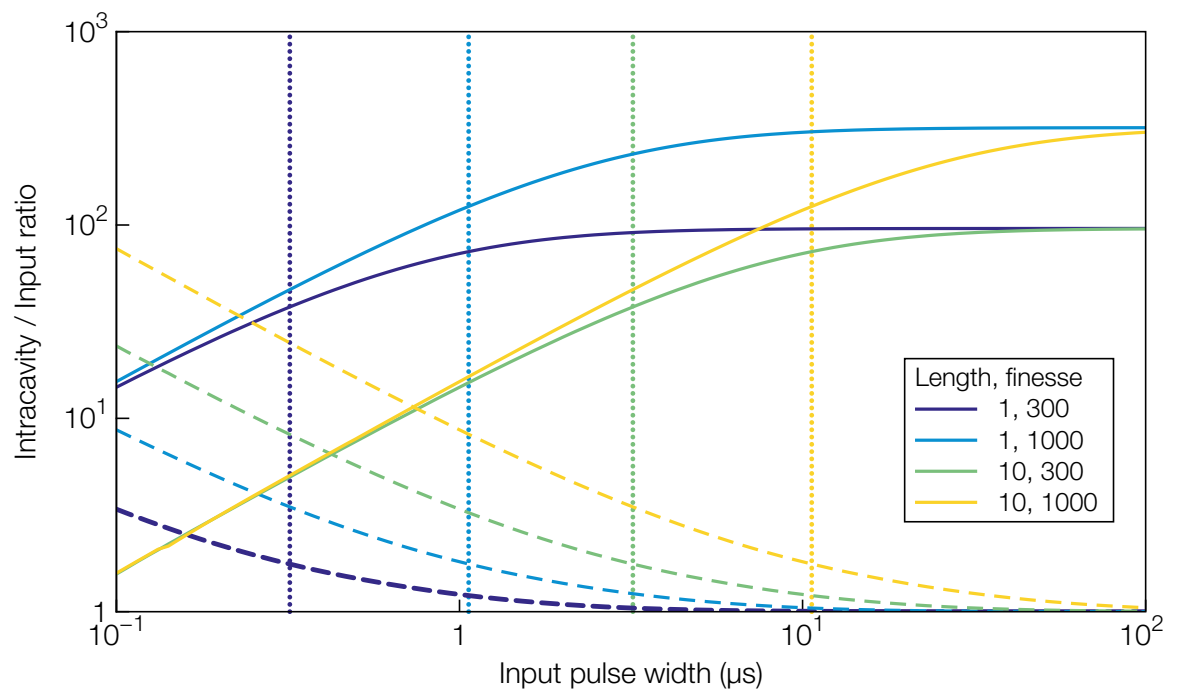

Figure 5.16: Transient response of the cavity to short pulses. Ratios of the intracavity field to the input pulse vs. the width of the input pulse, for both the pulse area (solid lines) and width (dashed lines), for four cavities of different length and finesse. The vertical lines represent the photon lifetime of each cavity. 
of $\tau_{p}=1 \mu \mathrm{s}$ injected with a short pulse of width $\delta t=1 \mu \mathrm{s}$, the intra-cavity field presents a deformed envelope of width $\approx 1.8 \mu \mathrm{s}, 80 \%$ longer than the input. A complete account of the photon-atom interactions in the optical cavity must include this effect.

Cavities having different photon lifetimes — or, equivalently, different bandwidth - respond differently to the same input (Figure 5.16). For input pulse widths much larger than the photon lifetime of the cavity, $\delta t \gg \tau_{p}$, the circulating field envelope tends to match the input envelope multiplied by the optical gain. In this scenario the cavity offers maximum power enhancement and does not cause any elongation, i.e., the temporal profile of the intra-cavity field is dominated by the input pulse. For pulse durations on the order of the photon lifetime or lower, power enhancement drops as the excitation does not have sufficient time to build up (the cavity does not reach a steady state), and the circulating field becomes elongated, with a temporal profile dominated by the cavity. For $\delta t \ll \tau_{p}$ the elongation becomes more severe. In the limit where the duration of the input pulse is of the order of the photon transit time or lower, Equation 5.74 also fails, and the only accurate method of computing the circulating field is by iterating the difference equations. In this limit, the cavity responds with a train of pulses (Figure 5.14).

\subsubsection{Effect of finesse and length on the atomic transitions}

Having constructed a time domain model of the cavity to compute the interrogation field $\Omega(t)$ the atoms will experience inside it, we are ready to explore the effect of the cavity parameters on the atomic transitions. We have already introduced the transfer efficiency of a $2 m$-photon transition as

$$
\lim _{t \rightarrow+\infty}|\langle m \mid \Psi(t)\rangle|^{2} \equiv \mathcal{P}(\bar{\Omega}, \delta t)
$$

where $|\Psi(t)\rangle=\left(\ldots g_{-m}(t), g_{-m+2}(t) \ldots\right)$ is the wave function describing the state of the atom (we use here the reduced wave function, assuming that the atom is only made up of either odd or even momentum states $|m\rangle)$. The shape of $\mathcal{P}(\bar{\Omega}, \delta t)$ will depend on the cavity parameters and on the shape of the envelope function of the input driving pulse. We have also defined the $\pi$-pulse duration within the first Rabi cycle as

$$
\delta t_{\pi}(\bar{\Omega}) \equiv \min \{\delta t: \mathcal{P}(\bar{\Omega}, \delta t)>0.95\}
$$

by deeming a population loss of $5 \%$ as acceptable for demonstration purposes. Restricting our computation to the first Rabi cycle - hence the 'min' — is done for the sake of simplicity. This is a reasonable restriction, however, as the interferometer requires the interaction time to be as short as possible. We perform the computation of $\delta t_{\pi}(\bar{\Omega})$ as we change the cavity parameters and measure the width of the elongated circulating cavity field $\tau_{\pi}(\bar{\Omega})$. Note that $\tau_{\pi}(\bar{\Omega})$ is the actual interaction time, and not $\delta t_{\pi}(\bar{\Omega})$ which refers to the width of the injected excitation. We are interested in the behaviour of $\tau_{\pi}(\bar{\Omega})$ with the cavity parameters of finesse $\mathcal{F}$ and length $L$.

Without loss of generality we consider a $10 \mathrm{~m}$ cavity injected with a pulse of Gaussian envelope function, and an ensemble of cold rubidium- 87 atoms interacting with the circulating field. For the purpose of this section, we regard the field in the cavity as uniform over the width of the cloud, and we shall make considerations 


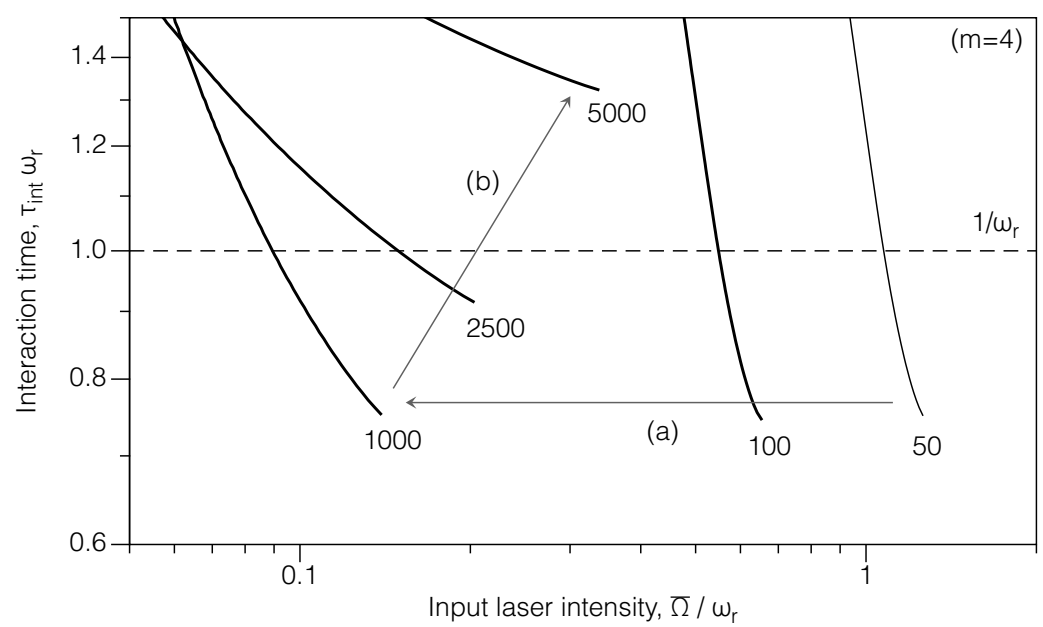

Figure 5.17: Duration vs intensity of the first mirror pulses in the $10 \mathrm{~m}$ cavity for different values of the cavity finesse, indicated at the bottom of each curve. As the cavity finesse increases, the curves shift left as the beam splitters require less input power due to the increased optical gain (a). After reaching a particular value of the finesse, $\mathcal{F}_{\max }$, the curves shift right and up, as the cavity-induced elongation becomes more severe and power enhancement of the beam splitters worsens (b).

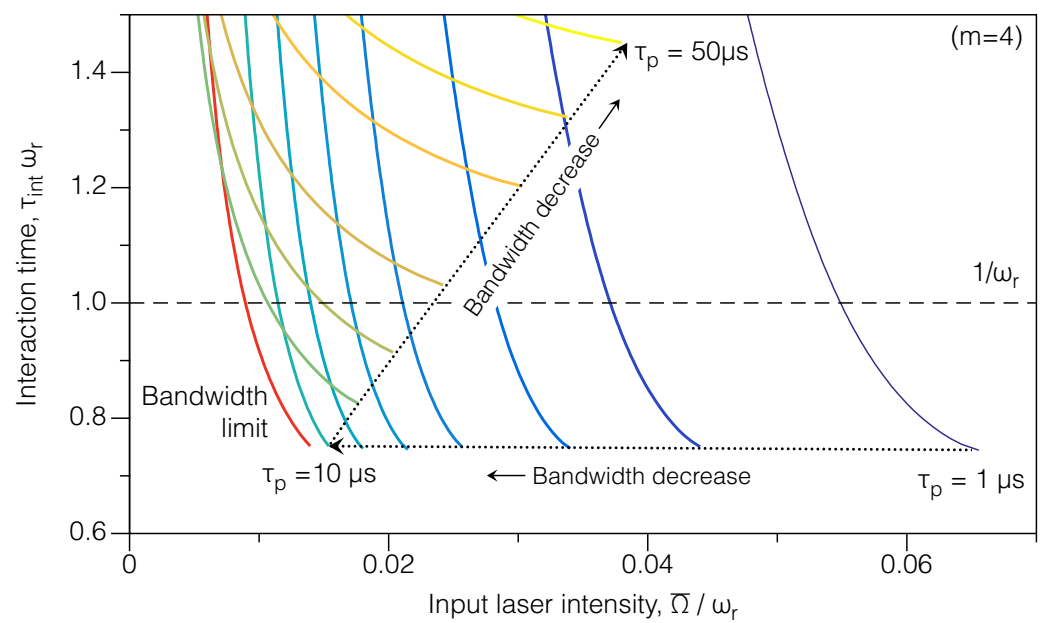

Figure 5.18: Duration vs intensity of the first mirror pulses for varying cavity photon lifetimes. The required input power is minimized for $\tau_{p}=\tau_{\max }$, in this case $(m=4)$ $\tau_{\max } \approx 10 \mu \mathrm{s}$. For $\tau_{p}>\tau_{\max }$, the minimum interaction time grows significantly. The input laser intensity scale shown here can be adjusted for any cavity length $L$ by applying a factor $L / 1 \mathrm{~m}$. 
about this approximation later on. We compute $\tau_{\pi}(\bar{\Omega})$ as we change the cavity finesse (Figure 5.17).

As we increase the cavity finesse we note three effects: (1) The required laser intensity of the beam splitters becomes considerably lower, as expected due to the cavity's buildup effect and highlighting the advantage of cavities for LMT beam splitting. (2) Power enhancement reaches a maximum for some value of the finesse, $\mathcal{F}_{\text {max }}$; increasing the finesse further comes at the price of increased intensity requirements for the short pulses. (3) The duration of the mirror pulses stays roughly the same as if there was no cavity up to $\mathcal{F}_{\text {max }}$; increasing the finesse further also comes at the price of increased interaction times, as the cavity's elongation effect becomes more severe.

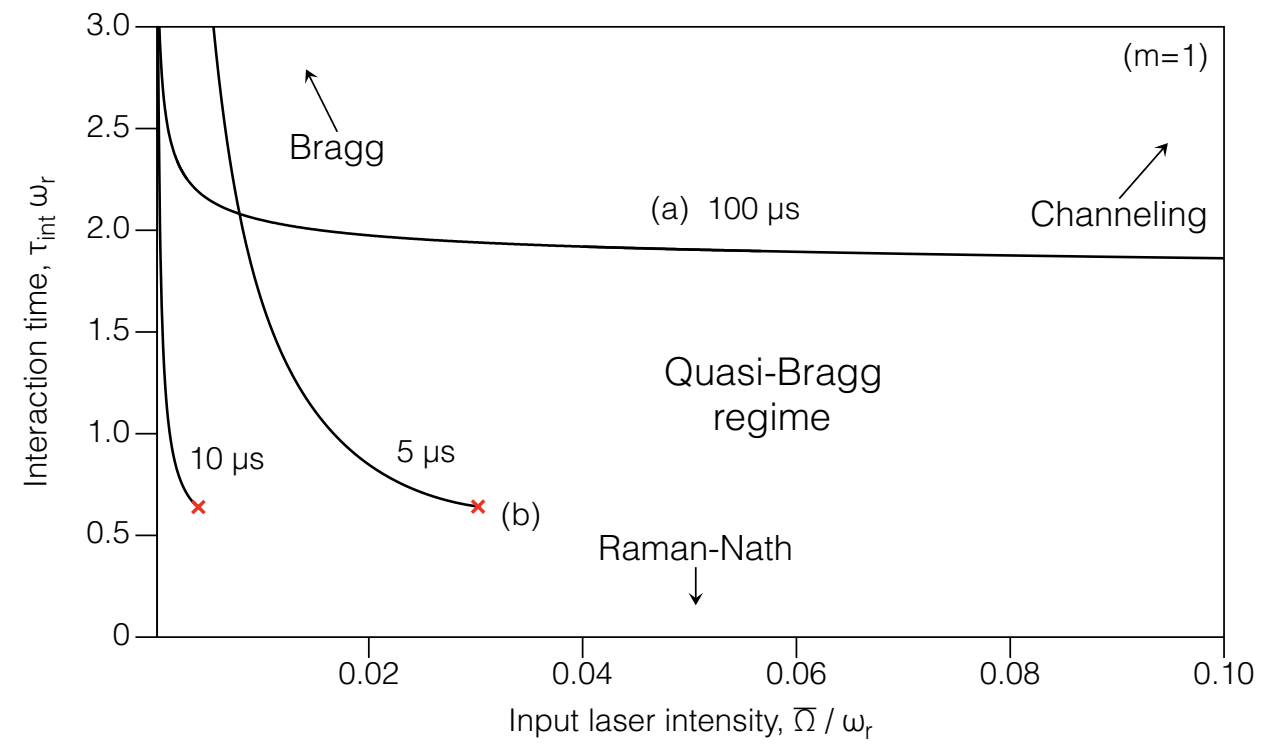

Figure 5.19: First Rabi cycle of a conventional $2 \hbar k$ beam splitter for three cavities with different photon lifetimes, indicated next to each curve. This transition is less lossy because of the direct coupling between the initial and final states. High-finesse cavities, with increased interaction times due to the severe elongation effect, exhibit a sharp transition between the adiabatic Bragg regime and the long-interaction steep-potential channeling regime, embodying the uncertainty relation between time and energy in the parameter space (a). The crosses represent the points where the transfer efficiency falls below $95 \%$ for the lower finesse cavities, as the interaction time approaches the Raman-Nath regime (b).

The cavity's effect on the pulse is therefore projected onto the transition probabilities by shifting both the required laser intensity and the photon-atom interaction time. Moreover, simulating more cavity length and finesse ranges and additional diffraction orders, we find that the shape of $\mathcal{P}(\bar{\Omega}, \delta t)$ is a function of the cavity bandwidth only. That is, there is no distinction between a length change and a finesse change with the exception of a linear shift in $\bar{\Omega}$ due to the scaling in optical 
gain. This is expected, as the cavity bandwidth univocally determines the shape of $G(t)$. Therefore, $\mathcal{F}_{\max }$ can be extrapolated for any cavity length from the value of the cavity photon lifetime $\tau_{\max }$. For example, cavities with length $L$ will exhibit the same behaviour depicted in Figure 5.17 for finesses adjusted by the ratio $10 \mathrm{~m} / L$ and intensities adjusted by $L / 10 \mathrm{~m}$.

In the absence of the cavity, the values of $\delta t_{\pi}(\bar{\Omega})$ decrease slightly with increasing $m$ for $m>2$, i.e., higher order processes yield shorter beam splitter pulses, which in turn have greater intensity requirements. The cavity deforms the pulse's envelope function $G(t)$, as determined solely by $\tau_{p}$, and the cavity with $\tau_{p}=\tau_{\text {max }}$ presents an optimal $G(t)$ that minimizes the required input power of the beam splitters. The value $\tau_{\max }$ is observed, through simulation, to be approximately $1 / 3$ the duration of the shortest beam splitter pulse in the absence of the cavity. We believe this is because the optimal $G(t)$ occurs at a certain ratio between the cavity photon lifetime and the input pulse width, before the pulse gets significantly distorted by the cavity. Hence, the dependence of $\tau_{\max }$ on $m$ is roughly the same as that of $\min \left\{\delta t_{\pi}(\bar{\Omega})\right\} / 3$, which is a rather slow dependence.

By increasing the interaction time, increasing the cavity finesse has the effect of parametrically pushing the photon-atom interactions towards the Bragg and channeling regimes. An evidence of this is the change in the slope of $\tau_{\pi}(\bar{\Omega})$ for cavities with $\tau_{p}>\tau_{\max }$, as can be seen in Figure 5.18. The very high finesse cavities have a slope $d \tau_{\pi} / d \bar{\Omega} \rightarrow+\infty$ for $\bar{\Omega} \rightarrow 0$, indicating adiabacity, and $d \tau_{\pi} / d \bar{\Omega} \rightarrow 0$ otherwise, indicating the channeling effect. As the cavity storage time becomes higher, the atomic interactions with the circulating cavity field become inevitably longer, and as they do so the diffraction process becomes more adiabatic. A high finesse cavity will transform a short input pulse with a large energy uncertainty into a long pulse with a well-defined energy. In doing so, energy conservation will favor transitions to the target state with low losses, unless the price is paid in terms of input power to drive efficient transitions that violate the adiabacity condition, thus operating in the long-interaction steep-potential channeling regime.

This is best seen in first order Bragg diffraction, $m=1$ (Figure 5.19). This case is unique because the initial and final states, $| \pm 1\rangle$, are coupled directly. While both states can transfer population to and from their other neighbour, the direct coupling between them makes the transition naturally less lossy. The adiabacity condition for this case is therefore much lower than for the $m=2$ case (note that in the $m=1$ case there are no intermediate states). For this reason the elongation effect appears to be less significant in this case when compared to, e.g., $m=2$, but in turn the intensity required for the shortest beam splitters grows more steeply. The higher finesse cavities with increased interaction times are parametrically "pushed" to transition sharply between the Bragg and channeling regimes. In Figure 5.19 note how the cavity with $\tau_{p}=100 \mu$ s produces efficient transitions with $d \tau_{\pi} / d \bar{\Omega} \rightarrow 0$ within the first Rabi cycle, whereas at lower $\tau_{p}$ efficiencies fall below $95 \%$ as expected in the quasi-Bragg regime. The curve for $\tau_{p}=100 \mu \mathrm{s}$ in Figure 5.19 is a good representation of the uncertainty relation between interaction time and energy in the parameter space.

Figure 5.20 presents $\min \left\{\tau_{\pi}\right\}$ and the corresponding $\bar{\Omega}$ as a function of the cavity photon lifetime for Bragg diffraction orders $m=1-9$. 

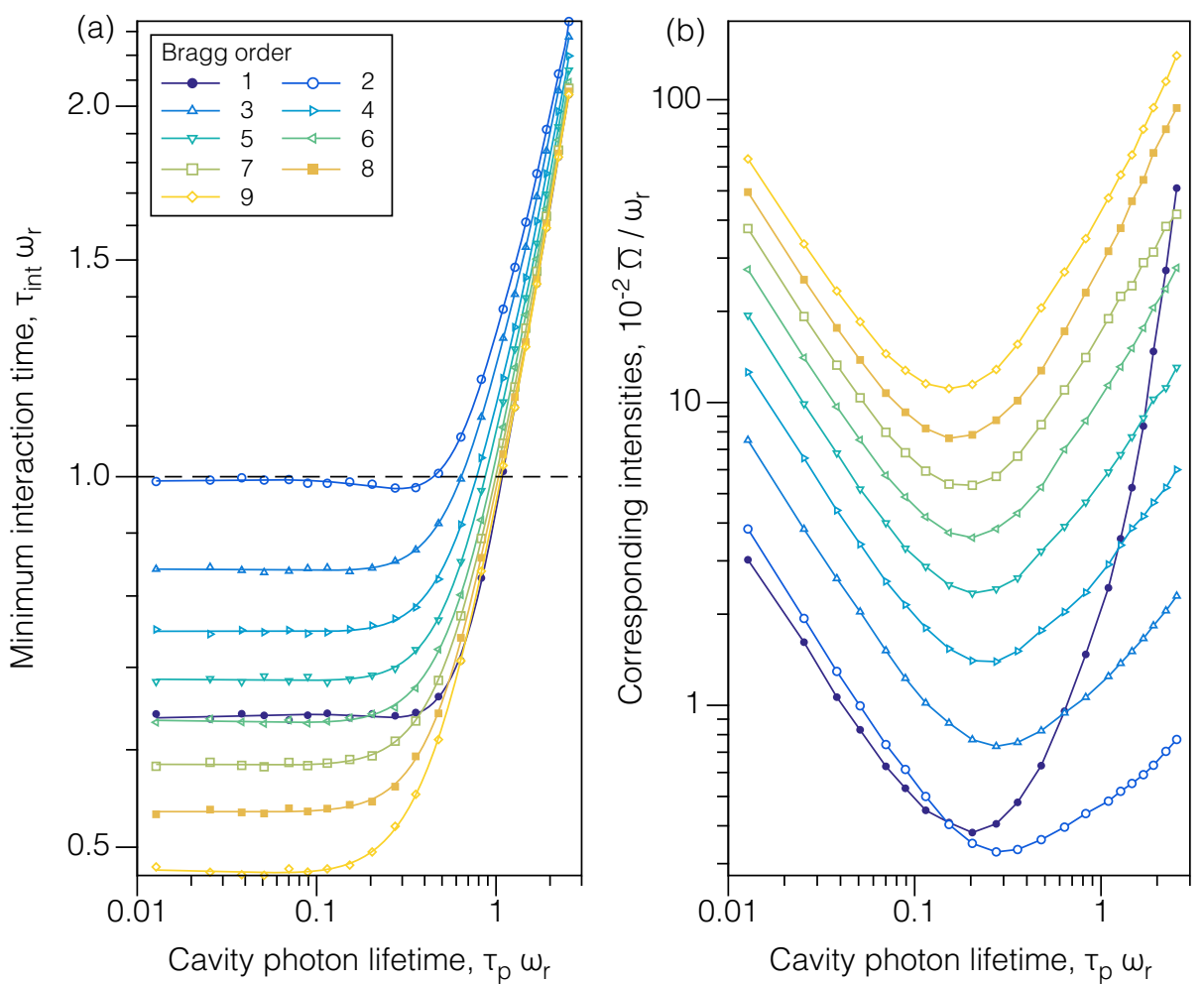

Figure 5.20: Effect of the cavity photon lifetime $\tau_{p}$ on the atomic transitions. The minimum photon-atom interaction time remains largely unaffected for cavities with $\tau_{p}<\tau_{\max }$, and increases linearly for $\tau_{p}>\tau_{\max }$ (a). The required intensity for the shortest beam splitter is minimal for $\tau_{p}=\tau_{\max }(\mathrm{b})$. Note that the transition features in the $n=1$ case are different from the higher diffraction orders due to the direct coupling between initial and final states. 

CHAPTER 6

\section{Fundamental limitations of cavity- assisted atom interferometry}

\section{Contents}

6.1 Towards low-power and low-noise large momentum transfer atom optics . . . . . . . . . . . . . . . . . 132

6.2 Cavity bandwidth limits . . . . . . . . . . . . 132

6.3 Constraints on spatial filtering and beam size . . . . . . . 135

6.4 Atom temperature and cavity length limits for interferometers $\mathbf{1 3 9}$

6.5 Summary and future work . . . . . . . . . . . . . 145 


\title{
6.1 Towards low-power and low-noise large momentum transfer atom optics
}

\begin{abstract}
A TOM interferometry inside an optical cavity was demonstrated in [5], where they A show a $\pi / 2-\pi-\pi / 2$ interferometer with caesium atoms loaded horizontally into a vertical $40 \mathrm{~cm}$ cavity (Figure 6.1). In this proof of principle experiment, the small cavity mode volume placed a tight constraint on the total measurement time, which was just $20 \mathrm{~ms}$. The same group was able to increase the total measurement time up to $130 \mathrm{~ms}$ [38-40]. A clever design of a marginally stable cavity with an intra-cavity lens was proposed in [110], also employing a perpendicular loading scheme (see Figure 6.5 e) but with a large mode volume capable of accommodating a $1 \mu \mathrm{K}$ cloud as it expands for up to $250 \mathrm{~ms}$. Cavity-assisted LMT beam splitters are also proposed for the gravitational-wave antenna MIGA [6,18], where the interferometric pulses resonate inside two horizontal $200 \mathrm{~m}$ cavities and interrogate three atom clouds launched vertically (see Figure 7.1) for a total measurement time of $250 \mathrm{~ms}$.
\end{abstract}

Despite its promising nature, the advantages and limitations of cavity-assisted atom interferometry have not yet been quantified. The cavity bandwidth plays a major role in the performance of the interferometer. Power enhancement and spatial filtering are both enhanced by increasing the cavity finesse. The maximal allowed beam size increases with cavity length. In atom interferometry, both good spatial filtering and large beam sizes are desired qualities. The cavity bandwidth scales inversely with the product of finesse and length. Thus, it would seem obvious that the narrower the bandwidth is, the better. We found, however, that there is a limit to the bandwidth below which the pulses suffer severe elongation - leading to undesirably long interaction times - and power enhancement of the beam splitter pulses worsens dramatically, nullifying the advantage of incorporating the cavity in the first place. Having realized this bandwidth limit, the task then becomes a balancing act between the quality of the cavity as a spatial filter of the interferometric beams and its ability to accommodate the size of the atomic cloud as it thermally expands during the measurement.

\subsection{Cavity bandwidth limits}

In the previous chapter we studied in detail the photon-atom interactions in a Bragg diffraction experiment inside an optical cavity, and specifically how the cavity parameters of finesse $\mathcal{F}$ and length $L$ affected the atomic transitions. For the short pulses that are needed in a Bragg-diffraction large momentum transfer atom interferometer, operation in the quasi-Bragg regime is essential, utilising the very first Rabi cycles of the interaction.

Analysing the behaviour of the mirror pulses in the parameter space of the interaction time and the input laser intensity, we found that there is a value of the cavity photon lifetime $\tau_{\max }$ or, equivalently, of the cavity bandwidth (in $\mathrm{rad} / \mathrm{s}$ )

$$
\Delta \omega_{\min } \equiv \frac{1}{\tau_{\max }}
$$




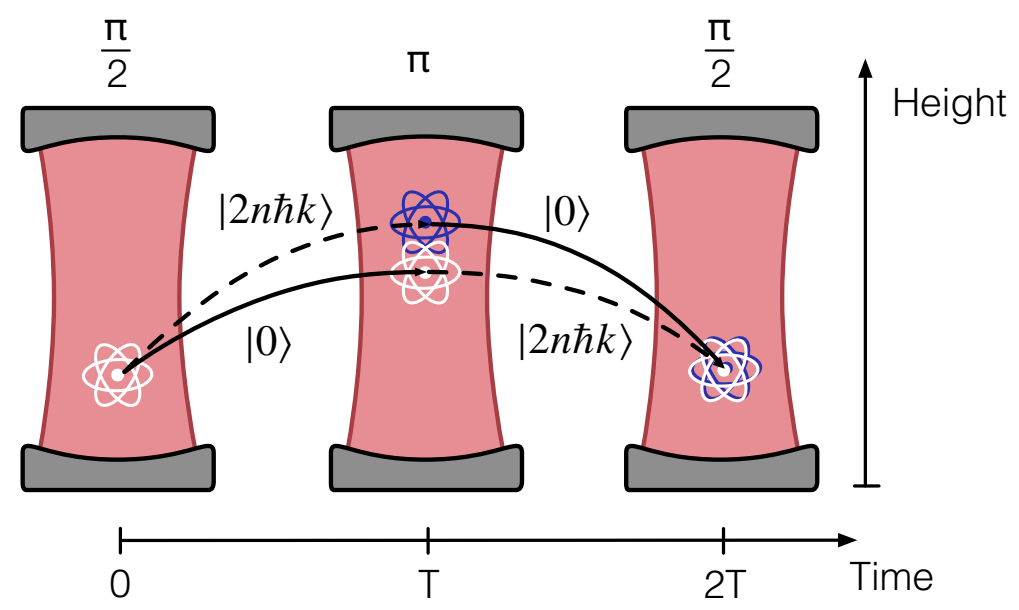

Figure 6.1: An interferometer sequence using cavity-assisted large momentum beam splitters. A cloud of atoms is coherently split $(t=0)$, deflected $(t=T)$, and recombined $(t=2 T)$ using light pulses that resonate in the cavity.

which guarantees that the cavity offers maximum power enhancement of the short beam splitter pulses while keeping the interaction time unaffected (equivalently, we also define $\Delta \nu_{\min } \equiv \Delta \omega_{\min } / 2 \pi$ in units of $\mathrm{Hz}$ ). Its value depends on the order of the diffraction process, with higher order processes leading to a higher bound on the bandwidth. A bandwidth higher or lower than this value will incur the requirement of a larger laser power, with lower bandwidths also incurring longer interaction times. It is thus reasonable to impose $\Delta \omega_{\min }$ as a lower bound to cavity bandwidth or, equivalently, $\tau_{\max }$ as an upper bound to the cavity photon lifetime. Note that these bandwidth limits are specially significant for long-baseline experiments, as the larger cavity length vastly reduces the feasible finesse range. Of course, it may be a design choice to use a bandwidth lower than $\Delta \omega_{\text {min }}$ and suffer the consequences (e.g., greater power requirements and a reduction of atomic flux due to the elongated pulses being able to resolve the velocity spread of the cloud). The bandwidth and photon lifetime limits defined here are not physical limits, but design limits based on optimising the performance of cavity-assisted atomic beam splitters.

The values of $\tau_{\max }$ for $m=1-9$ are depicted in Figure 6.2 and listed in Table 6.1. The values for rubidium- 87 for $m=1$ are easy to remember and very similar to the $m=4$ case: $10 \mu$ s photon lifetime $\left(16 \mathrm{kHz}\right.$ bandwidth), which translates into a $\mathcal{F}_{\text {max }}$ of roughly 10000, 1000 and 100 for cavity lengths of 1,10 and $100 \mathrm{~m}$ respectively. See Tables 6.1 and 6.2 for a complete set of values for $m=1-9$. We cannot predict the behaviour at higher diffraction orders, and computing these results gets increasingly computationally expensive as the system matrix grows. However, it looks as though the bandwidth limit will keep increasing as the transitions become faster due to the increasing $m$. It would therefore be possible for large momentum transfer beam splitters to be more efficient by employing a high power laser system 


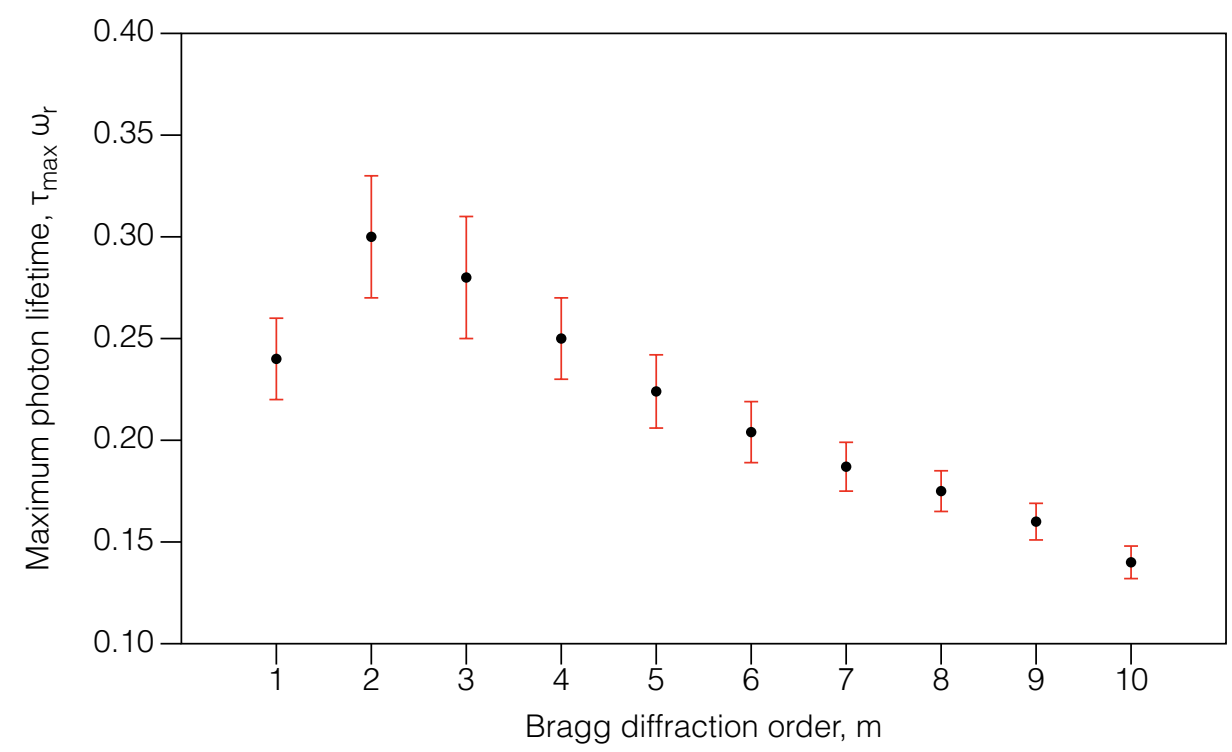

Figure 6.2: Variation of $\tau_{\max }$ with the order of the diffraction process. The error bars represent the statistical uncertainty yielded by propagation of error through a least squares fit of the data to calculate the photon lifetimes that minimize the required input intensity of the beam splitters.

instead of an optical cavity, depending on the order of the process and the cavity length.

\begin{tabular}{ccccccc}
\hline$m$ & $\tau_{\max } \omega_{r}$ & $\begin{array}{c}\tau_{\max }^{\mathrm{Rb} 87} \\
(\mu \mathrm{s})\end{array}$ & $\begin{array}{c}\tau_{\max }^{\mathrm{Cs} 133} \\
(\mu \mathrm{s})\end{array}$ & $\begin{array}{c}\Delta \omega_{\min } \\
\end{array}$ & $\begin{array}{c}\Delta \nu_{\min }^{\mathrm{Rb} 87} \\
(\mathrm{kHz})\end{array}$ & $\begin{array}{c}\Delta \nu_{\min }^{\mathrm{Cs} 133} \\
(\mathrm{kHz})\end{array}$ \\
\hline 1 & 0.24 & 10.0 & 18.5 & 4.2 & 16.0 & 8.6 \\
2 & 0.30 & 12.4 & 23.1 & 3.3 & 12.8 & 6.9 \\
3 & 0.28 & 11.6 & 21.5 & 3.6 & 13.7 & 7.4 \\
4 & 0.25 & 10.4 & 19.2 & 4.0 & 15.3 & 8.3 \\
5 & 0.22 & 9.1 & 16.9 & 4.5 & 17.4 & 9.4 \\
6 & 0.20 & 8.3 & 15.4 & 5.0 & 19.2 & 10.3 \\
7 & 0.19 & 7.8 & 14.4 & 5.3 & 20.5 & 11.1 \\
8 & 0.18 & 7.3 & 13.5 & 5.7 & 21.9 & 11.8 \\
9 & 0.16 & 6.6 & 12.3 & 6.3 & 24.0 & 12.9 \\
\hline
\end{tabular}

Table 6.1: Maximal cavity parameters for atom optics. A cavity with photon lifetime $\tau_{\max }$ (or bandwidth $\Delta \omega_{\min }$ ) minimises the required power of the atomic beam splitters and keeps the interaction time unaffected. The values given in $\mu \mathrm{s}$ and $\mathrm{kHz}$ correspond to the rubidium- $87 \mathrm{D}_{2}$ transition $\left(5^{2} \mathrm{~S}_{1 / 2} \rightarrow 5^{2} \mathrm{P}_{3 / 2}\right)$, and the caesium-133 $\mathrm{D}_{2}$ transition $\left(6^{2} \mathrm{~S}_{1 / 2} \rightarrow 6^{2} \mathrm{P}_{3 / 2}\right)$ 


\begin{tabular}{cccccccc}
\hline & \multicolumn{3}{c}{$\mathcal{F}_{\max }^{\mathrm{Rb} 8}$} & \multicolumn{3}{c}{$\mathcal{F}_{\max }^{\mathrm{Cs} 133}$} \\
$m$ & $1 \mathrm{~m}$ & $10 \mathrm{~m}$ & $100 \mathrm{~m}$ & $1 \mathrm{~m}$ & $10 \mathrm{~m}$ & $100 \mathrm{~m}$ \\
\hline 1 & 9373 & 934 & 91 & 17392 & 1736 & 171 \\
2 & 11717 & 1169 & 114 & 21741 & 2171 & 214 \\
3 & 10936 & 1091 & 106 & 20292 & 2026 & 200 \\
4 & 9763 & 974 & 94 & 18117 & 1809 & 178 \\
5 & 8592 & 856 & 83 & 15943 & 1591 & 156 \\
6 & 7810 & 778 & 75 & 14493 & 1446 & 142 \\
7 & 7302 & 727 & 70 & 13551 & 1352 & 132 \\
8 & 6834 & 681 & 65 & 12681 & 1265 & 124 \\
9 & 6248 & 622 & 59 & 11594 & 1157 & 113 \\
\hline
\end{tabular}

Table 6.2: The maximal finesse $\mathcal{F}_{\max }$ for cavity lengths of 1,10 and 100 metres corresponding to the rubidium- $87 \mathrm{D}_{2}$ transition $\left(5^{2} \mathrm{~S}_{1 / 2} \rightarrow 5^{2} \mathrm{P}_{3 / 2}\right)$, and the caesium$133 \mathrm{D}_{2}$ transition $\left(6^{2} \mathrm{~S}_{1 / 2} \rightarrow 6^{2} \mathrm{P}_{3 / 2}\right)$.

\subsection{Constraints on spatial filtering and beam size}

We shall now analyse the geometrical and optical properties of the full cavityassisted interferometer. The biggest constraint on the cavity parameters is set by the fact that its eigenmode (the fundamental Hermite-Gauss mode, $\mathrm{HG}_{00}$ ) must accommodate the size of the atomic cloud as it thermally expands during the measurement whilst maintaining a geometrically stable configuration. In this section we study the limitations of the cavity as a spatial filter under this constraint. The requirement of having a large waist may lead the cavity to be pushed very close to the edge of geometrical instability, which carries the consequent problems of increased sensitivity to alignment errors, mirror surface imperfections, and coupling to higher-order spatial modes. In addition, there is an incompatibility between having a cavity with a large beam size and simultaneously good spatial filtering (Figure 6.3). These findings, along with those from the previous section, allow us to establish upper bounds on the temperature of the atomic ensemble as a function of cavity length and three design parameters.

We assume here a cavity with a symmetric two-mirror configuration. Having the beam waist at the center of the cavity means that the curvature of the beam is symmetric with respect to it, allowing the possibility of running the interferometric sequence along the optical axis (on-axis sequence). In this configuration the cavity can be used to simultaneously interrogate two clouds launched vertically in a juggling atomic fountain. The cavity can also be used to interrogate several atom interferometers running in parallel along the optical axis with the clouds being loaded perpendicularly into the cavity, as proposed for MIGA (perpendicular sequence). In on-axis sequences the total measurement time scales with $\sqrt{L}$, as the atoms explore some fraction of the cavity length, whereas in perpendicular sequences the total measurement time is a parameter independent of cavity length.

Having established a lower bound on the cavity bandwidth on the previous section, we determine the maximum level of spatial filtering achievable by the cavity 

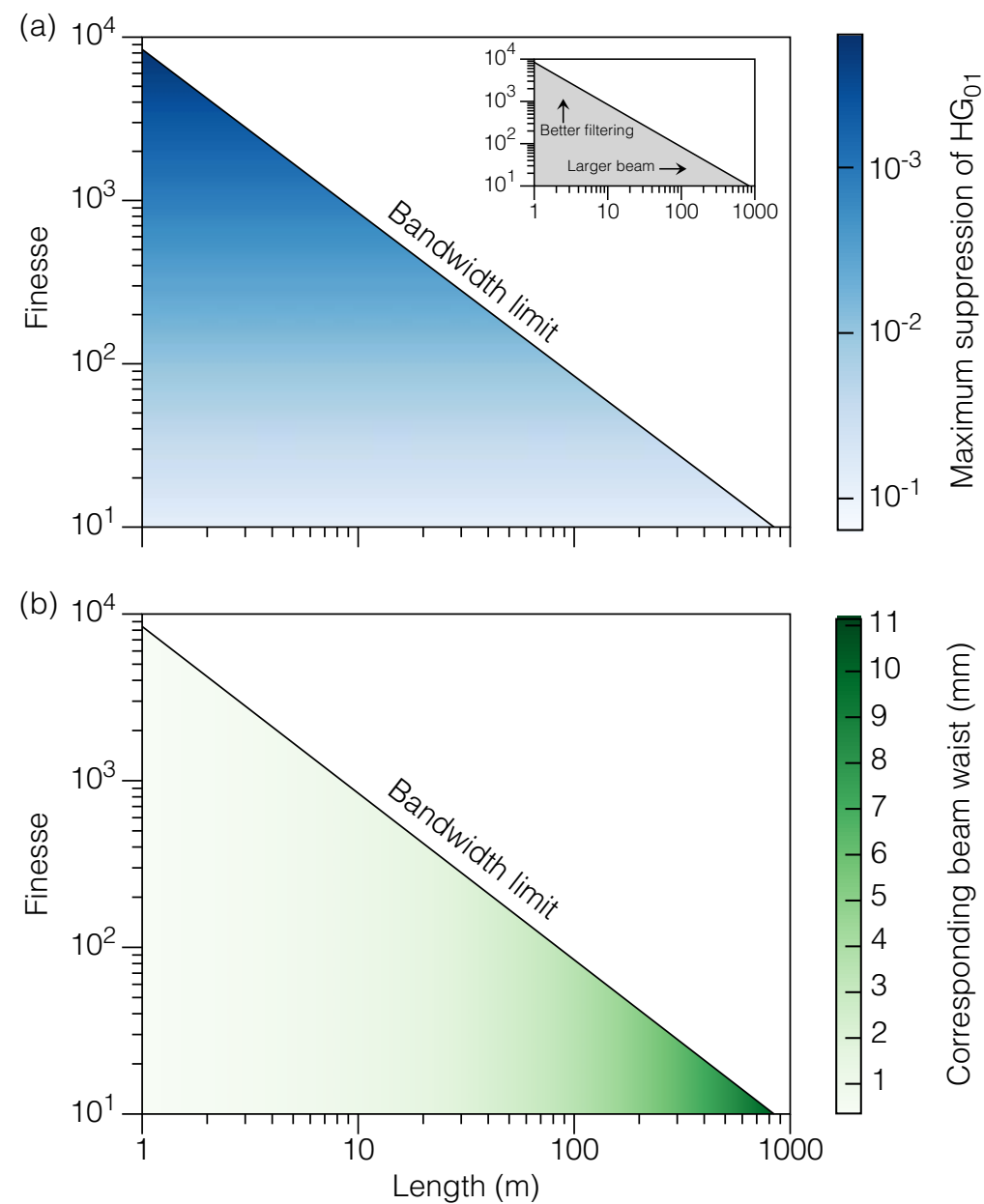

Figure 6.3: The bandwidth limit places a constraint on the cavity's $L-\mathcal{F}$ parameter space, depicted here for $m=1$. Higher finesses lead to better spatial filtering (a), while longer lengths allow for larger beams (b). In atom interferometry, both large beams and good spatial filtering are desired qualities.

as a function of beam size. This will help in understanding the limitations of the cavity given the constraint imposed on beam size by the expanding atomic cloud.

Let us recall that the round-trip Gouy phase shift in a Fabry-Perot cavity is given by (Equation 2.42)

$$
\zeta=2 \arccos \left( \pm \sqrt{g_{1} g_{2}}\right),
$$

where $g_{i}=1-L / \mathcal{R}_{i}$, with $\mathcal{R}_{i}$ the mirrors' radii of curvature, and the sign \pm is given by the sign of $g_{1}$. For symmetric cavities $\mathcal{R}_{1}=\mathcal{R}_{2} \Rightarrow g_{1}=g_{2}$. The total cavity $g$ factor $g=g_{1} g_{2}$ is an important parameter quantifying the cavity's geometrical stability. A strictly stable cavity verifies $0<g<1$, but in practice 
the cavity $g$ factor should not exceed a certain threshold $g_{\max }$ in order to maintain controllability [117]. The round-trip Gouy phase shift is an even more powerful parameter $(0<\zeta<2 \pi$ for a strictly stable cavity) because it is useful in quantifying if a particular set of higher order modes will be co-resonant with the fundamental mode. The additional Gouy phase accumulated by the $n m$ - $\mathrm{th}^{1}$ mode with respect to the fundamental mode is $(n+m) \zeta$ in a cavity round-trip, leading to an optical suppression factor of that mode of [118]

$$
S_{n m} \equiv\left[1+\left(\frac{2 \mathcal{F}}{\pi}\right)^{2} \sin ^{2}\left([n+m] \frac{\zeta}{2}\right)\right]^{-\frac{1}{2}} .
$$

By setting a constraint of the type $S_{01,02} \leq S_{\max }$, we introduce a design requirement on the quality of the cavity as a spatial filter, as a low suppression factor of the first and second order modes means that the cavity will present a cleaner wavefront.

The local Gouy phase shift of the cavity's fundamental Guassian mode, referenced to the location of the waist at the centre of the cavity, is given by (Equation 2.25 for $z_{0}=0$ )

$$
\zeta(z)=\arctan \left(\frac{z}{z_{R}}\right)
$$

where $z$ is the position along the optical axis with respect to the centre, $z_{R}=\pi w_{0}^{2} / \lambda$ is the Rayleigh range, and $w_{0}$ is the beam waist radius. Using Equations 6.2 and 6.3 we derive an expression for the optical suppression of higher-order modes in terms of the cavity finesse, length, and beam waist

$$
S_{n m}=\left[1+\left(\frac{2 \mathcal{F}}{\pi}\right)^{2} \sin ^{2}\left(2(n+m) \arctan \left[\frac{\lambda L}{2 \pi w_{0}^{2}}\right]\right)\right]^{-\frac{1}{2}},
$$

where we have used $\zeta=2\left[\zeta\left(\frac{L}{2}\right)-\zeta\left(-\frac{L}{2}\right)\right]$ (the factor 2 to compute the roundtrip phase shift). Note that $\zeta(z)-\zeta(-z)=2 \zeta(z)$.

We consider, as an example, three cavities of different length $-1,10$, and $100 \mathrm{~m}$ - all having the same bandwidth of $16 \mathrm{kHz}$, which is the lower limit for Bragg diffraction orders $m=1$ and $m=4$ (Figure 6.4). In the limit where the waist of the cavity is in the order of interest for atom interferometry, the suppression factors are approximately the same for all cavities having the same bandwidth,

$$
\lim _{\left(\begin{array}{c}
\text { large } \\
\text { waist }
\end{array}\right)} S_{n m}=1-\frac{(n+m)^{2} c^{2} \lambda^{2}}{2 \pi^{4} \Delta \nu_{\min }^{2} w_{0}^{4}}+O\left(\frac{1}{w_{0}}\right)^{8},
$$

as evidenced by the overlapping curves to the right of Figure 6.4 a. That is, for the large beam sizes needed in order to accommodate the thermally expanding clouds, the spatial filtering properties of cavities having the same bandwidth are approximately the same. When the cavity bandwidth is limited for design reasons,

\footnotetext{
${ }^{1}$ Note that the indices $n$ and $m$ used here refer to the order of the optical transverse mode, and not the electronic or momentum states of the atom $|n\rangle$ and $|m\rangle$ or the Bragg diffraction order $m$. It is assumed that the reader can infer the meaning of $n$ and $m$ from the context.
} 
(e)

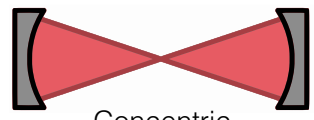

Concentric

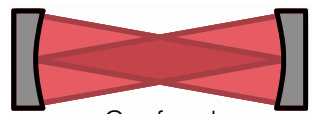

Confocal

Plane-parallel
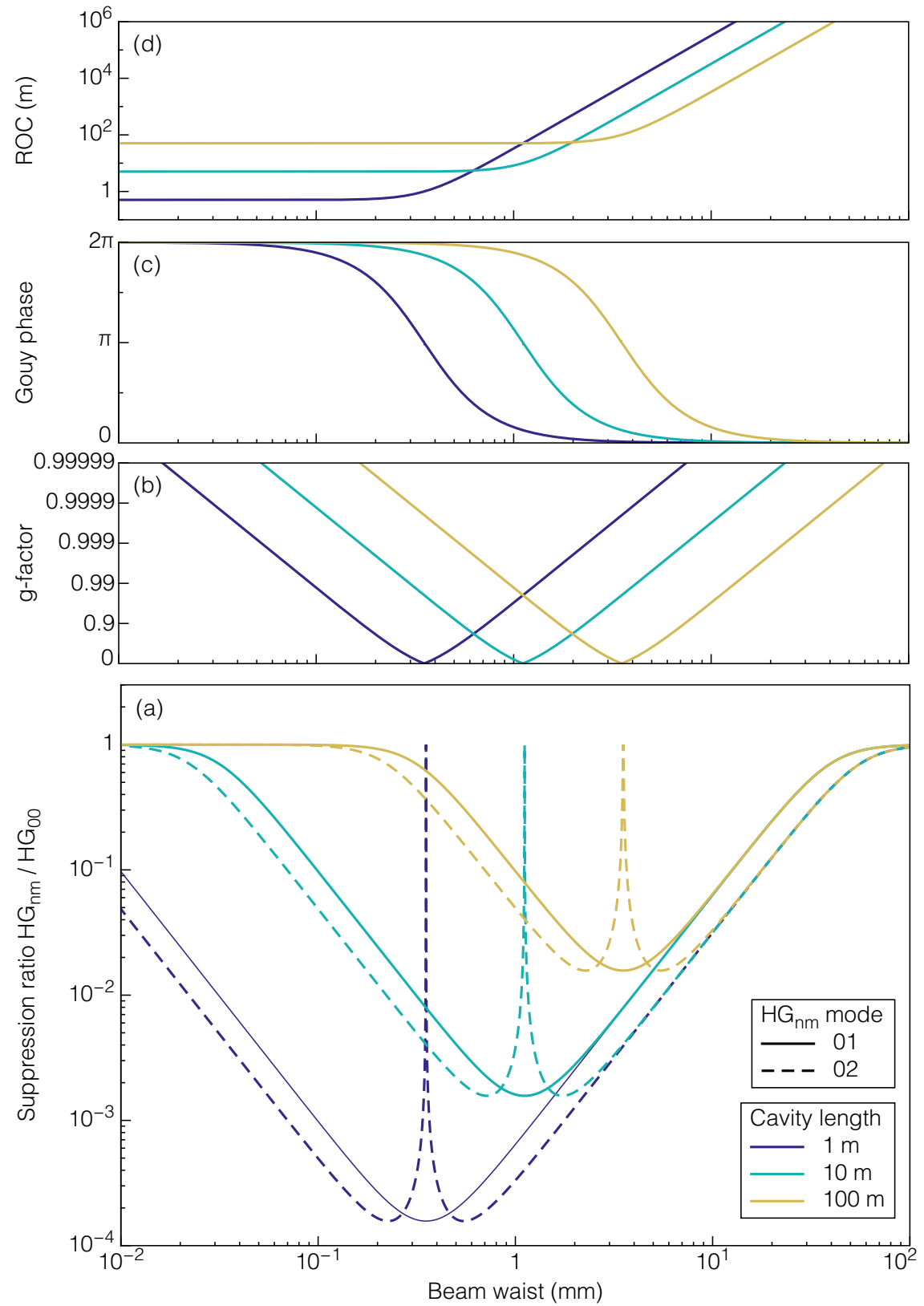

Figure 6.4: Continued on the following page. 
Figure 6.4: Spatial filtering and geometrical properties of the cavity. The optical suppression factor of the first and second TEM modes serve as indication of the quality of the laser wavefronts as a function of beam waist size (a). Cavities with the same bandwidth $(16 \mathrm{kHz}$ here, the limit for $m=1,4)$ have the same spatial filtering properties in the large waist limit. Also plotted: variation of the total cavity $g$ factor (b), the round-trip Gouy phase shift (c) and the mirror radii of curvature (d) of each cavity. As the beam waist varies from $10^{-2}$ to $10^{2} \mathrm{~mm}$, the cavity geometries (e) go from near-unstable concentric $\left(\mathcal{R} \rightarrow L / 2, g_{1,2} \rightarrow-1, \Delta \phi_{G} \rightarrow 2 \pi\right)$ through critically stable confocal $\left(\mathcal{R} \rightarrow L, g_{1,2} \rightarrow 0, \Delta \phi_{G} \rightarrow \pi\right)$ and up to near-unstable plane-parallel $\left(\mathcal{R} \rightarrow+\infty, g_{1,2} \rightarrow+1, \Delta \phi_{G} \rightarrow 0\right)$.

the wavefront quality is therefore also limited. Note that despite the fact that the bandwidth limits obtained in the previous section set a very high bar for the finesse of short cavities (e.g., roughly 10000 for $m=1,4$ at $L=1 \mathrm{~m}$ ), they have very similar performance as longer cavities with much smaller finesse (e.g., the $L=100 \mathrm{~m}, \mathcal{F}=100$ finesse cavity) for beam waist sizes on the order of a few millimetres. And of course, short cavities with finesses below the limit would have even worse performance in the region of interest than longer cavities operating at the limit.

One always has to ensure that the round-trip Gouy phase shift is not a ratio of $\pi$ so as to avoid bunching of higher-order modes. For example, $S_{02}$ peaks when $S_{01}$ is minimum indicating confocality for a round-trip Gouy phase shift of $\zeta=\pi$ [Figure 6.4(a)]; all even modes bunch together at this point. Note that, e.g., for a beam waist size of $w_{0}=5 \mathrm{~mm}$, the 1 and $10 \mathrm{~m}$ cavities are near-unstable planeparallel ( $\zeta \approx 0.006 \pi$ and $0.063 \pi$, respectively), while the $100 \mathrm{~m}$ cavity is clearly stable $(\zeta \approx 0.587 \pi)$ (Figure $6.4 \mathrm{~b})$.

The spatial filtering effect improves the quality of the beam inside the cavity regardless of the origin of the beam distortion, and applies in addition to other means of improving the input beam quality, such as pre-filtering, alignment, and mode matching. Here we do not consider specific input beam properties nor any fluctuations of the cavity parameters. Figure 6.4(a) indicates the relative difference in the intra-cavity build-up of the first and second order spatial modes, which represents the additional improvement in spatial filtering provided by the cavity.

\subsection{Atom temperature and cavity length limits for interferometers}

We distinguish two different limiting factors affecting the maximum allowed beam waist size in the cavity. The first one is the requirement of having a geometrically stable cavity, i.e., having a total $g$ factor of less than what would be experimentally unrealisable. The second one stems from the requirement of achieving a certain level of spatial filtering while staying within the bandwidth bound established in the previous section. We introduce this requirement by constraining the optical suppression factor of the first and second order spatial modes $S_{01}$ and $S_{02}$. 
1. Geometrical limit:

$$
g \leq g_{\max }
$$

2. Optical limit:

$$
\begin{array}{r}
S_{01,02} \leq S_{\max } \\
\Delta \nu \geq \Delta \nu_{\min }
\end{array}
$$

Short cavities will operate in the geometrical limit, as the smaller length comes at the price of putting the cavity very near the edge of geometrical instability. Larger cavities, on the other hand, will be optically limited, while easily maintaining a stable configuration despite the large waist. These upper bounds on beam waist size yield upper bounds on cloud temperature in order to keep the cloud within the confines of the beam (Figure 6.5).

Using Equations (6.3) and (2.42) we derive an expression for the maximum beam waist given a maximum cavity $g$ factor $g_{\max }$ :

$$
w_{0, \text { geo }}(L)=\sqrt{\frac{L \lambda}{2 \pi} \cot \left(\frac{\arccos \sqrt{g_{\max }}}{2}\right)} .
$$

Using Equation 6.4 we derive an expression for the maximum beam waist given a maximum suppression factor of the 1st order spatial mode $S_{\max }$ :

$$
\begin{aligned}
w_{0, \mathrm{opt}}(L)=\left[\frac{\lambda^{2}}{4 \pi^{4} \Delta \nu_{\min }^{2}}\right. & \left(\frac{2 c^{2} S_{\max }^{2}+\pi^{2} L^{2} \Delta \nu_{\min }^{2}\left(S_{\max }^{2}-1\right)}{1-S_{\max }^{2}}+\right. \\
& \left.\left.\frac{2 c S_{\max } \sqrt{c^{2} S_{\max }^{2}+\pi^{2} L^{2} \Delta \nu_{\min }^{2}\left(S_{\max }^{2}-1\right)}}{1-S_{\max }^{2}}\right)\right]^{1 / 4},
\end{aligned}
$$

where we have solved for $w_{0}$ in Equation 6.4 by setting $S_{01}=S_{\max }$, which gives eight complex solutions, four corresponding to real beams, and we have kept only the solution corresponding to the largest beam. In order to include the second order mode in our analysis, we repeat the same procedure setting $S_{02}=S_{\max }$ and solving for $w_{0}$ to find 16 complex solutions, eight corresponding to real beams, and we keep only one corresponding to the largest beam. We find that the largest beam possible under the $S_{02}$ constraint is always larger than that under the $S_{01}$ constraint within the interval

$$
L \in\left(0, \frac{\sqrt{3} c}{2 \pi \Delta \nu_{\min }} \frac{S_{\max }}{\sqrt{1-S_{\max }^{2}}}\right] .
$$

except at the upper limit, where they are the same size. In other words, the optical suppression of the first order mode for a given beam waist is better than that of the second order mode within this interval, except at the upper limit where the suppression for both modes is the same and equal to $S_{\max }$. At larger $L$ both $S_{01}$ and $S_{02}$ violate the imposed constraint, and thus this upper limit will constitute the cavity length limit under the optical constraints.

In the geometrical limit $w_{0, \text { geo }}<w_{0, \text { opt }}$, whereas in the optical limit $w_{0, \text { opt }}<$ $w_{0, \text { geo }}$. 
The size of the atomic ensemble after a time $t$ during the experiment is characterized by a Gaussian distribution of width

$$
\sigma_{t} \equiv \sqrt{\sigma_{0}^{2}+\sigma_{v}^{2} t^{2}}
$$

where $\sigma_{0}$ is the width of the initial position distribution, $\sigma_{v} \equiv\left(k_{B} \mathcal{T}_{e} / M\right)^{1 / 2}$ is the width of the velocity distribution of temperature $\mathcal{T}_{e}$ and mass $M$, and $k_{B}$ is the Boltzmann constant. Assuming that $\sigma_{v} t$ is much larger than $\sigma_{0}$, we can approximate Equation 6.12 by

$$
\sigma_{t} \approx \sqrt{\frac{k_{B} \mathcal{T}_{e}}{M}} t .
$$

The size of the cloud at time $t$ must be, at most, equal to the size of the cavity waist. Thus, the temperature of the atomic ensemble is limited by the maximum waist sizes in either the geometrical or the optical limits,

$$
\mathcal{T}_{e} \leq \frac{M w_{0}^{2}}{k_{B} t^{2}}
$$

A cavity-assisted interferometer operating in the geometrical limit will have the temperature of the atomic ensembles limited by

$$
\mathcal{T}_{e} \leq \frac{M \lambda L}{2 \pi k_{B} t^{2}} \cot \left(\frac{1}{2} \arccos \sqrt{g_{\max }}\right)
$$

whereas an interferometer operating in the optical limit requires, to first order in $L$,

$$
\mathcal{T}_{e} \lesssim \frac{M c \lambda}{\pi^{2} k_{B} t^{2} \Delta \nu_{\min }} \frac{S_{\max }}{\sqrt{1-S_{\max }^{2}}} .
$$

The approximation given here is valid to first order in $L$. For the exact expression, see Equations (6.10) and (6.14). Lastly, the maximum cavity length allowed under the optical constraints is given by

$$
L_{\max } \equiv \frac{\sqrt{3} c}{2 \pi \Delta \nu_{\min }} \frac{S_{\max }}{\sqrt{1-S_{\max }^{2}}} .
$$

This length limit is independent of the temperature of the atomic ensemble and is a consequence of the bandwidth and spatial filtering constraints. There are, thus, two factors at play limiting the length of cavities for atom interferometry, and when designing long cavities a sacrifice on either of these limits must be made: either the cavity bandwidth is chosen smaller than $\Delta \nu_{\text {min }}$, with the consequent problem of increased interaction times and higher power requirements; or the requirements on spatial filtering are relaxed, losing part of the advantage of incorporating the cavity in the first place.

Note that constraint set in Equation 6.14 is somewhat extreme: for a cloud of radius $\sigma_{t}$ being interrogated by a field of radius $w_{0}=\sigma_{t}$, the intensity distribution of the field across the cloud is far from uniform. Assuming that transition probabilities 

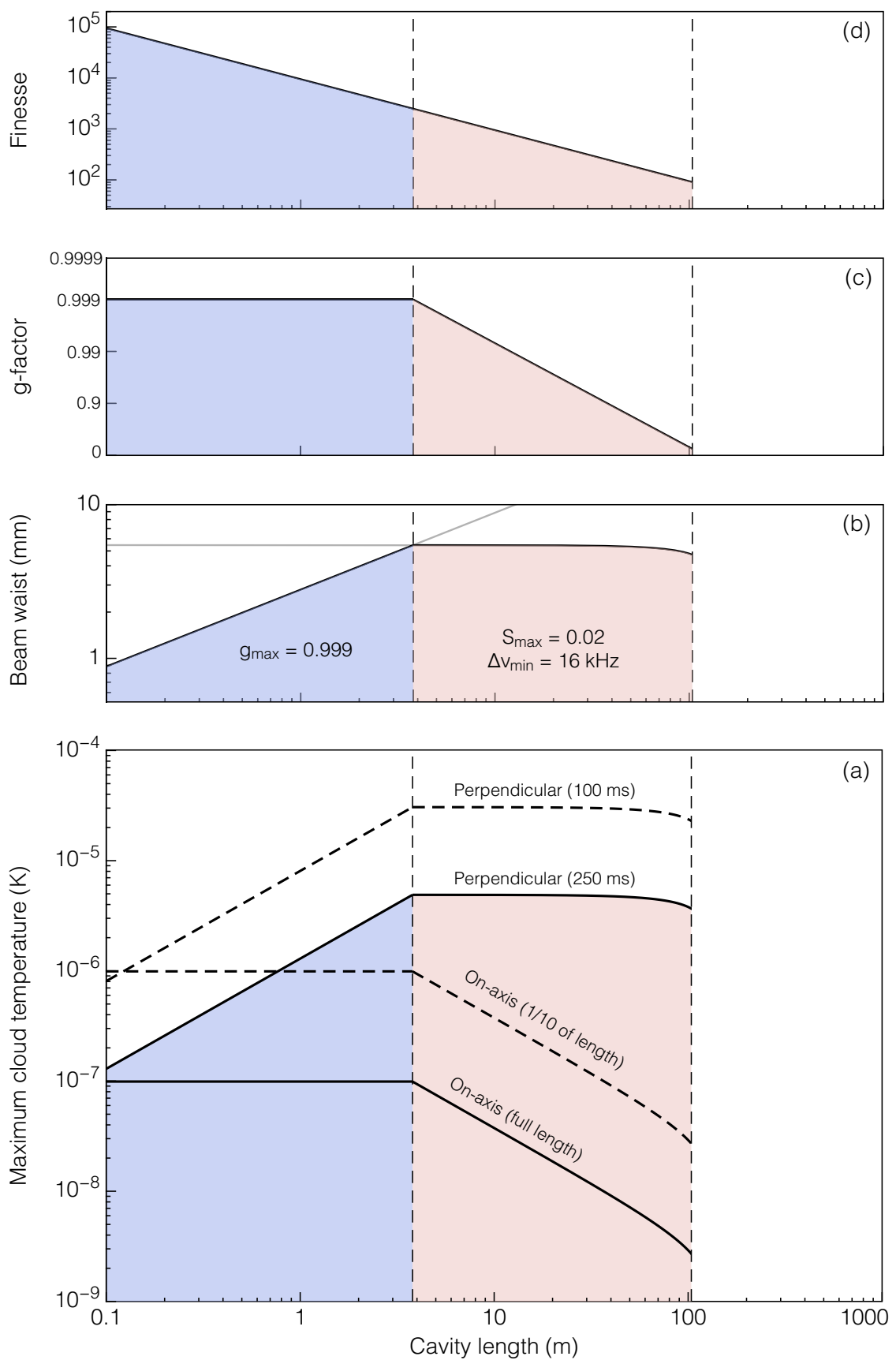

Figure 6.5: Continued on the following page. 

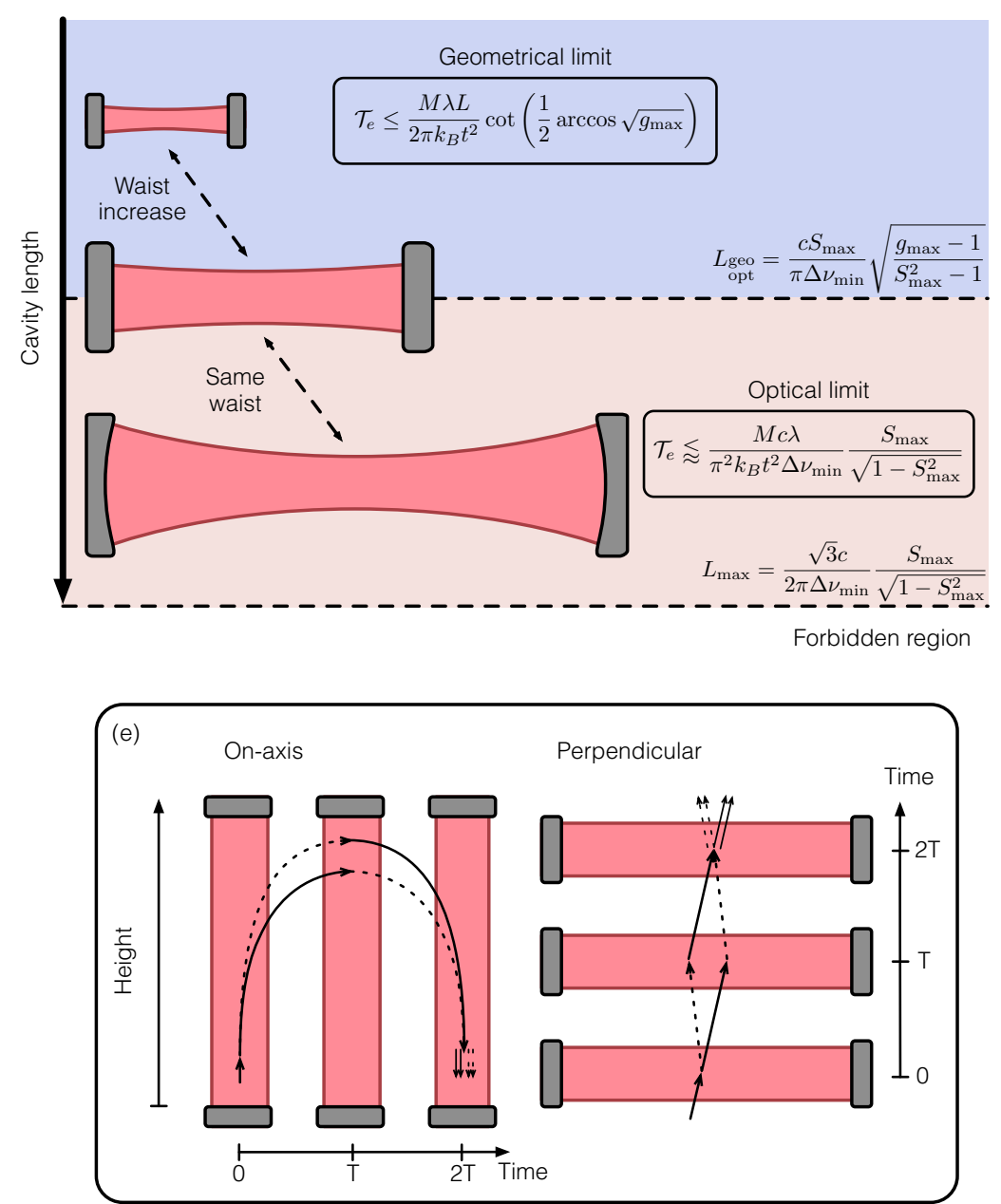

Figure 6.5: Geometrical and optical limits of the cavity-assisted atom interferometer. Beam waist size (b) and cloud temperature limits (a) are derived from a series of constraints. In the geometrical limit the upper bounds are set by the maximum cavity $g$ factor that is experimentally realisable (c). Longer cavities sit more comfortably within geometrical stability but in turn offer worse suppression of higher order spatial modes due to the bandwidth limit. In this region the upper bounds are set by the requirement of achieving a certain level of suppression of the first and second order spatial modes. The maximum cavity finesse is indicated in (d); cavities in the optical limit are by definition at the bandwidth limit, and thus also at the finesse limit. The temperature limits are calculated for two types of interferometric sequences (e). For on-axis sequences we show a case in which the atomic trajectories explore the entire cavity length and one in which they only use $1 / 10$ of the length. For the perpendicular sequence type we show the limits for total measurement times of 100 and $250 \mathrm{~ms}$. 
average out within the cloud (as considered in [110]), this will lead to a contrast reduction in the interferometer. If it is considered that the cloud size should instead be a fraction of the beam size $\sigma_{t}=w_{0} / a$, then the temperature limits would scale by a factor $1 / a^{2}$. For example, if we restrict the cloud size to be $\frac{1}{3}$ of the beam size, it would result in temperature limits nine times lower. A future iteration of this analysis should consider the impact of the $\sigma_{t} / w_{0}$ ratio on the performance of the interferometer, e.g., by including the transverse dimensions of the cloud and the optical field in the atom-optics model.

Owing to how the measurement time scales differently with cavity length for the two types of interferometers considered, the upper bound on cloud temperature scales differently too. In on-axis sequences it scales with 1 in the geometrical limit and $L^{-1}$ in the optical limit, whereas in perpendicular sequences it scales with $L$ and 1 respectively. The value $g_{\max }=0.999$ used here has been determined experimentally to be a safe choice in order to maintain controllability and good modefiltering behaviour of the cavity $[48,119]$. For this choice, an interferometer running on-axis sequences and using the entire cavity length requires sub- $\mu \mathrm{K}$ temperatures throughout the range. When restricting the atomic trajectories to use only a fraction of the total cavity length, these limits are relaxed: if the atoms explore a fraction $1 / \alpha$ of the length, the upper bound on cloud temperature scales with $\alpha$. Note that for on-axis interferometers the temperature limits in the optically-limited region are always lower than those in the geometrical limit, independent of the value of $S_{\max }$.

For running perpendicular sequences the temperature bounds are more forgiving, specially for larger, optically-limited cavities. We have presented two cases with total measurement times of 250 and $100 \mathrm{~ms}$, and the value $S_{\max }=0.02$ is chosen to obtain substantial suppression of higher order modes. This yields $w_{0, \text { opt }} \approx 5 \mathrm{~mm}$, which is a typical design value [110]. Under these constraints large cavities put an upper temperature limit of $4.9 \mu \mathrm{K}$ at $L=10 \mathrm{~m}$ and $3.8 \mu \mathrm{K}$ at $L=100 \mathrm{~m}$ for the $t=250 \mathrm{~ms}$ case, and $30.9 \mu \mathrm{K}$ at $L=10 \mathrm{~m}$ and $23.8 \mu \mathrm{K}$ at $L=100 \mathrm{~m}$ for the $t=100 \mathrm{~ms}$ case. For perpendicular sequences cavity stability yields $w_{0 \text {, geo }} \approx 2.8 \mathrm{~mm}$ at $L=1 \mathrm{~m}$, giving upper temperature limits of $1.3 \mu \mathrm{K}$ and $8.1 \mu \mathrm{K}$ for the $t=250 \mathrm{~ms}$ and $t=100 \mathrm{~ms}$ cases respectively. However, regardless of $t, \mathcal{T}_{e}$, or the sequence type, the maximum cavity length is $L_{\max } \approx 100 \mathrm{~m}$, and longer cavities would lie in the forbidden region under these constraints. The higher the order of the diffraction process, the more strict the temperature and length limits are due to the increasing value of $\Delta \nu_{\min }$.

These limits are conservative in the sense that the cavity is constrained to accommodate the size of the cloud at the centre, where the beam size is smallest. However, both in the geometrical limit and most of the optical limit (when the $g$ factor is close to 1), the cavity is in a near-unstable plane-parallel configuration and thus the size of the beam at the mirrors is approximately equal to the size of the beam waist.

We have assumed that Doppler shifts between atom states are less than the cavity bandwidth. If the cavity is subject to accelerations along the optical axis, the bandwidth must be large enough so as to compensate for the Doppler detuning of the interferometric pulses. The larger bandwidth will result in worse spatial filtering, and therefore in lower upper bounds to cloud temperature in order to maintain the same level of suppression of higher-order modes. To compensate for an increase 
over the minimum cavity bandwidth, the constraint on the optical suppression of the first and second order spatial modes would have to be relaxed by approximately the same amount. E.g., the situation presented in Figure 6.5 is analogous for a cavity with bandwidth $160 \mathrm{kHz}$ and $S_{\max }=0.2$.

\subsection{Summary and future work}

To summarise, we have shown that there is a lower bound on the cavity bandwidth below which the photon-atom interaction time increases substantially. Cavities with bandwidths below the minimum parametrically push the atomic diffraction process into the long-interaction Bragg and channeling regimes. A cavity operating at the bandwidth limit for the specific diffraction process provides: $(i)$ Maximum power enhancement of the atom optics pulses. (ii) Minimum elongation of the interaction time, potentially minimizing the degradation of atomic flux due to velocity selectivity. (iii) Best spatial filtering of the interferometric beams.

We have derived the spatial filtering properties of cavities operating at this bandwidth limit as a function of beam waist size. We have further derived beam waist size limits for the interferometer as a function of cavity length, which are divided into what we call the geometrical and optical limits. These limits allow us to determine the maximum temperatures of the atomic ensemble so that the atoms fit within the confines of the beam. A cavity operating with a beam waist size in these limits guarantees that: $(i)$ the cavity is stable, and (ii) the 1st and 2nd order spatial modes are suppressed below the desired threshold. In the geometrical limit - affecting short cavities - the maximum beam waist size scales with $\sqrt{L}$, whereas in the optical limit — affecting long cavities - it stays approximately constant.

A length limit emerges from the optical constraints, restricting the practicality of long-baseline detectors. This limit is independent of temperature, scales inversely with the cavity bandwidth and, to first order, scales directly with the maximum suppression factor of higher-order modes. These findings should assist the design of current and future detectors using two-mirror cavities (see Chapter 7), and pave the way towards alternative cavity designs (see Chapter 8). 

CHAPTER 7

\section{Gravitational-wave detection with cavity-assisted atom interferometry}

Contents

7.1 The MIGA large scale atom interferometer . . . . . . . . 148

7.2 Beam geometry and mirror radii of curvature in MIGA . . . 149

7.3 Cavity-enhanced interrogation in MIGA . . . . . . . 156

7.4 MIGA antenna design conclusions . . . . . . . . . . 163 


\subsection{The MIGA large scale atom interferometer}

7 He Matter wave-laser Interferometer Gravitation Antenna (MIGA) [6] is an 1 experiment being developed in France to study gravity at large scale. It aims at becoming a demonstrator for gravitational-wave detection in a frequency band where current optical interferometers have poor sensitivity [37].

Cavity-enhanced atom optics will be used to simultaneously interrogate several atomic clouds along the optical axis. By injecting the cavities with short pulses of specific durations, three atom interferometers are formed by Bragg scattering of pairs of photons from the standing cavity fields. Each atom interferometer's signal is linked to the phase of the cavity field. Hence, the atom interferometers become sensitive to any effect modifying the optical path length in the cavity, such as the strain of a passing gravitational wave.

The instrument consists of two horizontal $200 \mathrm{~m}$ cavities that are used to create enhanced Bragg beam splitter pulses for three atom interferometers running along the cavities' optical axes in a Mach-Zehnder configuration (Figure 7.1). The atomic clouds are launched vertically at three locations, two near the mirrors and one near the centre of the cavities. The lower cavity is used to perform the first and last $\frac{\pi}{2}$ pulses, and the upper cavity is used to perform the middle $\pi$ pulse (Figure 7.2). The combined action of the three pulses will create the three parallel interferometers.

Each interferometric pulse is obtained by injecting a time-modulated laser signal with a smooth temporal profile into the cavity. Bragg transitions are velocityselective and thus, in order to maximise atomic flux through the pulse sequence, the pulse durations must be kept as short as possible. MIGA will therefore operate in the quasi-Bragg regime, with pulse durations in the tens of $\mu$ s scale and a total

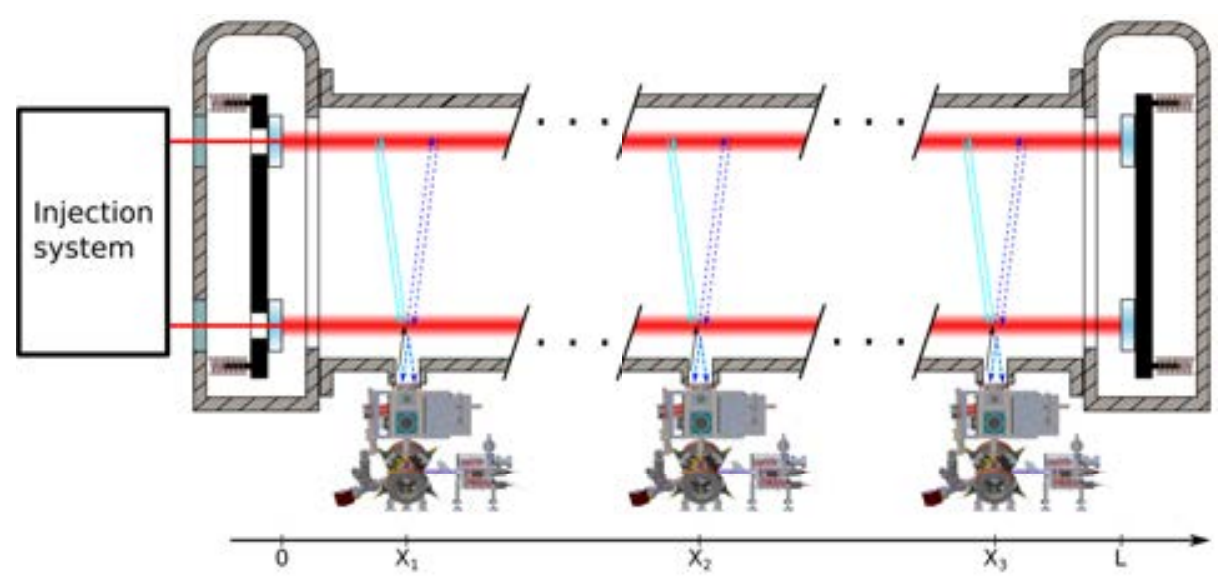

Figure 7.1: Scheme of the MIGA instrument, reproduced from [6]. Three atomic fountains launch ensembles of many cold atoms in almost vertical parabolic motion. The atoms then undergo a series of interactions with photons from the cavities' circulating fields, near the apogee of their trajectory. The diffracted atoms then interfere to give a signal that is linked to the phase of the cavity field. 
measurement time planned at $2 T=500 \mathrm{~ms}$.

The cavity offers two important advantages over using a retro-reflected highpower laser system: First, the cavity offers resonant enhancement of the interferometric beams, so that a high intra-cavity power may be achieved using a relatively low input power. Second, the cavity offers spatial filtering of the interferometric beams, effectively cleaning the laser phase fronts and thus enhancing the sensitivity of the detector.

But cavity-assisted atom interferometry also presents several technical challenges and limitations [41]. The injected excitation would ideally couple perfectly to the cavity's fundamental Hermite-Gaussian mode $\mathrm{HG}_{00}$. However, in practice, small alignment or mode matching errors, imperfect optical surfaces or fluctuating cavity parameters may cause phase front distortions that can be seen as coupling to the cavities' higher-order spatial modes $\mathrm{HG}_{n m}$. In addition, due to the cavities' relatively large storage time and the short duration of the interferometric pulses, the interrogation field's envelope function may be deformed, which can result in increased power requirements and interaction times.

In this chapter we explore the cavity parameters that will mitigate the shortcomings of the technique. This will influence the choice of the following parameters of the main cavities in the instrument:

1. Beam geometry ( $\rightarrow$ waist position).

2. Radii of curvature (ROC) of the mirrors ( $\rightarrow$ waist size).

3. Diameter of the mirrors.

4. Cavity finesse.

\subsection{Beam geometry and mirror radii of curvature in MIGA}

The primary atom interferometer constraint on the beam parameters is the fact that the clouds must fit within the confines of the beam as they thermally expand during the measurement. That is, the spread in position of the clouds in the directions transverse to the optical axes must at least be equal to the beam radius at the locations and times of the interrogations (See Figure 5.1). In MIGA the large cavity length will allow for relatively large beams with ease whilst maintaining a stable configuration.

This constraint can be expressed in terms of the temperature of the atomic ensemble as (assuming that the initial position dispersion of the source is negligible after a time $t$ )

$$
w(x) \geq t \sqrt{\frac{k_{B} \mathcal{T}_{e}}{M}},
$$

where $w(x)$ is the beam radius at the location of the cloud within the cavity, $t$ is the total evolution time up to the last pulse in that cavity, $\mathcal{T}_{e}$ is the temperature of the atomic ensemble, $k_{B}$ is the Boltzmann constant, and $M$ is the mass of the atom. 


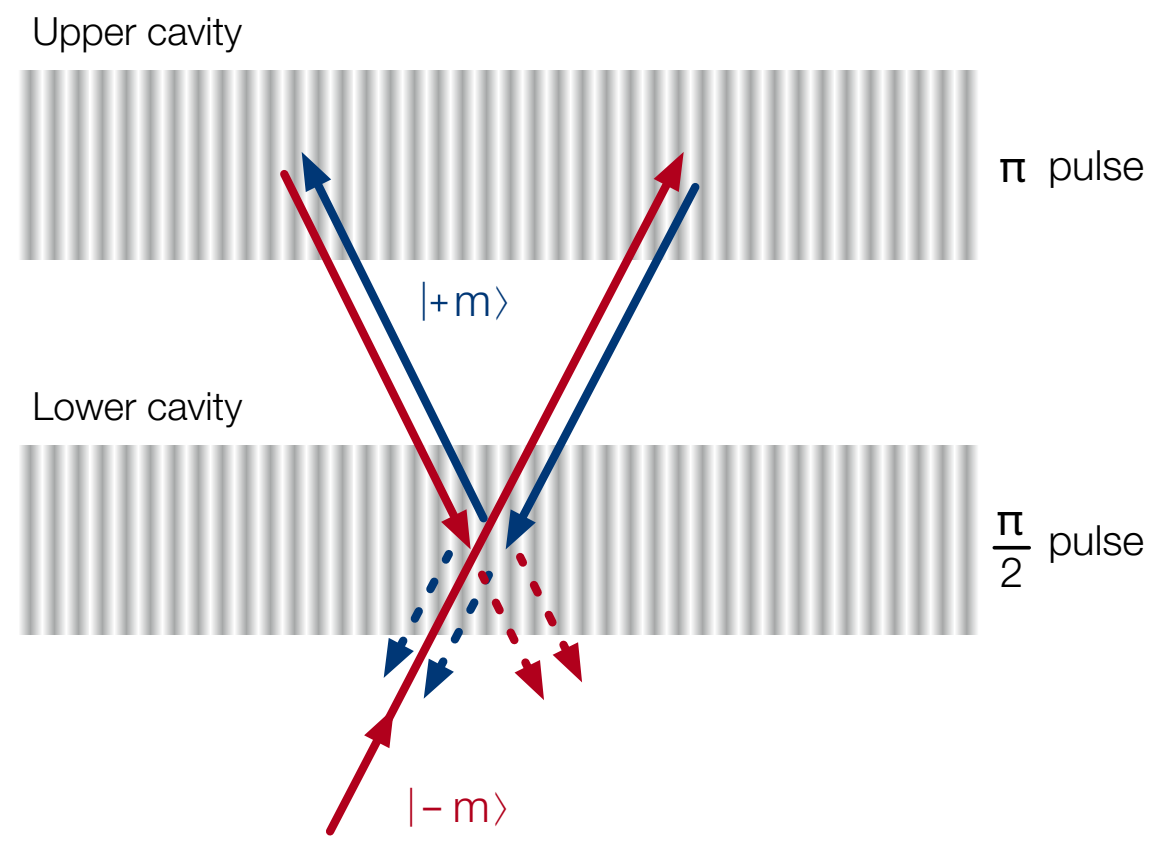

Figure 7.2: Bragg diffraction scheme in the MIGA instrument. A folded MachZehnder interferometer is formed by the combined action of the two cavities. The lower cavity sees the atoms first and last and performs a $\frac{\pi}{2}$ beam splitter pulse, while the upper cavity sees the atoms once at the apogee of their trajectory and performs a $\pi$ mirror pulse.

In addition to this minimal requirement set by the thermal expansion of the clouds, other considerations with respect to beam size are:

- A larger beam size to cloud size ratio results in the clouds probing a more uniform intensity profile ( $\rightarrow$ increased sensitivity).

- For a given cavity length and cloud position within the cavity, larger beams result in increased mode degeneracy ( $\rightarrow$ reduced sensitivity).

- Larger beams can make other things more complicated (e.g. higher clipping losses for a given mirror size $\rightarrow$ larger/more expensive optics, increased sensitivity to ROC deviations, etc).

The cavity geometries, in principle, do not need to be the same for both MIGA cavities. For example, the beam in the upper cavity could have a smaller waist because it will only see the clouds up to a time $T$, while the lower cavity will see them up to the final time of the sequence $2 T$. Since the constraint on beam size is more relaxed in the upper cavity, this cavity could use mirrors with a lower radius of curvature, yielding less susceptibility to mirror ROC deviations and slightly improved mode non-degeneracy than the lower cavity, and therefore potentially 
better spatial filtering. However, in order to simplify our analysis, for now we treat both cavities as having the same geometry.

The three atom interferometers are spread over the optical axis: One near the input mirror, one near the centre, and one near the end mirror. In a symmetric cavity configuration, the interferometers situated near the input and end mirrors see the same beam radius, whereas the central interferometer sees a slightly smaller beam. The difference in beam size experienced between the central interferometer and the input and end interferometers decreases with the overall size of the beam (i.e., with the mirror radii of curvature and the overall stability of the cavity). The alternative to the symmetric configuration is to have the waist position located slightly towards the input mirror or the end mirror, so that the central interferometer sees a slightly larger beam and, instead, either the input or the end interferometers see a slightly smaller one than in the symmetric case.

Assuming a completely symmetric configuration with both cavities having the same geometry, the beam waist will be at the centre of the cavity with a size constrained by Equation 7.1 with $t=2 T$. For the target temperature of the atomic sources of $\approx 1 \mu \mathrm{K}$, the minimal waist size is $\approx 5 \mathrm{~mm}$, which would make the cavities approximately confocal with mirror ROCs of $\approx 200 \mathrm{~m}$. Having a configuration close to the confocal would provide the maximum level of filtering of $\mathrm{HG}_{01}$, but on the other hand all even order modes would be nearly co-resonant with the fundamental mode.

When choosing the mirrors' radii of curvature, it is important to check whether in the resultant configuration any higher order spatial modes up to a certain order $N$ are co-resonant in the cavity with the fundamental mode, and ROC values that offer an optimal level of Gaussian mode non-degeneracy - taking into account manufacturing tolerances - should be chosen.

In order to choose an optimal ROC for the MIGA cavity mirrors, we introduce a figure of merit of Gaussian mode non-degeneracy up to order $N$,

$$
\Theta_{N} \equiv \frac{1}{\sqrt{\sum_{k=1}^{N} \frac{1}{k ! \Psi_{k}^{2}}}}
$$

where $\Psi_{k}$ is the mode non-degeneracy of the $k$-th order transverse mode

$$
\Psi_{k} \equiv \mid \frac{\Delta \phi_{k}}{2 \pi}-\text { round }\left(\frac{\Delta \phi_{\mathrm{k}}}{2 \pi}\right) \mid,
$$

and $\phi_{k}$ is the extra phase acquired by the $k$-th order mode with respect to the fundamental mode, given by the round-trip Gouy phase shift $\zeta$ of the cavity, i.e.,

$$
\Delta \phi_{k} \equiv k \zeta=2 k \arccos \sqrt{\left(1-\frac{L}{\mathcal{R}_{\mathrm{IM}}}\right)\left(1-\frac{L}{\mathcal{R}_{\mathrm{EM}}}\right)},
$$

where $\mathcal{R}_{\mathrm{IM}}$ and $\mathcal{R}_{\mathrm{EM}}$ are the ROC of the input and end mirrors respectively. When $\Psi_{k} \rightarrow 0$ the $k$-th order mode is co-resonant (i.e., degenerate) with the fundamental mode. The $\Theta_{N}$ function combines all modes up to order $N$, and each contribution is weighted so that lower order modes are more critical. 
Figure 7.3 shows $\Theta_{15}(\mathcal{R})$ for the MIGA cavities in symmetric configuration, with $\mathcal{R}_{\mathrm{IM}}=\mathcal{R}_{\mathrm{EM}}=\mathcal{R}$. Mode degeneracy gets gradually worse with $\mathcal{R}$ (hence, with the size of the beam) as expected. The function shows vertical dips when a particular mode or set of modes is co-resonant with the fundamental mode. Regions where $\Theta_{15}(\mathcal{R})$ is large and remains flat within the manufacturing tolerance represent a good choice of ROCs.

Table 7.1 gathers four possibilities for the ROC of the MIGA cavities that have been chosen with the aid of the figure of merit. The error in the ROC is obtained from the sagitta error, which can be specified when purchasing the mirrors,

$$
S \equiv \mathcal{R}-\sqrt{\mathcal{R}^{2}-(D / 2)^{2}} \approx \frac{D^{2}}{8 \mathcal{R}}
$$

where $S$ is the sag and $D$ is the diameter of the substrate. By differentiating and rearranging we can relate the error in the sag $\Delta S$ to the error in the ROC $\Delta \mathcal{R}$ as

$$
\Delta \mathcal{R} \approx-\frac{8 \mathcal{R}^{2}}{D^{2}} \Delta S
$$

The sag error can be as small as $\approx 5 \mathrm{~nm}$, but the ROC error scales with $\mathcal{R}^{2}$. For each choice of ROC in Table 7.1 the minimum polished area diameter to keep the clipping loss of the fundamental mode below $1 \mathrm{ppm}$ is calculated, and a figure with a small added margin in shown in the table. Figure 7.4 shows a detailed view of the figure of merit with the four choices of ROC and their corresponding uncertainty, indicating the higher order transverse modes that are closest in resonance frequency to the fundamental mode in each resulting configuration. Figure 7.5 shows the power in each transverse mode up to order 15 normalised to the power in the fundamental mode,

$$
\frac{\mathrm{HG}_{n m}}{\mathrm{HG}_{00}}=\left|\frac{t_{a}}{1-r_{a} r_{b} e^{-2 i \Delta \phi_{n m}}}\right|^{2},
$$

where $\mathrm{HG}_{n m}$ is the power in the $(n+m)$-th order transverse mode, $r_{a, b}$ and $t_{a}$ are the amplitude reflectivity and transmissivity coefficients of the input (subscript $a$ ) and end (subscript $b$ ) mirrors respectively, and $\Delta \phi_{n m} \equiv(n+m) \zeta$. This shows the amount of optical suppression of transverse modes provided by the MIGA cavities

\begin{tabular}{rcccc}
\hline & $\# 1$ & $\# 2$ & $\# 3$ & $\# 4$ \\
\hline Proposed mirror $\mathrm{ROC}^{\dagger}(\mathrm{m})$ & $555 \pm 7$ & $885 \pm 15$ & $1180 \pm 45$ & $1620 \pm 73$ \\
Sagitta error specification $(\mathrm{nm})$ & 5 & 5 & 10 & 10 \\
Safe polished area diameter ${ }^{\dagger \dagger}(\mathrm{mm})$ & 43 & 47 & 50 & 54 \\
Cavity $g$ factor & 0.64 & 0.77 & 0.83 & 0.88 \\
Mode non-degeneracy $\Theta_{15}\left(10^{-2}\right)$ & $20.0_{-0.02}^{-0.05}$ & $18.7_{+0.06}^{-0.11}$ & $16.7_{-0.35}^{-0.05}$ & $14.7_{-0.22}^{-0.24}$ \\
Closest higher order mode resonances & $\mathrm{HG}_{07,11,14}$ & $\mathrm{HG}_{09,11}$ & $\mathrm{HG}_{05,10,11}$ & $\mathrm{HG}_{06,12,13}$ \\
Beam waist $(\mathrm{mm})$ & 7.28 & 8.34 & 9.03 & 9.84 \\
Beam radius at mirror $(\mathrm{mm})$ & 8.04 & 8.82 & 9.36 & 10.16 \\
\hline
\end{tabular}

Table 7.1: Proposals of ROC for the MIGA cavity mirrors and relevant parameters. $\dagger$ Error based on the sag error and assuming the minimum substrate diameter. $\dagger \dagger$ Based on keeping the clipping loss of the fundamental mode below $1 \mathrm{ppm}$. 

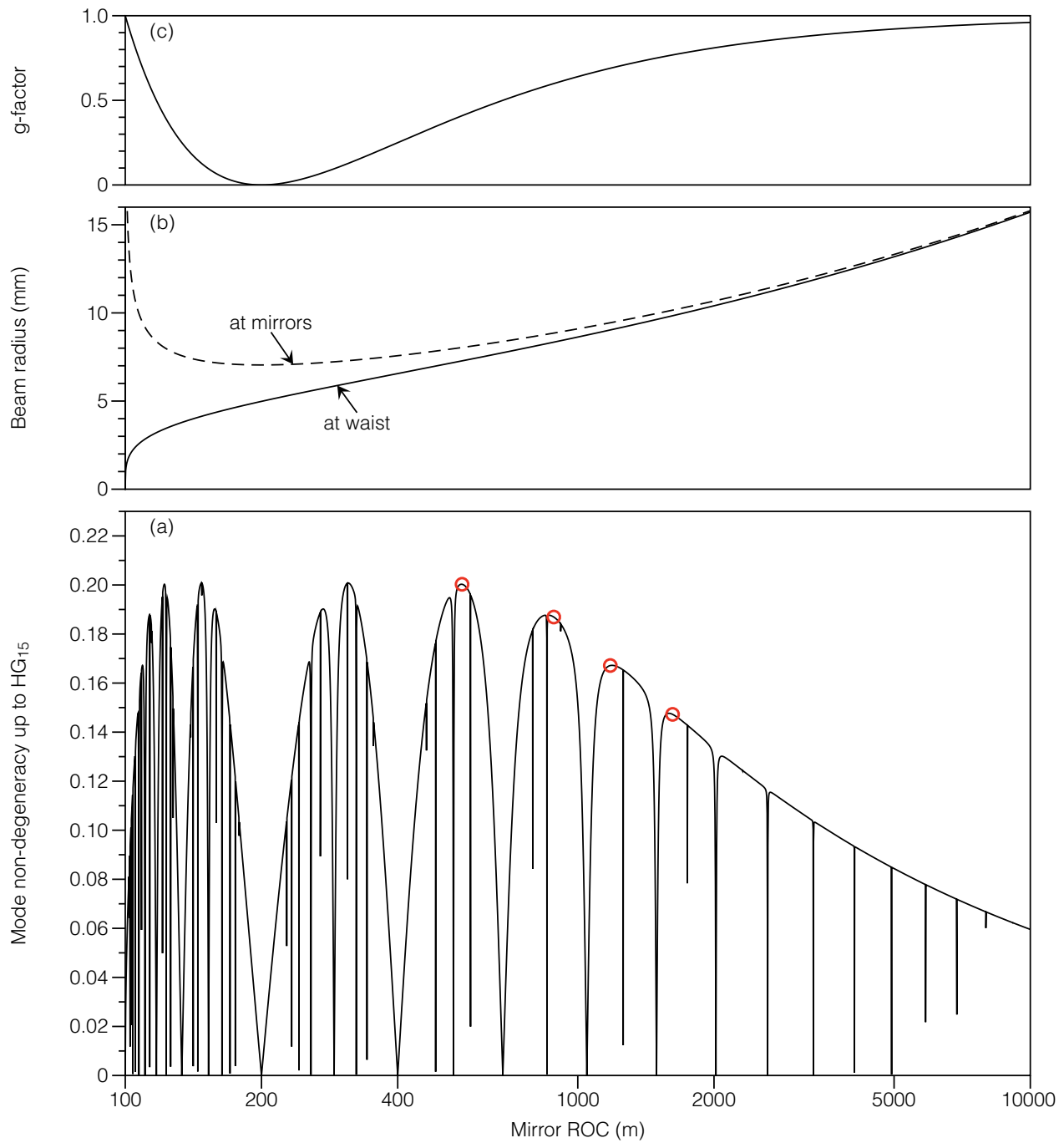

Figure 7.3: Figure of merit of Gaussian mode non-degeneracy up to order 15 as a function of mirror radii of curvature $(\mathcal{R})$ for a symmetric $200 \mathrm{~m}$ cavity (a). Also plotted: beam radius at the waist and at the mirrors (b), and total cavity $g$ factor (c). The cavities are concentric at $\mathcal{R}=100 \mathrm{~m}$, confocal at $\mathcal{R}=200$, and approach a plane parallel configuration for large $\mathcal{R}$ (e.g., $g=0.9$ at $\mathcal{R}=4000 \mathrm{~m}$ ). The red circles represent the four choices of ROC considered which result in a large Gaussian mode non-degeneracy. This ensures that the resulting cavity configuration provides optimal filtering of the input field.

in the already optimised configurations. Further narrowing down the choice of ROC would involve modelling of the cavity control and alignment signals. 

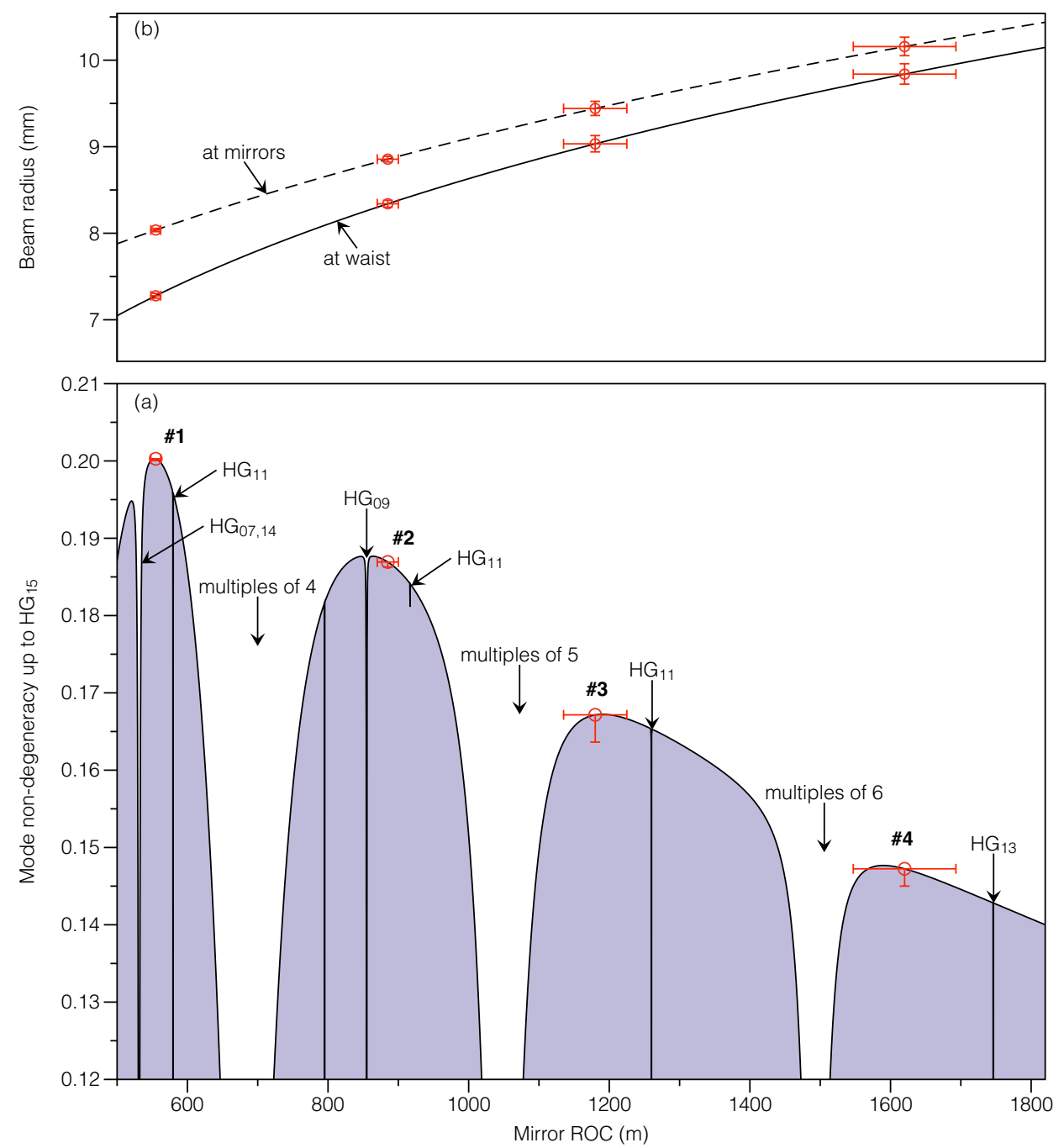

Figure 7.4: Figure of merit of Gaussian mode non-degeneracy showing the four choices of ROC considered and the resulting uncertainty (a), and beam size (b). The error bars are obtained from the estimated ROC error and the resultant uncertainty in the figure of merit and beam radius. Note that taking into account the manufacturing tolerances of the ROC is important to determine if a particular mode or set of modes could become co-resonant in the resulting cavity configuration. 

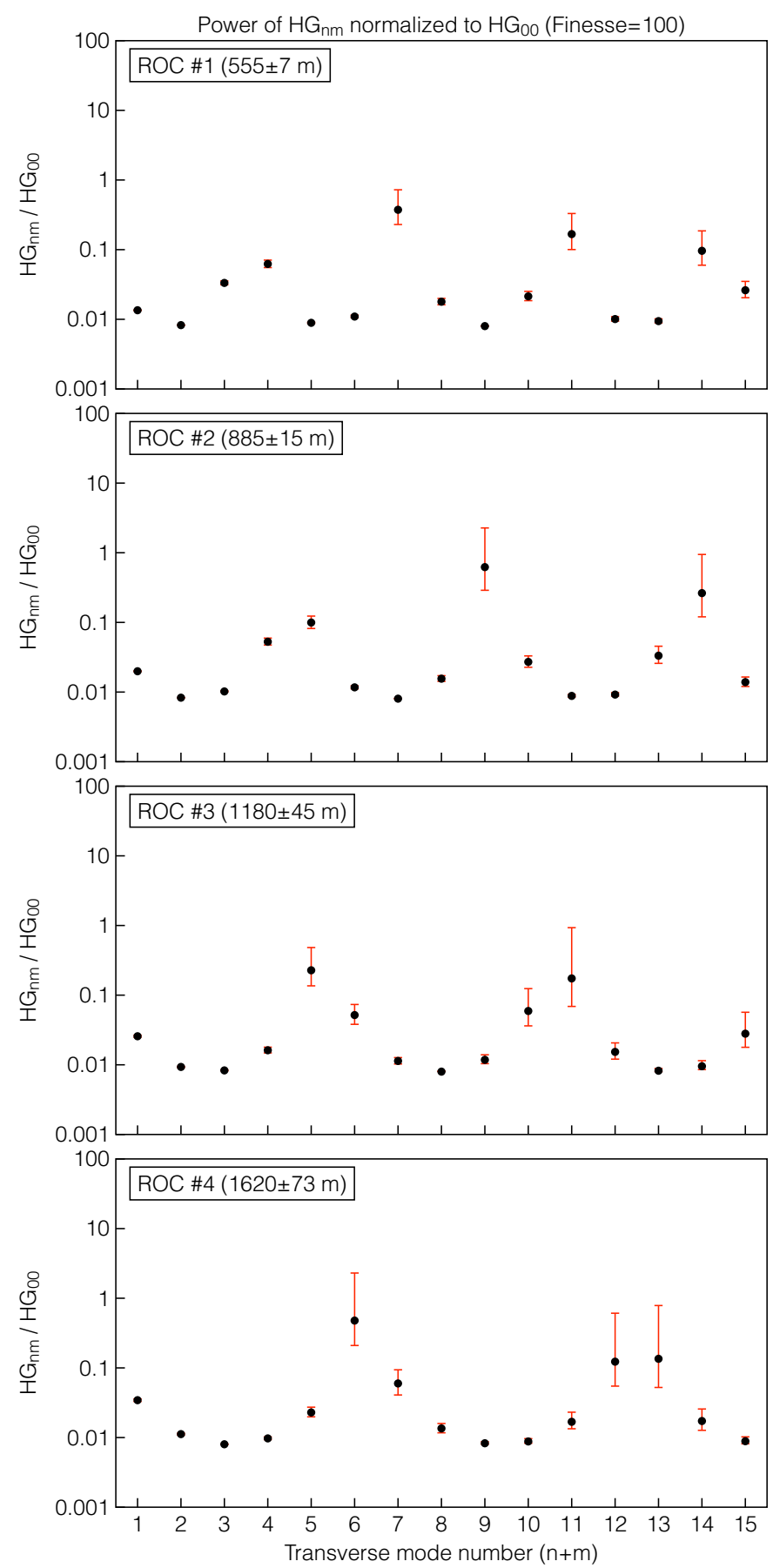

Figure 7.5: Power on each transverse mode up to order 15 for the 4 choices of ROC presented, normalised to the power of the fundamental mode and assuming a finesse of 100 . The level of spatial filtering provided by the cavities would be $10^{-1}$ to $10^{-2}$ overall except for some modes that are closer to resonance (e.g., modes with $n+m=9$ and 14 for the second choice of ROC, $\mathcal{R}=885 \pm 15 \mathrm{~m}$ ). 


\subsection{Cavity-enhanced interrogation in MIGA}

The cavities will deform the temporal envelope functions of the injected optical fields depending on the duration of the input pulses and the cavity bandwidth [41]. Optical cavities with photon lifetimes of the order of the pulse durations typical in the quasi-Bragg regime or lower, $\lesssim 1 / \omega_{r}$, where $\omega_{r}$ is the recoil frequency of the atomic transition, will exhibit some degree of pulse envelope deformation. This deformation may result in increased interaction times and power requirements for the short pulses needed for atom interferometry.

The transient response of the cavity to a time-varying input field can be described by (Equation 5.74)

$$
\dot{a}(t)=-\gamma a(t)+\sqrt{\frac{\gamma}{2 \tau}} a_{\mathrm{in}}(t),
$$

where $a(t)$ is the envelope function of the circulating field, $\dot{a}(t)$ is its time derivative, $a_{\text {in }}(t)$ is the envelope function of the input field, $\gamma=\pi c / 2 \mathcal{F} L$ is the cavity halfwidth, $\mathcal{F}$ is the finesse of the cavity, and $\tau=L / c$ is the photon transit time between the input and end mirrors. We consider an input field with a Gaussian envelope function of full width at half maximum $\delta t$,

$$
a_{\text {in }}(t)=a_{0} \exp \left(\frac{-2 t^{2} \log 2}{\delta t^{2}}\right) .
$$

Integrating Equation 5.74 for this input field and squaring to get the envelope function of the circulating intensity yields

$$
G(t)=\frac{\pi \gamma \delta t^{2} a_{0}^{2}}{16 \tau \log 2} \operatorname{erfc}\left(\frac{\gamma \delta t^{2}-4 t \log 2}{2 \delta t \sqrt{\log 4}}\right)^{2} \exp \left[-2 \gamma\left(t-\frac{\gamma \delta t^{2}}{\log 256}\right)\right],
$$

where we have used the condition $a(t \rightarrow-\infty)=0$, and erfc $(\mathrm{x})$ is the complementary error function $1-\operatorname{erf}(x)$, with

$$
\operatorname{erf}(x)=\frac{2}{\sqrt{\pi}} \int_{0}^{x} e^{-t^{2}} d t .
$$

The length of the MIGA cavities is already fixed at $200 \mathrm{~m}$ for design constraints, and thus the finesse is the only design parameter influencing the deformation. See Figure 7.7 for an example deformation of a $20 \mu$ s pulse for different values of the finesse if the MIGA cavities. Figure 7.8 quantifies the elongation and power enhancement effect as a function of the input pulse width for different values of the cavity finesse.

For a particular atomic transition and diffraction order, there is an optimal value of the finesse that maximises power enhancement in the quasi-Bragg regime and provides minimum elongation of the interaction time. Higher finesse cavities will parametrically push the process towards the Bragg and channeling regimes. Unfortunately the optimal value of the finesse changes with the Bragg order $n$, which means that a set of mirrors that is ideal, e.g., for a $n=1$ process, would not be so for a $n=10$ process. It would therefore be best to have different sets of mirrors to cover a range of cavity finesses for different configurations of the atom 


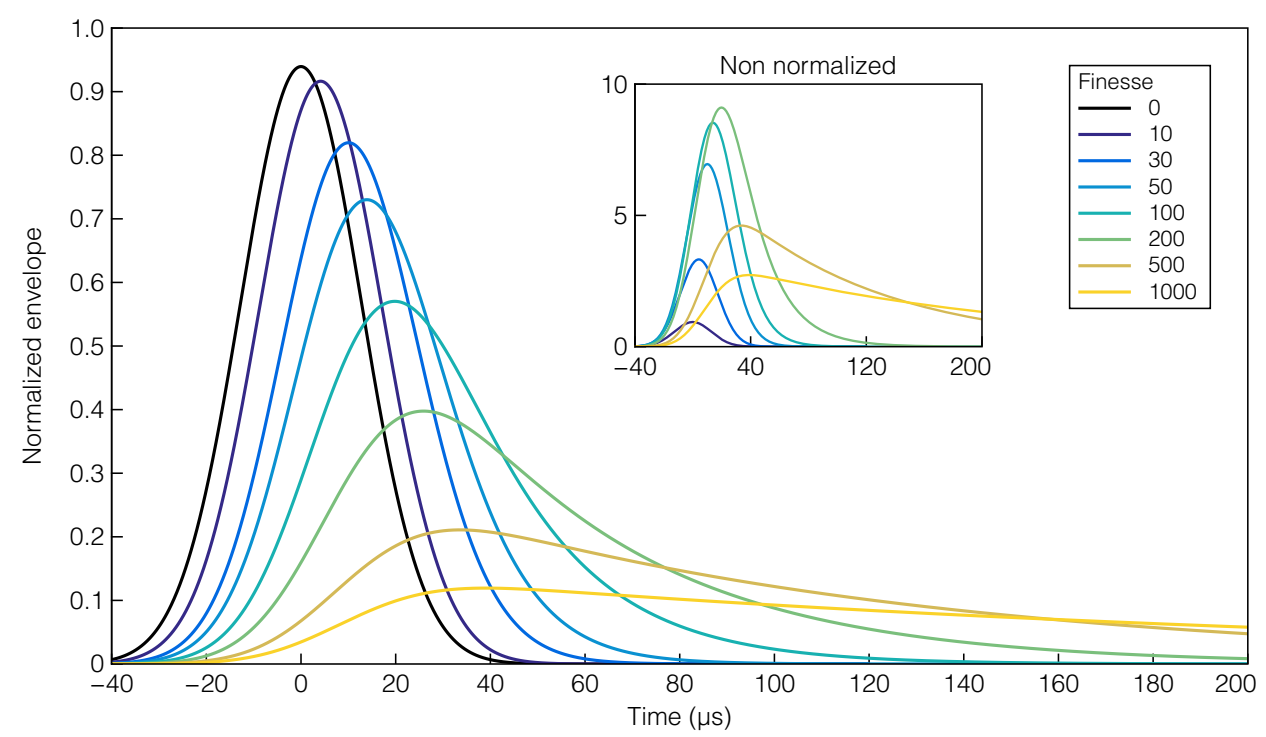

Figure 7.6: Deformation of a $30 \mu$ s pulse in MIGA for different values of the cavity finesse. Envelopes normalized to the input pulse area for comparison. The inset shows the envelopes without normalization (of increased area due to the gain of the cavity).

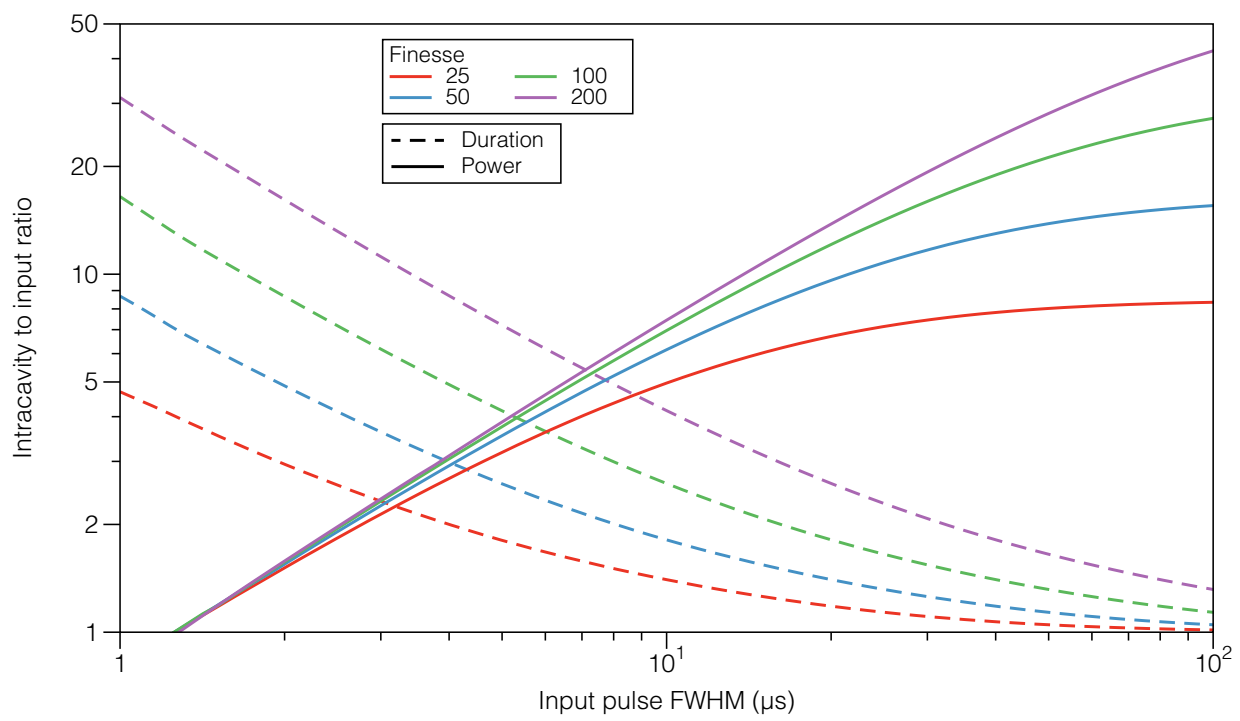

Figure 7.7: Characterisation of the response of the MIGA cavities to pulses of $1-100 \mu \mathrm{s}$ for different values of the cavity finesse. The dashed lines show the ratio of the duration of the intra-cavity pulse to the duration of the input. The solid lines show the ratio of the areas. For these values of the finesse the $200 \mathrm{~m}$ cavities would not reach a steady state in the range of input FWHM shown. 
interferometers, and a setup that allows for relatively easy swapping of the cavity mirrors.

See Table 7.2 for a list of optimal finesses for Bragg orders $n=1$ to 10 . For finesses greater than $\mathcal{F}_{\max }$ the interaction times increase and power enhancement worsens (see Figure 7.8). The higher the order of the process, the greater the difference in power requirements are for a given finesse change.

On the other hand, spatial filtering improves with $\mathcal{F}$. The optical suppression ratio of higher order modes is given by

$$
S_{n m}=\left[1+\left(\frac{2 \mathcal{F}}{\pi}\right)^{2} \sin ^{2}\left([n+m] \arccos \sqrt{\left(1-\frac{L}{\mathcal{R}_{\mathrm{IM}}}\right)\left(1-\frac{L}{\mathcal{R}_{\mathrm{EM}}}\right)}\right)\right]^{-\frac{1}{2}}
$$

A lower suppression ratio (specially of the lowest order modes) means that the interferometric beams will be "cleaned" from wavefront imperfections to a greater extent. The cavity finesse should then be chosen as a compromise between the sensitivity of MIGA [6], the desired level of suppression of higher order modes (affecting the sensitivity to wavefront distortions), and the degradation of the interrogation pulses in both interaction time and power requirements.

We performed a simulation of the photon-atom interactions in the MIGA cavities for Bragg orders 1 and 2 (Figures 7.9 and 7.10), in order to better visualise the effect of changing the cavity finesse within the vicinity of the optimal value. In both cases a finesse of 50 is closest to the optimal value. At $\mathcal{F}=100$ some elongation is noticeable but it is not critical in either case (the minimum interaction time for $n=1$ at $\mathcal{F}=100$ is still just about $\left.1 / \omega_{r}\right)$. At $\mathcal{F}=200$ and 400 the elongation effect is very significant in both cases. These low order processes do not exhibit large differences in power enhancement when using a finesse above the optimal, as higher orders would.

\begin{tabular}{ccc}
\hline$m$ & $\mathcal{F}_{\max }^{\mathrm{Rb} 87}$ & $\mathcal{F}_{\max }^{\mathrm{Cs} 133}$ \\
\hline 1 & 44 & 84 \\
2 & 55 & 106 \\
3 & 51 & 98 \\
4 & 46 & 87 \\
5 & 40 & 77 \\
6 & 36 & 69 \\
7 & 33 & 65 \\
8 & 31 & 60 \\
9 & 28 & 55 \\
10 & 24 & 48 \\
\hline
\end{tabular}

Table 7.2: Optimal finesse of a $200 \mathrm{~m}$ cavity for Bragg diffraction orders $1-10$. A cavity with the optimal finesse for the particular transition will offer maximum power enhancement in the quasi-Bragg regime whilst keeping the elongation effect minimum. 

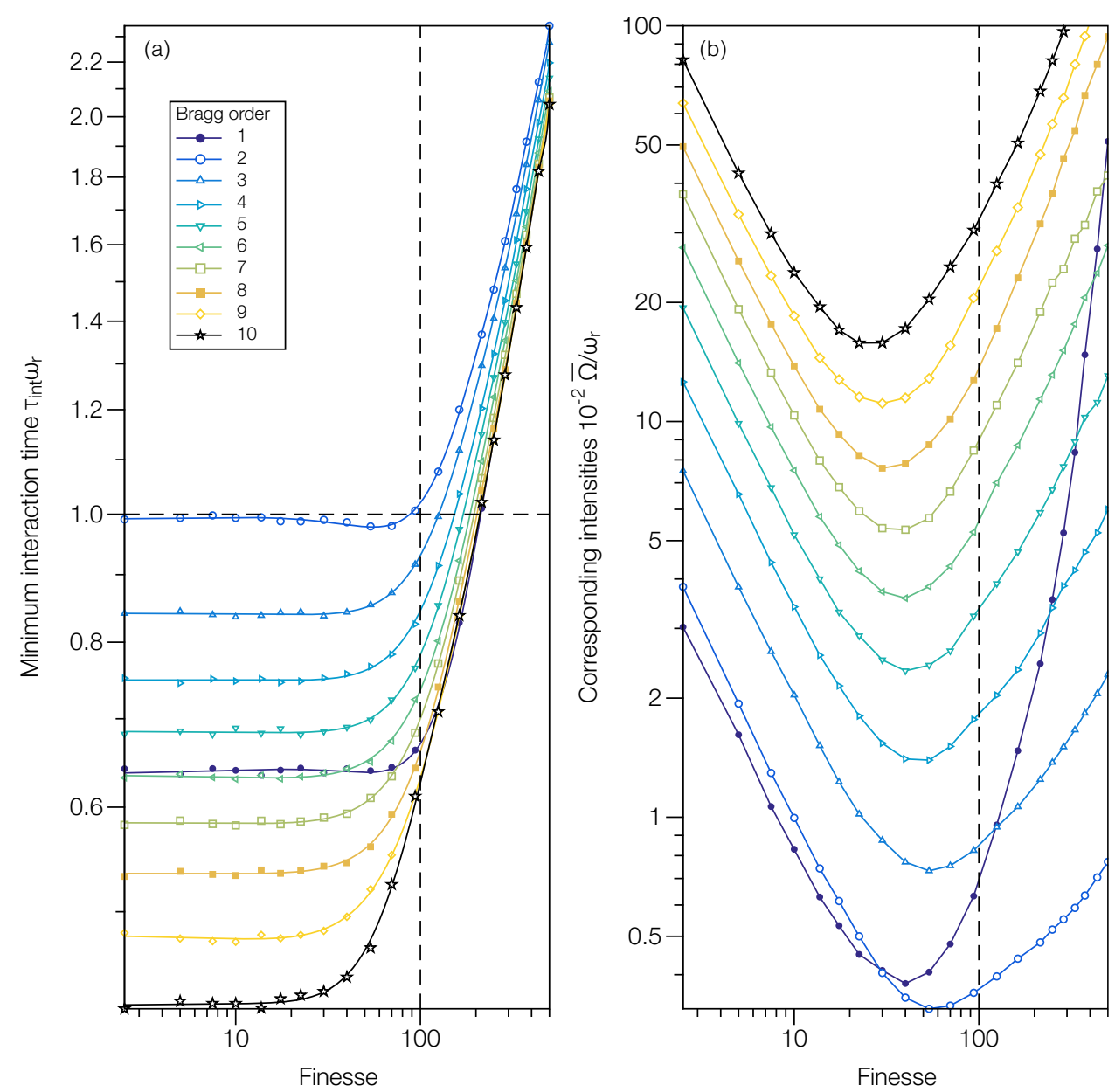

Figure 7.8: Minimum interaction times and corresponding intensities in MIGA for Bragg diffraction orders $n=1-10$, as a function of the cavity finesse. With a finesse of 100 the MIGA cavities would operate slightly above the optimal finesses for the Bragg orders considered (in the range $25-55$ ). However, this will not result in particularly worse performance for the lowest orders. Finesses higher than 100 are problematic, as the elongation effect becomes severe. 
Assuming relatively low-order Bragg diffraction $(n \leq 10)$ as a first step towards building MIGA, the initially proposed finesse of 100 is feasible. In the future, when implementing larger momentum beam splitters, it may be desirable to lower the finesse in order to allow for shorter interaction times and power requirements. For example, for $n=10$ it would be possible to gain a factor 2 power enhancement on the shortest $\pi$ pulse by using a finesse of $\approx 24$ instead of 100 . Finesses higher than 100 start to be a problem, e.g. at $\mathcal{F}=200$ orders $n \leq 10$ all have minimum interaction times $\gtrsim 1 / \omega_{r}$. We therefore propose $\mathcal{F}=100$ as a good starting point for MIGA, offering decent suppression of higher order modes as shown in Figure 7.5 whilst maintaining minimum interaction times $\lesssim 1 / \omega_{r}$ for all orders.

Note that for $n=10$ and $\mathcal{F}=100$, the minimum interaction time is 1.5 times larger than that in the absence of the cavity (although it still remains below the $1 / \omega_{r}$ level at $\left.0.6 / \omega_{r}\right)$. For this diffraction order the optimal finesse would be $\mathcal{F} \approx 24$, which would provide better power enhancement of the shortest beam splitter by nearly a factor 2, although it would provide worse optical suppression of higher order modes by a factor 4 . On the other hand, increasing the finesse to $\mathcal{F}=200$ would provide better suppression by a factor 2 , but the interaction time increase would then be significant (with minimum interaction times of $\approx 1 / \omega_{r}$ for $n=10$ and $\approx 1.32 / \omega_{r}$ for $n=2$ ). While power enhancement differences can be made up for by increasing the input power, the elongation effect cannot be compensated. It is therefore recommended that the finesse of the MIGA cavities does not exceed 100 . 
$m=1$
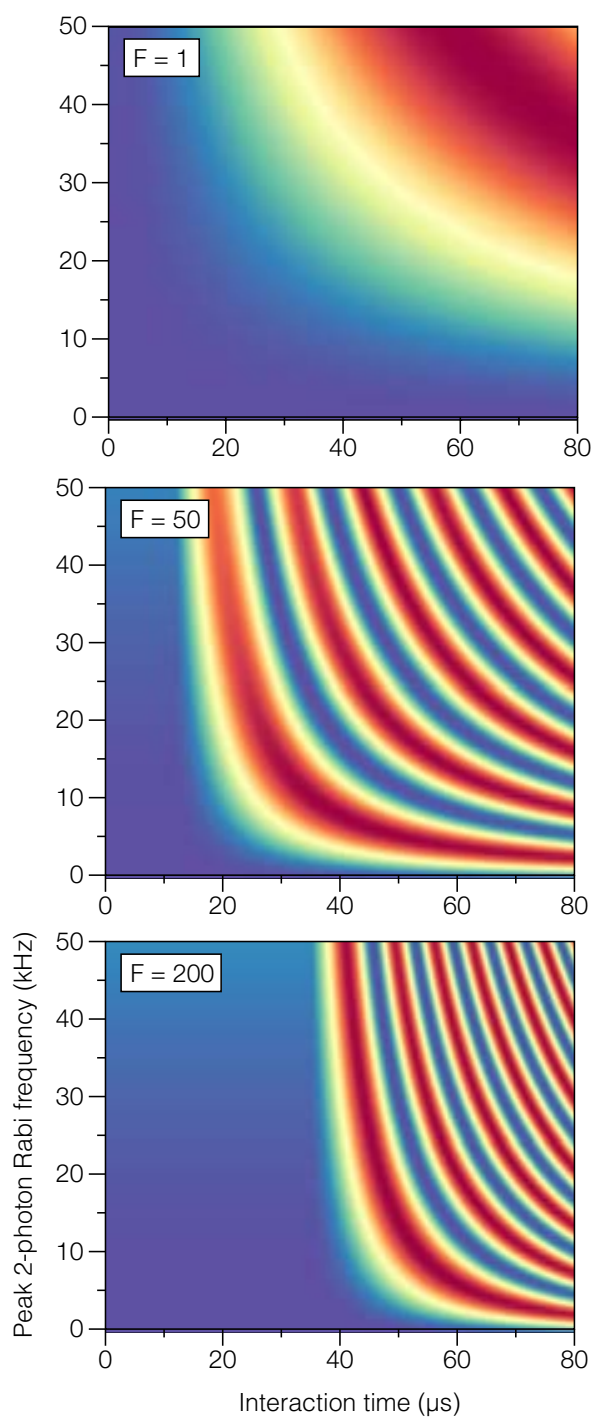

Transition Probability
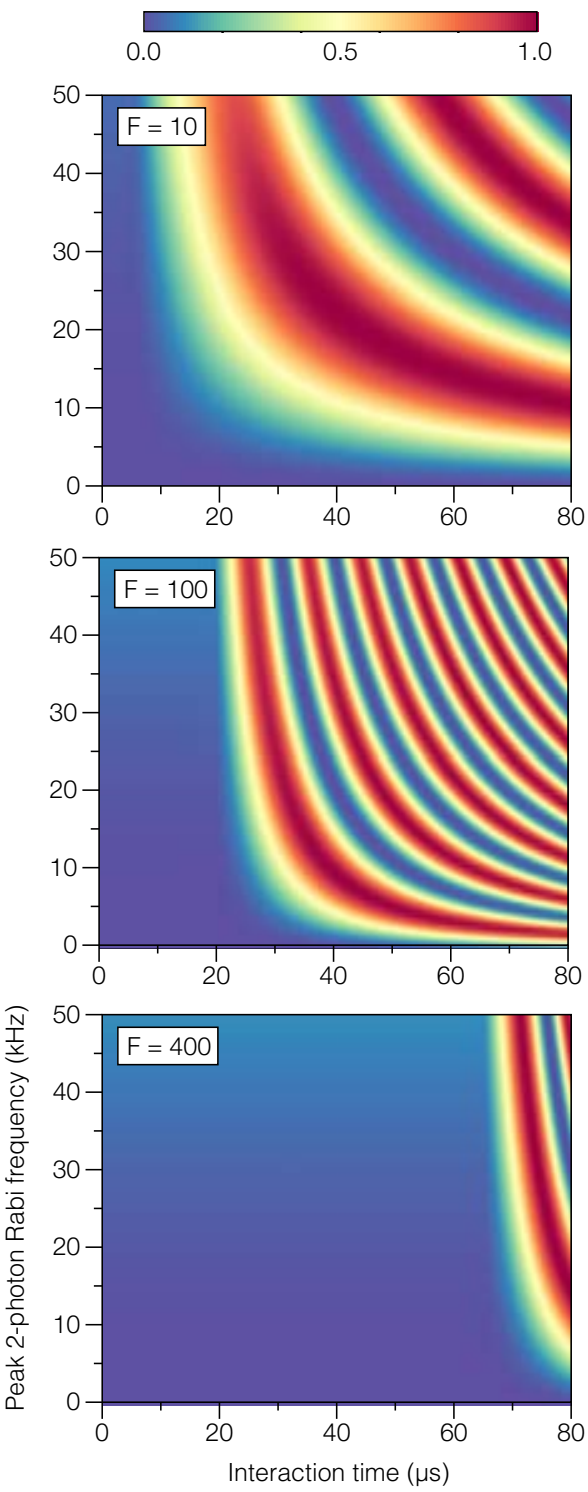

Figure 7.9: Transition probabilities in the time-intensity parameter space for the MIGA cavities at $n=1$. The finesse closest to optimal is $\mathcal{F}=50$. At $\mathcal{F}=100$ some elongation occurs but it is not yet significant. Differences in power enhancement between $\mathcal{F}=50$ and $\mathcal{F}=100$ are not significant for the lowest orders. 
$m=2$
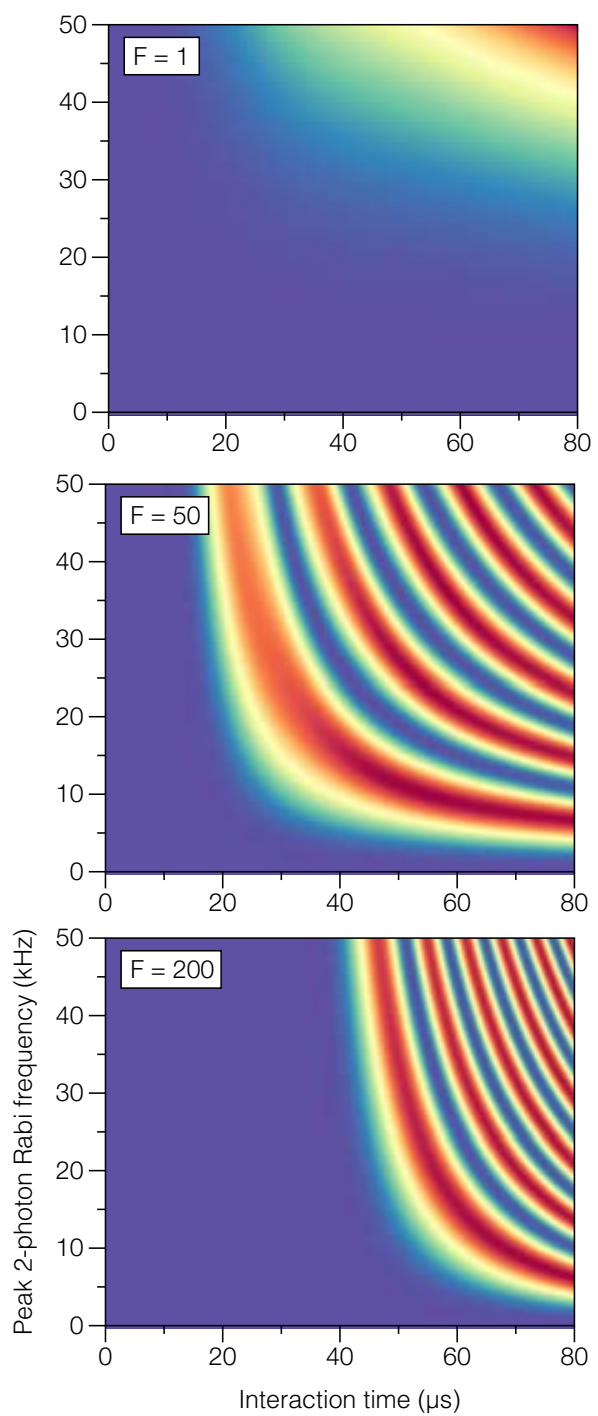
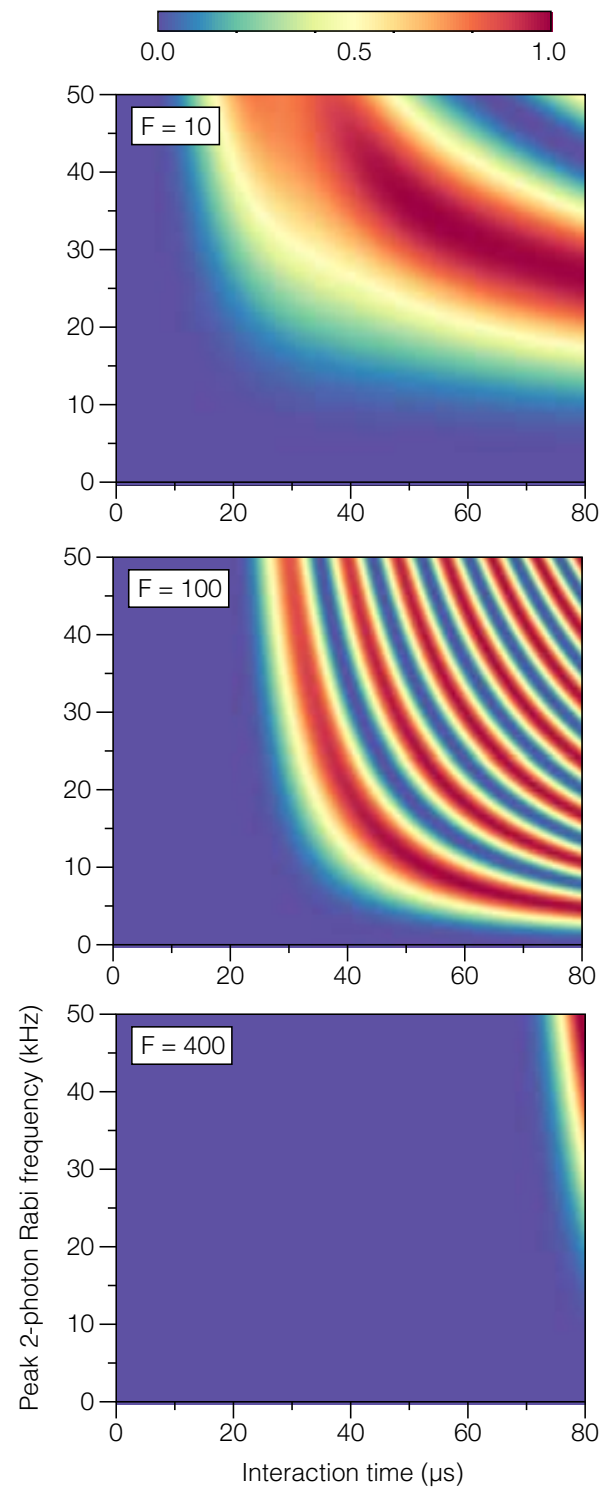

Figure 7.10: Transition probabilities in the time-intensity parameter space for the MIGA cavities at $n=2$. The finesse closest to optimal is $\mathcal{F}=50$. Conclusions same as Figure 7.9. 


\subsection{MIGA antenna design conclusions}

Due to the cavities' relatively large storage time with respect to the duration of the Bragg pulses, the interrogation fields will suffer some degree of deformation of their temporal amplitude profiles. This deformation scales with the cavity finesse, and it can have an adverse impact on the interferometers by increasing the minimum interaction time of the velocity-selective atomic transitions as well as their power requirements [41]. On the other hand, the cavities offer spatial filtering of the interferometric beams due to their frequency-dependent resonance conditions. This filtering, which also scales with the cavity finesse, effectively reduces the sensitivity of the interferometers to laser wavefront distortions, which are a leading source of noise in current state-of-the-art detectors [120]. The finesse of the cavities must therefore be chosen as a trade-off between these two cavity-induced effects on the atom optics pulses.

For the MIGA cavities a finesse of 100 is chosen to strike a balance between the two effects for Bragg orders $n \leq 10$. With this finesse, the minimum interaction time in a 20-photon diffraction process $(n=10)$ is 1.5 times larger than in the absence of the cavity whilst remaining below the $1 / \omega_{r}$ level (with $\omega_{r}=\hbar k^{2} / 2 M$ the 2-photon recoil frequency and $M$ the mass of the atom). In this configuration, most higher order spatial modes will be optically suppressed at the $10^{-2}$ level. A lower finesse would lead to less dilation of the interaction time and slightly improved power enhancement of the short beam splitter pulses, but would incur a worse optical suppression of higher order modes, partially negating the benefit of the cavity-assisted atom optics.

The radii of curvature (ROC) of the cavity mirrors are chosen to yield a beam waist large enough that the atomic clouds remain within the confines of the beam as they thermally expand during the measurement. Furthermore, the resulting cavity configuration provides sufficient mode non-degeneracy for Hermite-Gauss modes up to order 15, taking into account manufacturing tolerances of the ROC. For the chosen ROC of $555 \mathrm{~m}$ the beam radius is $7.28 \mathrm{~mm}$ at the waist and $9.10 \mathrm{~mm}$ at the mirrors, offering a good margin over the maximum cloud radius after the last pulse, which is expected to be roughly $5 \mathrm{~mm}$ for a $1 \mu \mathrm{K}$ rubidium- 87 ensemble after $2 T=500 \mathrm{~ms}$ of expansion. The resulting cavity configuration is robust to ROC deviations, although a reliable alignment sensing and control system will have to be implemented. 



\section{4-mirror large-waist cavity with tuneable stability for enhanced atom interferometry}

Contents

8.1 A novel resonator design for enhanced atom interferometry . . 166

8.2 Eigenmode size and stability . . . . . . . . . . . . . 168

8.3 Transient response . . . . . . . . . . . . . . . 173

8.4 Over-coupling and transverse mode filtering . . . . . . . . . 175

8.5 Gaussian mode non-degeneracy . . . . . . . . . . . . . . . 177

8.6 Summary and future work $\ldots \ldots \ldots \ldots$. . . . . . . . . 


\subsection{A novel resonator design for enhanced atom interferometry}

$\mathrm{T}^{\mathrm{N}}$ Chapters 5 and 6 we calculated a series of design constraints for employing 1 Fabry-Perot cavities to assist the large momentum transfer beam splitter pulses in an atom interferometer based on multi-photon Bragg diffraction. One of the fundamental limitations of cavities for this application, regardless of the number of elements in the cavity, is the cavity bandwidth limit, below which the cavity provides significant degradation of the interferometric pulses. The bandwidth limit, however, is of no concern in experiments employing short cavities, as the short length means that the cavity finesse can be made relatively large whilst maintaining the cavity bandwidth above such limit.

Short cavities, however, are challenged by the requirement of maintaining a stable configuration whilst supporting a fundamental Gaussian mode capable of accommodating the size of the atomic ensembles as they thermally expand during the measurement. By setting a maximum cavity $g$ factor $g_{\max }$ for a symmetric cavity, we derived the maximum allowed beam waist radius in the geometrical limit as a function of cavity length as (Equation 6.10)

$$
w_{0, \text { geo }}(L)=\sqrt{\frac{L \lambda}{2 \pi} \cot \left(\frac{\arccos \sqrt{g_{\max }}}{2}\right)},
$$

which in turn translates into a temperature limit for the atomic ensembles of (Equation 6.15)

$$
\mathcal{T}_{e} \leq \frac{M \lambda L}{2 \pi k_{B} t^{2}} \cot \left(\frac{1}{2} \arccos \sqrt{g_{\max }}\right)
$$

assuming that the radius of the optical field inside the cavity must match the size of the expanding atomic ensemble ${ }^{1}$. See Figure 8.1 for an example with $g$ factors of 0.999 and 0.99999 (the latter is an extreme example that would be exceedingly difficult to implement experimentally).

To get around this limitation, we present the design of an optical cavity capable of sustaining a very large beam $(w(z)>8 \mathrm{~mm}$ through a $1 \mathrm{~m}$ region) with tuneable stability. The ability to engineer the cavity's round-trip Gouy phase shift means that the cavity will provide improved Gaussian mode non-degeneracy up to a certain order. This experiment is a demonstrator for a large-waist cavity for enhanced atom interferometry with low wavefront distortions. The 4-mirror setup allows for a stable configuration with substantially greater beam sizes than those that can be obtained in a Fabry Perot cavity of the same path length whilst maintaining stability. The 4-mirror cavity can be used to interrogate clouds of up to $10 \mathrm{~mm}$ in size along a region of $1 \mathrm{~m}$.

The setup makes use of four mirrors in a "beam expander" configuration: $M_{1}$ (input convex mirror), $M_{2}$ (inner convex mirror), $M_{3}$ (inner concave mirror), and $M_{4}$ (end plane mirror). $M_{1}$ and $M_{2}$ expand the input beam, $M_{3}$ nearly collimates it,

\footnotetext{
${ }^{1}$ This is a very relaxed constraint, as in reality the radius $w_{0}$ of the beam should be larger than the radius of the atomic ensemble $\sigma_{t}$ to yield a more uniform intensity profile across the cloud. For a ratio $w_{0} / \sigma_{t}=a$, the temperature limit scales with $1 / a^{2}$.
} 


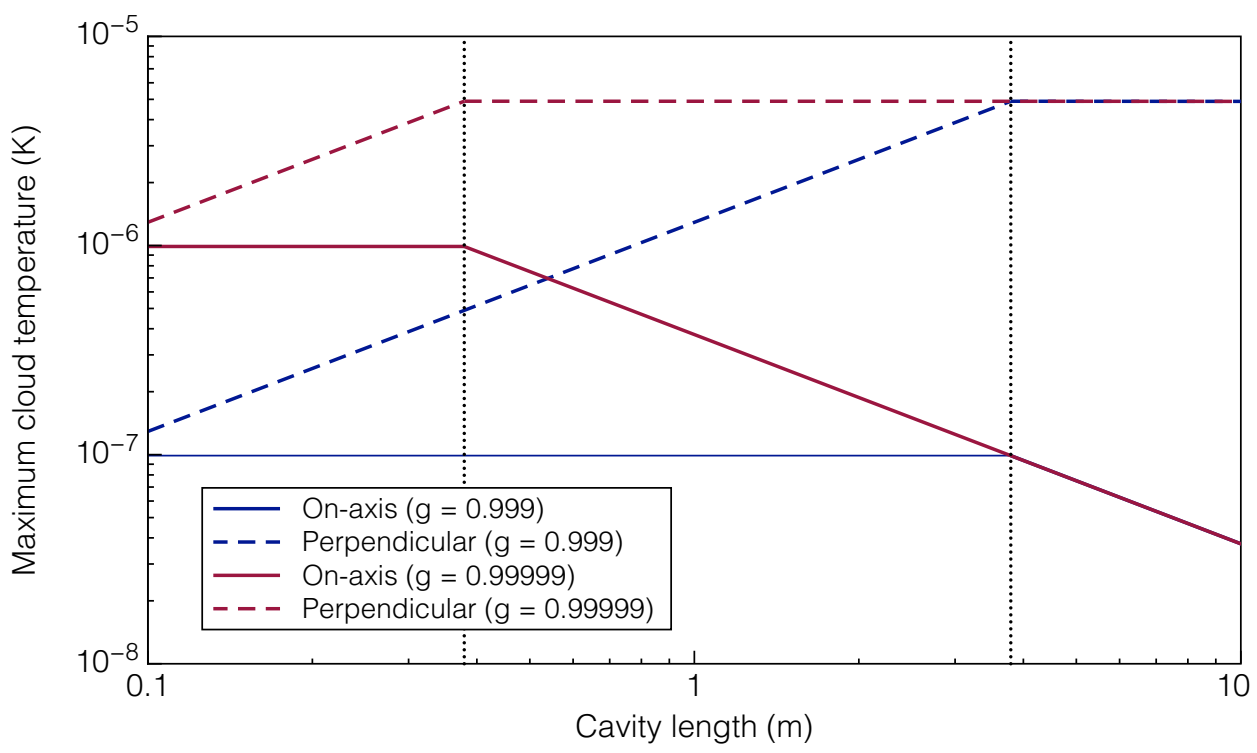

Figure 8.1: Atom temperature limits in Fabry-Perot cavities for $g$ factors of 0.999 (blue) and 0.99999 (red), for full-length on-axis sequences (solid lines) and perpendicular sequences with $2 T=250 \mathrm{~ms}$ (dashed lines). Short cavities are limited by the need to be configured near the edge of geometrical instability in order to accommodate the size of the atomic ensemble as it expands during the measurement. See Figure 6.5 e for a depiction of the two types of interferometer sequence considered. The vertical dotted lines represent the boundary between the geometrical limit and the optical limit, derived in Chapter 6. The red lines, corresponding to a near-unstable cavity with $g=0.99999$, represent an extreme case that is not experimentally feasible. These limits represent the somewhat extreme case where the size of the beam waist is constrained to be equal to the size of the cloud.

and $M_{4}$ closes the resonator. The region between $M_{4}$ and $M_{3}$ is the "science region", where the resulting resonator configuration yields a very large beam. The length $d_{3}$ of this region is set to $1 \mathrm{~m}$ to allow for sufficient space for running an interferometer sequence. For example, the cavity can be used to run several interferometers in parallel along the optical axis with the clouds being loaded perpendicularly into it. The distance $d_{1}$ between $M_{1}$ and $M_{2}$ can be modified to tune the round-trip Gouy phase shift between $0^{\circ}$ and $180^{\circ}$, hence effectively tuning the stability of the cavity and the amount of suppression of higher order transverse modes. The setup is illustrated in Figure 8.2.

To greatly simplify a possible experimental iteration of this design, we adopt a laser wavelength of $1064 \mathrm{~nm}$, which makes many of the required components readily available and/or cheaper. This will influence the cavity's design parameters, i.e., the distances between mirrors and the radii of curvature of the mirrors. Note that when designing a cavity for atom interferometry the laser wavelength will be different, according to the atomic transition of interest, and the cavity parameters 
will need to be re-computed.

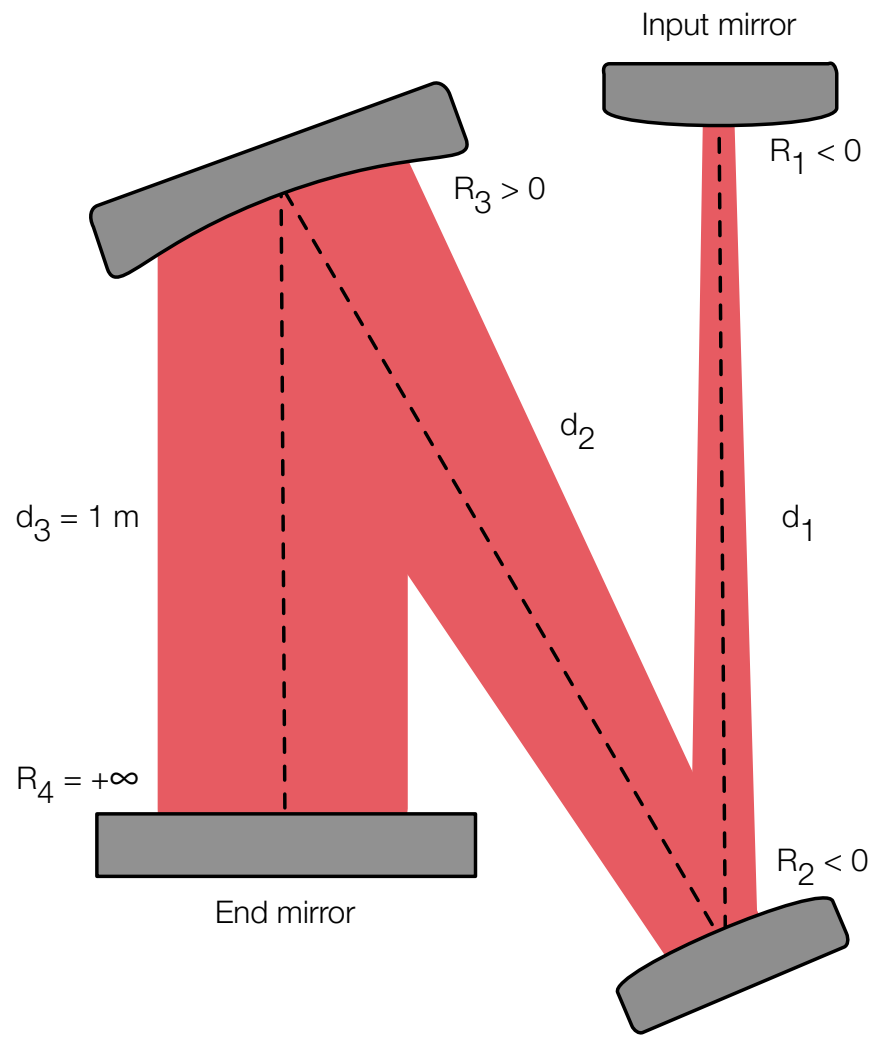

Figure 8.2: The 4-mirror cavity setup depicted here (not to scale) offers the possibility of accommodating a very large beam whilst maintaining a stable configuration in a relatively compact setup. The input and second mirrors expand the input beam, the third mirror nearly-collimates it, and the end mirror closes the resonator. The distance between the input mirror and the second mirror can be modified to tune the round-trip Gouy phase shift between $0^{\circ}$ and $180^{\circ}$.

\subsection{Eigenmode size and stability}

We start by computing the cavity's fundamental Gaussian mode by applying the self-consistency condition to a Gaussian beam of complex beam parameter $q(z)$. We recall from Section 2.2.5 that this condition can be expressed as

$$
\widehat{M}(z) \boldsymbol{q}(z)=\boldsymbol{q}(z),
$$

where $\boldsymbol{q}(z)$ is the column vector $(q(z), 1)^{T}$, and $\widehat{M}(z)$ is the resonator's round-trip transfer matrix referenced to a plane at position $z$ in the optical axis. Solving for 


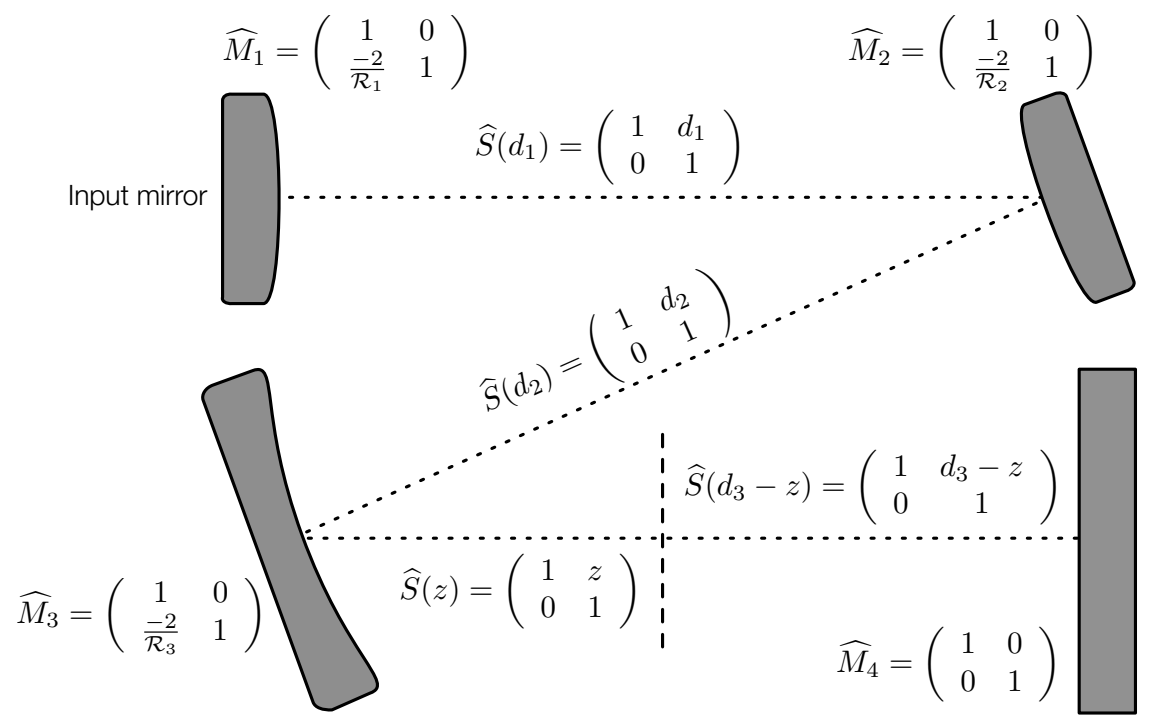

End mirror

Figure 8.3: Diagram of the 4-mirror cavity showing the corresponding transfer matrices for a complete round-trip referenced to an arbitrary point in the science region.

the complex beam parameter and imposing a confined solution yields

$$
\frac{1}{q(z)}=\frac{M_{22}(z)-M_{11}(z)}{2 M_{12}(z)}-\frac{\sqrt{1-m^{2}}}{\left|M_{12}(z)\right|}=\frac{1}{R(z)}-i \frac{\lambda}{\pi w^{2}(z)},
$$

where $R(z)$ is the radius of curvature of the beam, $w(z)$ is the beam radius, and $m=\operatorname{tr} \widehat{M}(z) / 2$ is known as the resonator's $m$ value, from which we define the $g$ factor as $g=(m+1) / 2$. If $m$ is real and $|m| \leq 1$ (i.e., if $0 \leq g \leq 1$ ), the resonator is real and geometrically stable and therefore the solution is confined and has a real and positive radius given by (Equation 2.30)

$$
w^{2}(z)=\frac{\lambda}{\pi} \frac{\left|M_{12}(z)\right|}{\sqrt{1-m^{2}}} .
$$

The cavity's round-trip Gouy phase shift can be cast in terms of $q(z)$ as

$$
\zeta=\arctan \left(\frac{\operatorname{Re}[q(z)]}{\operatorname{Im}[q(z)]}\right)=\operatorname{sgn}\left[M_{12}(z)\right] \arccos (m) .
$$

In a multi-element resonator the round-trip transfer matrix needs to be defined piecewise along the propagation direction, with each piece corresponding to a region between two elements. As such, in the 4-mirror cavity $\widehat{M}(z)$ is defined as

$$
\widehat{M}(z) \equiv\left\{\begin{array}{l}
\widehat{M}^{(a)}(z), \quad 0 \leq z<d_{1} \\
\widehat{M}^{(b)}\left(z-d_{1}\right), \quad d_{1} \leq z<d_{1}+d_{2} \\
\widehat{M}^{(c)}\left(z-d_{1}-d_{2}\right), \quad d_{1}+d_{2} \leq z \leq d_{1}+d_{2}+d_{3}
\end{array}\right.
$$




\begin{tabular}{cc}
\hline Parameter & metres \\
\hline$d_{2}$ & 1.6 \\
$d_{3}$ & 1 \\
$\mathcal{R}_{1}$ & -1 \\
$\mathcal{R}_{2}$ & -1 \\
$\mathcal{R}_{3}$ & 4 \\
$d_{1}^{(g=1)}$ & 1 \\
$d_{1}^{(g=0)}$ & 2 \\
$d_{1}+d_{2}+d_{3}$ & $3.6-4.6$ \\
\hline
\end{tabular}

Table 8.1: Cavity parameters for the eigenmode represented in Figure 8.4. By changing the distance $d_{1}$ between the input mirror and the second mirror from 1 to $2 \mathrm{~m}$, the cavity $g$ factor goes from 1 to 0 . The range of $d_{1}$ over which the cavity's round-trip Gouy phase changes by $180^{\circ}$ is exactly equal to $\left|\mathcal{R}_{1}\right|$

where the planes $z=0, z=d_{1}, z=d_{1}+d_{2}$, and $z=d_{1}+d_{2}+d_{3}$ correspond to the positions of the mirrors $M_{1}, M_{2}, M_{3}$, and $M_{4}$ respectively. The matrices $\widehat{M}^{(a)}(z), \widehat{M}^{(b)}(z)$, and $\widehat{M}^{(c)}(z)$ give the round-trip transfer to a point at a distance $z$ from mirrors $M_{1}, M_{2}$, and $M_{3}$ respectively. For example, the round-trip transfer matrix referenced to an arbitrary point in the science region, $\widehat{M}^{(c)}(z)$, is given by (see Figure 8.3)

$$
\widehat{M}^{(c)}(z) \equiv \widehat{S}\left(d_{3}-z\right) \widehat{M}_{4} \widehat{S}\left(d_{3}\right) \widehat{M}_{3} \widehat{S}\left(d_{2}\right) \widehat{M}_{2} \widehat{S}\left(d_{1}\right) \widehat{M}_{1} \widehat{S}\left(d_{1}\right) \widehat{M}_{2} \widehat{S}\left(d_{2}\right) \widehat{M}_{3} \widehat{S}(z),
$$

where $\widehat{M}_{i}$ is the transfer matrix of the $i$-th mirror with radius of curvature $\mathcal{R}_{i}$, given by

$$
\widehat{M}_{i} \equiv\left(\begin{array}{cc}
1 & 0 \\
-\frac{2}{\mathcal{R}_{i}} & 1
\end{array}\right)
$$

and $\widehat{S}(z)$ is the transfer matrix for a free space propagation of length $z$, given by

$$
\widehat{S}(z) \equiv\left(\begin{array}{cc}
1 & z \\
0 & 1
\end{array}\right)
$$

We can characterise the stability of the cavity as a function of the mirror's radii of curvature and the distances between mirrors by calculating the trace of $\widehat{M}(z)$, which is independent of $z$. The cavity's $g$ factor is given by

$$
\begin{aligned}
g= & \frac{4 d_{1} d_{2}+\mathcal{R}_{2}\left(\mathcal{R}_{3}-2 d_{2}\right)-2 d_{1}\left(\mathcal{R}_{2}+\mathcal{R}_{3}\right)}{\mathcal{R}_{1} \mathcal{R}_{2}^{2} \mathcal{R}_{3}^{2}} \\
& \begin{aligned}
{\left[4 d_{2}\left(\mathcal{R}_{1}-d_{1}\right)+\right.} & +2\left(d_{1}+d_{2}-\mathcal{R}_{1}\right) \mathcal{R}_{2} \\
& \left.+2\left(1+d_{2}\right)\left(d_{1}-\mathcal{R}_{1}\right) \mathcal{R}_{3}-\left(1+d_{1}+d_{2}-\mathcal{R}_{1}\right) \mathcal{R}_{2} \mathcal{R}_{3}\right]
\end{aligned}
\end{aligned}
$$

where we have set $d_{3}=1 \mathrm{~m}$ (all parameters given in metres).

Of special interest are the beam spots at each mirror, given by 


$$
\begin{array}{rlrl}
w_{1}^{2} & \equiv \frac{\lambda}{\pi} \frac{\left|M_{12}^{(a)}(0)\right|}{\sqrt{1-m^{2}}}, & \text { and } & w_{3}^{2} \equiv \frac{\lambda}{\pi} \frac{\left|M_{12}^{(c)}(0)\right|}{\sqrt{1-m^{2}}}, \\
w_{2}^{2} \equiv \frac{\lambda}{\pi} \frac{\left|M_{12}^{(b)}(0)\right|}{\sqrt{1-m^{2}}}, & w_{4}^{2} \equiv \frac{\lambda}{\pi} \frac{\left|M_{12}^{(c)}\left(d_{3}\right)\right|}{\sqrt{1-m^{2}}} .
\end{array}
$$

The complete expressions of $w_{1,2,3,4}$ as a function of the cavity parameters $\left\{d_{1}\right.$, $\left.d_{2}, d_{3}, \mathcal{R}_{1}, \mathcal{R}_{2}, \mathcal{R}_{3}\right\}$ are too long to be reproduced here. Note that due to the particularity of this setup $w_{3} \approx w_{4}$, and the beam maintains a large size in the $1 \mathrm{~m}$ long science region, which is one of the main features of this cavity.

In order to understand the behaviour of the setup it is useful to trace the beam back from $M_{4}$ to $M_{1}$, in the direction opposite to incidence. We start with a beam with a very large spot size at the end mirror, $w_{4} \approx 10 \mathrm{~mm}$. As the beam propagates freely to $M_{3}$, it accumulates only a small amount of Gouy phase due to the very large Rayleigh range in this region. Let us recall that the local Gouy phase of the beam is given by

$$
\eta(z)=\frac{\operatorname{Re}\{q(z)\}}{\operatorname{Im}\{q(z)\}}=\arctan \left(\frac{z-z_{0}}{z_{R}}\right),
$$

where $z_{R}=\operatorname{Im} q(z)=\pi w_{0}^{2} / \lambda$ is the Rayleigh range, which is independent of $z$ for any real Gaussian beam propagating in free space, $z_{0}$ is the location of the beam waist $\left(z_{0}=z: R(z) \rightarrow+\infty\right)$, and $w_{0}$ is the radius of the beam at the waist. Note that as the beam propagates in the cavity, $z_{R}$ suffers step changes at every interface,

\begin{tabular}{ccccccc}
\hline & & \multicolumn{5}{c}{ Beam spots $(\mathrm{mm})$} \\
$d_{1}$ & $g$ & $\zeta\left(^{\circ}\right)$ & $w_{1}$ & $w_{2}$ & $w_{4}$ & $w_{4} / w_{1}$ \\
\hline 1 & 1 & 0 & $+\infty$ & $+\infty$ & $+\infty$ & $\mathrm{n} / \mathrm{a}$ \\
1.001 & 0.999 & 2.78 & 2.87 & 5.74 & 28.7 & 10.0 \\
1.080 & 0.950 & 25.8 & 0.944 & 2.00 & 10.0 & 10.1 \\
1.100 & 0.937 & 29.1 & 0.889 & 1.91 & 9.57 & 10.8 \\
1.200 & 0.866 & 43.0 & 0.731 & 1.70 & 8.50 & 11.6 \\
1.315 & 0.773 & 56.8 & 0.631 & 1.62 & 8.11 & 12.8 \\
1.410 & 0.689 & 67.8 & 0.572 & 1.61 & 8.05 & 14.1 \\
1.500 & 0.602 & 78.2 & 0.524 & 1.63 & 8.14 & 15.5 \\
1.680 & 0.409 & 100 & 0.438 & 1.75 & 8.75 & 20.0 \\
1.720 & 0.363 & 106 & 0.418 & 1.80 & 8.99 & 21.5 \\
1.745 & 0.333 & 110 & 0.405 & 1.83 & 9.17 & 22.6 \\
1.828 & 0.230 & 123 & 0.359 & 2.00 & 10.0 & 27.9 \\
1.999 & 0.00141 & 176 & 0.0950 & 7.13 & 35.7 & 376 \\
2 & 0 & 180 & n/a & n/a & n/a & n/a \\
\hline
\end{tabular}

Table 8.2: Optical parameters of the cavity for different values of the distance $d_{1}$ between the input mirror and the second mirror, for the parameters listed in Table 8.1. The values in grey correspond to viable choices of $d_{1}$ with improved Guassian mode non-degeneracy (see Section 8.5). 

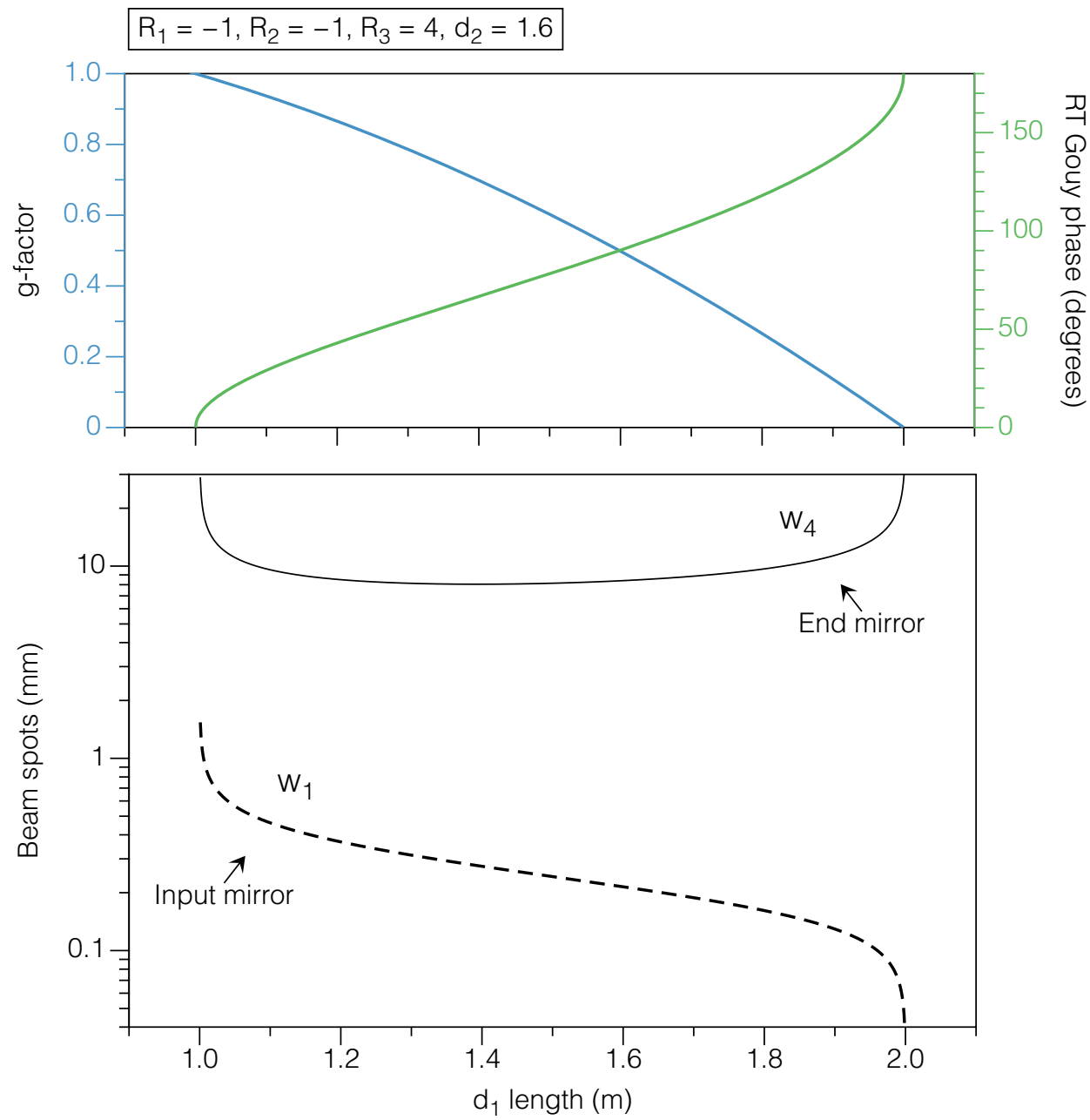

Figure 8.4: Eigenmode size (beam spots $w_{1}$ and $w_{4}$ at the input and end mirrors respectively) and stability (cavity's $g$ factor and round-trip Gouy phase shift $\zeta$ ) as a function of the distance $d_{1}$ between the input mirror and the second mirror, for the cavity parameters collected in Table 8.1.

which changes the rate of change of $\zeta$, but $\zeta$ itself never suffers a step change. A beam will accumulate a large amount of Gouy phase if it propagates close to the location of its waist.

The beam has a large Rayleigh range relative to $d_{3}$ as it propagates in the science region, so the accumulated Gouy phase in this region is only a small fraction of $\zeta$. The beam is then reflected from the concave mirror $M_{3}$, which places an image waist at a distance $\mathcal{R}_{3} / 2$ from that mirror. The convex mirror $M_{2}$ is then placed at a distance $d_{2}<\mathcal{R}_{3} / 2$ from $M_{3}$ so that, once again, the beam accumulates only a small amount of phase as it propagates far from the location of its waist. The 
reflection from $M_{2}$ of a beam with a virtual waist outside the cavity, expands the beam and places an image waist at a certain distance from it. Finally, $M_{1}$ is placed conveniently at a point before the image of $M_{2}$, where it will match the radius of curvature of the beam in order to close the resonator, yielding a beam spot $w_{1}$ at the position of $M_{1}$ much smaller than the beam spot $w_{4}$ at the position of $M_{4}$. In the resulting configuration, the distance $d_{1}$ between $M_{1}$ and $M_{2}$ can be modified to tune $\zeta$ from $\zeta=0^{\circ}(g=1)$ to $\zeta=180^{\circ}(g=0)$.

Under the set of assumptions used here, the critical points in $d_{1}$ are given by

$$
d_{1}^{(g=1)} \equiv \mathcal{R}_{1}+\frac{\mathcal{R}_{2}}{2}+\frac{\mathcal{R}_{2}^{2}}{4 d_{2}-2\left(\mathcal{R}_{2}+\mathcal{R}_{3}\right)}, \quad d_{1}^{(g=0)} \equiv \frac{\mathcal{R}_{2}\left(2 d_{2}-\mathcal{R}_{3}\right)}{4 d_{2}-2\left(\mathcal{R}_{2}+\mathcal{R}_{3}\right)},
$$

which reveals that the total tuneable $d_{1}$ range is

$$
\left|d_{1}^{(g=1)}-d_{1}^{(g=0)}\right|=\left|\mathcal{R}_{1}\right|
$$

Figure 8.4 shows $w_{1}, w_{4}, g$ and $\zeta$ for the set of parameters specified in Table 8.1. Note how very large values of $w_{4}$ can be obtained in a relatively compact setup and with a tuneable round-trip Gouy phase shift, starting from a relatively small spot size in the input mirror. In this example the total cavity length varies from 3.6 to $4.6 \mathrm{~m}$, with the largest section varying from 1.6 to $2 \mathrm{~m}$. More compact configurations may be achieved in future iterations of the experiment, at the expense of a lower magnification $w_{4} / w_{1}$ and less common mirror radii of curvature.

In this configuration the spot size at the end mirror $w_{4}$ never falls below $8 \mathrm{~mm}$

for any value of $d_{1}$. For values of $d_{1}$ close to instability $\left(d_{1} \rightarrow d_{1}^{(g=0)}\right.$ or $\left.d_{1} \rightarrow d_{1}^{(g=1)}\right)$ $w_{4}$ grows exponentially. We will consider the 'usable' $d_{1}$ range to be that where $w_{4}$ falls below $10 \mathrm{~mm}$, which for this configuration is the $(1.080,1.828) \mathrm{m}$ interval, corresponding to a $g$ interval of $(0.95,0.23)$ approximately. Table 8.2 displays the relevant optical parameters for several values of $d_{1}$.

\subsection{Transient response}

The 4-mirror cavity will have a response to short pulses similar to that of a FabryPerot cavity of the same optical path length, with the difference being that the cavity bandwidth (and hence the finesse) of the four mirror setup is different due to the four additional internal reflections per round-trip owned to the two extra mirrors.

We build a time domain model of the cavity and derive expressions for the reflected, transmitted and circulating field amplitudes. We then assume a constant driving field and derive expressions for the cavity's reflected, transmitted and circulating intensity response functions, the cavity bandwidth, and the finesse, as well as the optical suppression factor of higher order modes in the circulating field. We use the same notation and conventions as in Section 5.5.1, using complex phasors $a(t)$ in units of $\sqrt{\mathrm{W}}$ and $90^{\circ}$ phase shifts on transmission.

Assuming that the cavity is subject to an input excitation of time-varying amplitude $a_{\text {in }}(t)$ in near-normal incidence, and considering only the on-axis variation 


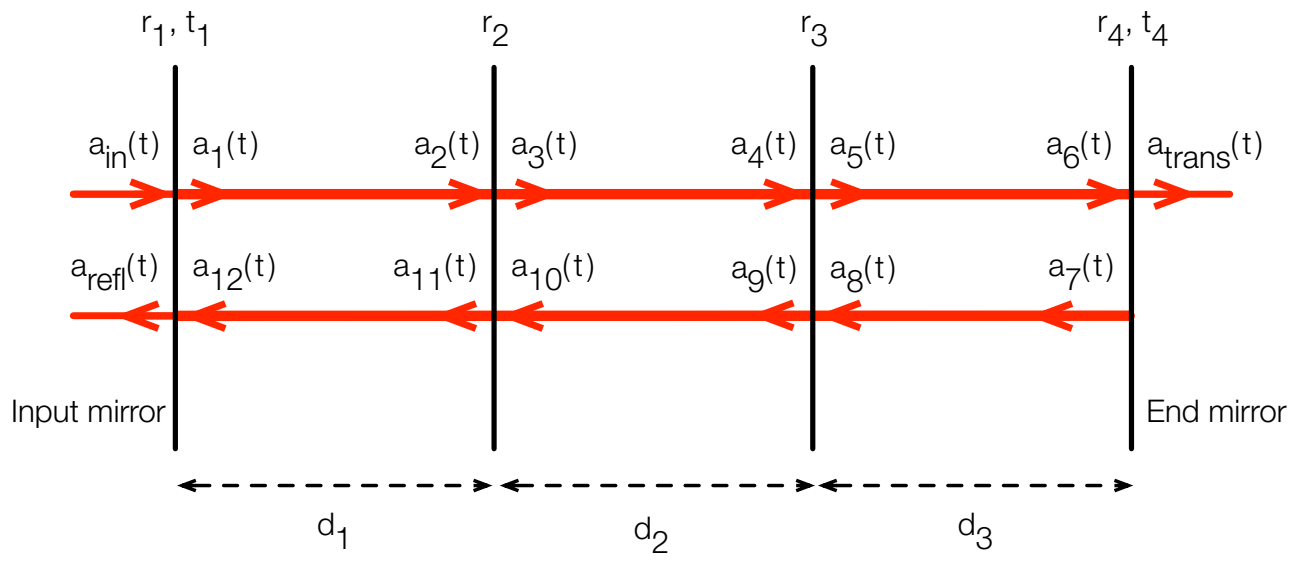

Figure 8.5: The incident field with amplitude $a_{\text {in }}(t)$ is reflected and/or transmitted by the cavity mirrors.

of the field, it is possible to write a series of difference equations by following the beam through a round-trip in the resonator (Figure 8.5),

$$
\begin{aligned}
a_{1}(t) & =i t_{1} a_{\text {in }}(t)+r_{1} a_{12}(t), \\
a_{2}(t) & =a_{1}\left(t-\tau_{1}\right) e^{-i \phi_{1}}, \\
a_{3}(t) & =r_{2} a_{2}(t), \\
a_{4}(t) & =a_{3}\left(t-\tau_{2}\right) e^{-i \phi_{2}}, \\
a_{5}(t) & =r_{3} a_{4}(t), \\
a_{6}(t) & =a_{5}\left(t-\tau_{3}\right) e^{-i \phi_{3}}, \\
a_{\text {trans }}(t) & =i t_{4} a_{6}(t), \\
a_{7}(t) & =r_{4} a_{6}(t), \\
a_{8}(t) & =a_{7}\left(t-\tau_{3}\right) e^{-i \phi_{3}}, \\
a_{9}(t) & =r_{3} a_{8}(t), \\
a_{10}(t) & =a_{9}\left(t-\tau_{2}\right) e^{-i \phi_{2}}, \\
a_{11}(t) & =r_{2} a_{10}(t), \\
a_{12}(t) & =a_{11}\left(t-\tau_{1}\right) e^{-i \phi_{1}}, \\
a_{\mathrm{refl}}(t) & =r_{1} a_{\mathrm{in}}(t)+i t_{1} a_{12}(t),
\end{aligned}
$$

where $\tau_{i}=d_{i} / c$ is the photon transit time between successive mirrors, and $\phi_{i}=k d_{i}$ the corresponding acquired phase. The total $M_{1}$ to $M_{4}$ photon transit time is $T=L / c$, and the total phase delay is $\phi=k L$, with $L=d_{1}+d_{2}+d_{3}$. The total forward propagating field inside the resonator at the position of the end mirror is

$$
a_{6}(t)=i t_{1} r_{2} r_{3} a_{\mathrm{in}}(t-T) e^{-i \phi}+r_{1} r_{2}^{2} r_{3}^{2} r_{4} a_{6}(t-2 T) e^{-i 2 \phi},
$$


and the transmitted and reflected field amplitudes are given by

$$
\begin{aligned}
a_{\mathrm{trans}}(t) & =-t_{1} t_{4} r_{2} r_{3} a_{\mathrm{in}}(t-T) e^{-i \phi}+r_{1} r_{2}^{2} r_{3}^{2} r_{4} a_{\mathrm{trans}}(t-2 T) e^{-i 2 \phi}, \\
a_{\mathrm{refl}}(t) & =r_{1} a_{\text {in }}(t)+r_{2}^{2} r_{3}^{2} r_{4}\left[-\left(t_{1}^{2}+r_{1}^{2}\right) a_{\text {in }}(t-2 T)+r_{1} a_{\mathrm{refl}}(t-2 T)\right] e^{-i 2 \phi}
\end{aligned}
$$

These equations can be easily iterated using a for-loop to compute the response of the cavity to an arbitrary time-varying input. The time step of the simulation should be chosen carefully (see Section 5.5.1).

\subsection{Over-coupling and transverse mode filtering}

When designing optical cavities for laser systems, an important consideration is whether the cavity should be under-coupled, over-coupled, or impedance matched for the particular application. These terms refer to the relationship between the cavity's input power transmissivity and all other resonator losses per round-trip, and the choice has a strong impact on the performance of the cavity. When the cavity is over-coupled, the circulating field power on resonance is enhanced, and thus spatial mode filtering is enhanced too. The impedance matching condition is found by calculating the reflection intensity response of the cavity, which is zero when the cavity is impedance matched and on resonance.

Taking the static solutions of the fields, considering a constant driving field $a_{\text {in }}=$ const, yields

$$
\begin{aligned}
a_{6} & =\frac{i t_{1} r_{2} r_{3} a_{\mathrm{in}} e^{-i \phi}}{1-r_{1} r_{2}^{2} r_{3}^{2} r_{4} e^{-i 2 \phi}}, \\
a_{\mathrm{trans}} & =\frac{-t_{1} t_{4} r_{2} r_{3} a_{\mathrm{in}} e^{-i \phi}}{1-r_{1} r_{2}^{2} r_{3}^{2} r_{4} e^{-i 2 \phi}} . \\
a_{\text {refl }} & =\frac{r_{1} a_{\text {in }}-r_{2}^{2} r_{3}^{2} r_{4}\left(r_{1}^{2}+t_{1}^{2}\right) a_{\text {in }} e^{-i 2 \phi}}{1-r_{1} r_{2}^{2} r_{3}^{2} r_{4} e^{-i 2 \phi}} .
\end{aligned}
$$

We can now calculate the transmission intensity response function by casting $\left|a_{\text {trans }} / a_{\text {in }}\right|^{2}$, which takes the form of the Airy distribution

$$
\mathcal{T}(\phi) \equiv\left|\frac{a_{\text {trans }}}{a_{\text {in }}}\right|^{2}=\left|\frac{-t_{1} t_{4} r_{2} r_{3} e^{-i \phi}}{1-r_{1} r_{2}^{2} r_{3}^{2} r_{4} e^{-i 2 \phi}}\right|^{2}=\frac{1}{\frac{\left(1-r_{1} r_{2}^{2} r_{3}^{2} r_{4}\right)^{2}}{t_{1}^{2} t_{4}^{2} r_{2}^{2} r_{3}^{2}}+\frac{4 r_{1} r_{4}}{t_{1}^{2} t_{4}^{2}} \sin ^{2}(\phi)} .
$$

This function peaks when the cavity is on resonance, i.e. when $\phi=k L=m \pi$, with $m \in \mathbb{Z}$. Let $\epsilon$ be the phase width of the resonance peaks,

$$
\mathcal{T}\left(m \pi \pm \frac{\epsilon}{2}\right)=\frac{1}{2} \Rightarrow \frac{\left(1-r_{1} r_{2}^{2} r_{3}^{2} r_{4}\right)^{2}}{t_{1}^{2} t_{4}^{2} r_{2}^{2} r_{3}^{2}}+\frac{4 r_{1} r_{4}}{t_{1}^{2} t_{4}^{2}} \sin ^{2}\left( \pm \frac{\epsilon}{2}\right)=2
$$

In the case of the Fabry-Perot cavity we obtain an explicit expression for $\epsilon$ as a function of the mirrors' electric parameters by expanding $\sin ^{2}\left( \pm \frac{\epsilon}{2}\right)$ to second order in $\epsilon$. In the 4-mirror resonator it is no longer practical to truncate to second order in $\epsilon$, as the obtained result is far from accurate. Including the fourth order term 
brings us closer to a meaningful solution. The cavity finesse is then obtained as the ratio of the separation of adjacent peaks to the peak width,

$$
\mathcal{F} \equiv \frac{\pi}{\epsilon} \text {. }
$$

The explicit expression of $\mathcal{F}$ as a function of the cavity parameters is too long to be reproduced here. Treating the four mirrors as identical and lossless, with amplitude reflection coefficients $r$, and truncating to fourth order in $\epsilon$, yields

$$
\mathcal{F} \approx \frac{\pi \sqrt{r^{5}+10 r^{11}+r^{17}}}{\left|r^{6}-1\right|\left(6 r^{5}+\sqrt{6} \sqrt{r^{20}+2 r^{18}+r^{16}+12 r^{14}+21 r^{12}+16 r^{10}+21 r^{8}+12 r^{6}+r^{4}+2 r^{2}+1}\right)} .
$$

However, this expression is still off by a factor of about 1.5 when compared to a FINESSE simulation [121] of the cavity. Therefore expanding to fourth order in $\epsilon$ is still inaccurate. When compared to a Fabry-Perot cavity with identical mirrors, the finesse of the 4-mirror cavity is a factor of $\approx 3$ lower.

The reflection amplitude transfer function is given by

$$
R(\phi) \equiv \frac{a_{\mathrm{refl}}}{a_{\mathrm{in}}}=\frac{r_{1}-r_{2}^{2} r_{3}^{2} r_{4}\left(r_{1}^{2}+t_{1}^{2}\right) e^{-i 2 \phi}}{1-r_{1} r_{2}^{2} r_{3}^{2} r_{4} e^{-i 2 \phi}} .
$$

Let $T_{i}=t_{i}^{2}, R_{i}=r_{i}^{2}$, and $L_{i}=1-r_{i}^{2}-t_{i}^{2}$ be the power transmitted, reflected, and lost at mirror $M_{i}$. For a cavity on resonance, $R(m \pi)$ takes the form

$$
R(m \pi)=\frac{\sqrt{1-T_{1}-L_{1}}-R_{2} R_{3} \sqrt{R_{4}}\left(1-L_{1}\right)}{1-\sqrt{R_{1} R_{4}} R_{2} R_{3}} .
$$

Solving $R(m \pi)=0$ for $T_{1}$ gives the condition for impedance matching,

$$
\begin{aligned}
T_{1}^{(\mathrm{im})} & \equiv L_{1}-1+\left(1-L_{1}\right)^{2} R_{2}^{2} R_{3}^{2} R_{4} \\
& =L_{1}-1+\left(1-L_{1}\right)^{2}\left(1-T_{2}-L_{2}\right)^{2}\left(1-T_{3}-L_{3}\right)^{2}\left(1-T_{4}-L_{4}\right) .
\end{aligned}
$$

The cavity is impedance matched if $T_{1}=T_{1}^{(\mathrm{im})}$, under-coupled if $T_{1}<T_{1}^{(\mathrm{im})}$, and over-coupled if $T_{1}>T_{1}^{(\mathrm{im})}$. The resonance effect is greater in over-coupled cavities, i.e., the cavity's circulating field power transfer function is greater for cavities with $T_{1}>T_{1}^{(\mathrm{im})}$. Therefore, the optical suppression of higher order transverse modes is also greater in over-coupled cavities.

The circulating field intensity transfer function at the end mirror differs from the transmitted field transfer function by a factor $1 / t_{4}^{2}$,

$$
\mathcal{C}(\phi) \equiv \frac{1}{t_{4}^{2}} \mathcal{T}(\phi)=\frac{1}{\frac{\left(1-r_{1} r_{2}^{2} r_{3}^{2} r_{4}\right)^{2}}{t_{1}^{2} r_{2}^{2} r_{3}^{2}}+\frac{4 r_{1} r_{4}}{t_{1}^{2}} \sin ^{2}(\phi)}
$$

The optical suppression ratio of the $n m$-th order transverse mode inside the cavity is therefore given by

$$
S_{n m} \equiv \frac{1}{\sqrt{\frac{\left(1-r_{1} r_{2}^{2} r_{3}^{2} r_{4}\right)^{2}}{t_{1}^{2} r_{2}^{2} r_{3}^{2}}+\frac{4 r_{1} r_{4}}{t_{1}^{2}} \sin ^{2}\left[(n+m) \frac{\zeta}{2}\right]}}
$$

which is lower (i.e., better suppression) for $T_{1}>T_{1}^{(\mathrm{I}-\mathrm{M})}$. 


\subsection{Gaussian mode non-degeneracy}

The round-trip Gouy phase shift of the cavity can be tuned from $0^{\circ}$ to $180^{\circ}$ by modifying the separation $d_{1}$ between mirrors $M_{1}$ and $M_{2}$. When choosing $d_{1}$ to complete the configuration of the cavity, it is important to check whether in the resultant configuration no higher order spatial modes up to a certain order $N$ are co-resonant with the fundamental mode. The problem of choosing $d_{1}$ to yield improved Guassian mode non-degeneracy can be simplified by introducing a figure of merit for mode non-degeneracy up to order $N$, as a function of $d_{1}$, with the rest of the cavity parameters as constants. As we saw in Chapter 7, we have defined the function $\Theta_{N}$ to help us do this (Equation 7.2),

$$
\Theta_{N} \equiv \frac{1}{\sqrt{\sum_{k=1}^{N} \frac{1}{k ! \Psi_{k}^{2}}}},
$$

where $\Psi_{k}$ is the mode non-degeneracy of the $k$-th order transverse mode

$$
\Psi_{k} \equiv\left|\frac{\Delta \phi_{k}}{2 \pi}-\operatorname{round}\left(\frac{\Delta \phi_{\mathrm{k}}}{2 \pi}\right)\right|
$$

and $\phi_{k}$ is the extra phase acquired by the $k$-th order mode with respect to the fundamental mode in a resonator round-trip, given by the round-trip Gouy phase shift

$$
\Delta \phi_{k} \equiv k \zeta=2 k \operatorname{sgn}\left[\widehat{M}_{12}(z)\right] \arccos (m) .
$$

Figure 8.6 shows $\Theta_{15}$ as a function of $d_{1}$ for the cavity parameters specified in Table 8.1. The function has dips when a certain mode (or set of modes) is co-resonant with the fundamental mode. We find that the cavity will generally be a worse mode filter for the lower values of $d_{1}\left(\right.$ as $\left.d_{1} \rightarrow d_{1}^{(g=1)}\right)$, and will perform better for larger values (as long as the value is chosen carefully). See Figure 8.7 for a more detailed view of a smaller $d_{1}$ interval, indicating the particular transverse modes that become co-resonant with the fundamental mode in the resulting cavity configuration.

\subsection{Summary and future work}

We have put forward the design of a large waist cavity with tuneable stability. The cavity features four mirrors in a beam expander configuration: two convex mirrors expand the input beam, a concave mirror nearly collimates it, and a flat end mirror closes the resonator. The cavity will provide a magnification of the input beam waist of a factor 10 to 30, always resulting in a beam radius larger than $8 \mathrm{~mm}$ in the science region of $1 \mathrm{~m}$. The distance $d_{1}$ between the input mirror and the second mirror can be modified to tune the cavity's round-trip Gouy phase shift from $0^{\circ}$ to $180^{\circ}$, and therefore it can be used to select a cavity configuration that yields improved Gaussian mode non-degeneracy. The cavity can thus act as a mode cleaner of the input beam, providing an interrogation field with low wavefront distortions, which are a leading source of noise in state-of-the-art atom interferometric sensors. 


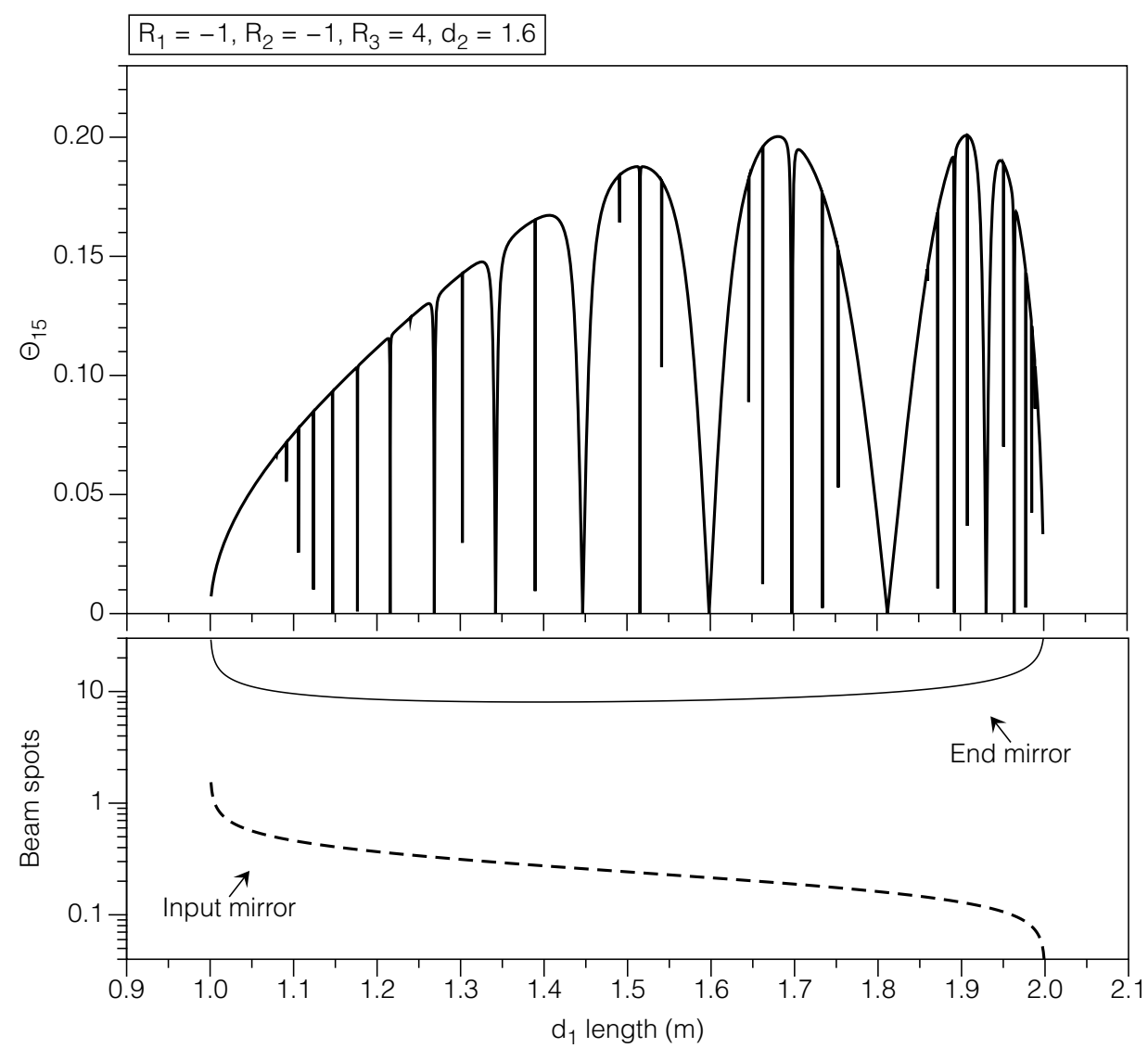

Figure 8.6: Figure of merit of mode non-degeneracy up to order 15 as a function of the distance $d_{1}$ between the input mirror and the second mirror. The function $\Theta_{15}$ indicates the level of Gaussian mode non-degeneracy, with larger values indicating that the resulting cavity configuration is better in terms of spatial filtering. The function presents dips when a particular mode or set of modes of order $\leq 15$ is co-resonant with the fundamental mode.

The proposed cavity gets around the geometrical limitations of Fabry-Perot cavities for atom interferometry, which place a tight constraint on the temperature of the atomic ensembles due to the requirement of maintaining a stable configuration [41]. Another solution to the limitation of the Fabry-Perot cavity is using an intra-cavity lens, as reported in [110]. However, the resulting cavity is marginally stable, and therefore performs poorly as a mode filter.

The $d_{1}$ range over which the cavity is stable is given by the absolute value of the radius of curvature of the input mirror $\left|\mathcal{R}_{1}\right|$, which is chosen to be $1 \mathrm{~m}$ for practical reasons. For the parameters specified in Table 8.1, the total cavity length is 3.6 to $4.6 \mathrm{~m}$ depending on the chosen setting of $d_{1} \in[1,2] \mathrm{m}$, and it is folded so that the longest section is 1.6 to $2 \mathrm{~m}$. More compact configurations can be obtained by using 
mirrors with lower radii of curvature. Due to the non-zero angles of incidence of the beam at mirrors $M_{2}$ and $M_{3}$ ( $\approx 3$ degrees for the parameters listed in Table 8.1), the cavity will suffer a certain degree of astigmatism, which leads to changes in the resonance conditions of higher order modes, as well as to warping of the spatial profiles of the modes $[122,123]$. These deviations from an ideal resonator need to be taken into consideration in future iterations of this design.

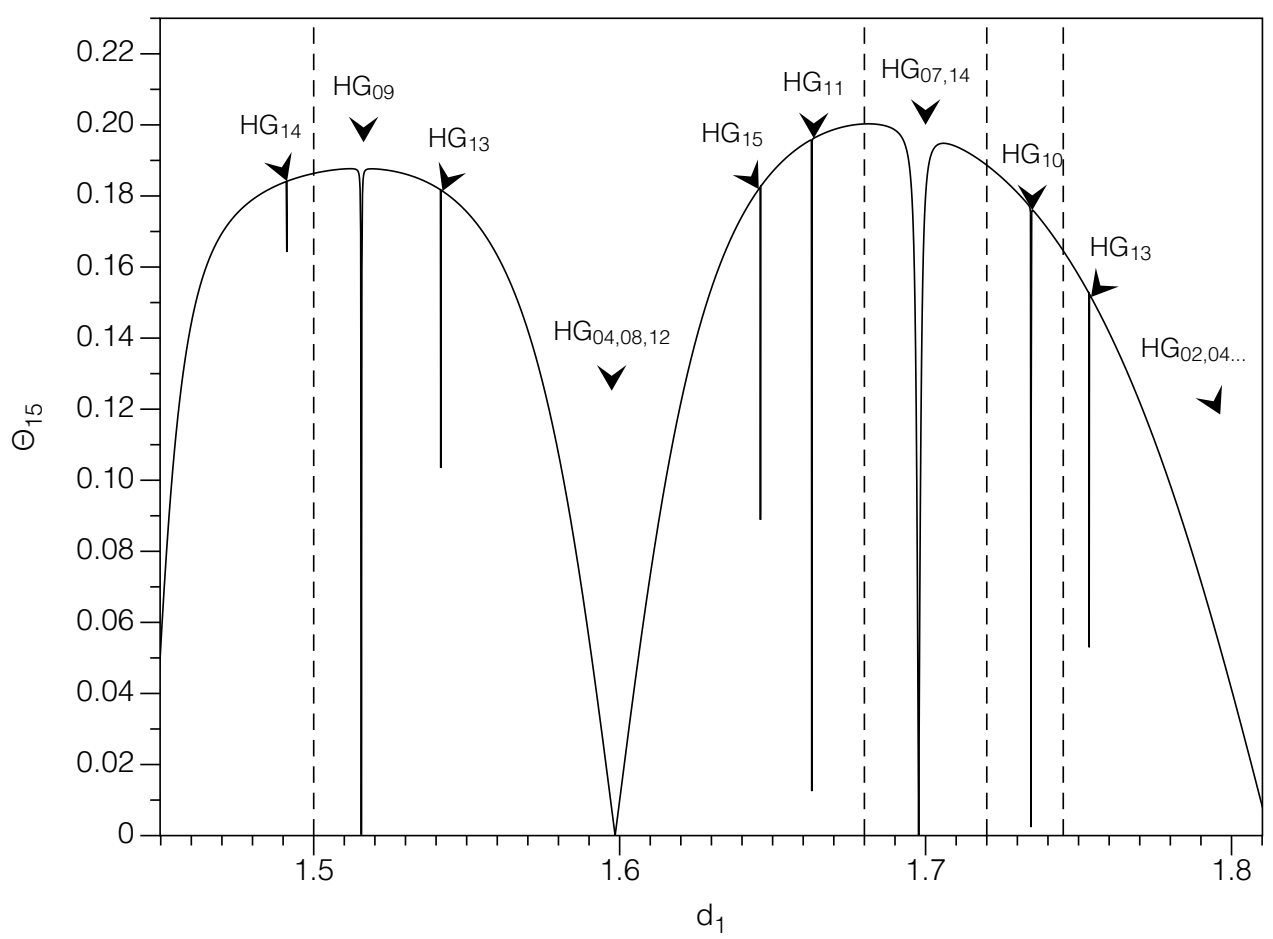

Figure 8.7: Same as Figure 8.6, indicating the higher order transverse modes that become co-resonant with the fundamental mode in the resulting cavity configuration. 

Cavities FOR

GravitationaL-WAVE

DETECTION 



\section{Near-unstable cavities for future gravitational wave detectors}

\section{Contents}

9.1 The hunt for gravitational wave signals . . . . . . . . . 184

9.1.1 Detectors and noise sources . . . . . . . . . . . . . 184

9.1.2 Thermal noise reduction . . . . . . . . . . . 186

9.2 Near-unstable cavities . . . . . . . . . . . . . . . . . . . . . 188

9.2.1 Thermal noise and resonator stability . . . . . . . . 188

9.2.2 Degenerate cavity issues . . . . . . . . . . . . . 190

9.3 Tabletop NUC optical design . . . . . . . . . . . . . . . . 191

9.3.1 Plane-concave cavity with tuneable stability . . . . . . 191

9.3.2 Precision characterisation of cavity stability . . . . . . 193

9.3.3 Alignment and frequency stabilisation systems . . . . . 194

9.4 Pushing the cavity over the edge . . . . . . . . . . . . 196

9.4.1 Influence of mirror imperfections . . . . . . . . . . 196

9.4.2 Characterising cavity stability . . . . . . . . . . . 197

9.4.3 Results and simple astigmatic model . . . . . . . . . 198

9.5 Summary and future work . . . . . . . . . . . . 202 


\subsection{The hunt for gravitational wave signals}

\subsubsection{Detectors and noise sources}

CRAVITATIONAL waves eluded science's best efforts to detect them for decades

T due to their extremely weak nature. The strength of a gravitational wave is parametrised by the so called strain $h$, which relates to the relative length change induced by the wave along one of its polarisation axes by [63]

$$
h(t) \equiv \frac{\Delta L(t)}{L},
$$

and is extremely small. Only the most violent astrophysical events, like the coalescence of two black holes, will emit gravitational waves generating a large enough strain that we may hope to detect here on Earth. For example, the first gravitational wave detected in 2015 by LIGO reached a peak strain of $1 \cdot 10^{-21}$, with the signal increasing in amplitude and frequency from $35 \mathrm{~Hz}$ to $250 \mathrm{~Hz}$ over a stretch of $0.2 \mathrm{~s}$.

In order to realise such sensitivity, we have developed over decades very sophisticated laser interferometer detectors. The Advanced LIGO second generation detectors are power- and signal-recycled Michelson interferometers with $4 \mathrm{~km}$ FabryPerot arm cavities (Figure 9.1). Each Fabry-Perot cavity features a pair of mirrors acting as test masses that can be considered effectively inertial above some frequency. The interferometer is operated at a dark fringe, which means that all the common mode optical power is reflected back to the laser port. Common mode refers to effects that are common to both arms of the interferometer and thus have equal and opposite effects on the two beams. On the other hand, differential mode refers to perturbations that alter the beam in a different way depending on the interferometer arm.

The passing through Earth of gravitational waves induces differential mode effects on the interferometer by effectively and orthogonally modifying each arm's length. The induced differential interferometric length for an optimally oriented detector is $\Delta L=h(t) L$, with the cavities providing substantially greater effective arm length $L$ (by a factor of approximately 300 ) due to their ability to store coherent light. This differential length translates into a differential phase of the light beams reflected by each arm cavity, which produces a signal at the beam splitter's output port that is proportional to the gravitational wave strain. The output port is also referred to as the dark port when the interferometer is operated at a dark fringe.

Power recycling is an interferometer technique for further increasing the power circulating in the arms by introducing a set of mirrors that form a pair of cavities with the input test masses: the power recycling cavities (PRC, or the PRX and PRY). The PRC's are designed and tuned so that almost no light is reflected back to the source, and when kept on resonance they enhance the power in the arms by a factor of about 40. Signal recycling, on the other hand, aims at enhancing differential mode signals in the interferometer by introducing a set of mirrors between the photodetector and the interferometer arms, forming the signal recycling cavity (SRC). The SRC can be tuned to either resonantly enhance the signal at particular frequencies or so that the cavity is detuned from the peak signal frequency to enhance the signal at sideband frequencies. The latter method is known as resonant sideband extraction, and is the current technique used in Advanced LIGO. 


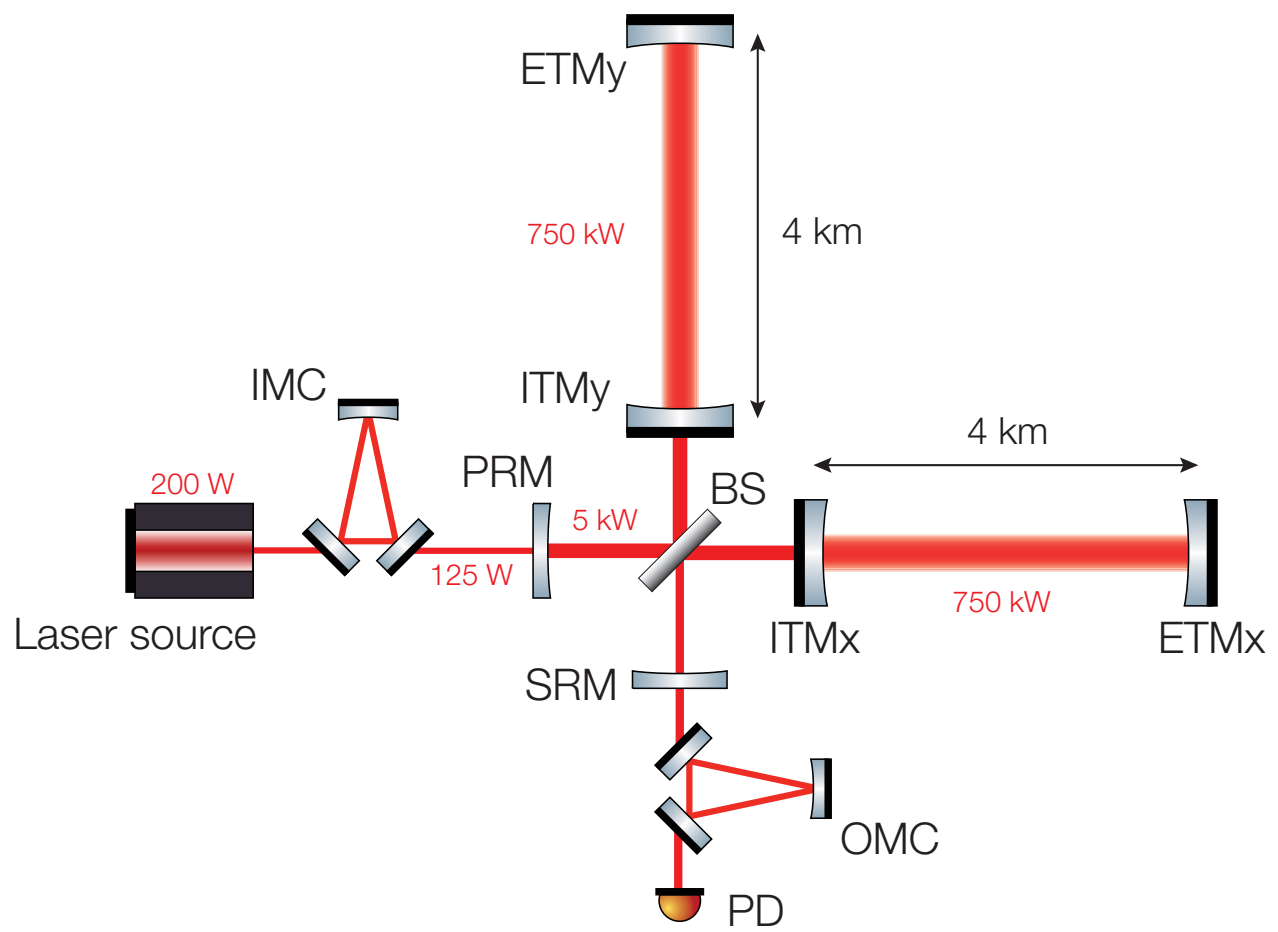

Figure 9.1: Simplified diagram of the Advanced LIGO detectors. Light from the laser source is split at the beam splitter (BS) and coupled into two giant orthogonal Fabry-Perot cavities, where it is resonantly enhanced before being recombined forming a cavity enhanced Michelson interferometer. Each cavity is formed by an input test mass (ITM) and an end test mass (ETM). The power recycling mirror (PRM) provides additional resonant buildup and the signal recycling mirror (SRM) optimises signal extraction. The input mode cleaner (IMC) provides spatial filtering of the beam before entering the interferometer core optics. The output mode cleaner (OMC) is used to optically suppress the modes that do not carry the gravitational wave signal before impinging on the photodetector. A gravitational wave propagating perpendicularly to the detector plane will cause an elongation of one arm and shortening of the other, which generates a signal that is measured at the photodetector $(\mathrm{PD})$. The powers indicated are design values.

Two identical detectors were built in Hanford (Washington) and Livingston (Louisiana) in order to provide rejection of local instrument and environment noise, and to provide sky localisation of the source as well as a measurement of wave polarisation. 
The length and phase changes induced by passing gravitational waves are tiny, and many technical and fundamental noise sources affect the measurement (Figure 9.2):

- Seismic noise: Ground vibration is the main source of noise at low frequency (from DC to a few hertz), as the local motion of the ground at the detector site - originating from both natural sources, such as wind, earthquakes or ocean waves, and human activity - couples to the test masses and causes a differential signal. To reduce seismic noise in the detection band, each test mass is mounted on a passive quadruple suspension system using silica fibres, which is in turn mounted on an active vibration isolation system. In addition to ground motion, changes over time of the local acceleration due to gravity also induce random displacements of the test masses, the so called Newtonian noise.

- Quantum noise: The quantum fluctuations of light limit the sensitivity of the detectors over much of the detection band, and are the limiting source of noise at high frequency $(>100 \mathrm{~Hz})$. At low frequencies, radiation pressure noise due to the mechanical interaction of the circulating field in the cavity and the test masses causes random changes in the arm lengths. At higher frequencies, shot noise at the photodetector due to random fluctuations of the phase of light at the detection port couples directly to the signal and limits the sensitivity.

- Thermal noise: The random motion of the atoms forming the test masses and the suspension fibres also causes arbitrary modifications of the arm length. Thermal noise is the limiting source of noise at mid-frequency [71,124]. Lowloss high-quality-factor materials with resonance frequencies much higher or lower than the detection bandwidth are used to reduce this source of noise. This noise scales inversely with the beam radius at the position of the test masses, and therefore using configurations with larger beam sizes helps reduce thermal noise.

\subsubsection{Thermal noise reduction}

Mirror thermal noise is a major source of noise in advanced gravitational-wave detectors over much of the detection band (from a few tens of hertz to a few hundreds). The amplitude of the induced random length changes in the interferometer scales inversely with the radius of the beam at the optic $w$. One straightforward way to reduce thermal noise is to use cavity configurations with an increased beam radius $w$ at the test masses. This means, however, that the cavity will be nearer its region of geometrical instability. A cavity that is stable but that lies parametrically close to the boundary of instability is referred to as a near-unstable cavity (NUC). These devices have found many applications, for example in experiments where a relatively large or small mode volume is required $[125,126]$. In this work we discuss their application in advanced gravitational-wave detectors.

Near unstable cavities may be driven into the unstable region via small perturbations, such as any effects that may cause small cavity length changes or mirror 


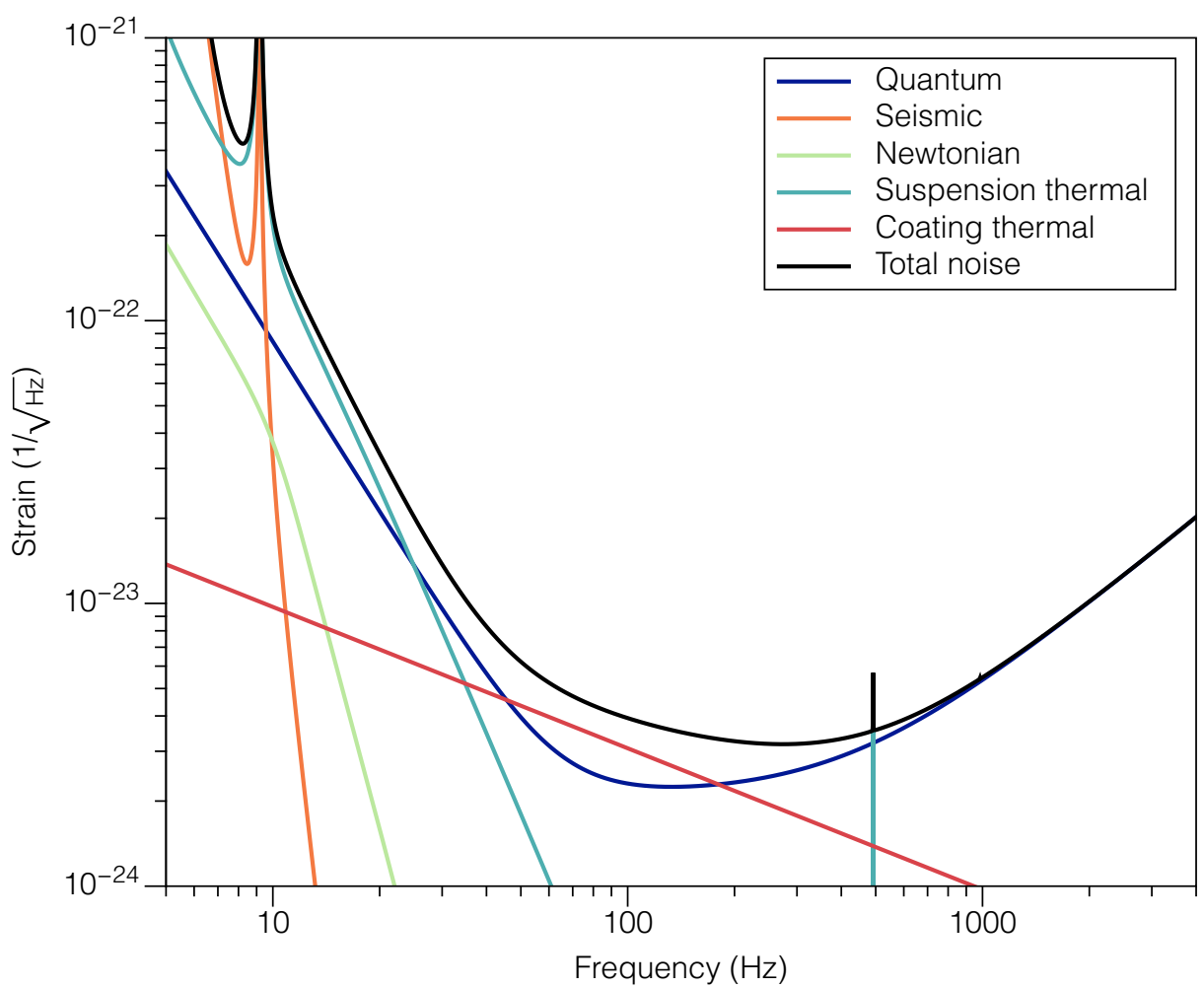

Figure 9.2: Main sources of noise affecting the sensitivity of laser interferometer gravitational-wave detectors, such as the Advanced LIGO detectors.

surface distortions. For example, thermal transients of the mirror substrates can result in small changes of the radius of curvature of the optic and render the cavity unstable. NUCs are therefore at risk of suffering from a variety of problems, namely high optical loss and Gaussian mode degeneracy [127,128]. The increased mode degeneracy coupled with a higher sensitivity to mirror surface imperfections and alignment errors means that the light field in a NUC is prone to become scattered into higher order modes. This will reduce the measured signal as light leaving the fundamental Gaussian mode will not reach the detection port.

In order to investigate NUC issues in a feasible scale, we have developed a tabletop experiment featuring a near-hemispherical cavity whose stability can be tuned by adjusting the position of the concave end mirror. In this chapter we present the experiment, detailing both its design and operation, as well as the results obtained as the cavity is parametrically pushed into the near-unstable and unstable regions. This work aims to provide a detailed account of the design challenges and technical hurdles associated with this type of cavity, as well as an insight into how far the cavity parameters can be pushed towards instability whilst maintaining controllability and spatial filtering. 


\subsection{Near-unstable cavities}

\subsubsection{Thermal noise and resonator stability}

As stated in Chapter 2, the Brownian motion of the atoms in the coated mirrors induces $1 / f$ displacement noise in the cavity, and is approximated by

$$
S_{\text {mirror }}(f) \equiv S_{\mathrm{sb}}(f)+S_{\mathrm{ct}}(f) \text {, }
$$

with (Equations 2.51 and 2.52)

$$
\begin{aligned}
S_{\mathrm{sb}}(f) & =\frac{4 k_{B} T}{2 \pi f} \frac{1-\sigma_{\mathrm{FS}}^{2}}{\sqrt{\pi} Y_{\mathrm{FS}} w} \phi_{\mathrm{FS}}, \\
S_{\mathrm{ct}}(f) & =\frac{4 k_{B} T}{2 \pi f} \frac{2\left(1+\sigma_{\mathrm{FS}}\right)\left(1-2 \sigma_{\mathrm{FS}}\right) d_{\mathrm{ct}}}{\pi Y_{\mathrm{FS}} w^{2}} \phi_{\mathrm{ct}},
\end{aligned}
$$

where $f$ is the frequency of the noise, $k_{B}$ is Boltzmann's constant, $T$ is the temperature of the mirror, $w$ is the beam radius at the optic, $Y_{\mathrm{FS}}$ is fused silica's Young's modulus, $\sigma_{\mathrm{FS}}$ is its Poisson's ratio, $d_{\mathrm{ct}}$ is the coating's thickness, and $\phi_{\mathrm{FS}}$ and $\phi_{\mathrm{ct}}$ are fused silica's and the coating's mechanical loss angles respectively. This is the main source of noise limiting the sensitivity of laser interferometer gravitational-wave detectors at mid-frequency. The substrate contribution scales with $1 / w$, and the coating contribution with $1 / w^{2}$. In order to reduce this source of noise, using resonator configurations with a large beam radius $w$ at the mirrors is a straightforward solution. However the size of the beam in the cavity and the resonator's geometrical stability are fundamentally linked.

On the one hand, the beam radii at the mirrors in a Fabry-Perot resonator are given by (Equation 2.39)

$$
w_{1,2}^{2} \equiv \frac{\lambda L}{\pi} \sqrt{\frac{g_{2,1}}{g_{1,2}\left(1-g_{1} g_{2}\right)}}
$$

where $g_{1,2} \equiv 1-L / \mathcal{R}_{1,2}$ are the $g$-factors of the mirrors, $L$ is the cavity length, and $\mathcal{R}_{1,2}$ are the radii of curvature (ROC) of the mirrors. The sign criterion for the ROC is that it is positive for mirrors concave towards the cavity centre and negative otherwise. On the other hand, the geometrical stability of the cavity is parametrised by the total $g$-factor $g=g_{1} g_{2}$, which can be interpreted as the magnification experienced by an incident collimated beam upon completing a resonator round-trip. If the magnification is such that the beam maintains a finite size over an arbitrarily large number of round-trips, the cavity is said to be stable. If on the other hand the beam size diverges, the cavity is unstable. The condition for stability translates to

$$
0 \leq g_{1} g_{2} \leq 1
$$

Figure 9.3 shows the stability diagram of the cavity as parametrised by $g_{1}$ and $g_{2}$. The arm cavities of current gravitational-wave detectors Advanced LIGO and Advanced VIRGO are configured close to the near-concentric regime, but with $g$-factors well within stability $(g \approx 0.83$ in Advanced LIGO and $g \approx 0.87$ in Advanced VIRGO). Pushing Advanced LIGO or Advanced VIRGO's arm cavities 


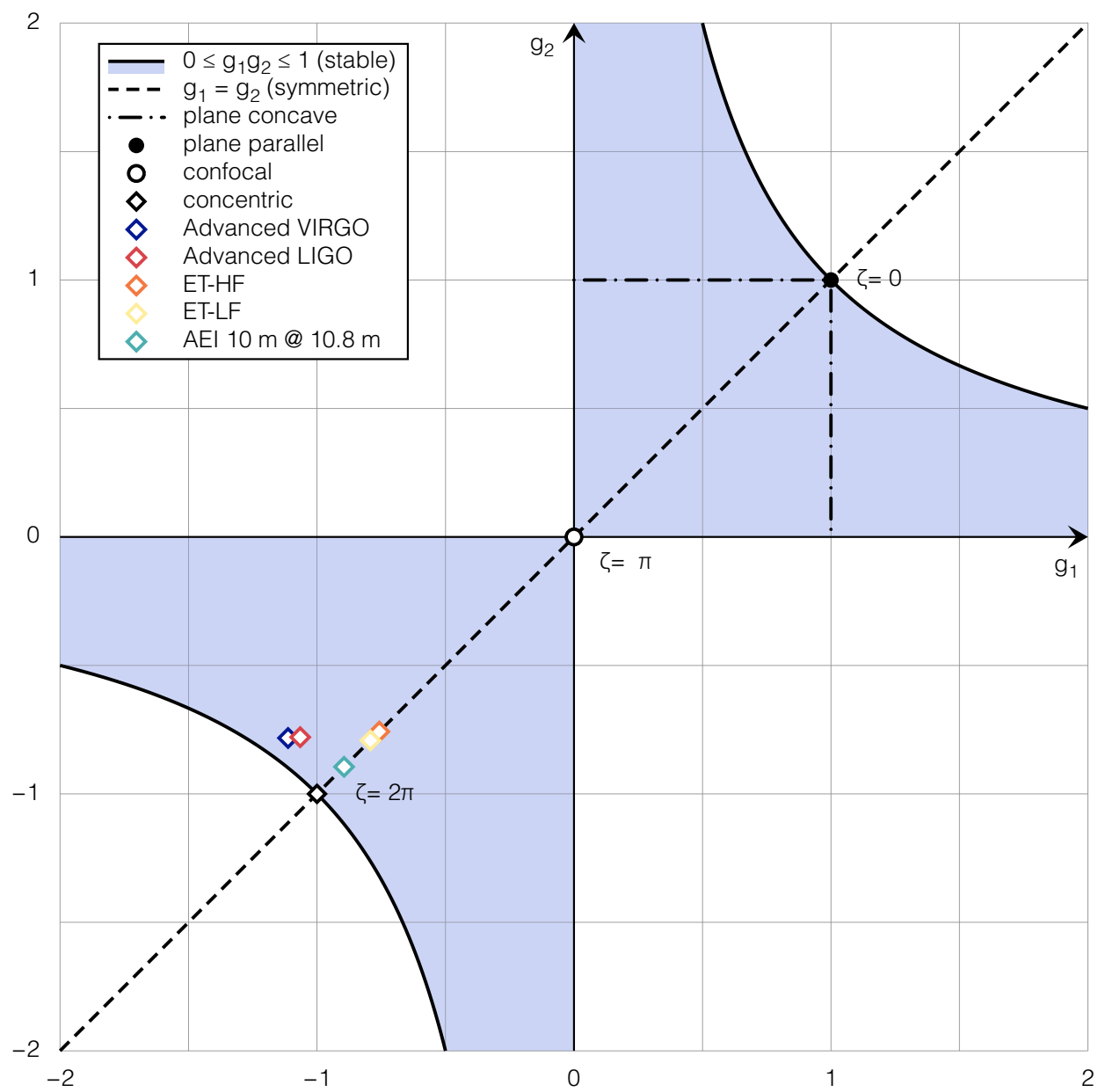

Figure 9.3: Fabry-Perot resonator stability in the $g_{1}-g_{2}$ parameter space, showing the stability region $\left(0 \leq g_{1} g_{2} \leq 1\right)$ and other features. Cavities are stable if the size of the circulating beam does not diverge after an arbitrary number of internal reflections. Cavities with configurations near the region of instability suffer from a variety of issues, but in turn offer the advantage of producing a large beam size at the position of the mirrors, which can help reduce the thermal noise floor of gravitational-wave detectors.

further towards instability (e.g., $g>0.98$ ) would result in substantially larger beam sizes on the test masses, the high power circulating beams would then sample a larger surface area of the mirror, resulting in reduced displacement noise of the mirror substrates and the mirror coatings, ultimately improving the sensitivity of the detector.

The tabletop cavity in our experiment is a plane-concave cavity that we push towards the hemispherical limit, so that the beam becomes large on the concave 
mirror and small on the flat mirror. In many ways it is equivalent to a concentric cavity of twice the length.

Reducing the thermal noise by changing the intensity distribution of the beam can also be achieved by using alternative beam shapes, such as Laguerre-Gauss modes [129-131]. Alternative beam shapes present unique difficulties that make them challenging to implement in gravitational-wave detectors. For an overview of alternative beam shapes in interferometry see Chapter 13 "Beam Shaping" in [124].

\subsubsection{Degenerate cavity issues}

The major issue of using a cavity configuration very close to geometrical instability is that higher order modes can become co-resonant with the fundamental mode. The phase shift accumulated by higher order modes after a resonator round-trip is determined by the round-trip Gouy phase shift as (Equation 2.43)

$$
\Delta \phi_{n m}=(n+m) \zeta
$$

where $n+m$ is the order of the mode. Thus, the offset in resonance frequency of the $\mathrm{nm}$-th order mode with respect to the fundamental mode is given by (Equation 2.44)

$$
\Delta \nu_{n m}=\frac{\Delta \phi_{n m}}{2 \pi} \Delta \nu_{\mathrm{FSR}}=(n+m) \frac{\zeta}{2 \pi} \Delta \nu_{\mathrm{FSR}} .
$$

where $\Delta \nu_{\mathrm{FSR}}=c /(2 L)$ is the free spectral range, as seen on Chapter 2, and for a Fabry-Perot cavity the round-trip Gouy phase shift is given by (Equation 2.42)

$$
\zeta=2 \arccos \left( \pm \sqrt{g_{1} g_{2}}\right),
$$

and the sign \pm is determined by the sign of $g_{1}$. For a near-concentric cavity the round-trip Gouy phase of the $n m$-th order spatial mode is close to $(n+m) 2 \pi$, and in consequence its offset in resonance frequency is close to $(n+m) \Delta \nu_{\mathrm{FSR}}$ (i.e., all higher order modes are co-resonant with the fundamental mode). A near-hemispherical cavity has half the round-trip Gouy phase of a near-concentric, and hence the $n m$-th order mode is offset by $\frac{1}{2}(n+m) \Delta \nu_{\mathrm{FSR}}$ (i.e., even higher order modes are co-resonant).

In order to quantify the separation in resonance frequency of higher order modes relative to the fundamental mode and in terms of the cavity bandwidth, it is useful to introduce the separation factor as

$$
\delta_{n m} \equiv \frac{\left|\frac{\Delta \nu_{n m}}{\Delta \nu_{\mathrm{FSR}}}-\operatorname{round}\left(\frac{\Delta \nu_{n m}}{\Delta \nu_{\mathrm{FSR}}}\right)\right| \Delta \nu_{\mathrm{FSR}}}{\Delta \nu_{\mathrm{FWHM}}},
$$

where $\Delta \nu_{\mathrm{FWHM}}=\Delta \nu_{\mathrm{FSR}} / \mathcal{F}$ is the cavity bandwidth and $\mathcal{F}$ is the finesse. Substituting we find the more readable expression

$$
\delta_{n m}=\mathcal{F}\left|(n+m) \frac{\zeta}{2 \pi}-\operatorname{round}\left([n+m] \frac{\zeta}{2 \pi}\right)\right| .
$$

In practice, the separation factor of the first few higher order modes should be much greater than 1 in order to avoid degeneracy. Figure 9.4 shows the separation 


\begin{tabular}{ccccccc}
\hline Detector & $L(\mathrm{~m})$ & $\mathcal{R}_{1}(\mathrm{~m})$ & $\mathcal{R}_{2}(\mathrm{~m})$ & $\mathcal{F}$ & $g$ & $\delta_{01}$ \\
\hline Advanced LIGO & 3994.5 & 1934 & 2245 & 446 & 0.830 & 60.3 \\
Advanced VIRGO & 3000 & 1420 & 1683 & 443 & 0.871 & 51.8 \\
ET-HF & 10000 & 5690 & 5690 & 880 & 0.574 & 199.2 \\
ET-LF & 10000 & 5580 & 5580 & 880 & 0.627 & 184.0 \\
AEI 10 m prototype & 10.8 & 5.7 & 5.7 & 675 & 0.800 & 99.6 \\
& 11.3952 & & & & 0.998 & 9.6 \\
\hline
\end{tabular}

Table 9.1: Parameters pertaining to the stability of current and future gravitationalwave detectors. The Advanced LIGO and Advanced VIRGO detectors are dualrecycled Michelson interferometers with Fabry-Perot arm cavities, as depicted in Figure 9.1. The Albert Einstein Institute's $10 \mathrm{~m}$ prototype experiment also consists of a Michelson interferometer with Fabry-Perot arm cavities, and is designed so that it can be commissioned with high stability ( $L=10.8 \mathrm{~m}, g=0.8$ configuration) and later pushed towards the concentric limit ( $L=11.3952 \mathrm{~m}, g=0.998$ configuration). The Einstein Telescope (ET) is a future detector currently in design phase, employing giant $10 \mathrm{~km}$ Fabry-Perot arm cavities in 3 nested detectors, each in turn composed of 2 dual-recycled Michelson interferometers, one devoted to low-frequency signals (ET-LF) and one to high-frequency signals (ET-HF).

factor of the first order mode as a function of cavity stability in Advanced LIGO [4], Advanced Virgo [132,133], the AEI $10 \mathrm{~m}$ prototype [134,135], and the Einstein Telescope [46]. The separation factor can be useful to determine the value of cavity parameters in order to avoid higher order mode degeneracy.

\subsection{Tabletop NUC optical design}

\subsubsection{Plane-concave cavity with tuneable stability}

The following optical setup is implemented to investigate the behaviour of an optical cavity as it is parametrically pushed towards geometrical instability. We showed that near instability the cavity's resonance conditions become very sensitive to mirror surface imperfections, leading to changes in the resonant and spatial structure of the beam. We started by setting up a cavity with a flat input mirror and a concave end mirror with a radius of curvature $\mathcal{R}_{2} \approx 1 \mathrm{~m}$. We chose this plane-concave mirror combination over a symmetric configuration for the ease of mode matching and length stabilisation. The beam waist will therefore be located at the flat mirror, and the beam size will grow towards the curved mirror. For these mirror parameters, the cavity will be exactly hemispherical when the cavity length matches the radius of curvature of the concave mirror. In the near-unstable regime, with $L \rightarrow 1 \mathrm{~m}$, we have $g \rightarrow 0$ with $g_{1}=1$ and $g_{2} \rightarrow 0$.

Since the total $g$-factor of our cavity approaches zero in the near unstable region, the round-trip Gouy phase shift $\zeta=2 \arccos ( \pm \sqrt{g})$ approaches $\pi$. This means that in our experiment even order modes will become co-resonant with the fundamental mode, and odd order modes will become anti-resonant (meaning that their resonant 


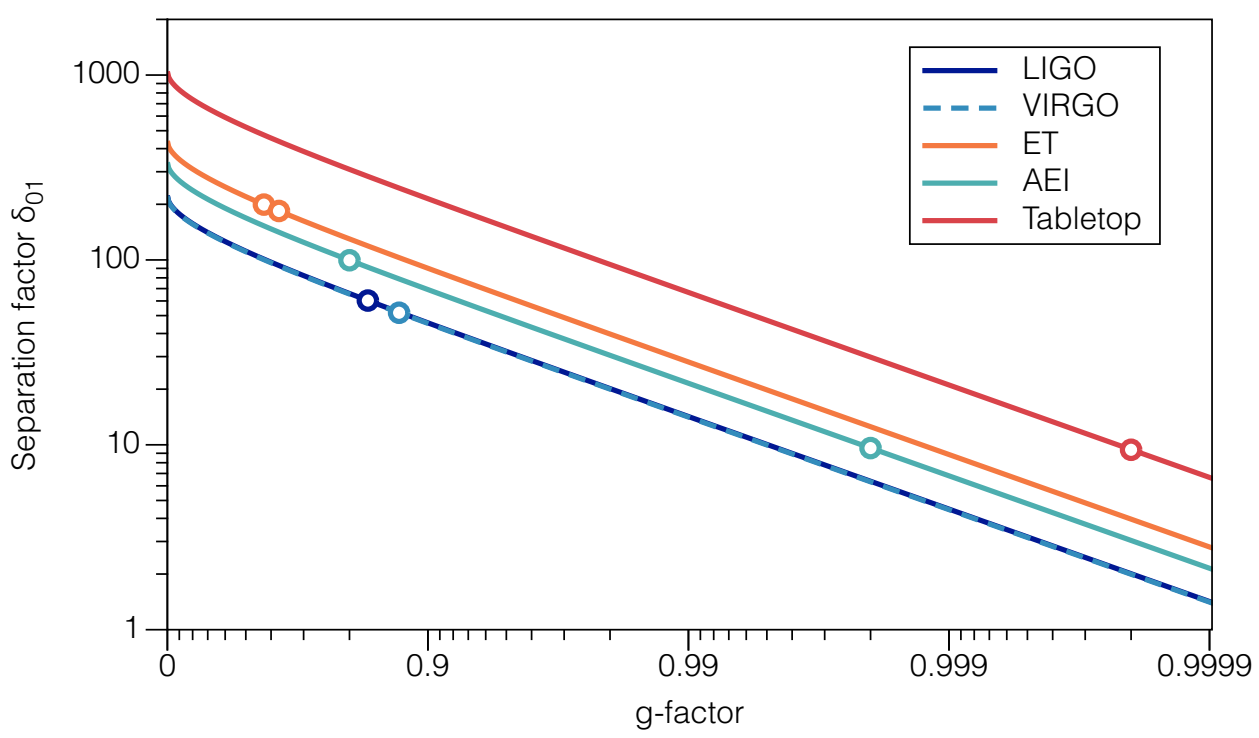

Figure 9.4: The separation factor indicates the offset in resonance frequency of a higher order mode to the closest fundamental mode resonance, in multiples of the cavity bandwidth. The separation factor of the first order mode $\delta_{01}$ is depicted as a function of the cavity $g$-factor in different gravitational-wave detectors. The dots represent the current or planned configuration. They all use separation factors between 60 and 200, except the AEI $10 \mathrm{~m}$ prototype with $\delta_{01} \approx 10$ when pushed to its maximum design $g$-factor of 0.998. For comparison we have included our tabletop cavity as the equivalent near-concentric cavity of twice the length, which would have a separation factor similar to the prototype at $g=0.9998$.

frequency will approach half the free spectral range). This is the fundamental difference with respect to the near-concentric cavity, where the round-trip Gouy phase shift is nearly $2 \pi$ and both even and odd order modes become co-resonant. Setting this difference aside, our $1 \mathrm{~m}$ near-hemispherical cavity is equivalent to a $2 \mathrm{~m}$ near-concentric cavity (i.e., they have the same eigenmodes).

In our experiment, cavity stability is tuneable by adjusting the position of the curved mirror, which is mounted on a translation stage, hence modifying the total resonator length. We chose a finesse of approximately 2000, which will provide a large separation factor even at $g$-factors very close to 0 . At $g=0.0001$ the separation factor of the first order mode will be similar to that of the AEI $10 \mathrm{~m}$ prototype at its maximum target $g$-factor of 0.998 . See Table 9.2 for a list of some of the design parameters of the tabletop cavity at several targeted stabilities. The cavity is first set up to lie well within the stable region, with a $g$-factor similar to that of the arm cavities in Advanced LIGO and Advanced Virgo. Once the cavity is aligned and locked, its length is incrementally adjusted to change the overall stability. 


\subsubsection{Precision characterisation of cavity stability}

Measuring the cavity's free spectral range provides a very accurate measurement of the total resonator length. However, we do not have an accurate measurement of the radius of curvature of the end mirror, just the manufacturer's specification and tolerances. When characterising the cavity's stability, we cannot trust this specification. In order to carry out a precise measurement of the $g$-factor, we used the measured resonant frequencies of higher order modes, which can be related to the $g$-factor via the round-trip Gouy phase shift. In our case, we used the second order mode $\mathrm{HG}_{02}$, which is second in amplitude after the fundamental mode. The phase shift acquired by $\mathrm{HG}_{02}$ in a resonator round-trip is given by

$$
\Delta \phi_{02}=2 \zeta
$$

where we have substituted $n+m=2$ in Equation 2.43. In a plane-concave cavity, with $g_{1}=1$, the round-trip Gouy phase is given by

$$
\zeta=2 \arccos \left( \pm \sqrt{g_{1} g_{2}}\right)=2 \arccos \left(\sqrt{g_{2}}\right)
$$

or, alternatively,

$$
\zeta=2 \arctan \left(\frac{L}{z_{R}}\right),
$$

where $z_{R}$ is the Rayleigh range, and for a plane-concave cavity it is given by

$$
z_{R}=L \sqrt{\frac{g_{2}}{1-g_{2}}}
$$

Therefore, the offset in resonant frequency of the second order mode with respect to the fundamental mode is given by

$$
\Delta \nu_{02}=2 \Delta \nu_{\mathrm{FSR}} \frac{\arctan \left(\frac{L}{z_{R}}\right)}{\pi},
$$

which is something we can measure with as much precision as the free spectral range. From this equation we can derive equations for both $g_{2}$ and $L$ in terms of this observable:

$$
g_{2}=\frac{1}{2}\left[1+\cos \left(\frac{\Delta \nu_{02}}{\Delta \nu_{\mathrm{FSR}}} \pi\right)\right]
$$

and

$$
L=\mathcal{R}_{2}\left(1-g_{2}\right)=\frac{\mathcal{R}_{2}}{2}\left[1-\cos \left(\frac{\Delta \nu_{02}}{\Delta \nu_{\mathrm{FSR}}} \pi\right)\right] .
$$

Equation 9.10 can be used to characterise cavity stability given a mirror configuration. Equation 9.11 can be used following a number of measurements at different cavity lengths to obtain an accurate measurement of the radius of curvature of the concave mirror $\mathcal{R}_{2}$. Thus, the resonance frequency of $\mathrm{HG}_{02}$, together with the cavity's free spectral range, becomes an accurate probe of the overall geometrical stability of the cavity. As we will see in the next section, it turns out that as we approach instability, the degeneracy between the second order mode resonances is broken, and they appear at different frequencies. This indicates that the phase shift acquired by these higher order modes is dependent on the orientation of their spatial profile. 


\subsubsection{Alignment and frequency stabilisation systems}

The optical setup (Figure 9.5) consists of two commercial Nd:YAG lasers from Innolight: the "control laser" (Mephisto $300 \mathrm{NE}$ ), and the "probe laser" (Mephisto $1200 \mathrm{NE}$ ), both coupled to the NUC with orthogonal polarisations via the flat input mirror. Both lasers are $1064 \mathrm{~nm}$ tuneable over $30 \mathrm{GHz}$ with a narrow linewidth of $1 \mathrm{kHz}$ over $100 \mathrm{~ms}$. Before combination at the central beam splitter, each beam goes through its own set of polarising optics and mode matching telescopes (not shown) and an electro-optic modulator (Newport Corp. Model 4004) to generate rf sidebands for control. The control laser is locked to the cavity via a Pound-DreverHall (PDH) loop [54] using $15 \mathrm{MHz}$ sidebands. The probe laser, in turn, is locked to the control laser via an offset phase locking loop. The offset frequency between the probe laser and the control laser can then be tuned to scan the cavity.

After combination at the central beam splitter, both lasers have the same parameters but orthogonal polarisation. Both beams then pass through the same group of lenses to be identically mode matched to the cavity. The alignment of this lens group is critical, as any offset of the beams from the centre of the lenses would cause an error on the transverse position of the beam on the input mirror, dependent on the longitudinal positions of the lenses. When mode matching, we find that it is best to first carry out an initial approximation using the position and size of the beam waist, and then use the cavity itself for fine adjustment. A mode mismatch couples to the second order mode, so mode matching can be optimised by minimising the amplitude of $\mathrm{HG}_{02}$ as the cavity is being scanned. Unfortunately, the amplitude of $\mathrm{HG}_{02}$ depends non-linearly on the position of the lenses, so this fine adjustment is not always easy to perform. This method, however, is more precise than just using the beam parameters, as it is often difficult to measure accurately the size and position of the resulting beam waist.

In order to measure the offset in resonance frequency of higher order modes with respect to the fundamental mode with accuracy and precision, it is imperative that the control laser remains locked to the cavity, and both lasers remain well aligned. To facilitate alignment, an alignment sensing system based on quadrant photodiodes (QPD) is implemented, using the technique proposed in $[136,137]$. The probe laser is modulated at $1 \mathrm{MHz}$ for this purpose. For small misalignments, translation of the cavity axis or the input beam axis couples linearly to the first order mode. Tilt also couples to the first order mode, but with a phase shift of 90 degrees. The beam reflected by the cavity is sensed at the QPD's, which we set with a relative Gouy phase shift of 90 degrees. The QPD's make a differential measurement of the beam profile and produce a signal that, once demodulated, and for small misalignments, is proportional to the amplitude of the translation or tilt error. The signals could then be fed back to the system to provide automatic alignment. However, we choose to align the system manually, with the aid of these signals, which we find is already an easy and powerful alignment method.

Besides precise alignment of both lasers, the other fundamental requirement for precision measurement of the resonant structure of the cavity, is that the control laser's frequency remains well stabilised to the length of the cavity. For this purpose the control laser is modulated at $15 \mathrm{MHz}$. The beam reflected by the cavity is detected at a photodiode $\left(\mathrm{PD}_{\mathrm{R}}\right)$ and demodulated with a $7 \mathrm{dBm}$ mixer circuit (ZAD-1-1+ from MiniCircuits) to produce the PDH error signal which is then fed to 
a digital servo system produced in house. The servo's control signal passes through a high-voltage amplifier and then is fed to the laser's piezo drive. The servo also automates the initial frequency scan and lock acquisition.

The final component of the experiment is the probe laser, which we use to scan the cavity. In order to do this, this laser is phase-locked to the control laser after the latter is locked to the cavity. This is done in two steps: First, a fraction of the $\mathrm{PDH}$ signal from the control laser is fed to the piezo drive of the probe laser to greatly reduce common mode frequency noise. Subsequently, we engage an offset phase locking loop between the two lasers: Both lasers are made to interfere at a

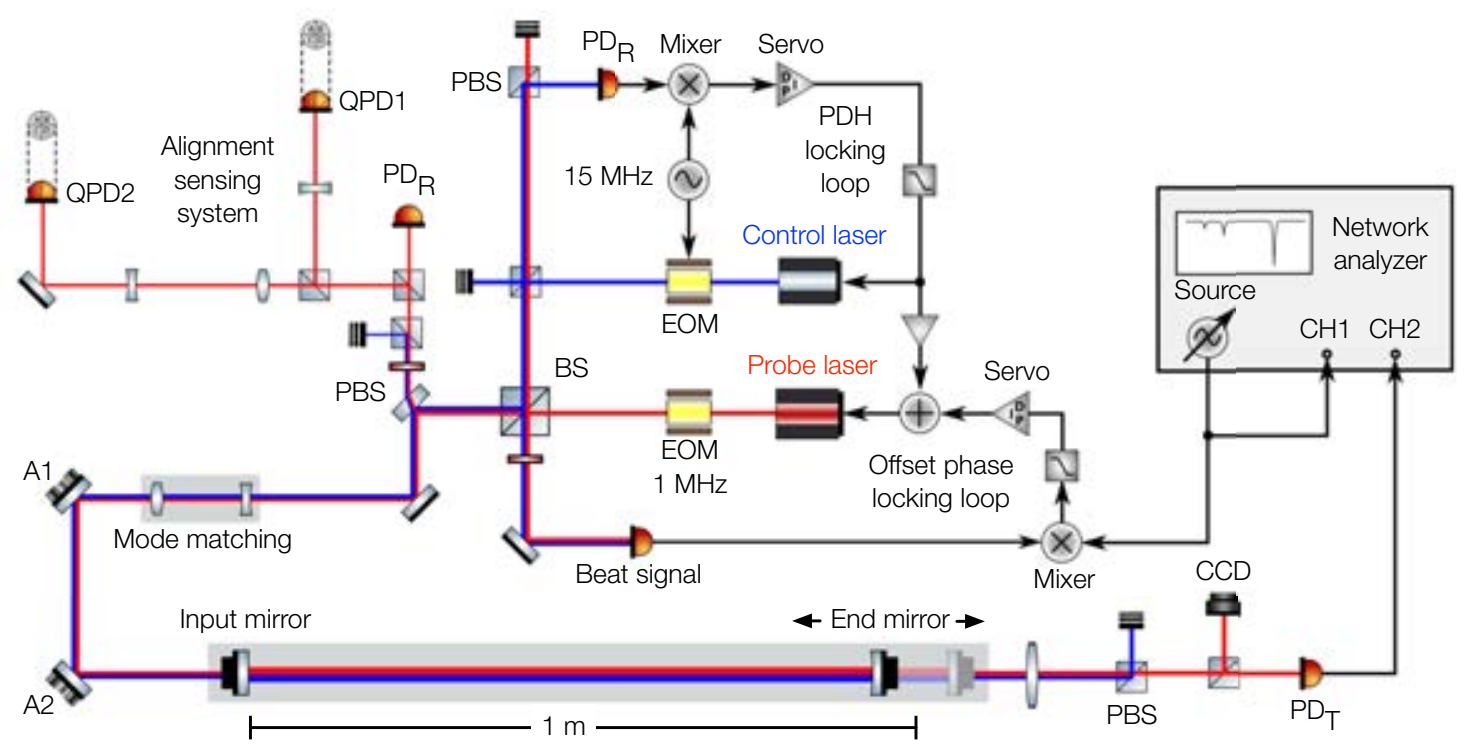

Figure 9.5: Schematic of the near-unstable cavity setup. The blue and red lines represent the beams from the $1064 \mathrm{~nm}$ control and probe lasers respectively. They pass through phase modulators (EOM) that generate the sidebands used for control purposes. The beams are combined at the central beam splitter (BS) and then coupled into the cavity through two mode matching lenses. The beams have orthogonal polarisations upon combination to ensure that they do not interfere. The control laser is locked to the cavity via a Pound-Drever-Hall loop (PDH). A fraction of the PDH signal is fed back to the probe laser to reduce common mode frequency noise. The probe laser is then locked to the control laser with a tuneable frequency offset via an offset phase locking loop. The frequency offset is controlled using the source of a network analyser. The probe laser is then used to study the cavity. A camera $(\mathrm{CCD})$ and a photodetector $\left(\mathrm{PD}_{\mathrm{T}}\right)$ are placed at the cavity's transmission port to observe the output. The subscripts $\mathrm{R}$ and $\mathrm{T}$ signal the cavity's reflection and transmission ports respectively. An alignment system based on wavefront sensing is used to help maintain accurate alignment of the cavity. 


\begin{tabular}{ccccc}
\hline & \multicolumn{4}{c}{ Cavity length $(\mathrm{m})$} \\
& 0.956 & 0.993 & 0.999 & 0.9999 \\
\hline Beam waist $(\mu \mathrm{m})$ & 263.56 & 168.04 & 103.46 & 58.19 \\
Beam spot at EM $(\mathrm{mm})$ & 1.26 & 2.01 & 3.27 & 5.82 \\
Rayleigh range $(\mathrm{mm})$ & 205.10 & 83.37 & 31.61 & 10.00 \\
Divergent angle $(\mathrm{mrad})$ & 1.29 & 2.02 & 3.27 & 5.82 \\
FSR $(\mathrm{MHz})$ & 156.80 & 150.95 & 150.05 & 149.91 \\
$g$ & 0.044 & 0.007 & 0.001 & 0.0001 \\
$g^{*}$ & 0.832 & 0.972 & 0.996 & 0.9996 \\
$\mathrm{HG}_{01}-\mathrm{HG}_{00}$ offset $\left(\times \Delta \nu_{\mathrm{FSR}}\right)$ & 0.433 & 0.474 & 0.490 & 0.497 \\
$\mathrm{HG}_{02}-\mathrm{HG}_{00}$ offset $\left(\times \Delta \nu_{\mathrm{FSR}}\right)$ & 0.135 & 0.053 & 0.020 & 0.006 \\
$\delta_{02}$ & 281.3 & 112.0 & 42.2 & 13.3 \\
\hline
\end{tabular}

Table 9.2: Parameters of the tabletop cavity at four different values of the cavity length. The cavity $g$-factor is calculated assuming an ROC of $1 \mathrm{~m}$ of the curved mirror, as specified by the manufacturer. The value $g^{*}$ corresponds to the $g$-factor of the near-concentric cavity that has the same eigenmodes but twice the length.

photodetector to produce an rf beat signal of frequency equal to their frequency difference. This beat signal is then combined with a local oscillator signal at a mixer to produce an error signal proportional to the difference between the frequency offset and the reference signal. This signal is then used to actuate on the probe laser and maintain the offset frequency at a target value. By adjusting the frequency of the local oscillator, which can be tuned over several free spectral ranges of the cavity, we can scan the cavity while maintaining its length stability relative to both lasers.

\subsection{Pushing the cavity over the edge}

\subsubsection{Influence of mirror imperfections}

We scan the cavity as we push it to the edge of geometrical stability. With the help of a polarising beam splitter and a CCD sensor, we observe the transmitted probe laser beam, which displays the resonance structure of the cavity (i.e., its HermiteGauss eigenmodes $\mathrm{HG}_{n m}$ ). We set the cavity initially in a stable configuration, and it becomes immediately apparent that the second order modes, as well as the fourth order modes, resonate at different frequencies. Usually we would expect that resonances of the same order appear degenerate in frequency, since ideally they should experience the same Gouy phase shift in a resonator round-trip. However, we observe a structure like the one depicted in Figure 9.6. This observation is consistent with the effect that an imperfect mirror surface can have on the cavity's eigenmodes. If the deformation is such that the modes experience a slightly different effective radius of curvature (or, equivalently, a slightly different cavity length) depending on the orientation of their spatial profile relative to the mirror surface, then they would acquire different Gouy phase shifts and resonate at slightly different frequencies. 


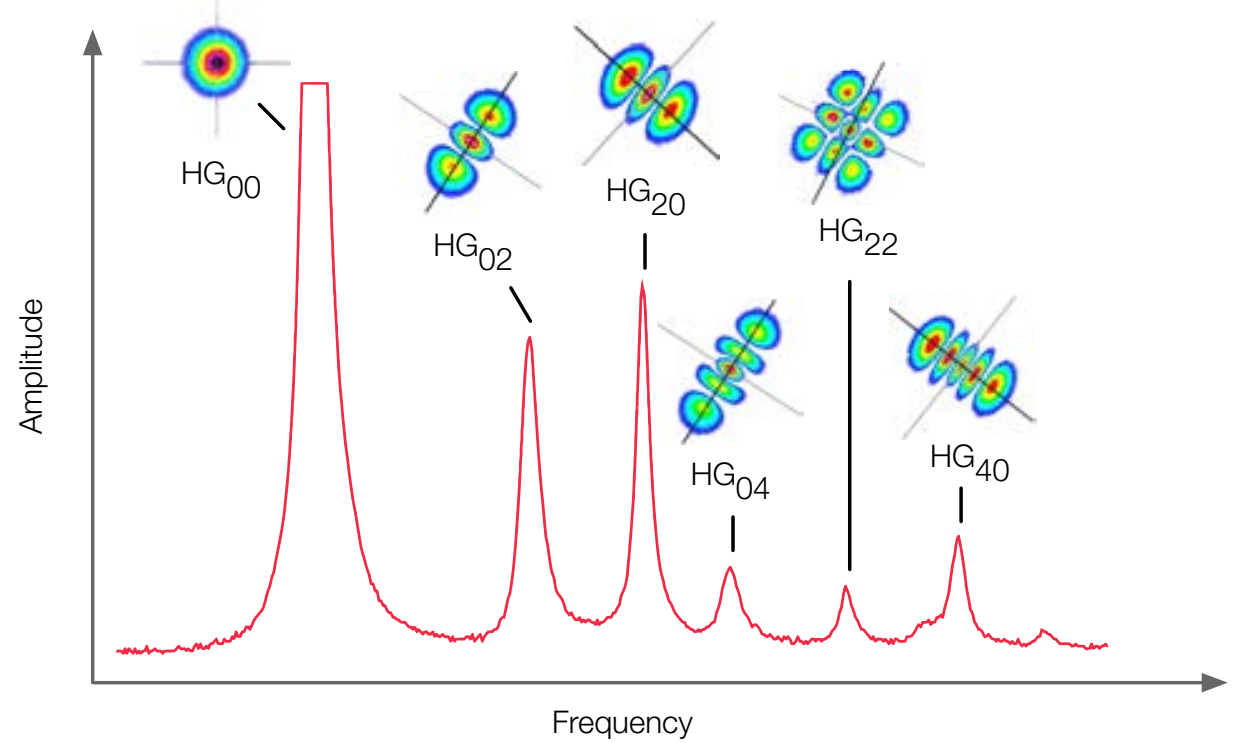

Figure 9.6: A cavity scan measurement showing the resonant frequencies of the fundamental mode, and the second and fourth order spatial modes. The resonances of modes of the same order but orthogonal spatial profile appear separate due to the imperfect surface of the spherical mirror. The separation can be reduced by increasing the stress of the screw holding the spherical mirror, thus compensating the surface deformation.

Notice that the eigenmode shapes captured by the CCD (Figure 9.6) appear with their symmetry axis oriented at 45 degrees with the vertical. We found that the offset between eigenmodes of the same order could be compensated by tightening the screw holding the curved mirror, which was also at 45 degrees with the vertical. That is, the resonance peaks that appeared separate due to the imperfect mirror surface could be made to overlap by applying structural stress to the mirror in a way that compensated for its default deformation. This compensation affected the shapes of the second and fourth order modes, making them resemble circularly symmetric Laguerre-Gauss modes. The sensitivity of the cavity's modal structure to mirror surface imperfections is usually negligible for cavities lying well within geometrical stability, but becomes significant in NUC's. Before continuing with our measurements, we rotate the end mirror by about 45 degrees to attempt to align the eigenmodes' symmetry axes with the transverse axes.

\subsubsection{Characterising cavity stability}

To characterise the stability of the cavity, we carry out precision measurements of $\Delta \nu_{02}$ and $\Delta \nu_{\mathrm{FSR}}$, as explained in Section 9.3.2, with the exception that we will measure $\Delta \nu_{20}$ as well, which is slightly different from $\Delta \nu_{02}$. We do so as we adjust the position of the end mirror, which is mounted on a Newport Corporation M-460A 
translation stage with a differential micrometer (DM-13), enabling position control of the mirror with a resolution of $0.5 \mu \mathrm{m}$.

We set the position of the end mirror manually to roughly $999 \mathrm{~mm}$ from the input mirror. From this starting position, we adjust the translation stage 18 times to increase and decrease the cavity length (i.e., pushing the cavity towards and away from geometrical instability). Each time the cavity length changes we carry out the following procedure: (1) rough mode matching using the beam waist size and position, (2) fine mode matching using the second order mode content of the reflected beam ${ }^{1}$, (3) fine alignment using the QPD's, and (4) PDH lock of the control laser. The cavity is then scanned using the offset phase locked probe laser with a swept sinusoidal signal as the tuneable oscillator. Lastly, the transmitted light of the probe laser is captured at the CCD and at the photodetector $\mathrm{PD}_{\mathrm{T}}$, revealing the resonance structure of the cavity. Each scan consists of 800 points in a $2.5 \mathrm{MHz}$ range, resulting in $3.125 \mathrm{kHz}$ resolution. The cavity length is adjusted from its initial position, $L_{0}$, from $L_{1}=L_{0}-1200.0 \mu \mathrm{m}$ to $L_{18}=L_{0}+1400.0 \mu \mathrm{m}$.

The amplitude of the first order mode can be minimised to a large extent at cavity lengths $L_{1}$ to $L_{7}$ during mode matching and alignment, but after $L_{7}$ we find that there is always some residual amplitude in $\mathrm{HG}_{01}$ that we cannot get rid of, and it increases with cavity length from $L_{7}$ to $L_{18}$. This is thought to be due to the large beam ( $\sim 5-10 \mathrm{~mm}$ radius) suffering clipping loss at the end mirror, which is a 1 inch diameter optic. As a result, the reflected beam is no longer axially symmetric, and when sensed at the QPD's the difference signal between the two halves of the beam can no longer be zero. Precise alignment is still possible by trying to reduce the amplitude of $\mathrm{HG}_{01}$ as much as possible.

\subsubsection{Results and simple astigmatic model}

The resonance of the fundamental mode $\mathrm{HG}_{00}$ is used to measure $\Delta \nu_{\mathrm{FSR}}$ and calibrate the translation stage. A linear fit of all 18 data points $\left(L_{1}\right.$ to $\left.L_{18}\right)$ yields the true value of the starting cavity length $L_{0}=1000166.8 \pm 7.7 \mu \mathrm{m}$, which is just over $1 \mathrm{~mm}$ away from the result of the measurement made using a ruler. Subsequently, the offset frequency between $\mathrm{HG}_{00}$ and $\mathrm{HG}_{02,20}$ is used to measure $\Delta \nu_{02,20}$. This offset is reduced as the cavity length increases, as is expected due to the cavity's Gouy phase shift approaching $\pi$. However, after a certain point, the modes start to deviate significantly from this expected behaviour, with the offset frequencies increasing with cavity length. The images of the spatial profiles of the modes also show substantial deviation from the expected shapes (Figure 9.7).

We perform fits of the measured cavity length to the measured second order mode resonance frequencies, according to a simple astigmatic mirror model based

\footnotetext{
${ }^{1}$ Fine mode matching is not always possible. At large part of the cavity length range explored, the amplitudes of $\mathrm{HG}_{02}$ and $\mathrm{HG}_{20}$ could not be simultaneously reduced. However, since we are not interested in the amplitudes of the modes but only on their resonance frequencies, mode matching does not affect the measurement, and the mode matching lenses are left at the optimum position for the initial cavity length.
} 
on Equation 9.11:

$$
\begin{aligned}
& L_{0}+\Delta L=\frac{\mathcal{R}_{2+}}{2}\left[1-\cos \left(\frac{\Delta \nu_{20}}{\Delta \nu_{\mathrm{FSR}}} \pi\right)\right], \\
& L_{0}+\Delta L=\frac{\mathcal{R}_{2-}}{2}\left[1-\cos \left(\frac{\Delta \nu_{02}}{\Delta \nu_{\mathrm{FSR}}} \pi\right)\right]
\end{aligned}
$$

where $\Delta L$ is the translation stage reading with respect to the initial position $L_{0}$. The model assumes that the extra relative Gouy phase shift between eigenmodes $\mathrm{HG}_{02}$ and $\mathrm{HG}_{20}$ is due to an astigmatic end mirror that provides slightly different surface curvature on its transverse axes. When the light in the cavity interacts with this imperfect mirror, each eigenmode probes a different section of the mirror surface, and hence experiences a slightly different curvature and phase shift. The model's assumptions are not unreasonable, as most of the Gouy phase shift in the cavity is due to the reflection at this mirror (the flat mirror's contribution is negligible). By fitting this model to the data, we obtain the results presented in Figure 9.8 and Table 9.3. With the obtained values of $\mathcal{R}_{2+}$ and $\mathcal{R}_{2-}$, we can characterise the overall stability of the cavity by defining a $g$-factor with the average mirror curvature,

$$
\bar{g} \equiv 1-\frac{2 L}{\mathcal{R}_{2+}+\mathcal{R}_{2-}} .
$$

We find good agreement between the measurements and the mode for cavity lengths up to a certain threshold, after which the measurements start deviating significantly from prediction. The two second order mode resonances $\mathrm{HG}_{20}$ and $\mathrm{HG}_{02}$ start deviating by more than $1 \%$ at $L_{7}$ and $L_{10}$ respectively, at which point their spatial profiles also begin showing distortions. From $L_{7}$ to $L_{13}$ the spatial profile of $\mathrm{HG}_{02}$ can be seen to gradually rotate clockwise. At $L_{13}$ the second order mode resonance should have overlapped completely with the fundamental mode, as the cavity would be almost exactly hemispherical at this point. The resonance of $\mathrm{HG}_{20}$ follows the model for a few additional length increments, and starts deviating at $L_{10}$, at which point its spatial profile suffers clockwise rotation as well.

At some point we appear to be tracking resonances which no longer seem to belong to pure Hermite-Gauss modes. The fundamental mode also suffers significant distortions after $L_{13}$, going from the Gaussian shape to a two-spot shape. We believe this is due to the cavity becoming essentially an amplifier for beam distortions due

\begin{tabular}{ccc}
\hline Parameter & Value $(\mu \mathrm{m})$ & Error $(\mu \mathrm{m})$ \\
\hline$L_{0}$ & 1000166.8 & 7.7 \\
$\mathcal{R}_{2+}$ & 1001284.9 & 4.6 \\
$\mathcal{R}_{2-}$ & 1001140.0 & 15.7 \\
\hline
\end{tabular}

Table 9.3: Results of fitting the simple astigmatic model (Equation 9.12) to the data. $L_{0}$ is obtained from a fit using all 18 data points. $\mathcal{R}_{2+}$ and $\mathcal{R}_{2-}$ are obtained from fits using the first 12 and 9 data points respectively, before the eigenmodes's behaviour starts to deviate. 


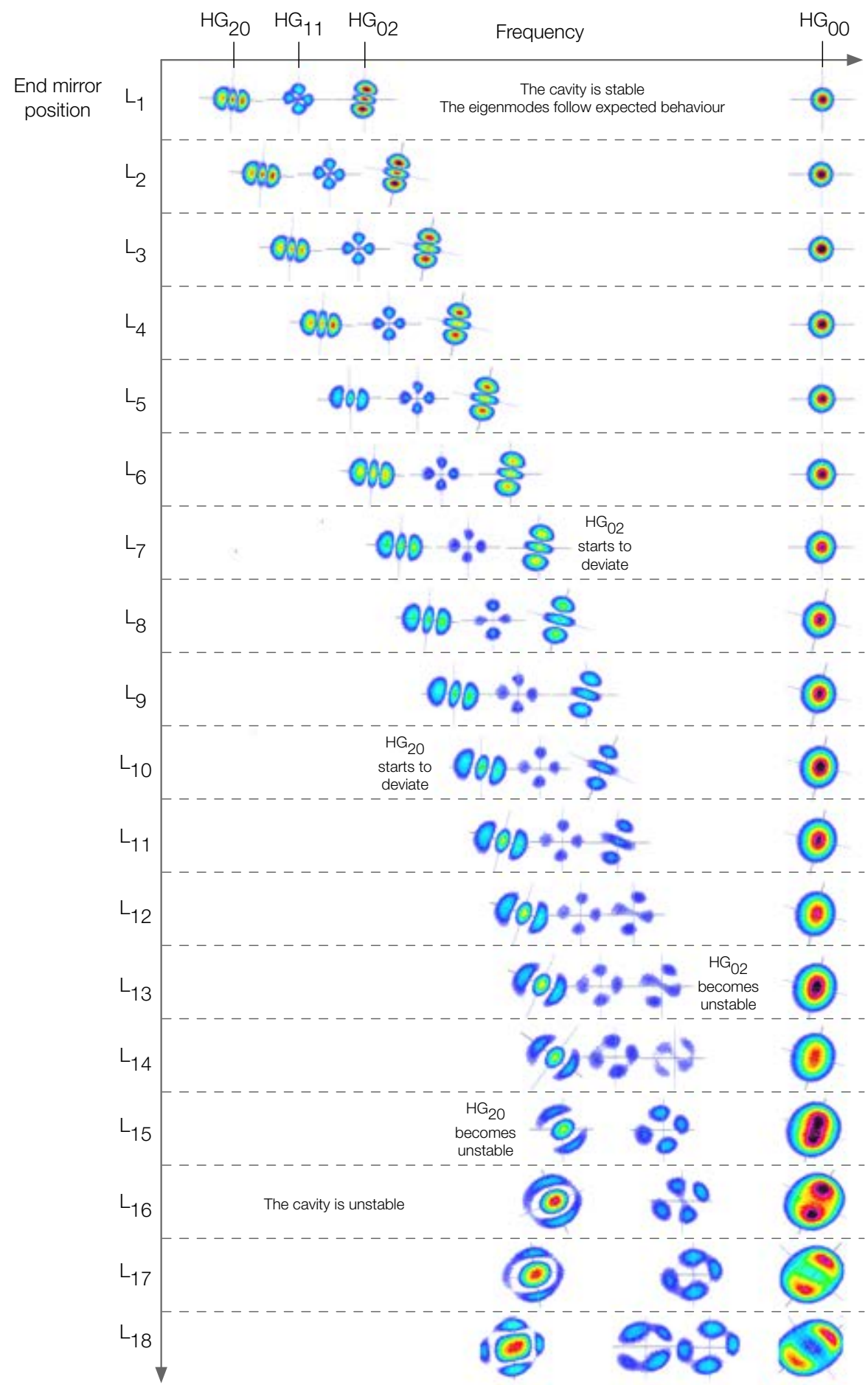

Figure 9.7: Continued on the following page. 
Figure 9.7: Spatial profile of the transmitted beam as a function of cavity length. The horizontal position of the image indicates the measured resonance frequency. The two second order spatial modes $\mathrm{HG}_{02}$ and $\mathrm{HG}_{20}$ resonate at different frequency due to a slightly astigmatic end mirror. Both the resonance structure of the cavity and the spatial profile of the beam suffer significant distortion in the near-unstable region, starting at $L_{7}$ and culminating at $L_{15}$ where the cavity is approximately hemispherical. The PDH error signal is still reasonably clear and the control laser can still be locked until the cavity is pushed $500 \mu \mathrm{m}$ beyond $L_{15}$.

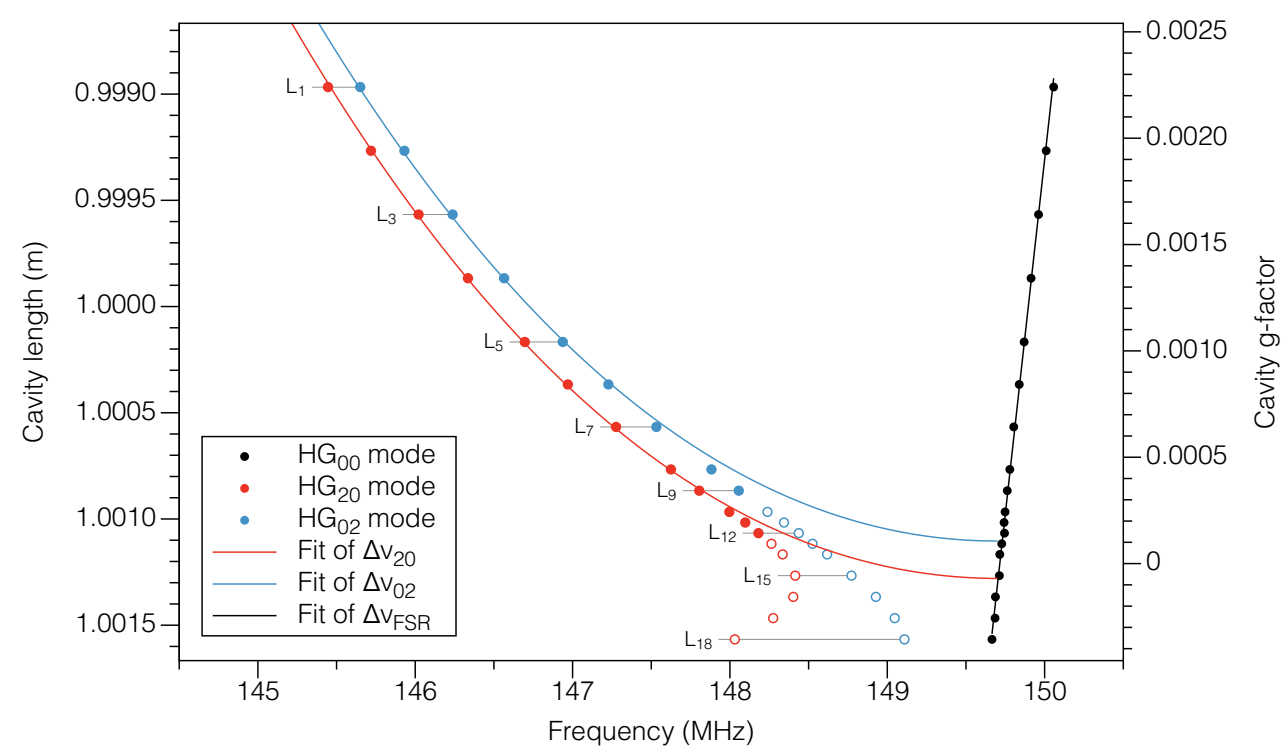

Figure 9.8: Fits of the measured resonance frequencies of the two second order mode resonances $\mathrm{HG}_{02}$ and $\mathrm{HG}_{20}$ to a simple model accounting for a slightly astigmatic end mirror. The fits yield two values for the radius of curvature of the end mirror, differing by about $145 \mu \mathrm{m}$, as probed by the two orthogonal modes. From the fits we derive an average cavity $g$-factor, which predicts that the cavity is hemispherical at approximately $L_{15}$. Also shown is a fit of the fundamental mode resonance $\mathrm{HG}_{00}$ which is used to calibrate the translation stage and provide a measurement of the cavity's free spectral range. The second order mode resonance frequencies start deviating from the model in the near-unstable region $(g<0.001)$. 
to it resonantly enhancing even higher order modes just as much as the fundamental mode. From the average $g$-factor we can tell that the cavity is approximately hemispherical at $L_{15}$, which is consistent with our observation of high optical loss. In the $700 \mu \mathrm{m}$ region from $L_{7}$ to $L_{15}$ the spatial profiles of the eigenmodes undergo a series of changes, with small deviations in both spatial structure and resonance frequency. Beyond about $L_{12}$ the modes no longer resemble pure Gaussian modes. This is coincident with high optical loss. We found, however, that in this nearunstable region, a reasonably clear PDH error signal could still be obtained, and the control laser could still be stabilised to the cavity without noticeable issues. The PDH error signal finally starts to degenerate after the cavity is pushed $500 \mu \mathrm{m}$ further beyond $L_{15}$.

\subsection{Summary and future work}

We have constructed an optical cavity which we can use to probe an as-yet untested extreme near-unstable region, and demonstrated experimentally that the cavity eigenmode behaviour in such a cavity departs significantly from the model based on ideal mirrors and input beams. This involves a $1 \mathrm{~m}$ cavity which is pushed to the edge of stability by incrementally increasing its length. We have measured a series of frequencies and shapes of resonant transverse modes, including the fundamental mode, and the separated $\mathrm{HG}_{02}$ and $\mathrm{HG}_{20}$ modes. The measured resonant frequencies are compared with our fitting results, and the stability of the cavity is quantified.

Immediately upon setting up the experiment, scanning the cavity for the first time, and capturing the transmitted beam at the CCD sensor, we observed that the second order modes $\mathrm{HG}_{02}$ and $\mathrm{HG}_{20}$ resonated at different frequencies, indicating that these modes acquire different phases in a resonator round-trip. We believe this is attributed to the interaction of the circulating beam with an imperfect end mirror, which is effectively astigmatic, and hence the two orthogonal modes "see" a different radius of curvature. By fitting the measurements of the resonance frequencies of these modes and the fundamental mode to a simple model accounting for the different radii of curvature depending on the mode which is probing it, we have derived an ROC difference of $\approx 145 \mu \mathrm{m}$, indicating that the mirror is slightly more curved in the vertical direction. As a result, $\mathrm{HG}_{02}$ becomes co-resonant with the fundamental mode prior to $\mathrm{HG}_{20}$ as we increase the cavity length and the cavity becomes hemispherical.

We found that the two modes started deviating from the model at slightly different values of the cavity length, but overall the breakdown point occurred at a $g$-factor of $\bar{g} \approx 3 \cdot 10^{-4}$, corresponding to a second order mode separation factor $\delta_{02} \approx 22$. This is a relatively large separation factor: it means that the second order mode resonance is 22 linewidths away from the closest fundamental mode resonance. Therefore we believe that the root cause of the issues observed as the cavity becomes unstable is not the overlapping of modes, but rather the result of the interaction of the beam with the distorted mirror surface. This is, in our opinion, the key factor that will ultimately limit the feasibility of this type of cavity in future gravitational-wave detectors. Thermal control of the mirror could provide some compensation for these effects, e.g., to correct an overall error of the ROC. 
However, it is the small imperfections, such as a slight astigmatism, that ultimately become more important. Near-unstable cavities are very sensitive to such small imperfections.

It is found that for $g$-factors down to about $3 \cdot 10^{-4}$, the cavity eigenmodes' behaviour is close to that predicted by an idealised model of a perfectly aligned cavity, with resonance spacing between the higher order modes and the fundamental mode decreasing as predicted. For smaller $g$-factors, new behaviour is observed. The resonance gap between the fundamental mode and the closest higher order modes begins to grow again, while the profile of these modes deviates from Gaussian modes such as Hermite-Gauss or Laguerre-Gauss modes. This latter effect can be understood as being due to the eigenmodes becoming much more sensitive to mirror surface distortions, which strongly breaks the cylindrical symmetry normally exhibited by the cavity. Additional modelling and simulation is required to fully understand these observations, and to determine the requirements in mirror flatness and in the angular control systems for a given $g$-factor to be feasible in a gravitational-wave detector cavity.

The work carried out in this paper motivates further experimental activity, for instance using mirrors with known significant figure errors or with thermally or otherwise deformable mirrors. It also motivates simulation work, which could eventually be used to help design and understand NUCs for gravitational-wave detector cavities. The FINESSE code [121], which is used to model cavities and related optical systems in the frequency domain, represents an ideal tool with which to carry out this program of investigation, and this work is underway within the group. 



\section{Modelling parametric instabilities at Advanced LIGO and ET}

\section{Contents}

10.1 Parametric instabilities . . . . . . . . . . . . 206

10.1.1 Optomechanical interaction of light and mirrors . . . . 206

10.1.2 Parametric instability in a Fabry-Perot cavity . . . . . 207

10.1.3 Observation of parametric instabilities and mitigation strategies . . . . . . . . . . . . . . . . . . . . . . . . . . . . . . . . .

10.2 Finite element modelling . . . . . . . . . . . . . 210

10.2.1 Introduction to eigenmode analysis using the finite element method . . . . . . . . . . . . . . 2 210

10.2.2 Accuracy of the model . . . . . . . . . . . . . . 212

10.3 Eigenmode analysis of Advanced LIGO and ET test masses . . 218

10.3.1 Advanced LIGO test masses . . . . . . . . . . . . . . . . . . . . . . 218

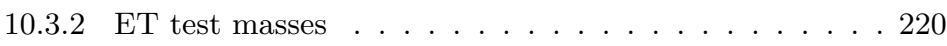

10.4 Summary and future work $\ldots \ldots \ldots \ldots \ldots \ldots$ 


\subsection{Parametric instabilities}

\subsubsection{Optomechanical interaction of light and mirrors}

DARAmetric instabilities in Fabry-Perot cavities were first introduced in 2001 1 as a potential pitfall and serious problem for laser interferometer gravitationalwave detectors $[138,139]$. Parametric instabilities emerge from the optomechanical interaction between the high power optical field resonating in the cavity and the cavity mirrors which act as test masses. In Advanced LIGO each test mass is a $\sim 40 \mathrm{~kg}$ fused silica cylinder, and at design levels they are subject to $\sim 800 \mathrm{~kW}$ of optical power. Under some conditions the optical field will yield energy to the test mass in a way that excites a particular mechanical resonance. If the amplitude of such excitation grows exponentially in time, it is referred to as an oscillatory parametric instability (PI). These perturbations are of purely dynamical nonlinear origin. If not addressed, they can saturate the interferometer control systems and displace the instrument away from a suitable operating point.

The optomechanical interaction can be explained in terms of coupling between optical and mechanical waves or quanta (Figure 10.1). A quantum $\hbar \omega_{0}$ from the optical field will be scattered by a quantum $\hbar \omega_{m}$ belonging to a mechanical resonance of the test mass, and give rise to a so-called Stokes sideband $\hbar \omega_{s}$ (Stokes process). In this process the optical field yields energy to the mechanical wave (energy conservation yields $\left.\hbar \omega_{0}=\hbar \omega_{s}+\hbar \omega_{m}=\hbar\left(\omega_{0}-\omega_{m}\right)+\hbar \omega_{m}\right)$. In other words, the circulating $\mathrm{HG}_{00}$ field in the cavity can scatter inelastically from the acoustic motion of the test mass, dividing its energy between the test mass and a lower frequency transverse mode of the optical field. The inverse process, in which the optical field absorbs energy from the mechanical wave, will also take

(a) Stokes mode

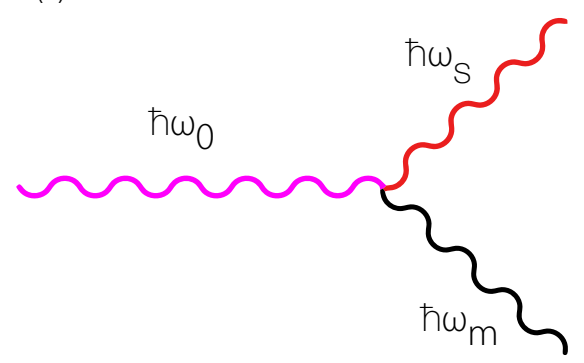

(b) Anti-Stokes mode

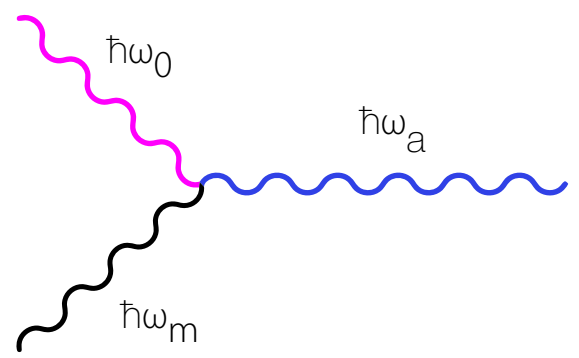

Figure 10.1: Quantum picture of the optomechanical interaction between the cavity field and the test mass. The optical field can yield energy to the test mass by scattering a phonon belonging to a particular mechanical resonance (a). It is also possible for the optical field to absorb energy from the test mass in the inverse process (b). 
place (anti-Stokes process). The parametric gain of the mechanical mode is defined as $[140]$

$$
R_{m} \equiv-\frac{\gamma_{\mathrm{opt}}}{\gamma_{m}}
$$

where $\gamma_{\text {opt }}$ is the optical-to-mechanical damping rate, and $\gamma_{m}$ is the mechanical damping rate. If the Stokes process takes preference over the anti-Stokes process, $\gamma_{\text {opt }}>\gamma_{m}$, and therefore $R>1$, the amplitude of the mechanical wave grows without bounds and the system is unstable (PI). If these perturbations are not mitigated in some way, they will limit the detector sensitivity by limiting the maximum circulating power.

\subsubsection{Parametric instability in a Fabry-Perot cavity}

The parametric gain of a mechanical mode $m$ interacting with a single optical mode $n$ of a Fabry-Perot cavity is given by [141]

$$
R_{m, n}=\frac{4 P_{c}}{M c L} \frac{Q_{m} Q_{n}}{\omega_{m}^{2}} \Lambda_{\omega} \Lambda_{s}
$$

where $P_{c}$ is the power circulating in the cavity of length $L$ and mirrors of mass $M$, $Q_{m}$ is the mirror material's mechanical quality factor associated with that particular resonance, $Q_{n}=\omega_{n} / 2 \gamma_{n}$ is the optical $Q$-factor associated with the optical mode of order $n$, frequency $\omega_{n}$, and halfwidth $\gamma_{n}$, and $\Lambda_{\omega}$ and $\Lambda_{s}$ are dimensionless factors describing the frequency and the spatial overlap between the mechanical mode and the optical mode.

The frequency overlap factor is given by

$$
\Lambda_{\omega}=\frac{1}{1+\frac{\Delta \omega}{\gamma_{n}}}
$$

where $\Delta \omega=\omega_{0}-\omega_{n}-\omega_{m}$ is the detuning, and $\gamma_{n}=\pi c / 2 L \mathcal{F}_{n}$ is the halfwidth of the $n$-th order optical mode with finesse $\mathcal{F}_{n}$. In general the finesse $\mathcal{F}_{n}$ of a higher order mode will be different from the cavity finesse associated with the fundamental mode due to the clipping loss at the finite mirror surfaces, which depends on the spatial distribution of the mode.

The spatial overlap factor for a mirror with its surface at $z=0$ in the optical axis $\boldsymbol{e}_{z}$ is given by

$$
\Lambda_{s}=\frac{V\left|\iint u_{0} u_{n} w_{m} d x d y\right|^{2}}{\iint\left|u_{0}\right|^{2} d x d y \iint\left|u_{n}\right|^{2} d x d y \iiint\left|\left(u_{m}, v_{m}, w_{m}\right)\right|^{2} d x d y d z}
$$

where $V$ is the volume of the test mass, $u_{0}=u_{0}(x, y, z=0)$ and $u_{n}=u_{n}(x, y, z=0)$ are the spatial profiles of the fundamental and the $n$-th order optical modes at the mirror surface, and $u_{m}=u_{m}(x, y, z), v_{m}=v_{m}(x, y, z)$ and $w_{m}=w_{m}(x, y, z)$ are the $x, y$ and $z$ components respectively of the displacement field of the mirror vibrating in the $m$-th mechanical resonance. The surface and volume integrals are over the entirety of the test mass' surface and volume respectively. 
The total parametric gain of each mechanical mode is obtained by adding the contributions of all the optical modes. Hence, the condition of parametric instability for the $m$-th mechanical resonance is given by

$$
\sum_{n=0}^{+\infty} R_{m, n}>1 .
$$

\subsubsection{Observation of parametric instabilities and mitigation strategies}

The observation of parametric instabilities at Advanced LIGO was first reported in 2015 [142]. The $15538 \mathrm{~Hz}$ mechanical resonance of one of the test masses was observed ringing up and saturating the primary output electronics at the LIGO Livingston Observatory. At the time of the observation the circulating power in the arms was $\sim 50 \mathrm{~kW}$. The growth time of the excitation was long enough (e-fold time constant of 240 seconds) that operators were able to lower the power to $16 \mathrm{~kW}$ and observe the excitation ringing down. The threshold power (i.e., the circulating arm power to yield a parametric gain of unity for that mechanical mode) was estimated to be $\sim 25 \mathrm{~kW}$. Parametric instabilities have since become a regular occurrence at both LIGO sites, and as the detectors shift towards higher power they are expected to become more common and disruptive.

Parametric instabilities depend on a number of parameters. As such, the parameter space has been explored and some mitigation strategies have been proposed for reducing the impact of PI's on the detectors:

- Optical power: A trivial solution is using lower power, as the total parametric gain of each mechanical resonance $R_{m}$ scales directly with the total circulating power in the cavity $R_{m} \propto P_{c}$. Of course, this solution comes at the price of decreased detector sensitivity due to increased shot noise.

- Optical wavelength: The parametric gain scales with the $Q$-factor of the optical modes $Q_{n}$, which in turn scale inversely with the optical wavelength $\lambda$. Using a larger wavelength (e.g., $1550 \mathrm{~nm}$ as planned for LIGO Voyager) would in principle help mitigate parametric instabilities. However, the increase in wavelength would also incur an increase in shot noise, which could only be averted by the same increase in optical power.

- Cavity length and mirror ROC: The parametric gain seems at first only weakly dependent on the cavity length $L$, as $R_{m} \propto Q_{n} \Lambda_{\omega} / L$ with $Q_{n} \propto L$ and $\Lambda_{\omega} \propto 1 /(1+L)$, so that $R_{m} \propto 1 /(1+L)$. However, a change of the cavity length — or similarly a change of the radii of curvature (ROC) of the mirrors - would induce changes to the modal structure of the optical field in the cavity, the overall geometrical stability of the cavity, and the size of the beam. Hence, it is not at all trivial how these changes could affect the total parametric gains of mechanical modes. Some studies have shown that thermal tuning of the mirror ROC can help mitigate parametric instabilities [143, 144]. 
- Higher order mode finesse: We have briefly introduced the finesse of a higher order mode $\mathcal{F}_{n}$, and seen that $R_{m} \propto \mathcal{F}_{n}$. Decreasing $\mathcal{F}_{n}$ by increasing the loss of higher order modes is an effective way of reducing the impact of parametric instabilities. This idea is currently being explored in our group, and a possible mitigation strategy is using an extraction cavity to effectively increase the bandwidth of higher order modes [145].

- Spatial overlap: The parametric gain is directly proportional to the spatial overlap factor, which measures the amount of overlap between the transverse profile of the optical mode at the mirror surface and the vibrating test mass deformation field in the direction of the optical axis. Some work has been made into analysing the possibility of using beams with alternative shapes, such as Laguerre-Gauss modes [146] and even non-Gaussian beams [147].

- Test mass dimensions: The parametric gain is inversely proportional to the mass $M$ of the test mass. The mass can be altered by modifying the test mass dimensions, and hence the total test mass volume. However, increasing the volume of the test mass will yield a larger number of mechanical modes in the frequency range of interest. In addition, depending on the test mass' radius to thickness ratio $r / h$ the mechanical resonances can shift up or down in frequency. In general the test mass structure - which will deviate from a perfect cylinder - needs to be analysed, and its vibrational modes calculated, by means of a numerical model. The test mass' flats and suspension ears break the axial symmetry of the cylinder and introduce extra modes with azimuth displacement components. The density of mechanical modes increases with frequency, and along with it so does the probability of the onset of PI's.

- Test mass material: The magnitude of the test mass' resonant frequencies scales with the speed $c_{t}$ of the transverse elastic waves in the material, which in turn scales with the material's mechanical parameters (i.e., its Young's modulus $Y$, Poisson's ratio $\sigma$, and density $\rho$ ). To add to the complexity of the problem, these parameters will also have a slight dependency with the overall temperature of the test mass. Alternative materials to fused silica, such as sapphire, have been proposed $[143,144]$. The parametric gain is also directly proportional to the mechanical quality factor $Q_{m}$, and both passive and active damping strategies have been proposed in order to significantly reduce it [147-149]. The dependence of fused silica's Young's modulus with temperature has been used to use mechanical resonances as accurate test mass thermometers [150].

Parametric instabilities are also expected to impact next generation detectors, such as the Einstein Telescope (ET) [151,152], which is currently in design phase [46]. In order to achieve an order of magnitude greater sensitivity than previous generation detectors, ET will employ $10 \mathrm{~km}$ Fabry-Perot arm cavities, much larger and heavier $200 \mathrm{~kg}$ test masses, and up to $3 \mathrm{MW}$ of circulating power. As a result of these technology improvements, ET is expected to experience more parametric instabilities than Advanced LIGO. The detector will also operate at cryogenic temperatures $(\sim 10 \mathrm{~K})$ in order to reduce various sources of thermal noise of the test masses and 


\begin{tabular}{cccc}
\hline Mesh size & $\begin{array}{c}\text { Number of } \\
\text { elements }\end{array}$ & $\begin{array}{c}\text { Degrees of } \\
\text { freedom }\end{array}$ & $\begin{array}{c}\text { Solution } \\
\text { time (s) }\end{array}$ \\
\hline Extremely Coarse & 97 & 606 & 1 \\
Extra Coarse & 311 & 1752 & 2 \\
Coarser & 1067 & 5166 & 4 \\
Coarse & 2300 & 10767 & 9 \\
Normal & 7377 & 32358 & 29 \\
Fine & 14972 & 64419 & 75 \\
Finer & 46636 & 195123 & 100 \\
Extra Fine & 185328 & 763053 & $\dagger$ \\
Extremely Fine & 1003425 & 4075812 & $\ddagger$ \\
\hline
\end{tabular}

Table 10.1: Simulation statistics of a finite element model of a 3D cylinder with the same dimensions and material parameters as an Advanced LIGO test mass. Results are given for tetrahedral meshes of the 9 standardised mesh sizes available in COMSOL. The solution time corresponds to a simulation job that finds the 3 mechanical modes closest to $10 \mathrm{kHz}$ with $\omega_{m}<2 \pi \cdot 10 \mathrm{kHz}$. Machine used: Intel Core i7-7700 at $2.80 \mathrm{GHz}$ (turbo boost to $3.4 \mathrm{GHz}$ ) quad-core processor with 16 GB of physical memory and 100 GB of virtual memory. $\dagger$ The solution time is not given because it is greatly influenced by disk speed due to the large amount of virtual memory used during the simulation. $\ddagger$ The solution time is not given because the simulation could not complete due to insufficient system memory.

suspension fibres. Silicon is chosen over fused silica for the cryogenic test masses because of its superior mechanical qualities at low temperature.

\subsection{Finite element modelling}

\subsubsection{Introduction to eigenmode analysis using the finite element method}

A key part of the problem of analysing PI's in a gravitational-wave detector is performing an accurate eigenmode analysis of the test masses. The analysis is carried out in order to compute the mechanical mode frequencies $\omega_{m}$, as well as the amplitude of the displacement field $\boldsymbol{\psi}_{m}=\left(u_{m}, v_{m}, w_{m}\right)$ which is needed to compute the spatial overlap factors $\Lambda_{s}$ between the cavity's optical field and the test mass' mechanical modes.

If the test masses were ideal cylinders (perfectly symmetrical and isotropic), analytic expressions for $\omega_{m}$ and $\boldsymbol{\psi}_{m}$ would be readily available [153]. Moreover, exact solutions are known for certain values of the cylinder's radius to thickness ratio in the form of Chree-Lamb modes $[154,155]$. However, the test mass' geometry deviates from the ideal cylinder due to the flats and ears for suspension. These features break the cylindrical symmetry of the test mass and introduce additional mechanical modes with azimuth displacement components. Therefore, carrying out an eigenmode analysis of the test masses requires using the finite element method 


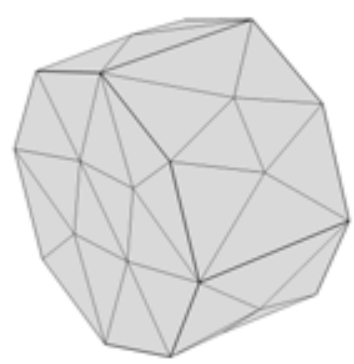

Extremely Coarse

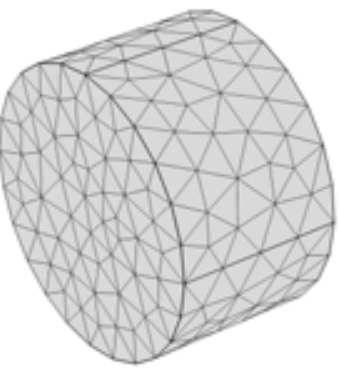

Coarse

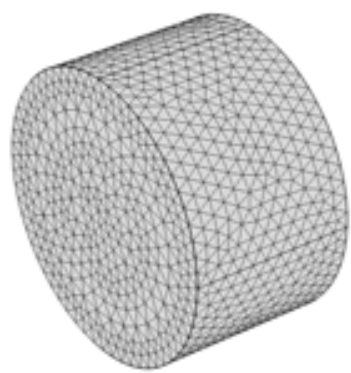

Finer

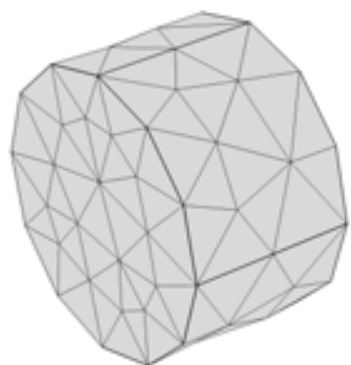

Extra Coarse

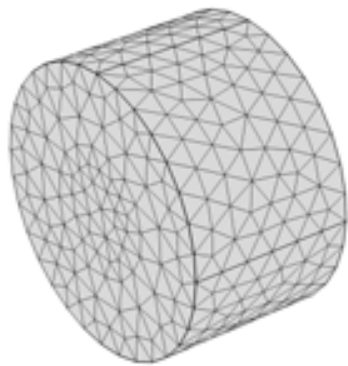

Normal

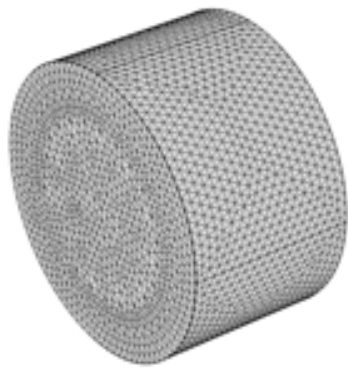

Extra Fine

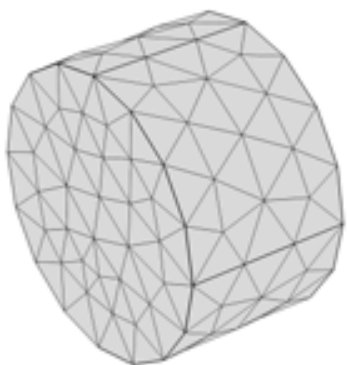

Coarser

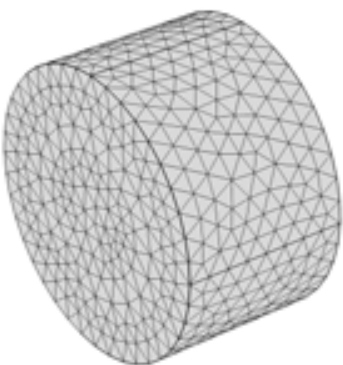

Fine

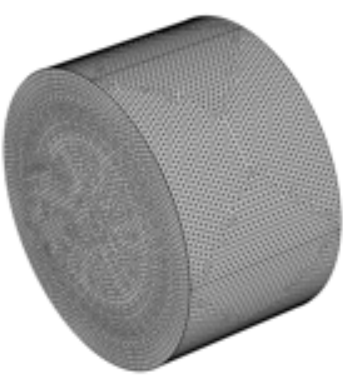

Extremely Fine

Figure 10.2: Meshed 3D geometry of a cylinder with the same dimensions as an Advanced LIGO test mass. The 9 meshes depicted are different standard size options of the tetrahedral mesh type. The total number of degrees of freedom of the system scales with the total number of nodes in the mesh and the number of independent variables. With the number of degrees of freedom scale both the model accuracy and the required computational resources of time and memory.

(FEM), where the geometry of the structure under study is discretised into small elements, and the equations of motion of the system are formed by the coupled equations of motion of the constituting elements. A finite element model of the test masses can be developed using a computer and commercially available FEM 
software, such as COMSOL Multiphysics [75].

In the finite element model, the geometry of the test mass can be defined or imported using a CAD interface. The 3D geometry is subsequently meshed using one of the available mesh types, typically a tetrahedral mesh. The meshed geometry contains a number of nodes that depends on the number of mesh elements that form the whole structure (hence depending on the size of each element and the dimensions of the structure) as well as the element type, and if the element is a boundary element or an edge element. For each mechanical mode, the motion of the test mass at an arbitrary point is described by the displacement vector $\boldsymbol{r}(t)=\boldsymbol{\psi}_{m} e^{i \omega_{m} t}$. The model will solve the equations of motion and evaluate the displacement vector at each node in the mesh $\left\{\boldsymbol{r}_{i}(t)\right\}_{i=1}^{N}$ [156]. Therefore, the number of degrees of freedom of the system is given by $\Sigma_{i=1}^{N} \Theta_{i}$, where $N$ is the total number of mesh nodes and $\Theta_{i}$ is the number of independent variables at the $i$-th node. In our problem, $\Theta_{i}$ is equal to 3 ( $x, y$ and $z$ displacement) for all nodes except for those that may be constrained by added symmetry boundary conditions. Without symmetry boundary conditions, the number of degrees of freedom would be $3 N$. The number of degrees of freedom of the system will have an impact on both the accuracy of the model and the computational resources needed to perform the simulation. However, the number of degrees of freedom is not the only factor affecting computational resources. Other factors of the model, notably the solver configurations, will have a big impact on both the simulation time and memory required to perform specific jobs. See Table 10.1 and Figure 10.2 for a comparative of different mesh sizes and simulation statistics.

\subsubsection{Accuracy of the model}

When developing a finite element model of Advanced LIGO test masses for simulating parametric instabilities — or for any other application — we must determine the accuracy of the model. This will help us establish whether this method is suitable for the task at hand, as well as the requirements in terms of mesh size in order to maintain accuracy throughout the parameter space. In an eigenmode analysis, model accuracy for a given mesh size will be a function of the eigenfrequency, with higher frequencies leading to lower accuracy. This is in part due to the higher order modes featuring more complex spatial shapes, which require denser meshes in order to be resolved by the model. For analysing parametric instabilities the tightest accuracy requirement is in the value of the eigenfrequencies $\omega_{m}$, and not in the shapes of the eigenmodes $[157,158]$.

Normally the accuracy of FEM models is estimated by running simulations with varying number of mesh elements, and hence varying number of degrees of freedom of the system, and comparing the differences in the results. The differences become smaller as the number of mesh elements and degrees of freedom is increased (Figure 10.7). It is also useful to compare simulations using regular (uniform) meshes with simulations using adaptive mesh refinement, which adjusts the mesh element size dynamically in those regions where the displacement field is greater. However, the best method of determining the accuracy of the model is by comparing the results against known solutions.

To estimate the accuracy of our finite element model, we will make use of the 


\begin{tabular}{cccccccc}
\hline$k$ & 0 & 1 & 2 & 3 & 4 & 5 & 6 \\
\hline$a_{k}$ & 0 & 1.8412 & 5.3314 & 8.5363 & 11.7060 & 14.8636 & 18.0155 \\
$b_{k}$ & 3.1416 & 9.4248 & 15.7080 & 21.9911 & 28.2743 & 34.5575 & 40.8407 \\
\hline
\end{tabular}

Table 10.2: Approximate values of the first 7 Chree-Lamb coefficients $a_{i}$ and $b_{j}$.

known analytical solutions for the Chree-Lamb waves of an elastic cylinder. We can compare our results with the known exact solutions for cylinders with given ratios of radius to thickness. For a cylinder with radius $r$ and thickness $h$, the eigenfrequencies of Chree-Lamb eigenmodes are given by [157]

$$
\omega_{m} \equiv \sqrt{2} \beta c_{t}, \quad \beta r \equiv a_{i}, \quad \beta h \equiv b_{j},
$$

where $\beta$ is an arbitrarily introduced variable, $a_{i}$ and $b_{j}$ are parameters taking restricted values,

$$
a_{i} J_{0}\left(a_{i}\right)-J_{1}\left(a_{i}\right)=0, \quad b_{j} \equiv \pi+2 \pi j,
$$

where $i, j \in \mathbb{N}$, and $J_{0}$ and $J_{1}$ are the zeroth and first order Bessel functions of the first kind (see Figure 10.3 and Table 10.2), and

$$
c_{t} \equiv \sqrt{\frac{Y}{2 \rho(1+\sigma)}}
$$

is the speed of the acoustic wave, $Y$ is Young's modulus, $\sigma$ is Poisson's ratio, and $\rho$ is the density of the material. Finally, the shape of the eigenmodes is given by

$$
\boldsymbol{\psi}_{m} \equiv A_{m}\left(\begin{array}{c}
-J_{1}(\beta \varrho) \cos \beta \zeta \\
J_{0}(\beta \varrho) \sin \beta \zeta \\
0
\end{array}\right)
$$

in cylindrical coordinates $(\varrho, \zeta, \phi)$, where $A_{m}$ is the amplitude of the displacement of the $m$-th mode (arbitrarily scalable). Note that there is no azimuth displacement due to the complete cylindrical symmetry.

We simulate Chree-Lamb modes in 7 cylinders with the radii and thicknesses listed in Table 10.3. These cylinders have $r / h$ ratios close to the ratio for Advanced LIGO test masses, as well as the same volume and material parameters. The resulting mechanical mode frequencies $f_{m}=\omega_{m} / 2 \pi$ and eigenmode shapes $\boldsymbol{\psi}_{m}$ are presented in Figure 10.4, and the relative error between the COMSOL model and the known analytical solutions are depicted in Figure 10.5. For each cylinder we developed 2 models: a 2D model using an axisymmetric boundary condition, and a full $3 \mathrm{D}$ model. The $2 \mathrm{D}$ model is considerably more accurate than the $3 \mathrm{D}$ model when using mesh elements of comparable size, in addition to being many times more efficient due to the reduced number of degrees of freedom modelled.

We find that using the Normal mesh in the 2D model is about as accurate as using the Extra Fine mesh in the 3D model, but many times more efficient. Unfortunately it is not possible to model Advanced LIGO test masses using a 2D 


\begin{tabular}{cccccccc}
\hline$\#$ & $a_{i}$ & $b_{j}$ & $r / h=a_{i} / b_{j}$ & $r(\mathrm{~m})$ & $h(\mathrm{~m})$ & $\beta=a_{i} / r=b_{j} / h$ & $f_{m}=\omega_{m} / 2 \pi(\mathrm{Hz})$ \\
\hline 1 & $a_{1}$ & $b_{0}$ & 0.58606700 & 0.14763852 & 0.25191407 & 12.47088993 & 10570.0267660 \\
2 & $a_{2}$ & $b_{0}$ & 1.69705094 & 0.21043383 & 0.123999715 & 25.33548294 & 21473.7467984 \\
3 & $a_{3}$ & $b_{1}$ & 1.24204570 & 0.18963993 & 0.15268354 & 61.72753086 & 52318.7724940 \\
4 & $a_{4}$ & $b_{1}$ & 1.57707574 & 0.20535319 & 0.13021137 & 72.38060724 & 61348.0641516 \\
5 & $a_{5}$ & $b_{2}$ & 1.14690412 & 0.18466857 & 0.16101482 & 97.55600677 & 82686.1280879 \\
6 & $a_{6}$ & $b_{2}$ & 1.34736563 & 0.19485539 & 0.14461954 & 108.61577750 & 92060.1241844 \\
7 & $a_{7}$ & $b_{3}$ & 1.24855009 & 0.18997040 & 0.15215280 & 144.53331200 & 122502.9637160 \\
\hline
\end{tabular}

Table 10.3: Parameters relevant to the Chree-Lamb modes simulations presented in Figure 10.4. To produce test models that are as close to the Advanced LIGO test masses as possible, we model cylinders with $r / h$ ratios close to the value for Advanced LIGO (0.85), as well as the same volume $\left(0.0172505 \mathrm{~m}^{3}\right)$ and material parameters.

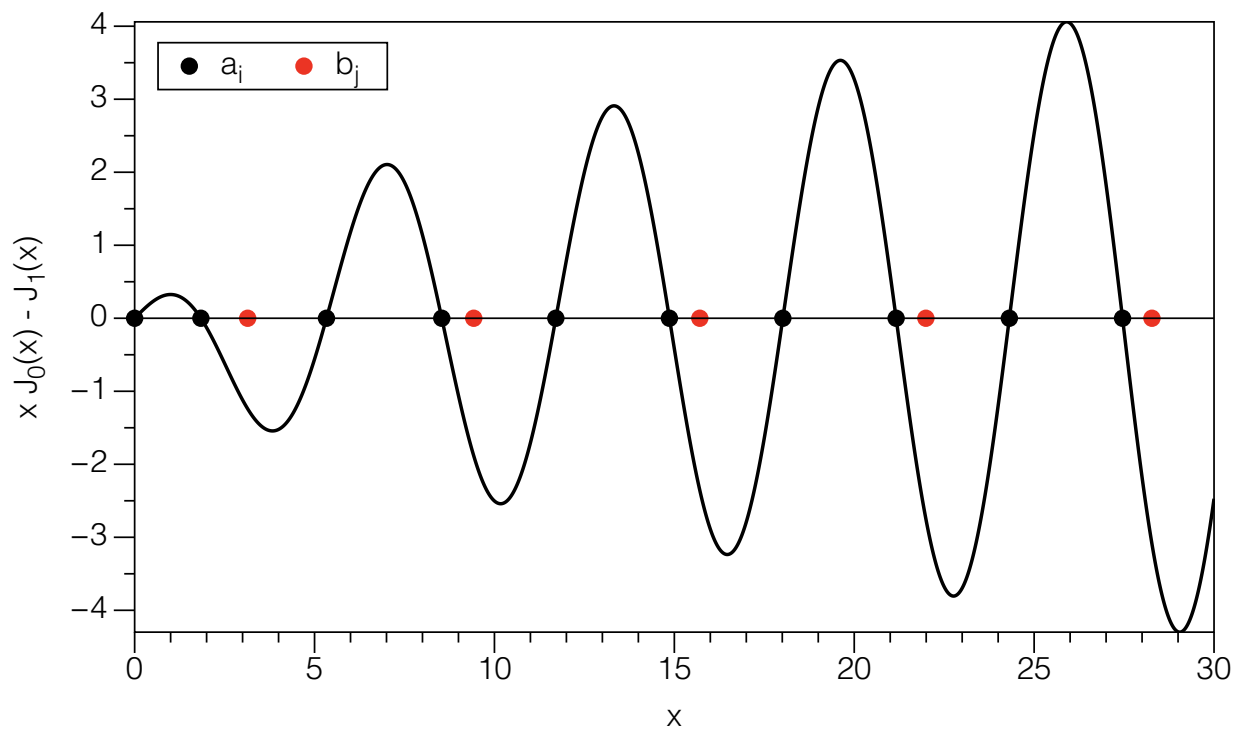

Figure 10.3: Chree-Lamb coefficients $a_{i}$ and $b_{j}$ : For cylinders with certain radius to thickness ratios $r / h=a_{i} / b_{j}$ there exist a series of mechanical modes known as Chree-Lamb modes with eigenfrequencies proportional to $\beta=a_{i} / r=b_{j} / h$. The coefficients $a_{i}$ are obtained by numerically solving $a_{i} J_{0}\left(a_{i}\right)-J_{1}\left(a_{i}\right)=0$, whereas $b_{j}=\pi+2 \pi j$. 

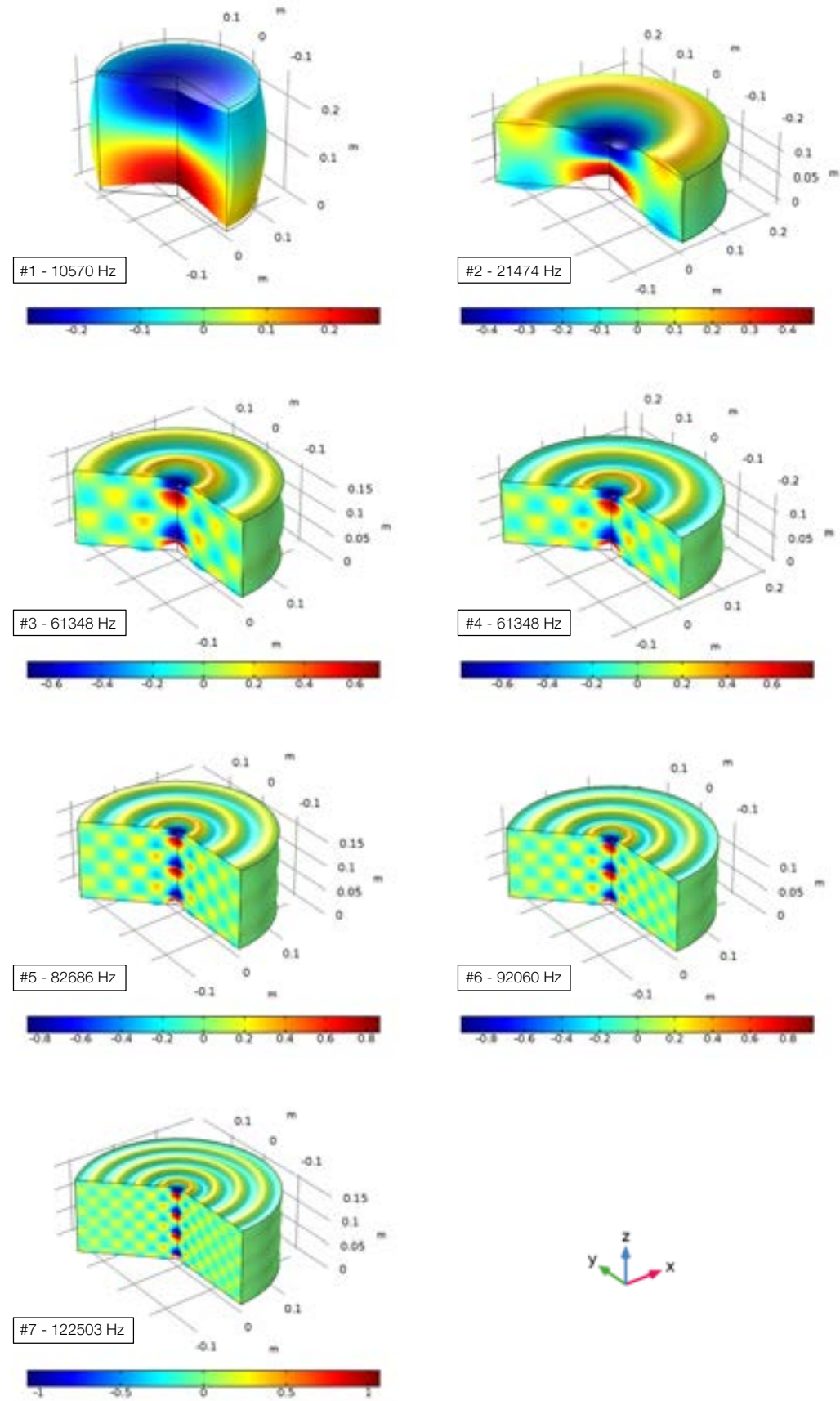

Figure 10.4: Chree-Lamb modes in ideal cylinders of the radii and thicknesses specified in Table 10.3 as modelled in COMSOL. These results can be compared against the known analytical solutions for Chree-Lamb modes. 
model due to the flats made for suspension of the optic. In both cases the model accuracy scales inversely with eigenfrequency, with higher order mechanical modes leading to a larger relative error between the COMSOL results and the analytical solution. Note that in the 3D case we are only able to simulate the first $3-4$ modes; the higher order modes featured a more intricate displacement field that the 3D meshes were not able to resolve. Perhaps the 3D model with the Extremely Fine mesh size would have been able to resolve those modes with an accuracy comparable to the Fine or Finer meshes in the 2D model, but we are not able to run the 3D model with that mesh option and our available computational resources. All the 3D models are able to compute the eigenfrequencies below $50 \mathrm{kHz}$ with relative errors better than $10^{-2}$, with the Extra Fine mesh size giving the best results at $10 \mathrm{kHz}$ (relative error $<10^{-6}$ ). While the Normal mesh option should provide enough accuracy for the first few mechanical modes of the Advanced LIGO test masses, we recommend using the Extra Fine or Extremely Fine mesh options for anything above $50 \mathrm{kHz}$. For even higher order mechanical modes (e.g., up to $f_{m}=100 \mathrm{kHz}$ ), a custom mesh with an even smaller mesh element size may be necessary. 


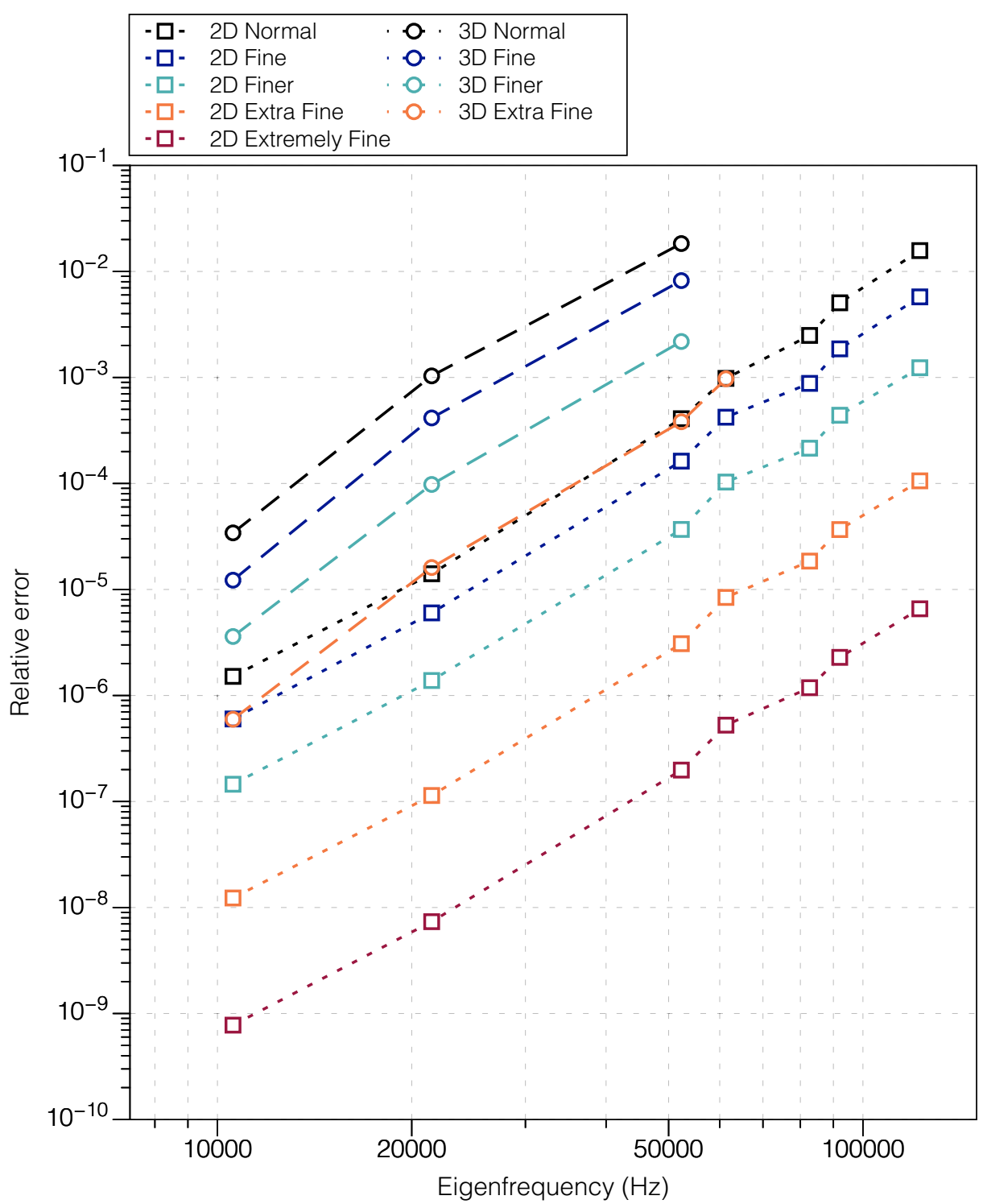

Figure 10.5: Accuracy of 2D and 3D finite element models of Chree-Lamb modes in ideal cylinders as a function of eigenfrequency and for different mesh sizes. The 2D models are significantly more accurate than the $3 \mathrm{D}$ models with mesh elements of comparable size. In both the $2 \mathrm{D}$ and $3 \mathrm{D}$ cases the accuracy decreases linearly with increasing eigenfrequency (in this plot the abscissa axis is $f_{m}=\omega_{m} / 2 \pi$ ). 


\subsection{Eigenmode analysis of Advanced LIGO and ET test masses}

\subsubsection{Advanced LIGO test masses}

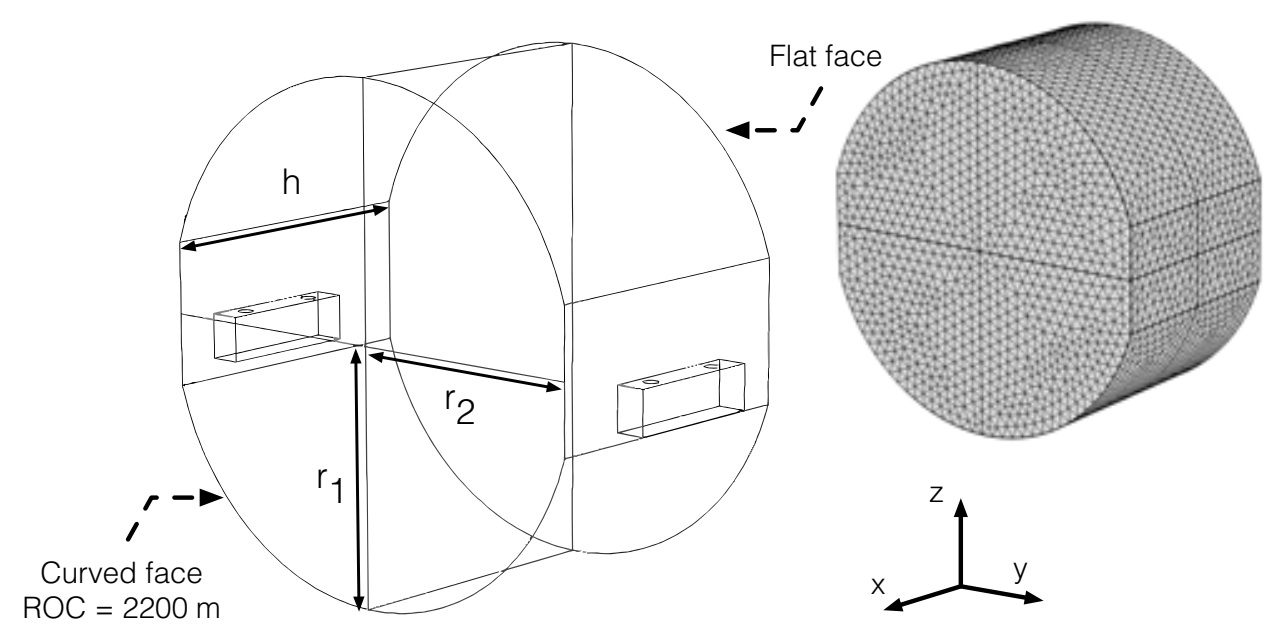

Figure 10.6: Geometry of an Advanced LIGO test mass. The test mass consist of a $\sim 40 \mathrm{~kg}$ cylinder made of fused silica, with the sides flattened in order to provide a surface to bond the suspension to $\left(r_{1}=17 \mathrm{~cm}, r_{2}=16.325 \mathrm{~cm}, h=20 \mathrm{~cm}\right)$. Mock suspension ears are depicted for reference, but not included in the model. The geometry is divided by the middle $x z$ symmetry plane; the model only considers solutions with zero displacement normal to this plane. Also depicted is the meshed geometry using COMSOL's Extra Fine tetrahedral mesh option.

The geometry of an Advanced LIGO test mass is depicted in Figure 10.6. It deviates from an ideal cylinder due to the flats and ears for suspension, which break the cylindrical symmetry of the structure. The material properties of fused silica used in the model are listed in Table 10.4. The mirror is symmetric with respect to the middle $x z$ plane, and thus a symmetry boundary condition is defined in that plane which discards solutions which have nonzero displacement normal to that boundary. The flats and ears introduce additional modes with respect to the ideal cylinder with azimuth displacement components, therefore increasing the total number of modes and the mode density, specially at higher frequency.

\begin{tabular}{cc}
\hline Poisson's ratio & 0.17 \\
Young's modulus & $72.6 \mathrm{GPa}$ \\
Density & $2203 \mathrm{~kg} / \mathrm{m}^{3}$ \\
\hline
\end{tabular}

Table 10.4: Mechanical properties of fused silica at room temperature. 
We carry out a modal analysis of the mirror using COMSOL Multiphysics' structural mechanics module. The model solves for the eigenfrequencies and the shape of the eigenmodes of the unperturbed 3D mirror geometry governed by the equations of linear elasticity. Because only the shape and not the size of the modes have physical significance, the computed modes can be scaled arbitrarily. The most common scaling method is modal mass normalisation, which means that the eigenmodes are orthogonalised with respect to the mass matrix.

We restrict our computation to eigenfrequencies between $1 \mathrm{~Hz}$ and $60 \mathrm{kHz}$, where we are able to achieve relative accuracies between $10^{-6}$ and $10^{-2}$ with mesh sizes up to the Extra Fine mesh option, according to our estimates from the previous section. To further test the accuracy of the model, we track the frequency of the well known "butterfly" mode at $\sim 15.54 \mathrm{kHz}$ as we change the size of the mesh and use adaptive mesh refinement (Figure 10.7). This is the mode responsible for the first observed parametric instability at Advanced LIGO [142]. The corresponding mode shape is depicted in Figure 10.8. A concise note on how to export surface displacement data from COMSOL can be found in [159]. This mode has a large spatial overlap with the $\mathrm{HG}_{03}$ and $\mathrm{HG}_{21}$ optical modes.

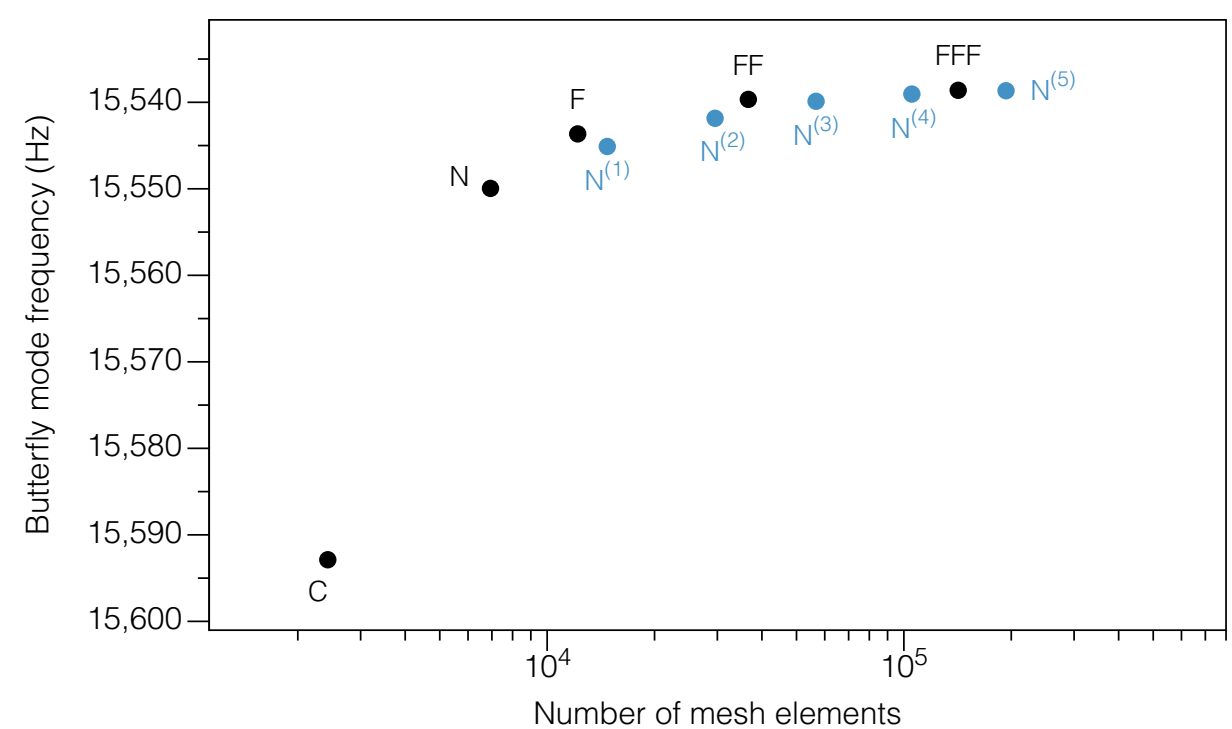

Figure 10.7: Eigenfrequency of the butterfly mode (related to the first observed parametric instability in Advanced LIGO) obtained from our finite element model of the test mass as a function of the number of mesh elements in the model. Mesh sizes: C: Coarse, N: Normal, F: Fine, FF: Finer, FFF: Extra Fine, $\mathrm{N}^{(k)}$ : Normal mesh $+k$ iterations of adaptive mesh refinement. Usually the accuracy of finite element models is estimated by varying the number of mesh elements and measuring the difference in the results. Where possible, it is always better to compare the model against known analytical solutions. 

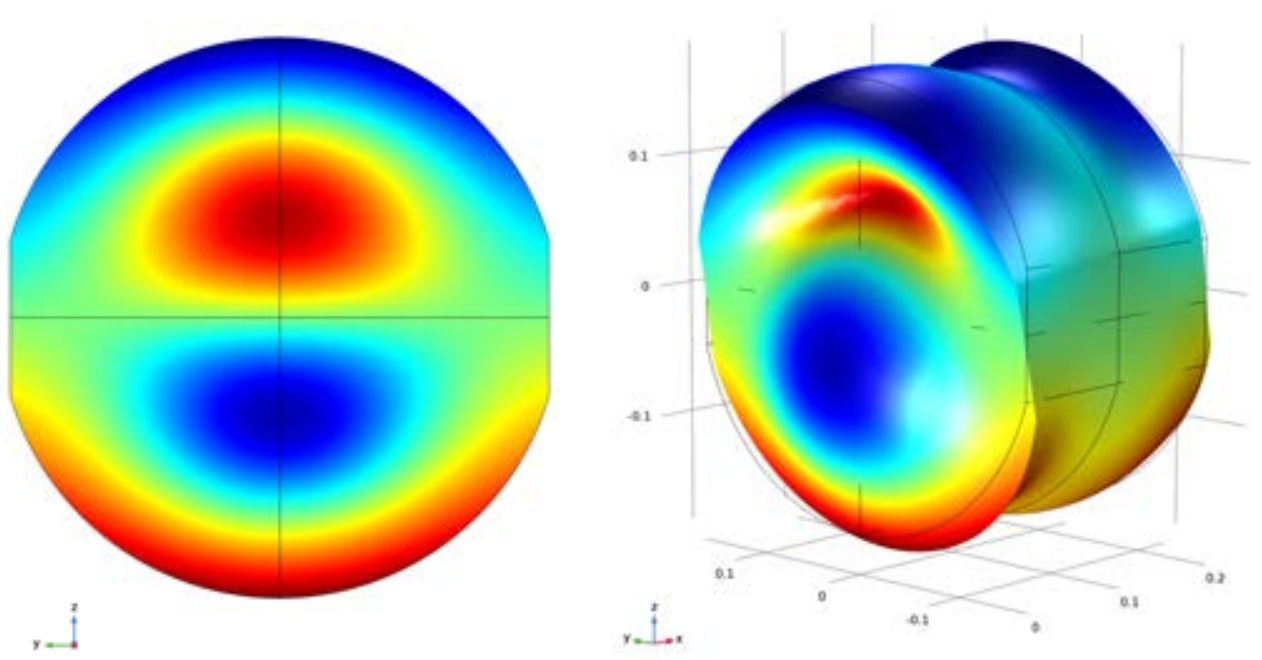

Figure 10.8: Mode shape of the $15538 \mathrm{~Hz}$ eigenmode associated with the first observed parametric instability in Advanced LIGO. Colour indicates relative displacement (arbitrarily scalable) in the direction of the optical axis.

We found a total of 800 mechanical modes between between $1 \mathrm{~Hz}$ and $60 \mathrm{kHz}$, with the first "drumhead" mode at approximately $8.1 \mathrm{kHz}$. To compute the parametric gain of each mechanical mode, the test mass' eigenmode data (i.e., its eigenfrequencies and corresponding mode shapes) is exported from COMSOL and input into a FINESSE model of the interferometer that includes both power and signal recycling $[121,141]$. The inclusion of the power- and signal-recycling cavities shapes the interferometer's response to parametric instabilities and results in up to four times as many PI's. We found that the round-trip Gouy phase shift in the signal-recycling cavity has a particularly significant impact on the parametric gains of mechanical modes, and therefore on which modes require suppression.

\subsubsection{ET test masses}

The Einstein Telescope is a European proposal for developing an underground gravitational-wave detector with a sensitivity roughly 10 times better than current second generation detectors. The ET design study [46] proposed a "xylophone" configuration using 3 nested detectors, each in turn composed of 2 dual-recycled Michelson interferometers with $10 \mathrm{~km}$ Fabry-Perot arms, one devoted to lowfrequency signals (ET-LF) and one to high frequency signals (ET-HF). More recently, another proposal for ET to build a single broadband interferometer (ET$120 \mathrm{~K}$ ) with a bandwidth similar to the xylophone configuration at the expense of a lower peak sensitivity has been put forward, with plans to later advance towards ET's design sensitivity over future upgrades. 


\begin{tabular}{ccc}
\hline & ET-HF & ET-120K \\
\hline Temperature $(\mathrm{K})$ & 293 & 123 \\
Material & Fused silica & c-Silicon \\
Poisson's ratio & 0.17 & 0.22 \\
Young's modulus $(\mathrm{GPa})$ & 72.6 & 156 \\
Density $\left(\mathrm{kg} / \mathrm{m}^{3}\right)$ & 2203 & 2330 \\
Diameter $(\mathrm{cm})$ & 62 & 45 \\
Thickness $(\mathrm{cm})$ & 30 & 55 \\
\hline
\end{tabular}

Table 10.5: Geometrical and material parameters of the ET-HF and ET-120K test masses.

Parametric instabilities are a main concern in both the ET-HF and the ET-120K designs, where the circulating intra-cavity power is planned at $3 \mathrm{MW}$ to reduce quantum shot noise, which is the limiting source of noise at high frequency. This is in contrast with the relatively low power of ET-LF, planned at $18 \mathrm{~kW}$, which in conjunction with cryogenic cooling of the test masses to $10 \mathrm{~K}$ will reduce thermal noise and radiation pressure noise, which are the limiting sources of noise at low frequency in addition to seismic noise.

The geometry of the proposed ET-120K test masses is very similar to that of ET-LF, also employing crystalline silicon as the substrate material, which provides better thermal performance at low temperature compared to fused silica. The ET-120K test masses will be cooled to around $123 \mathrm{~K}$ instead of $10 \mathrm{~K}$ as proposed for ET-LF, and hence the ET-120K design can be viewed as a "warmer ET-LF". The geometrical and material parameters of the proposed ET-HF and ET-120K test masses are collected in Table 10.5 . Both test masses are $\approx 200 \mathrm{~kg}$, in contrast with Advanced LIGO's $40 \mathrm{~kg}$ test masses, which will improve the radiation pressure noise performance.

Crystalline silicon is an anisotropic material, meaning that its mechanical properties depend on the orientation relative to the crystal lattice. Its Young's modulus varies from 130 to $169 \mathrm{GPa}$ [160]. We simplify our analysis by treating the material as isotropic, with a Young's modulus of $156 \mathrm{GPa}$, as it is done for the LIGO Voyager design [161]. Future models should include the material's variation of Young's modulus with direction.

The exact geometry of the ET test masses (i.e., the size and shape of the flats and ears for suspension) is still unknown, and as such they are modelled as simple cylinders (Figure 10.9). However, they are modelled in 3D in order to allow solutions with non-zero azimuth displacement. The models should be updated in the future once the final geometrical parameters are known. The ET-HF test masses have the same radius to thickness ratio as Advanced LIGO test masses, while the ET-120K test masses are slightly thicker due to restrictions of the manufacturing techniques.

The accuracy of the ET models should be analogous to that of the Advanced LIGO models, with the caveat that the ET models are preliminary because $(i)$ the geometry of the test masses is not final, and ( $i i)$ we did not model the variation of Young's modulus with orientation in crystalline silicon. We simulate the same $1 \mathrm{~Hz}$ to $60 \mathrm{kHz}$ frequency range in order to compare results with the Advanced LIGO 

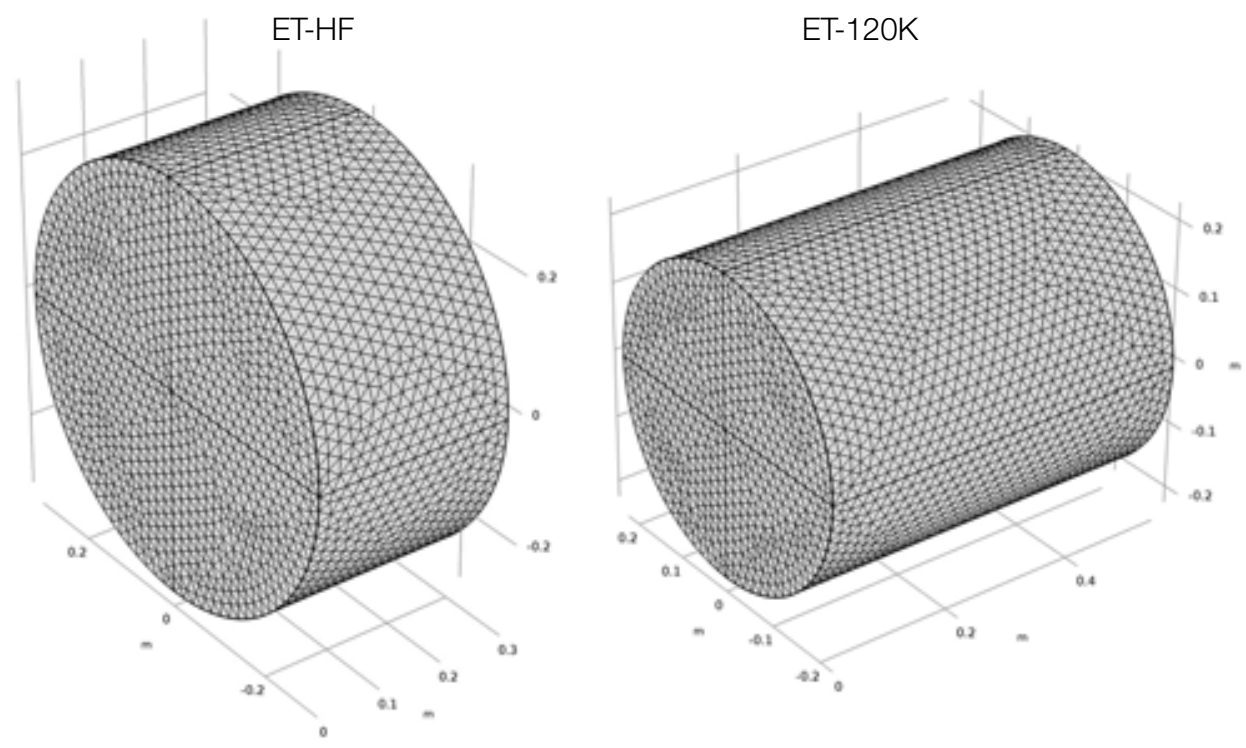

Figure 10.9: Geometry of the proposed test masses in the ET-HF and ET-120K designs. The ET-HF fused silica test mass has the same radius to thickness ratio as the Advanced LIGO test masses, but with larger dimensions to increase the mass to $200 \mathrm{~kg}$. The ET- $120 \mathrm{~K}$ silicon test mass is also $200 \mathrm{~kg}$, but is made much thicker due to manufacturing limitations.

model. The results show 3838 modes in ET-HF and 1497 in ET-120K, in contrast with Advanced LIGO's 800 modes in the same frequency range.

The increased number of mechanical modes within the measured interval is primarily due to the increase of mass, which yields an overall decrease in the values of the eigenfrequencies. While Advanced LIGO's first drumhead mode is measured at $8.1 \mathrm{kHz}$, in ET-HF it is measured at $4.2 \mathrm{kHz}$, and in ET-120K at $7.2 \mathrm{kHz}$. The increased eigenmode density will result in more mechanical modes overlapping with an optical resonance, and hence an increased risk of parametric instabilities. The ET-120K test masses display a much lower number of modes compared to the ET-HF test masses within the measured range, despite having the same mass. This is due to the increase of Young's modulus in crystalline silicon with respect to fused silica (see Equation 10.8).

Note that in ET-HF the frequency of the first drumhead mode is particularly low at $4.2 \mathrm{kHz}$, and in fact it falls within the gravitational-wave detection band for probing the neutron star equation of state $(1-5 \mathrm{kHz})$ [162]. This type of mode (Figure 10.10) is of great importance for parametric instabilities because the resulting displacement of the test mass cannot be subtracted by using linear or angular alignment control systems, unlike the fundamental DC motion (i.e., the "piston" mode). 


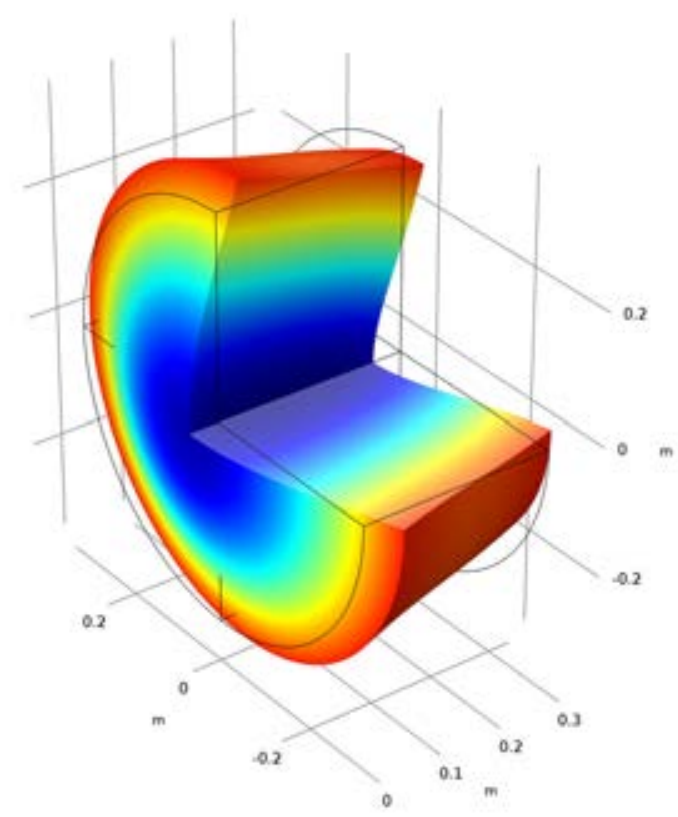

Figure 10.10: Mode shape of the $4170 \mathrm{~Hz}$ drumhead mode of the ET-HF test masses. Colour indicates surface displacement in the direction of the optical axis (arbitrarily scalable). The large $200 \mathrm{~kg}$ masses that will be used in ET will incur a reduction of the radiation pressure noise performance with respect to Advanced LIGO. However, the increased mass means that the values of the eigenfrequencies will decrease overall, and the higher eigenmode density will result in an increased risk of parametric instabilities. In ET-HF, the fundamental drumhead mode depicted here is particularly low in frequency and falls within the gravitational-wave detection band.

\subsection{Summary and future work}

Parametric instabilities are a main concern in the design of gravitational-wave detectors employing optical cavities with suspended mirrors and very high laser power. They originate from the optomechanical coupling between the high power optical field circulating in the cavity and the cavity mirrors which act as test masses for gravitational-wave detection. Under some conditions, the cavity's optical field will excite a mechanical resonance of the test mass and result in a parametric oscillatory instability, which can saturate the interferometer control systems. Parametric instabilities can therefore effectively reduce the sensitivity of the detectors by limiting the amount of power that is feasible to adopt.

Simulating parametric instabilities in the detectors is a crucial step towards developing mitigation strategies to reduce their impact in current and future experiments. A critical part of this effort is carrying out an accurate eigenmode analysis of the test masses, which requires developing finite element models to 
compute the eigenfrequencies and the shape of the eigenmodes of the unperturbed structure. This chapter discussed the simulation techniques that we have developed in order to do this, as well as their accuracy. We carried out analysis of Advanced LIGO test masses, as well as the proposed ET-HF and ET-120K test masses, which we then incorporated into realistic FINESSE models of the interferometers to simulate parametric instabilities $[49,141]$. 


\section{Summary and conclusions}

7 Hroughout this work we have extensively explored several applications of 1 optical cavities in the frontier of experimental physics. Part I of the thesis described the development of a novel ultra-stable optical reference cavity for use in optical atomic clocks, which is shown to be mainly limited by fundamental thermal noise at a few seconds of integration time, with a relative stability better than 1 part in $10^{16}$ at 2 seconds of integration. Achieving such stability required designing a long $\sim 0.5 \mathrm{~m}$ ULE cavity, which put very tight constraints on the vibration, temperature and pressure noise levels of the system. We have detailed the modelling and experimental techniques that are developed in order to realise this device (Chapter 2), and to keep all sources of noise below the required levels (Chapters 3 and 4).

This next-generation reference cavity consists of a cylindrical spacer made of ULE glass, with fused silica mirrors with high-reflectivity coatings optically contacted to the end faces. The spacer rests on four supports that are attached to a self-balancing mount. When a laser is locked to the cavity, the structural stability of the cavity is transferred to the frequency stability of the laser. The influence of external forces and any effects modifying the optical path length of the cavity compromise its structural stability. Hence, all efforts are directed towards isolating the cavity from external perturbations.

We designed a cavity spacer and support structure that make even this long cavity virtually insensitive to accelerations in all directions. This task required developing finite element models of the cavity and the mounting system, and several iterations of parameter optimisation. The cavity is housed in a triple-heat-shield system that minimises temperature fluctuations, a vacuum chamber that minimises pressure fluctuations, and an active vibration isolation platform that minimises vibrations. The heat shield system is designed to reduce conductive heat transfer, with ventilation holes that reduce radiative heat transfer, and which results in a thermal time constant of around ten days. The final device has achieved thermalnoise-limited performance, with its structural stability fundamentally limited by the thermal motion of its constituents, with the major contribution arising from the high-reflectivity optical coatings of the mirrors. Despite a few mishaps with the machining of the spacer that caused a few minor cracks and dents to the structure, the system met its design expectations. The acceleration response is measured in 
all directions and is within the required levels for the amount of vibration isolation provided by the AVI system, despite not having optimised the positions of the cavity supports experimentally.

This device currently serves as the master local oscillator at the National Physical Laboratory, delivering its stability to the network of optical atomic clocks being developed as part of the effort towards redefining the base SI unit of time - the second - in terms of an optical frequency standard. A second system of the same design is being built, with several improvements based on what we have learned from the first iteration, and is expected to surpass the stability displayed by the initial system.

In Part II of the thesis we described the development of time-domain models of atomic Bragg diffraction inside an optical cavity (Chapter 5). This new tool allowed us to realise the potential as well as the fundamental limitations of using optical cavities to assist the beam splitter pulses in large momentum transfer atom interferometers (Chapter 6). Before the publication of this work, atom interferometry inside an optical cavity was demonstrated in a proof of principle experiment, but no quantitative analysis of how the cavity parameters affected the interferometer was available. We applied our findings to aid the design of the MIGA experiment in France (Chapter 7), which will use cavity-enhanced interrogation to address an array of atom interferometers, and aims at becoming a demonstrator for ground-based gravitational-wave detection using this technology. We also described the design of a multi-mirror resonator with tuneable stability for enhanced atom interferometry with low wavefront distortions (Chapter 8).

To increase the sensitivity of light-pulse atom interferometers, the ensembles of many cold atoms are subjected to multi-photon interactions that impart a large momentum difference between the two arms of the interferometer. However, this type of atomic transitions come at the price of increased sensitivity to laser wavefront distortions and high power requirements. Optical cavities are proposed as the key enabling technology for this technique, as they provide both power enhancement and spatial filtering of the interferometric beams, thus helping mitigate the two main drawbacks of large momentum transfer interactions.

The cavity however introduces some technical challenges that need to be addressed. The biggest constraint on the cavity parameters is set by the size of the atomic ensembles as they thermally expand during the measurement: the cavity's optical field must accommodate the ensembles as they expand, and at the same time the cavity must maintain a geometrically stable configuration. In addition, depending on the cavity parameters of finesse and length, the cavity may shape the envelope function of the interrogation pulse in the time domain, which will increase the photon-atom interaction time and impact negatively on the interferometer. Power enhancement and spatial filtering are both enhanced by increasing the cavity finesse, while the maximal allowed beam size in the cavity scales with cavity length. The cavity bandwidth scales inversely with the product of finesse and length, and hence it would seem that the narrower the cavity bandwidth is, the better the cavity will perform for atom interferometry.

Our analysis, however, allowed us to establish a cavity bandwidth limit, which avoids the problem of elongation of the interrogation field in the time domain and maximises power enhancement. Having realised this bandwidth limit, we obtained 
atom temperature limits as a function of cavity length for the cavity-assisted interferometer, as well as a cavity length limit, as a function of two additional design parameters: the maximum cavity $g$-factor that would be experimentally realisable to maintain the cavity's geometrical stability, and the maximum optical suppression ratio of the first and second order spatial modes, which we have used as a probe of the level of spatial filtering of the beam splitter beams provided by the cavity. The interferometer temperature limits are divided into two regions: short cavities are limited by the constraint of having a stable configuration (the geometrical limit), while long cavities are limited by the constraint imposed on their quality as a spatial mode filter (the optical limit). These findings have important repercussions in experiments planning to use cavity-assisted beam splitters, specially in experiments planning to employ very long cavities.

Shortly after publishing our analysis [41] we started a collaboration with the MIGA consortium to aid the design of their cavity-enhanced interrogation system for an underground antenna that combines laser and atom interferometry [6]. The MIGA antenna will feature two large $200 \mathrm{~m}$ cavities that will be used to interrogate several atom interferometers simultaneously. The antenna will be used to measure gravity and gravity gradients, and will become a demonstrator for gravitational-wave detection at low frequency using atom interferometry. Chapter 7 shows the work being carried out in the design of the MIGA cavities.

In Chapter 8 we presented the design of a four-mirror cavity with tuneable stability. The proposed cavity will support a large beam capable of accommodating the size of the atomic ensembles as they expand up to about $10 \mathrm{~mm}$, all whilst maintaining a strictly stable configuration, getting around the geometrical limitations of Fabry-Perot cavities. Moreover, due to the ability to completely tune its stability, the cavity can be configured as a mode filter, providing rejection of wavefront distortions, which are a leading source of noise in current state-of-the-art detectors, and ultimately leading to a sensitivity increase of atom interferometric sensors.

Part III described our research in instrumentation science for second and next generation laser interferometer gravitational-wave detectors, such as Advanced LIGO or ET. The first detection of gravitational waves in late 2015 by the LIGO Hanford and Livingston observatories was a historic event. However it was but the beginning of a new era in astrophysics, the era of gravitational-wave astronomy, and for the years to come we will see major updates to existing detectors - as well as all new detectors - being designed and commissioned, increasing the sensitivity and range of our cosmic exploration, and being driven by constant progress in instrumentation science.

One of the major sources of noise in gravitational-wave detectors over much of the signal band is thermal noise, particularly originating at the optical coatings of the test masses. In Chapter 9 we presented our study towards reducing the thermal noise floor in future detectors by using near-unstable cavities [48], and described the development of a table-top near-unstable cavity with tuneable stability to investigate its behaviour as the cavity parameters are pushed towards geometrical instability.

Mirror coating thermal noise scales inversely with the size of the optical beam at the mirror, and thus is minimised in cavities with configurations close to the 
boundary of geometrical instability where the size of the beam at the mirrors is maximised. However, operating a cavity near instability involves many technical challenges that need to be studied and addressed. By carrying out precise measurements of the cavity's resonance structure as a function of its stability, we demonstrated how the resonance conditions become very sensitive to mirror surface distortions. This investigation offers an insight into how far the cavity parameters can be pushed towards instability, providing a support for future detectors that will use this technology.

As Advanced LIGO progresses towards using higher power in order to reach its design sensitivity, parametric oscillatory instabilities originating from the mechanical interaction between the cavity's optical field and the test mass' vibrational modes are expected to become problematic. These perturbations can effectively limit the sensitivity of the detectors by restricting the maximum power that can be reached in the interferometer whilst maintaining controllability.

In order to aid the development of mitigation strategies for parametric instabilities in current and future detectors, we developed realistic models of the Advanced LIGO core optics with inherent imperfections, for the first time including both the power- and signal-recycling cavities [49]. A fundamental part of this modelling effort is carrying out an accurate analysis of the test mass' vibrational modes, which required developing finite element models of these structures. Chapter 10 described our work in such models, detailing the simulation techniques that are developed for this purpose and their level of accuracy. This work paves the way towards further investigation in parametric instabilities, particularly to design future detectors such as ET - that can be designed taking parametric instabilities into account. 


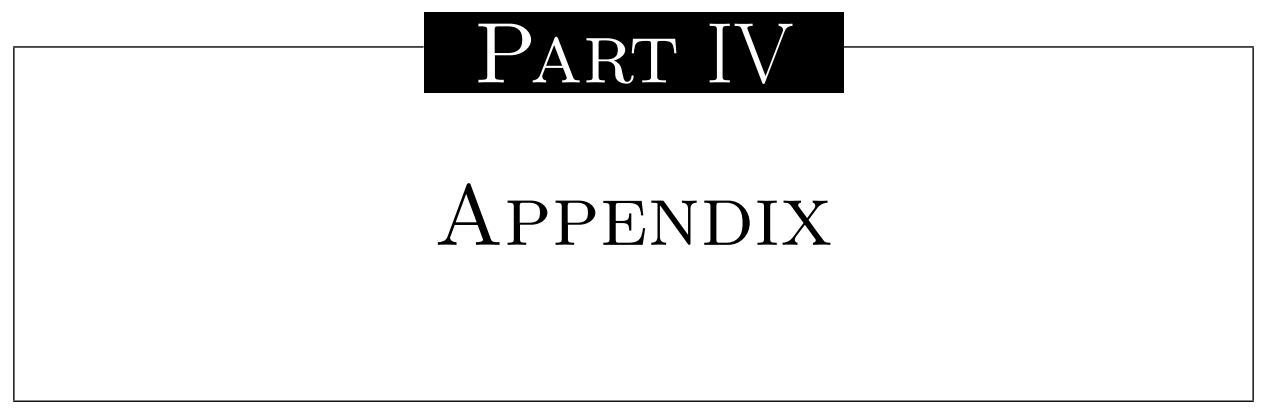





\section{Characterisation of frequency stability}

In Part I of the thesis (Chapters 2-4) the design and development of an ultra-stable optical cavity is presented. The cavity is used to transfer the frequency stability of its resonance structure (determined by its structural stability) to a laser. The ultra-stable laser system is then used as master local oscillator to transfer stability through a frequency comb to the network of microwave and optical frequency standards being developed at NPL.

An essential part of both the design and experimental characterisation of the cavity involves producing a measurement or an estimate of its frequency stability. The characterisation of the frequency stability of the cavity resonance (or equivalently of its length) serves two purposes: $(i)$ in the design phase, it allows the evaluation and comparison of the impact of different noise sources; ( $i i)$ in the experimental phase, it allows the evaluation of the achieved performance. The frequency stability characterisation of oscillators is a very extensive field [163].

Note that we never measure directly the cavity's resonance frequency: we measure the frequency of a laser that is locked to the cavity using a set of servo electronics. In order to experimentally characterise the frequency stability of the cavity resonance (i.e., of the laser locked to it), a second, independent system is necessary. Measurements using only one laser and one reference cavity do not yield information about the laser's frequency stability, as the behaviour of the laser is determined by the coupled action of the cavity and servo electronics. To fully characterise the laser, we need to obtain a beat note signal with a second system. This implies the need to build two systems and two sets of control electronics. Moreover, the second system should be completely independent of the first one in order to avoid any common mode noise rejection. That is, the cavities should be mounted on independent vacuum chambers and separate vibration isolation platforms.

The rf beat signal between the two systems can then be downshifted to a countable frequency. A frequency counter can be used to record a time series data $\nu_{i}$ by sampling the instantaneous value of the signal frequency $\nu(t)$. From this time series we can extract a lot of information about the laser, and fully characterise its stability. In the following, we show the methods used to extract the power spectral 
density and Allan deviation of the measured signal. We follow the notation of [164].

Fluctuating signals are commonly decomposed into a purely fluctuating contribution and a mean value. Moreover, stability is commonly expressed using adimensional quantities. For these purposes, we define the normalised frequency fluctuation,

$$
y(t) \equiv \frac{\Delta \nu(t)}{\nu_{0}}
$$

where $\Delta \nu(t) \equiv \nu(t)-\nu_{0}$ is the fluctuation of the instantaneous frequency of the beat note signal, and $\nu_{0}$ is the mean value of this frequency. An autocorrelation function of the signal fluctuation can be defined as

$$
R_{y}(\tau) \equiv \overline{\Delta \nu(t+\tau) \Delta \nu(t)}=\lim _{T \rightarrow+\infty} \frac{1}{2 T} \int_{-\infty}^{+\infty} \Delta \nu(t+\tau) \Delta \nu(t) d t
$$

which measures the distribution of the frequency excursions $\Delta \nu(t)$ as a function of the averaging time $\tau$. Note that for completely uncorrelated fluctuations (i.e., a purely random system) the autocorrelation function is zero because $\overline{\Delta \nu(t+\tau) \Delta \nu(t)}$ cancels for any $\tau$. On the other hand, we assume that the oscillator is highly stable (i.e., $\left.\Delta \nu(t) \ll \nu_{0}\right)$ and that the distribution of $\Delta \nu(t)$ is time independent. The autocorrelation function and the power spectral density function $S_{y}^{2 \text {-sided }}(f)$ form a Fourier transform pair,

$$
\begin{aligned}
S_{y}^{2 \text {-sided }}(f) & \equiv \int_{-\infty}^{+\infty} R_{y}(\tau) \exp (-i 2 \pi f \tau) d \tau \\
R_{y}(\tau) & \equiv \int_{-\infty}^{+\infty} S_{y}(f) \exp (i 2 \pi f \tau) d f
\end{aligned}
$$

The power spectral density measures the distribution of signal energy in frequency space, and has units of $y^{2} / \mathrm{Hz}$. Note that the definition given here extends to both sides of the frequency spectrum, $f \in(-\infty,+\infty)$. Experimentally only positive frequencies are of interest, and thus the one-sided power spectral density is introduced as

$$
S_{y}(f) \equiv 2 S_{y}^{2 \text {-sided }}(f)
$$

The power spectral density gives information about the root mean square of the measured quantity through

$$
y_{\mathrm{rms}}^{2} \equiv \lim _{T \rightarrow+\infty}\left[\frac{1}{T} \int_{0}^{T} y^{2}(t) d t\right]^{\frac{1}{2}}=\int_{0}^{+\infty} S_{y}(f) d f
$$

We can relate the power spectral density of the normalised frequency fluctuation to that of the frequency fluctuation, $S_{\nu}(f)\left(\mathrm{Hz}^{2} / \mathrm{Hz}\right)$, via

$$
S_{y}(f)=\frac{S_{\nu}(f)}{\nu_{0}^{2}} .
$$


In frequency standards the spectral density is usually used to characterise the short-term stability of the system. In highly-stable systems, the power spectral density of the normalised frequency fluctuation can be approximated by a power law,

$$
S_{y}(f) \sim \sum_{\alpha=-2}^{2} h_{\alpha} f^{\alpha},
$$

with different regions of the spectrum corresponding to different types of noise. For example, $\alpha=-1$ is flicker frequency noise (i.e., $1 / f$ noise), $\alpha=0$ is white frequency noise (i.e., frequency-independent noise), etc.

The more common measure of oscillator stability in time and frequency standards is the Allan variance (or equivalently the Allan deviation). It is used to characterise the stability of an oscillator over a time interval $\tau$. For a system whose normalised frequency fluctuation $y(t)$ has been measured by taking $N$ samples $\left\{\bar{y}_{i}\right\}_{i=1}^{N}$, each sample averaged over a $\tau$ interval, with a time $T$ between successive measurements (i.e., with $T-\tau$ dead time), the $N$-sample variance is defined as

$$
\sigma_{y}^{2}(N, T, \tau) \equiv \frac{1}{N-1} \sum_{i=1}^{N}\left(\bar{y}_{i}-\frac{1}{N} \sum_{j=1}^{N} \bar{y}_{j}\right)^{2}
$$

and the Allan variance is simply defined as a two-sample variance with zero dead time,

$$
\sigma_{y}^{2}(\tau) \equiv\left\langle\sigma_{y}^{2}(2, \tau, \tau)\right\rangle=\frac{1}{2}\left\langle\left(\bar{y}_{2}-\bar{y}_{1}\right)^{2}\right\rangle
$$

or, equivalently,

$$
\sigma_{y}^{2}(\tau)=\left\langle\frac{1}{2}\left(\frac{1}{\tau} \int_{t}^{t+\tau} y\left(t^{\prime}\right) d t^{\prime}-\frac{1}{\tau} \int_{t-\tau}^{t} y\left(t^{\prime}\right) d t^{\prime}\right)^{2}\right\rangle .
$$

We can read the Allan variance as "one half of the time average of the squares of the differences between successive values of the normalised frequency fluctuation sampled over the averaging time". Therefore, the Allan variance is based on differences between adjacent values of the time series data, instead of differences from the mean value. The Allan variance was introduced to account for certain types of noise commonly seen in frequency standards for which the classical variance diverges. The Allan deviation is then simply

$$
\sigma_{y}(\tau) \equiv \sqrt{\sigma_{y}^{2}(\tau)}
$$

Given a fixed $\tau$ for $m$ measurements we can give one estimate of the Allan variance as

$$
\sigma_{y}^{2}=\frac{1}{2(m-1)} \sum_{i=1}^{m-1}\left(\bar{y}_{i+1}-\bar{y}_{i}\right)^{2} .
$$

Like the power spectral density, for highly-stable systems the Allan variance can be modelled by a power law,

$$
\sigma_{y}^{2}(\tau) \sim \tau^{\mu}
$$


with $\mu$ relating the obtained variance with a certain type of noise. For example, $\mu=-1$ for white frequency noise, $\mu=0$ for flicker frequency noise, etc. We can compute the Allan variance from the one-sided power spectral density as

$$
\sigma_{y}^{2}(\tau)=2 \int_{0}^{+\infty} S_{y}(f) \frac{\sin ^{4}(\pi f \tau)^{2}}{\pi f \tau} d f
$$

However, it is not possible in general to compute the power spectral density from the Allan variance.

In practice, there are many alternative definitions of the Allan variance, each designed to provide advantages for a particular type of noise or measurement. For example, the Allan variance does not allow to distinguish between flicker phase noise and white phase noise (i.e., $f$ and $f^{2}$ noise respectively, which both yield $\left.\sigma_{y}(\tau) \sim \tau^{-2}\right)$. The modified Allan variance $\operatorname{Mod} \sigma_{y}^{2}(\tau)$ is introduced to get around this limitation, which gives the same variance as $\sigma_{y}^{2}(\tau)$ but with improved sensitivity for white phase noise (it is the one used in Chapter 4). For more details on Mod $\sigma_{y}^{2}(\tau)$, the overlapping Allan variance, and other definitions, see [165]. 


\section{Müller-Chiow-Chu method}

Chapter 5 describes the physical model used to solve the problem of the deflection of atoms from an optical cavity's standing wave field. The problem was approached using a semiclassical treatment of the atom-field interaction. First, the excited electronic state is adiabatically elliminated by assuming a large detuning compared to the linewidth of the excited state. The atoms in the ground electronic state are then described by an equation of motion with a periodic potential $\propto \cos ^{2}(k z)$, i.e., invariant under a spatial translation by an integer multiple of $1 / k$. The ground electronic state is then decomposed into an infinite superposition of $|m \hbar k\rangle \equiv|m\rangle$ momentum plane waves with amplitudes $g_{m}(t) e^{i m k z}$. The term $\cos ^{2}(k z)$ acts as a $2 \hbar k$-momentum shift operator over the wave function of the atom, leading to an infinite set of coupled equations of motion of the momentum eigenstates. Lastly, the system is truncated and the Schrödinger wave equation is solved numerically by means of a computer model.

The cavity is modelled in the time domain and enters the problem through its effect on the envelope function of the interrogation pulse, i.e., through a modulation of the potential term in the Schrödinger equation. There are multiple degrees of freedom in the problem. Intrinsic to the photon-atom interaction problem are the interaction time and intensity, as well as the shape of the envelope function of the interrogation field. Then, indirectly, the cavity parameters (e.g., the cavity finesse and length), determine the cavity-induced deformation of the interrogation field, thus shaping all three intrinsic degrees of freedom of the interaction. As the evolution of the atomic state presents very different behaviour depending on the relationship between time and energy in the parameter space (i.e., depending on the region of the space spanned by the interaction time and intensity where the interaction takes place), as well as depending on the particular shape of the envelope function of the interrogation field, the effect of the cavity on these degrees of freedom is paramount to the problem, and its projection onto the transition probabilities of the atom is all that was done in Chapter 5.

During the development of the numerical models to solve this problem, a parallel effort was invested into finding ways of solving the problem analytically without having to resort to numerical integration of the equations of motion. This could be very useful to gain a more explicit understanding of how the cavity parameters affect the atomic transitions without the need to run expensive simulations, which can take 
very long and also require a decent amount of data analysis. The Müller-Chiow-Chu method [112] was attempted. It consists in taking the adiabatic approximation that is valid in the Bragg regime as a starting point, eliminating the intermediate states and obtaining an effective Rabi frequency, and then using adiabatic expansion. That is, reinserting the result into the Schrödinger equation to yield higher-order corrections to the effective Rabi frequency. By employing this method, Müller et al. obtain a closed expression for $\Omega_{\text {eff }}$ for diffraction orders $m \leq 29$ (Equation 48 in [112]). Solving for $g_{ \pm m}$ then requires doing the integral $\int_{-\infty}^{t} \Omega_{\mathrm{eff}}\left(t^{\prime}\right) d t^{\prime}$, which they do for pulses having square and Gaussian envelope functions.

In this section we describe more concisely and clearly the method of adiabatic expansion presented in [112]. This method is very difficult to apply in situations where the envelope function of the interrogation field is not a simple function (e.g., a Gaussian or a square function), due to having to integrate $\Omega_{\text {eff }}(t)$, which includes a term $\propto \Omega^{m}$ as well as higher order corrections proportional to $\Omega$ and its time derivatives to arbitrary powers. When considering the field circulating in an optical cavity this method proves prohibitively difficult, and numerical integration of the equations of motion remains the method of choice for solving such systems.

It is assumed that the evolution of all states other than $| \pm m\rangle$ can be described by the following equation

$$
\widehat{\mathcal{H}}^{\prime}|\psi\rangle \equiv|c\rangle+i \frac{d}{d t}|\psi\rangle
$$

where $|\psi\rangle$ is $|\Psi\rangle$ with $| \pm m\rangle$ removed, $\widehat{\mathcal{H}}^{\prime}$ is $\widehat{\mathcal{H}}$ with the rows and columns of $| \pm m\rangle$ removed, and $|c\rangle$ is a vector describing the coupling of states $| \pm m\rangle$ with states $| \pm m \pm 2\rangle$

$$
\begin{gathered}
|\psi\rangle \equiv\left(\ldots, g_{-m-2}, g_{-m+2}, \ldots, g_{+m-2}, g_{+m+2}, \ldots\right), \\
|c\rangle \equiv-\frac{1}{2}\left(\ldots, \Omega g_{-m}, \Omega^{*} g_{-m}, \ldots, \Omega g_{+m}, \Omega^{*} g_{+m} \ldots\right) .
\end{gathered}
$$

We expand $|\psi\rangle$ and its time derivative as an infinite series, each element an order of magnitude smaller than the previous

$$
\begin{gathered}
|\psi\rangle \equiv|\psi\rangle^{(0)}+|\psi\rangle^{(1)}+|\psi\rangle^{(2)} \ldots=\sum_{q=0}^{\infty}|\psi\rangle^{(q)} \\
\frac{d}{d t}|\psi\rangle \equiv|\kappa\rangle^{(0)}+|\kappa\rangle^{(1)}+|\kappa\rangle^{(2)} \ldots=\sum_{q=0}^{\infty}|\kappa\rangle^{(q)}
\end{gathered}
$$

where $|\psi\rangle^{(q)}$ and $|\kappa\rangle^{(q)}$ are functions of $g_{ \pm m}$ and of $\Omega$ and its time derivatives only. Here $\Omega=\Omega(t)$ is a smooth and infinitely differentiable function of time. We express these vectors as

$$
|\psi\rangle^{(q)} \equiv|D\rangle^{(q)} g_{-m}+|E\rangle^{(q)} g_{+m},
$$

where the vectors $|D\rangle^{(q)}$ and $|E\rangle^{(q)}$ contain the dependency of $|\psi\rangle^{(q)}$ in $\Omega, \dot{\Omega}, \ddot{\Omega} \ldots$ etc. The $q$-th order amplitude of a state is given by

$$
\langle k \mid \psi\rangle^{(q)}=\langle k \mid D\rangle^{(q)} g_{-m}+\langle k \mid E\rangle^{(q)} g_{+m},
$$


where $\langle k \mid \psi\rangle^{(q)}=g_{-m+2 k}^{(q)}$ with $k=\ldots-2,-1,+1,+2 \ldots$ We denote $\langle k \mid D\rangle^{(q)}$ and $\langle k \mid E\rangle^{(q)}$ with $D_{k}^{(q)}$ and $E_{k}^{(q)}$ respectively. Thus

$$
g_{-m+2 k}^{(q)}=D_{k}^{(q)} g_{-m}+E_{k}^{(q)} g_{+m} .
$$

Note that here $k$ points to the state with momentum $(-m+2 k) \hbar k$.

We first define $|\psi\rangle^{(0)}$ and then establish recursion relations for every other term. The zeroth order term in $|\psi\rangle$ is obtained by setting $\frac{d}{d t}|\psi\rangle=0$ in Equation B.1

$$
|\psi\rangle^{(0)} \equiv \widehat{\mathcal{H}}^{\prime-1}|c\rangle
$$

This vector is a function of $g_{ \pm m}$ and $\Omega$ only. To obtain $|\kappa\rangle^{(q)}$ we differentiate $|\psi\rangle^{(q)}$

$$
|\kappa\rangle^{(q)}=\frac{d}{d t}|\psi\rangle^{(q)}
$$

For the zeroth order,

$$
|\kappa\rangle^{(0)}=\frac{d}{d t}\left(|D\rangle^{(0)}\right) g_{-m}+|D\rangle^{(0)} \dot{g}_{-m}+\frac{d}{d t}\left(|E\rangle^{(0)}\right) g_{+m}+|E\rangle^{(0)} \dot{g}_{+m}
$$

Lastly, to obtain $|\psi\rangle^{(q+1)}$,

$$
\begin{aligned}
\widehat{\mathcal{H}}^{\prime}|\psi\rangle^{(q+1)}=i|\kappa\rangle^{(q)}, & \\
& \Rightarrow|\psi\rangle^{(q+1)}=i \widehat{\mathcal{H}}^{\prime-1}|\kappa\rangle^{(q)} .
\end{aligned}
$$

By iterating this process we can therefore obtain $|\psi\rangle$ as a function of $g_{ \pm m}$ and of $\Omega$ and its time derivatives, to arbitrary accuracy ${ }^{1}$.

Now we obtain the corrected effective Rabi frequency. We begin by writing the equations of motion of $g_{ \pm m}$ in the Bragg regime,

$$
\left\{\begin{array}{l}
i \dot{g}_{-m}=\frac{\Omega^{*}}{2} g_{-m-2}+\frac{\Omega}{2} g_{-m+2} \\
i \dot{g}_{+m}=\frac{\Omega^{*}}{2} g_{+m-2}+\frac{\Omega}{2} g_{+m+2}
\end{array}\right.
$$

\footnotetext{
${ }^{1}$ Example of iteration

$|\psi\rangle^{(0)}=\widehat{\mathcal{H}}^{\prime-1}|c\rangle$

$|\kappa\rangle^{(0)}=\frac{d}{d t}|\psi\rangle^{(0)}$

$|\psi\rangle^{(1)}=i \widehat{\mathcal{H}}^{\prime-1}|\kappa\rangle^{(0)}$

$|\kappa\rangle^{(1)}=\frac{d}{d t}|\psi\rangle^{(1)}$

$|\psi\rangle^{(2)}=i \widehat{\mathcal{H}}^{\prime-1}|\kappa\rangle^{(1)}$
} 
and then replace $g_{-m \pm 2}$ and $g_{+m \pm 2}$ with the corresponding series of elements of $|\psi\rangle$,

$$
\begin{aligned}
& g_{-m \pm 2}=\langle \pm 1 \mid \psi\rangle=\sum_{q=0}^{\infty} g_{-m \pm 2}^{(q)}=\sum_{q=0}^{\infty}\left(D_{ \pm 1}^{(q)} g_{-m}+E_{ \pm 1}^{(q)} g_{+m}\right) \\
& g_{+m \pm 2}=\langle m \pm 1 \mid \psi\rangle=\sum_{q=0}^{\infty} g_{+m \pm 2}^{(q)}=\sum_{q=0}^{\infty}\left(D_{m \pm 1}^{(q)} g_{+m}+E_{m \pm 1}^{(q)} g_{-m}\right) .
\end{aligned}
$$

Substituting this into Equation B.13 we obtain a pair of coupled differential equations for $g_{ \pm m}$, with the corrected effective Rabi frequency being the cross-coupling term.

To zeroth order,

$$
\begin{aligned}
& g_{-m \pm 2} \approx g_{-m \pm 2}^{(0)}=D_{ \pm 1}^{(0)} g_{-m}+E_{ \pm 1}^{(0)} g_{+m} \\
& g_{+m \pm 2} \approx g_{+m \pm 2}^{(0)}=D_{m+ \pm 1}^{(0)} g_{-m}+E_{m+ \pm 1}^{(0)} g_{+m} .
\end{aligned}
$$

Substituting into Equation B.13,

$$
\left\{\begin{array}{l}
i \dot{g}_{-m} \approx \frac{\Omega^{*}}{2}\left(D_{-1}^{(0)} g_{-m}+E_{-1}^{(0)}\right) g_{+m}+\frac{\Omega}{2}\left(D_{+1}^{(0)} g_{-m}+E_{+1}^{(0)} g_{+m}\right) \\
i \dot{g}_{+m} \approx \frac{\Omega^{*}}{2}\left(D_{m-1}^{(0)} g_{-m}+E_{m-1}^{(0)}\right) g_{+m}+\frac{\Omega}{2}\left(D_{m+1}^{(0)} g_{-m}+E_{m+1}^{(0)} g_{+m}\right),
\end{array}\right.
$$

that is,

$$
\left\{\begin{array}{l}
i \dot{g}_{-m} \approx \frac{1}{2}\left[\left(\Omega^{*} D_{-1}^{(0)}+\Omega D_{+1}^{(0)}\right) g_{-m}+\left(\Omega^{*} E_{-1}^{(0)}+\Omega E_{+1}^{(0)}\right) g_{+m}\right] \\
i \dot{g}_{+m} \approx \frac{1}{2}\left[\left(\Omega^{*} D_{m-1}^{(0)}+\Omega D_{m+1}^{(0)}\right) g_{-m}+\left(\Omega^{*} E_{m-1}^{(0)}+\Omega E_{m+1}^{(0)}\right) g_{+m}\right] .
\end{array}\right.
$$

The effective Rabi frequency (to zeroth order in this case) corresponds to the cross-coupling terms, with the other terms representing the AC stark shifts,

$$
\left\{\begin{array}{l}
i \dot{g}_{-m} \approx \Omega_{\mathrm{ac}}^{(0)} g_{-m}+\Omega_{\mathrm{eff}}^{(0)} g_{+m}, \\
i \dot{g}_{+m} \approx \Omega_{\mathrm{ac}}^{(0)} g_{+m}+\Omega_{\mathrm{eff}}^{*(0)} g_{-m},
\end{array}\right.
$$

where

$$
\Omega_{\mathrm{eff}}^{(0)}=\frac{1}{2}\left(\Omega^{*} E_{-1}^{(0)}+\Omega E_{+1}^{(0)}\right), \quad \Omega_{\mathrm{ac}}^{(0)}=\frac{1}{2}\left(\Omega^{*} D_{-1}^{(0)}+\Omega D_{+1}^{(0)}\right) .
$$

The leading order in $\Omega$ of $\Omega_{\text {eff }}^{(0)}$ is identical to Equation 5.51. Note that in this zeroth order calculation we have taken the fast varying $|\kappa\rangle$ to be zero, thus ignoring its slowly varying part due to the adiabatic following. This is taken into account when carrying out the next order calculation of $|\psi\rangle$, which sets $|\kappa\rangle \approx|\kappa\rangle^{(0)}$.

The main challenge presented by this method is evident now, as we try to solve for $g_{ \pm m}$ by integrating the resulting differential equations. Doing so requires being able to integrate the resulting effective Rabi frequency, which is a function of $\Omega$ and $\dot{\Omega}$ to arbitrary power. Doing so proves challenging enough for pulses having square or Gaussian envelope functions, and extremely challenging (if at all possible) for the pulses temporally-distorted by an optical cavity. Numerical integration of the equations of motion remains the main method used throughout this work. 


\section{List of publications and conferences}

\section{List of publications}

Journal abbreviations:

ApJ: The Astrophysical Journal

ApJL: The Astrophysical Journal Letters

CQG: Classical and Quantum Gravity

PRA: Physical Review A

PRD: Physical Review D

PRL: Physical Review Letters

Sci. Rep.: Scientific Reports

1. Exploring gravity with the MIGA large scale atom interferometer

B. Canuel, A. Bertoldi, L. Amand, E. Borgo di Pozzo, T. Chantrait, C. Danquigny, M. Dovale Álvarez, B. Fang, A. Freise, R. Geiger, J. Gillot, S. Henry, J. Hinderer, D. Holleville, J. Junca, G. Lefèvre, M. Merzougui, N. Mielec, T. Monfret, S. Pelisson, M. Prevedelli, S. Reynaud, I. Riou, Y. Rogister, S. Rosat, E. Cormier, A. Landragin, W. Chaibi, S. Gaffet, P. Bouyer

Sci. Rep. 8, 14064 (2018) | DOI:10.1038/s41598-018-32165-Z

2. GW170817: Implications for the Stochastic Gravitational-Wave Background from Compact Binary Coalescences

The LIGO Scientific Collaboration and The Virgo Collaboration, including M. Dovale-Álvarez

PRL 120, 091101 (2018) | arXiv:1710.05837 | DOI:10.1103/PhysRevLett.120.091101

3. Constraints on cosmic strings using data from the first Advanced LIGO observing run

The LIGO Scientific Collaboration and The Virgo Collaboration, including M. Dovale-Álvarez

PRD 97, 102002 (2018) | arXiv:1712.01168 | DOI:10.1103/PhysRevD.97.102002

4. Feasibility of near-unstable cavities for future gravitational wave detectors

H. Wang, M. Dovale Álvarez, C. Collins, D. D. Brown, M. Wang, C. M. MowLowry and A. Freise

PRD 97, 022001 (2018) | arXiv:1711.05177 | DOI:10.1103/PhysRevD.97.022001 
5. GW170608: Observation of a 19-solar-mass Binary Black Hole Coalescence The LIGO Scientific Collaboration and The Virgo Collaboration, including M. Dovale Álvarez ApJL 851, L35 (2017) | arXiv:1711.05578 | DOI:10.3847/2041-8213/aa9f0c

6. Fundamental limitations of cavity-assisted atom interferometry M. Dovale Álvarez, D. D. Brown, A. W. Jones, C. M. Mow-Lowry, H. Miao and A. Freise

PRA 96, 053820 (2017) | arXiv:1710.02448 | DOI:10.1103/PhysRevA.96.053820

7. Search for post-merger gravitational waves from the remnant of the binary neutron star merger GW170817

The LIGO Scientific Collaboration and The Virgo Collaboration, including M. Dovale Álvarez

ApJL 851, L16 (2017) | arXiv:1710.09320 | DOI:10.3847/2041-8213/aa9a35

8. First narrow-band search for continuous gravitational waves from known pulsars in advanced detector data

The LIGO Scientific Collaboration and The Virgo Collaboration, including M. Dovale Álvarez

PRD 96, 122006 (2017) | arXiv:1710.02327 | DOI:10.1103/PhysRevD.96.122006

9. $\quad$ On the Progenitor of Binary Neutron Star Merger GW170817

The LIGO Scientific Collaboration and The Virgo Collaboration, including M. Dovale Álvarez

ApJL 850, L40 (2017) | arXiv:1710.05838 | DOI:10.3847/2041-8213/aa93fc

10. Estimating the Contribution of Dynamical Ejecta in the Kilonova Associated with GW170817

The LIGO Scientific Collaboration and The Virgo Collaboration, including M. Dovale Álvarez

ApJL 850, L39 (2017) | arXiv:1710.05836 | DOI:10.3847/2041-8213/aa9478

11. A gravitational-wave standard siren measurement of the Hubble constant

The LIGO Scientific Collaboration, The Virgo Collaboration, The 1M2H Collaboration, The Dark Energy Camera GW-EM Collaboration and the DES Collaboration, The DLT40 Collaboration, The Las Cumbres Observatory Collaboration, The VINROUGE Collaboration and The MASTER Collaboration, including M. Dovale Álvarez

Nature 551, 85-88 (2017) | arXiv:1710.05835 | DOI:10.1038/Nature24471

12. Gravitational Waves and Gamma-Rays from a Binary Neutron Star Merger: GW170817 and GRB 170817A

The LIGO Scientific Collaboration, The Virgo Collaboration, The Fermi GammaRay Burst Monitor and INTEGRAL, including M. Dovale Álvarez ApJL 848, L13 (2017) | arXiv:1710.05834 | DOI:10.3847/2041-8213/aa920c

13. GW17081\%: Observation of Gravitational Waves from a Binary Neutron Star Inspiral

The LIGO Scientific Collaboration and The Virgo Collaboration, 
including M. Dovale Álvarez

PRL 119, 161101 (2017) | arXiv:1710.05832 | DOI:10.1103/PhysRevLett.119.161101

14. Multi-messenger Observations of a Binary Neutron Star Merger

The LIGO Scientific Collaboration, The Virgo Collaboration, et al., including

M. Dovale Álvarez

ApJL 848, L12 (2017) | arXiv:1710.05833 | DOI:10.3847/2041-8213/aa91c9

15. GW170814: A Three-Detector Observation of Gravitational Waves from a Binary Black Hole Coalescence

The LIGO Scientific Collaboration and The Virgo Collaboration, including M. Dovale Álvarez

PRL 119, 141101 (2017) | arXiv:1709.09660 | DOI:10.1103/PhysRevLett.119.141101

16. First search for nontensorial gravitational waves from known pulsars

The LIGO Scientific Collaboration and The Virgo Collaboration, including M. Dovale Álvarez

PRL 120, 031104 (2018) | arXiv:1709.09203 | DOI:10.1103/PhysRevLett.120.031104

17. First low-frequency Einstein@Home all-sky search for continuous gravitational waves in Advanced LIGO data

The LIGO Scientific Collaboration and The Virgo Collaboration, including M. Dovale Álvarez

PRD 96, 122004 (2017) | arXiv:1707.02669 | DOI:10.1103/PhysRevD.96.122004

18. All-sky Search for Periodic Gravitational Waves in the O1 LIGO Data

The LIGO Scientific Collaboration and The Virgo Collaboration, including M. Dovale Álvarez

PRD 96, 062002 (2017) | arXiv:1707.02667 | DOI:10.1103/PhysRevD.96.062002

19. Upper Limits on Gravitational Waves from Scorpius X-1 from a Model-Based Cross-Correlation Search in Advanced LIGO Data

The LIGO Scientific Collaboration and The Virgo Collaboration, including M. Dovale Álvarez

ApJ 847, 47 (2017) | arXiv:1706.03119 | DOI:10.3847/1538-4357/aa86f0

20. The influence of dual-recycling on parametric instabilities at Advanced LIGO

A. C. Green, D. D. Brown, M. Dovale Álvarez, C. Collins, H. Miao, C. M. MowLowry, A. Freise

CQG 34, 205004 (2017) | arXiv:1704.08595 | DOI:10.1088/1361-6382/aa8af8

21. Search for intermediate mass black hole binaries in the first observing run of Advanced LIGO

The LIGO Scientific Collaboration and The Virgo Collaboration, including M. Dovale Álvarez

PRD 96, 022001 (2017) | arXiv:1704.04628 | DOI:10.1103/PhysRevD.96.022001

22. Search for gravitational waves from Scorpius $X-1$ in the first Advanced LIGO observing run with a hidden Markov model

The LIGO Scientific Collaboration and The Virgo Collaboration, 
including M. Dovale Álvarez

PRL 95, 122003 (2017) | arXiv:1704.03719 | DOI:10.1103/PhysRevD.95.122003

23. Search for high-energy neutrinos from gravitational wave event GW151226 and candidate LVT151012 with ANTARES and IceCube

The ANTARES Collaboration, The IceCube Collaboration, The LIGO Scientific Collaboration and The Virgo Collaboration, including M. Dovale Álvarez PRD 96, 022005 (2017) | arXiv:1703.06298 | DOI:10.1103/PhysRevD.96.022005

24. GW170104: Observation of a 50-Solar-Mass Binary Black Hole Coalescence at Redshift 0.2

The LIGO Scientific Collaboration and The Virgo Collaboration, including M. Dovale Álvarez

PRL 118, 221101 (2017) | arXiv:1703.06298 | DOI:10.1103/PhysRevLett.118.221101

25. First Search for Gravitational Waves from Known Pulsars with Advanced LIGO

The LIGO Scientific Collaboration and The Virgo Collaboration, including M. Dovale Álvarez ApJ 839, 12 (2017) | arXiv:1701.07709 | DOI:10.3847/1538-4357/aa677f

26. Directional limits on persistent gravitational waves from Advanced LIGO's first observing run

The LIGO Scientific Collaboration and The Virgo Collaboration, including M. Dovale Álvarez

PRL 118, 121102 (2017) | arXiv:1612.02030 | DOI:10.1103/PhysRevLett.118.121102

27. Thermal modelling of Advanced LIGO test masses

H. Wang, C. Blair, M. Dovale Álvarez, A. Brooks, M. F. Kasprzack, J. Ramette, P. M. Meyers, S. Kaufer, B. O'Reilly, C. M. Mow-Lowry and A. Freise CQG 34, 115001 (2016) | arXiv:1612.02866 | DOI:10.1088/1361-6382/aa6e60

28. Upper Limits on the Stochastic Gravitational-Wave Background from Advanced LIGO's First Observing Run

The LIGO Scientific Collaboration and The Virgo Collaboration, including M. Dovale Álvarez

PRL 118, 121101 (2017) | arXiv:1612.02029 | DOI:10.1103/PhysRevLett.118.121101

29. Search for Gravitational Waves Associated with Gamma-Ray Bursts during the First Advanced LIGO Observing Run and Implications for the Origin of GRB $150906 B$

The LIGO Scientific Collaboration, The Virgo Collaboration and The IPN Collaboration, including M. Dovale Álvarez

ApJ 841, 89 (2017) | arXiv:1611.07947 | DOI:10.3847/1538-4357/aa6c47

30. Effects of waveform model systematics on the interpretation of GW150914

The LIGO Scientific Collaboration and The Virgo Collaboration, including M. Dovale Álvarez

CQG 34, 104002 (2017) | arXiv:1611.07531 | DOI:10.1088/1361-6382/aa6854

31. All-sky search for short gravitational-wave bursts in the first Advanced LIGO run 
The LIGO Scientific Collaboration and The Virgo Collaboration, including M. Dovale Álvarez

PRD 95, 042003 (2017) | arXiv:1611.02972 | DOI:10.1103/PhysRevD.95.042003

\title{
Technical documents
}

32. MIGA cavities design document

M. Dovale Álvarez and A. Freise

2017 | Institute of Gravitational Wave Astronomy, University of Birmingham

33. Exporting surface displacement data from COMSOL Multiphysics

M. Dovale Álvarez

2017 | LIGO Scientific Collaboration

34. Simulating atomic Bragg diffraction

M. Dovale Álvarez

2017 | Institute of Gravitational Wave Astronomy, University of Birmingham

35. Digital temperature control system for optical reference cavities

M. Dovale Álvarez

2016 | Quantum Metrology Institute, National Physical Laboratory

36. Passive thermal isolation system for optical reference cavities

M. Dovale Álvarez and R. Williams

2015 | Quantum Metrology Institute, National Physical Laboratory

37. An ultra-high vacuum chamber for optical reference cavities

M. Dovale Álvarez and R. Williams

2015 | Quantum Metrology Institute, National Physical Laboratory

38. Next-generation ultra-stable optical reference cavity design document

M. Dovale Álvarez and R. Williams

2015 | Quantum Metrology Institute, National Physical Laboratory

\section{Invited talks and conferences}

\author{
March 2018 GRAvitational-waves Science\&technology Sympo- \\ sium (Padova, Italy) \\ Talk: Gravitational-wave detection using cavity-assisted \\ atom interferometry.
}

August 2017 LIGO-Virgo Collaboration Meeting (Geneva, Switzerland) 
July 2017

September 2016

May 2016

May 2016

April 2016

August 2015

March 2015
Poster: Fundamental limitations of cavity-assisted atom interferometry.

Poster: Modelling dual-recycled parametric instabilities at LIGO.

Conference of the European Group on Atomic Systems (Durham, UK)

Poster: Fundamental limitations of cavity-assisted atom interferometry.

LIGO-Virgo Collaboration Meeting (Glasgow, UK) Poster: Near-unstable Fabry-Perot cavities for future gravitational-wave detectors.

Invited talk at University of Santiago de Compostela (Santiago, Spain)

Talk: Optical cavities - from gravitational-wave detection to atom interferometry.

Gravitational-wave Advanced Detector Workshop (Isola d'Elba, Italy)

Poster: FINESSE 2.1 with multimode squeezing.

DSTL's Introduction to the Defence and Security Sector Spring Workshop at QinetiQ (Farnborough, UK)

Talk: Development of new technologies for defence applications.

DSTL's National Quantum Technology Summer School (Birmingham, UK)

Talk: Development of business strategies in the UK national quantum technologies landscape.

Defence Science and Technology Laboratory Meeting (Glasgow, UK)

Talk: Ultra-stable optical cavities for use in optical atomic clocks. 


\section{References}

[1] P. Meystre, Atom Optics. Springer-Verlag Berlin Heidelberg, 2001.

[2] A. Perot and C. Fabry, On the Application of Interference Phenomena to the Solution of Various Problems of Spectroscopy and Metrology, The Astrophysical Journal 9 (1899) 87.

[3] C. Fabry and A. Perot, On a New Form of Interferometer, The Astrophysical Journal 13 (1901) 265.

[4] L. S. Collaboration, Advanced ligo, Class. Quantum Grav. 32 (Apr., 2015) 074001 .

[5] P. Hamilton, M. Jaffe, J. M. Brown, L. Maisenbacher, B. Estey, and H. Müller, Atom interferometry in an optical cavity, Phys. Rev. Lett. 114 (2015), no. 10100405.

[6] B. Canuel, A. Bertoldi, L. Amand, E. P. di Borgo, T. Chantrait, C. Danquigny, M. Dovale Álvarez, B. Fang, A. Freise, R. Geiger, J. Gillot, S. Henry, J. Hinderer, D. Holleville, J. Junca, G. Lefèvre, M. Merzougui, N. Mielec, T. Monfret, S. Pelisson, M. Prevedelli, S. Reynaud, I. Riou, Y. Rogister, S. Rosat, E. Cormier, A. Landragin, W. Chaibi, S. Gaffet, and P. Bouyer, Exploring gravity with the MIGA large scale atom interferometer, Scientific Reports 8 (Sept., 2018) 1-23.

[7] "National Physical Laboratory." http://npl.co.uk/. Accessed: 16.07.2018.

[8] S. R. Jefferts, J. Shirley, T. E. Parker, T. P. Heavner, D. M. Meekhof, C. Nelson, F. Levi, G. Costanzo, A. D. Marchi, R. Drullinger, L. Hollberg, W. D. Lee, and F. L. Walls, Accuracy evaluation of NIST-F1, Metrologia 39 (Feb., 2002) 321-336.

[9] "Bureau International des Poids et Mesures." https://www.bipm.org/en/ bipm-services/timescales/time-ftp/data.html\#nohref. Accessed: 16.11.2018.

[10] P. Gill, Optical frequency standards, Metrologia 42 (2005), no. 3 S125-S137.

[11] P. Gill, When should we change the definition of the second?, Philos. Trans. R. Soc. A Math. Phys. Eng. Sci. 369 (2011), no. 1953 4109-4130. 
[12] M. Dovale Álvarez and R. A. Williams, Design of NPL's new long optical cavity, tech. rep., National Physical Laboratory, 2015.

[13] S. Dimopoulos, P. W. Graham, J. M. Hogan, and M. A. Kasevich, Testing general relativity with atom interferometry, Phys. Rev. Lett. 98 (2007), no. 11 111102.

[14] S. Dimopoulos, P. W. Graham, J. M. Hogan, and M. A. Kasevich, General relativistic effects in atom interferometry, Phys. Rev. D 78 (2008), no. 4 042003.

[15] S. Dimopoulos, P. W. Graham, J. M. Hogan, M. A. Kasevich, and

S. Rajendran, Atomic gravitational wave interferometric sensor, Phys. Rev.

D 78 (2008), no. 12122002.

[16] P. W. Graham, J. M. Hogan, M. A. Kasevich, and S. Rajendran, New method for gravitational wave detection with atomic sensors, Phys. Rev. Lett. 110 (2013), no. 17171102.

[17] W. Chaibi, R. Geiger, B. Canuel, A. Bertoldi, A. Landragin, and P. Bouyer, Low frequency gravitational wave detection with ground based atom interferometer arrays, Phys. Rev. D 93 (2016), no. 2021101.

[18] B. Canuel, S. Pelisson, L. Amand, A. Bertoldi, E. Cormier, B. Fang, S. Gaffet, R. Geiger, J. Harms, D. Holleville, A. Landragin, G. Lefèvre, J. Lhermite, N. Mielec, M. Prevedelli, I. Riou, and P. Bouyer, Miga: Combining laser and matter wave interferometry for mass distribution monitoring and advanced geodesy, Proc. SPIE 9900, Quantum Optics 990008 (Apr., 2016).

[19] M. Kasevich and S. Chu, Atomic interferometry using stimulated raman transitions, Phys. Rev. Lett. 67 (1991), no. 2181.

[20] J. B. Fixler, G. T. Foster, J. M. McGuirk, and M. A. Kasevich, Atom interferometer measurement of the newtonian constant of gravity, Science 315 (2007), no. 5808 74-77.

[21] G. Rosi, F. Sorrentino, L. Cacciapuoti, M. Prevedelli, and G. M. Tino, Precision measurement of the newtonian gravitational constant using cold atoms, Nature 510 (2014), no. 7506 518-521.

[22] D. S. Weiss, B. C. Young, and S. Chu, Precision measurement of $\hbar / m_{\mathrm{cs}}$ based on photon recoil using laser-cooled atoms and atomic interferometry, Appl. Phys. B 59 (1994), no. 3 217-256.

[23] M. Cadoret, E. de Mirandes, P. Cladé, S. Guellati-Khélifa, C. Schwob, F. Nez, L. Julien, and F. Biraben, Combination of bloch oscillations with a ramsey-bordé interferometer: new determination of the fine structure constant, Phys. Rev. Lett. 101 (2008), no. 23230801.

[24] R. Bouchendira, P. Cladé, S. Guellati-Khélifa, F. Nez, and F. Biraben, New determination of the fine structure constant and test of the quantum electrodynamics, Phys. Rev. Lett. 106 (2011), no. 8080801. 
[25] D. Schlippert, J. Hartwig, H. Albers, L. L. Richardson, C. Schubert, A. Roura, W. P. Schleich, W. Ertmer, and E. M. Rasel, Quantum test of the universality of free fall, Phys. Rev. Lett. 112 (2014), no. 20203002.

[26] M. G. Tarallo, T. Mazzoni, N. Poli, D. V. Sutyrin, X. Zhang, and G. M. Tino, Test of einstein equivalence principle for 0-spin and half-integer-spin atoms: Search for spin-gravity coupling effects, Phys. Rev. Lett. 113 (2014), no. 2023005 .

[27] A. Peters, K. Y. Chung, and S. Chu, Measurement of gravitational acceleration by dropping atoms, Nature 400 (1999), no. 6747849.

[28] Z. Hu, B. L. Sun, X. C. Duan, M. K. Zhou, L. L. Chen, S. Zhan, Q. Z. Zhang, and J. Luo, Demonstration of an ultrahigh-sensitivity atom-interferometry absolute gravimeter, Phys. Rev. A 88 (2013), no. 4043610.

[29] J. M. McGuirk, G. T. Foster, J. B. Fixler, M. J. Snadden, and M. A. Kasevich, Sensitive absolute-gravity gradiometry using atom interferometry, Phys. Rev. A 65 (2002), no. 3033608.

[30] F. Sorrentino, Q. Bodart, L. Cacciapuoti, Y.-H. Lien, M. Prevedelli, G. Rosi, L. Salvi, and G. M. Tino, Sensitivity limits of a raman atom interferometer as a gravity gradiometer, Phys. Rev. A 89 (2014), no. 2023607.

[31] T. L. Gustavson, P. Bouyer, and M. A. Kasevich, Precision rotation measurements with an atom interferometer gyroscope, Phys. Rev. Lett. 78 (1997), no. 112046.

[32] B. Canuel, F. Leduc, D. Holleville, A. Gauguet, J. Fils, A. Virdis, A. Clairon, N. Dimarcq, C. J. Borde, A. Landragin, and P. Bouyer, Six-axis inertial sensor using cold-atom interferometry, Phys. Rev. Lett. 97 (2006), no. 1 010402 .

[33] J. K. Stockton, K. Takase, and M. A. Kasevich, Absolute geodetic rotation measurement using atom interferometry, Phys. Rev. Lett. 107 (2011), no. 13 133001.

[34] I. Dutta, D. Savoie, B. Fang, B. Venon, C. L. Garrido Alzar, R. Geiger, and A. Landragin, Continuous cold-atom inertial sensor with 1 nrad/sec rotation stability, Phys. Rev. Lett. 116 (2016), no. 18183003.

[35] P. Wolf, P. Lemonde, A. Lambrecht, S. Bize, A. Landragin, and A. Clairon, From optical lattice clocks to the measurement of forces in the casimir regime, Phys. Rev. A 75 (June, 2007).

[36] T. Kovachy, P. Asenbaum, C. Overstreet, C. A. Donnelly, S. M. Dickerson, A. Sugarbaker, J. M. Hogan, and M. A. Kasevich, Quantum superposition at the half-metre scale, Nature 528 (Dec., 2015) 530-533.

[37] D. V. Martynov et al., Sensitivity of the Advanced LIGO detectors at the beginning of gravitational wave astronomy, Physical Review D 93 (June, 2016) 433-19. 
[38] P. Hamilton, M. Jaffe, P. Haslinger, Q. Simmons, H. Müller, and J. Khoury, Atom-interferometry constraints on dark energy, Science 349 (2015) 849-851.

[39] M. Jaffe, P. Haslinger, V. Xu, P. Hamilton, A. Upadhye, B. Elder, J. Khoury, and H. Müller, Testing sub-gravitational forces on atoms from a miniature, in-vacuum source mass, Nat. Phys. 13 (2017) 938.

[40] P. Haslinger, M. Jaffe, V. Xu, O. Schwartz, M. Sonnleitner, M. Ritsch-Marte, H. Ritsch, and H. Müller, Attractive force on atoms due to blackbody radiation, Nature Physics (Mar., 2018) 1-5.

[41] M. Dovale Álvarez, D. D. Brown, A. W. Jones, C. M. Mow-Lowry, H. Miao, and A. Freise, Fundamental limitations of cavity-assisted atom interferometry, Physical Review A 96 (Nov., 2017) 053820-10.

[42] M. Dovale Álvarez, C. M. Mow-Lowry, and A. Freise, 4-mirror large-waist stable cavity for enhanced atom interferometry, tech. rep., University of Birmingham, 2018.

[43] B. P. Abbott et al., Observation of Gravitational Waves from a Binary Black Hole Merger, Physical Review Letters 116 (Feb., 2016) 061102-16.

[44] B. P. Abbott et al., GW170817: Observation of Gravitational Waves from a Binary Neutron Star Inspiral, Physical Review Letters 119 (Oct., 2017) 61-18.

[45] B. P. Abbott et al., Multi-messenger Observations of a Binary Neutron Star Merger, The Astrophysical Journal Letters 848 (Oct., 2017).

[46] M. Abernathy, F. Acernese, P. Ajith, B. Allen, P. Amaro-Seoane, et al., Einstein gravitational wave Telescope conceptual design study, available from European Gravitational Observatory, document number ET-0106C-10 (2011).

[47] P. Amaro-Seoane et al., Laser Interferometer Space Antenna, arXiv.org (Feb., 2017) [arXiv:1702.00786].

[48] H. Wang, M. Dovale Álvarez, D. D. Brown, S. Cooper, A. Green, D. Töyrä, H. Miao, C. Mow-Lowry, and A. Freise, Near-unstable fabry-perot cavities for future gravitational wave detectors, Manuscript in preparation (2017).

[49] A. C. Green, D. D. Brown, M. Dovale Álvarez, C. Collins, H. Miao, C. M. Mow-Lowry, and A. Freise, The influence of dual-recycling on parametric instabilities at Advanced LIGO, Classical and Quantum Gravity 34 (Sept., 2017) 205004-17.

[50] T. W. Hänsch, Nobel Lecture: Passion for precision, Reviews of Modern Physics 78 (Nov., 2006) 1297-1309.

[51] L. Essen, Atomic Time and the Definition of the Second, Nature 178 (July, 1956) 34-35.

[52] M. A. Kasevich, E. Riis, S. Chu, and R. G. Devoe, Rf spectroscopy in an atomic fountain, Phys. Rev. Lett. 63 (1989), no. 6 612-615. 
[53] A. Clairon, C. Salomon, S. Guellati, and W. D. Phillips, Ramsey Resonance in a Zacharias Fountain, Europhys. Lett. 16 (1991), no. 2 165-170.

[54] R. W. P. Drever, J. L. Hall, F. V. Kowalski, J. Hough, G. M. Ford, A. J. Munley, and H. Ward, Laser phase and frequency stabilization using an optical resonator, Appl. Phys. B Photophysics Laser Chem. 31 (1983), no. 2 97-105.

[55] S. Häfner, S. Falke, C. Grebing, S. Vogt, T. Legero, M. Merimaa, C. Lisdat, and U. Sterr, 8e-17 fractional laser frequency instability with a long room-temperature cavity, Opt. Lett. 40 (2015), no. 2112-2115 17-21, [arXiv: 1502.02608].

[56] M. Born and E. Wolf, Principles of Optics. Cambridge University Press, 1999.

[57] M. Rakhmanov, R. L. Savage, D. H. Reitze, and D. B. Tanner, Dynamic resonance of light in Fabry-Perot cavities, Physics Letters A 305 (2002), no. 5 239-244.

[58] L. L. Sánchez-Soto, J. J. Monzón, and G. Leuchs, The many facets of the Fabry-Perot, European Journal of Physics 37 (Sept., 2016) 1-15.

[59] A. G. Fox and T. Li, Resonant Modes in a Maser Interferometer, Bell System Technical Journal 40 (1961), no. 2 453-488.

[60] A. G. Fox and T. Li, Modes in a maser interferometer with curved and tilted mirrors, Proceedings of the IEEE 51 (1963), no. 1 80-89.

[61] H. Kogelnik and T. Li, Laser Beams and Resonators, Applied optics 5 (Oct., 1966) 1550-1567.

[62] S. Feng and H. G. Winful, Physical origin of the gouy phase shift, Opt. Lett. 26 (2001), no. 8 485-487.

[63] C. Bond, D. D. Brown, A. Freise, and K. A. Strain, Interferometer techniques for gravitational-wave detection, Living Reviews in Relativity 19 (Apr., 2017) 1-217.

[64] A. E. Siegman, Lasers. University Science Books, 1986.

[65] F. Cardarelli, Materials Handbook - a concise desktop reference. Springer-Verlag London, 2 ed., 2008.

[66] H. B. Callen and R. F. Greene, On a theorem of irreversible thermodynamics, Phys. Rev. 86 (1952), no. 5 702-710.

[67] K. Numata, A. Kemery, and J. Camp, Thermal-noise limit in the frequency stabilization of lasers with rigid cavities, Phys. Rev. Lett. 93 (2004), no. 25.

[68] T. Kessler, T. Legero, and U. Sterr, Thermal noise in optical cavities revisited, JOSA B 29 (Jan., 2012) 178-184. 
[69] S. Amairi, T. Legero, T. Kessler, U. Sterr, J. B. Wübbena, O. Mandel, and P. O. Schmidt, Reducing the effect of thermal noise in optical cavities, Appl. Phys. B 113 (2013), no. 2 233-242, [arXiv:1212.3461v].

[70] Y. Levin, Internal thermal noise in the LIGO test masses: A direct approach, Physical Review D 57 (Jan., 1998) 659-663.

[71] G. M. Harry et al., Thermal noise in interferometric gravitational wave detectors due to dielectric optical coatings, Classical and Quantum Gravity $\mathbf{1 9}$ (2002), no. 5897.

[72] S. a. Webster, M. Oxborrow, and P. Gill, Vibration insensitive optical cavity, Phys. Rev. A - At. Mol. Opt. Phys. 75 (2007), no. 1 1-4.

[73] S. A. Webster, M. Oxborrow, S. Pugla, J. Millo, and P. Gill, Thermal-noise-limited optical cavity, Physical Review A $7 \mathbf{7}$ (Mar., 2008) 033847-6.

[74] S. Webster and P. Gill, Force-insensitive optical cavity, Optics Letters 36 (2011), no. 183572 .

[75] "COMSOL Multiphysics Reference Manual, version 5.3, COMSOL Inc." http://www. COMSOL. com/.

[76] G. B. Airy, I. On the Flexure of a uniform Bar supported by a number of equal Pressures applied at equi-distant points, and on the Positions proper for the Applications of these Pressures, in order to prevent any sensible Alteration of the Length of the Bar by small Flexure, Monthly Notices of the Royal Astronomical Society 6 (1845), no. 12 143-146.

[77] F. M. Phelps III, Airy Points of a Meter Bar, American Journal of Physics 34 (May, 1966) 419-422.

[78] J. Millo, D. V. Magalhäes, C. Mandache, Y. Le Coq, E. M. L. English, P. G. Westergaard, J. Lodewyck, S. Bize, P. Lemonde, and G. Santarelli, Ultrastable lasers based on vibration insensitive cavities, Phys. Rev. A - At. Mol. Opt. Phys. 79 (2009), no. 5 1-7, [arXiv:0901.4717].

[79] T. Nazarova, F. Riehle, and U. Sterr, Vibration-insensitive reference cavity for an ultra-narrow-linewidth laser, Appl. Phys. B Lasers Opt. 83 (2006), no. $4531-536$.

[80] M. Roberts, P. Taylor, and P. Gill, CLM 8 Laser Linewidty at the Sub-Hertz Level, tech. rep., National Physical Laboratory, 1999.

[81] Y. Liu and P. H. Daum, Relationship of refractive index to mass density and self-consistency of mixing rules for multicomponent mixtures like ambient aerosols, Journal of Aerosol Science 39 (Nov., 2008) 974-986.

[82] T. Legero, T. Kessler, and U. Sterr, Tuning the Thermal Expansion Properties of Optical Reference Cavities with Fused Silica Mirrors, J. Opt. Soc. Am. B 27 (2010), no. 5 914-919, [arXiv:1002.2070]. 
[83] D. B. Newell, S. J. Richman, P. G. Nelson, R. T. Stebbins, P. L. Bender, J. E. Faller, and J. Mason, An ultra-low-noise, low-frequency, six degrees of freedom active vibration isolator, Review of Scientific Instruments 68 (Aug., 1997) 3211-3219.

[84] "ATLAS Technologies, Leaders in Aluminium Vacuum Technology." http://atlasuhv.com/. Accessed: 01.03.2015.

[85] “Thorlabs GmbH." http://thorlabs.de/. Accessed: 01.03.2015.

[86] M. F. Modest, Radiative Heat Transfer. Academic Press, 3 ed., 2003.

[87] J. D. Harvey and D. F. Walls, Quantum Optics IV, Proceedings of the Fourth International Symposium, Hamilton, New Zealand, February 10-15, 1986. Springer-Verlag Berlin Heidelberg, 1986.

[88] D. Nicolodi, B. Argence, W. Zhang, R. Le Targat, G. Santarelli, and Y. Le Coq, Spectral purity transfer between optical wavelengths at the $10^{-18}$ level, Nature Photonics 8 (Jan., 2014) 219-223.

[89] W. Zhang, M. J. Martin, C. Benko, J. L. Hall, J. Ye, C. Hagemann, T. Legero, U. Sterr, F. Riehle, G. D. Cole, and M. Aspelmeyer, Reduction of residual amplitude modulation to $1 \times 10^{-6}$ for frequency modulation and laser stabilization, Optics Letters 39 (2014), no. 7 1980-4.

[90] L. A. M. Johnson, P. Gill, and H. S. Margolis, Evaluating the performance of the NPL femtosecond frequency combs: agreement at the $10^{-21}$ level, Metrologia 52 (Jan., 2015) 62-71.

[91] J. E. Gray and D. W. Allan, A Method for Estimating the Frequency Stability of an Individual Oscillator, in 28th Annual Symposium on Frequency Control, pp. 243-246, IEEE, 1974.

[92] H. R. Telle, B. Lipphardt, and J. Stenger, Kerr-lens, mode-locked lasers as transfer oscillators for optical frequency measurements, Applied Physics B $\mathbf{7 4}$ (Jan., 2002) 1-6.

[93] L. S. Ma, P. Jungner, J. Ye, and J. L. Hall, Delivering the same optical frequency at two places: accurate cancellation of phase noise introduced by an optical fiber or other time-varying path, Optics Letters 19 (1994), no. 211777.

[94] D. G. Matei, T. Legero, S. Häfner, C. Grebing, R. Weyrich, W. Zhang, L. Sonderhouse, J. M. Robinson, J. Ye, F. Riehle, and U. Sterr, $1.5 \mu \mathrm{m}$ Lasers with Sub-10 mHz Linewidth, Physical Review Letters 118 (June, 2017) 263202-6.

[95] C. Lisdat, N. Quintin, C. Shi, S. M. F. Raupach, C. Grebing, D. Nicolodi, F. Stefani, A. Al-Masoudi, S. D. o. rscher, S. H. a. fner, J. L. Robyr, N. Chiodo, S. Bilicki, E. Bookjans, A. Koczwara, S. Koke, A. Kuhl, F. Wiotte, F. Meynadier, E. Camisard, M. Abgrall, M. Lours, T. Legero, H. Schnatz, U. Sterr, H. Denker, C. Chardonnet, Y. Le Coq, G. Santarelli, 
A. Amy-Klein, R. Le Targat, J. Lodewyck, O. Lopez, G. Grosche, and P. E. Pottie, A clock network for geodesy and fundamental science, Nature Communications 7 (Aug., 2016) 1-7.

[96] M. Schioppo, R. C. Brown, W. F. McGrew, N. Hinkley, R. J. Fasano, K. Beloy, T. H. Yoon, G. Milani, D. Nicolodi, J. A. Sherman, N. B. Phillips, C. W. Oates, and A. D. Ludlow, Ultrastable optical clock with two cold-atom ensembles, Nature Photonics (Nov., 2016) 1-6.

[97] M. Kasevich, D. S. Weiss, E. Riis, K. Moler, S. Kasapi, and S. Chu, Atomic velocity selection using stimulated Raman transitions, Physical Review Letters 66 (May, 1991) 2297-2300.

[98] K. Moler, D. S. Weiss, M. Kasevich, and S. Chu, Theoretical analysis of velocity-selective Raman transitions, Physical Review A 45 (Jan., 1992) $342-348$.

[99] A. Sugarbaker, S. M. Dickerson, J. M. Hogan, D. M. S. Johnson, and M. A. Kasevich, Enhanced atom interferometer readout through the application of phase shear, Phys. Rev. Lett. 111 (2013), no. 11113002.

[100] L. Zhou, Z. Xiong, W. Yang, B. Tang, W. Peng, K. Hao, R. Li, M. Liu, J. Wang, and M. Zhan, Development of an atom gravimeter and status of the 10-meter atom interferometer for precision gravity measurement, Gen. Rel. Gravit. 43 (2011), no. 7 1931-1942.

[101] J. M. McGuirk, M. J. Snadden, and M. A. Kasevich, Large area light-pulse atom interferometry, Phys. Rev. Lett. 85 (2000), no. 214498.

[102] S. Chiow, T. Kovachy, H. C. Chien, and M. A. Kasevich, 102ћk large area atom interferometers, Phys. Rev. Lett. 107 (2011), no. 13130403.

[103] H. Müller, S. Chiow, Q. Long, S. Herrmann, and S. Chu, Atom interferometry with up to 24-photon-momentum-transfer beam splitters, Phys. Rev. Lett. 100 (2008), no. 18180405.

[104] P. L. Kapitza and P. A. M. Dirac, The reflection of electrons from standing light waves, Mathematical Proceedings of the Cambridge Philosophical Society 29 (Oct., 2008) 297-5.

[105] P. Meystre, E. Schumacher, and S. Stenholm, Atomic beam deflection in a quantum field, Optics communications 73 (1989), no. 6 443-447.

[106] P. Meystre, E. Schumacher, and E. M. Wright, Quantum Pendellösung in Atom Diffraction by a Light Grating, Annalen der Physik 503 (1991), no. 1-3 141-148.

[107] P. Meystre and M. Sargent, Elements of Quantum Optics. Springer-Verlag Berlin Heidelberg, 2007.

[108] P. L. Gould, P. J. Martin, G. A. Ruff, R. E. Stoner, J.-L. Picqué, and D. E. Pritchard, Momentum transfer to atoms by a standing light wave: Transition from diffraction to diffusion, Physical Review A 43 (1991), no. 1 585-588. 
[109] G. C. Kokkorakis and J. A. Roumeliotis, Power series expansions for Mathieu functions with small arguments, Mathematics of Computation 70 (2000), no. 235 1221-1236.

[110] I. Riou, N. Mielec, G. Lefèvre, M. Prevedelli, A. Landragin, P. Bouyer, A. Bertoldi, R. Geiger, and B. Canuel, A marginally stable optical resonator for enhanced atom interferometry, J. Phys. B 50 (2017) 155002.

[111] C. Keller, J. Schmiedmayer, A. Zeilinger, T. Nonn, S. Dürr, and G. Rempe, Adiabatic following in standing-wave diffraction of atoms, Appl. Phys. B 69 (1999), no. 4 303-309.

[112] H. Müller, S. Chiow, and S. Chu, Atom-wave diffraction between the raman-nath and the bragg regime: Effective rabi frequency, losses, and phase shifts, Phys. Rev. A 77 (2008), no. 2023609.

[113] P. R. Berman, L. Yan, K.-H. Chiam, and R. Sung, Nonadiabatic transitions in a two-level quantum system: Pulse-shape dependence of the transition probability for a two-level atom driven by a pulsed radiation field, Phys. Rev. A 57 (1998), no. 179.

[114] C. W. S. Conover, Effects of pulse shape on strongly driven two-level systems, Phys. Rev. A 84 (2011), no. 6063416.

[115] M. J. Lawrence, B. Willke, M. E. Husman, E. K. Gustafson, and R. L. Byer, Dynamic response of a Fabry-Perot interferometer, Journal of the Optical Society of America B 16 (1999), no. 4523.

[116] D. F. Walls and G. J. Milburn, Quantum Optics. Springer-Verlag Berlin Heidelberg, 2008.

[117] H. Wang, M. Dovale Álvarez, C. Collins, D. D. Brown, M. Wang, C. M. Mow-Lowry, S. Han, and A. Freise, Feasibility of near-unstable cavities for future gravitational wave detectors, Physical Review D 97 (Jan., 2018) 022001 .

[118] A. Araya, N. Mio, K. Tsubono, K. Suehiro, S. Telada, M. Ohashi, and M. Fujimoto, Optical mode cleaner with suspended mirrors, Appl. Opt. 36 (1997), no. 7 1446-1453.

[119] H. Wang, Beware of warped surfaces: near-unstable cavities for future gravitational wave detectors. PhD thesis, University of Birmingham, 2017.

[120] T. Kovachy, J. M. Hogan, A. Sugarbaker, S. M. Dickerson, C. A. Donnelly, C. Overstreet, and M. A. Kasevich, Matter wave lensing to picokelvin temperatures, Phys. Rev. Lett. 114 (2015), no. 14143004.

[121] D. D. Brown and A. Freise, Finesse, May, 2014. You can download the binaries and source code at http://www.gwoptics.org/finesse. 
[122] H. Abitan and T. Skettrup, Laser resonators with several mirrors and lenses with the bow-tie laser resonator with compensation for astigmatism and thermal lens effects as an example, Journal of Optics A: Pure and Applied Optics 7 (Nov., 2004) 7-20.

[123] S. J. M. Habraken and G. Nienhuis, Modes of a twisted optical cavity, Physical Review A $\mathbf{7 5}$ (Mar., 2007) 219-11.

[124] G. Harry, T. P. Bodiya, and R. DeSalvo, Optical coatings and thermal noise in precision measurement. Cambridge University Press, 2012.

[125] P. Bertet, S. Osnaghi, A. Rauschenbeutel, G. Nogues, A. Auffeves, M. Brune, J. Raimond, and S. Haroche, A complementarity experiment with an interferometer at the quantum-classical boundary, Nature 411 (2001), no. $6834166-170$.

[126] K. Durak, C. H. Nguyen, V. Leong, S. Straupe, and C. Kurtsiefer, Diffraction-limited fabry-perot cavity in the near concentric regime, New Journal of Physics 16 (2014), no. 10103002.

[127] A. Siegman and R. Arrathoon, Modes in unstable optical resonators and lens waveguides, IEEE Journal of Quantum electronics 3 (1967), no. 4 156-163.

[128] A. M. Gretarsson, E. D'Ambrosio, V. Frolov, B. O'Reilly, and P. K. Fritschel, Effects of mode degeneracy in the LIGO Livingston Observatory recycling cavity, JOSA B 24 (2007), no. 11 2821-2828.

[129] B. Mours, E. Tournefier, and J.-Y. Vinet, Thermal noise reduction in interferometric gravitational wave antennas: using high order TEM modes, Classical and Quantum Gravity 23 (Oct., 2006) 5777-5784.

[130] C. Bond, P. Fulda, L. Carbone, K. Kokeyama, and A. Freise, Higher order laguerre-gauss mode degeneracy in realistic, high finesse cavities, Phys. Rev. D 84 (Nov., 2011) 102002.

[131] B. Sorazu, P. J. Fulda, B. W. Barr, A. S. Bell, C. Bond, L. Carbone, A. Freise, S. Hild, S. H. Huttner, J. Macarthur, and K. A. Strain, Experimental test of higher-order laguerre-gauss modes in the $10 \mathrm{~m}$ glasgow prototype interferometer, Classical and Quantum Gravity 30 (2013), no. 3 035004 .

[132] The Virgo Collaboration, Advanced Virgo Technical Design Report, Virgo Internal Document VIR-0128A-12 (2012). https : //tds . virgo-gw . eu/? content=3\&r=9317.

[133] F. Acernese et al., Advanced Virgo: a second-generation interferometric gravitational wave detector, Classical and Quantum Gravity 32 (2015), no. 2 024001 .

[134] S. Goßler et al., The AEI 10 m prototype interferometer, Classical and Quantum Gravity 27 (Apr., 2010) 084023. 
[135] C. Gräf, S. Hild, H. Lück, B. Willke, K. A. Strain, S. Goßler, and K. Danzmann, Optical layout for a 10 m Fabry-Perot Michelson interferometer with tunable stability, Classical and quantum gravity 29 (2012), no. 7075003 .

[136] D. Z. Anderson, Alignment of resonant optical cavities, Appl. Opt. 23 (Sept., 1984) 2944-2949.

[137] E. Morrison, B. J. Meers, D. I. Robertson, and H. Ward, Automatic alignment of optical interferometers, Applied Optics 33 (1994), no. 22 5041-5049.

[138] V. B. Braginsky, S. E. Strigin, S. V. P. L. A, and 2001, Parametric oscillatory instability in Fabry-Perot interferometer, Physics Letters A 287 (Sept., 2001) 331-338.

[139] V. B. Braginsky, S. E. Strigin, and S. P. Vyatchanin, Analysis of Parametric Oscillatory Instability in Power Recycled LIGO Interferometer, arXiv.org (Sept., 2002) 111-124, [gr-qc/0209064v1].

[140] M. Evans, L. Barsotti, and P. Fritschel, A general approach to optomechanical parametric instabilities, Physics Letters A 374 (Jan., 2010) 665-671.

[141] A. Green, When Light Gets Pushy: Radiation Pressure Effects in Interferometric Gravitational Wave Detectors. PhD thesis, University of Birmingham, 2018.

[142] M. Evans, S. Gras, P. Fritschel, J. Miller, L. B. P. review, and 2015, Observation of parametric instability in Advanced LIGO, APS.

[143] L. Ju, C. Zhao, S. Gras, J. Degallaix, D. G. Blair, J. Munch, and D. H. Reitze, Comparison of parametric instabilities for different test mass materials in advanced gravitational wave interferometers, Physics Letters A 355 (July, 2006) 419-426.

[144] C. Zhao, L. Ju, J. Degallaix, S. Gras, and D. G. Blair, Parametric Instabilities and Their Control in Advanced Interferometer Gravitational-Wave Detectors, Physical Review Letters 94 (Apr., 2005) 325-4.

[145] D. D. Brown, A. Green, and M. Dovale Álvarez, Optical suppression of parametric instabili- ties with extraction cavities, In preparation.

[146] S. E. Strigin and S. P. Vyatchanin, Analysis of parametric oscillatory instability in Fabry-Perot cavity with Gauss and Laguerre-Gauss main mode profile, Physics Letters A 374 (Feb., 2010) 1101-1104.

[147] S. Gras, P. Fritschel, L. Barsotti, and M. Evans, Resonant dampers for parametric instabilities in gravitational wave detectors, Physical Review D 92 (Oct., 2015) 082001-10.

[148] S. Gras, D. G. Blair, and L. Ju, Test mass ring dampers with minimum thermal noise, Physics Letters A 372 (Feb., 2008) 1348-1356. 
[149] J. Miller, M. Evans, L. Barsotti, P. Fritschel, M. MacInnis, R. Mittleman, B. Shapiro, J. Soto, and C. Torrie, Damping parametric instabilities in future gravitational wave detectors by means of electrostatic actuators, Physics Letters A 375 (Jan., 2011) 788-794.

[150] H. Wang, C. Blair, M. D. Álvarez, A. Brooks, M. F. Kasprzack, J. Ramette, P. M. Meyers, S. Kaufer, B. OŔeilly, C. M. Mow-Lowry, and A. Freise, Thermal modelling of Advanced LIGO test masses, Classical and Quantum Gravity 34 (2017), no. 11115001.

[151] S. E. Strigin, The effect of parametric oscillatory instability in a Fabry-Perot cavity of the Einstein telescope, Optics and Spectroscopy 112 (Mar., 2012) 373-376.

[152] S. E. Strigin, Suppression of parametric oscillatory instability in third generation gravitational wave detectors, Physics Letters A 379 (Aug., 2015) 1671-1674.

[153] J. R. Hutchinson, Vibrations of Solid Cylinders, Journal of Applied Mechanics 47 (Dec., 1980) 901-907.

[154] C. Chree, Longitudinal vibrations of a circular bar, Quarterly Journal of Mathematics 21 (1886), no. 83/84 287-288.

[155] H. Lamb, On Waves in an Elastic Plate., Proccedings of the Royal Society of London 93 (1917) 114-128.

[156] D. D. Brown, Interactions of light and mirrors: Advanced techniques for modelling future gravitational wave detectors. PhD thesis, University of Birmingham, 2015.

[157] S. E. Strigin, D. G. Blair, S. Gras, and S. P. Vyatchanin, Numerical calculations of elastic modes frequencies for parametric oscillatory instability in Advanced LIGO interferometer, Physics Letters A 372 (Aug., 2008) $5727-5731$.

[158] V. V. Meleshko, S. E. Strigin, and M. S. Yakymenko, Parametric oscillatory instability on axially-symmetrical test mass elastic modes in Advanced LIGO interferometer, Physics Letters A 373 (Oct., 2009) 3701-3704.

[159] M. Dovale Álvarez, D. Brown, and A. Freise, Exporting Surface Displacement Data from COMSOL Multiphysics, LIGO Document T1700268 (2017).

[160] M. A. Hopcroft, W. D. Nix, and T. W. Kenny, What is the Young's Modulus of Silicon?, Journal of Microelectromechanical Systems 19 (Mar., 2010) 229-238.

[161] R. X. Adhikari, N. Smith, A. Brooks, L. Barsotti, B. Shapiro, B. Lantz, D. McClelland, E. K. Gustafson, D. V. Martynov, V. Mitrofanov, D. Coyne, K. Arai, C. Torrie, and C. Wipf, LIGO voyager upgrade: Design concept, LIGO Document T1400226-v9 (2017). 
[162] J. S. Read, C. Markakis, M. Shibata, K. Uryū, J. D. E. Creighton, and J. L. Friedman, Measuring the neutron star equation of state with gravitational wave observations, Physical Review D 79 (June, 2009) 124032-12.

[163] NIST Technical Note, Characterisation of Clocks and Oscillators, US Govt Printing Office 1337 (1990).

[164] F. Riehle, Frequency Standards: Basics and Applications. Wiley-VCH, 2005.

[165] W. Riley and D. Howe, Handbook of Frequency Stability Analysis, Special Publication (NIST SP) - 1065 (2008). 


\section{Optical Cavities for Optical Atomic Clocks, Atom Interferometry and Gravitational wave Detection}

$\mathrm{T}^{\mathrm{T}}$ is an extremely exciting time for physics. In the last 100 years we have moved from the 1 formulation of Einstein's general relativity to the first direct observation of gravitational waves in late 2015 by the Laser Interferometer Gravitational-wave Observatory (LIGO). Within that time science and technology have come a long way: we have learned to use light to cool atoms to nearly absolute zero temperature, and to use atomic transitions in the microwave and optical regimes to devise the most accurate time and frequency references. We have observed the wave-like behaviour of cold atoms in diffraction experiments using both micro-fabricated structures and the periodic structure of light beams. Exploiting this wave-like behaviour, we have constructed atom interferometers which allow us to test and measure gravity in a new scale. All of these amazing experiments have one thing in common, from LIGO's giant $4 \mathrm{~km}$ arms to the transportable atomic clocks sent to space or the atom interferometers that will someday replace current navigation systems, they all make use of a device that has become essential in many areas of science and technology: the Fabry-Perot optical cavity.

This thesis delves deeply into the application of optical cavities at the forefront of experimental physics, and it is divided into three parts, each pertaining to a different field where optical cavities are a key technology. Part I of the thesis follows the development of a next-generation, thermal-noise-limited, ultra-stable optical cavity for use as the reference oscillator in optical atomic clocks. This is part of the effort being carried out at the National Physical Laboratory in the UK in the field of precision metrology of time and frequency towards redefining the base SI unit of time, the second, in terms of an optical frequency standard. Part II presents work in the application of optical cavities for enhancing the sensitivity of atom interferometers, with an analysis of multi-photon Bragg diffraction inside an optical cavity. This led to an exploration of the true potential and limitations of the technique. The findings were used to aid the design of the MIGA experiment in France, and design a multi-mirror cavity for enhanced atom interferometry that overcomes some of the limitations of two-mirror cavities. Lastly, Part III presents work towards enhancing current and future laser interferometer gravitational-wave detectors by using near-unstable optical cavities in order to reduce the mirror coating thermal-noise floor, and by modelling parametric oscillatory instabilities that arise in the interferometers from the coupling between the optical field and the mechanical resonances of the test masses. The work carried out during the extent of this $\mathrm{PhD}$, and the variety of contexts, accounts for the relevance and versatility of such an elegant setup as the Fabry-Perot optical cavity is.

Keywords: optical cavities, cold atoms, atomic clocks, atom interferometry, gravitational waves. 
What I cannot create, I do not understand. - Richard Feynman.

What do you care what other people think? - Arline Greenbaum.

Thou, nature, art my goddess; to thy law My services are bound.

— William Shakespeare. 